INEEL/EXT-99-01008

January 2000

\title{
ENVIRONMENTAL SYSTEMS RESEARCH FY-99 ANNUAL REPORT
}

D. L. Miller 


\title{
Environmental Systems Research, FY-99 Annual Report
}

\author{
D. L. Miller, Program Manager
}

Published January 2000

Idaho National Engineering and Environmental Laboratory Idaho Falls, Idaho 83415

Prepared for the

U.S. Department of Energy

Office of Environmental Management

Under DOE Idaho Operations Office

Contract DE-AC07-99ID13727 


\begin{abstract}
The Environmental Systems Research (ESR) Program, a part of the Environmental Systems Research and Analysis (ESRA) Program, was implemented to enhance and augment the technical capabilities of the INEEL. The program has now completed its second year. The purpose for strengthening technical capabilities of the INEEL is to provide the technical base to serve effectively as the Environmental Management Laboratory for the Office of Environmental Management (EM). The program is starting into its third year of the initial three-year program content cycle. The original portfolio of research activities was assembled after an analysis of the EM technology development and science needs as gathered by the Site Technology Coordination Groups (STCGs) complex-wide. Current EM investments in science and technology throughout the research community were also included in this analysis to avoid duplication of efforts. The content of the portfolio was modified at the end of the first year to respond to comments from external review panels brought in to help strengthen the program.
\end{abstract}

This is a progress report for the second year of the ESR Program (Fiscal Year 99). A report of activities is presented for the five ESR research investment areas: (a) Transport Aspects of Selective Mass Transport Agents, (b) Chemistry of Environmental Surfaces, (c) Materials Dynamics, (d) Characterization Science, and (e) Computational Simulation of Mechanical and Chemical Systems. In addition to the five technical areas activities in the Science and Technology Foundations element of the program, e.g., interfaces between ESR and the EM Science Program (EMSP) and the EM Focus Areas, are described. 


\section{CONTENTS}

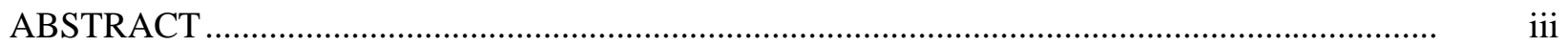

INTRODUCTION

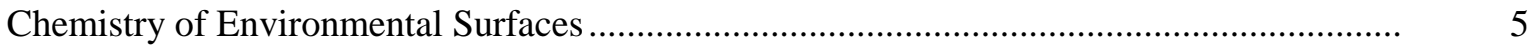

Advanced Strategy for Probing Structure and Reactivity at the Top Monolayer.......... 7

Biochemical and Geochemical Reactions on Environmental Surfaces ........................ $\quad 35$

Reactive Transport in Variably Saturated Heterogeneous Media ................................ $\quad 67$

Transport Phenomena in Porous Media …................................................................. 101

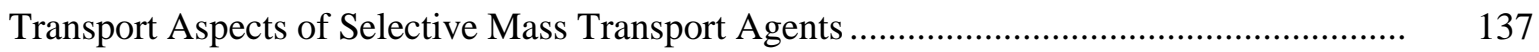

Selective Mass Transport in Polymers .......................................................................... 139

Pore Size and Morphology Control for Solid and Polymer Matrices............................ 153

Adsorption and Absorption Materials of Molecular Separations .................................. 169

Computational Simulation of Mechanical and Chemical Systems ....................................... 189

Computational Simulation Project ……............................................................... 191

Computational Infrastructure ................................................................................ 219

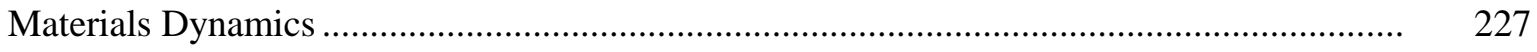

Coatings for Environmental Applications ……........................................................ 229

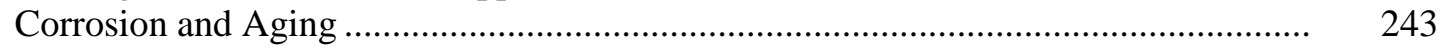

Transport in Solid and Liquid Media ................................................................. 261

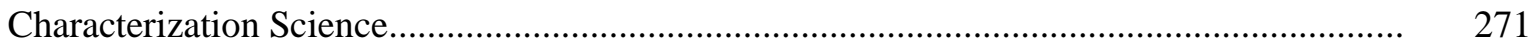

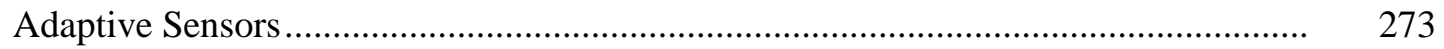

Integrated Instruments for In Situ Chemical Measurements ....................................... 293

Intelligent Nonintrusive Methods for Characterization............................................ $\quad 317$

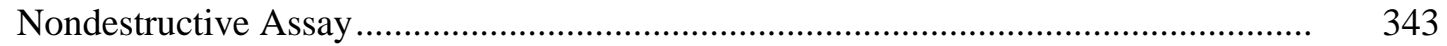

Nuclear Structure Research ................................................................................ 361

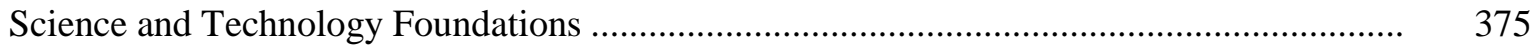

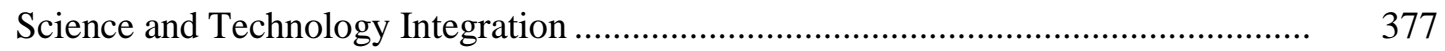

Appendix —Peer Reviewed Publications ...................................................................... A-1 


\section{Environmental Systems Research FY-99 Annual Report}

\section{INTRODUCTION}

Welcome to the 1999 fiscal year annual report for the Environmental Systems Research (ESR) Program. The ESR program was initiated in mid-fiscal year 1998 and is focused on science and engineering research that supports EM's technical needs. In particular the ESR program is looking forward in EM's mission and performing the groundwork to provide the technical foundations for longterm environmental stewardship. To this end, the program is organized around five main research themes (five of the six top line boxes in Figure 1) that support the central premise adopted for the program. This premise, "long-term stewardship starts when waste disposition begins," acknowledges the fact that decisions made on the method of dispositioning waste streams and performing cleanup have consequences for the way long-term stewardship is addressed. Each of the five main research themes, aimed at reducing technical uncertainty, supports one or more aspects pertinent to long-term stewardship. These aspects are 1) preparing for disposition, 2) understanding environmental fate and transport, 3 ) developing monitoring methods for the long-term, and 4) measuring ecological health. The relationships of these research themes to these four aspects are shown in Figure 2. These areas are particularly important in relationship to long-term stewardship in the geologic subsurface.

The Environmental Systems Research program is structured to perform two types of research activities. The first type is core research, which is more basic in tone and is focused on understanding environmental processes, developing new tools, and collecting data. The second type is focused on problem-driven research and thus is more applied in nature. The program composition has been structured to achieve balance and interaction between these two areas.

It was an exciting and productive year for the program in 1999. Research projects initiated in mid FY-98 continued to make solid technical progress. New staff, focused on applying their particular research expertise to environmental problems, was added to the skill mix at the Idaho National Engineering and Environmental Laboratory, EM's multiprogram national laboratory. New research tools acquired in FY-98 were brought online and made available to the research staff. Capabilities in computation modeling and tools for studying contaminant surface interactions were added. Additional tools were acquired in FY-99 to support the exploration of fission processes that might lead to new methods for nondestructive assay. The Science and Technology Integration (STI) activity arranged for external peer reviews that were held on two of the five research themes during the latter part of FY-99. The peer reviews were positive and provided constructive suggestions that have been incorporated into program planning for FY 2000. With the completion of these reviews, 90\% of the research projects comprising the ESR program have been subjected to external peer review. Research conducted as part of the ESR program has resulted in 45 technical papers that are in print, in press, in review, or in preparation. Additionally, numerous presentations were made at professional society meetings. The ESR program participants have also been proactive in working with the Waste Management and Spent Nuclear Fuels programs at the INEEL to understand particular programmatic science needs. A relationship facilitated by STI was also established with the Subsurface Contaminant Focus Area for reviewing EMSP projects that might be carried forward through the ESR program in FY 2000 and later years. 


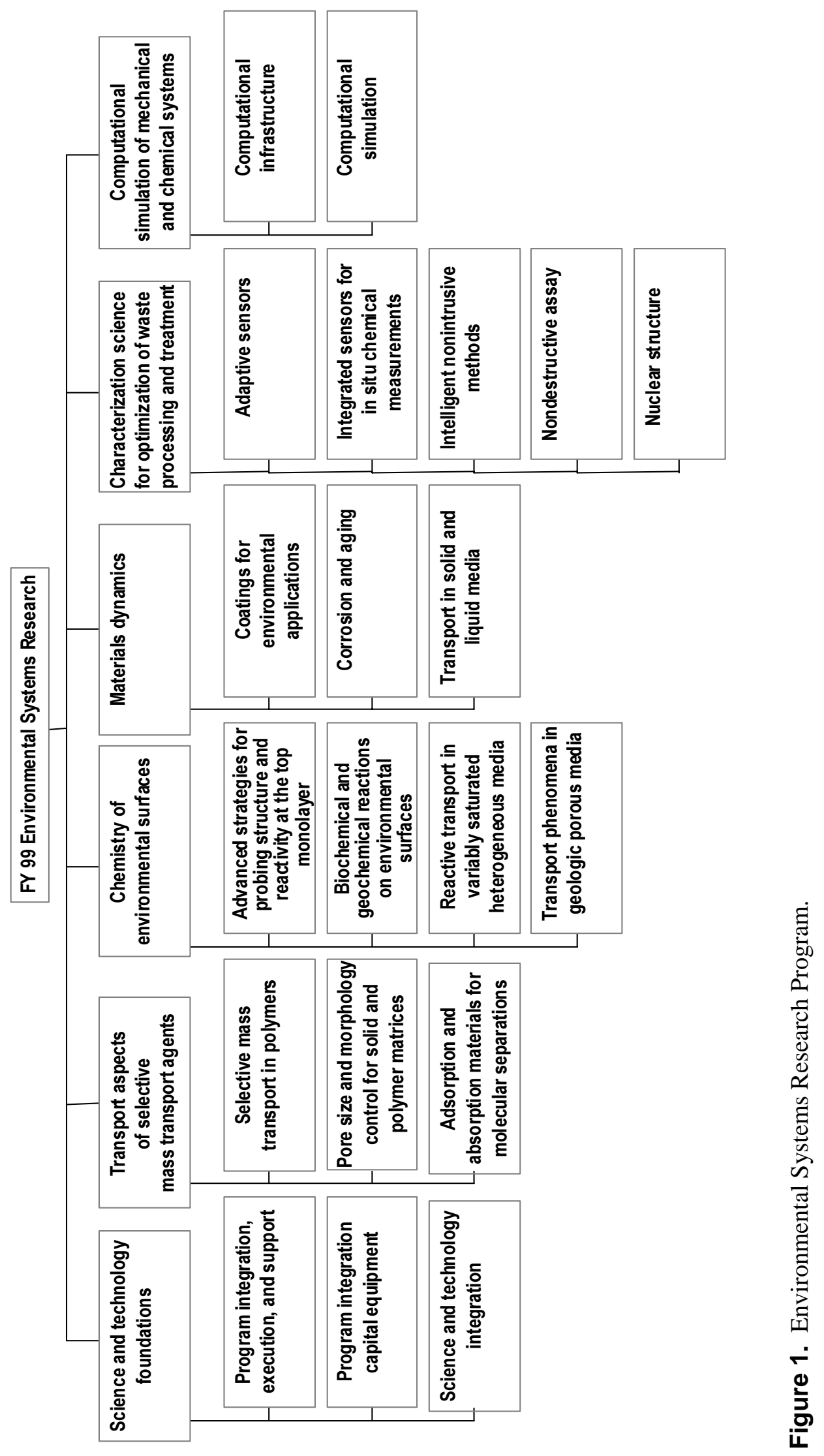




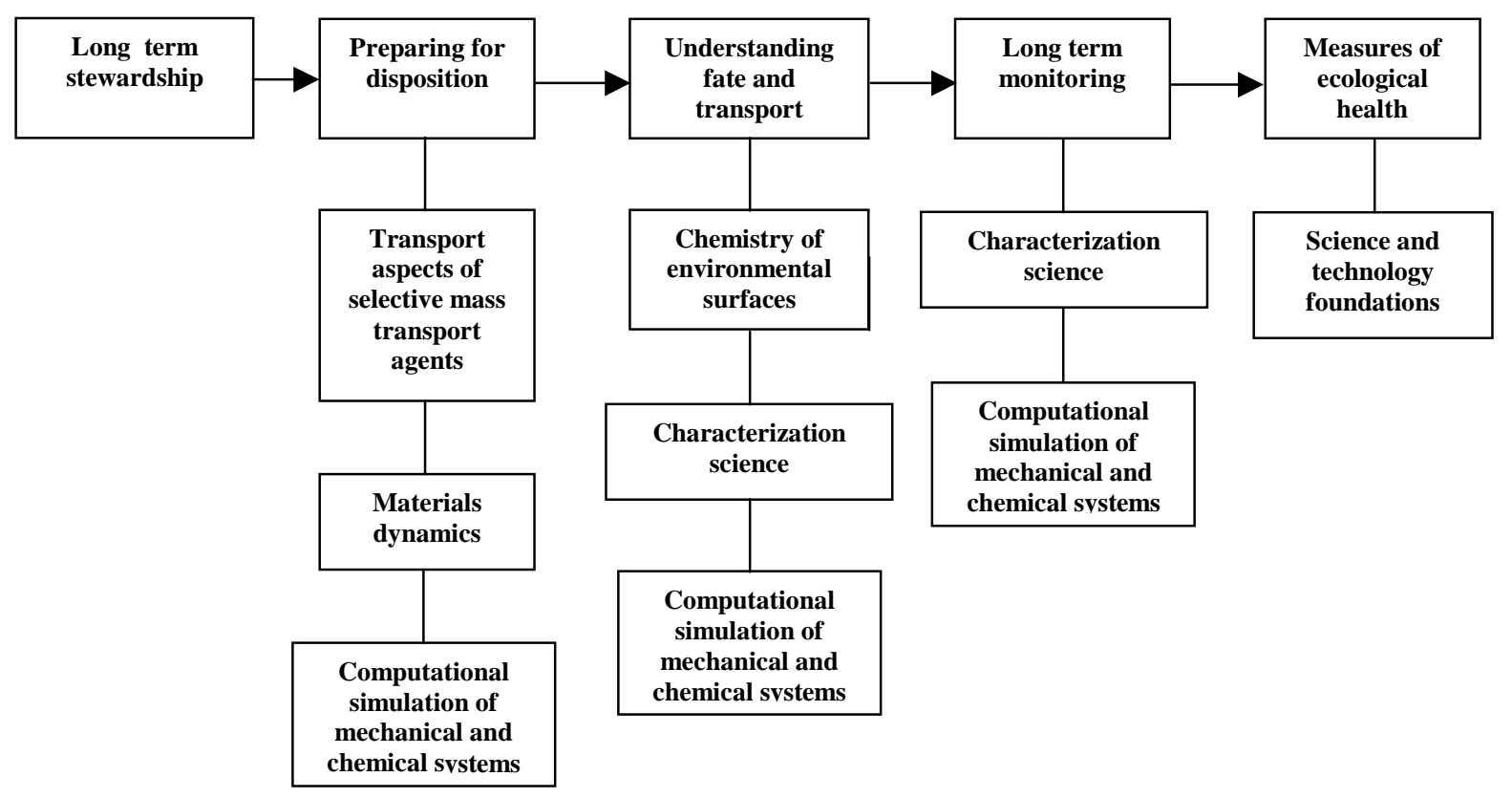

Figure 2. Relationship of ESR research themes to aspects of long-term stewardship. 


\section{Chemistry of Environmental Surfaces}

Many of the technical needs for long-term environmental stewardship are either directly, or indirectly, associated with surface chemistry. Large information gaps exist in the surface chemistry area, as a consequence of the complexity of real world systems, and the inherent difficulty encountered when probing contaminant surface structure and reactivity. The complexity of real world environmental surfaces is derived from micron-scale heterogeneity, a wide spectrum of possible chemical speciation, and the presence of microorganisms, which are perhaps the salient agents for chemical change in the subsurface.

In order to predict environmental fate and transport, various modeling approaches have been employed. However, modeling results are usually substantially different from observed phenomena. The underlying hypothesis of the research performed in the Environmental Surfaces focus area is that if contaminant structure and reactivity behavior were better understood, then improved models could be developed that would enable accurate prediction of contaminant fate and transport. In one sense, the Environmental Surfaces research is premature, because even if chemical, biochemical and geochemical structure and reaction dynamics were perfectly understood, no current modeling approaches are sufficiently sophisticated to effectively utilize the information. Nevertheless, research in the Environmental Surfaces focus area is proceeding in anticipation of the fact that long-term environmental stewardship will eventually compel modeling at a much higher level, that will require molecular-level structure and reactivity information.

For these reasons, four tasks were executed in FY-99. The first task is entitled Advanced Strategies for Probing the Structure and Reactivity of the Top Monolayer. The objective is to generate contaminant structure and reactivity information on real world environmental surfaces and to develop improved surface interrogation techniques. Research in the general area of trapped ion mass spectrometry resulted in a detailed understanding of the dissociative adsorption of water on aluminate moieties and in the fabrication and demonstration of a laser desorption Fourier transform mass spectrometer (FTMS). The FTMS will be applicable to characterization of biologicals on surfaces.

The second task is entitled Biological and Geochemical Reactions on Environmental Surfaces. The objective of this task is to understand the roles of adsorbed Fe and Fe-reducing bacteria on contaminant adsorption. Fe is the focus of this effort because it is responsible for biologically mediated uranyl adsorption. The task has resulted in the identification of uranyl sorption structures and implementation of an environmental scanning electron microscope at the lab.

The third task is entitled Reactive Transport in Variably Saturated Media. The objective of the task is to understand how contaminant migration occurs in the vadose zone in order to enable modeling to be scaleable from the cubic meter realm to cubic kilometers. More specifically, information is sought on how pore-scale mechanisms are expressed in field-scale processes, on the effect of reactive gases on radionuclide movement and on the relationship between subsurface saturation and microbial activity. Progress for FY-99 describes the distribution of FeO coatings in relationship to grain size and pore distribution, uranyl adsorption mechanisms, and reactions of $\mathrm{CO}_{2}$ with surfaces.

The fourth task is entitled Transport Phenomena in Geologic Porous Media. The objective of this task is to investigate temporal and spatial variations in ecological microbes on bacterial structure function. Specifically, the partitioning of bacteria between mobile and immobile phases has been investigated, to better understand biologically mediated actinide transport. Modeling and soil water potential measurements are also included in this task. 


\title{
Advanced Strategies for Probing Structure and Reactivity at the Top Monolayer
}

\author{
Gary S. Groenewold and Jill R. Scott \\ SUMMARY
}

Interactions of adsorbate molecules with oxide surfaces are not well understood at the molecular level, because of limitations of surface spectroscopy techniques ${ }^{1,2}$ and because of the complexities of oxide surfaces. Yet these interactions are controlling with respect to contaminant fate and transport in the geologic subsurface. ${ }^{3,5,6}$ Hence, the molecular understanding of these phenomena, which critically impact human and environmental toxicity, is inadequate. The Advanced Strategies Program has developed an approach for interrogating these interactions that is based on sputter desorption of ionic oxide species, followed by reaction with gas-phase adsorbates. Direct observation of reaction pathways and measurement of reaction kinetics is achieved. Ab initio calculations provide structural and thermodynamic information. Initial studies focused on the interactions of $\mathrm{Al}, \mathrm{Si}$, and $\mathrm{Al}+\mathrm{Si}$ oxyanions with water, which is the most ubiquitous surface interaction occurring in the subsurface, and consequently is a prerequisite for understanding interactions of contaminant species. It was found that water would dissociatively add to dehydrated $\mathrm{Al}$ and $\mathrm{Al}+\mathrm{Si}$ oligomeric oxyanions at the collision rate. Si oligomeric oxyanions reacted somewhat more slowly, and monomeric species were practically unreactive. The results show that reaction occurs with dangling oxygen atoms in species where $\mathrm{Al}$ is present.

Complementing this research, a Fourier transform mass spectrometer ${ }^{7}$ was designed and fabricated for the purpose of performing enhanced surface characterization and reactivity studies. The instrument was equipped with laser desorption capabilities, ${ }^{8,9,10,11,12,13,14}$ and is designed to accommodate particle desorption and electron ionization. The multiple ion formation modes will significantly expand the systems amenable to surface characterization and ion reactivity studies. In addition, the instrument has a mass range of $2 \times 10^{4} \mathrm{amu}$, which will enable the study of biological molecules participating in biogeochemical sequestration or degradation reactions.

The combination of tasks is generating methodology and instrumentation that will provide direct information on oxide-adsorbate interactions at a molecular level; previously, this information has been impossible to obtain. Improved knowledge of molecular interactions will ultimately result in enhanced predictive capability at the meso- and macro-scale.

\section{PROJECT DESCRIPTION}

\section{Introduction}

The information contained in this report describes investigations of silicon, aluminum and aluminosilicate oxide materials using secondary ion mass spectrometry (SIMS) techniques. The study has emphasized the characterization of these materials and their subsequent reactivity with water. The project has also had a focus on the design and fabrication of a novel Fourier transform mass spectrometer (FTMS), which will enable greatly augmented investigation of geochemical and biogeochemical systems. The FTMS enjoys a number of analytical advantages compared to existing mass spectrometers, which will make the resulting instrument applicable to a broad spectrum of analytical and physical chemistry measurements.

The project is directed toward both general and specific objectives. The current state of knowledge regarding the interactions of surface-bound oxyanions with adsorbate molecules is inadequate. This lack 
of knowledge stems from the difficulty encountered in attempting to interrogate the surfaces of oxide mineral forms. Of specific interest is the reactivity of silicate- and aluminate-based minerals, because these comprise the most abundant forms encountered in the subsurface; and hence, are significant for their interactions with adsorbates. ${ }^{15,16}$ However, it is acknowledged that generally, methods for interrogating naturally occurring oxide surfaces are inadequate. Therefore, another general thrust is the development of next generation instrumentation, that is capable of enhanced surface sensitivity and better molecular speciation capability. An FTMS, capable of ultra high mass resolution and measurement accuracy, with laser desorption and ion bombardment capability, has been designed and largely fabricated. Pursuit of FTMS testing and refinement was a major objective for the program for 1999.

On the largest scale, the program was motivated by long term remediation and facility stewardship issues facing the Department of Energy. Cold war weapons and fuel production have resulted in largescale subsurface and industrial contamination problems. Relative to the subsurface, it is known that in many cases, natural attenuation processes can effectively sequester, or even degrade contaminants. On the other hand, particulate and colloidal matter can result in facilitated transport of contaminants beyond site boundaries, where they can impact the general public.

The salient interactions that govern contaminant sequestration, or facilitated transport occur on the molecular level, at the top-most monolayer of mineral oxide surfaces. Both biochemical and geochemical processes are significant. In general, these interactions are poorly understood. In certain instances where the matrix material is highly homogeneous, contaminant behavior can be successfully predicted based on simple column tests to determine partitioning coefficients. More often, however, behavior in the environment is significantly different than predicted, which directly impacts decisionmaking.

If the chemical reactions between contaminants and surfaces were understood at the molecular level, it is believed that improved models could be constructed, which in turn would result in better estimates of fate and transport. Ideally, knowledge of contaminant interaction at the molecular level would lead to the ability to manipulate adsorption reactions so that contaminant retention, mobilization, or perhaps even degradation could be deliberately controlled.

\section{Background}

Contaminant problems that are directly addressed by this project include toxic and radioactive metals and solvents in the subsurface. ${ }^{17,18,19}$ Metals of particular interest include $\mathrm{Pu}, \mathrm{U}, \mathrm{Tc}, \mathrm{Cs}, \mathrm{Am}, \mathrm{Pb}$, $\mathrm{Cr}$, and $\mathrm{Hg}$. The organics of interest include the chlorinated solvents such as perchloroethylene, trichloroethylene, dichloroethylene, 1,1,1-trichloroethane, and carbon tetrachloride. The subsurface environment to which these compounds and metal species are subjected is variably saturated and is an oxide matrix dominated by silicon, with substantial quantities of aluminum, iron, and sometimes titanium. Calcium is the dominant counter cation, although magnesium and group I alkali metals are also commonly encountered. These facts define which contaminant-surface systems must be understood.

INEEL has had an ongoing program in the area of detection of environmental surface contaminants. During the course of adsorbate analysis, it was noted that large oxyanions could be sputtered into the gas phase. Specifically, $\mathrm{Al}, \mathrm{Si}$, and combination $\mathrm{Al}+\mathrm{Si}$ species could be generated in the gas phase by bombarding the mineral oxide surface with an energetic $\mathrm{ReO}_{4}{ }^{-}$projectile. ${ }^{20,21}$ Once in the gas phase, the oxyanions could be trapped and reacted through either decomposition or condensation reactions. In particular, condensation reactions with $\mathrm{H}_{2} \mathrm{O}$ were observed, and these comprise a strong emphasis for the technical scope for the program in FY-99. While $\mathrm{H}_{2} \mathrm{O}$ is not a contaminant, it is a ubiquitous adsorbate at the molecular level (particularly in the variably saturated vadose zone). 
Therefore, understanding the interactions of $\mathrm{H}_{2} \mathrm{O}$ with oxides, and the resulting reaction products, is a prerequisite for further understanding of the interactions of oxides with other contaminant adsorbates.

The program in FY-99 capitalized on previous investments in instrumentation design and fabrication, which resulted in an ion trap secondary ion mass spectrometer (IT-SIMS) ${ }^{22,23}$ capable of sputter desorption, trapping, and reaction of oligomeric oxyanions. The instrument employs a perrhenate $\left(\mathrm{ReO}_{4}{ }^{-}\right)$primary projectile, which promotes enhanced production of molecular (versus atomic) secondary ions from the sample surface. As the name of the instrument implies, the secondary ions may be trapped for variable, millisecond time periods (Figure 1). By monitoring the disappearance of the reactant and appearance of the product ions as a function of time, reaction kinetics can be measured, and rate constants can be calculated if the concentration of the neutral adsorbate is known. ${ }^{24}$ The rate constant describes the intrinsic reactivity of the oxide/adsorbate system, which cannot be directly measured using standard surface-phase techniques.

The drawback to the gas-phase approach is that it will not produce information on the structures of the gas-phase oxyanions. To generate ion structures, computational approaches have been used, specifically Hartree-Fock and density functional theory. Using this combined approach, a detailed picture of $\mathrm{Si}, \mathrm{Al}$ oxyanion reactivity is emerging. The general method will be applicable to the study of a wide range of oxyanion adsorbate interactions.
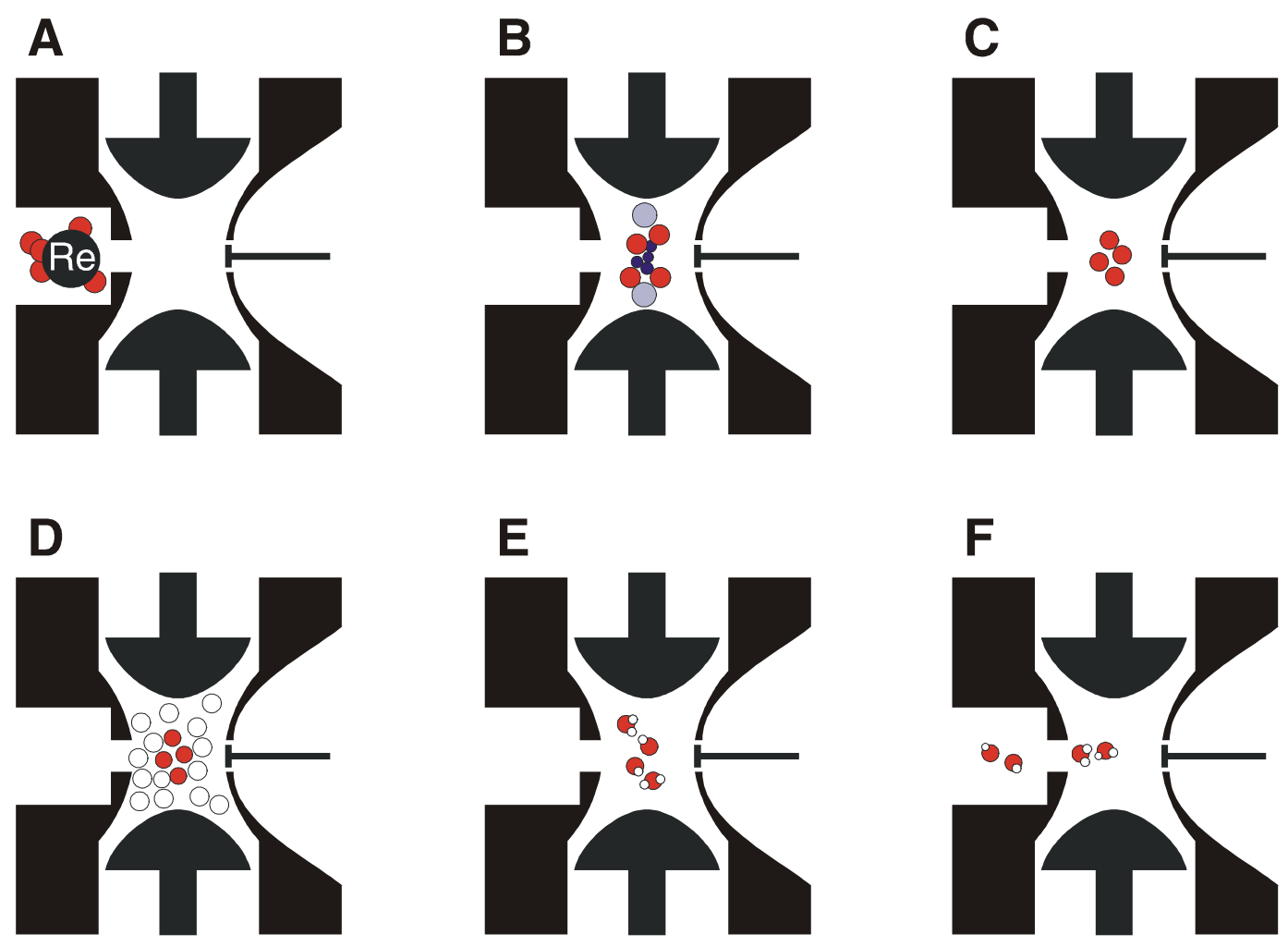

Figure 1. Schematic representation of oxyanion production and reaction in the IT-SIMS. A. Oxide surface is bombarded by energetic projectile $\left(\mathrm{ReO}_{4}^{-}\right)$. B. Si, $\mathrm{Al}$ oxyanions of various sizes are sputtered into the gas phase, and trapped by the IT-SIMS. C. Oxyanions of a selected mass are isolated (other masses are ejected from the IT-SIMS). D. Adsorbate molecules (i.e., $\mathrm{H}_{2} \mathrm{O}$ ) are admitted to the IT-SIMS. E. Adsorbate molecules react with oxyanions. F. Reaction product ions are scanned out of the IT-SIMS. 


\section{Overview: Chemical Investigations and Instrument Development}

Using the IT-SIMS approach described above, oxide surfaces were characterized based on the oxyanions generated, and the oxyanion reactivity was investigated. Specifically, oxides of silicon, aluminum, and aluminun+silicon were studied. Interest in these systems was prompted by the observation of oxyanion oligomeric species in the SIMS spectra of soil matrices, which are predominantly silicate in nature, but also have substantial quantities of $\mathrm{Al}$ present in their oxide lattices. The anion SIMS spectrum (Figure 2) of soil particles contains substantial 'monomeric' species $\mathrm{SiO}_{2}{ }^{-}, \mathrm{SiO}_{3}{ }^{-}$and $\mathrm{HSiO}_{3}^{-}$, but also contains substantial low abundance oligomeric species which most likely correspond to $\mathrm{AlSiO}_{4}^{-}(\mathrm{m} / z, 119)$ and $\mathrm{AlSi}_{2} \mathrm{O}_{6}^{-}(\mathrm{m} / z, 179)$.

It was believed that these oligomeric species could be utilized to characterize oxide surfaces and to directly probe reactivity. However, analysis of soil particles represented an unknown substrate, which made spectral interpretation difficult. Consequently, the experiment was initially simplified by analyzing silicon and aluminum oxides, based on the rationale that the oligomer compositions would be known with a relatively high degree of certainty. These analyses resulted in the observation of oligomeric oxyanions consisting of only silicon or aluminum. Using the ion isolation/reaction capability of the IT-SIMS, 'monomer' and 'dimer' species were isolated and reacted with water: this allowed the reaction pathways to be identified and the pseudo-first-order kinetics to be measured. To understand observed reactivity in terms of structure, $a b$ initio and Hartree-Fock level calculations were performed, which resulted in energy minimized structures and postulated reaction mechanisms. ${ }^{25,26}$ Separate sections in the following text are devoted to describing these studies.

Zeolite materials were analyzed in order to begin investigations of mixed aluminum-silicon systems. Mixed Al+Si systems are of interest, because studies performed in our laboratory and elsewhere have indicated that $\mathrm{Al}$ inserted into the silicate lattice induces anionic sites, which are largely responsible for cation adsorption. ${ }^{27,28}$ Zeolites were chosen because they are well understood in terms of composition and structure, and they can be manipulated easily by varying the counter cation. It was observed that oligomeric species up to $n=9$ were present in the ion trap. Under constant reaction conditions, the relative tendency of the oligomers to undergo dissociative hydration reactions could be assessed. As in the case of the aluminate and silicate anions, the IT-SIMS was used to identify reaction pathways and kinetics. ${ }^{29,30,31,32}$ Computational studies using Hartree-Fock and density functional approaches were also performed. A subheading in the following text is devoted to these studies.

Metal oxides in numerous instances are implicated in human and environmental toxicity, which is highly sensitive to the specific metal species present. ${ }^{33,34,35,36,37,38}$ Those species which are most responsible for toxic manifestation exist on the top-most layer of environmental surfaces, because they are most susceptible to

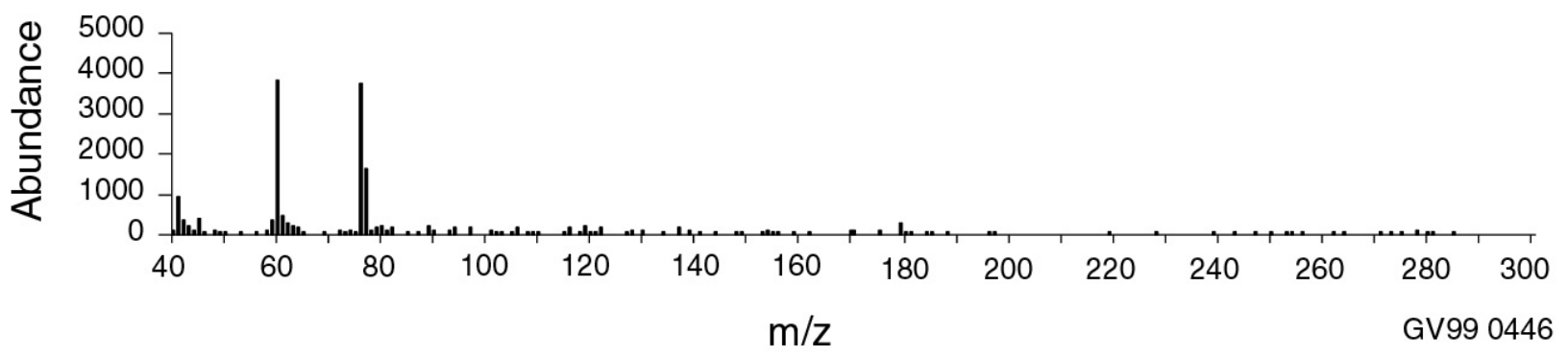

Figure 2. Anion SIMS spectrum of soil particles acquired using a low pressure SIMS instrument. The molecular species are dominated by $\mathrm{SiO}_{2}{ }^{-}, \mathrm{SiO}_{3}{ }^{-}$, and $\mathrm{HSiO}_{3}{ }^{-}(\mathrm{m} / \mathrm{z} 60,76$, and 77), but low abundance oligomers can be observed at $\mathrm{m} / \mathrm{z}, 119$ and 179 . 
solubilization, and consequent mobility and uptake. For this reason, a goal is to understand metal speciation at mineral surfaces. The program undertook an initial investigation of chromium speciation that showed substantial spectral differences resulting from oxide form and counter ion present. This research is described below under a separate subheading.

Throughout the course of the oxide research performed thus far, the IT-SIMS has demonstrated remarkable utility for performing analytical physical chemistry investigations on surfaces that are difficult to interrogate using other methods. However, the studies performed have also pointed to shortcomings in the instrumentation and analytical strategy, viz., the compositions of the ions cannot be unequivocally identified, and the vacuum system in the ion trap is decidedly non-ideal. For these reasons, the design and fabrication of a new-generation instrument was undertaken, which will enable a more detailed analysis of surface chemistry, gas-phase reactivity/structure, and the relationship between the two. The new instrumentation is based on a combination of Fourier transform mass spectrometry, laser desorption/ionization, and secondary ion mass spectrometry. Design, fabrication, and initial studies are presented below under the appropriate subheading.

\section{Generation and Reactions of Aluminum Oxyanions from Alumina Surfaces}

The secondary anion mass spectrum of alumina was acquired using a low pressure SIMS instrument (quadrupole analyzer) in order to ascertain the major ions produced. The two salient ions corresponded to $\mathrm{AlO}_{2}{ }^{-}$and $\mathrm{Al}_{2} \mathrm{O}_{4} \mathrm{H}^{-}$at $\mathrm{m} / z 59$ and 119 (Figure 3). ${ }^{39}$ The 'dimeric' ion $\mathrm{m} / z, 119$ is relatively more abundant when compared to data acquired using an atomic bombarding projectile. The instrumentation used in acquisition of Figure 3 utilizes a polyatomic projectile, which is more efficient for generating oligomeric secondary ions. The experiment shows that $\mathrm{m} / \mathrm{z} 119 \mathrm{can}$ be formed without the presence of $\mathrm{SiO}_{2}$ in the sample material. Next, the analysis was performed using the IT-SIMS. The low pressure spectrum can, to some degree, be replicated if the alumina target is evacuated for $12 \mathrm{H}$ prior to analysis (Figure 4). The additional ions observed in the IT-SIMS spectrum are the result of reaction with one and two $\mathrm{H}_{2} \mathrm{O}$ molecules: for example, $\mathrm{AlO}_{2}{ }^{-}\left(\mathrm{m} / z\right.$ 59) reacts with one and two $\mathrm{H}_{2} \mathrm{O}$ to form $\mathrm{m} / z 77$ and 95, respectively. Similarly, $\mathrm{m} / z, 137$ and 155 are the mono- and dihydrates of $\mathrm{Al}_{2} \mathrm{O}_{4} \mathrm{H}^{-}$. A trimeric ion is observed at $m / z, 179$, and the mono- and dihydrates are observed at $m / z, 197$ and 215 . These observations highlight one of the salient differences between the IT-SIMS and more conventional low pressure mass spectrometers: in the IT-SIMS, the pressure is high $\left(3 \times 10^{-5}\right.$ torr $\mathrm{He}$, with a low $\mathrm{H}_{2} \mathrm{O}$

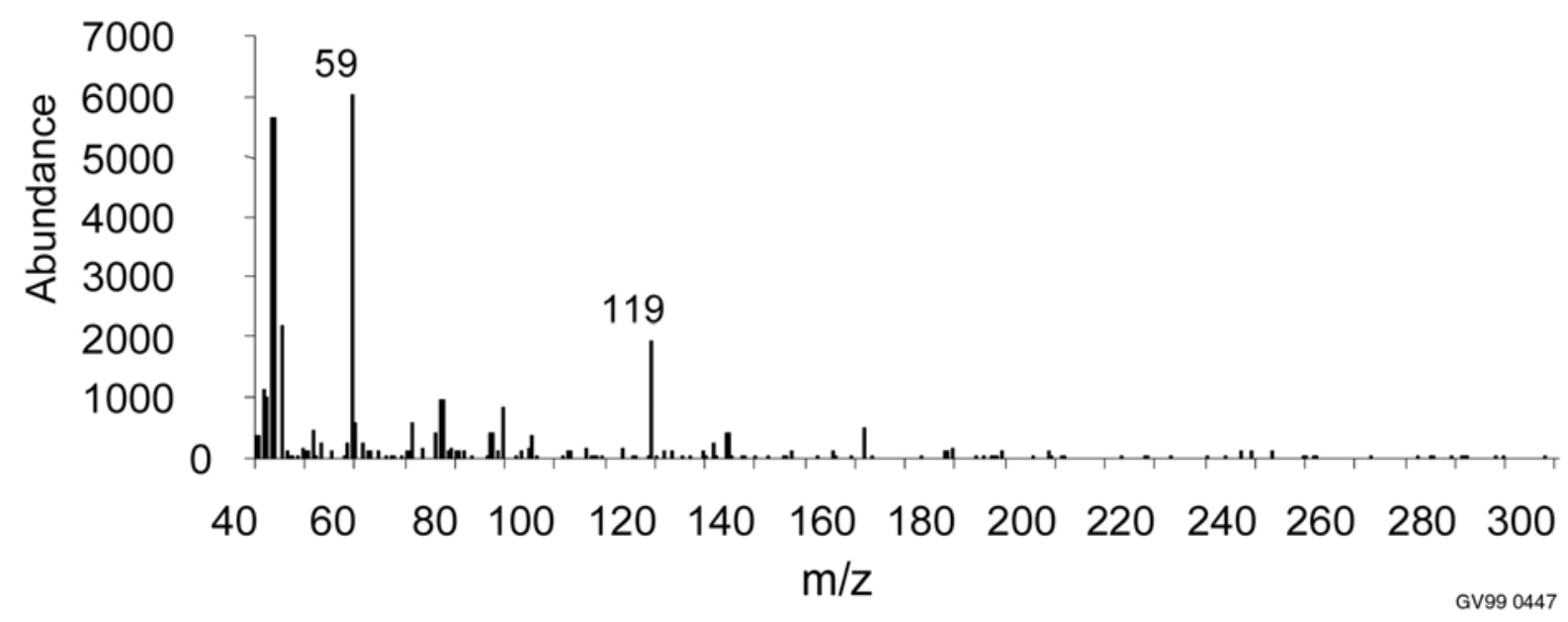

Figure 3. Anion SIMS spectrum of alumina acquired using a low pressure SIMS instrument. 


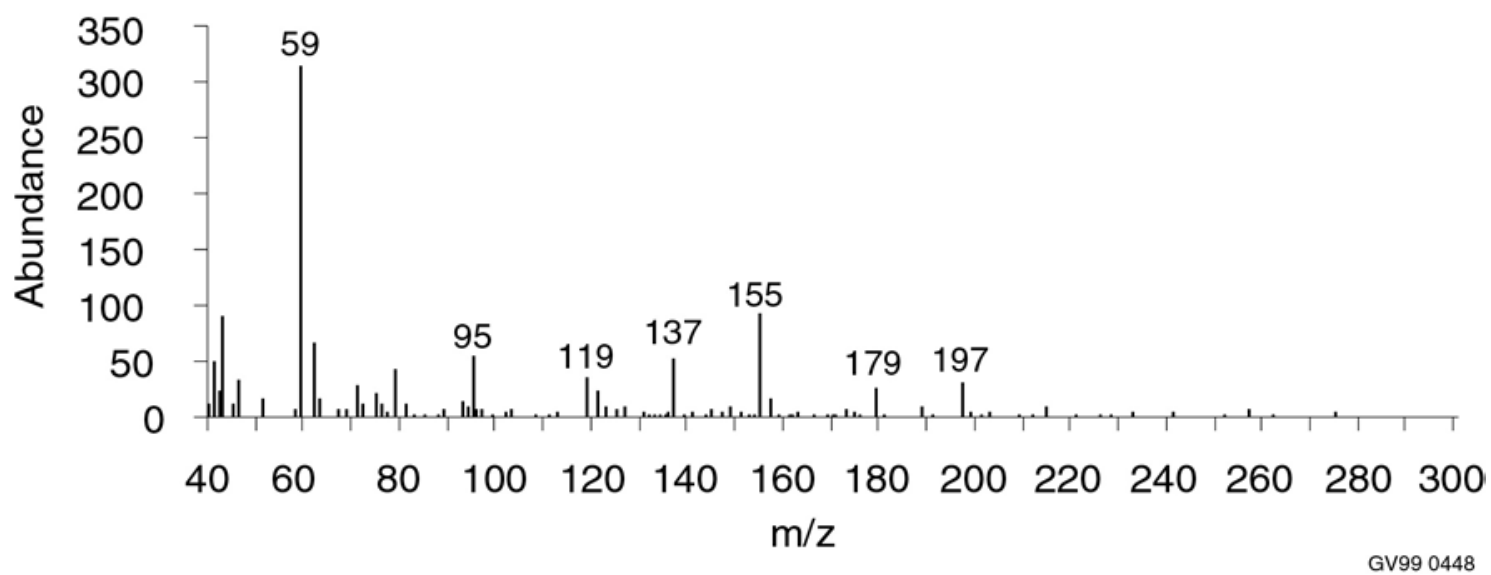

Figure 4. Anion SIMS spectrum of alumina acquired using the IT-SIMS. Alumina was evacuated at $9 \times 10^{-8}$ torr for $12 \mathrm{H}$ prior to analysis.

background), and ion lifetimes are long. For these reasons, the IT-SIMS functions as a gas-phase reaction device, and the hydrates observed are formed as a result of gas-phase ion molecule reactions. The presence of higher mass oligomeric species is also a unique feature of the IT-SIMS: in the case of alumina, the presence of the trimeric species $(\mathrm{m} / \mathrm{z} 179$ and 197) may be the result of direct sputtering of the trimeric species into the gas phase, followed by collisional stabilization from the He bath gas. ${ }^{40}$ Alternatively, the high pressure conditions of the IT-SIMS may facilitate formation of condensation ions between, for example, $\mathrm{AlO}_{2}{ }^{-}$and $\mathrm{AlO}_{2} \mathrm{H}$. This question is still open.

When alumina is analyzed immediately after insertion into the IT-SIMS, the appearance of the spectrum is markedly changed (Figure 5). At low mass, the dihydrated $\mathrm{m} / \mathrm{z} 95$ is more abundant the dehydrated $\mathrm{m} / \mathrm{z}$ 59 and monohydrate $m / z$ 77. The dehydrated and monohydrated dimer and trimer species are either not observed, or are low abundance. Instead, the ion abundance is concentrated in the dihydrated, and trihydrated species, i.e., $\mathrm{m} / \mathrm{z} 155,173$, and 233. In addition, hydrated oligomers are observed up to $\mathrm{m} / \mathrm{z}$ 593, which corresponds to the trihydrated nine-mer, generally the compositions can be written $\mathrm{H}\left(\mathrm{AlO}_{2}\right)_{\mathrm{x}}\left(\mathrm{H}_{2} \mathrm{O}\right)_{\mathrm{y}}^{-}$(Table 1). The reason behind the appearance of the higher mass oligomers has not been established. As presented above, they could be sputtered from the surface intact, and appear preferentially from a more highly hydrated surface (which had spent minimal time under vacuum). Alternatively, they may form in the desorption plume, and the condensation reactions may be facilitated by the presence of gaseous $\mathrm{H}_{2} \mathrm{O}$. Certainly, the presence of gaseous $\mathrm{H}_{2} \mathrm{O}$ is responsible for the lower mass hydrates.

Water most likely bonds to the aluminosilicate anions in a dissociative fashion..$^{41,42,43,44}$ This conclusion derives from the observation that the hydration reactions are fast, and do not appear to proceed in reverse to any appreciable extent. The dihydrated dimer $\mathrm{m} / \mathrm{z} 155$ was isolated in the IT-SIMS, and subjected to collisionally induced excitation. No dissociation to the monohydrate (or any other fragment ion) could be observed at the energies accessible in the IT-SIMS. This observation is consistent with the idea that $\mathrm{H}_{2} \mathrm{O}$ is dissociatively bound to the aluminosilicates: in related experiments, it was learned that hydrogen bound species (such as an ion with molecularly bound $\mathrm{H}_{2} \mathrm{O}$ ) could be dissociated with a few tens of millivolts. This energy level is only a fraction of what the dihydrated dimer was subjected to.

A detailed description of the reactivity and kinetics $\mathrm{m} / \mathrm{z} 59$ was generated by isolating this ion and following its reaction in a $\mathrm{He}$ atmosphere containing a controlled quantity of $\mathrm{H}_{2} \mathrm{O}$. Initially, only the isolated $\mathrm{AlO}_{2}{ }^{-}$is observed, with a tiny amount of the monohydrate at $m / z 77$ (Figure 6). After 100 milliseconds, $m / z 95$ has appeared, and is as abundant as $m / z 59$. M/z 77 remains low abundance 


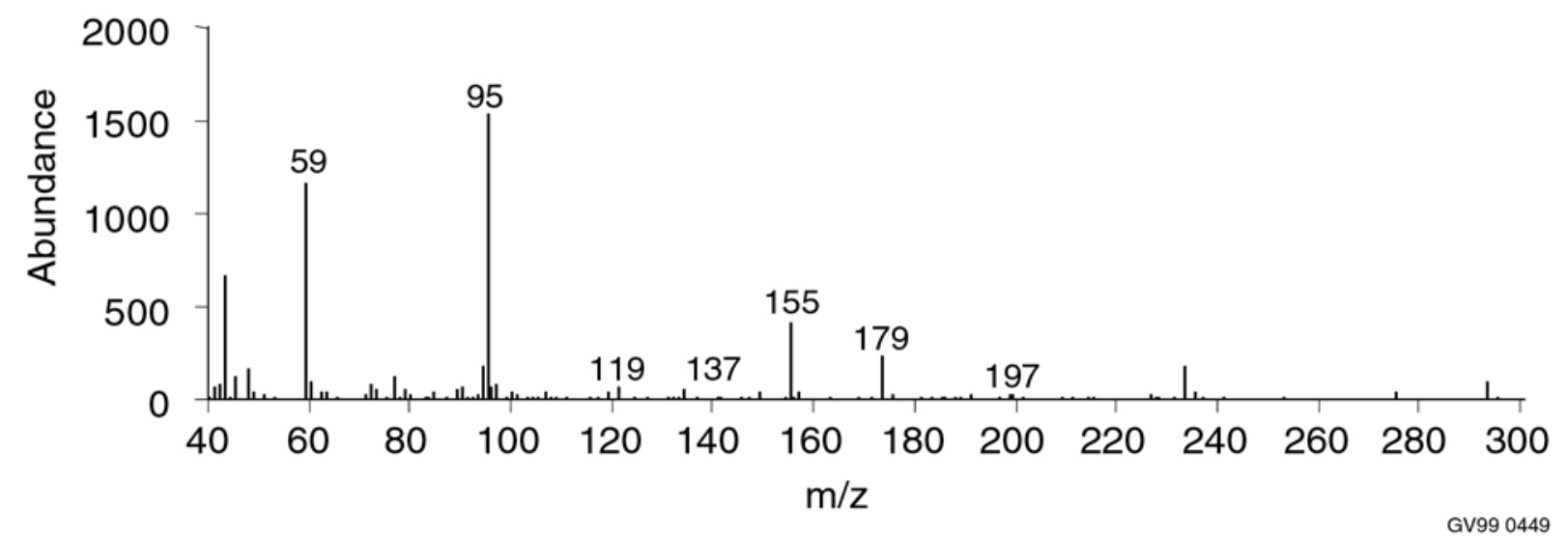

Figure 5. Anion SIMS spectrum of alumina acquired using the IT-SIMS. Alumina was evacuated to $3 \times 10^{-5}$ torr and analyzed immediately.

Table 1. Masses and compositions of aluminum oxyanions having the general composition $\mathrm{H}\left(\mathrm{AlO}_{2}\right)_{\mathrm{x}}\left(\mathrm{H}_{2} \mathrm{O}\right)_{\mathrm{y}}{ }^{-}$. (Ions not listed were not observed with abundance.)

\begin{tabular}{crrrr}
\hline $\mathrm{H}\left(\mathrm{AlO}_{2}\right)_{\mathrm{x}}\left(\mathrm{H}_{2} \mathrm{O}\right)_{\mathrm{y}}^{-}$ & \multicolumn{3}{c}{$\mathrm{y}$} \\
$\mathrm{x}$ & 0 & 1 & 2 & 3 \\
2 & 119 & 137 & 155 & 173 \\
3 & 179 & 197 & 215 & 233 \\
4 & & 257 & 275 & 293 \\
5 & & & 335 & \\
6 & & 395 & \\
7 & & 455 & 473 \\
8 & 497 & 515 & \\
9 & & & 593 \\
\hline
\end{tabular}
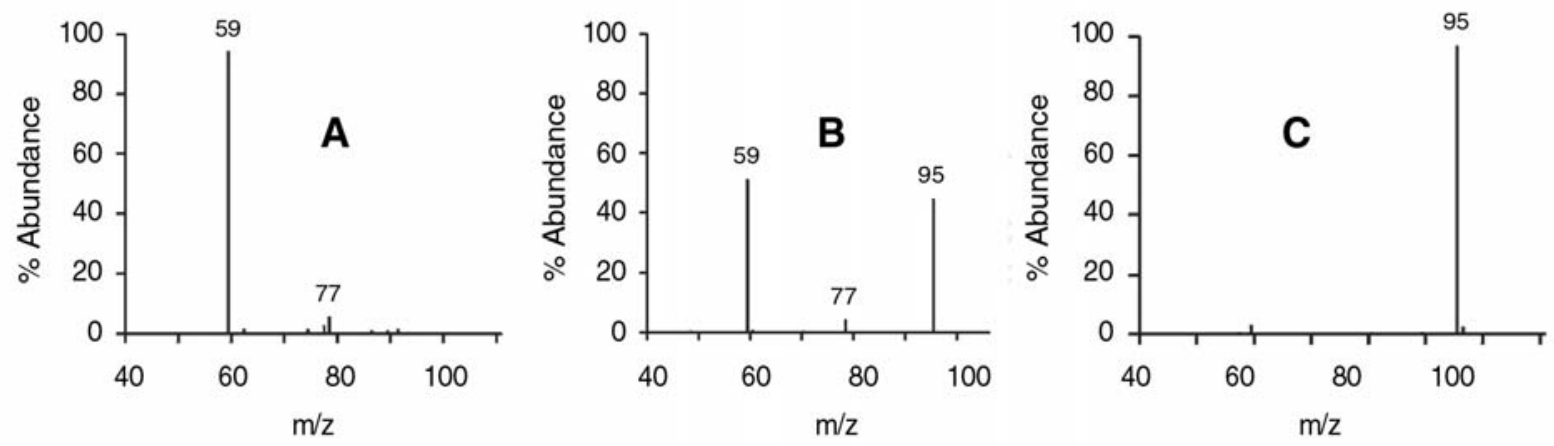

GV99 0450

Figure 6. Isolation of $\mathrm{m} / z$, 59 in $1 \times 10^{-6}$ torr $\mathrm{H}_{2} \mathrm{O} / 3 \times 10^{-5}$ torr He. A. Reaction time of 0 milliseconds. B. Reaction time of 100 milliseconds. C. Reaction time of 600 milliseconds. 
throughout these experiments. After 600 milliseconds, all of the ions have been converted to the dihydrate $(\mathrm{m} / \mathrm{z}, 95)$. These results are consistent with a set of consecutive reactions in which the initial reaction $(\mathrm{m} / \mathrm{z} 59 \rightarrow 77)$ is slow, and the consecutive reaction $(\mathrm{m} / \mathrm{z} 77 \rightarrow 95)$ is fast. A plot of reaction progress versus time (Figure 7), confirms this conclusion, and allows the pseudo-first order kinetics for the initial reaction to be calculated. The rate constant for the reaction of $\mathrm{AlO}_{2}{ }^{-}$with $\mathrm{H}_{2} \mathrm{O}$ was calculated at $3 \times 10^{-11} \mathrm{~cm}^{3} \mathrm{sec}^{-1} \mathrm{molecule}^{-1}$, which is only $1 \%$ of the collision rate (reparameterized average dipole orientation model).$^{45}$ Hence the reaction would be characterized as inefficient. By kinetic modeling of the data, it was found that the second reaction, $\mathrm{AlO}_{3} \mathrm{H}_{2}{ }^{-}+\mathrm{H}_{2} \mathrm{O} \rightarrow \mathrm{Al}(\mathrm{OH})_{4}{ }^{-}$was 10 times faster than the initial hydration step.

Insights into ion structure, reactive sites, and transition states of the $\mathrm{AlO}_{2}{ }^{-}+\mathrm{H}_{2} \mathrm{O}$ system were generated using $a b$ initio calculations, performed using the General Atomic and Molecular Electronic Structure System (GAMESS) program. Ion geometries were calculated at the Hartree-Fock (HF) level using a 6-311G basis set with a d-type polarization function added to the $\mathrm{Al}$ (and later to $\mathrm{Si}$ ), a p-type polarization function added to $\mathrm{H}$, and a diffuse sp shell added to the $\mathrm{O}, \mathrm{Al}$, and $\mathrm{Si}$ to account for the negative charge. Vibrational frequencies were calculated to identify minima and transition states. The intrinsic reaction coordinate (IRC) was calculated for each reaction to insure that reactant, transition state, and product all fall on the same reaction path. Energies were calculated using the Møller-Plesset second order perturbation theory (MP2) at the HF optimized geometry, to include the correlation contribution. Energies are corrected for the zero point energy and from absolute zero to $298.15 \mathrm{~K}$ (using HF vibrational frequencies). To date, all of the systems studied have been calculated accounting for all electrons (core and outer shell).

Initial approach of the $\mathrm{H}_{2} \mathrm{O}$ molecule to $\mathrm{AlO}_{2}{ }^{-}$resulted in the formation of a hydrogen-bound adduct (Figure 8). The reaction proceeds through a transition state in which the water $\mathrm{O}$ atom is brought into proximity with the $\mathrm{Al}$ atom. Cleavage of the water $\mathrm{O}-\mathrm{H}$ bond produces $\mathrm{AlO}_{3} \mathrm{H}_{2}^{-}$, which is calculated to be $57 \mathrm{kcal} \mathrm{mol}^{-1}$ exothermic. Note that the minimized structures are consistent with dissociative $\mathrm{H}_{2} \mathrm{O}$ adsorption.

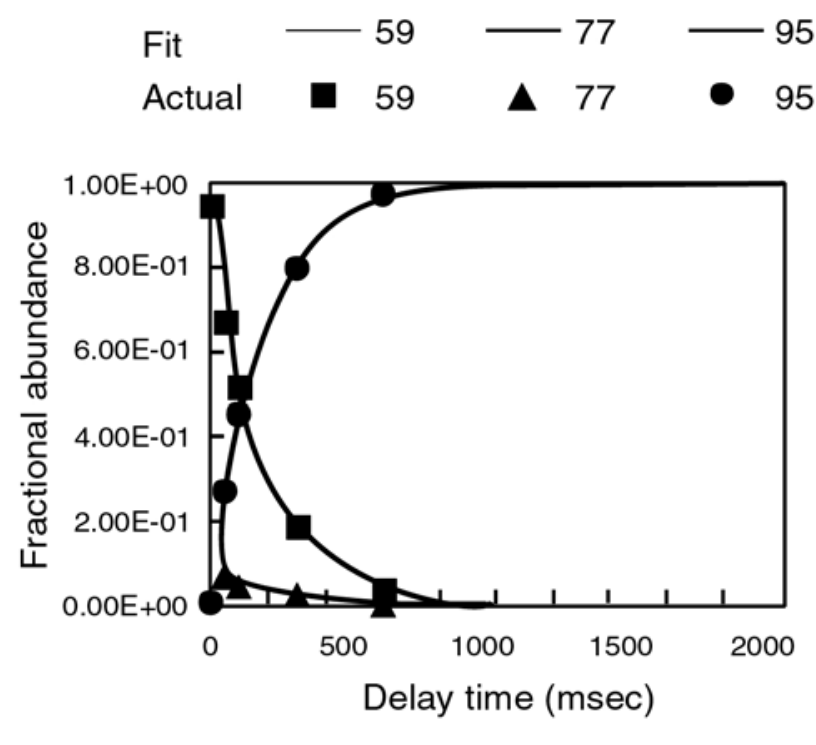

GV99 0451

Figure 7. Kinetic plot for the reaction of isolated $\mathrm{AlO}_{2}{ }^{-}(\mathrm{m} / z, 59)$ with $\mathrm{H}_{2} \mathrm{O}$. Consecutive reaction with $\mathrm{H}_{2} \mathrm{O}$ to form $m / z 95$ from 77 . 


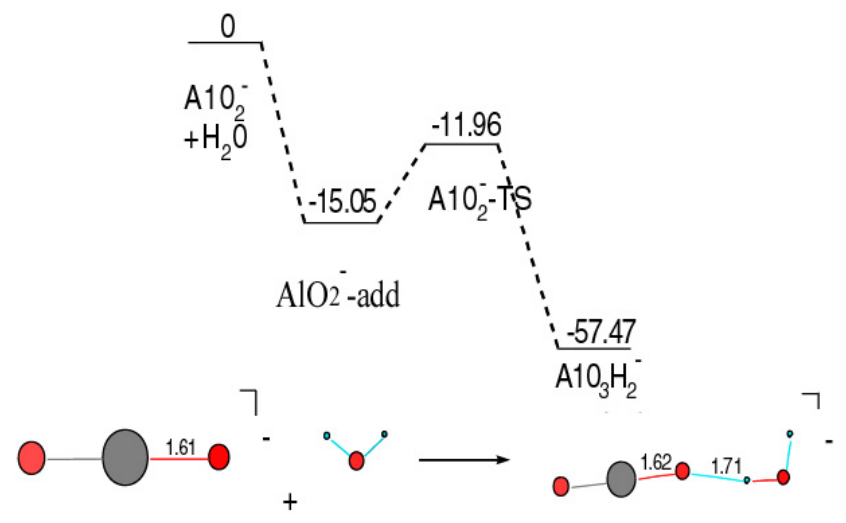

$\mathrm{A} 10_{2}^{-}$

A102-add
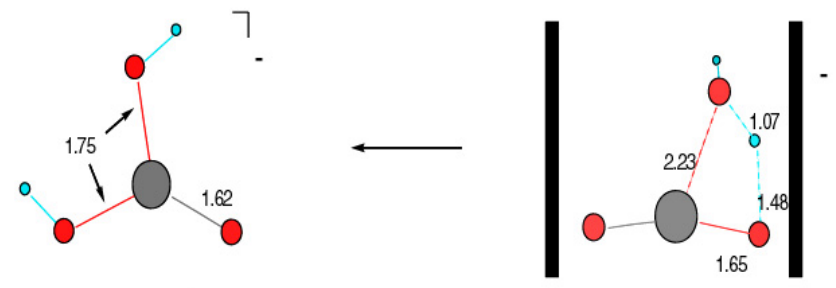

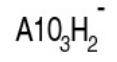

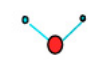

A10 ${ }_{2}^{-T S}$
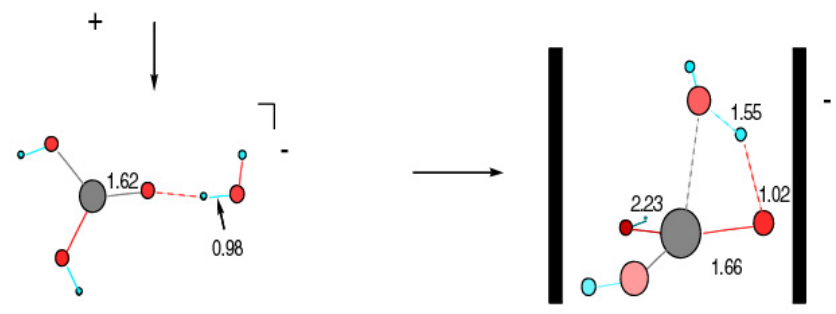

$\mathrm{A} 10_{3} \mathrm{H}^{-}$-add
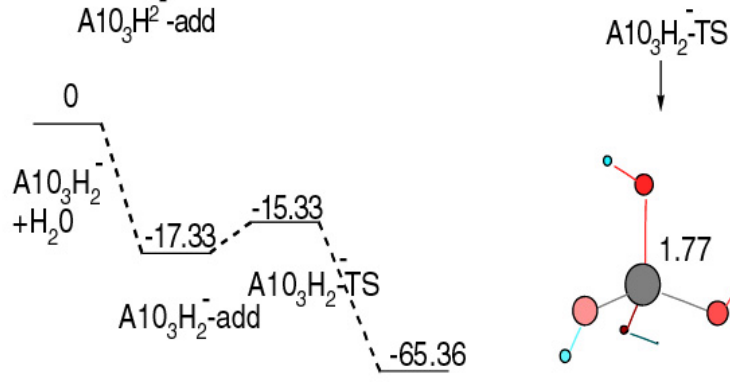

$\mathrm{A} \mathrm{O}_{4} \mathrm{H}_{4}^{-}$

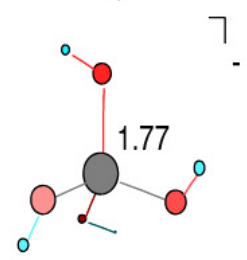

$\mathrm{A}^{1} \mathrm{O}_{4} \mathrm{H}_{4}$

GV990452

Figure 8. Calculated thermodynamics (density functional theory) for the reaction of $\mathrm{AlO}_{2}^{-}$plus one and two $\mathrm{H}_{2} \mathrm{O}$ molecules. 
The addition of the second $\mathrm{H}_{2} \mathrm{O}$ molecule proceeds by a mechanism that is similar: initial formation of a hydrogen bond, a transition state in which the water $\mathrm{O}$ atom is brought close to the $\mathrm{Al}$ atom, and finally cleavage of the water $\mathrm{O}-\mathrm{H}$ bond to produce $\mathrm{Al}(\mathrm{OH})_{4}{ }_{4}^{-}$. The reaction is calculated to be net exothermic by $65 \mathrm{kcal} \mathrm{mol}^{-1}$. The computational studies give us few hints as to why the second hydration reaction is faster than the first. We speculate that the hydroxyl groups of $\mathrm{AlO}_{3} \mathrm{H}_{2}{ }^{-}$may facilitate formation of the initial complex in the latter case, but there is no computational evidence to substantiate this position. The transition state barrier is somewhat smaller in the case of the addition of the second $\mathrm{H}_{2} \mathrm{O}$ molecule.

A similar strategy was used to investigate the reaction of $\mathrm{Al}_{2} \mathrm{O}_{4} \mathrm{H}^{-}$with $\mathrm{H}_{2} \mathrm{O} . M / 2119$ was isolated in an atmosphere containing a small but measurable quantity of $\mathrm{H}_{2} \mathrm{O}$, and the reaction was monitored as a function of time in the IT-SIMS (Figure 9, 10). The ion could be observed to undergo two sequential hydration reactions (but not a third) to form $\mathrm{m} / \mathrm{z} 137$ and 155 . The data enabled the rate constant for the reaction of $\mathrm{Al}_{2} \mathrm{O}_{4} \mathrm{H}^{-}+\mathrm{H}_{2} \mathrm{O}$ to be calculated at $2 \times 10^{-9} \mathrm{~cm}^{3} \mathrm{sec}^{-1}$ molecule ${ }^{-1}$, which agrees with the collision constant within experimental error. Clearly, this reaction is highly efficient compared to the
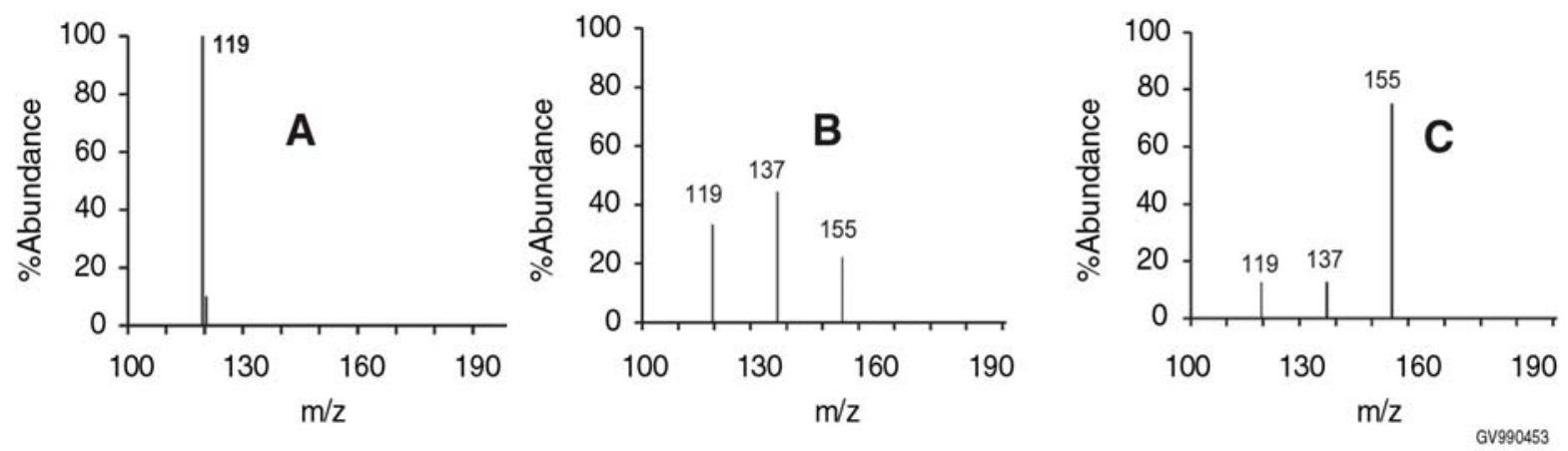

Figure 9. Isolation of $\mathrm{m} / \mathrm{z} 119$ in $1 \times 10^{-7}$ torr $\mathrm{H}_{2} \mathrm{O} / 3 \times 10^{-5}$ torr He. A. Reaction time of 0 milliseconds. B. Reaction time of 280 milliseconds. C. Reaction time of 1880 milliseconds.

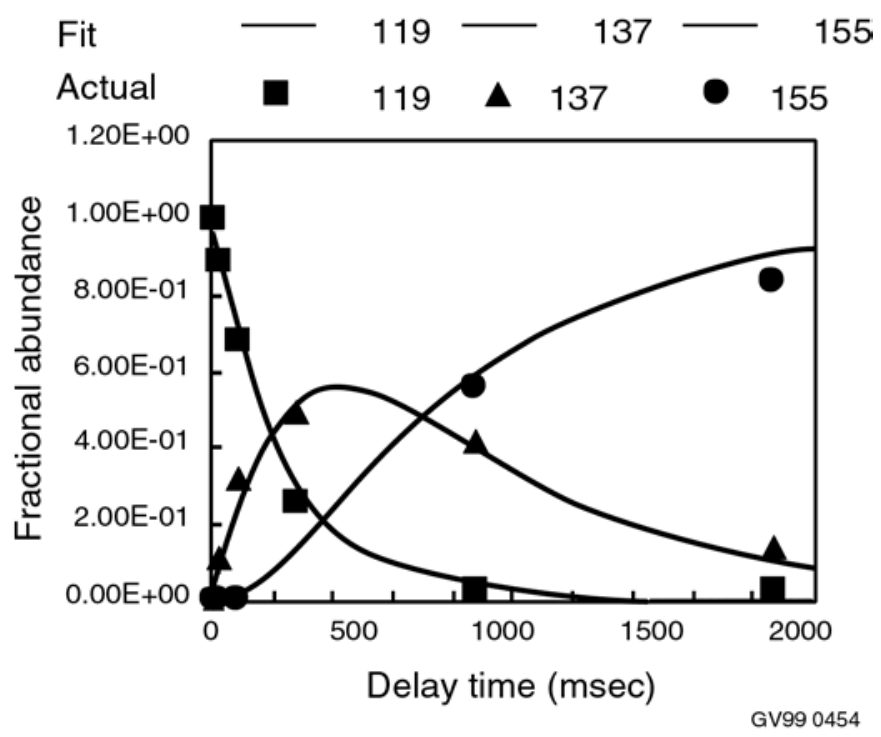

Figure 10. Kinetic plot for the reaction of isolated $\mathrm{Al}_{2} \mathrm{O}_{4} \mathrm{H}^{-}\left(\mathrm{m} / \mathrm{z}\right.$ 119) with $\mathrm{H}_{2} \mathrm{O}$. Consecutive reaction with $\mathrm{H}_{2} \mathrm{O}$ form $m / z, 155$ from 137 . 
reaction of $\mathrm{AlO}_{2}^{-}+\mathrm{H}_{2} \mathrm{O}$. By modeling the data using a consecutive reaction approach, a rate constant of $8 \times 10^{-10} \mathrm{~cm}^{3} \mathrm{sec}^{-1}$ molecule ${ }^{-1}$ was calculated for the reaction $\mathrm{Al}_{2} \mathrm{O}_{5} \mathrm{H}_{3}^{-}+\mathrm{H}_{2} \mathrm{O} \rightarrow \mathrm{Al}_{2} \mathrm{O}_{6} \mathrm{H}_{5}{ }^{-}$. This reaction, while somewhat slower than that of $\mathrm{Al}_{2} \mathrm{O}_{4} \mathrm{H}^{-}$, is still very fast compared to the monomeric species.

Computational modeling of $\mathrm{Al}_{2} \mathrm{O}_{4} \mathrm{H}^{-}$generated a cyclic structure with dangling $\mathrm{Al}-\mathrm{O}^{-}$and $\mathrm{Al}-\mathrm{OH}$ moieties (Figure 11). The initially formed complex involved formation of an Al-O bond, which implies nucleophilic attack on the aluminum by the $\mathrm{H}_{2} \mathrm{O}$ lone pairs. The initial complex proceeds to the product by transferring a water-H atom to the gem-O atom. No thermodynamic transition state could be identified, and the reaction was net exothermic by $67 \mathrm{kcal} \mathrm{mol}^{-1}$.

Higher aluminum oxyanion oligomers have not yet been modeled. Based on the phenomenological behavior of the $\mathrm{Al}_{\mathrm{x}} \mathrm{O}_{\mathrm{y}}{ }^{-}$oligomers in the IT-SIMS, it is clear that reactivity is fast, and that there is a preference for the addition of two (but frequently not additional) $\mathrm{H}_{2} \mathrm{O}$ molecules. The study suggests that only dehydrated species of $\mathrm{Al}_{\mathrm{x}} \mathrm{O}_{\mathrm{y}}{ }^{-}$are capable of dissociative adsorption.

\section{Generation and Reactions of Silicon Oxyanions from Silica Surfaces}

The anion spectrum of silica acquired using a low pressure SIMS instrument contains relatively abundant 'monomeric' ions corresponding to $\mathrm{SiO}_{2}{ }^{-}, \mathrm{SiO}_{3}{ }^{-}$, and $\mathrm{SiO}_{3} \mathrm{H}^{-}$at $\mathrm{m} / \mathrm{z} 60,76$, and 77 (Figure 12) ${ }^{46}$ Dimeric and trimeric species can also be observed at low abundance, at $m / z 136,137$, and 197. These masses correspond to $\mathrm{Si}_{2} \mathrm{O}_{5}^{-}, \mathrm{Si}_{2} \mathrm{O}_{5} \mathrm{H}^{-}$, and $\mathrm{Si}_{3} \mathrm{O}_{7} \mathrm{H}^{-}$. When silica was analyzed using the IT-SIMS, similar ions can be observed, except that the oligomer abundances are much greater. Figure 13 shows the anion spectrum collected after an average ion lifetime of 44 milliseconds; by limiting the ion lifetime, gas-phase condensation reactions with $\mathrm{H}_{2} \mathrm{O}$ are minimized. The dehydrated ions $\mathrm{Si}_{2} \mathrm{O}_{5} \mathrm{H}^{-}$and $\mathrm{Si}_{3} \mathrm{O}_{7} \mathrm{H}^{-}$are clearly observed, as are low abundance monohydrates. A low abundance ion is observed at $\mathrm{m} / \mathrm{z} 2 \mathrm{275}$, which corresponds to $\mathrm{Si}_{4} \mathrm{O}_{10} \mathrm{H}_{3}^{-}$.

When the ion lifetime is lengthened, the $\mathrm{H}_{2} \mathrm{O}$ condensation reactions have the opportunity to proceed further, and ion abundance is shifted toward the mono- and di-hydrated ions (Figure 14). The monohydrates are observed at $m / z, 95,155,215$, and 275, dihydrates at $m / z 173$ and 233 (Table 2). The dihydrate of the tetramer is not readily formed.

The reaction pathways and kinetics for the reactions of the silica oxyanions were studied by following the same strategy used for alumina. Reactant ions were isolated in a gaseous atmosphere containing a trace of $\mathrm{H}_{2} \mathrm{O}$ and allowed to react for variable periods of time. It was found that $\mathrm{SiO}_{2}{ }^{-}$ reacted slowly (if at all) with the $\mathrm{H}_{2} \mathrm{O}$ atmosphere to produce $m / z 77$ (addition of an $\mathrm{OH}$ radical). The rate constant for this reaction was measured at $6 \times 10^{-12} \mathrm{~cm}^{3} \mathrm{sec}^{-1}$ molecule $\mathrm{e}^{-1}$, which is at the limit of what can be reliably measured using the IT-SIMS. The reaction is extremely inefficient, with reaction occurring perhaps only one of every two hundred collisions. The $\mathrm{SiO}_{3} \mathrm{H}^{-}$ion could be observed to react slowly with $\mathrm{H}_{2} \mathrm{O}$ to produce $\mathrm{SiO}_{4} \mathrm{H}_{3}{ }^{-}, \mathrm{m} / z$ 95. Computational modeling predicted that the initially formed complex was a hydrogen-bound structure, which proceeded over a substantial energetic barrier to reach a minimized $\mathrm{SiO}_{4} \mathrm{H}_{3}{ }^{-}$structure. This is consistent with the slow rate of the reaction.

When the dimeric ion $\mathrm{Si}_{2} \mathrm{O}_{5} \mathrm{H}^{-}$was isolated, it was observed to react with a single $\mathrm{H}_{2} \mathrm{O}$ to produce $\mathrm{m} / \mathrm{z} 155$, at a rate which was about $50 \%$ of the collision rate. As in the case of the aluminate oxyanions, the dimeric species was highly reactive compared to the monomers. Ab initio calculations showed that a cyclic structure for $\mathrm{Si}_{2} \mathrm{O}_{5} \mathrm{H}^{-}$was the most stable by $23 \mathrm{kcal} \mathrm{mol}^{-1}$ (Figure 15). The initial water adduct was coordinated through both a hydrogen bond and interaction of the water $\mathrm{O}$ with Si. The reaction proceeds over a $20 \mathrm{kcal} \mathrm{mol}^{-1}$ barrier to form the monohydrate, with a net exothermicity of $23 \mathrm{kcal} \mathrm{mol}^{-1}$. This result is not in good agreement with the observed fast reaction kinetics, and additional studies are being planned to reconcile the work. 


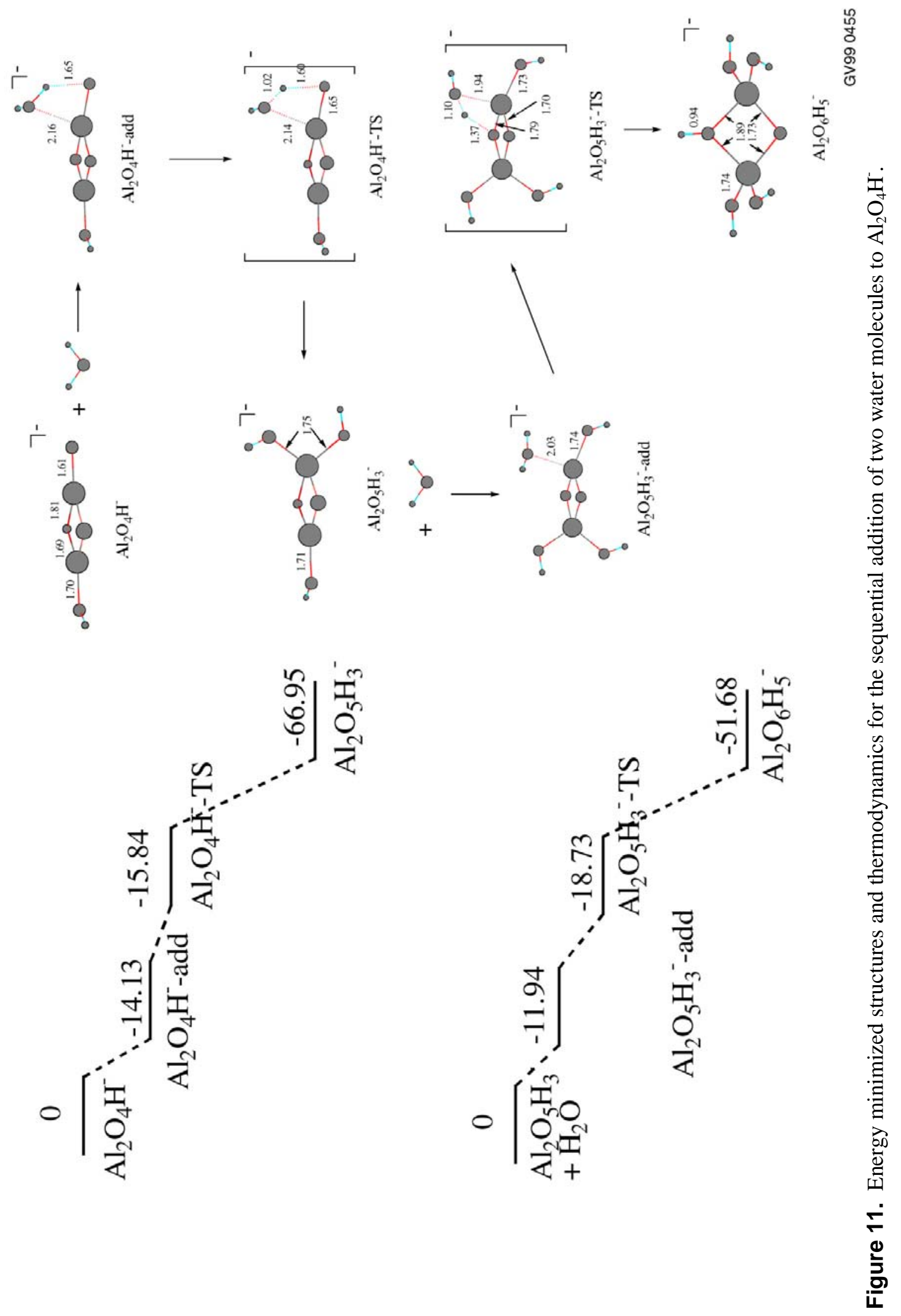




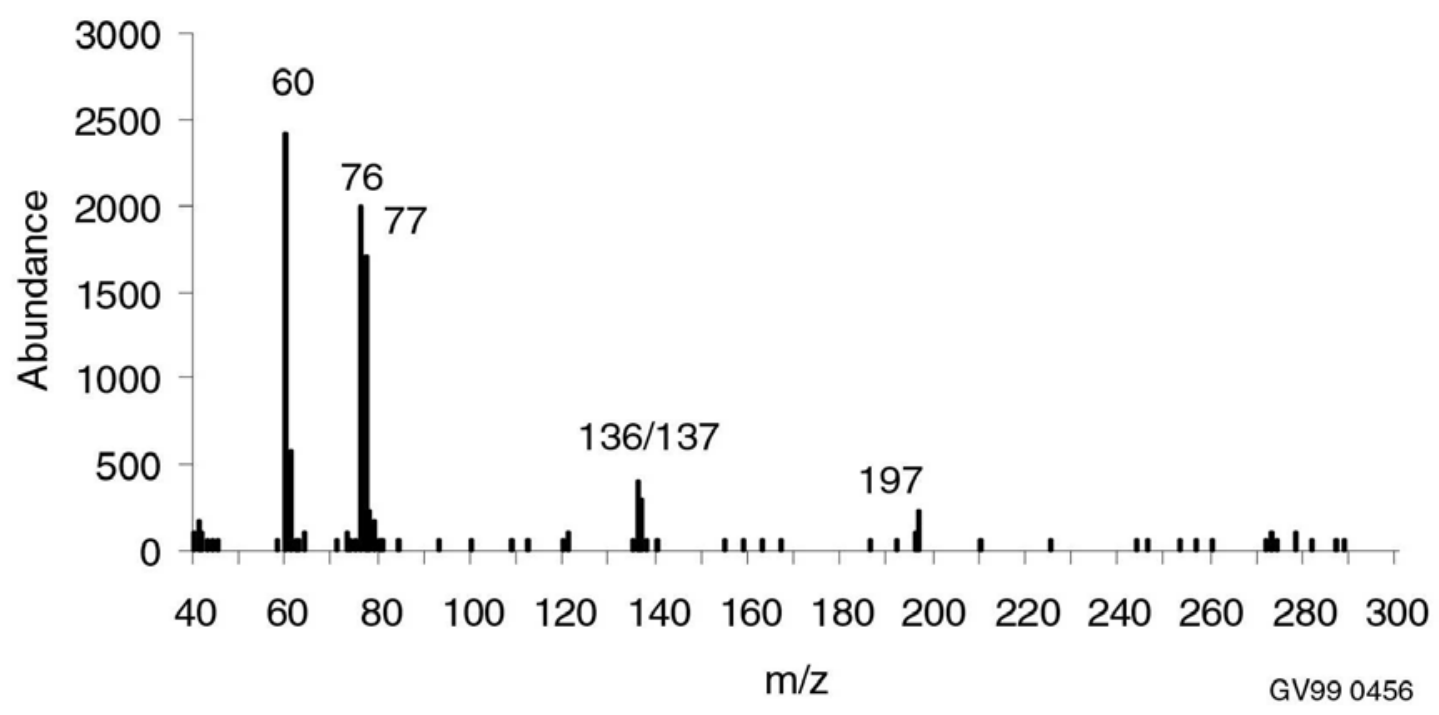

Figure 12. Anion SIMS spectrum of silica, acquired using a low pressure SIMS instrument.

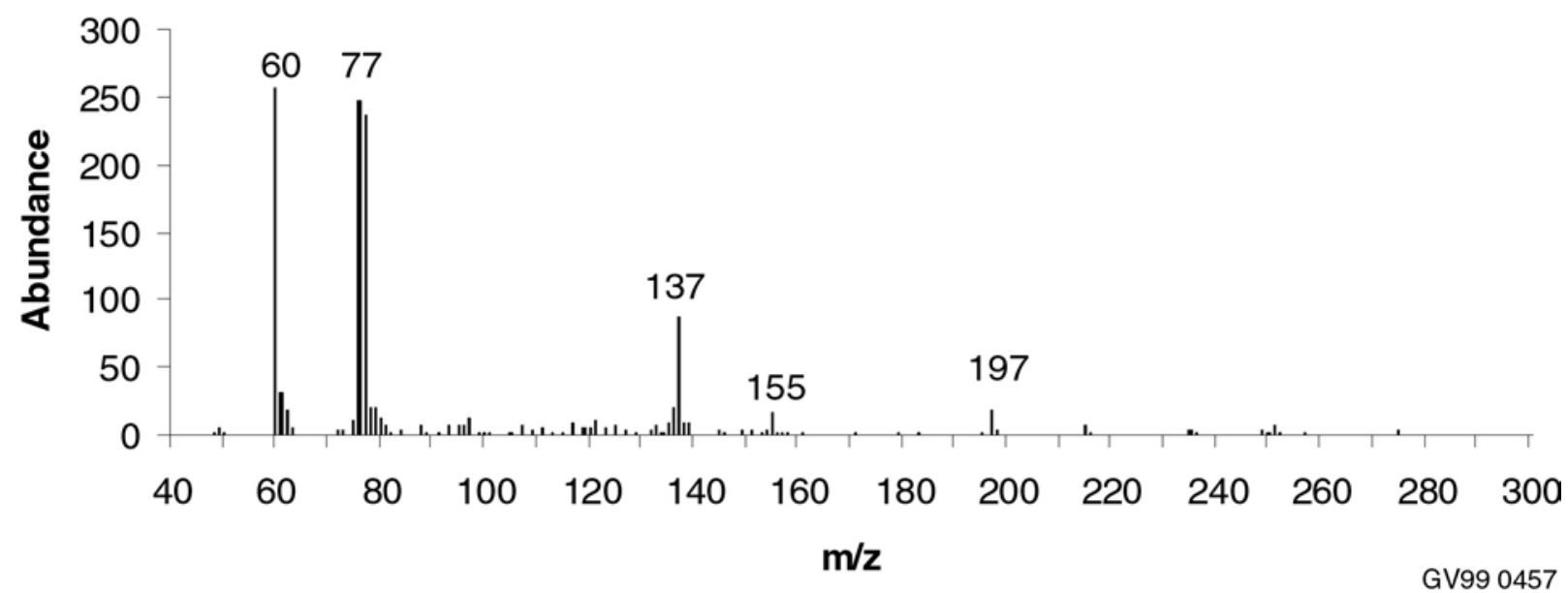

Figure 13. Anion SIMS spectrum of silica, acquired using the IT-SIMS, with an average ion lifetime of 44 milliseconds. Low abundance ions at 251 and 235 are from the $\mathrm{ReO}_{4}$ projectile.

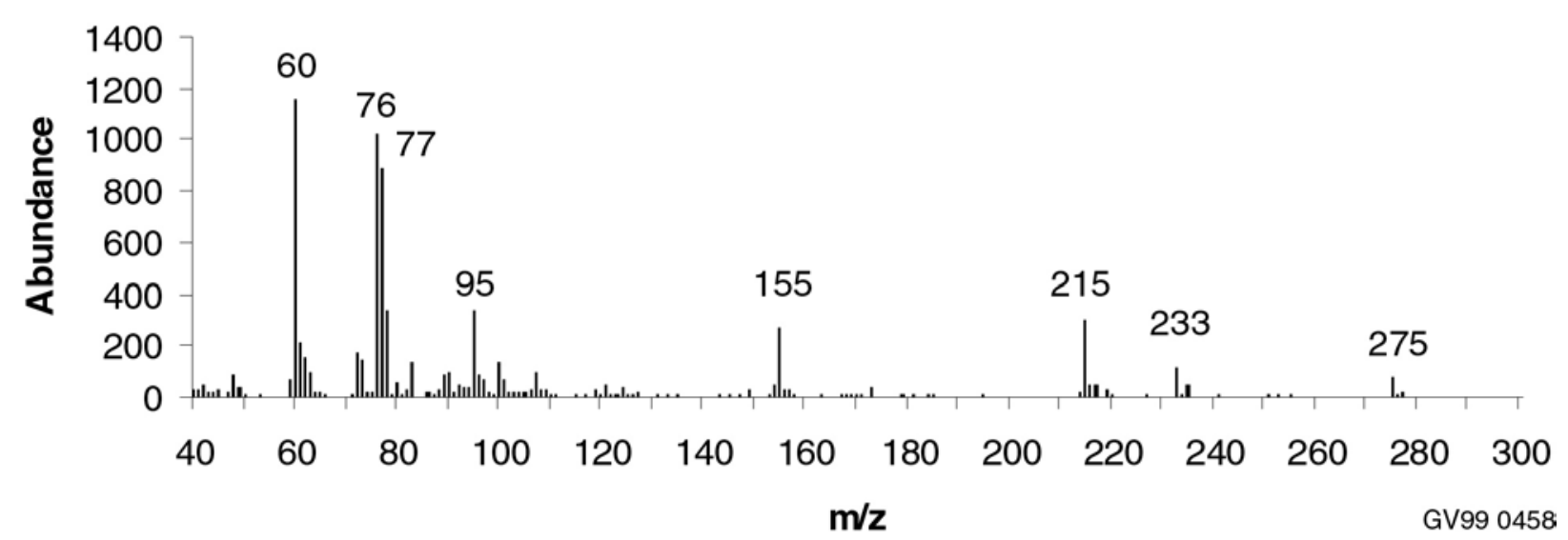

Figure 14. Anion SIMS spectrum of silica acquired using the IT-SIMS, with an average ion lifetime of 175 milliseconds. Ion abundance is shifted toward the mono- and di-hydrated silicate oxyanion oligomers. 
Table 2. Masses and compositions of silicon oxyanions having the general composition $\mathrm{HO}\left(\mathrm{SiO}_{2}\right)_{\mathrm{x}}\left(\mathrm{H}_{2} \mathrm{O}\right)_{\mathrm{y}}{ }^{-}$. (Ions not listed were not observed with abundance.)

\begin{tabular}{crrrr}
$\mathrm{HO}\left(\mathrm{SiO}_{2}\right)_{\mathrm{x}}\left(\mathrm{H}_{2} \mathrm{O}\right)_{\mathrm{y}}{ }^{-}$ & \multicolumn{5}{c}{$\mathrm{y}$} \\
$\mathrm{x}$ & 0 & 1 & 2 & 3 \\
2 & 137 & 155 & 173 & \\
3 & 197 & 215 & 233 & \\
4 & & 275 & & \\
\hline
\end{tabular}
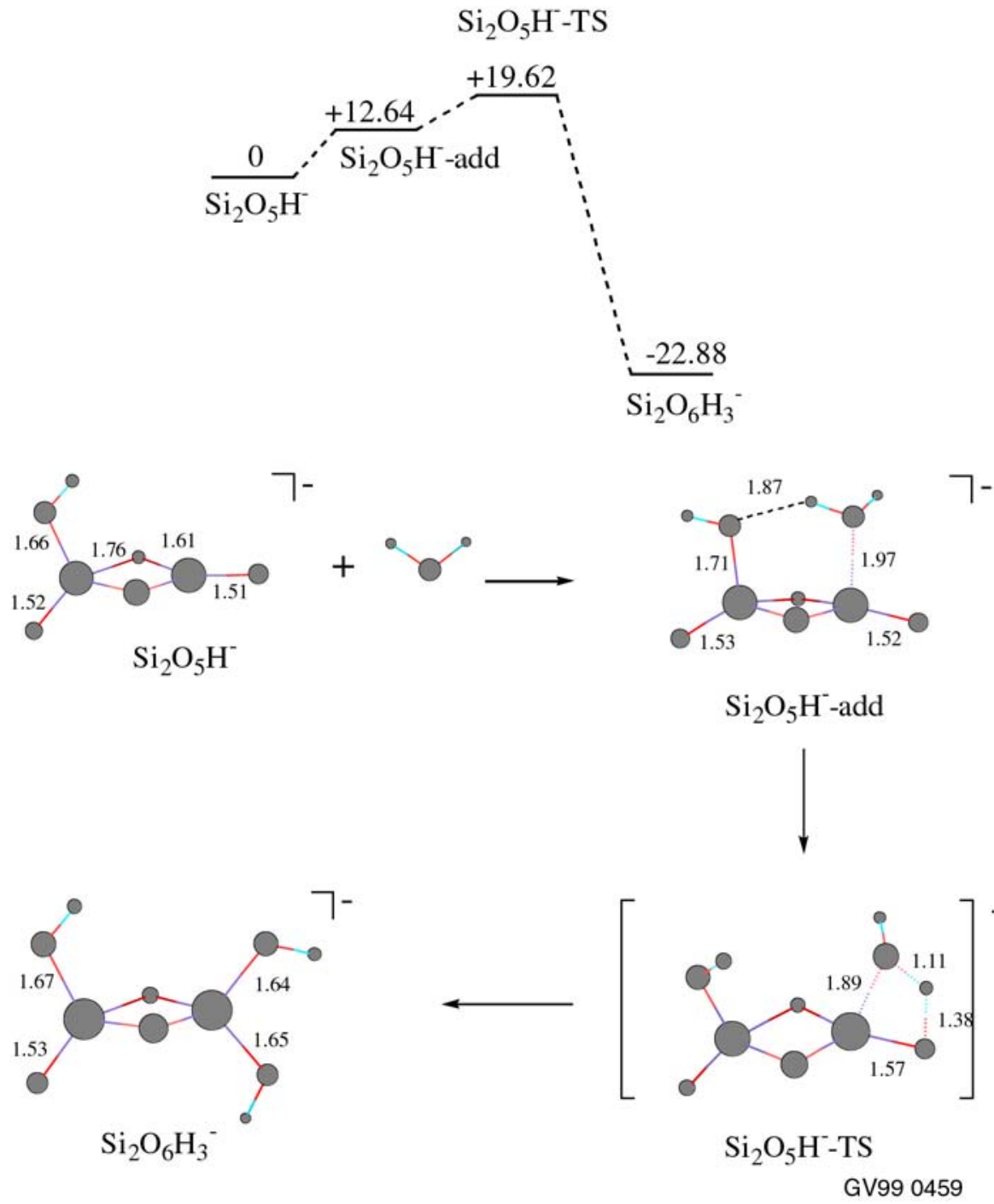

Figure 15. Ab initio calculations showing the energy minimized structure for $\mathrm{Si}_{2} \mathrm{O}_{5} \mathrm{H}^{-}$and its reaction with water, together with reaction coordinate with thermodynamic calculations. 
These initial studies of silicon and aluminum oxyanions are significant because the reactions of water with these oxide surfaces are among the most ubiquitous in environmental chemistry. The studies show that, at least from a compositional perspective, characteristic ions can be generated from the oxide surfaces. Clearly, the major challenge ahead is to relate the surface structure to that of the sputtered gasphase ion species. If this can be accomplished, then it will be possible to utilize the IT-SIMS to directly investigate relevant interaction chemistry.

\section{Generation and Reactions of Aluminosilicate Oxyanions from Zeolite Surfaces}

An investigation of zeolite materials was undertaken, because in general, secondary ion abundances derived from alumina and silica were low, which hampered attempts to understand reaction pathways and measure kinetics. It was felt that these aluminosilicate materials would be better for emission of secondary anions, because aluminum incorporated into a silicate matrix has the effect of inducing anionic sites on the surface. Thus, the zeolites could be viewed as a surface containing substantial 'preformed' ions, which are known to facilitate secondary ion production.

Analysis of zeolite sample revealed abundant, higher mass oligomeric ions (Figure 16). The compositions of these ions can be interpreted in terms of either the aluminate or the silicate series mentioned above. At low $\mathrm{H}_{2} \mathrm{O}$ pressure and short ion lifetimes, the ions revert back to the dehydrated masses (see Tables 1,2), which indicates that compositions containing only silicon cannot account for these ions. Compositions containing only aluminum are also unlikely, because substantial ' $\mathrm{M}+1$ ' and ' $\mathrm{M}+2$ ' ions are clearly observed for all the abundant ions, which are certainly ${ }^{29} \mathrm{Si}$ and ${ }^{30} \mathrm{Si}$ isotopic peaks - these patterns are not observed in the alumina analyses. The composition that best fits the data can be written $\mathrm{AlO}_{2}\left(\mathrm{SiO}_{2}\right)_{\mathrm{x}}\left(\mathrm{H}_{2} \mathrm{O}\right)_{\mathrm{y}}{ }^{-}$(see Table 3). A single $\mathrm{Al}$ atom is favored in these compositions because of the low abundance of $\mathrm{Al}$ in the zeolite $(\sim 14 \%$ of the $\Sigma$ (metal + metalloid)), and because preliminary deuterium labeling experiments were consistent with the number of exchangeable $\mathrm{H}$ expected (data not shown).

Generally, the secondary ion abundances of the oligomers generated from the zeolites were greater than abundances from either soil, alumina, or silica. It was found that secondary ion abundance was dependent on the counter cation on the zeolite surface. Secondary ion abundances of the oligomeric ions increased as the cation was varied from $\mathrm{Na}$ to $\mathrm{K}$ to $\mathrm{Rb} \sim \mathrm{Cs}$ (Figure 17). The origin of this enhancement

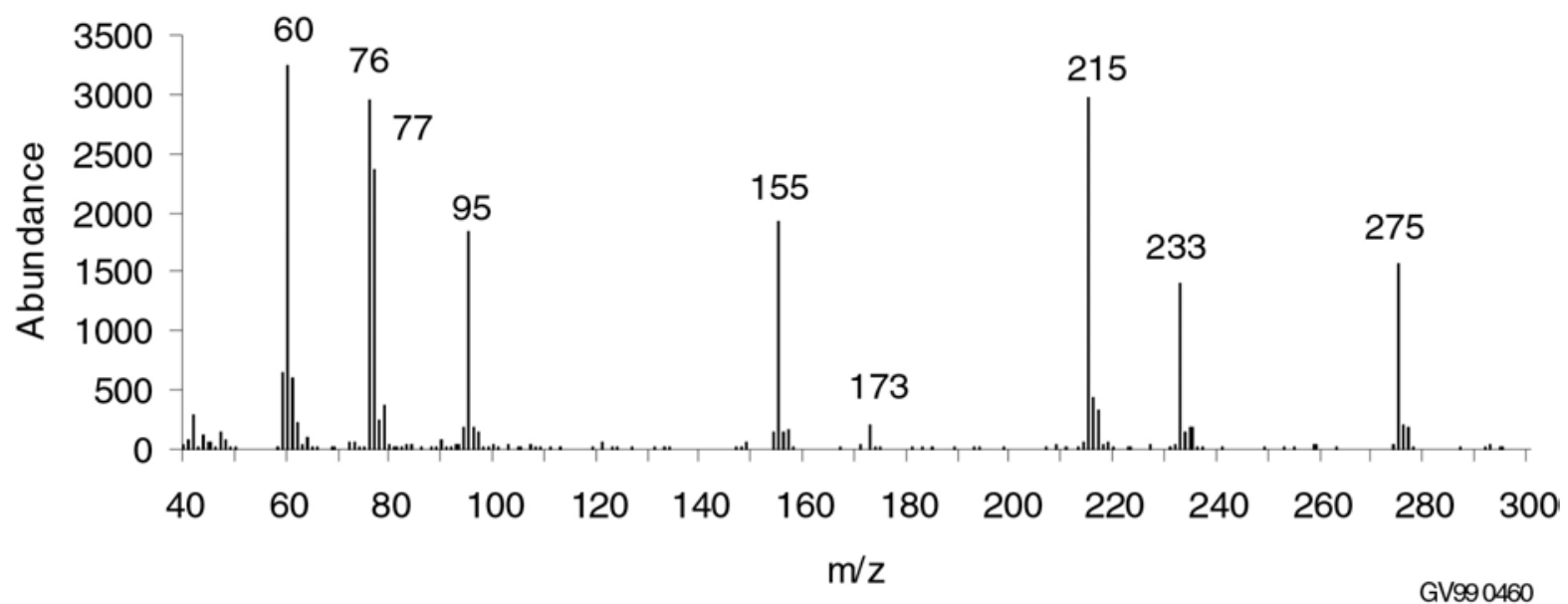

Figure 16. Anion SIMS spectrum of Cs zeolite, acquired with an average ion lifetime of 175 milliseconds. 
Table 3. Masses and compositions of aluminosilicate oxyanions having the general composition $\mathrm{AlO}_{2}\left(\mathrm{SiO}_{2}\right)_{\mathrm{x}}\left(\mathrm{H}_{2} \mathrm{O}\right)_{\mathrm{y}}{ }^{-}$. (Ions not listed were not observed with abundance.)

\begin{tabular}{crrrr}
\hline $\mathrm{AlO}_{2}\left(\mathrm{SiO}_{2}\right)_{\mathrm{x}}\left(\mathrm{H}_{2} \mathrm{O}\right)_{\mathrm{y}}{ }_{\mathrm{y}}^{-}$ & \multicolumn{1}{c}{$\mathrm{y}$} \\
\hline $\mathrm{x}$ & 0 & 1 & 2 & 3 \\
2 & & 137 & 155 & 173 \\
3 & & & 215 & 233 \\
4 & & 275 & 293 \\
5 & 317 & 335 & 353 \\
6 & 377 & 395 & 413 \\
7 & & 455 & 473 \\
8 & 497 & 515 & 533 \\
\hline
\end{tabular}

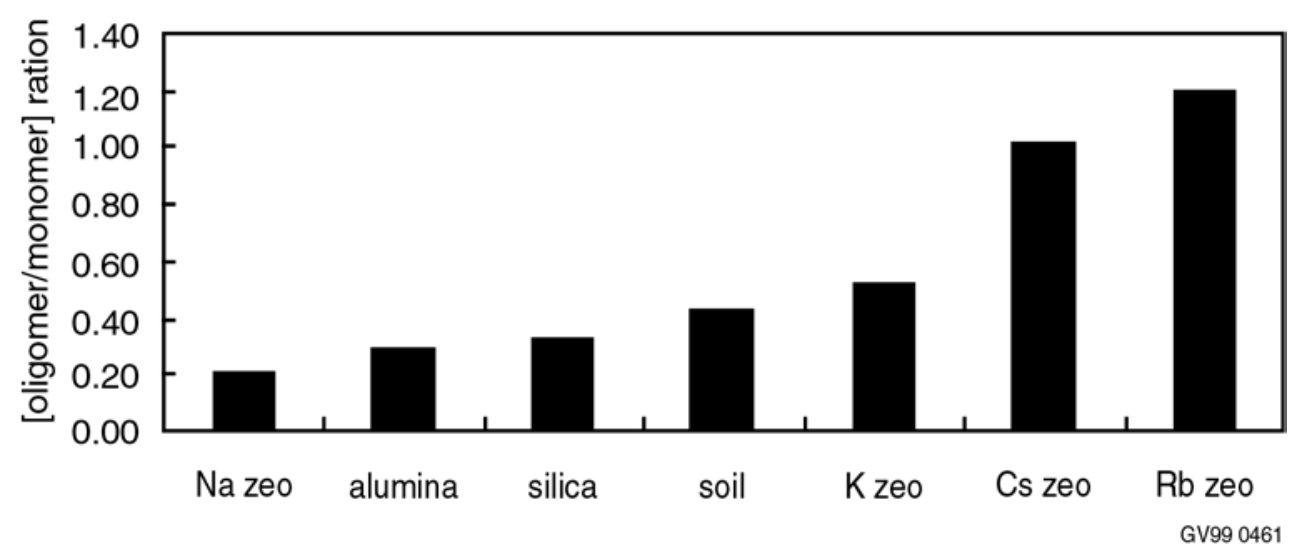

Figure 17. Chart of the ratio [oligomer abundance/monomer abundance] versus changing counter ion.

may be due to a reduced work function on the zeolite surface, which likely occurs when the larger alkali metals are substituted. This may have the effect of maintaining charge balance during primary ion bombardment, which would mitigate deleterious surface charging, which is known to prevent emission of the larger secondary ions. Alternatively, the alkali may alter the zeolite surface in such a fashion that emission of oligomeric oxyanions is facilitated. Irrespective of the mechanism of action, alkali substitution is an effective means for augmenting emission of large secondary ions, and has been used effectively with silica and alumina.

The data collected under conditions conducive to reaction with $\mathrm{H}_{2} \mathrm{O}$ show a clear preference for addition of up to three $\mathrm{H}_{2} \mathrm{O}$ molecules. Under normal conditions in the IT-SIMS, addition of two water molecules is preferred, regardless of the size of the oligomer. The number of $\mathrm{H}_{2} \mathrm{O}$ molecules does not increase with increasing oligomer size. This fact may be indicative of structural factors, which are discussed below. To verify the reaction pathways and measure kinetics, $\mathrm{AlO}_{2} \mathrm{SiO}_{2}{ }^{-} \mathrm{m} / z, 119$ was isolated and reacted with $\mathrm{H}_{2} \mathrm{O}$. The rate constant was measured at $2 \times 10^{-9} \mathrm{~cm}^{3} \mathrm{sec}^{-1}$ molecule ${ }^{-1}$, which is within experimental error of the collision constant; i.e., the reaction proceeds at unit efficiency. In a similar manner, the rate constant for the trimer $\mathrm{AlO}_{2}\left(\mathrm{SiO}_{2}\right)_{2}{ }^{-}$was measured and found to be equal to the collision rate.

Ab initio calculations were performed for both the $\mathrm{AlO}_{2} \mathrm{SiO}_{2}{ }^{-}$and $\mathrm{AlO}_{2}\left(\mathrm{SiO}_{2}\right)_{2}{ }^{-}$systems. The minimized structure derived for the $\mathrm{AlSiO}_{4}{ }^{-}$system is square planar with dangling oxygen atoms 
(Figure 18). Initial attack is nucleophilic by the water $\mathrm{O}$ atom on the silicon. No transition state could be identified, but the system proceeds thermodynamically downhill $\left(-45 \mathrm{kcal} \mathrm{mol}^{-1}\right)$ to the $\mathrm{gem}$-silandiol structure for $\mathrm{AlSiO}_{5} \mathrm{H}_{2}{ }_{2}^{-}$. Addition of a second $\mathrm{H}_{2} \mathrm{O}$ proceeds by attack at the $\mathrm{Al}$ atom, resulting in the dihydrated ion $\mathrm{AlSiO}_{6} \mathrm{H}_{4}$ at $m / z$ 155. The energetics for the second $\mathrm{H}_{2} \mathrm{O}$ addition have not yet been calculated.

Four stable structures of the trimeric species $\mathrm{AlSi}_{2} \mathrm{O}_{6}{ }^{-}$were also generated using ab initio methods (Figure 19). Structures 1 and 3 are very similar, consisting of two planar four-membered rings, joined in a spiro fashion, with two dangling oxygen atoms. Structure 2 consists of a six-membered ring, with three dangling oxygen atoms. Structure 4 is a basket structure with two dangling oxygens. The reaction of structure 1 with one $\mathrm{H}_{2} \mathrm{O}$ has been modeled to date. The reaction shows that the initially formed adduct involves coordination of the water $\mathrm{O}$ atom with the silylyl, and coordination of a water $\mathrm{H}$ atom with a cyclic oxygen (Figure 20). As the new $\mathrm{Si}-\mathrm{O}$ bond is formed, the water $\mathrm{H}$ is transferred to a geminal position. The reaction is predicted to be $55 \mathrm{kcal} \mathrm{mol}^{-1}$ exothermic.

$A b$ initio modeling of oligomeric oxyanions to date has shown that reaction occurs exclusively at sites possessing a dangling $\mathrm{O}$ atom. If this finding is correct, then it may explain hydration patterns observed for the trimeric species, which can exist as structures 1,3, and 4 with two, or as structure 2 with three dangling $\mathrm{O}$ atoms. Presumably, those structures with two dangling $\mathrm{O}$ atoms would be able to undergo two hydration reactions, as opposed to structure 2 with three dangling $\mathrm{O}$ atoms, which would be able to undergo three hydration reactions. For the trimer, significant amounts of the dihydrate and the trihydrate are observed at $\mathrm{m} / \mathrm{z} 215$ and 233 (Figure 16), which may be indicative of multiple structures.

The aluminosilicate investigations show that it is possible to generate large $\mathrm{AlSi}_{\mathrm{x}} \mathrm{O}_{\mathrm{y}}{ }^{-}$species. The fact that oligomers are observed near the mass limit of the IT-SIMS indicates that higher mass species may be observable if an instrument having a higher mass range were employed. Identifying the elemental composition of the oligomers is also difficult with the current instrumentation. These difficulties point to the need to develop advanced instrumentation capable of large oligomer ion generation, high mass measurement accuracy, and careful control of vacuum environment. Nevertheless, the results show that the approach is applicable for the facile characterization of oxyanion reactivity.

\section{Generation of Chromium Oxyanions from Salt Surfaces}

Initial studies were performed to understand the nature of chromium oxide species sputtered from the surfaces of chromium salts. Chromium is a significant environmental problem because of its carcinogenicity in the (VI) oxidation state. ${ }^{47,48,49,50,51,52,53}$ For this reason, speciation at the surface is a significant issue is assessing remediation options. ${ }^{54,55,56}$ The anion spectrum of many of the $\mathrm{Cr}(\mathrm{VI})$ oxides were rich with abundant oxide species (Figure 21, Table 4). What is observed in the anion spectra are $\mathrm{Cr}$ species with a variety of oxidation states, which arise primarily from reduction of the Cr metal during the bombardment process. In a few cases $(\mathrm{m} / \mathrm{z} 117,200)$, the oxidation state of the original material is preserved, [in the case of $m / z 116$, the VI state is preserved, and a radical anion is formed]; however, in most cases in the anion spectrum, the Cr species undergo reduction. $M / z 100$ is invariably the most abundant anion in these spectra, indicating that $\mathrm{Cr}(\mathrm{V})$ is the most stable oxidation state in the gas phase subsequent to bombardment.

Many of the Cr oxide species yielded similar ions when interrogated using a SIMS approach. In particular, it was difficult to reproducibly distinguish between the monochromate and dichromate species, particularly if the counter cation was $\mathrm{Na} . \mathrm{K}_{2} \mathrm{Cr}_{2} \mathrm{O}_{7}$ yielded more abundant $\mathrm{Cr}_{2} \mathrm{O}_{\mathrm{x}}{ }^{-}$species than did the corresponding monochromate, however. In terms of identifying the counter cation, this was readily 


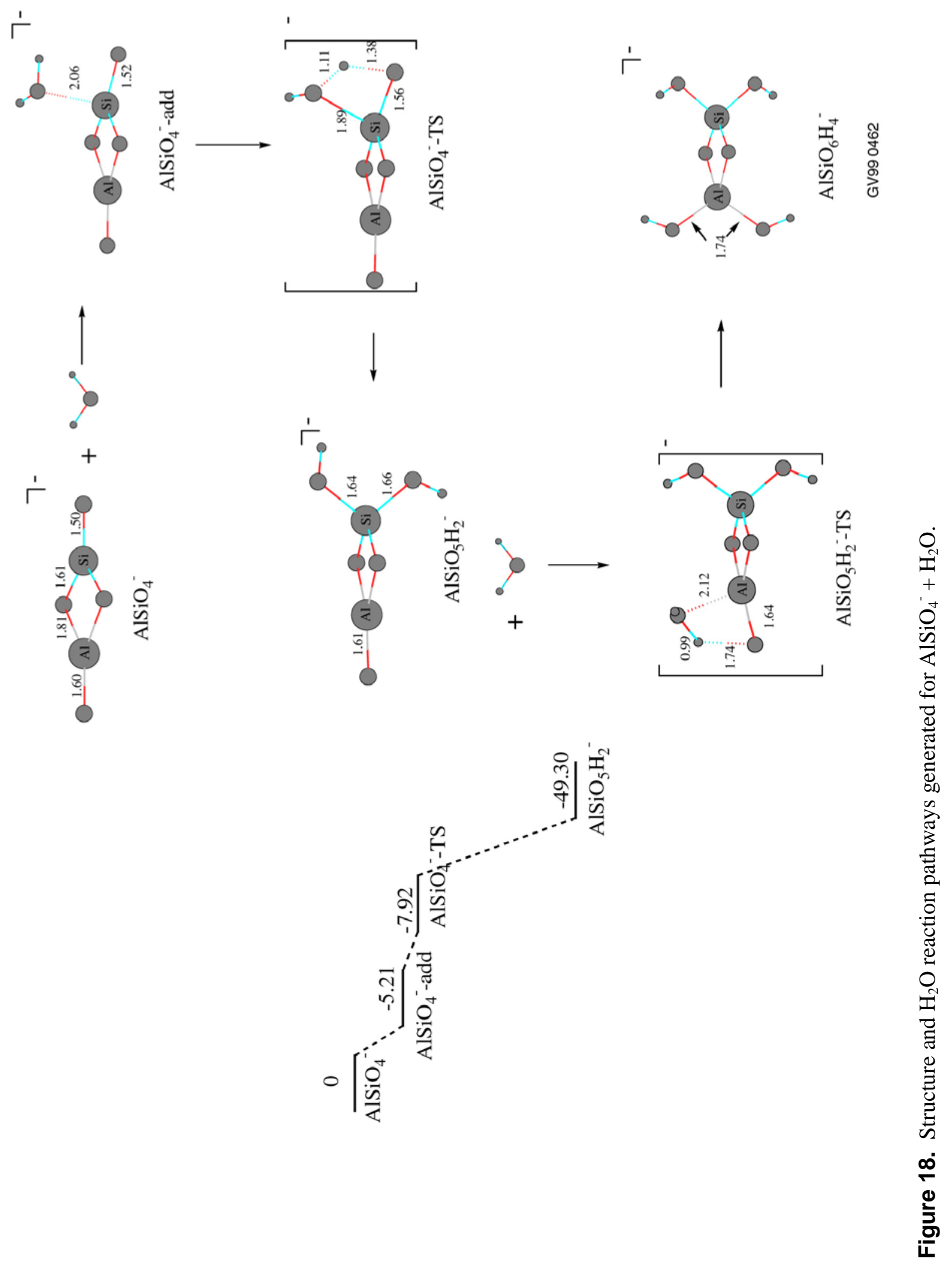



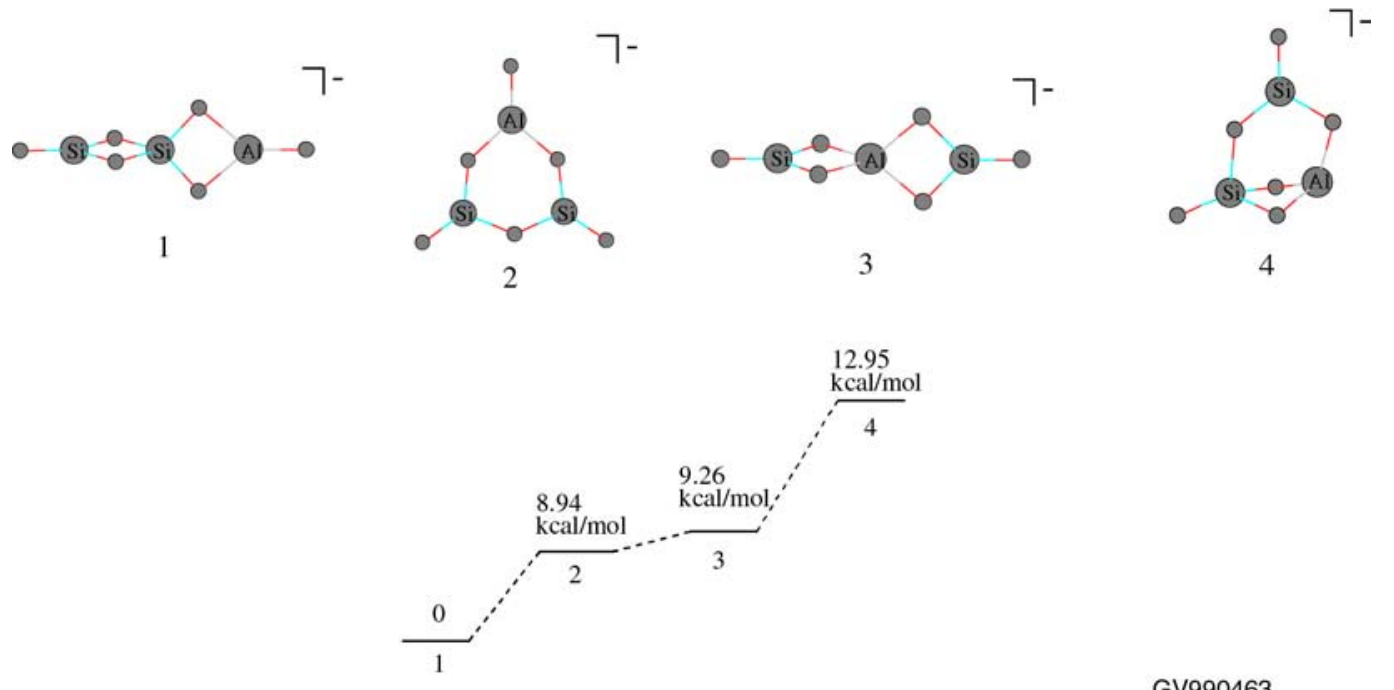

GV990463

Figure 19. Minimized structures of $\mathrm{AlSi}_{2} \mathrm{O}_{6}{ }^{-}$.
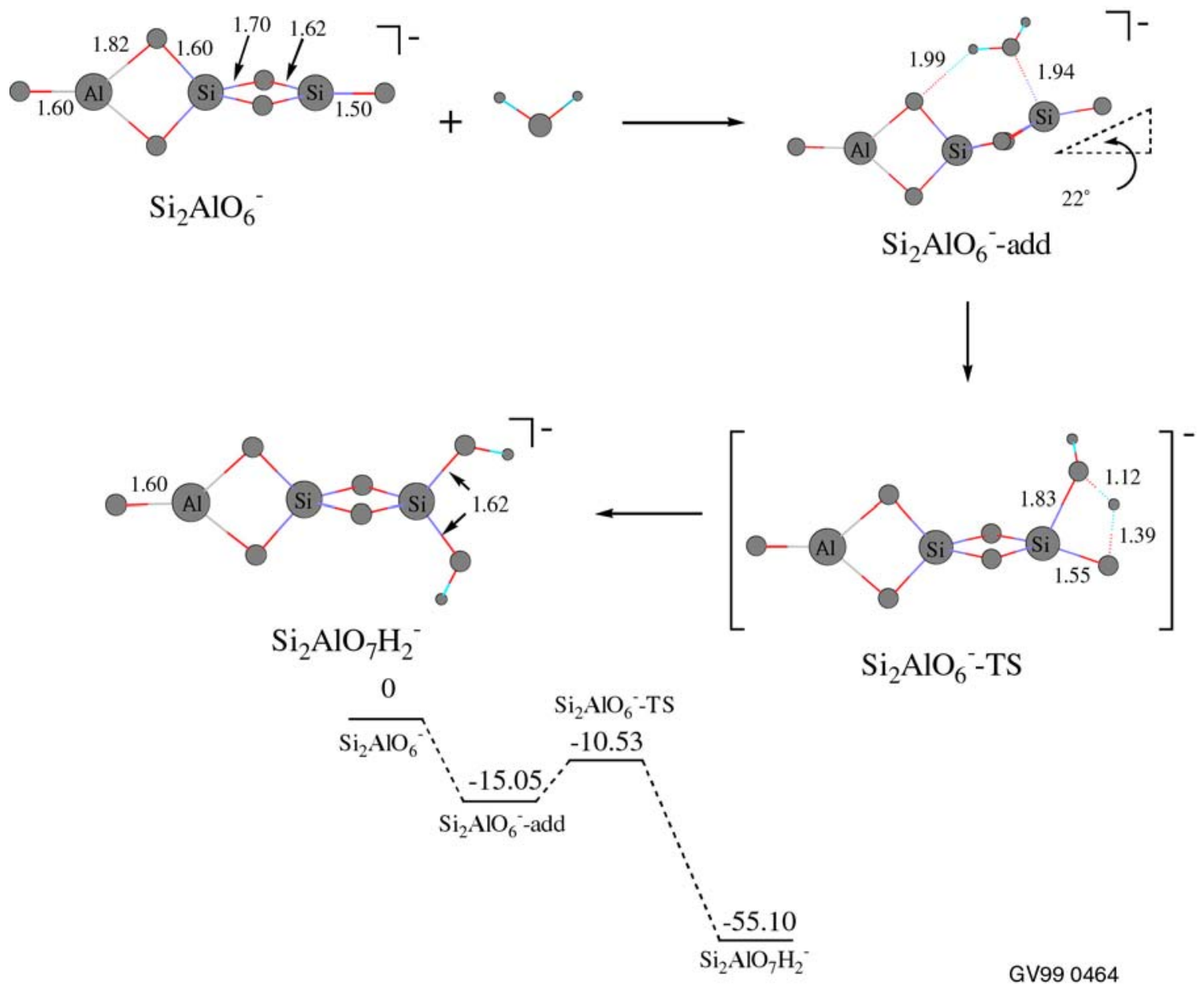

Figure 20. Reaction of $\mathrm{AlSi}_{2} \mathrm{O}_{6}{ }^{-}$structure 1 (Figure 19), with $\mathrm{H}_{2} \mathrm{O}$. 


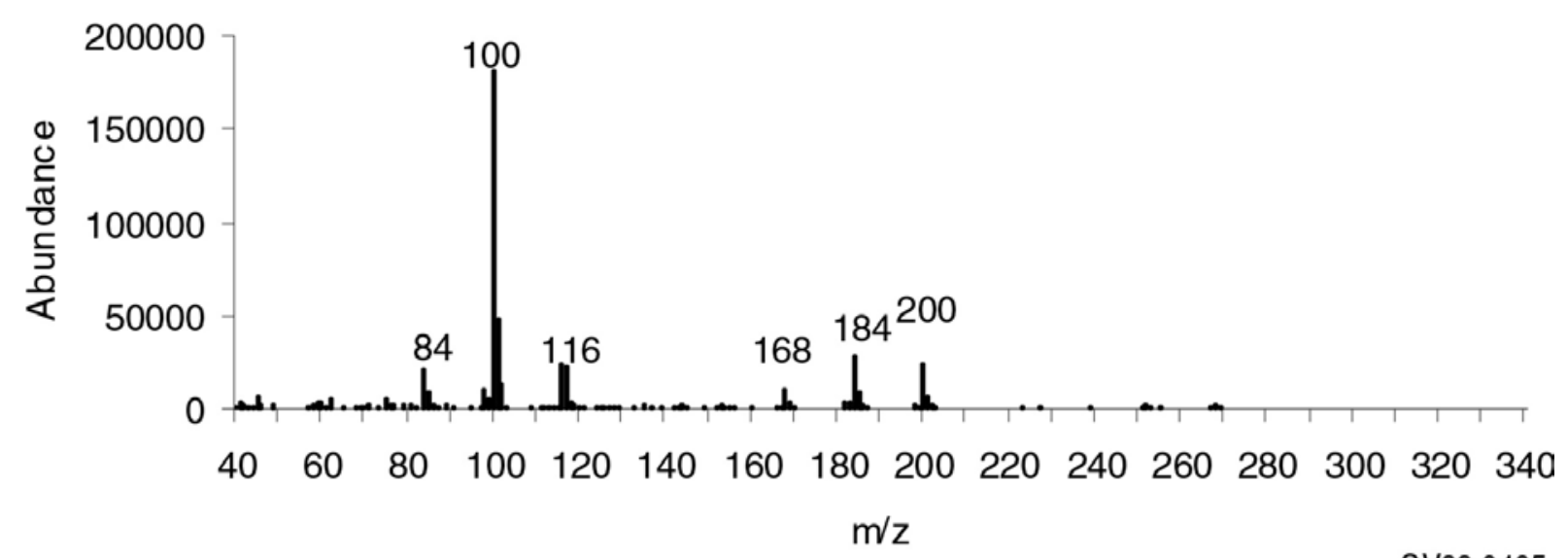

GV99 0465

Figure 21. Anion spectrum of $\mathrm{K}_{2} \mathrm{Cr}_{2} \mathrm{O}_{7}$, acquired using a low pressure SIMS instrument.

Table 4. Masses, compositions, and Cr oxidation states of ions formed from SIMS analysis of Cr oxide species.

\begin{tabular}{rcllll}
\hline $\mathrm{M} / \mathrm{z}$ & Composition & Cr Oxidation States & $\mathrm{M} / \mathrm{z}$ & \multicolumn{2}{c}{ Composition Cr Oxidation States } \\
\hline 84 & $\mathrm{CrO}_{2}^{-}$ & $\mathrm{III}$ & 168 & $\mathrm{Cr}_{2} \mathrm{O}_{4}^{-}$ & III, IV \\
100 & $\mathrm{CrO}_{3}^{-}$ & V & 184 & $\mathrm{Cr}_{2} \mathrm{O}_{5}^{-}$ & IV, V \\
101 & $\mathrm{HCrO}_{3}^{-}$ & $\mathrm{IV}$ & 200 & $\mathrm{Cr}_{2} \mathrm{O}_{6}^{-}$ & V, VI \\
116 & $\mathrm{CrO}_{4}^{-}$ & VI, radical species & 252 & $\mathrm{Cr}_{3} \mathrm{O}_{6}^{-}$ & III, IV, IV \\
117 & $\mathrm{HCrO}_{4}^{-}$ & VI & 268 & $\mathrm{Cr}_{3} \mathrm{O}_{7}^{-}$ & IV, IV, V \\
& & & 284 & $\mathrm{Cr}_{3} \mathrm{O}_{8}^{-}$ & V, V, V \\
\hline
\end{tabular}

accomplished by observation of $\mathrm{M}_{3} \mathrm{CrO}_{\mathrm{x}=3,4}{ }^{+}$species in the cation spectra. These were particularly pronounced in the case of $\mathrm{Cs}$, which generated very abundant $\mathrm{Cs}_{3} \mathrm{CrO}_{\mathrm{x}=3,4}{ }^{+}$ions. The tendency of $\mathrm{Cs}^{+}$to form complexes with these species appeared to shut off anion formation channels, because only a modestly abundant $\mathrm{CrO}_{3}{ }^{-}$peak is observed in the anion spectrum. The result suggests that $\mathrm{Cs}^{+}$doping combined with SIMS may constitute an approach for $\mathrm{Cr}(\mathrm{VI})$ differentiation on the surface.

Several other $\mathrm{Cr}$ salts were also interrogated. When $\mathrm{Cr}_{2} \mathrm{O}_{3}(\mathrm{Cr}(\mathrm{III}))$ was analyzed, no $\mathrm{Cr}$ ions could be identified in either the positive or negative spectrum. This reluctance to produce significant secondary ions has been observed in other non-ionic metal oxides. $\mathrm{Cr}\left(\mathrm{NO}_{3}\right)_{3}$, on the other hand, produced abundant $\mathrm{Cr}_{\mathrm{x}} \mathrm{O}_{\mathrm{y}}{ }^{-}$species identical to those described in Table 4. This indicates that metal reduction processes must be operative in this compound also, and that the $\mathrm{NO}_{3}{ }^{-}$ligand is decomposing and leaving $\mathrm{O}$ attached to the $\mathrm{Cr}$ metal. The behavior of $\mathrm{Cr}\left(\mathrm{NO}_{3}\right)_{3}$ represents a problem, because it will obfuscate species and oxidation state identification in cases where either chromate, dichromate or nitrate salts are present. Finally, $\mathrm{Cr}(\mathrm{III})$ chloride was analyzed, and found to generate abundant $\mathrm{CrCl}_{3}{ }^{-}$, which represents reduction of $\mathrm{Cr}(\mathrm{III})$ to $\mathrm{Cr}(\mathrm{II})$, and permits easy identification. 


\section{Laser Desorption Fourier Transform Mass Spectrometer: Design and Fabrication}

A laser desorption (LD) FTMS instrument was designed and fabricated. The motivation for the instrument development effort was several fold:

- $\quad$ Overcome ion identification problems using high resolution, high accuracy mass measurement for assignment of elemental composition. This would permit distinction of compositions (for example) containing $\mathrm{Al}+\mathrm{H}$ from those containing $\mathrm{Si}$.

- $\quad$ Overcome mass limitations of the current IT-SIMS and quadrupole (i.e., low pressure) SIMS instruments. Current mass ranges are in the $600 \mathrm{amu}$ regime, which is not sufficient to investigate larger oligomers which are likely to be most representative of the surface. The expanded mass range will also enable the investigation of biomolecules on oxide surfaces.

- Implement and evaluate high energy LD as a complement, or perhaps replacement, for secondary ion mass spectrometry. It is known that in several cases LD is capable of generating ions that have not, to date, been generated using SIMS. It is intended that the FTMS instrument be equipped with both LD and SIMS capability, which will permit direct comparison of the two techniques.

- Improve control of atmosphere in the vacuum environment. Current instrumentation is capable of only rudimentary control of reactant and buffer gases. FTMS instrumentation will implement pulsed gas inlets for fast pressure rise events and rapid pump out.

- Implement nondestructive ion detection, which will enable ion mass remeasurement, which will improve signal-to-noise and detectability. This will also enable $\mathrm{MS}^{\mathrm{n}}$ experiments to be performed on the same ion population.

The FTMS (Figure 22) is composed of four main parts: a superconducting magnet, vacuum system, data acquisition/control system, and ionization source(s). There were three main goals in the design of the FTMS at INEEL: 1) incorporate a high enough magnetic field to ensure high resolution, mass accuracy ( $<10 \mathrm{ppm})$, and wide $\mathrm{m} / \mathrm{z}$ range, 2 ) utilize an oil-free vacuum system to maintain pristine samples and enable operation over a range of pressures $\left(10^{-4}\right.$ to $10^{-9}$ torr $)$ to facilitate reaction-pathway studies, and 3) include a variety of ionization methods, including LD.

The FTMS employs a 7 Tesla superconducting magnet (Oxford Instruments, UK). The $7 \mathrm{~T}$ superconducting magnet provides high resolution, high mass accuracy $(<10 \mathrm{ppm})$, and an effective massto-charge $(\mathrm{m} / \mathrm{z})$ range of $\mathrm{m} / \mathrm{z} 20-20,000$. The data acquisition and control system was acquired from Finnigan FT/MS (Madison, WI) and uses Odyssey software version 4.1.1 (Finnigan FT/MS, Madison, WI). The nondestructive detection of the ions is accomplished by monitoring image currents induced on the detection plates of the cell by the cyclotron motion of the ions. Because the detection is nondestructive, $\mathrm{MS}^{\mathrm{n}}$ can be performed on the same ion population. This system provides control over a variety of options such as pulsed valves and auxiliary electronics, which facilitate implementation of multifaceted $\mathrm{MS}^{\mathrm{n}}$ experiments for the study of complex gas-phase reactions.

The FTMS instrument utilizes two CT-8 cryogenic pumps (CTI-Cryogenics, Waltham, MA) for the vacuum chamber and a dry scroll pump (300DS, Varian, Lexington, MA) for the sample inlet port. Cryopumps were chosen because they provide oil-free pumping and very low pressures. An oil-free vacuum environment is necessary to avoid hydrocarbon contamination of samples; contamination is common in vacuum chambers that utilize oil roughing pumps to evacuate sample inlet systems or to back 


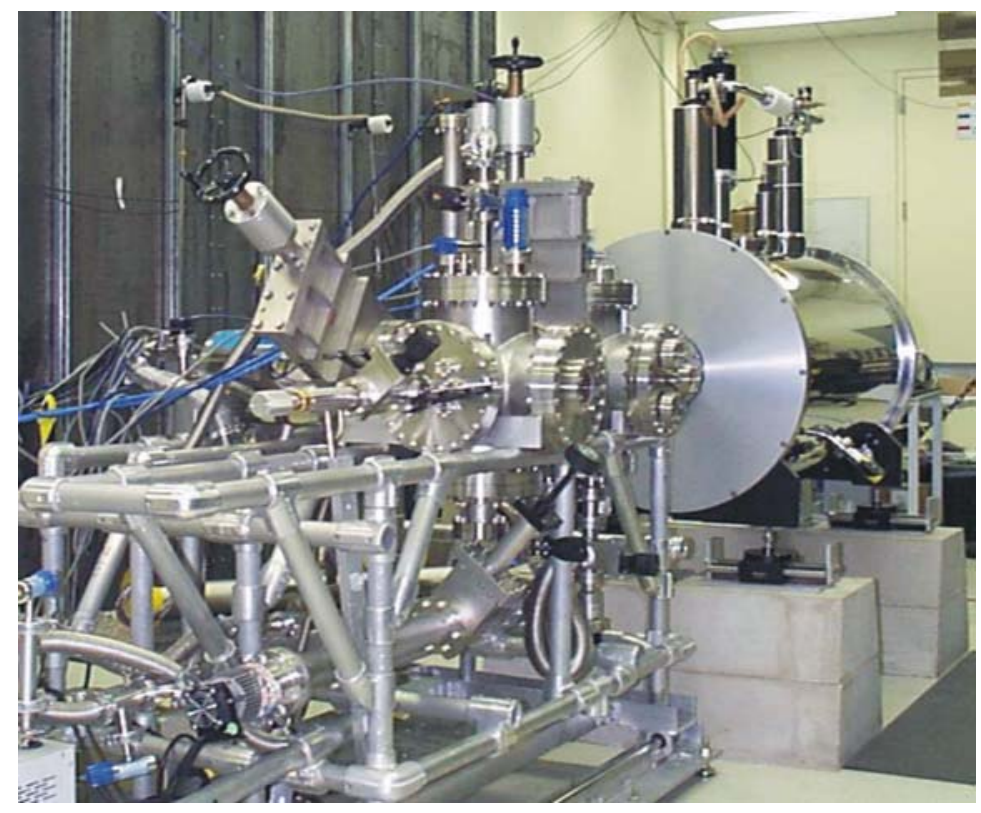

Figure 22. Photograph of FTMS instrument. Sample probe and vacuum system are shown in the foreground, 7 Tesla superconducting magnet in background.

turbo pumps. Contamination on samples can cause ionization of the actual sample to be inefficient, obscure signals of interest with chemical noise, and can permit undesired reactions to occur with the sample. The pressure range for operation of the cryopumps is from $10^{-3}$ to $10^{-11}$ torr. Currently, the FTMS base pressure is $8 \times 10^{-10}$ torr. Incorporation of UV lamps into the vacuum chamber will provide a more efficient method for baking out the system, which should allow pressures in the $10^{-11}$ torr range to be obtained.

An important goal for the FTMS is incorporation of multiple ionization sources that include LD, atomic particle beam $\left(\mathrm{Cs}^{+}\right)$, polyatomic particle beam $\left(\mathrm{ReO}_{4}{ }^{-}\right)$, and electron impact. Ideally, two or more ionization techniques will be simultaneously available for sample analysis. This will allow direct comparison of different ionization techniques on the same sample, so that a direct comparison of ionization methods for a given type of sample type is possible and straightforward. Currently, the choice of ionization technique requires interpretation of published literature. This approach is inherently flawed because there are too many variables. For example, sample preparation often varies between literature reports. Additionally, the literature reports not only utilize different ionization techniques, but also different mass spectrometers, which introduces variable conditions such as pressure and cleanliness of the vacuum chamber (e.g., sample contamination occurring in systems with oil roughing pumps). To overcome these problems, design of custom FTMS cells that will allow multiple ionization techniques to be applied to the same sample is currently being pursued. An example of a first generation, custom FTMS cell with a particle beam gun mounted on movable doors is pictured in Figure 23. Final design of the particle beam sources and door manipulators is currently underway. The FTMS system is designed to always have laser desorption available for sample analysis. A Nd:YAG (Continuum, Santa Clara, CA), which is capable of operating at wavelengths of $1064,532,355$, and $266 \mathrm{~nm}$ has been incorporated into the FTMS system. Utilization of the four wavelengths that span the UV (266 nm) to IR (1064 nm) will allow the wavelength dependence of sample analysis to be studied. 


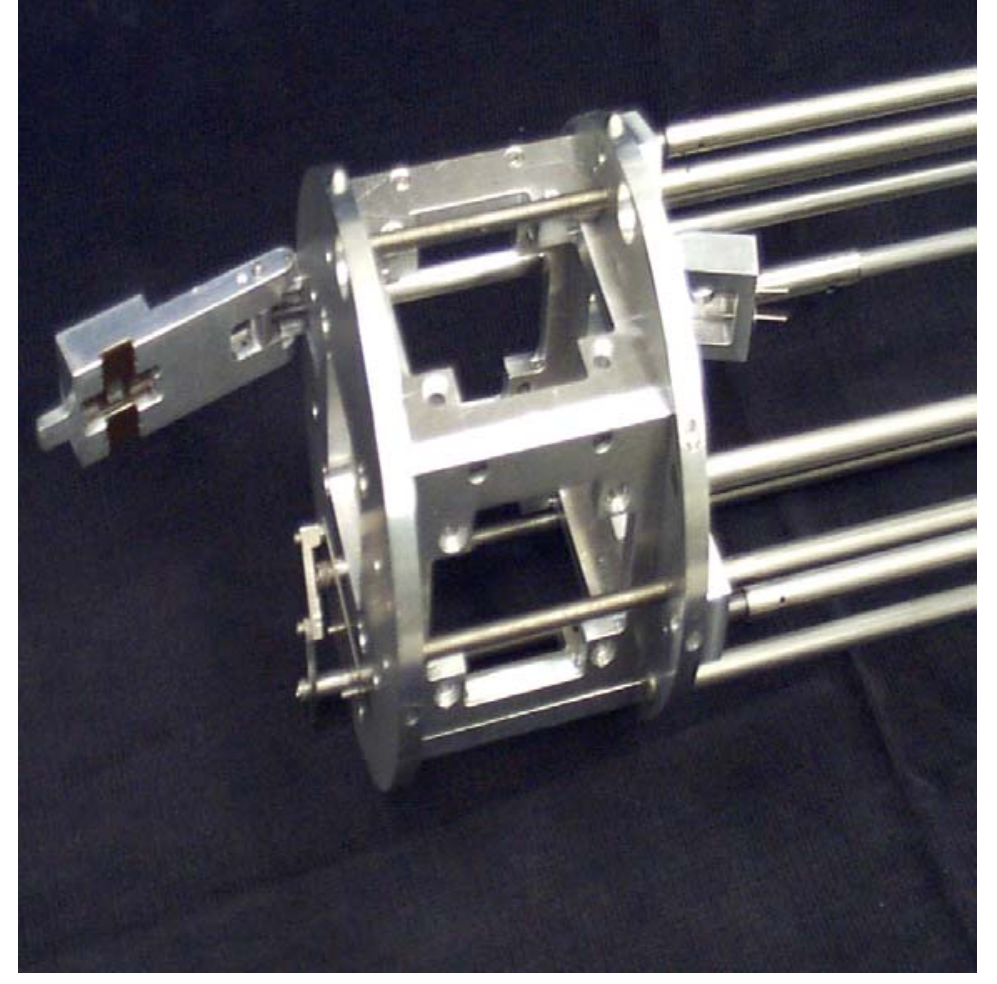

Figure 23. Two-inch cubic FTMS Cell.

The primary motivation for developing the FTMS is to move beyond the limitations of the ITMS. Typical spectra from the FTMS and the ITMS are shown in Figure 24. The resolution for the ITMS spectrum (Figure 24a) is less than 500 as compared to a resolution of 35,700 for the FTMS spectrum (Figure 24b). The observed mass of $\mathrm{Au}^{-}$in the FTMS spectrum is $196.966043 \mathrm{Da}$, which compares very well with the theoretical mass of $\mathrm{Au}^{-}$of $196.965994 \mathrm{Da}$. The resulting mass accuracy is $0.25 \mathrm{ppm}$. The resolution and mass accuracy of the FTMS are more than sufficient to provide unambiguous identification.

\section{ACCOMPLISHMENTS}

The Advanced Strategies Program has developed a new approach for studying reactions of adsorbates with oxides. The approach enjoys the advantage that it allows reaction pathways and kinetics to be measured directly; consequently, the fundamental reactions of adsorbate molecules with oxide moieties can be evaluated. Reactive moieties identified in this manner can be correlated with adsorption or degradation observed on meso- and macro-scales.

The approach for studying reactions of adsorbates with oxides is based on sputter desorption of ionic oxide moieties into the gas phase, where the are trapped and reacted with adsorbates in an ion trap secondary ion mass spectrometer (IT-SIMS). Reaction pathway and kinetic studies are complemented with $a b$ initio calculations, which provide structural and thermodynamic information. This combined strategy has been applied to the study of aluminum, silicon, and aluminosilicate oxyanions with the adsorbate water. The study is relevant because water adsorption is perhaps the most fundamental of environmental adsorption reactions, is ubiquitous, and hence must be understood before studies of more complex systems (i.e., contaminant adsorbates) are undertaken. It was found that water dissociatively adds to the oxyanions of $\mathrm{Al}, \mathrm{Si}$, and mixed $\mathrm{Al}+\mathrm{Si}$ systems. Multiple metal/metalloid atoms are required for the reaction to proceed efficiently; put another way, monomeric oxyanions are relatively unreactive, but oligomeric oxyanions are highly reactive. The research strongly suggests that dangling oxygen atoms are responsible for the oxyanion reactivity. The research resulted in the generation of four presentations at the National Mass Spectrometry meeting in Dallas, which are being written as full journal articles. 

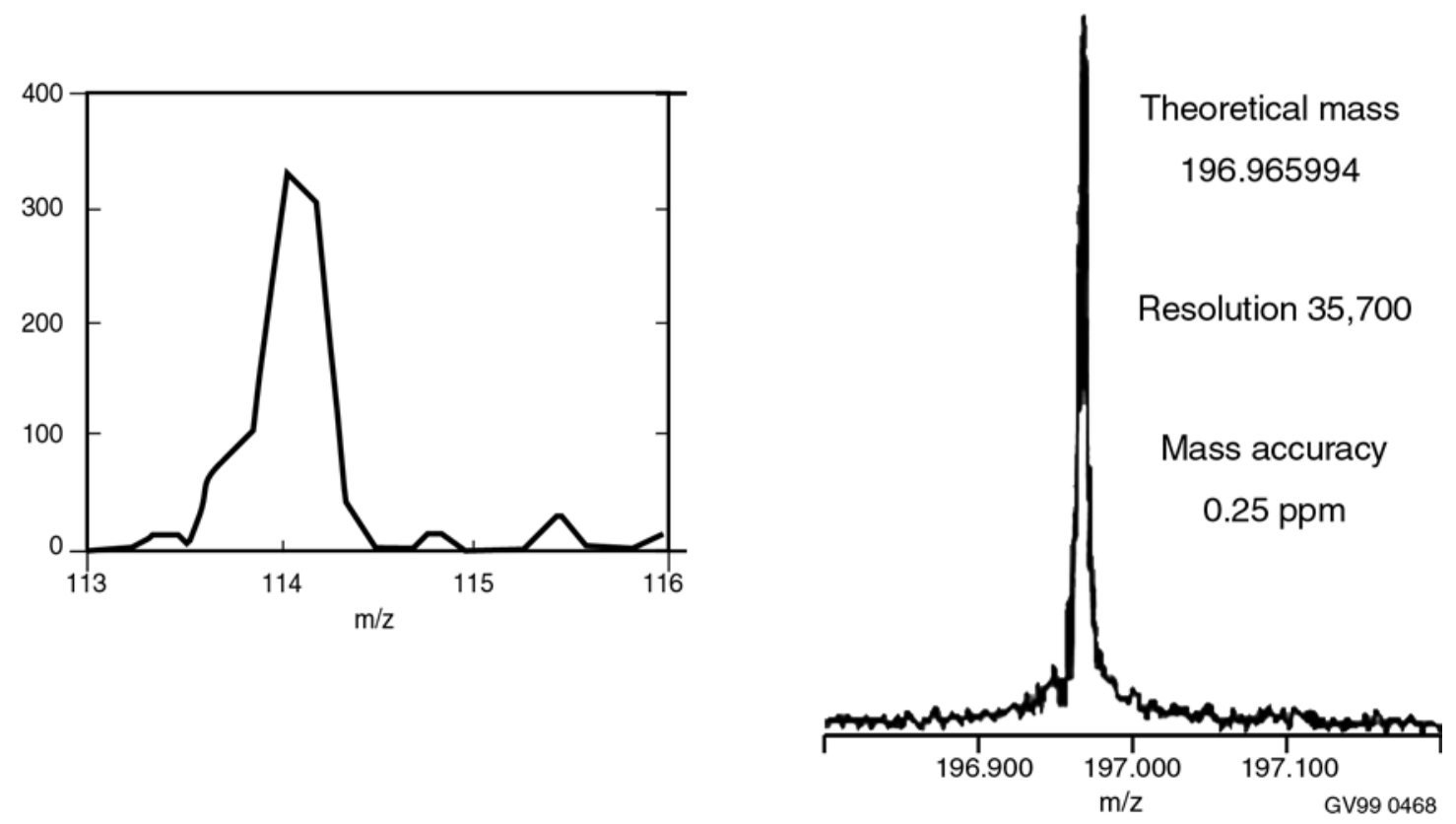

Figure 24. Comparison of mass resolution achievable, using the IT-SIMS (left), and the FTMS (right). The FTMS spectrum was acquired using laser desorption, in the anion mode; the ion displayed is $\mathrm{Au}^{-}$.

The approach for studying reactions of adsorbates with oxides is applicable to a wide range of systems: any oxide which can be desorbed as an ion, can be reacted with any adsorbate that can be volatilized. Initial studies showed that chromium oxide species can be desorbed, which contain a variety of oxidation states. These can be trapped and reacted with adsorbate molecules.

A specific goal for the oxide surfaces is to gain an understanding of their reactivity with toxic adsorbates. Hence, the direction of the oxide research will move toward understanding interactions of chlorinated organic species.

It is widely recognized that many, if not most environmentally significant interactions (e.g., sequestration, degradation), involve microorganisms acting on contaminant molecules or surfaces. These interactions occur either directly through microbial action, or involve participation of excreted biomolecules. Thus an instrumental approach capable of investigating these types of systems is desired. A combined LD / secondary ion MS / FTMS instrument has been designed and completed, to the extent that LD and FTMS capability have been demonstrated. Initial studies have demonstrated the ability of the instrument to perform LD and trapping of metal cations and anions. Superior mass measurement accuracy and mass resolution were demonstrated; these are capabilities that have obvious applications to the $\mathrm{Al}$ and $\mathrm{Si}$ oxide research. The implementation of a 7 Tesla superconducting magnet will enable the analysis of singly charged ions to a mass of approximately $2 \times 10^{4} \mathrm{amu}$. This mass range encompasses a wide range of biologically significant molecules, which opens a new realm of biogeochemical interactions to surface and reactivity study. 


\section{REFERENCES}

1. W. C. Mackrodt, J. Chem. Soc., Faraday Trans., 2, 1989, 85, 541.

2. P. W. Tasker, Adv. Ceram., 1988, 10, 176.

3. "Basic Research for Environmental Restoration," U.S. Department of Energy, Office of Energy Research, Report DOE/ER-0482-T, December, 1990, p. 43.

4. J. A. Davis and K. F. Hayes, "Geochemical Processes at Mineral Surfaces: An Overview," in Geochemical Processes at Mineral Surfaces, Davis, J. A.; Hayes, K. F., eds., ACS Symposium Series 323, American Chemical Society, Washington, D. C., 1986, p. 2-18.

5. M. Kriegman-King and M. Reinhard, "Abiotic Transformation of Carbon Tetrachloride at Mineral Surfaces,” U.S. Environmental Protection Agency Report EPA/60/SR-94-018, March, 1994.

6. R. C. Knox and D. A. Sabatini, in Transport and Remediation of Subsurface Contaminants, D. A. Sabatini and R. C. Knox, eds., ACS Symposium Series 491, American Chemical Society, Washington, D.C., 1992, pp. 1-11.

7. S. A. Lorenz, E. P. Maziarz III, and T. D. Wood, Appl. Spectrosc., 1999, 53, 18A-36A.

8. P. E. Lafargue, J. J. Gaumet, and J. F. Muller, Chem. Phys. Lett., 1998, 288, 494-8.

9. P. E. Lafargue, J. J. Gaumet, J. F. Muller, and A. Labrosse, J. Mass Spectrom., 1996, 31, 623-32.

10. A. Pramann and K. Rademann, Int. J. Mass Spectrom. Ion Proc., 1999, 185/186/187, 673-83.

11. A. Thierry de Ville d'Avray, E. E. Carpenter, C. J. O'Connor, and R. B. Cole, Eur. Mass Spectrom., 1998, 4, 441-9.

12. E. A. Michiels, A. Celis, and R. Gijbels, Int. J. Mass Spectrom. Ion Phys., 1983, 47, 23-26.

13. J. Perez, C. J. Petzold, M. A. Watkins, W. E. Vaughn, and H. I. Kenttamaa, J. Amer. Soc. Mass Spectrom., 1999, 11, 1105-1110.

14. J. Liu, C. Han, W. Zheng, Z. Gao, and Q. Zhu, Int. J. Mass Spectrom., 1999, 189, 147-56.

15. G. S. Groenewold, J. C. Ingram, J. E. Delmore, A. D. Appelhans, and D. A. Dahl, J. Haz. Mats., 1995, 41, 359-70.

16. J. M. Zachara and J. P. McKinley, Aquatic Sciences 1993, 55, 250.

17. J. F. Kaminsky and A. H. Wylie, Ground Water Monitoring \& Remediation, 1995, 15, 97-103.

18. Hanford Site Sampling and Analysis Data Document, Report EGG-ES-7953, U.S. Department of Energy: Washington, D.C., August, 1988.

19. "Environmental Management Research and Development Plan, Solution-Based Investments in Science and Technology," United States Department of Energy, draft report, August 28, 1998. 
20. G. S. Groenewold, J. E. Delmore, J. E. Olson, A. D. Appelhans, J. C. Ingram, and D. A. Dahl, Int. J. Mass Spectrom. Ion Proc., 1997, 163, 185-95.

21. J. F. J. Todd, in "Practical Aspects of Ion Trap Mass Spectrometry," Vol. 1, R. E. March and J. F. J. Todd, eds., CRC Press, New York, 1995, p. 13.

22. G. S. Groenewold, A. D. Appelhans, and J. C. Ingram, J. Amer. Soc. Mass Spectrom., 1998, 9, $35-41$.

23. G. S. Groenewold, A. D. Appelhans, J. C. Ingram, G. L. Gresham, and A. K. Gianotto, Talanta, 1998, 47, 981-6.

24. J. F. J. Todd, in "Practical Aspects of Ion Trap Mass Spectrometry," Vol. 1, R. E. March, J. F. J. Todd, J. F. J., eds., CRC Press, New York, 1995, p. 13.

25. M. W. Schmidt, K. K. Baldridge, J. A. Boatz, J. H. Jensen, S. Koseki, N. Matsunaga, M. S. Gordon, K. A. Nguyen, S. Su, T. L. Windus, S. T. Elbert, J. Montgomery, and M. Dupuis, J. Comput. Chem., 1993, 14, 1347.

26. C. Møller and M. S. Plesset, Phys. Rev., 1934, 46, 618.

27. G. S. Groenewold, J. C. Ingram, T. McLing, A. K. Gianotto, and R. Avci, "Cs ${ }^{+}$Speciation on Soil Particles by TOF-SIMS Imaging," Anal. Chem. 1998, 70, 534-9.

28. G. S. Groenewold, A. K. Gianotto, J. C. Ingram, and A. D. Appelhans, "Analysis of Environmental Surfaces using Static Secondary Ion Mass Spectrometry," Current Topics in Analytical Chemistry, 1998, 1, 73-91.

29. G. Innorta, S. Torroni, A. Foffani, L. Pontoni, J. Mass Spectrom., 1995, 30, 1633-5.

30. L. Operti, M. Splendore, G. A. Vaglio, A. M. Franklin, and J. F. J. Todd, Int. J. Mass Spectrom. Ion Proc., 1994, 136, 25-33.

31. G. Centini, L. Operti, R. Rabezzana, G. A. Vaglio, and P. Volpe, J. Organomet. Chem., 1996, 519, 169-75.

32. J. F. Gal, R. Grover, P. C. Maria, L. Operti, R. Rabezzana, G. A. Vaglio, and P. Volpe, J. Phys. Chem., 1994, 98, 11978-87.

33. M. E. Farmer and R. W. Linton, Environ. Sci. Technol., 1984, 18, 319-26.

34. R. Van Grieken and C. Xhoffer, J. Anal. Atom. Spectrom., 1992, 7, 81-8.

35. T. R. Keyser, D. F. S. Natusch, C. A. Evans, Jr., and R. W. Linton, Environ. Sci. Technol., 1978, 12, 768-73.

36. M. E. Farmer and R. W. Linton, Environ. Sci. Technol., 1984, 18, 319-26

37. T. M. Williams, Environmental Geology, 1993, 21, 62-69

38. L. J. Evans, Environ. Sci. Technol., 1989, 23, 1046-56 
39. “The Static SIMS Library,” Surface Spectra Ltd., Manchester, UK: 1999, p. 1:3.13.

40. G. S. Groenewold, A. D. Appelhans, and J. C. Ingram, "Characterizations of bis(alkylamine)mercury Cations from Mercury Nitrate Surfaces using an Ion Trap SIMS," J. Amer. Soc. Mass Spectrom. 1998, 9, 35-41.

41. M. A. Johnson, E. V. Stefanovich, T. N. Truong, J. Gunster, and D. W. Goodman, J. Phys. Chem. $B, \mathbf{1 9 9 9}, 103,3391-8$

42. J. M. Wittbrodt, W. L. Hase, and H. B. Schlegel, J. Phys. Chem. B, 1998, 102, 6539-48.

43. E. V. Stefanovich and T. N. Truong, Chem. Phys. Lett., 1999, 299, 623-9.

44. M. A. Johnson, E. V. Stefanovich, and T. N. Truong, J. Phys. Chem. B, 1998, 102, 6391-6.

45. T. Su and M. T. Bowers, Int. J. Mass Spectrom. Ion Proc., 1975, 17, 211-2.

46. “The Static SIMS Library,” Surface Spectra Ltd., Manchester, UK: 1999, p. 1:3.3.

47. X. Zhang and J. A. Koropchak, Anal. Chem., 1999, 71, 3046-53.

48. M. Derbyshire, A. Lamberty, and P. H. E. Gardiner, Anal. Chem., 1999, 71, 4203-7.

49. "Environmental Management Research and Development Program Plan, Solution-Based Investments in Science and Technology, United States Department of Energy Draft Report, August 28, 1998.

50. "Groundwater/Vadose Zone Integration Project Specification,” Report DOE/RL-98-48 Draft C, United States Department of Energy, December 17, 1998.

51. M. Soma, H. Seyama, and K. Okamoto, Talanta, 1985, 32, 177-81.

52. M. H. Koppelman, and J. G. Dillard, ACS Symp. Ser., 1975, 18, 186-201.

53. M. E. Farmer and R. W. Linton, Environ. Sci. Technol., 1984, 18, 319-26.

54. L. J. Evans, Environ. Sci. Technol., 1989, 23, 1046-56.

55. K. Poels, L. Van Vaeck, and R. Gijbels, Anal. Chem., 1998, 70, 504-12.

56. K. R. Neubauer, M. V. Johnston, and A. S. Wexler, Int. J. Mass Spectrom. Ion Proc., 1995, 151, $77-87$.

\section{APPENDIX}

\section{Presentations}

A. K. Gianotto, Groenewold, J. R. Scott, G. F. Kessinger, M. T. Benson, J. B. Wright, "Condensation Reactions of Aluminosilicate Oxyanions With Water," Proceedings of the $47^{\text {th }}$ American Society Mass Spectrometry Conference on Mass Spectrometry and Allied Topics, Dallas, Texas, June 13-17, 1999. 
J. R. Scott, G. S. Groenewold, A. K. Gianotto, G. F. Kessinger, M. T. Benson, J. B. Wright, "Reactions of Gas-Phase $\mathrm{Al}_{\mathrm{x}} \mathrm{O}_{\mathrm{y}}{ }^{-}$and $\mathrm{Si}_{\mathrm{x}} \mathrm{O}_{\mathrm{y}}{ }^{-}$Anions With Water," Proceedings of the $47^{\text {th }}$ American Society Mass Spectrometry Conference on Mass Spectrometry and Allied Topics, Dallas, Texas, June 13-17, 1999.

G. S. Groenewold, G.F. Kessinger, J. E. Delmore, G. L. Gresham, A. Shaw, "Enhancement of SIMS-Generated Oligomeric Oxyanions from Refractory Oxide Surfaces Using Cs ${ }^{+}$Substitution," Proceedings of the $47^{\text {th }}$ American Society Mass Spectrometry Conference on Mass Spectrometry and Allied Topics, Dallas, Texas, June 13-17, 1999.

G. L. Gresham, G. S. Groenewold, J. E. Olson, A. D. Appelhans, "Formation of Alkali Halide Cluster Ions and Assessment of Their Stability," Proceedings of the $47^{\text {th }}$ American Society Mass Spectrometry Conference on Mass Spectrometry and Allied Topics, Dallas, Texas, June 13-17, 1999.

G. S. Groenewold, A. D. Appelhans, and J. C. Ingram, "Characterization of bis(alkylamine)mercury Cations from Mercury Nitrate surfaces using an Ion Trap SIMS," J. Amer. Soc. Mass Spectrom. 1998, 9 , $35-41$. 


\title{
Biochemical and Geochemical Reactions on Environmental Surfaces
}

\author{
William F. Bauer, George D. Redden, Joni M. Barnes, Gracy Elias, and Peter A. Pryfogle \\ SUMMARY
}

In response to an assessment of Department of Energy Environmental Management (DOE EM) research needs, a project has been developed to examine the importance of geo-chemical and bio-chemical reactions at environmental surfaces. The environmental surfaces being studied are minerals common to DOE subsurface environments. The program has progressed upon three parallel lines, namely surface chemistry associated with biological activity, methods to identify microbial heterogeneity on mineralogically heterogeneous surfaces, and the direct chemical interaction of contaminant species with other chemical species and mineral surfaces. The results of the program will be relevant to understanding how these chemical and spatial variations affect the transport and fate of many different contaminant species of concern to DOE. Since iron oxides are among the most prevalent minerals that significantly influence subsurface chemistry, they have been selected as the primary mineral surface to be studied initially. Some specific accomplishments include: (1) improvements to a procedure to measure intracellular $\mathrm{pH}$ variations of microbes in direct contact with mineral surfaces and in biofilms covering a mineral surface, (2) tentative identification of extracellular compounds that dissimilatory iron-reducing bacteria may produce to solubilize and reduce highly insoluble iron from iron oxide surfaces, (3) completion of studies using extended X-ray absorption fine structure spectroscopy (EXAFS) and attenuated total reflectance Fourier transform infrared spectroscopy (ATR-FTIR) to determine the effect of the multidentate ligand citrate upon the interaction of uranyl ions with geothite (FeOOH) surfaces, and (4) assembling the instrumentation for and conducting some initial studies to develop spectroscopic and spectroscopic imaging techniques to identify microbial phylotypes in suspension and on surfaces using fluorescent in situ hybridization.

\section{PROJECT DESCRIPTION}

\section{Introduction}

An assessment of DOE EM research needs ${ }^{1,2}$ has identified many problems that are related, either directly or indirectly, to interactions of chemical species with surfaces. At almost all DOE sites, contamination by toxic and radioactive chemicals is a problem. The contamination issues are mainly associated with the many years of use and subsequent disposal of organic chemicals, radionuclides, and other metals related to processing nuclear fuel and related materials. Surface chemistry plays a very important role in the fate of these contaminants.

At the Idaho National Engineering and Environmental Laboratory (INEEL), contamination issues associated with the subsurface environment are primarily associated with the presence of the radionuclides, including ${ }^{90} \mathrm{Sr},{ }^{3} \mathrm{H},{ }^{137} \mathrm{Cs},{ }^{234} \mathrm{U}$, heavy metals, and chlorinated solvents. Many of these contaminants reached the subsurface following their disposal in infiltration ponds and injection wells. ${ }^{3}$ Recently, ${ }^{241} \mathrm{Am}$ and isotopes of Pu have been detected in the subsurface surrounding the burial ground at the Radioactive Waste Management Complex (RWMC). ${ }^{4}$ At the Idaho Chemical Processing Plant (ICPP), renamed Idaho Nuclear Technology Engineering Center (INTEC), low-level radioactive waste was discharged directly to the Snake River Plain Aquifer via an injection well resulting in very low contamination levels of ${ }^{99} \mathrm{Tc},{ }^{236} \mathrm{U},{ }^{237} \mathrm{~Np},{ }^{3} \mathrm{H},{ }^{36} \mathrm{Cl},{ }^{90} \mathrm{Sr},{ }^{137} \mathrm{Cs},{ }^{129} \mathrm{I}$, and isotopes of $\mathrm{Pu} .{ }^{5}$ Some of these 
radioactive contaminants are transported freely in the aquifer and others like I, $\mathrm{U}$, and $\mathrm{Np}$ are apparently retarded by interactions with the subsurface mineralogy.

The subsurface environment at the INEEL is quite complex as it consists of basaltic lava flows interbedded with terrestrial sediment layers. The basaltic lava flows have an average thickness of 6-8 $\mathrm{m}$ with an aggregate thickness of up to $1,000 \mathrm{~m}$ or more. There are as many as 38 major basalt flows interbedded with as many as 15 major sediment horizons from $3-10 \mathrm{~m}$ thick. ${ }^{6}$ The sediments range from gravel and sand in alluvial deposits to silts and clays where streams were damned by basalt flows. ${ }^{3}$ The basalt flows generally fractured during cooling, thus creating channels. Rubble zones are also created at the edges and beneath the flows. The basalt flows and interbedded sediment layers under the INEEL are a portion of the Snake River Plain Aquifer, which is the main source of water for agricultural and domestic uses on the plain.

Basalt is generally a dark-colored, fine-grained rock consisting primarily of a groundmass of labradorite feldspar with other plagioclase $\left(\mathrm{NaAlSi}_{3} \mathrm{O}_{8}\right.$ to $\left.\mathrm{CaAl}_{2} \mathrm{Si}_{2} \mathrm{O}_{8}\right)$ phases present as phenocrysts. ${ }^{7}$ Augite (a pyroxene of the general formula $\left.(\mathrm{Ca}, \mathrm{Na})(\mathrm{Mg}, \mathrm{Fe}, \mathrm{Al})(\mathrm{Si}, \mathrm{Al})_{2} \mathrm{O}_{6}\right)$ and olivine $\left((\mathrm{Mg}, \mathrm{Fe}) \mathrm{SiO}_{4}\right)$ are also found as phenocrysts. Augite can also be found as part of the groundmass. Some glass may be present in the groundmass as well. Magnetite $\left(\mathrm{Fe}_{3} \mathrm{O}_{4}\right)$ and ilmenite $(\mathrm{FeTiO})$ are also found in basalts common to the INEEL. ${ }^{8}$ The tops of basalt flows commonly have enough gas cavities to be vesicular, creating areas of very high surface area. Rubble zones at the edges and beneath the flows also have a very large available surface area.

The mineralogy of the interbedded sediment layers separating the basalt flows at the INEEL has been examined. ${ }^{9,10}$ It is expected that the source of much of these sediments would arise from the Big Lost River, Little Lost River, and Birch Creek drainages, which drain the Pioneer, White Knob, Lost River, Lemhi, and Bitterroot ranges. Quartz $\left(\mathrm{SiO}_{2}\right)$, plagioclase feldspar, potassium feldspar, and clays are the most common minerals comprising the sediment interbeds. Calcite $\left(\mathrm{CaCO}_{3}\right)$ is also abundant, particularly in the Birch Creek basin where it comprises $>70 \%$ of the sediment in many of the samples. Pyroxene was also found in many of the samples. Smectite, illite, and kaolinite $\left(\mathrm{Al}_{2} \mathrm{Si}_{2} \mathrm{O}_{5}(\mathrm{OH})_{4}\right)$ were the most abundant clay minerals found.

Other DOE sites have significant subsurface contamination problems as well. The behavior of the contaminants in the subsurface will be dictated by the mineralogy of these sites. At Hanford, the subsurface consists primarily of sediments of coarse to fine grained sand and gravel. ${ }^{11}$ These sediments contain many iron-bearing minerals such as illemenite, magnetite, pyroxene, amphiboles, and basaltic glass. Calcium carbonate is also quite prevalent and responsible for the majority of the buffering capacity. At Savannah River, the subsurface consists primarily of iron oxide coated clays.

Because of this wide variety of different mineral surfaces that exist in the DOE subsurface environments, the number and kinds of contaminants that may interact with them, and the potential for very large surface areas, there are a large number of possible surface interactions that can occur to varying degrees within small volumes. Absorption, adsorption, and chemical reactions will change as the surfaces a contaminant encounters change. The chemical characteristics of the surfaces may also be altered after contact with certain contaminants. Much of the transport/mobility modeling work is performed with only common physical and chemical parameters as inputs, but biogeochemistry is also a very important factor influencing the fate of chemical species in the subsurface environment. Microbes influence redox chemistry, $\mathrm{pH}$, produce chemicals that affect transport properties, mineralize organic chemicals, and affect the solubility of inorganic chemical species. Microbes use minerals and mineral surfaces as nutrient sources or as chemical substrates to facilitate metabolic processes like respiration. 
The presence of iron and iron-bearing minerals is very important in determining the fate of chemical contaminants in subsurface environments as iron is the fourth most abundant element in the earth's crust at 5\%. Reduced iron has been proposed for direct chemical remediation of chlorinated organic compounds by reductive dehalogenation. ${ }^{12-14}$ The general reaction for this process can be described with the following reactions:

$$
\begin{array}{cl}
\mathrm{Fe}^{0} \rightarrow \mathrm{Fe}^{2+}+2 \mathrm{e}^{-} & \mathrm{E}^{0}=-0.441 \mathrm{~V} \\
\mathrm{RX}+2 \mathrm{e}^{-}+\mathrm{H}^{+} \rightarrow \mathrm{RH}+\mathrm{X}^{-} & \mathrm{E}^{0}=0.5-1.5 \mathrm{~V}
\end{array}
$$

$$
\mathrm{RX}+\mathrm{Fe}^{0}+\mathrm{H}^{+} \rightarrow \mathrm{RH}+\mathrm{Fe}^{2+}+\mathrm{X}^{-}
$$

No indication has been given as to the presence or concentration of $\mathrm{Fe}^{2+}$ or $\mathrm{Fe}^{3+}$ in the solution that would confirm the above reaction sequence. However, attempts at reductive dehalogenation under anaerobic conditions with just $\mathrm{Fe}^{2+}$ or $\mathrm{H}_{2}$ in solution did not occur, indicating that direct interaction of the halogenated organic with the iron surface was the predominant pathway for this reaction. ${ }^{12}$

Recent work indicates that $\mathrm{Fe}^{2+}$ bearing minerals such as olivine, wüstite $(\mathrm{FeO})$, and ilmenite can participate in reductive dechlorination under anoxic conditions. ${ }^{15}$ Little or no reaction was noted for $\mathrm{Fe}^{3+}$ bearing minerals. Because of the previous work indicating that $\mathrm{Fe}^{2+}$ in solution did not enhance reductive dehalogenation, it can be concluded that the reductive dehalogenation with highly insoluble $\mathrm{Fe}^{2+}$ minerals is also a surface mediated process. In the case of wüstite, the following reaction equations might describe the pertinent pathway to dehalogenation:

$$
\begin{array}{cl}
2 \mathrm{FeO}+\mathrm{H}_{2} \mathrm{O} \rightarrow \mathrm{Fe}_{2} \mathrm{O}_{3}+2 \mathrm{H}^{+}+2 \mathrm{e}^{-} & \mathrm{E}^{0}=0.271 \mathrm{~V}-0.0591(\mathrm{pH}) \\
\mathrm{RX}+2 \mathrm{e}^{-}+\mathrm{H}^{+} \rightarrow \mathrm{RH}+\mathrm{X}^{-} & \mathrm{E}^{0}=0.5-1.5 \mathrm{~V}
\end{array}
$$$$
2 \mathrm{FeO}+\mathrm{RX}+\mathrm{H}_{2} \mathrm{O} \rightarrow \mathrm{Fe}_{2} \mathrm{O}_{3}+\mathrm{RH}+\mathrm{H}^{+}+\mathrm{X}^{-}
$$

It can be postulated that since the $\mathrm{Fe}^{0}$ surface in the first case was in contact with water that some $\mathrm{FeO}$ or $\mathrm{FeOH}$ terminal groups were present on the surface of the iron and that these groups ultimately played the major part in the reductive dehalogenation process. This implies that significant natural attenuation of halogenated organic contaminants and other contaminants susceptible to reduction by iron ${ }^{16}$ may occur in natural systems where there is a significant presence of $\mathrm{Fe}^{2+}$ bearing minerals.

One important factor that influences the redox chemistry of iron in the subsurface environment is the presence and action of bacteria. Bacteria that can either oxidize or reduce iron are known to exist in natural systems. ${ }^{17}$ Iron-reducing bacteria in subsurface environments have been suggested to play a large role in the formation of $\mathrm{Fe}^{2+}$ mineral deposits ${ }^{18-20}$ and in the general microbial oxidation of organic compounds and hydrogen. ${ }^{21}$ Iron-reducing bacteria also can play significant roles in the fate of chemical contaminants such as some organic compounds, ${ }^{22,23}$ plutonium, technetium, ${ }^{24}$ chromium,$~^{25}$ cobalt,$~^{26}$ and uranium $^{27}$ by using them, either directly or indirectly, as alternative electron acceptors to iron.

Based upon the preceding discussion, it becomes clear that understanding the mechanisms of microbial iron activity and its relationship to other abiotic chemical reactions that occur in subsurface environments is very important to understanding and predicting the chemical fates of many contaminants in the subsurface. Because of this, a program with three basic components and associated tasks has been 
developed to examine some of these relationships. One task is largely focused upon microbiological interactions with mineral surfaces. A second task is designed to develop an analytical tool that may be used to study heterogeneous microbial populations on heterogeneous mineral surfaces. The third task is to examine direct chemical processes that occur at mineral surfaces. This final task will ultimately tie to the first two tasks to examine how specific biological chemicals affect chemistry at environmental surfaces.

\section{Microbial Interactions with Environmental Surfaces}

\section{Objective}

The main objective of this task has been to develop methods to examine the microchemical environments and surface interactions of microbes with mineral surfaces. Iron-reducing bacteria have been selected as a model organism because of their importance in understanding the iron cycle in an environmental system and the resulting effects of their activity in contaminated environments. The subtasks consist of the following:

1. Since microbial iron reduction can be influenced by the $\mathrm{pH}$ and redox potential inside the cell, in the cell membrane, and outside of the cell. Fluorescence spectroscopy, microspectroscopy, and confocal microscopy methods with appropriate fluorescent indicators for $\mathrm{pH}$, redox potential, and possibly metal ions are being developed to attempt to measure these parameters in living cells under specific and changing conditions. Initially, this work is being carried out with bacterial cultures of Shewanella sp., fluorescent probes, and simple $\mathrm{Fe}^{3+}$ bearing oxide surfaces.

2. Atomic force microscopy (AFM) and environmental scanning electron microscopy (ESEM) are being used to identify surface attachment features and patterns of the bacteria and other alterations to the surface that may significantly alter the surface chemical characteristics of the mineral.

3. Literature suggests that iron-reducing bacteria produce enzymes specifically for iron reduction and/or complexation, but give little in the way of a clear identification of these enzymes or complexants. The most likely class of protein/enzyme responsible for the reduction chemistry is cytochromes. This subtask will focus upon identifying and isolating cytochrome-like compounds and siderophores from iron-reducing bacteria using spectroscopic and chromatographic techniques. Isolation of any compounds with ironreducing or complexing potential will be attempted so that the direct interaction of that compounds with various iron-bearing mineral surfaces, iron species in solution, and other contaminant species such as $\mathrm{Co}$ and $\mathrm{UO}_{2}{ }^{2+}$ may be examined.

4. Maintenance of the bacterial cultures, determination of appropriate growing conditions for the experiments outlined in the other subtasks, and characterization of the changes to the bacteria under the differing growing conditions.

\section{Background}

Iron and sulfate reducing bacteria fall into a number of phylogenetic groups. Two well-known genera of dissimilatory iron reducers are Geobacter and Shewanella. Dissimilatory metal reduction by bacteria is a very important process in subsurface environments; however, the mechanisms involved are still relatively poorly understood. Microbial oxidation of organic compounds and elemental hydrogen 
(electron donors) is coupled with metal reduction where the oxidized form of the metal serves as the terminal electron acceptor. The possible mechanisms might include:

- The microbe controls the redox potential and $\mathrm{pH}$ in the microenvironment surrounding the cell or in the interfacial zone between the cell and mineral surface such that $\mathrm{Fe}^{3+}$ is reduced as a natural chemical consequence of the conditions created by close contact with the bacterium.

- The cell uses an electron shuttle to transfer electrons to $\mathrm{Fe}^{3+}$ species extracellularly, i.e., iron reduction is via a secondary enzymatic or chemical process used to regenerate the electron transport cofactors or is simply just a cofactor.

- Iron is chelated and transported to the cell where the reduction occurs, once again, either by chemical or enzymatic means either on the surface of or within the cell and the reduced iron is subsequently released.

The predominant evidence suggests that enzymatic processes dictate the iron reduction and may involve a nitrate reductase, ${ }^{17}$ a ferric reductase, ${ }^{28}$ or some other yet unidentified enzyme. ${ }^{29}$ The nature of the "iron-reducing enzyme" is a curiosity since not only can $\mathrm{Fe}^{3+}$ be reduced, but also so can many other complex species, including metal chelates like $\mathrm{Co}^{\mathrm{III}} \mathrm{EDTA}^{-26}$ and vitamin $\mathrm{B}_{12}{ }^{30}$ Much of the evidence for the presence of an enzymatic process is based upon "enzyme inhibition" by chemical species that may be stronger oxidizers than $\mathrm{Fe}^{3+}$ in its various chemical forms. In many of these cases, the interpretation of the presence of an "enzyme" is questionable because even though the enzyme may be more likely to react with the stronger oxidizer, so would many other chemical species that would not necessarily be considered enzymes. It is also not clear whether the $\mathrm{Fe}^{3+}$, either free or chelated, is reduced enzymatically at the cell wall surface, in the cell wall or within the cell.

The best evidence as to the actual presence and identity of an enzyme is in the recent work of Myers and Myers ${ }^{31}$ who identified a 561 base-pair gene in Shewanella putrefaciens MR-1 coding a protein of 187 amino acids with a mass of $20.98 \mathrm{kDa}$. The amino acid sequence was consistent with sequences of other multiheme c-type cytochromes found in other bacteria. These types of cytochromes are typically involved in the transfer of electrons from the cytoplasmic membrane to acceptors in the periplasm. The protein was found in the membrane and soluble cell fractions suggesting that the transfer of electrons does indeed occur on or in the cell membrane. The utilization of a cytochrome compound that is present in the cell membrane would be a logical explanation based upon a normal respiratory chain. Indeed, iron reduction has been shown to occur on or near the surfaces of cells of other microorganisms including Listeria spp, ${ }^{32}$ Neisseria gonorrhoeae, ${ }^{33}$ Saccharomyces cerevisiae, ${ }^{34,35}$ and Vibrio (Listonella) anguillarum $^{28}$ using enzymes most frequently identified as cytochromes. Cytochromes have also been identified that may play a role in the reductive dechlorination activity on or near the surface of certain other types of bacteria.

The available iron in the subsurface environment is generally present as an oxide with very low solubility, therefore the iron-reducing bacteria must either act directly on the iron oxide or must first release the iron as complexed $\mathrm{Fe}^{3+}$ ions, which can then be used by the enzymatic process. It appears that it is necessary for the iron-reducing bacteria to come into direct contact with and adhere to the iron oxide surface in order to utilize the iron. ${ }^{17,21}$ This suggests that the iron oxide might be used directly by reduction at the cell wall surface. It may also mean that the cell contact with the mineral surface chemically releases $\mathrm{Fe}^{3+}$ ions that are then used by the reducing enzymatic process. However, recent work $^{37}$ suggests that that strains of Shewanella alga (RAD20) that do not strongly attach to the oxide surface can use an iron oxide surface just as effectively as a strain which adheres strongly (BrY). This 
result implies that simple contact or attachment of the bacterial cell to the surface is an oversimplification of a more complex reaction sequence. This work seems to relate well to some studies using atomic force microscopy (AFM) ${ }^{38,39}$ that correlated bacterial mobility and adhesion to the mineral surface with nutrient and anaerobic or aerobic conditions. Generally, under anaerobic conditions, the bacteria remained passively mobile as relatively uniform deterioration of the iron oxide surface was observed, while under aerobic conditions more localized pitting of the surface was observed indicating a stronger localized interaction with the surface.

Biofilms have been shown to display variations in $\mathrm{pH}$ with depth, ${ }^{40}$ therefore, it is reasonable to expect that localized control of Eh and $\mathrm{pH}$ by the microorganisms are also likely to play a role in iron reduction by making more iron soluble and thus available for reduction. The production and use of chelating agents, i.e., siderophores, by microorganisms to help solubilize iron from ferric oxides is also an important function that microbes play in the environment. The dissolution may be a natural by-product of the normal microbial function or may result from the physiological need for iron by the microbe. Dissolution of ferric oxides can be enhanced by lowering the $\mathrm{pH}$ to less than 5 and by the presence of complexing ligands such as humic acids, simple organic acids or more specific chelating agents. The dissolution of some iron oxide materials by simple complexing organic acids, ${ }^{41}$ ascorbate, ${ }^{42}$ and ferric ion specific siderophores ${ }^{43}$ has been investigated. The surface dissolution aided by siderophores is thought to be a surface mediated process where the organic ligand first forms a chelate on the surface that is then released to solution under appropriate conditions. ${ }^{41}$ For some iron-reducing bacteria, simply having chelated iron available may still not necessarily mean that it can easily be reduced if the complex formation constants are quite high. ${ }^{44}$ In a study using Shewanella putrefaciens, ${ }^{45}$ the size of the solvated clusters of chelated iron appeared to have an affect on the ability of iron to be reduced, possibly because the large clusters could not enter the periplasm compartment. In addition to ironreducing bacteria, iron solubility, transport, and reduction are important for many other microbes. Ferric iron siderophores are known to collect iron for transport and storage in fungal cells. ${ }^{46,47}$ Pseudomonas sp. have been shown to acquire iron from iron oxides by a process employing siderophores that they produce in the absence of any other soluble ferric species. ${ }^{48}$

References such as Pourbaix ${ }^{49}$ list reduction potentials of some chemical species of iron pertinent to iron-reducing bacteria, as well as some relevant subsurface contaminants and biological compounds. If a cytochrome $\mathrm{C}$ type compound is the active "enzyme" responsible for $\mathrm{Fe}^{3+}$ reduction as suggested by Myers and Myers, one would expect its formal reduction potential to be in the range of 0.20 to $0.25 \mathrm{~V}$. From the tabulated reduction potentials one can explain why much of the reduction activity of Shewanella sp. occurs. However, because of the construction of the cell, it is most likely that the iron must be solubilized to make it through the cell wall to be reduced in the periplasm or at the cell membrane surface.

With all of the work that has been performed using iron-reducing bacteria, it appears that the following questions have not yet been conclusively answered and may simply vary from species to species:

- $\quad$ Must iron-reducing bacteria come into direct contact with the surface Fe(III) oxides to use them as electron acceptors and, if so, how long must contact be maintained and what is the physical/chemical nature of that contact?

- $\quad$ Do iron-reducing bacteria provide chelators (siderophores) or local $\mathrm{pH}$ extremes that help solubilize the Fe(III) oxides to make the Fe(III) more bioavailable? 
- $\quad$ Alternatively, do iron-reducing bacteria produce electron shuttling compounds that interact directly with the Fe(III) oxides on the surface?

- $\quad$ Are the electron shuttling or metal chelating compounds a part of the cell membrane structure or are they released to the surrounding solution and return to the cell surface?

- Where does the actual reduction take place, at the mineral surface, in the bulk solution, at the cellular surface or within the cell and is it dependent upon what the available source of iron is?

- If a class of compounds that reduce iron can be isolated, how do they specifically interact, if at all, with iron-bearing mineral surfaces?

The activities in this task are geared toward developing the methodologies that can be used to begin to answer these questions more directly.

\section{Microbes, Bioreactor, and Surface Attachment Activities}

A major problem that had to be dealt with in our laboratory was the irreproducibility of the experiments involving the Shewanella alga culture that was used for all of our previous studies. This culture had apparently mutated or been unwittingly selectively cultured to such a point that each time an experiment was initiated, a slightly different strain was used and reproducibility became a serious issue. Therefore, this culture was abandoned in favor of a Shewanella putrefaciens culture obtained from the American Type Culture Collection (ATCC). Once received in the laboratory, the culture was split and stored in such a way that when doubts arise as to the nature of the culture, it is always possible to start a new experiment series with exactly the same initial culture. For continuity and assurance of similar exposure conditions in long-term experiments on biofilm development on mineral surfaces, a continuous flow bioreactor was also set up. Figure 1 is a schematic of this bioreactor.

The continuous flow bioreactor was set up for long-term anaerobic growth of $S$. putrefaciens. The reactor is operated at a flow rate of $10 \mathrm{~mL} /$ day and contains approximately $300 \mathrm{~mL}$ of basal salts medium containing lactate $(20 \mathrm{mM})$ as a carbon source for the bacteria and $3 \mathrm{~g}$ of $\mathrm{Fe}_{2} \mathrm{O}_{3}$ particles for use as a

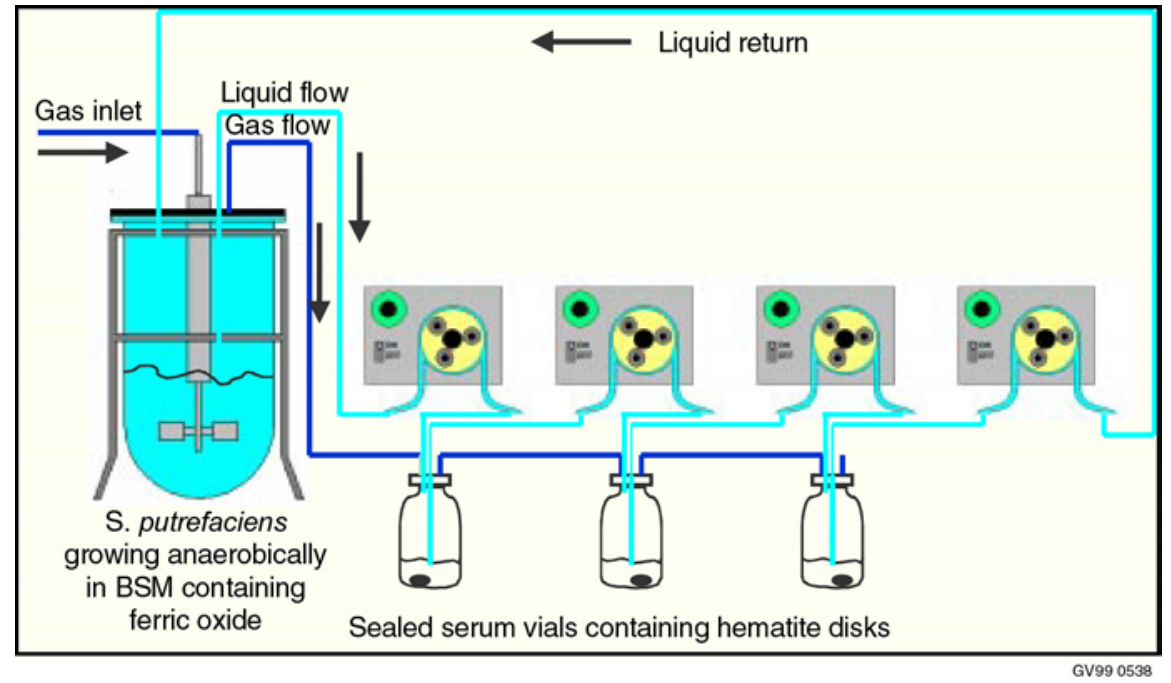

Figure 1. Schematic of continuous flow bioreactor for continuity in long-term studies on biofilm development. 
terminal electron acceptor. Basal growth medium is similar in salt and metal content to naturally occurring waters that contain iron-reducing bacteria. To maintain the growth conditions as constant as possible, $35 \mathrm{~mL}$ of medium are removed every 3 days and replaced with $35 \mathrm{~mL}$ of fresh anaerobic medium. The reactor and satellites are maintained anaerobic with a continuous purge of anaerobic gas mix (90\% nitrogen, $5 \%$ carbon dioxide, $5 \%$ hydrogen).

At the time of start up, $\mathrm{Fe}^{2+}$ and cell concentrations in the bioreactor were monitored to ensure that the bacteria were growing. During this period it was discovered that a significant number of bacteria were associated with the $\mathrm{Fe}_{2} \mathrm{O}_{3}$ particles, this in turn, interfered with accurate determination of cell number. A technique to free the cells from the iron oxide that uses glutaraldehyde (30\%) to fix the cells and then hydroxylamine hydrochloride $(0.25 \mathrm{M}$ in $0.25 \mathrm{M} \mathrm{HCl})$ to dissolve amorphous $\mathrm{Fe}(\mathrm{III})^{37}$ was evaluated for application in this work. However, even with modifications to the technique $(\mathrm{pH}$, time, temperature) complete dissolution of $\mathrm{Fe}_{2} \mathrm{O}_{3}$ particles was not achieved and low cell recoveries were observed. Accurate determination of cell number remains a problem and work to develop an acceptable procedure continues.

An experiment was conducted using the continuous flow bioreactor to reexamine the surface activity of Shewanella putrefaciens on hematite disks. Previous studies indicated a rather high degree of "colonization" on the surface without any apparent surface attachment structures, however, we wanted to make sure that this was not simply an artifact of the sample preparation. In this experiment, hematite pellets were placed in the flow path of the bioreactor and two disks were removed at various times points. One disk was treated with a gentle wash procedure as in previous experiments and the other was treated more harshly with a rather strong rinse stream. The disks were stained with a fluorescent dye (DAPI, 15 minutes), air-dried, and examined with AFM and optical microscopy. This experiment has only recently been concluded and the results have not yet been fully evaluated.

A smaller continuous flow bioreactor was also set up using a hematite disk as a $\mathrm{Fe}^{3+}$ source for $S$. putrefaciens. To generate high cell numbers, the culture was initiated under aerobic conditions and at log phase $\left(10^{8}\right.$ cells $\left./ \mathrm{ml}\right)$ was converted to anaerobic conditions by flushing the headspace with the anaerobic gas mix. The medium from a reservoir vial containing a single hematite disk was circulated by a peristaltic pump at $\sim 0.1 \mathrm{~mL} / \mathrm{min}$ through a microscope flow cell containing a second hematite disk and returned to the reservoir vial in a closed loop. The anaerobic conditions were maintained by replacing the headspace of the reservoir biweekly with anaerobic gas mix. As in the large reactor, aliquots were also periodically removed and replaced with fresh media. Cell counts and $\mathrm{Fe}^{2+}$ concentrations were monitored as indicators of biological activity. The hematite disk in the vial was periodically removed for examination by AFM and optical microcopy for microbial surface activity and replaced with a clean disk. The disk in the cell was periodically examined by optical microscopy in a reflectance mode to verify the presence of cells on the surface of the disk.

Figure 2 is a series of typical AFM images from one of the disks removed from the small continuous flow bioreactor reservoir after just 24 hours. Overall, the images show no clear attachment features to the surface, however, the lack of flagella seems to indicate that there is a relatively strong surface association. Cells tend to be grouped tightly, which creates the possibility for them to control and derive benefit from a localized chemical environment over which they can collectively assert some control.

\section{pH Measurement Activities}

Microbes have been found to play a significant role in many subsurface geochemical processes. This role is widely variable and may affect the mineralogy by direct interaction with the mineral surface or via indirect processes. The indirect processes may result from the production of chemical species (e.g., organic acids) that subsequently interact with the mineral surface or cause precipitation of other chemicals 

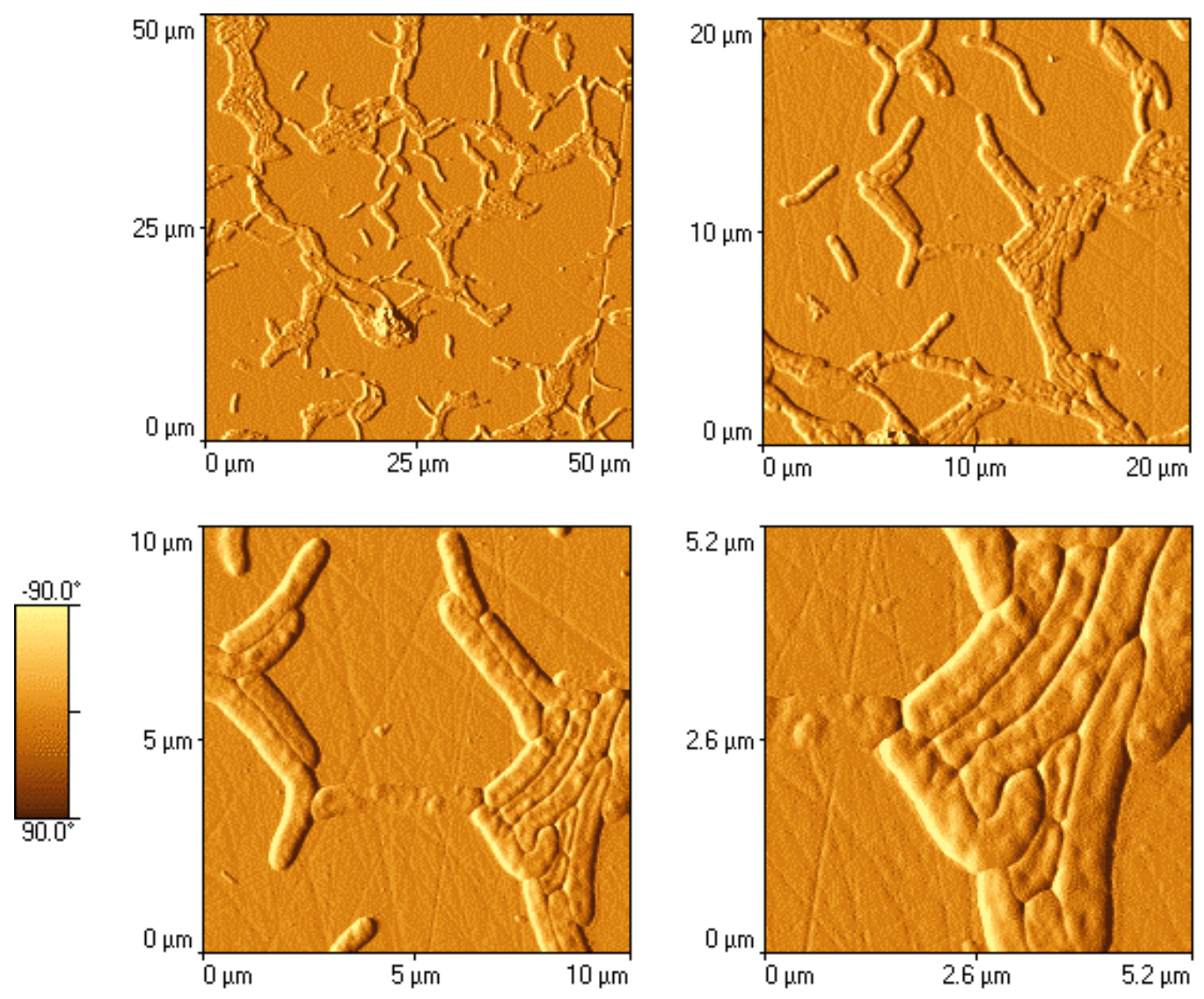

Figure 2. Series of AFM images showing the "colonization" of a hematite surface by Shewanella putrefaciens.

from solution. Redox chemistry, dissolution or precipitation at mineral surfaces can be greatly influenced by $\mathrm{pH}$. Microbes in intimate contact with a mineral surface, individually and especially in groups ${ }^{50}$ like those seen in Figure 2, can create microchemical environments where the chemistry can be controlled by the microbe or microbial colony. One of the parameters in these microenvironments that the microbes may control is the $\mathrm{pH}$. Normally, the intracellular $\mathrm{pH}$ is maintained to within a few tenths of a $\mathrm{pH}$ unit of the extracellular $\mathrm{pH}^{51}$ For microbes within a "biofilm" or in direct contact with a surface, intracellular $\mathrm{pH}$ variations beyond those expected from the bulk solution should therefore reflect the $\mathrm{pH}$ in the microchemical environments within the biofilm or in the interfacial zones between the microbe and the surface.

Measurement of intracellular $\mathrm{pH}$ and $\mathrm{pH}$ gradients within biofilms can be performed with various fluorescent probes. ${ }^{52}$ For this work, they are primarily the seminaphthorhodafluor (SNARF) dyes that exhibit a $\mathrm{pH}$ dependent emission ratio at $580 / 640 \mathrm{~nm}$. The SNARF dyes are commercially available (Molecular Probes, Inc.) and are available as succinimidyl and calcein derivatives to enhance cellular retention and as acetoxymethyl (AM) esters to make them cell membrane permeable. Microscopic identification of bacteria via $\mathrm{pH}$ sensitive fluorescent probes is optimal because individual bacteria can be identified and the response separated from extracellular fluorescence, i.e., fluorescence in the bulk solution. 
SNARF, SNARF Calcein, and their AM esters were obtained from Molecular Probes, Inc., and stock solutions prepared in dimethyl sulfoxide (DMSO). Cells (Shewanella alga and S. putrefacians) were grown in dilute tryptic soy broth (dTSB) or basal salts medium (BSM) and harvested by centrifugation. The resulting cell pellet was washed and the cells resuspended in media containing the fluorescent $\mathrm{pH}$ probe at concentrations of $1-20 \mu \mathrm{M}$. Cells were incubated for 2 hours in the media containing the fluorescent probe at either ambient or elevated $\left(\sim 35^{\circ} \mathrm{C}\right)$ temperature to load the probe. The cells were again centrifuged to a pellet, washed to remove excess dye, and resuspended in growth media or buffer for any additional incubation times. For spectroscopic measurements, the cells were again harvested by centrifugation, washed, and resuspended in $0.5-1 \mathrm{~mL}$ of appropriate media/buffer. For microscopic measurements, cells were collected by filtration onto a polycarbonate filter with $0.2-\mu \mathrm{m}$ pores and mounted on a microscope slide. Microscopic images were recorded with a Nikon PCM 2000 confocal scanning laser microscope system (CLSM) using excitation wavelengths of 488 or $514 \mathrm{~nm}$ from an $\mathrm{Ar}^{+}$laser or the green $543 \mathrm{~nm}$ line of a HeNe laser. Two channel detection was performed using a $580 \pm 16-\mathrm{nm}$ band pass filter for the green channel and a $630 \pm 30-\mathrm{nm}$ band pass filter for the red channel. Fluorescence spectra were recorded using a PTI DeltaScan 4000 spectrofluorometer with excitation at $488 \pm 10 \mathrm{~nm}, 514 \pm 10 \mathrm{~nm}$ or $543 \pm 10 \mathrm{~nm}$. Alternatively, a laser based fluorescence system consisting of the laser sources from the PCM 2000 CLSM were fiberoptically coupled to an emission cell via a $100-\mu \mathrm{m}$ fiber. Fluorescence emission was collected into a 500- $\mu \mathrm{m}$ fiber and focused onto the entrance slit of a Acton Research Corporation, SpectraPro 300i polychrometer equipped with a Princeton Instruments TEA $1152 \mathrm{CCD}$ detector. Chemometric analysis of spectral data was performed using Grams/32 with the PLSplus/IQ add-on package (Galactic Industries, Inc.).

The use of $\mathrm{pH}$ sensitive fluorescent dyes generally requires the measurement of the fluorescent emission intensity at two emission or excitation wavelengths. In the case of the SNARF dyes, the fluorescence emission intensity at $\sim 580$ and $640 \mathrm{~nm}$ is used. The $\mathrm{pH}$ is determined via the following equation:

$$
\mathrm{pH}=\mathrm{pK}_{\mathrm{a}}+\log \left(\frac{\mathrm{R}_{\mathrm{B}}-\mathrm{R}}{\mathrm{R}-\mathrm{R}_{\mathrm{A}}} * \frac{\mathrm{FI}_{\mathrm{b}, 640}}{\mathrm{FI}_{\mathrm{a}, 640}}\right)
$$

Where $R$ is the ratio of emission intensity at $640 \mathrm{~nm}$ to that at $580 \mathrm{~nm} . \mathrm{R}_{\mathrm{B}}$ and $\mathrm{R}_{\mathrm{A}}$ are the emission intensity ratios at the basic and acidic limits, respectively. The fluorescence intensity ratio at $640 \mathrm{~nm}$ at the basic and acidic limits is intended as a correction factor. Calibrations can be obtained employing this equation to spectroscopic or microscopic data. However, the uncertainty in the determinations of $R_{B}, R_{A}$, and the correction factor can contribute heavily to the overall uncertainty of the $\mathrm{pH}$ determination making the measurement of small $\mathrm{pH}$ differentials difficult. Correlation coefficients are frequently in the 0.93-0.99 range using this methodology.

To limit this uncertainty, full spectrum methods have been attempted using principal components regression (PCR) ${ }^{53}$ and partial least squares (PLS). ${ }^{54}$ Spectral data were of SNARF and SNARF-calcein in the $\mathrm{pH}$ range of 4-9. Pretreatment of the spectra consisted of normalizing to the total area or normalizing to the total area and then taking the natural logarithm of the spectrum. Using the spectra normalized only, produced calibrations with 2-4 factors and correlation coefficients of at least 0.98 and usually $>0.99$ using either PCR or PLS. With the log of the normalized spectra, calibrations consistently required only one or two factors and correlation coefficients were always $>0.99$. Accuracy and precision of microbial $\mathrm{pH}$ measurements will be considerably improved using this full spectrum measurement technique.

Retention of SNARF-Calcein in the microbes at times beyond 20 hours has been demonstrated making it very desirable for long-term experiments. Cells are loaded with SNARF-Calcein as the AM ester. As the AM ester, the fluorescence intensity is quite small and intracellular esterase activity is 
required to cleave the acetoxymethyl group and return SNARF-Calcein to its highly fluorescent, $\mathrm{pH}$ sensitive state. This can take several hours as net fluorescent intensity appears to continuously increase after loading with a slight increase in intracellular $\mathrm{pH}$. Several attempts to calibrate and measure $\mathrm{pH}$ in Shewanella putrefaciens on a hematite disk under aerobic and anaerobic conditions using CLSM have met with limited success. The reasons are two-fold: esterase activity has been lower than expected and somewhat erratic. Speculating that this was due to the nutrients that were available to the cells, loading, washing, and postloading incubating conditions were varied. Generally, loading, washing, and postloading conditions employing deionized water as the medium most consistently produce the brightest cells. The esterase activity is demonstrated in Figure 3 showing cells 0.5 and 20 hours after loading with SNARF-Calcein AM ester in deioinzed water. The cells are generally brighter after 20 hours.

Figure 3 also demonstrates the second problem with $\mathrm{pH}$ measurements using the CLSM. Even though the cells are noticeable, even in the 0.5 -hour postloading image, the signal-to-noise $(\mathrm{S} / \mathrm{N})$ ratio is quite poor and the measurable light in the green $(580 \pm 15 \mathrm{~nm})$ channel is very low compared to the red $(630 \pm 30 \mathrm{~nm})$ channel. In fact, finding bacteria with green channel intensities greater than the $2 \mathrm{x}$ the noise level is extremely difficult. The lower signal on the green channel can be partially attributed to the spectral bandpass of the respective filters.
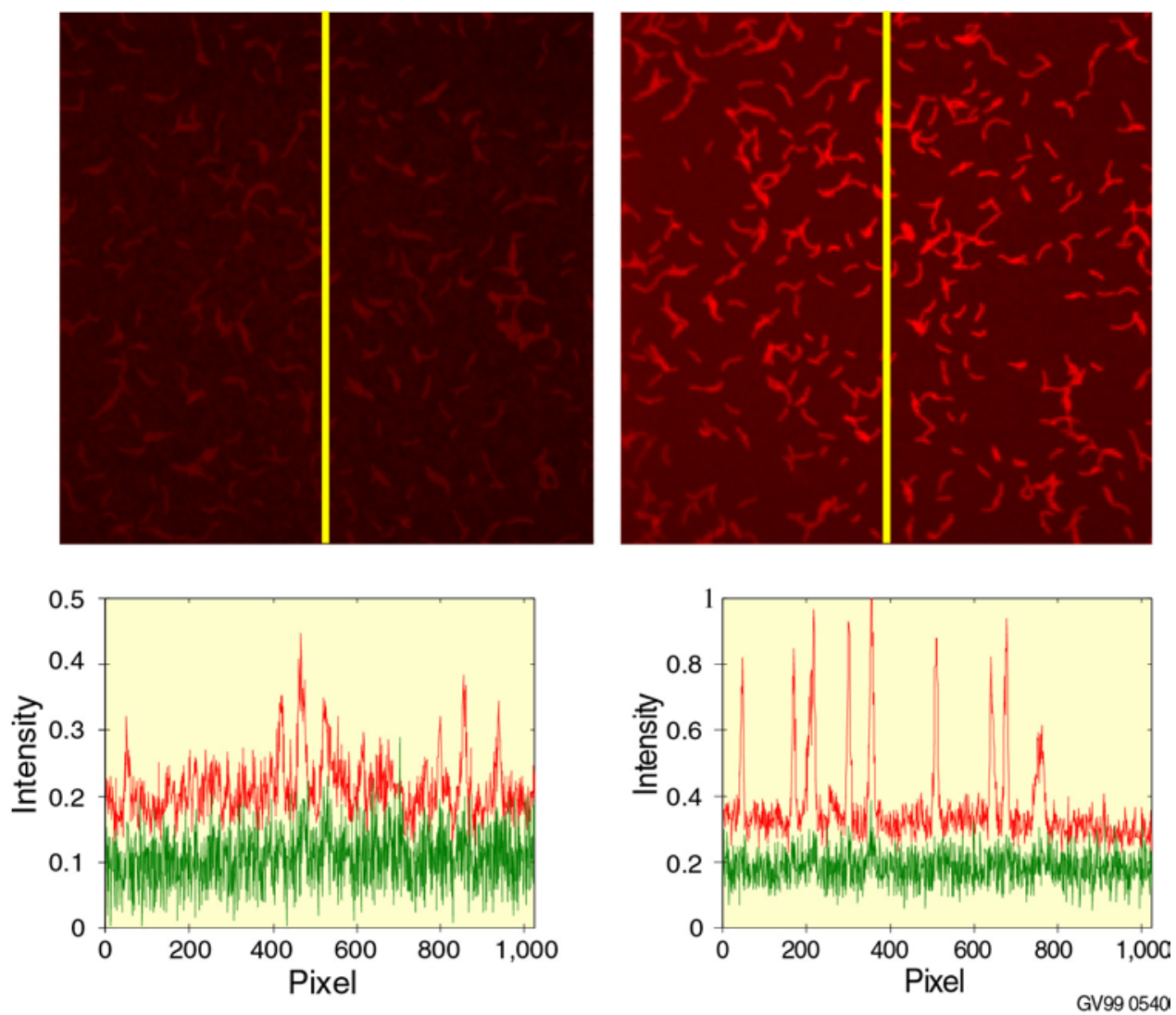

Figure 3. Confocal laser scanning microscopic images of Shewanella putrefaciens loaded with SNARFCalcein AM ester $0.5 \mathrm{hr}$ (left) and $20 \mathrm{hr}$ (right) after loading for $2 \mathrm{hr}$ in water. Traces underneath images are from a single pixel line dissecting the image showing the magnitude of the noise and signals on the red channel $(630 \pm 30 \mathrm{~nm})$ and the green channel $(580 \pm 15 \mathrm{~nm})$.

In order to make the images like those in Figure 3 useful for $\mathrm{pH}$ measurement, a $590 \pm 15$-nm filter has been acquired to replace the $580 \pm 16-\mathrm{nm}$ filter, since the true maximum of the acidic form is closer to 
$590 \mathrm{~nm}$ and work had been initiated to digitally process the images to enhance the overall S/N. The images in Figure 4 are the same images as in Figure 3, but have been Fourier filtered sequentially in the two spatial dimensions using a Gaussian cutoff function to eliminate the high frequency random noise. The $\mathrm{S} / \mathrm{N}$ ratio has increased by orders of magnitude in both images. In both images, the green channel now clearly shows microbes with fluorescent intensities above background. In fact, the rise in $\mathrm{pH}$ with time is noticeable in the selected traces as the fluorescent intensity ratio of the red to green channel for bacteria is larger in the 20-hour postloading trace.

\section{Identification of Siderophores and Iron-reducing Agents}

As stated above, the exact nature of the iron reduction mechanism by dissimilatory iron-reducing bacteria is not clear from the literature. Aspects such as the influence of localized chemical regimes have not been significantly investigated. Closely associated groups of cells near an iron oxide surface can release chemicals to either free iron from surface or send an "electron shuttle" or enzyme to the iron oxide surface with a higher probability of return. In order to do more involved studies to assess the actual chemical reactions occurring at mineral surfaces by chemicals actually produced by DIRBs, something must be known about what these chemicals are. To address this, an effort has been underway to determine the presence and nature of some siderophores and "iron-reducing enzymes" produced by Shewanella putrefaciens. Reversed phase-high pressure liquid chromatography (RP-HPLC) had been employed to separate the extracellular organic compounds produced by cells grown in batches.
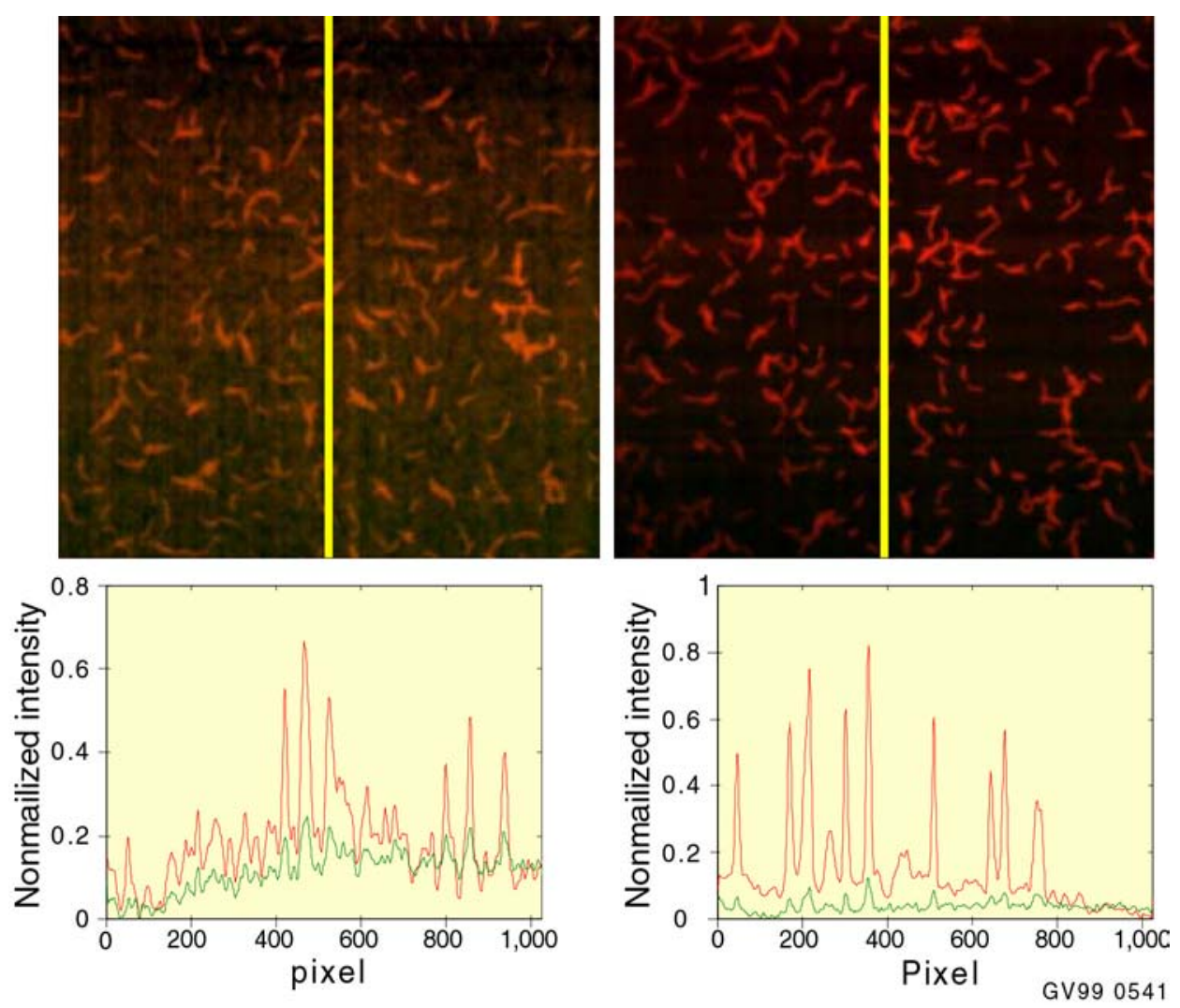

Figure 4. The same CLSM images as in Figure 3 after 2D FFT with a Gaussian cutoff function. Significant improvement in $\mathrm{S} / \mathrm{N}$ allows more accurate determination of Red/Green ratios associated with bacteria. 
Shewanella putrefaciens was grown under aerobic and anaerobic conditions in BSM with lactate as a carbon source. Iron in the form of powdered $\mathrm{Fe}_{2} \mathrm{O}_{3}, \mathrm{Fe}$ (III)EDTA or Fe(III) citrate was added to both aerobic and anaerobic cultures. Some aerobic cultures also containing iron were allowed to go anaerobic. Five-mL samples were collected as the cultures progressed, filtered to remove cells and other particulates, and lyophilized. The residue from the lyphilization was reconstituted in $0.5-1 \mathrm{~mL}$ of deionized water or HPLC mobile phase and 30-50 $\mu \mathrm{L}$ injected directly onto the column. The HPLC consisted of a Waters 600E solvent delivery system, Model 991 photodiode array detector, and 717 autosampler. Detection was via UV-Vis absorption spectroscopy with a full spectrum from 200-600 nm collected every 3 seconds. The analytical column was a Machery Nagel 250x3 mm Nucleosil 120-8 $\mathrm{C}_{18}$. For preparative-scale separations, an Alltech $250 \times 22 \mathrm{~mm}$ Econosil $\mathrm{C}_{18}$ with $10-\mu \mathrm{m}$ particles was used. The mobile phase consisted of $100 \% 5-50 \mathrm{mM}$ phoshpate buffer at $\mathrm{pH} 2$ for the siderophore separations. For separation of additional components, the initial mobile phase was $100 \% 50 \mathrm{mM}$ phosphate buffer at $\mathrm{pH} 2$ held for 4 minutes then ramped to a $50 \%$ mixture of the buffer with acetonitrile and held for 25 minutes. For the preparative-scale separations, fractions were collected manually and submitted to an iron chelating assay using chrome azurol S (CAS).

The CAS assay is based upon the competitive binding between the ligand to be tested and a highly colored (blue) iron III chrome azurol S (CAS) complex. If the iron III ligand complex is stronger than the colored complex, CAS is released from the iron III and the solution changes to orange according to the following reaction:

$\mathrm{FeCAS}^{3-\lambda}+\mathrm{L}^{\alpha-} \rightarrow \mathrm{FeL}^{3-\alpha}+\mathrm{CAS}^{\lambda-}$

Fractions were analyzed for the presence of siderophores by mixing with an equal volume of CAS solution ( $\mathrm{pH}$ 5.6) containing $0.6 \mathrm{mM}$ hexadecyltrimethylammonium bromide, $0.015 \mathrm{mM} \mathrm{FeCl}_{3}, 0.15 \mathrm{mM}$ chrome azurol $\mathrm{S}$, and $0.1 \mathrm{M}$ piperazine (sodium salt). After equilibration, absorbance of the test solution is measured at $630 \mathrm{~nm}$. Phosphate in the HPLC mobile phase was found to interfere slightly with the CAS assay and the procedure had to be adjusted to dilute the phosphate to a noninterfering level. Future work will employ trifluoroacetic acid (TFA) at $\mathrm{pH} 2$ to eliminate this problem. Preliminary results indicate no change in the chromatography using TFA.

Figure 5 is a series of chromatograms showing the difference in extracellular components of Shewanella putrefaciens grown aerobically without iron oxide and anaerobically with iron oxide for 1 day. All cultures grown with iron oxides had the same pattern: four peaks that elute at 9.4, 10.4, 13.1, and 21.5 minutes in this chromatogram. These components do not appear in any aerobic or anaerobic cultures containing Fe(III) EDTA or Fe(III) citrate, indicating that they are only produced when insoluble $\mathrm{Fe}(\mathrm{III})$ oxides are present. Fractions were collected from similar preparative-scale separations and submitted to the CAS assay. The compound corresponding to the peak at 9.4 minutes tested positive as a siderophore by this assay. Additional experiments have been performed in which $40 \mathrm{~mL}$ of culture media have been lyophilized have been performed. These samples are now awaiting preparative chromatography.

The chromatograms in Figure 6 were obtained via gradient elution RP-HPLC of similar extracts as in Figure 5. The UV-Vis spectra of the compounds corresponding to the peaks at 34.9 and 41.0 minutes of the chromatograms Shewanella putrefaciens culture medium correlate well with the characteristic spectrum of cytochrome $\mathrm{c}$ in a $\mathrm{pH} 2$ solution confirmed by the mammalian cytochrome c chromatogram. While the compounds have not been conclusively identified as cytochromes, the available evidence to date implies that they are. If future experiments confirm this finding, it is a potentially important result because it suggests that more than one cytochrome may be produced: one in response to available oxygen and one for iron. It will also be important because it could confirm similar observations of extracellular cytochromes produced by iron reducers ${ }^{50}$ and "catalysts" ${ }^{156}$ produced by other organisms. 


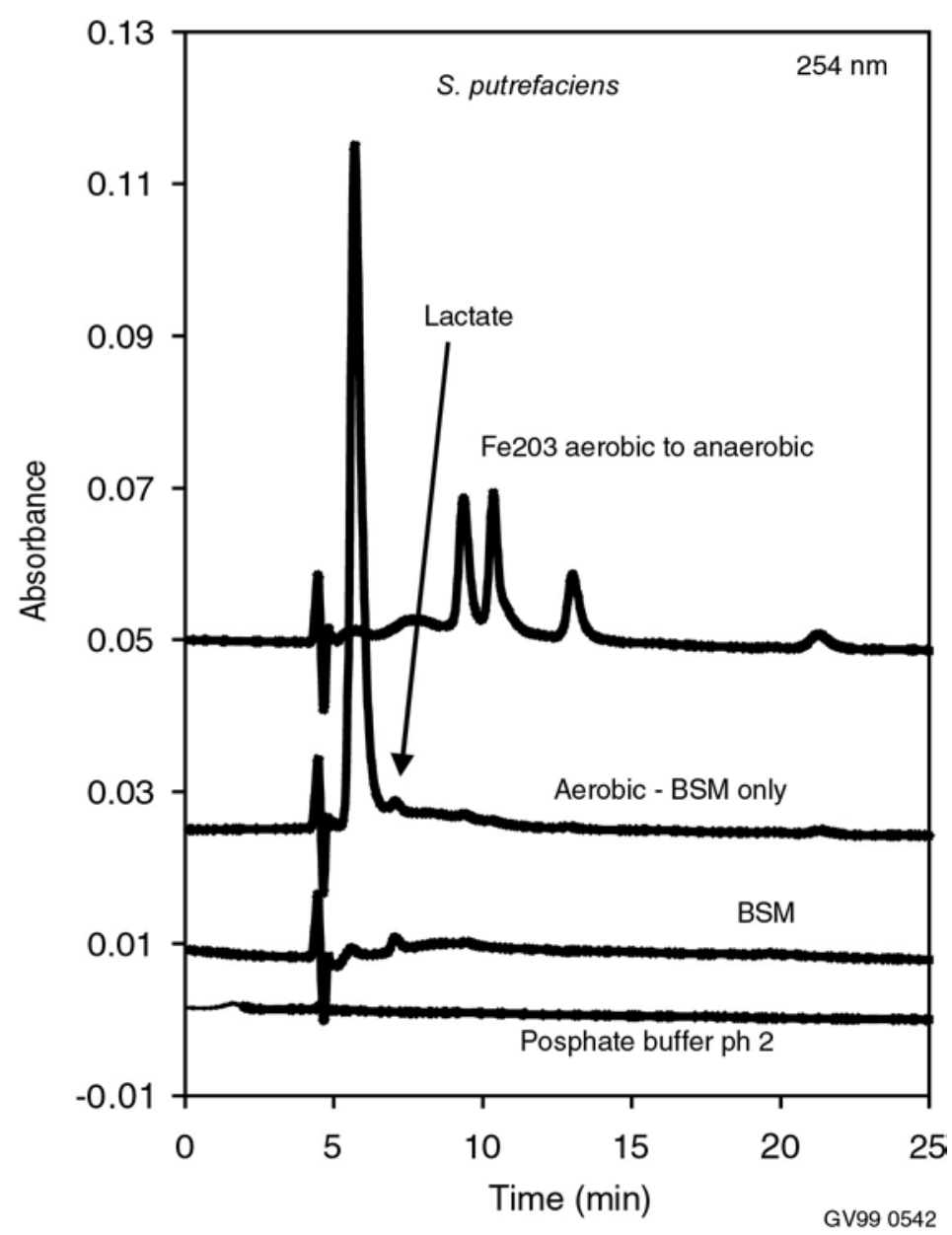

Figure 5. RP-HPLC chromatographs showing the differences between a Shewanella putrefaciens culture grown aerobically and one allowed to go anaerobic in the presence of an iron oxide. Conditions: isocratic with $5 \mathrm{mM}$ phosphate buffer at $\mathrm{pH} 2,0.3 \mathrm{~mL} / \mathrm{min}$ with detection at $254 \mathrm{~nm}$. 


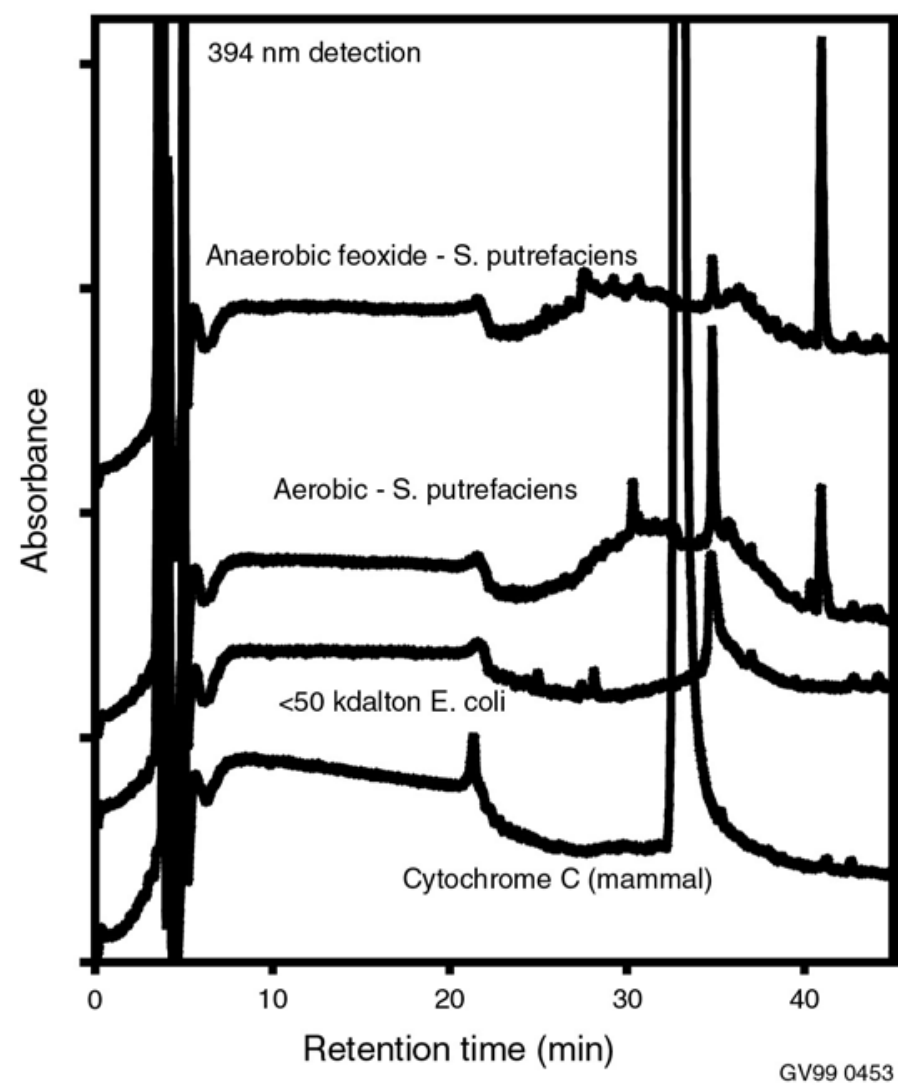

Figure 6. RP-HPLC chromatograms of media from aerobic and anaerobic cultures of Shewanella putrefaciens compared to and extract from Eschericia coli cells and a mammalian cytochrome C. HPLC conditions: $100 \%$ solvent A for 4 min. then ramped to 50:50 A:B over $20 \mathrm{~min}$ and held for 20 min with detection at $394 \mathrm{~nm}$ where A was $100 \% 50 \mathrm{mM}$ phosphate buffer at $\mathrm{pH} 2$ and , B was $100 \%$ acetonitrile.

\section{Method Development to Examine Microbial Grouping and Their Effects on Environmental Surfaces}

\section{Objective}

The objective of this task is to develop analytical methodologies for the identification of bacterial types in mixed communities on mineral and other surfaces using optical techniques. This is a collaborative task associated with Mike Lehman of the Microbial Partitioning Between Mobile and Immobile Phases in Geologic Media task within the Porous Media Project. Ultimately, the techniques developed in this task will be used to identify and quantify relative microbial population abundances based upon phylogenic groupings. The relationships of bacteria with each other and with specific mineral phases will be discernable. The overall approach will be to use fluorescently labeled oligionucleotides as probes for fluorescent in situ hybridization (FISH) of phylogenic groups based upon their 16S rRNA. Ultimately, each phylogenic probe will be labeled with a different fluorophor. Using a simple spectroscopic approach, fluorescence excitation, and/or emission spectra will be obtained and the relative contributions of each phylogenic group quantitated using chemometric techniques such as principal components regression (PCR) ${ }^{53}$ or partial least squares (PLS). ${ }^{54}$ Using hyperspectral imaging techniques where images of the same image field obtained at several excitation or emission wavelengths, preferably simultaneously, similar chemometric methods can be used to process the set of spectral images to produce 
a new set of images, one for each phylogenic group. Fluorescence spectroscopy, microscopy and microspectroscopy, and confocal microscopy are being employed in this task to develop the most appropriate methods. Specifically, the approach will include:

1. Selection of the optimum fluorescent probes to be used as taggants and the best wavelengths to create the minimal data sets from solution studies using fluorescence spectroscopy and chemometric processing of the spectral data.

2. FISH labeled bacteria will be suspended in solution, fluorescence spectra collected, and the chemometric techniques adjusted to accommodate any background fluorescence or fluorescence quenching from the bacteria. Quantitation or at least semiquantitation of population densities by phylogenic group should be achievable.

3. Methodologies developed above will be applied to fluorescence microspectroscopic images of FISH labeled bacteria collected from solution on polycarbonate disks and then from other experimental samples using real and fabricated surfaces.

4. A similar approach to the fluorescence microspectroscopy will be taken with the confocal microscope. Initially, images of the same field will be collected using different filters and different excitation lasers. Later a monochrometer may be added between the confocal collection fiber-optic and the photomultiplier tube to allow the collection of images at different wavelengths with a much narrower bandpass and unlimited wavelength options than are currently available with filters. This should help to improve the specificity and accuracy of the analyses. Images will need to be processed using similar techniques as in the fluorescence spectroscopy applications above, necessitating some effort to convert the image data to a format that can be easily processed.

Using the above approach, the techniques being developed should overcome several of the problems associated with the use of $16 \mathrm{~S}$ rRNA directed oligionucleotide probes to detect environmental microorganisms. In particular, because of the low cell densities and low number of ribosomes associated with environmental microbes, ${ }^{57}$ use of the intense laser excitation sources should correspond to an increase in measurable fluorescence emission. Additionally, the digital processing of the data will serve to enhance the signal-to-noise ratios and eliminate the ambiguities of identification.

\section{Experimental Activities}

Work was initiated on developing spectral and hyperspectral imaging methods for determining multiple microbial phylotypes in a single sample spectrum or hyperspectral image after labeling with FISH probes. Each of the FISH probes will ultimately be labeled with a different fluorophore with spectral characteristics different than any of the other probes in the assay. Ideally, each probe would be completely resolved from the other probes and single excitation or emission wavelengths could be used for each probe. However, excitation and emission spectra for the available probes are very broad and overlap can be a severe problem, particularly with the 10-60-nm bandpass filters typically used in microscopy. Figure 7 can serve to demonstrate the potential nature of the problem. In Figure 7 there are the fluorescence spectra of oligionucleotides labeled with fluorescein and the indodicarbocyanine dye Cy-3 excited with the $488 \mathrm{~nm} \mathrm{Ar}{ }^{+}$line. While Cy-3 will not interfere with the detection of fluorescein, the fluorescein emission spectrum extends well beyond the optimal wavelengths for Cy-3 detection. If only a single image were available with the typical bandpass filters depicted in the figure, one could conclusively determine the relative number of fluorescein labeled microbes, but may have trouble identifying the relative number of Cy-3 labeled microbes. A second image with a different excitation wavelength would be required to eliminate the contributions of fluorescein. As the number of microbes 


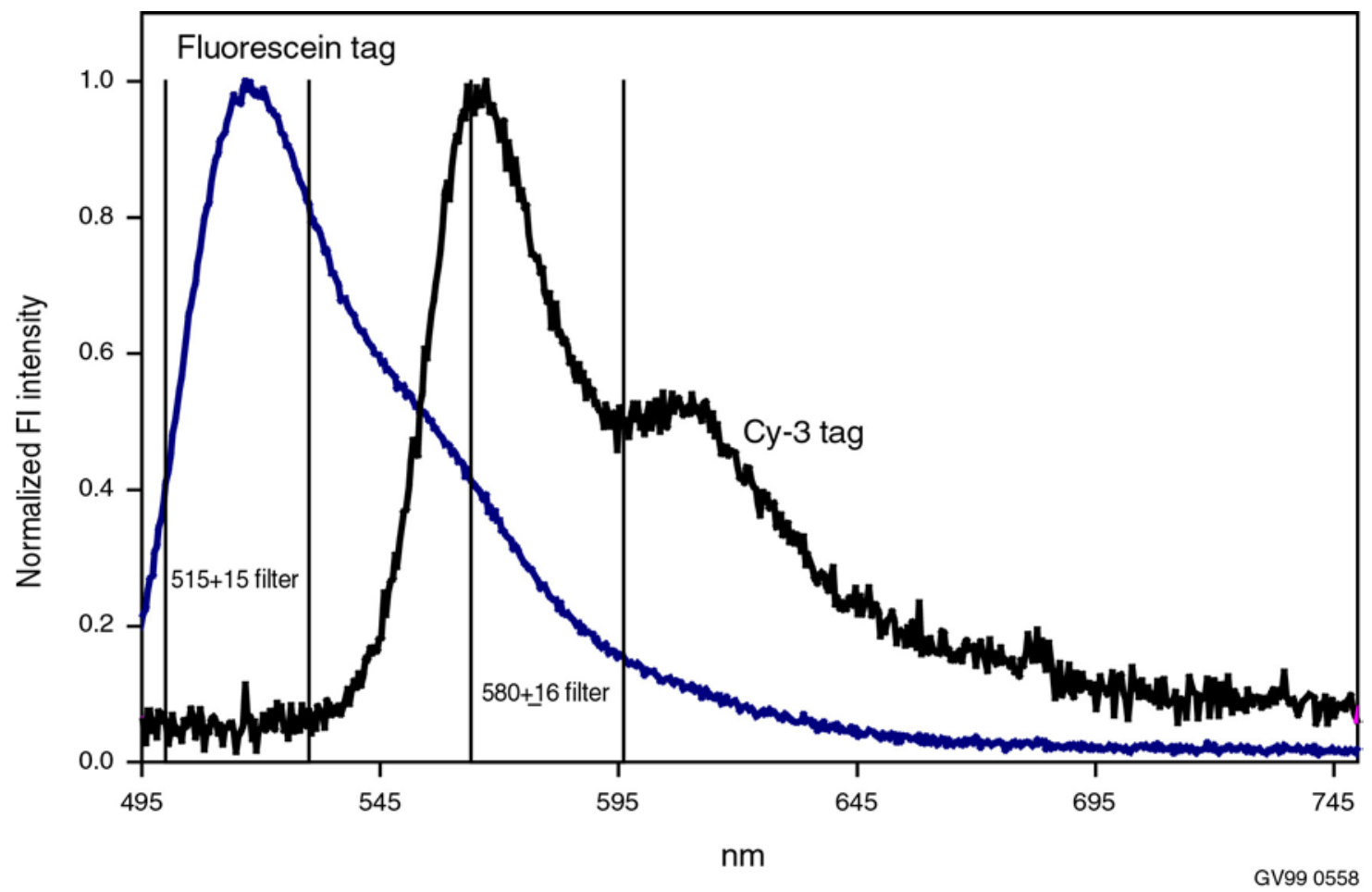

Figure 7. Fluorescence emission spectra of fluorescein and Cy-3 labeled oligionuclides excited with the $488 \mathrm{~nm} \mathrm{Ar}{ }^{+}$ion line. Vertical lines represent the bandpasses of two common filters used for microscopy.

to be labeled with different probes increases, the problem becomes even more complex as a simple change in excitation wavelength will no longer give a clear separation.

This spectral overlap problem can be overcome mathematically by collecting, at a minimum, images (i.e., spectral data points) at $\mathrm{n}$ excitation or emission wavelengths for $\mathrm{n}$ FISH probes and solving for the contributions of each of the probes. Ideally, the images at the $\mathrm{n}$ excitation or emission wavelengths will be recorded simultaneously to limit translocation problems. Images at multiple wavelengths will be collected using the CLSM or a microspectroscopy system equipped with an appropriate CCD camera to collect spatially resolved spectra of cells along the entrance slit.

A laser fluorescence spectroscopy system was built using the $\mathrm{Ar}^{+}$ion and $\mathrm{HeNe}(543 \mathrm{~nm})$ lasers from the CLSM as excitation sources. The output of these lasers was focussed onto a 100- $\mu \mathrm{m}$ optical fiber to transfer the light to a standard 1-cm fluorescence cell. Emitted light was collected and focused on a 500- $\mu \mathrm{m}$ optical fiber and transferred to a $0.3 \mathrm{~m}$ SpectraPro 300i polychrometer (Acton Research Corporation) equipped with a Princeton Instruments TEA1152 CCD detector. This system will be used to design the optical detection schemes to be applied using the CLSM and neat probes and suspensions of FISH labeled cells. The PARISS microspectrometer system mounted on a Nikon E600 microscope was also upgraded by adding a more sensitive Dage DC-330 CCD camera to the spectrometer. The CLSM (Nikon PCM2000) is also currently being upgraded to allow use of laser excitation wavelengths of $488 \mathrm{~nm}(\mathrm{Ar}+), 514 \mathrm{~nm}(\mathrm{Ar}+), 543(\mathrm{HeNe})$, and $633 \mathrm{~nm}(\mathrm{HeNe})$ and simultaneous detection on three channels. With the upgraded CLSM system, it will be possible to spatially and spectrally resolve three probes with each image (i.e., one excitation wavelength and three emission wavelengths), even if they are not completely spectrally resolved. 
A limited number of experiments have been performed using probes labeled with fluorescein, Cy-3, and Cy-5. Fluorescein emission overlaps significantly with Cy-3 when excited at 488 or $514 \mathrm{~nm}$ as the fluorescein emission spectrum tails out to beyond $600 \mathrm{~nm}$. Labeled cell suspensions of Pseudomonas stutzeri and Alcaligenes xylosidans were prepared from cultures grown overnight in nutrient broth. Cells were harvested by centrifugation, washed with phosphate buffered saline (PBS), and fixed by resuspending them in PBS with 3.7\% formalin for 30 minutes at room temperature. The cells were again centrifuged, washed twice with deionized water, resuspended in $200 \mu \mathrm{L}$ of hybridization buffer ${ }^{58}$ containing $5 \mathrm{ng} / \mu \mathrm{L}$ of the probe, and incubated for 2 hours at $46^{\circ} \mathrm{C}$ to "anneal" the probe. After centrifugation, excess probe was removed by washing twice with hybridization wash buffer ${ }^{58}$ and then resuspending the cells in this buffer and incubating for an additional 15 minutes at $48^{\circ} \mathrm{C}$. Cells were once again retrieved by centrifugation and finally resuspended in DI water. In the initial experiment using the laser fluorescence spectrometer, no emission from cells with either the Cy-3 or fluorescein probes were noted. At the time, a 100- $\mu \mathrm{m}$ fiber was being used for collecting the fluorescence emission from the cell. It is not known whether the cells did not accumulate the probe or if the light collection efficiency was too low to record a reasonable spectrum. In a second experiment, the cells were collected on the polycarbonate filters and examined using the PARISS microspectrometer system. Pseudomonas stutzeri did not appear to be hybridized with the fluorescein labeled eubacter DNA probe, but the Alcaligenes xylosidans cells hybridized with the $\mathrm{Cy}-3$ labeled beta probe, fluoresced brightly, and spatially resolved spectra were recorded. Figure 8 is a sample plot showing a spectral image of a mass of cells labeled with $\mathrm{Cy}-3$ positioned on the entrance slit of the spectrometer.

Future work will continue to refine the instrumental configurations and the preparation of the FISH labeling of cells. Once confident that cells can be consistently labeled and detected, we will move to mixed cultures and labeling with multiple FISH probes. Probes will be selected for maximum spectral differences while minimizing the excitation wavelengths that must be used on the CLSM. For example, using the probes fluorescein, Cy-3 and HEX (5-hexachloro-fluorescein), the 488-nm excitation line, and emission filters at 515,540 , and $580 \mathrm{~nm}$, three phylotypes can be unambiguously resolved from a single image. Collecting a second image with a different excitation wavelength with either the same or different set of emission filters should extend the analysis to six phylogenic groups.

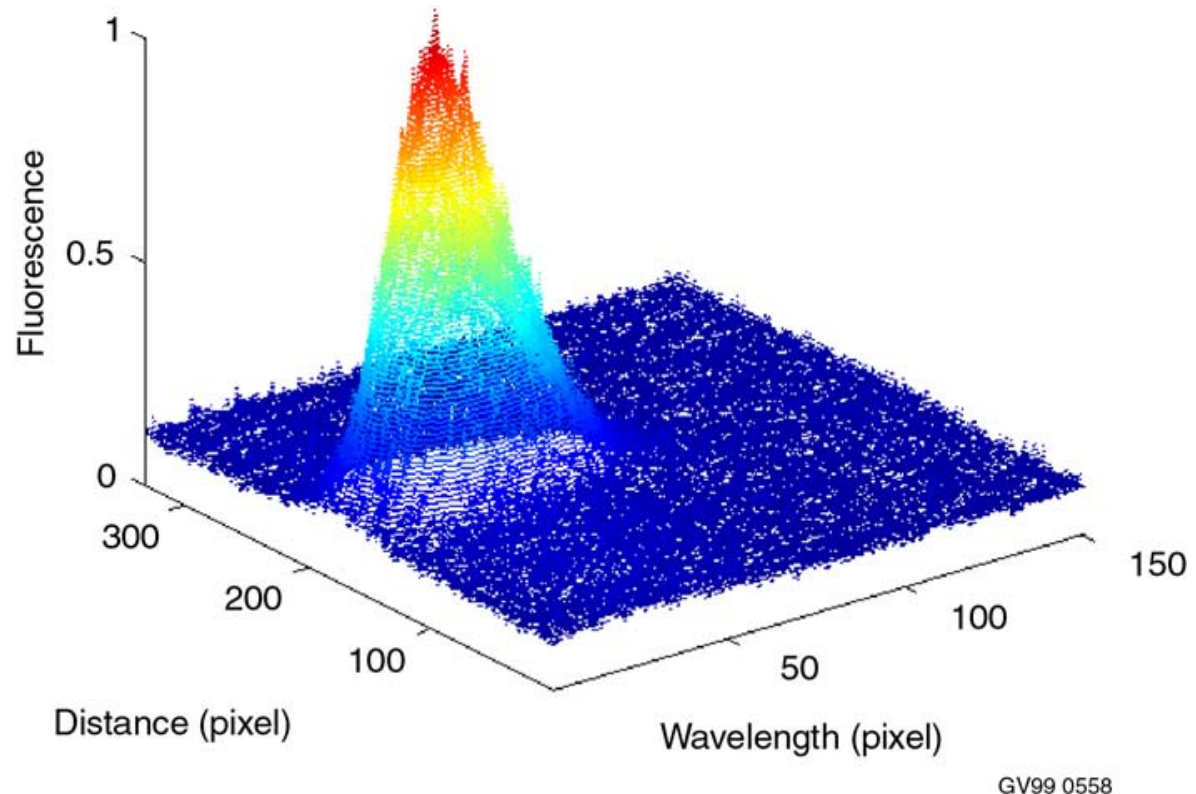

Figure 8. Spectral image showing the placement of Cy-3 labeled cells along the entrance slit of the spectrometer. 
Ultimately, biofilms on actual mineral surfaces will be studied. Because the surfaces will be irregular and 3-D information will be desired, a technique is required to allow this 3-D examination. Such a procedure has been developed at Montana State University using cryosampling and cryosectioning techniques. ${ }^{59}$ This technique, graphically depicted in Figure 9, was evaluated using a biofilm established on a stainless steel coupon obtained from a biocorrosion project at the INEEL. The film was stained with acridine orange, air dried, coated with tissue embedding medium, and rapidly frozen in $-80^{\circ} \mathrm{C}$ freezer. The metal coupon was then removed from the frozen medium leaving the biofilm on the medium. The exposed base of the biofilm was coated with more tissue embedding medium and rapidly frozen again. The sandwiched biofilm was sectioned at $-25^{\circ} \mathrm{C}$ using a cryostat at Idaho State University. The thin sections were mounted onto glass slides and viewed by CLSM. Images from these sections appear similar to the images obtained from the surface films directly; however, some sections indicated a microbial contamination. The possible contamination structures present were not detected on the surface of the biofilm or in the biocorrosion liquid. At this point we do not have enough data to determine if the structures represent a microbial component of the biofilm or an artifact introduced during cryosampling. Future work will use biofilms of known cell populations.

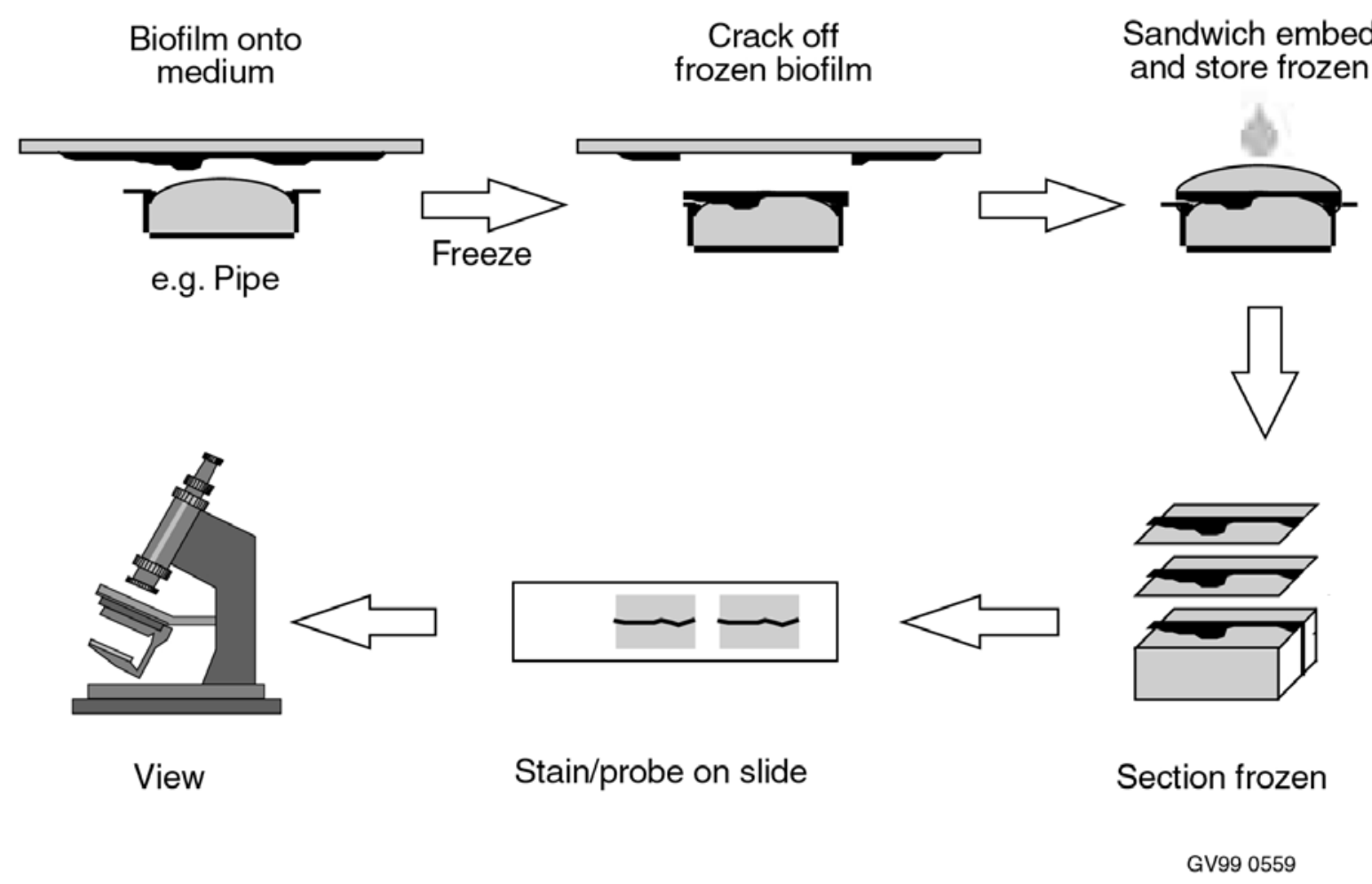

Figure 9. Schematic of process for removing biofilms from solid surfaces. ${ }^{59}$ 


\section{Task 3. Metal and Metal-Ligand Interactions with Mineral Surfaces}

\section{Objective}

The activities in this task can be summed up with the schematic of the model system shown in Figure 10 consisting of a metal contaminant, a multidentate ligand, and an environmentally relevant environmental surface. In this case, the uranyl ion, citrate, and the surface of goethite $(\alpha-\mathrm{FeOOH})$, respectively. Predictive models for solute sorption vary from highly empirical, system-specific approaches, to mechanistic approaches that are based on generalized principles with additive components. Although mechanistic models are difficult to apply due to the extensive parameterization, they are important for identifying how chemical constituents of systems are involved in sorption and to what degree chemical gradients will affect sorption.

Ternary surface complexes that involve multidentate organic ligands, metal species and metal oxides, such as depicted in Figure 10, are generally not well understood. The arrangement of the individual components, the type of bonds, and the distribution of electrostatic charge will be important when attempting to represent the sorption reactions using conceptual models. In the case of the uranyl, citrate, geothite system, high concentrations of citrate have been shown to increase uranyl sorption onto goethite. There are several plausible mechanisms by which citrate could increase uranyl sorption. Each of these potential mechanisms has implications for the types of reaction stoichiometries that can be written to model the ternary interaction, and for the location of electrostatic charge. Providing structural and stoichiometric information for a surface reaction can help constrain the application of complexation models for simulating experimental observations.

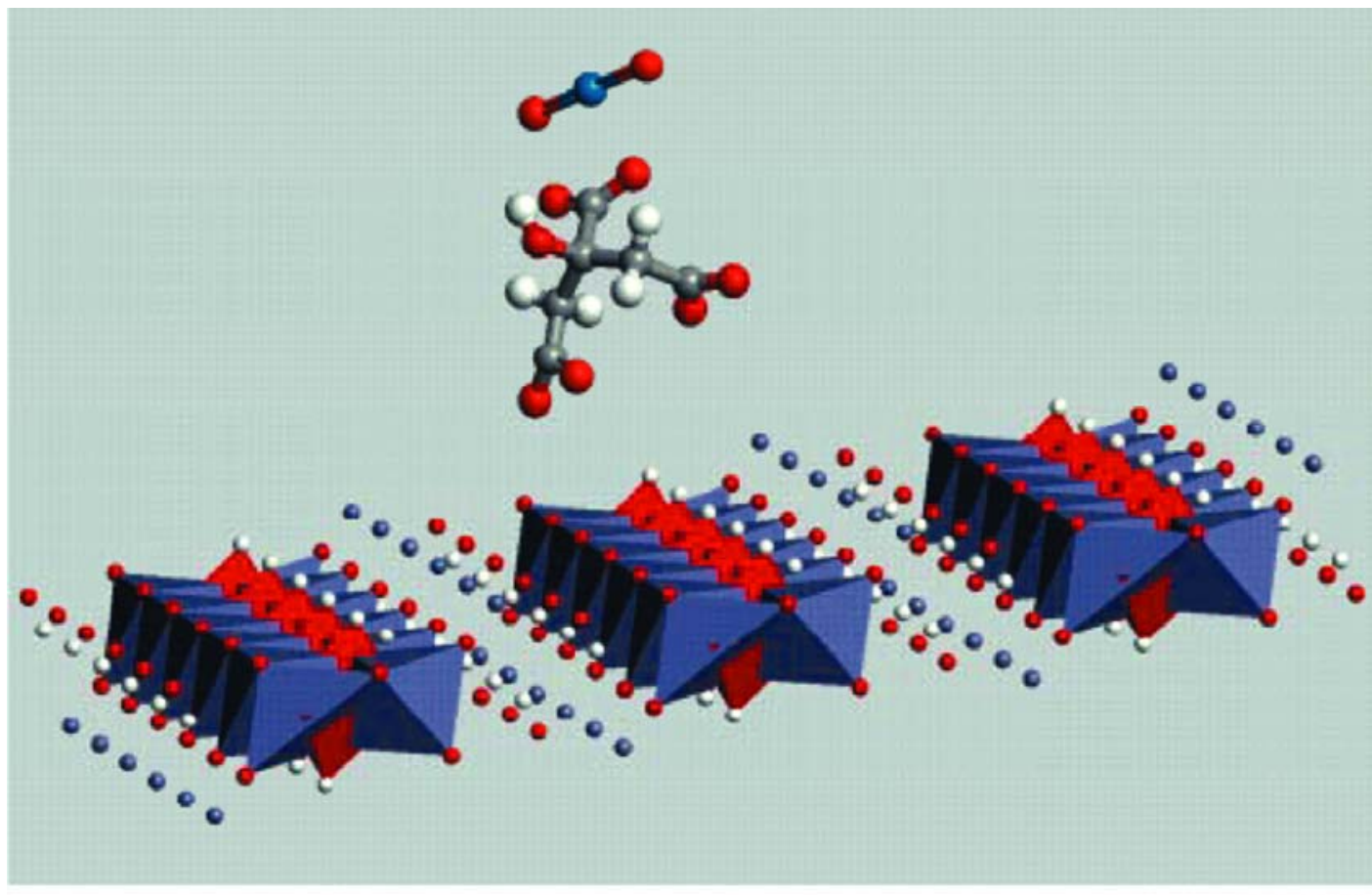

GV99 0537

Figure 10. Schematic of the uranyl, citrate, goethite model system. 
The overall purpose of this task is to study the interactions of metal contaminants and metal-ligand complexes with various mineral surfaces beginning with iron oxides. The approach includes the following activities:

1. Simulation of multidentate ligand complexation on a planar surface to evaluate mass action expression (partition coefficient). This project is designed to evaluate the nature (constant or variable) of a mass action coefficient for multidentate complexes on a planar surface with different arrangements of reactive sites. A statistical dynamics simulation will be used and the work will be in collaboration with Randall LaViolette in the Computational Simulation Project. The result will provide an essential constraint on model simulations of surface complexes at mineral surfaces.

2. Evaluation of the kinetics and reversibility of metal and metal-ligand surface complexes. The rate and reversibility of metal complexation is being evaluated in parallel for field and model substrates and representative solutes. This includes monitoring proton stoichiometry and the variable of solute addition sequence in the case of metal-ligand systems. Data are being collected from batch reactors controlled with an automated chemostat/titrator. The results will address specific contaminant transport modeling issues, especially in high flow vadose zones and where colloid transport is significant. Carbonate (and carbon dioxide concentrations) is one variable given its significant and variable concentration in the vadose zone. $^{9-11}$ This work is being performed in collaboration with Larry Hall and Bob Smith of the Reactive Transport in Variably Saturated Heterogeneous Media Project.

3. Laboratory studies of ternary complexation. Studies of metal complexation in the presence of organic complexing ligands using extended X-ray absorption fine structure spectroscopy (EXAFS) and attenuated total reflectance Fourier transform infrared spectroscopy (ATR-FTIR) have been continued. The EXAFS and ATR-FTIR work was done in collaboration with researchers at the Stanford Synchrotron Light Source and the Umea University, respectively. One specific study involving citrate and uranyl has been concluded and the study is now being extended to at least one new system that involves EDTA and ultimately actual microbially produced ligands. This activity represents a basic science investigation of the nature of ternary complexes and will be used for comparison with measured complexation stoichiometry and results from the evaluation of the mass action expression for multidentate surface complexation.

\section{Experimental Activities}

Two customized chemostats were assembled for conducting experiments where precise control of electrode-sensitive solutes, or potentiometric titrations are required. Commercially available chemostats are not suitable for the type of control needed in this project and are not easily modified for different research applications. These chemostats are expected to have a broad range of applications for the environmental research conducted at INEEL. For this project, the chemostats are initially being used to characterize the surface charging characteristics of model substrates (e.g., iron oxides and aluminosilicates), colloids, and more complex field samples. They will also be used to characterize the stoichiometries of surface complexation reactions involving heavy metals and organic chelating ligands. A schematic of the titrators is shown in Figure 11.

An analysis of the citrate + uranyl + goethite system using EXAFS was concluded. The EXAFS technique, in this case, probes the nearest neighbor atomic environment of uranium and provides information about the bonding environment of uranyl. For example, by comparison with model systems, and from theoretical modeling, it is possible to distinguish oxygen from water of hydration, axial oxygen 


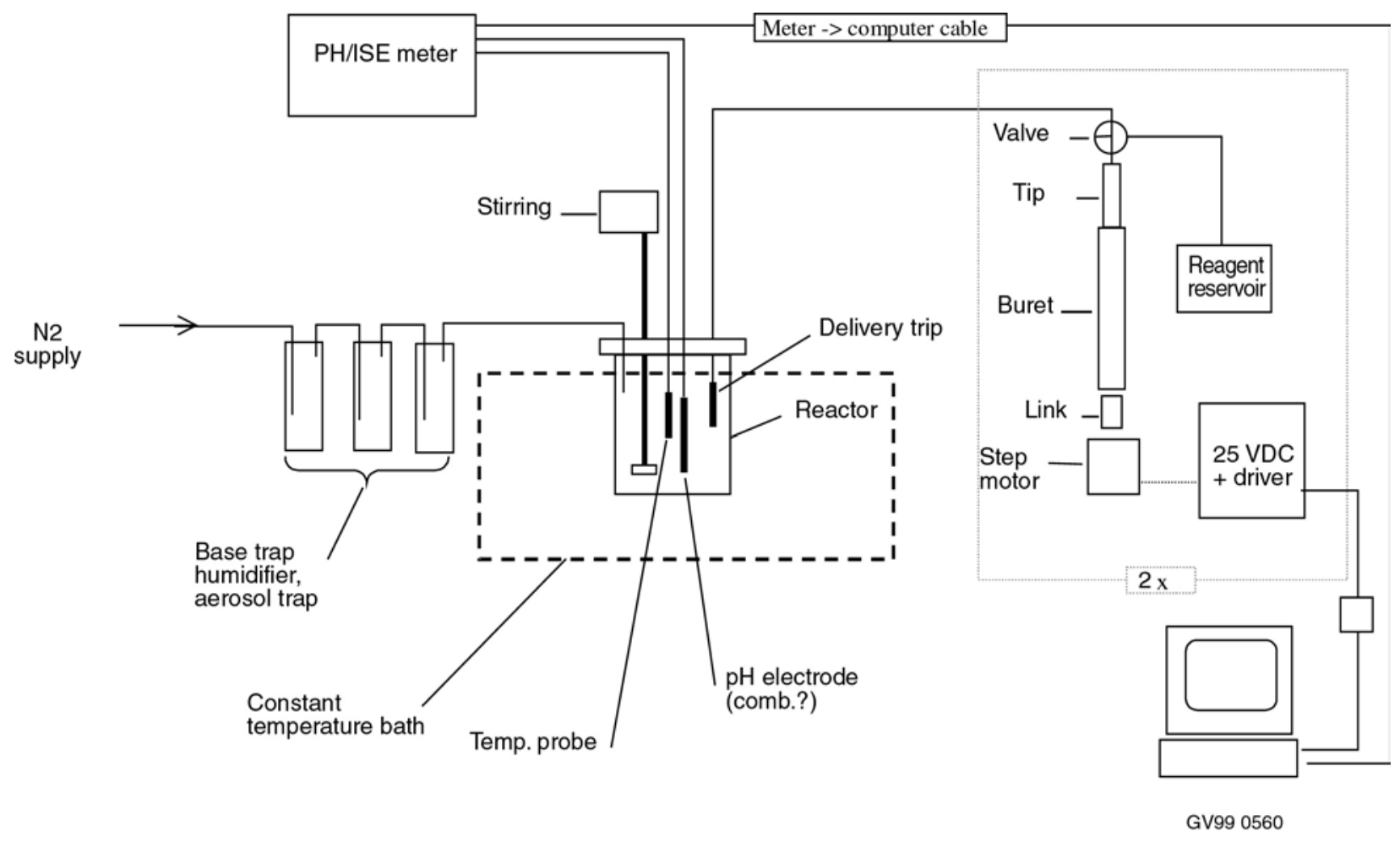

Figure 11. Schematic of chemostat designed for precise measurement and control of experimental conditions.

of the UO22+ complex, oxygen from coordinated carboxylic acid groups, etc. An example of the EXAFS spectra that were collected for our system is shown in Figure 12.

The intention in this work was to provide information that will help constrain the development of predictive models that describe surface complexation in ternary systems (metal + ligand + oxide). The current models employ electrostatic fields in describing the nature and stability of surface complexes, and location of charge sites has become critical. The summary conclusion from this work is that uranyl forms an inner-sphere coordination with carboxylate groups of citrate and that citrate is also coordinated with the oxide surface. At higher $\mathrm{pH}$ values, the speciation changes with uranyl directly complexed to the surface and citrate forming a weaker outer-sphere association with either the charged uranyl sites, or the protonated oxide surface sites. What does not take place at lower $\mathrm{pH}$ values is the formation of a weak outer-sphere complexation between the charged oxide surface and a uranyl-citrate anion. The weaker outer-sphere complexes would be expected to undergo more rapid sorption/desorption reactions. With respect to modeling, this work will help constrain the location of charge sites and the influence of electrostatic interactions in the free energy of sorption reactions.

In future work, the relation between the surface speciation and bioavailability of the metal and ligand will be explored. This work is now being extended to a study of the EDTA + uranyl + iron oxide system. The engineering and remediation applications are in the area of contaminant transport in the presence of co-contaminants or complexing agents.

In addition to EXAFS, an ATR-FTIRS study has been completed on this system in collaboration with the Inorganic Chemistry Department at the University of Umeå, Sweden. EXAFS provides information on the bonding environment of the metal atom, but does not provide information about the organic ligand. The ATR-FTIRS study provides the additional information needed to establish 


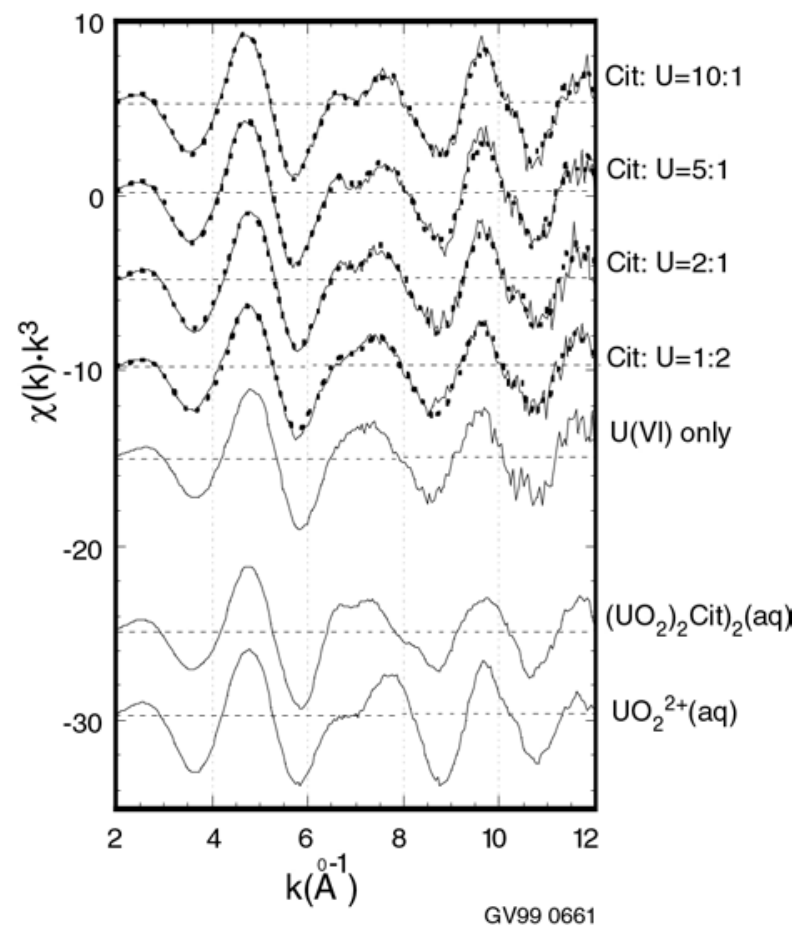

Figure 12. EXAFS spectra for the citrate + uranyl + goethite system at $\mathrm{pH}$ 5.5. Two model solution species are included at the bottom of the plot.

consistency in the spectroscopic interpretations. The goals of the study are the same as described for the EXAFS work with respect to complexation model development, and understanding issues of contaminant transport and bioavailability. An sample set of spectra from this study is shown in Figure 13.

Based on the ATR-FTIRS data, we are able to conclude that citrate is directly coordinated with the iron oxide surface. Combined with the sorption and surface charge data that have already been collected, we can conclude that citrate acts, in a sense, to modify the iron oxide surface and provide sites for complexing Lewis acids such as heavy metals. The direct coordination with uranyl is confirmed and the nature of the surface complex has been shown to be distinct from the structures of soluble species that exist under the same chemical conditions. In addition, the shift in speciation has been confirmed. This last observation is considered very important since the change in speciation occurs where there is no chemical signature in the macroscopic sorption data (both ligand and metal are 100\% sorbed). Therefore, this is a demonstration that the sorption models cannot, under certain conditions, be used for molecular structure interpretation (as is frequently done).

In support of the environmental concerns of the INEEL, a project is being conducted to study the sorption of uranium(VI) on soil samples from the INEEL. There are two primary objectives in this work. One is to provide, at a minimum, empirical data describing the chemical parameters controlling uranyl migration in the soil. The second is to illustrate the extent to which the behavior of the soil can be described as a set of component sorption reactions on individual mineral constituents. There is currently a great deal of work being done to refine the models that describe surface reactions between mineral oxides and contaminant solutes. However, employing the more fundamental models requires a level of parameterization that is not practical on a field scale. Therefore, this project is attempting to define the limitations of the reductionist approach, but at the same time to explore alternative approaches that, in the 


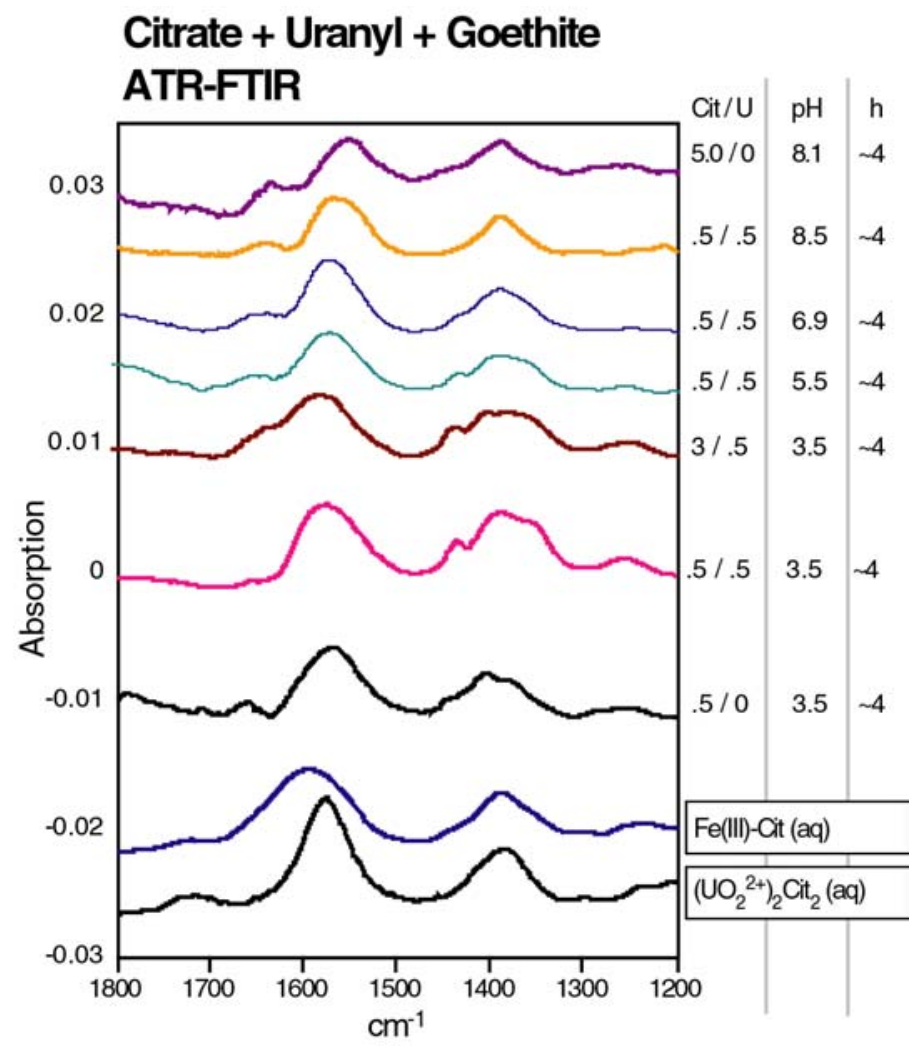

Figure 13. ATR-FTIR spectra from an iron oxide surface with uranyl sorbed in the presence of citric acid.

absence of complete parameterization, can still capture the chemical processes that are important for describing contaminant transport and therefore the feasibility of remediation techniques. This project is still in the initial stages with both column studies of uranyl migration in soil samples plus "calibration" exercises that are required to validate the experimental methods that will be used to characterize surface charge and sorption on mineral isolates from the soil samples.

\section{ACCOMPLISHMENTS}

\section{Microbial Interactions with Environmental Surfaces}

- $\quad$ To overcome reproducibility problems associated with the bacterial cultures used in our previous experiments, a pure culture of Shewanella putrefaciens was acquired from the American Type Culture Collection and experiments using the Shewanella alga MR1 culture were terminated. To ensure that all samples in long exposure experiments were incubated under identical conditions, a continuous flow bioreactor was constructed for use in all future experiments.

- $\quad$ Experiments were conducted using the continuous flow bioreactor to expose hematite surfaces to Shewanella putrefaciens with the goal of identifying physical attachment features of the bacteria to the surface under anaerobic conditions. Analysis of the exposed hematite surfaces with AFM revealed no obvious physical attachment features. Although no specific attachment structures were noted, cells were noted to be associated with the surface in relatively well-organized groups. In such groups, the bacteria may be able to control their immediate chemical environment more effectively. 
- To probe the microchemical environments that may be controlled by environmental microbes, method development continued for examining intracellular $\mathrm{pH}$ with fluorescent dyes. Cells are loaded with a $\mathrm{pH}$ sensitive dye as an acetoxymethyl ester and intracellular esterase activity converts the dye back to the membrane impermeable form. Under certain growing conditions, the intracellular enzyme activity in $S$. spp. appears to be low and results in minimal fluorescent dye in the cells. By loading the cells in an extremely nutrient limiting environment, e.g., deionized water, the enzyme activity appears to be slightly enhanced and the cells fluoresce brighter. It was found that using a full spectrum approach with multivariate analysis, more precise determinations of $\mathrm{pH}$ could be obtained from cells in suspension. Attempts at quantitation using the confocal microscope were not routinely successful, partially because of the issues related to loading the dye, but primarily due to the poor signal-to-noise-ratio on the green channel and the limitation of the 8-bit A/D. We are currently working on methods to effectively filter the noise from the many images on file. Images have been obtained of bacteria on membrane filters and on a hematite surface under varying conditions.

- While pH may play an important role in microbial utilization of iron oxide surfaces, iron chelating and reducing compounds produced by the bacteria also play a vital role in the iron reduction mechanism. Work was continued on identifying and isolating siderophores and "iron-reducing enzymes" so that the chemical interaction of these compounds directly with an iron oxide surface could be observed. A series of compounds only found when Shewanella was grown in the presence of insoluble iron oxides have been observed with HPLC. One of these compounds was positively identified as a siderophore. Spectroscopic evidence indicates that two additional compounds identified in a separate HPLC procedure are likely to be extracellular cytochromes. The quantities of each appear to be dependent on the aerobic condition of the medium.

\section{Method Development to Examine Microbial Grouping and Their Effects on Environmental Surfaces}

- Work was initiated to develop a method for the identification of multiple microbial phylotypes in/on a sample using fluorescent in situ hybridization with multiple dyes. Fluorescence spectroscopy and hyperspectral imaging will be used to spectrally and spatially resolve the microbial phylotypes. A laser fluorescence spectroscopy system was built using $\mathrm{Ar}^{+}$and $\mathrm{HeNe}$ lasers as illumination sources and a polychrometer with a CCD detector for spectral data acquisition. Hyperspectral images were collected using an epiluminescence microscope equipped with a PARISS microspectrometer equipped with a CCD for collection of spatially resolved spectra from a microscope slide.

- $\quad$ Preliminary experiments to demonstrate the potential methodology were conducted using both the spectrometer and the hyperspectral imaging system on bacteria labeled with probes having fluorescein, $\mathrm{Cy}-3$ or $\mathrm{Cy}-5$ fluorescent labels.

\section{Metal and Metal-Ligand Interactions with Mineral Surfaces}

- $\quad$ Extended X-ray absorption fine structure and attenuated total reflectance Fourier transform infrared spectroscopic studies were performed on a ternary system of citrate + uranyl + goethite. The conclusions of these studies were consistent and indicate that at low to moderate $\mathrm{pH}$, citrate is directly complexed to the oxide surface and the uranyl is complexed to this. At higher $\mathrm{pH}$, uranyl is complexed directly to the surface. 
- Two customized chemostats were assembled for conducting experiments where precise control of electrode-sensitive solutes, or potentiometric titrations are required. These chemostats are being used in experiments that were initiated to study the sorption of uranium(VI) on soil samples from the INEEL and to illustrate the extent to which the behavior of the soil can be described as a set of component sorption reactions on individual mineral constituents.

\section{REFERENCES}

1. D. L. Miller, Environmental Management Core Competency Enhancement Technical Capabilities Enhancement Program Definition, INEEL, 1998.

2. INEEL, Systems Engineering Analysis for the Environmental Management Science Programs, INEEL, 1997.

3. C. H. Hemming, R. L. Bunde, M. J. Liszewski, J. J. Rosentreter, and J. Welhan, "Effect of Experimental Technique on the Determination of Strontium Distribution Coefficients of a Surficial Sediment from the Idaho National Engineering Laboratory, Idaho," Water Research, 31, 1997, pp. 1629-1636.

4. S. A. Ibrahim and R. C. Morris, "Distribution of Plutonium Among Soil Phases Near a Subsurface Disposal Area in Southeastern Idaho, USA," Journal of Radioanalytical and Nuclear Chemistry, 226, 1997, pp. 217-220.

5. T. M. Beasley, P. R. Dixon, and L. J. Mann, " 99 Tc, ${ }^{236} \mathrm{U},{ }^{237} \mathrm{~Np}$ in the Snake River Plain Aquifer at the Idaho National Engineering and Environmental Laboratory, Idaho Falls, Idaho," Environmental Science and Technology, 32, 1998, pp. 3875-3881.

6. J. Shervais, S. Vetter, and W. Hackett, "Chemical Stratigraphy of Basalt in Coreholes NPR-E \& WO-2, Idaho National Engineering Laboratory, Idaho: Implications for Plume Dynamics in the Snake River Plain," Proceedings VII ${ }^{\text {th }}$ International Symposium on the Observation of the Continental Crust Through Drilling, 1994.

7. C. Klein, C. and J. C. S Hurlbut, Manual of Mineralogy, $20^{\text {th }}$ ed., New York: John Wiley and Sons, 1985 , p. 485.

8. L. A. Morgan, Lithologic Log of Site E Corehole, Idaho National Engineering Laboratory, Letter Report from USGS to W.F. Downs, EGG Idaho, USGS, NPR-DEIS-C-059, 1985.

9. R. C. Bartholomay, L. L. Knobel, and L. C. Davis, Mineralogy and Grain Size of Surficial Sediment from the Big Lost River Drainage and Vicinity with Chemical and Physical Characteristics of Geologic Materials from Selected Sites at the Idaho National Engineering Laboratory, Idaho, USDOE/USGS, USGS Open-file Report 89-384, DOE/ID-22081, 1989.

10. M. R. R. Reed and R. C. Bartholomay, Mineralogy of Selected Sedimentary Interbeds At or Near the Idaho National Engineering Laboratory, Idaho, USDOE/USGS, USGS Open-File Report 94-374, DOE/ID-22116, 1994.

11. U.S. DOE, Groundwater/Vadose Zone Integration Project Specification: Appendix G Current State of Technical Knowledge, DOE/RL-98-48, Draft C, 1998. 
12. L. J. M. Matheson and P. G. Tratnyek, "Reductive Dehalogenation of Chlorinated Methanes by Iron Metal,” Environmental Science and Technology, 28, 1994, pp. 2045-2053.

13. W. S. O. Orth and R. W. Gillham, "Dechlorination of Trichloroethene in Aqueous Solution Using $\mathrm{Fe}^{0}$," Environmental Science and Technology, 30, 1996, pp. 66-71.

14. D. I. Kaplan, K. J. Cantrell, T. W. W. Wietsma, and M. A. Potter, "Retention of Zero-Valent Iron Colloid by Sand Columns: Application to Chemical Barrier Formation," Journal of Environmental Quality, 25, 1996.

15. J. C. Ingram, personal communication, 1998.

16. D. R. Lovley and D. R. Lonergan, "Anaerobic Oxidation of Toluene, Phenol, and p-cresol by the Dissimilatory Iron-Reducing Organism, GS-15,” Applied and Environmental Microbiology, 1990, pp. 1858-1864.

17. H. L. Ehrlich, "Geomicrobiology, $2^{\text {nd }}$ ed., rev. and expanded," in: Geomicrobiology, $2^{\text {nd }}$ ed., rev. and expanded New York: Marcel Dekker, Inc., 1990, pp. 282-346.

18. K. H. Nealson and C. R. Myers, "Iron Reduction by Bacteria: A Potential Role in the Genesis of Banded Iron Formations," American Journal of Science, 290A, 1990, pp. 35-45.

19. C. Zhang, H. Vali, S. Siu, R. Roh, D. Cole, J. L. Kirschvink, T. Onsttot, D. Mckay, and T. Phelps, "Formation of Magnetite and Iron-Rich Carbonates by Thermophilic Iron-Reducing Bacteria," Proceedings of SPIE-International Society for Optical Engineering, 3111, 1997, pp. 61-68.

20. C. Zhang, S. Liu, T. J. Phelps, D. R. Cole, J. Horita, S. M. Fortier, M. Elless, and J. W. Valley, "Physiochemical, Mineralogical, and Isotopic Characterization of Magnetite-Rich Iron Oxided Formed by Thermophilic Iron-Reducing Bacteria," Geochimica et Cosmochimica Acta, 61, 1997, pp. 4621-4632.

21. D. R. Lovley, "Microbial Fe(III) Reduction in Subsurface Environments," FEMS Microbiology Reviews, 20, 1997, pp. 305-313.

22. P. M. Bradley and F. H. Chapelle, "Anaerobic Mineralization of Vinyl Chloride in Fe(III) Reducing Aquifer Sediments,” Environmental Science and Technology, 30, 1996, pp. 2084-2086.

23. C. Heijman, G., C. Holliger, M. A. Glaus, R. P. Scharzenbach, and J. Zeyer, "Abiotic Reduction of 4-chloronitrobenzene to 4-chloroaniline in a Dissimilatory Iron-Reducing Enrichment Culture," Applied and Environmental Microbiology, 59, 1993, pp. 4350-4353.

24. J. R. Lloyd and L. E. Macaskie, "A Novel PhosphorImager-Based Technique for Monitoring the Microbial Reduction of Technetium," Applied and Environmental Microbiology, 62, 1996, pp. $578-582$.

25. y.-T. Wang and H. Shen, "Modelling Cr(VI) Reduction by Pure Bacterial Cultures," Water Research, 31, 1997, pp. 727-732.

26. Y. A. Gorby, J. Caccavo, and Frank and J. H. Bolton, "Microbial Reduction of Cobalt $\mathrm{III}_{\mathrm{EDTA}}$ - in the Presence and Absence of Manganese(IV) Oxide," Environmental Science and Technology, 32, 1998, pp. 244-250. 
27. R. Ganesh, K. G. Robinson, G. D. Reed, and G. S. Sayler, "Reduction of Hexavalent Uranium from Organic Complexes by Sulfate- and Iron-Reducing Bacteria," Applied and Environmental Microbiology, 1997, p. 11.

28. R. Mazoy and M. L. Lemos, "Ferric-Reductase Activities in Whole Cells and Cell Fractions of Vibrio (Listonella) anguillarum," Microbiology, 142, 1996, pp. 3187-3193.

29. D. R. Lovely, E. J. P. Phillips, and D. J. Lonergan, "Enzymatic versus Nonenzymatic Mechanisms for Fe(III) Reduction in Aquatic Sediments," Environmental Science and Technology, 25, 1991, pp. 1062-1067.

30. D. J. Workman, S. L. Woods, Y. A. Gorby, J. K. Fredrickson, and M. J. Truex, "Microbial Reduction of Vitamin $\mathrm{B}_{12}$ by Shewanella alga Strain BrY with Subsequent Transformation of Carbon Tetrachloride," Environmental Science and Technology, 31, 1997, pp. 2292-2297.

31. C. R. Myers and J. M. Myers, "Cloning and Sequence of cymA, a Gene Encoding a Tetraheme Cytochrome $c$ Required for Reduction of Iron(III), Fumarate and Nitrate by Shewanella putrefaciens MR-1," Journal of Bacteriology, 179, 1997, pp. 1143-1152.

32. H. G. Deneer, V. Healey, and I. Boychuk, "Reduction of Exogenous Ferric Iron by a SurfaceAssociated Ferric Reductase of Listeria spp.," Microbiology, 141, 1995, pp. 1985-1992.

33. A. E. Le Faou and S. A. Morse, "Characterization of a Soluble Ferric Reductase from Neisseria gonorrhoeae," Biology of Metals, 4, 1991, pp. 126-131.

34. A. Dancis, D. G. Roman, G. J. Anderson, A. G. Hinnebusch, and R. D. Klausner, "Ferric reductase of Saccharomyces cerevisiae: Molecular Characterization, Role in Iron Uptake and Transcriptional Control by Iron," Proceedings of the National Academy of Sciences USA, 89, 1992, pp. 3869-3873.

35. K. P. Shatwell, A. Dancis, A. R. Cross, R. D. Klausner, and A. W. Segal, "The FRE1 Ferric Reductase of Saccharomyces cerevisiae Is a Cytochrome b Similar to That of NADPH Oxidase," The Journal of Biological Chemistry, 271, 1996, pp. 14240-14244.

36. T. M. Louie, S. Ni, L. Xun, and W. W. Mohn, "Purification, Characterization, and Gene Sequence Analysis of a Novel Cytochrome $c$ Co-Induced with Reductive Dechlorination Activity in Desulfomonile tiedjei DCB-1," Archives in Microbiology, 168, 1997, pp. 520-527.

37. J. Caccavo, P. Frank, C. Schamberger, K. Keiding, and P. H. Nielsen, "Role of Hydrophobicity in Adhesion of the Dissimilatory Fe(III)-Reducing Bacterium Shewanella alga to Amorphous Fe(III) Oxide," Applied and Environmental Microbiology, 63, 1997, pp. 3837-3843.

38. M.C. Grantham, P. M. Dove, and T. J. DiChristina, "Microbially Catalyzed Dissolution of Iron and Aluminum Oxyhydroxide Mineral Surface Coatings," Geochimica et Cosmochimica Acta, 61, 1997, pp. 4467-4477.

39. M. C. Grantham and P. M. Dove, "Investigation of Bacterial-Mineral Interactions Using Fluid Tapping Mode Atomic Force Microscopy," Geochimica et Cosmochimica Acta, 60, 1996, pp. 2473-2480. 
40. J. M. Vroom, K. J. De Grauw, H. C. Gerritsen, D. J. Bradshaw, P. D. Marsh, G. K. Watson, J. J. Birmingham, and C. Allison, "Depth Penetration and Detection of pH Gradients in Biofilms by Two-Photon Excitation Microscopy," Applied and Environmental Microbiology, 65, 1999, pp. 3502-3511.

41. P. A. Maurice, M. F. Hochella Jr., G. A. Parks, G. Sposito, and U. Schwertmann, "Evolution of Hematite Surface Microtopography Upon Dissolution with Simple Organic Acids," Clays and Clay Minerals, 43, 1995, pp. 29-38.

42. Y. Deng, "Effect of pH on the Reductive Dissolution Rates of Iron(III) Hydroxide by Ascorbate," Langmuir, 13, 1997, pp. 1835-1839.

43. J. B. Neilands, "Siderophores: Structure and Function of Microbial Transport Compounds," Journal of Biological Chemistry, 270, 1995, pp. 26723-26726.

44. B. A. Holmén, J. D. Sison, D. C. Nelson, and W. H. Casey, "Hydroxamate Siderophores, Cell Growth and Fe(III) Cycling in Two Anaerobic Iron Oxidemedia Containing Geobacter metallireducens," Geochimica et Cosmochimica Acta, 63, 1999, pp. 227-239.

45. P. S. Dobbin, L. M. R. Burmeister, S. L. Heath, A. K. Powek, A. G. McEwan, and D. J. Richardson, "The Influence of Chelating Agents Upon the Dissimilatory Reduction of Fe(III) by Shewanella putrifaciens. Part 2. Oxo- and hydroxo-bridged polynuclear Fe(III) complexes," Biometals, 9, 1996, pp. 291-301.

46. O. Ardon, H. Weizman, J. Libman, A. Shanzer, Y. Chen, and Y. Hadar, "Iron Uptake in Ustilago maydis: Studies with Fluorescent Ferrichrome Analogue," Microbiology, 143, 1997, pp. 3625-3631.

47. B. F. Matzanke, E. Bill, A. X. Trautwein, and G. Winkelmann, "Ferricrocin Functions as the Main Intracellular Iron-Storage Compound in Mycelia of Neurospora crassa," Biology of Metals, 1, 1988, pp. 18-25.

48. L. Hersman, P. Maurice, and G. Sposito, Chem. Geol., 132, 1996, pp. 25-31, "Iron Acquisition from Hydrous Fe(III)-Oxides by Aerobic Pseudomonas sp," Chemical Geology, 132, 1996, pp. 25-31.

49. M. Pourbaix, Atlas of Electrochemical Equilibria in Aqueous Solutions, $2^{\text {nd }}$ ed., Houston: National Association of Corrosion Engineers, 1974.

50. S. Seeliger, R. Cord-Ruwisch, and B. Schink, "A Periplasmic and Extracellular c-Type Cytochrome of Geobacter sulfurreducens Acts as a Ferric Iron Reductase and as an Electron Carrier to Other Acceptors or to Partner Bacteria,” Journal of Bacteriology, 180, 1998, pp. 3686-3691.

51. H. Siegumfeldt, K. B. Rechinger, and M. Jakobsen, "Use of Fluorescence Ratio Imaging for Intracellular $\mathrm{pH}$ Determinations of Individual Bacterial Cells in Mixed Cultures," Microbiology, 145, 1999, pp. 1703-1709.

52. R. P. Haugland, Handbook of Fluorescent Probes and Research Chemicals, 6th ed., Eugene, OR.: Molecular Probes, Inc., 1996. 
53. R. Henrion, G. Henrion, M. Böhme, and H. Behrendt, "Three-Way Principal Components Analysis for Fluorescence Spectroscopic Classification of algae species," Fresenius' Journal of Analytical Chemistry, 357, 1997, pp. 522-526.

54. D. M. Haaland and E. V. T. Thomas, "Partial Least-Squares Methods for Spectral Analyses. 1. Relation to Other Quantitative Calibration Methods and the Extraction of Qualitative Information," Analytical Chemistry, 60, 1988, pp. 1193-1201.

55. B. Schwyn and J. B. Neilands, "Universal Chemical Assay for the Detection and Determination of Siderophores," Analytical Biochemistry, 160, 1987, pp. 47-56.

56. C.-H. Lee, T. A. Lewis, A. Paszcynski, and R. L. Crawford, "Identification of an Extracellular Catylst of Carbon Tetrachloride Dehalogenation from Pseudomonas stutzeri Strain KC as Pyridine-2,6-bis(thiocarboxylate)," Biochemical and Biophysical Research Communications, 261, 1999, pp. 562-566.

57. R. Amann, W. Ludwig, and K. Schleifer, "Phylogentic Identification and In Situ Detection of Individual Microbial Cells without Cultivation," Microbiological Reviews, 59, 1995, pp. 143-169.

58. W. Manz, R. Amann, W. Lugwig, M. Wagner, and K. Schleifer, "Phylogenetic Oligodeoxynucleotide Probes for the Major Subclasses of Proteobacteria: Problems and Solutions," Systematic Applied Microbiology, 15, 1992, pp. 593-600.

59. J. A. Flood, N. J. Ashbolt, and P. J. Beatson, "Simultaneous Morphological and Population Analyses of Environmental Biofilms," Investigation of Biofilms., 1999, in press.

\section{APPENDIX}

\section{Peer Reviewed Publications}

W. F. Bauer, J. M. Barnes, G. Elias, and P. Pryfogle, “An Improved Method for Determination of Intracellular pH Using Fluorescence Spectroscopy," in preparation of Analytical Biochemistry.

W. F. Bauer, J. M. Barnes, G. Elias, G. Redden, and P. Pryfogle, "Identification of Siderophores and Cytochromes Released by Microbial Cells," in progress.

G. Redden, J. Bargar, and R. Bencheikh-Latmani, 1999, Citrate Enhanced Sorption of U(VI) on Goethite: Characteristics Of A Ternary Complex Using EXAFS, submitted to Environmental Science and Technology.

\section{Presentations}

M. Vierkorn, P. Maurice and W.F. Bauer, "Assessing the Importance of Microbial Attachment in Mineral Dissolution: TMAFM of Interactions Between Shewanella alga Bacteria and Fe Oxide Surfaces," Meeting of the Geological Society of America, October 26-29, 1998, Toronto, Canada.

G. Redden, J. Bargar, and Per Persson, 1999, "Structure of a Ternary Goethite-Citrate-Uranyl Surface Complex," $217^{\text {th }}$ ACS National Meeting, May 1999, Anaheim, California.

G. Redden, "A Sorption Experiment and Approaches to Complexation Modeling: A Discussion on Goals, Model Applications, Model Constraints, and Environmental Applications," Lecture given at 
Stanford University for CEE 373: Special Topics In Aquatic Chemistry Solute Interactions At Solid-Solution Interfaces, 1999, Stanford, California.

W.F. Bauer, J.M. Barnes, G. Elias, and P.A. Pryfogle, "Measurement of pH in Microbial Microenvironments Near Mineral Surfaces," XIV ${ }^{\mathrm{th}}$ International Symposium on Environmental Biogeochemistry, September 26-30, 1999, Toronto, Canada.

G. Redden, J. Bargar, R. Bencheikh-Latmani, and W. Bauer, "Surface Interactions Between Goethite, Citrate and Uranyl: Linking Surface Chemistry to Issues of Microbial Influences on Metal Partitioning and Bioavailability," XIV ${ }^{\text {th }}$ International Symposium on Environmental Biogeochemistry, September 26-30, 1999, Toronto, Canada.

Dr. Larry Hull, Dr. George Redden, and Ms. Mary Pace, "Adsorption Parameters for Raffinate Migration," to be presented at the Materials Research Society Fall Meeting, November 29-December 3, 1999, Boston, Massachusetts.

George Redden and James A. Davis, 1999, "Surface Complexation Models in Solute Transport: Issues of Practicality and Applicability for Transport Modeling," to be presented at the Materials Research Society Fall Meeting, November 29-December 3, 1999, Boston, Massachusetts. 


\title{
Reactive Transport in Variably Saturated Heterogeneous Media
}

\author{
R. W. Smith, R. W. Johnson, L. C. Hull, E. D. Mattson, A. L. Schafer, \\ J. B. Sisson, D. N. Thompson, M. N. Pace, and D. L. Suarez \\ SUMMARY
}

The Idaho National Engineering and Environmental Laboratory (INEEL) through the Environmental Systems Research Program embarked in FY 1998 on a three-year project with the objectives of developing a better understanding of the movement and transformation of reactive constituents in heterogeneous, variably saturated subsurface media (e.g., vadose zone) and parlaying this better understanding into improved estimates of the fate and mobility of reactive constituents in the vadose zone. The Environmental Systems Research Program is organized into five technical theme areas. This project along with "Advanced Strategies for Probing Structure and Reactivity at the Top Monolayer (fundamental analytical surface chemistry)," "Biochemical and Geochemical Reactions on Environmental Surfaces (interfacial processes research)," and "Transport Phenomena in Geologic Porous Media (experimental-based evaluation of biogeochemical processes in porous media)" comprise the Chemistry of Environmental Surfaces theme area. There are two specific goals of this project :

The first is to build upon existing INEEL expertise in vadose zone processes through laboratory and field investigations and by developing mechanistically oriented models that can adequately simulate the complex behavior of contaminants in the vadose zone.

The second is to transfer the expertise and models developed to DOE environmental remediation programs. To date, instruments and techniques developed in part through the Reactive Transport in Variably Saturated Heterogeneous Media project have been deployed and utilized at Savannah River, and are nearing deployment at Hanford.

Although the Environmental Systems Research Program activities directly support the environmental management research needs of the DOE, the accomplishment of these goals will have implications beyond the INEEL and DOE. For example, the modeling framework and expertise developed in this project will be transferable to private industries concerned with vadose zone contamination.

\section{PROJECT DESCRIPTION}

Throughout arid portions of the world, as exemplified by the Western United States, water quantity and water quality are areas of major concern to the general population, agricultural communities, mining and energy resource interests, and local and national governments. Water supplies are becoming critically impacted as population and water demands increase. In many locations, water supplies have been contaminated either by accident or as a result of past waste-disposal practices. For example, within the United States, activities of the U.S. Department of Energy (DOE) have resulted in the contamination of large tracts of land with complex mixtures of radionuclides and other contaminants. Much of this contamination results from the migration of wastes disposed at the land surface into the region between the surface and underlying aquifer. Other activities that occur in and may result in contamination of the vadose zone include the following:

- $\quad$ Disposal of municipal and hazardous waste in landfills 
- Using septic systems

- $\quad$ Mining and milling of ores

- $\quad$ Production and refining of petroleum products

- $\quad$ Application of agricultural chemicals such as fertilizers, pesticides, and herbicides to crops.

Although the vadose zone is often relied on as the primary protective barrier to prevent groundwater contamination, it is often the conduit for the transport of contamination from the surface to subsurface water supplies. However, our current understanding of the movement and transformation of contaminants is not adequate to accurately predict their behavior in the vadose zone. For example, simplistic chemical characterizations and supporting modeling of the behavior of actinides and other radionuclides in the vadose zone suggest that these contaminants are immobile. However, at some locations (e.g., the INEEL Radioactive Waste Management Complex [RWMC] and the Hanford Tank Farms), actinides and other radionuclides have migrated hundreds of feet through the vadose zone and are influenced by poorly understood physical, chemical, and biological processes. Because centuries of human activities have led to water use and disposal practices that are not environmentally sustainable, new coordinated research and technology development activities are needed for improved and sustainable stewardship of the vadose zone.

Within the science and engineering community numerous disciplinary investigations exist relevant to understanding and modeling the behavior of contaminants in the vadose zone. However, the ability to predict contaminant fate and transport in the vadose zone requires the ability to represent the important physical, biological, and geochemical processes and properties of vadose zone soils and sediments in a modeling framework that allows the transport, retention, and transformation of contaminants and other solutes (nutrients, electron acceptor/donors, etc.) to be accurately assessed. This framework must include

- A high degree of temporal resolution so effects on contaminant transport and transformation of events such as snow melt can be evaluated

- A high degree of spatial resolution so effects on contaminant transport and transformation of physical, chemical, and biological heterogeneities can be evaluated

- $\quad$ Appropriate coupling of the important biological, geochemical, and transport processes so synergistic and antagonistic effects can be evaluated.

Theoretical derivations, experimental investigations, and field observation will be needed to fill key data or understanding gaps required to successfully develop improved vadose zone predictive capabilities. The unifying theme for Reactive Transport in Variably Saturated Heterogeneous Media Project research is to develop an enhanced understanding of the cycling of carbon in the spatially and temporally heterogeneous vadose zone and the effect that this cycling has on the mobility of radionuclides and other DOE contaminants. In the vadose zone, carbon can occur as particulate (e.g., solid organic waste) and dissolved organic carbon and as solid (e.g., calcite), dissolved (bicarbonate), and gaseous (carbon dioxide) inorganic carbon. Because of the existence of a discrete and mobile gas phase in the vadose zone, transport of inorganic carbon in both the aqueous and gas phase can occur. The rapid local equilibration between dissolved, solid, and gaseous inorganic carbon can lead to the development of spatially variable $\mathrm{pH}$ in the vadose zone and associated variability in contaminant behavior. The presence of organic carbon as either disposed waste or as natural materials can promote microbial mineralization providing an additional subsurface source for inorganic carbon. Microbial activity in turn will be a function of available electron acceptors, such as oxygen in the vadose zone gas phase, and free water 
(water that microorganism can access). The nonlinear interrelationship between movement of the gas and aqueous phase and their effect on microbial activity and ultimately contaminant mobility is the focus of the Reactive Transport in Variably Saturated Heterogeneous Media Project research. This research is embodied in the five technical tasks described below.

\section{Overview}

\section{Up-Scaled Effective Reactive Transport Properties in Variably Saturated Heterogeneity Porous Media}

Field-scale transport through geologically heterogeneous media occurs at scales smaller than the discretization level practical in numerical simulators. This demands the use of upscaled parameters to capture the effects of smaller scale heterogeneity at each discretization level. While evaluation of fieldscale transport has widely recognized the importance of the smaller scale hydraulic heterogeneity in controlling fluid migration, the influence of geochemical heterogeneity at similar scales has not been acknowledged.

\section{Propagation of Geochemical Environment in the Vadose Zone}

Unlike groundwater systems, in the vadose zone, both advection of water and diffusion/advection of gas can transport volatile reactive species. This task will focus on the interaction of processes that control the geochemical environment and the migration of contaminants. Inorganic carbon often controls $\mathrm{pH}$ in natural waters through the coexistence of three phases: carbon dioxide gas, solid calcium carbonate, and dissolved carbonate in soil water. Inorganic carbon can be transported by advective water movement [bicarbonate] or by gaseous diffusion or advection [carbon dioxide]. Ultimately, $\mathrm{pH}$ is one of the most important master variables in controlling reactive transport and other biogeochemical processes. The $\mathrm{pH}$ of soil water affects the surface charge on soil minerals that can retard the migration of contaminants by specific adsorption reactions. The $\mathrm{pH}$ can also affect the formation of aqueous complexes of contaminants with ligands such as carbonate ion. The generation of carbon dioxide by the biological degradation of organic matter in radioactive or hazardous waste disposal sites can affect the mobility of metals and radionuclides by altering the vadose zone gas phase and aqueous phase $\mathrm{pH}$.

\section{Relationships Between Moisture Content, Tension, and Biogeochemical Activity in the Vadose Zone}

The objective of this task is to clarify the role that water content plays in soil microbial activity. The availability of water and water-borne nutrients affects microbial activity in the subsurface, which in turn affects water availability and mobility in soil. This interrelationship, as well as interactions between surface area, total pore volume, and microbiologically available pore volume can be very important in determining the remediation potential in the subsurface. For example, hydraulic conductivity (a measure of water mobility in soil) is strongly influenced by the concentration of $\mathrm{Ca}^{2+}$ (through precipitation and dissolution of calcium carbonate) and the $\mathrm{CO}_{2}$ level influences the $\mathrm{Ca}^{2+}$ concentration. In addition, the influences of $\mathrm{Ca}^{2+}$ and $\mathrm{CO}_{2}$ levels on the water retention curve are also unknown. Finally, many microorganisms produce biofilms that may modify a flow path. Furthermore, the production and migration of $\mathrm{CO}_{2}$ significantly influences the migration of radionuclides in the vadose zone.

\section{Vadose Zone Moisture Movement}

Understanding the distribution and movement of water in the vadose zone is paramount to assessing subsurface bioactivity and its effects on contaminant fate and transport. Although substantial research to elucidate moisture characteristics and relationships for vadose zone materials has been 
conducted in controlled repacked laboratory column experiments, evaluation of important in situ processes at the field scale are limited. The objective of this task is to develop, test, and promote field-based measurements of in situ vadose zone properties to support the evaluation of the spatial variability of these properties and their effects on moisture/gas transport at the field scale.

Accomplishment of these technical objectives provides field-based observations that are required for the translations of observations and results from the modeling and laboratory tasks to understanding of vadose processes occurring at the field-scale. Enhanced understanding of moisture movement is being accomplished by establishing a number of field locations for long-term monitoring (relationship of moisture content and movement to natural meteoric fluxes) and field experiments (system response to specific, controlled manipulations).

\section{Enhanced Computational Methods}

The B-spline collocation method shows potential to increase dramatically the speed of finding numerical solutions of differential equations compared to standard finite element/volume/difference methods. In this method, B-spline curves/surfaces are fit to the differential equation at discrete points called collocation points. Because the B-spline curve/surface has continuous derivatives to the order desired, the approximate solution not only does an excellent job of fitting the solution, it also uses far fewer points or nodes that the other standard methods. This feature means that the numerical solution can be found by solving far smaller matrix equations. Another important feature of the B-spline method is that the function, which represents the solution and its derivative, is continuous everywhere in the solution domain. By contrast, finite elements/volumes only have discrete solutions at points and do not have continuous derivatives from cell to cell.

\section{Up-Scaled Effective Reactive Transport Properties in Variably Saturated Heterogeneity Porous Media}

Numerous biogeochemical processes occurring in the subsurface at mineral-water interfaces influence the mobility of contaminants in groundwater systems. One of these processes, adsorption, has been the subject of extensive laboratory investigations over the previous two decades (Jenne 1998). These investigations have given rise to surface complexation models that describe the adsorption behavior of metals and radionuclides as a function of surface area, $\mathrm{pH}$, ionic strength, and competing ion and ligand concentrations. In addition, databases of reaction constants for these models have been tabulated for common subsurface phases (Dzombak and Morel 1990, Smith and Jenne 1991). However, the use of surface complexation models in field-scale transport calculations has been limited because of inadequate characterization of the subsurface medium (e.g., surface area of reactive phases) and incomplete understanding of groundwater chemistry. Many transport codes account for all interfacial processes using linear equilibrium adsorption as represented by a mass-based distribution coefficient $\left(K_{d, m}\right)$. Although it may be convenient to formulate transport calculations in terms of $K_{d, m}$, it is the abundance and characteristics of reactive surface area of subsurface materials that control the adsorption of contaminants. We have previously shown (Smith et al. 1996, Tompson et al. 1996) that systems with spatial variability in permeability exhibit variability in $K_{d, m}$ even for materials that are compositionally homogeneous. In this paper, we demonstrate through the concept of effective reactive surface area that systems with permeability anisotropy will also exhibit anisotropy in $K_{d, m}$.

\section{Relationship Between Surface Area and Permeability}

Depositional processes exert close controls on mineralogy, grain size, grain shape, sorting, and other sedimentalogical properties of sand bodies. Because the primary porosity $(\theta)$ of sand and sandstone bodies is a function of sedimentalogical variables, permeability $(k)$ and $\theta$ should, in part, be functions of 
facies distribution. As an example, glacial till deposits are characterized by poor sorting with respect to size and shape and commonly consist of a fair amount of clay and silt, resulting in low $k$. Beach processes are more effective in removing silt to clay-sized grains and in grain sorting, resulting in comparatively high $k$. Variations of these properties permit recognition of sediments from various parts of the depositional environment in which different processes predominated during accumulation. We have previously shown that for monomineralic uniformly packed sediments that reactive surface area is inversely related to permeability via (Smith et al. 1996).

$$
\mathrm{A}_{\mathrm{S}}=\alpha_{\mathrm{S}} \frac{1-\theta}{\theta}=\sqrt{\frac{\theta}{2 \tau \mathrm{k}}}
$$

where $A_{S}$ is the surface area per unit volume of saturated porosity $\left(A_{\text {surface }} / V_{\text {pores }}\right), \alpha_{S}$ is the surface area per unit volume of media matrix, $\theta$ is the porosity, $\tau$ is the tortuosity, and $k$ is the permeability. The above equation links the reactive surface area to the medium $k$ and provides a theoretical basis for the expected correlation between heterogeneity in reactive properties and the heterogeneity in hydraulic properties for saturated aquifers. Taking the logarithm of equation 1 ,

$\ln \mathrm{A}_{\mathrm{S}}=0.5 \ln \theta-0.5 \ln 2 \tau-0.5 \ln \mathrm{k}$

shows that $\ln A_{s}$ and $\ln k$ for unconsolidated sediments should be correlated with a slope of - 0.5 . In addition, both $\theta$ and $\tau$ should influence the relationship between $A_{S}$ and $k$. In the simple case where both $\theta$ and $\tau$ are constant, Equation 2 reduces to a straight line. In the case where $\tau$ is constant, variations in $\theta$ should not significantly affect the expected relationship between $A_{S}$ and $k$. However, the assumption of constant $\tau$ is probably valid for only limited ranges of $\theta$. Panda and Lake (1994) discussed the importance of sediment sorting and packing on $\tau$ and indicated that $\tau$ is a power function of sorting. In addition, they suggested that small amounts of secondary minerals could significantly influence the value of $\tau$. Because of potential variation of $\tau$ in an aquifer and of the difficulties in quantifying $\tau$, the direct application of Equation 2 for developing correlated aquifer models is limited. However, we have shown (Smith et al. 1996, Tompson et al. 1996, Smith et al. 1997) that expressions of the form of Equation 2 can be used to describe the relationships between surface-area-based biogeochemical reactivity and medium permeability for compositionally homogeneous sediments and naturally occurring oxide-coated sediments.

Equations 1 and 2 were derived for isotropic packing of compositionally homogeneous sand grains - that is, the value of $k$ is the same in all directions. Isotropic packing is often strived for in column experiments and is usually obtainable only in repacked columns. Naturally occurring sedimentary aquifers exhibit anisotropic $k$ (with $k_{\text {horizontal }}>k_{\text {vertical }}$ ) because of heterogeneities in packing and in grain size at scales smaller than the scale of interest for flow and transport. An example of the relationship between anisotropy in $k$ and reactive surface area is presented below for a simple but important type of heterogeneity characteristic of horizontal banding (Figure 1).

The average (effective) horizontal permeability of a unit-thick packet of sediments with two layers of high $\left(k_{1}\right)$ and low $\left(k_{2}\right)$ permeability with relative thicknesses of $1-m_{2}$ and $m_{2}$, respectively, is given by the thickness-weighted arithmetic average of permeability,

$$
\mathrm{k}_{\mathrm{H}}=\left(1-\mathrm{m}_{2}\right) \mathrm{k}_{1}+\mathrm{m}_{2} \mathrm{k}_{2}
$$




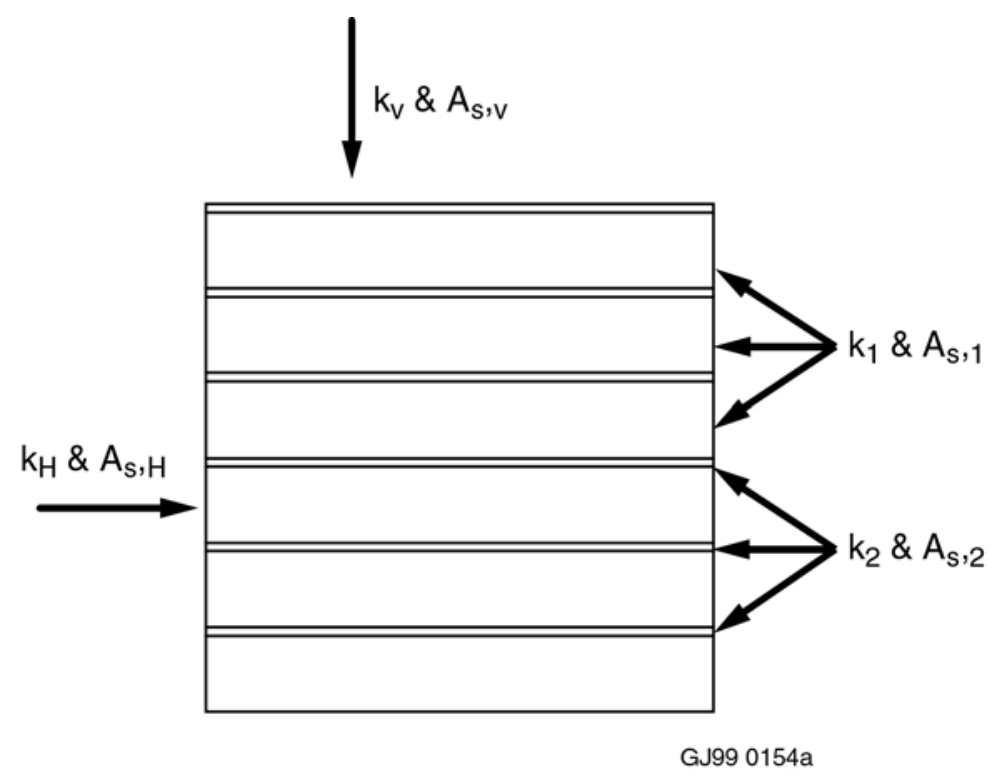

Figure 1. Anisotropic heterogeneous packet of sediments composed of two isotropic homogeneous materials.

and the effective vertical permeability is given by the thickness-weighted harmonic average of permeability:

$$
\mathrm{k}_{\mathrm{V}}=\frac{1}{\frac{1-\mathrm{m}_{2}}{\mathrm{k}_{1}}+\frac{\mathrm{m}_{2}}{\mathrm{k}_{2}}}
$$

The ratio of the horizontal to vertical permeability is the anisotropy ratio, from which it is apparent that the anisotropy ratio will be greater than, or equal to, 1 regardless of the absolute values of $k_{1}, k_{2}$, and $m_{2}$ :

$$
\frac{\mathrm{k}_{\mathrm{H}}}{\mathrm{k}_{\mathrm{V}}}=\frac{\left(1-\mathrm{m}_{2}+\frac{\mathrm{k}_{1}}{\mathrm{k}_{2}} \mathrm{~m}_{2}\right)\left(\frac{\mathrm{k}_{1}}{\mathrm{k}_{2}}+\mathrm{m}_{2}-\frac{\mathrm{k}_{1}}{\mathrm{k}_{2}} \mathrm{~m}_{2}\right)}{\frac{\mathrm{k}_{1}}{\mathrm{k}_{2}}}
$$

Similar, although more complex, equations can be developed to describe the effective reactive surface area as a function of direction by using Equation 1 and appropriate volume and flux weighting for the bands. Effective surface area in the horizontal direction is calculated from the surface areas of the individual bands weighted by their relative thicknesses and fluxes,

$$
A_{S, H}=\frac{\left(1-m_{2}\right) k_{1} A_{S, 1}+m_{2} k_{2} A_{S, 2}}{\left(1-m_{2}\right) k_{1}+m_{2} k_{2}}
$$


and the effective surface area in the vertical direction is equivalent to the total surface area in the sediment packet:

$$
\mathrm{A}_{\mathrm{S}, \mathrm{V}}=\left(1-\mathrm{m}_{2}\right) \mathrm{A}_{\mathrm{S}, 1}+\mathrm{m}_{2} \mathrm{~A}_{\mathrm{S}, 2}
$$

The anisotropy ratio for effective reactive surface area under conditions of uniform $\theta$ and $\tau$, $A_{S, H} / A_{S, V}$, is given by:

$$
\frac{A_{\mathrm{S}, \mathrm{H}}}{\mathrm{A}_{\mathrm{S}, \mathrm{V}}}=\frac{\mathrm{m}_{2}\left(\frac{\mathrm{k}_{1}}{\mathrm{k}_{2}}-\sqrt{\frac{\mathrm{k}_{1}}{\mathrm{k}_{2}}}\right)-\frac{\mathrm{k}_{1}}{\mathrm{k}_{2}}}{\left(1-\mathrm{m}_{2}+\mathrm{m}_{2} \sqrt{\frac{\mathrm{k}_{1}}{\mathrm{k}_{2}}}\right)\left(\mathrm{m}_{2} \frac{\mathrm{k}_{1}}{\mathrm{k}_{2}}-\mathrm{m}_{2}-\frac{\mathrm{k}_{1}}{\mathrm{k}_{2}}\right)}
$$

Inspection of Equations 5 and 8 reveals that both $k_{H} / k_{V}$ and $A_{S, H} / A_{S, V}$ are independent of the absolute values of the permeability of the individual layers and that they depend only on the ratio of the permeability and on the relative thicknesses of the beds. Analysis of Equation 8 indicates that $A_{S, H} / A_{S, V}$ will always be less than or equal to 1 regardless of the absolute values of $k_{1}, k_{2}$, and $m_{2}$. A value of less than 1 indicates that the effective surface area in the horizontal direction will always be less than in the vertical direction, illustrating the importance of the high permeability, low surface area bands in transmitting water. Figure 2 depicts the relationship between $k_{H} / k_{V}$ and $A_{S, H} / A_{S, V}$ for several values of $m_{2}$. From this figure, $\ln k_{H} / k_{V}$ and $\ln A_{S, H} / A_{S, V}$ are inversely related (i.e., negative slope) with a slope that becomes more negative as the fraction of the low permeability bands increases. The implications to reactive transport are profound. A key concept is that the effective reactive surface area and any property or process (e.g., $\left.K_{d, m}\right)$ that is related to reactive surface area will have directionally dependent values.

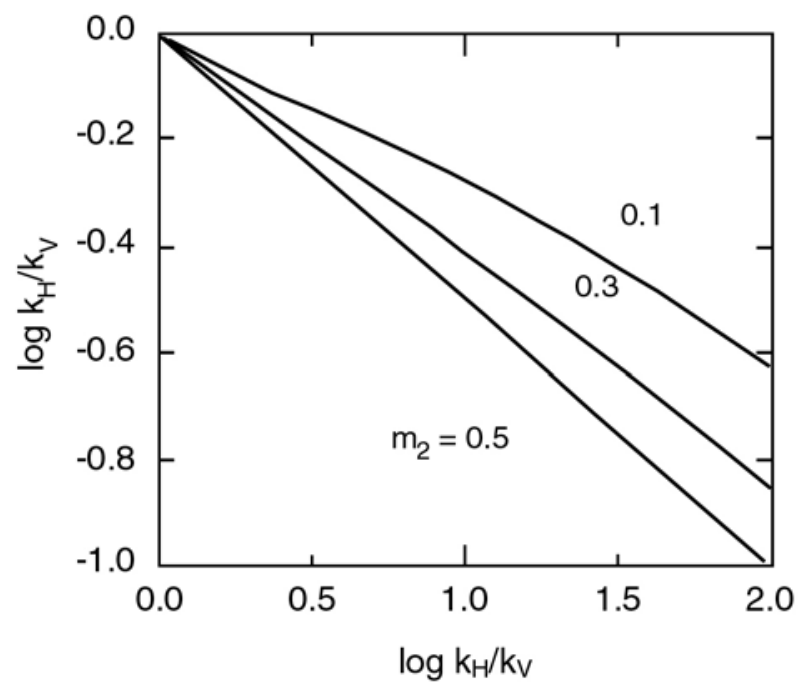

Figure 2. Predicted relationship between anisotropy in effective reactive surface area and permeability for a two-layer system. 
Because the effective reactive surface area is a function of both material properties and advective fluxes, it cannot be readily characterized in a static system. Characterization is best accomplished by using reactive tracers and the relationship between the reactive surface area and the retardation factor $\left(R_{f}\right)$

$$
\mathrm{R}_{\mathrm{f}}=1+\mathrm{K}_{\mathrm{d}, \mathrm{m}} \rho \frac{1-\theta}{\theta}=1+\mathrm{K}_{\mathrm{d}, \mathrm{A}} \mathrm{A}_{\mathrm{S}}
$$

where $\rho$ is the grain density of the medium and $K_{d, A}$ is the surface-area-based distribution coefficient.

Results of an example numerical experiment to evaluate the appropriateness of the approach described above to estimate effective properties of layered media as illustrated in Figure 1 is given as Figure 3 (Smith and Schafer 1999).

Figure 3 shows the results of the simulations for the two-dimensional layered system (symbols) and the equivalent system with effective properties (solid lines). As may be seen in the figure, there is relatively good agreement between the calculated breakthrough for the layered system and the equivalent effective system, although the layered system (symbols) exhibits slightly earlier arrivals than the equivalent system. The derivations used to estimate effective parameters did not consider dispersion. However, examination of the breakthrough curves for a conservative tracer (not shown) revealed the same slightly early arrival in the layered simulation as for the reactive tracer, suggesting that dispersion may be important and cannot be ignored in the development of approaches to estimate effective properties. The specific reason for the early arrival is beyond the scope of this paper and is the subject of ongoing research that includes both numerical simulations as well as tracer experiments on oriented intact cores of layered materials.

The simulations and the derivations presented here suggest that systems that are anisotropic with respect to permeability will also be anisotropic with respect to surface-area-based reactive properties. This anisotropy exists even for systems that are compositionally homogeneous. The implications for characterizing reactive transport are profound. First, laboratory batch characterization of surface-areabased reactive properties (e.g., $K_{d, m}$ ) will give values that are appropriate estimates for transport in the

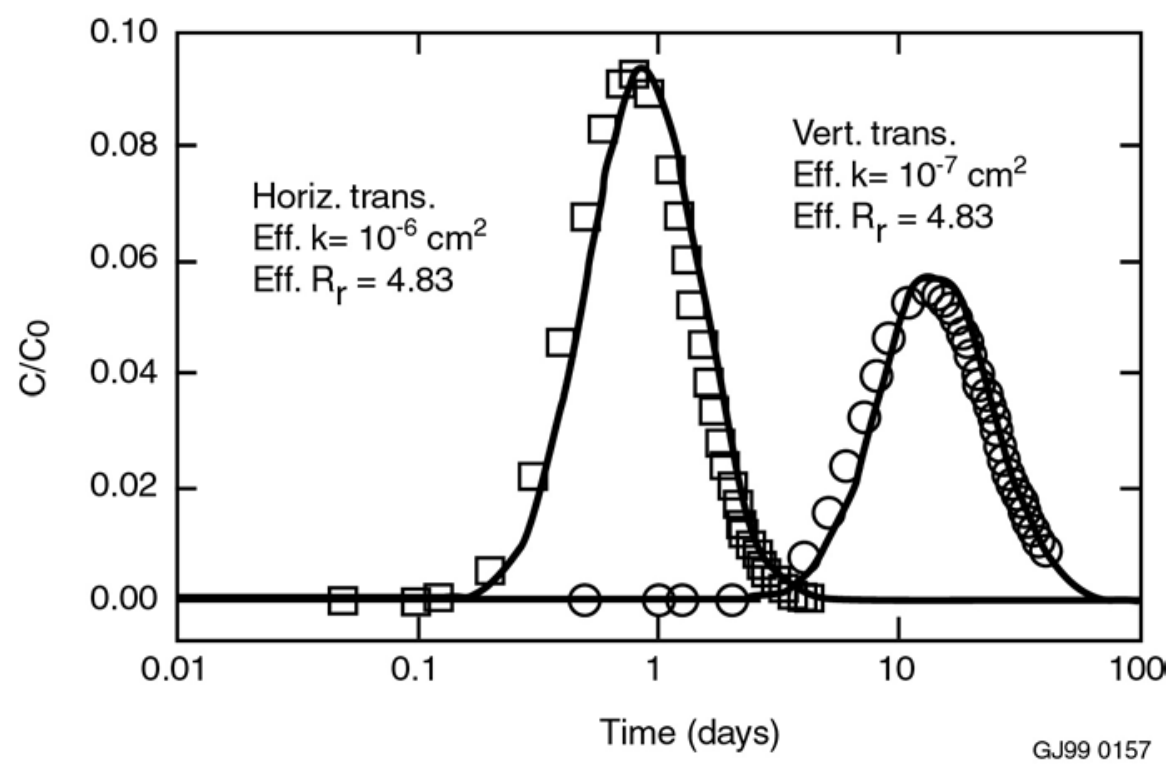

Figure 3. Calculated reactive tracer breakthrough curves in the vertical and horizontal direction for heterogeneous porous media composed of two layers (symbols) and equivalent effective homogeneous media (solid lines). 
direction of lowest permeability (i.e., vertical) and will overestimate the retardation that occurs along horizontal flow paths. Second, the formulations of transport models, many of which consider anisotropy in permeability, are not adequate to describe the interfacial processes in advecting systems, regardless of the sophistication of the chemical interaction model, because they assume that the interfacial area is not a directional property. Third, real systems may have more pronounced anisotropy than the simplified systems presented here because the minerals that compose low permeability bands are intrinsically more reactive than are the minerals that compose high permeability bands.

The concepts outlined above for a fully saturated system formed the basis for a successful Natural and Accelerated Bioremediation Research Program (NABIR) proposal. The focus of this task was shifted to the more complex case of variably saturated media and formal collaborative relationship was established with Dr. Jim Yeh (Univ. of Arizona) and Dr. Gary Pope (Univ. of Texas).

\section{Propagation of Geochemical Environments}

\section{Task Summary}

The objective of this project is to increase our understanding of the development of geochemical environments in the vadose zone and the role those environments play in the fate and transport of components through the vadose zone. The geochemical environment includes four phases: the soil gas phase, the soil water phase, the solid phases that form the soil or rock matrix in the vadose zone, and a biological phase that lives in the soil. An improved understanding of the geochemical environment will lead to an improved ability to predict the fate and transport of components through the vadose zone.

To focus the effort, the specific goal of this task is to develop our ability to perform reactive transport modeling of uranium in the vadose zone. Uranium poses a long-term chemical and radiological risk where it has been concentrated in the environment by man's activities. Significant quantities of uranium have been used in the U.S. Department Of Energy system and have been disposed to nearsurface burial grounds where vadose zone transport is of particular concern. Uranium transport is selected in this investigation because it is very dependent on the vadose zone geochemical environment. Vadose zone factors such as $\mathrm{pH}$, the partial pressure of carbon dioxide, and the redox conditions all play very significant roles in the mobility of uranium. Activities for the fiscal year have included conducting experiments to evaluate procedures for modeling adsorption on soil minerals, improving models of plant and microbe carbon dioxide production, and finding efficient algorithms for modeling reactive transport.

This research is being conducted in collaboration with Dr. Donald Suarez of the U.S. Salinity Laboratory. We also collaborate with Dr. George D. Redden (Biochemical and Geochemical Reactions on Environmental Surfaces Project) on experimental aspects of the interfacial biogeochemistry of uranium.

In the vadose zone, both advection of water and diffusion/advection of gas can transport volatile reactive species. This task will focus on the interaction of processes that control the geochemical environment and the migration of contaminants. Inorganic carbon often controls $\mathrm{pH}$ in natural waters through the coexistence of three phases: carbon dioxide gas, solid calcium carbonate, and dissolved carbonate in soil water. Inorganic carbon can be transported by advective water movement as bicarbonate or by gaseous diffusion or advection as carbon dioxide. Ultimately, $\mathrm{pH}$ is one of the most important master variables in controlling reactive transport and other biogeochemical processes. The $\mathrm{pH}$ of soil water affects the surface charge on soil minerals that can retard the migration of contaminants by specific adsorption reactions. The $\mathrm{pH}$ can also affect the formation of aqueous complexes of contaminants with ligands such as carbonate ion. The generation of carbon dioxide by the biological degradation of organic 
matter in radioactive or hazardous waste disposal sites can affect the mobility of metals and radionuclides by altering the vadose zone gas phase and aqueous phase $\mathrm{pH}$.

Microbes oxidize organic matter $\left[\mathrm{CH}_{2} \mathrm{O}\right]$ to water $\left[\mathrm{H}_{2} \mathrm{O}\right]$ and carbon dioxide $\left[\mathrm{CO}_{2}\right]$ using dissolved oxygen $\left[\mathrm{O}_{2}\right]$ and releasing energy that the microbes use to live.

$$
\begin{aligned}
\mathrm{CH}_{2} \mathrm{O}+\mathrm{O}_{2}(\mathrm{aq}) \underset{\text { microbes }}{\longleftrightarrow} \mathrm{CO}_{2}(\mathrm{aq})+\mathrm{H}_{2} \mathrm{O} \\
\mathrm{O}_{2}(\mathrm{~g}) \stackrel{\mathrm{O}_{2}(\mathrm{aq})}{\mathrm{CO}_{2}(\mathrm{aq})} \longleftrightarrow \mathrm{CO}_{2}(\mathrm{~g})
\end{aligned}
$$

Oxygen is dissolved from the vadose zone vapor phase and carbon dioxide released to the vapor phase to compensate for the changes in aqueous concentrations from the reaction. The presence of a vapor phase is important to this reaction, and so vadose zone biogeochemical activity is tied to hydrology as the water content controls the gas-filled porosity and the transport of nutrients.

Carbon dioxide in the vadose zone reacts with water to form carbonic acid $\left[\mathrm{H}_{2} \mathrm{CO}_{3}\right]$. Carbonic acid reacts with soil minerals such as calcite $\left[\mathrm{CaCO}_{3}(\mathrm{~s})\right]$, dissolving the minerals and releasing solutes to the aqueous phase. Since these reactions are reversible, minerals can precipitate from solution when vadose zone water is lost by evapotranspiration.

$$
\begin{aligned}
& \mathrm{CO}_{2}(\mathrm{aq})+\mathrm{H}_{2} \mathrm{O} \longleftrightarrow \mathrm{H}_{2} \mathrm{CO}_{3} \\
& \mathrm{CaCO}_{3}(\mathrm{~s})+\mathrm{H}_{2} \mathrm{CO}_{3} \longleftrightarrow \mathrm{Ca}^{+2}+2 \mathrm{HCO}_{3}^{-}
\end{aligned}
$$

The dissolution of minerals by carbonic acid consumes hydrogen ion, raises the $\mathrm{pH}$, and increases the concentration of dissolved carbonate $\left[\mathrm{HCO}_{3}^{-}\right]$.

Dissolved carbonate forms aqueous complexes with metals such as uranyl $\left[\mathrm{UO}_{2}^{+2}\right]$. Geochemical reactions depend on the concentration of the free ions in solution. As carbonate complexes the uranyl ion, the concentration of free uranyl ion in solution decreases, although the total amount of uranium in solution remains the same.

$$
\begin{aligned}
& \mathrm{UO}_{2}^{+2}+\mathrm{nHCO}_{3}^{-} \longleftrightarrow \mathrm{UO}_{2}\left(\mathrm{CO}_{3}\right)_{\mathrm{n}}^{-2(\mathrm{n}-1)}+\mathrm{nH}^{+} \\
& \mathrm{UO}_{2}^{+2}+\equiv \mathrm{SOH}^{0} \longleftrightarrow \equiv \mathrm{SOUO}_{2}^{+}+\mathrm{H}^{+}
\end{aligned}
$$

By decreasing the free uranyl concentration in solution, carbonate complexing decreases the amount of uranyl that can be removed by forming surface complexes on mineral adsorption sites in the soil $\left[\equiv \mathrm{SOH}^{0}\right]$.

The important processes in biogeochemistry are surface adsorption, dissolution-precipitation, oxidation-reduction, complexation, volatilization, and biotransformation. Biogeochemical reactions are described by the law of mass action that indicates that chemicals react in definite proportions and that matter is conserved during reactions. The applicability of the law of mass action to a wide range of biogeochemical reactions is illustrated by the decrease in uranyl ion $(\mathrm{UO} 2+2)$ adsorption above a $\mathrm{pH}$ of 8.5 as a side effect of the consumption of organic matter by microbes. 


\section{Task Accomplishments}

Two approaches can be taken to quantifying the adsorption of uranium to soils. One is to treat the soil as a black box and measure an adsorption isotherm on the soil. The adsorption isotherm is fitted to the specific adsorption model by empirically creating sites. The alternative approach is to treat the soil as a mixture of individual minerals and model adsorption as the physical mixing of the individual mineral phases. Both approaches will be used in this study. Whole-soil adsorption isotherms will be compared to isotherms computed by summing the mineral isotherms using the calculated number of surface sites for each mineral as the weighting factor. The ability to fit the adsorption data with either model will be considered a success. However, fitting the whole-soil isotherm with an empirical set of sites is not as mechanistically satisfying as being able to assign those sites to specific minerals. The ability to treat the soil as a mixture of ideal minerals provides a possible approach to predicting adsorption behavior rather than just quantifying adsorption behavior. Either approach is a significant improvement on the current widely used $\mathrm{K}_{\mathrm{d}, \mathrm{m}}$ approach.

Many laboratory experiments have been conducted on the adsorption of uranium on single, synthetic minerals (Redden et al. 1998; Payne et al. 1998). However, little work has been done to expand these studies to adsorption on mixtures of minerals or on natural soil materials. Proton titration experiments will be conducted on minerals and on soil to quantify surface charge and to evaluate buffer capacity. The minerals used will be goethite and smectite clay. Experiments will also be conducted on soils high in smectite and iron oxyhydroxides. The mineralogy, surface area, and chemistry of the soil will be quantified. Batch adsorption experiments will be conducted to measure uranyl adsorption isotherms on minerals and on soil. Experiments will be conducted over a range of carbonate contents to evaluate the formation of aqueous uranyl-carbonate complexes on adsorption. Adsorption parameters for adsorption isotherms for individual minerals and for soil will be determined using FITEQL (Westall 1982). The results will be used to assess the performance of radioactive and hazardous waste disposal sites and remediation actions.

A number of computer codes have been developed that deal with propagation of $\mathrm{pH}$ in the vadose zone under a set of limiting assumptions. Suarez and Simunek $(1996,1997)$ use empirical $\mathrm{CO}_{2}$ production curves for agricultural soils and include diffusion of $\mathrm{CO}_{2}$ in the gas phase. This handles the transport of $\mathrm{CO}_{2}$ in the gas and aqueous phases, but does not permit prediction of contaminant transport. HYDROGEOCHEM (Yeh and Salvage 1997) has a very open geochemical architecture that would permit inclusion of many geochemical and microbial reactions, both equilibrium and kinetic. However, this code does not include a gas phase so that diffusion of $\mathrm{CO}_{2}$ as a mechanism for propagation of $\mathrm{pH}$ cannot be included. The INEEL will use and adapt both of these codes for investigations into geochemical reactions in the vadose zone based on applicability. The INEEL is collaborating with Dr. Suarez to incorporate the following enhancements into the UNSATCHEM code.

- A generalized chemistry "matrix" solver allowing the modeling of trace constituents (contaminants and trace nutrients).

- A generalized kinetic solver for microbially mediated reactions (e.g., Monod kinetics, Michaelis-Menton kinetics).

- A more rigorous mass balance on carbon.

- A multisite nonelectrostatic adsorption model. 


\section{Laboratory Experiments}

The focus of this year's activities was the development of laboratory capabilities to conduct rigorous sorption experiments. To this end, two autotitrator systems were hand built, debugged, and set up to run proton stoichiometry titrations from which surface charge can be calculated and to determine the sorption capacity of synthetic and natural materials. These instruments were used to titrate synthetic goethite and INEEL soils, in preparation for detailed uranium adsorption experiments to be conducted in FY-00. Although experimental methods are still being developed, an example of an early titration curve for goethite is shown as Figure 4.

The goethite $(\alpha-\mathrm{FeOOH})$ used in this project was prepared by neutralizing a solution of ferric nitrate and characterizing the precipitate by X-ray diffraction. INEEL soil from spreading area B (SAB) soil was collected and treated with sodium acetate $(\mathrm{pH} 5)$ to dissolve carbonates and other soluble salts (Klute, 1986). Surface areas of $34.2 \mathrm{~m} 2 / \mathrm{g}$ and $30.3 \mathrm{~m} 2 / \mathrm{g}$ were measured for the treated and untreated soil samples, respectively using the BET method. (Brunauer et al. 1938). The soil was additional characterized for extractable metal oxides using the reducing dithionite-bicarbonate-citrate extraction (DCB, Klute 1986). This extraction, which removes crystalline and amorphous hydrous metal oxides,

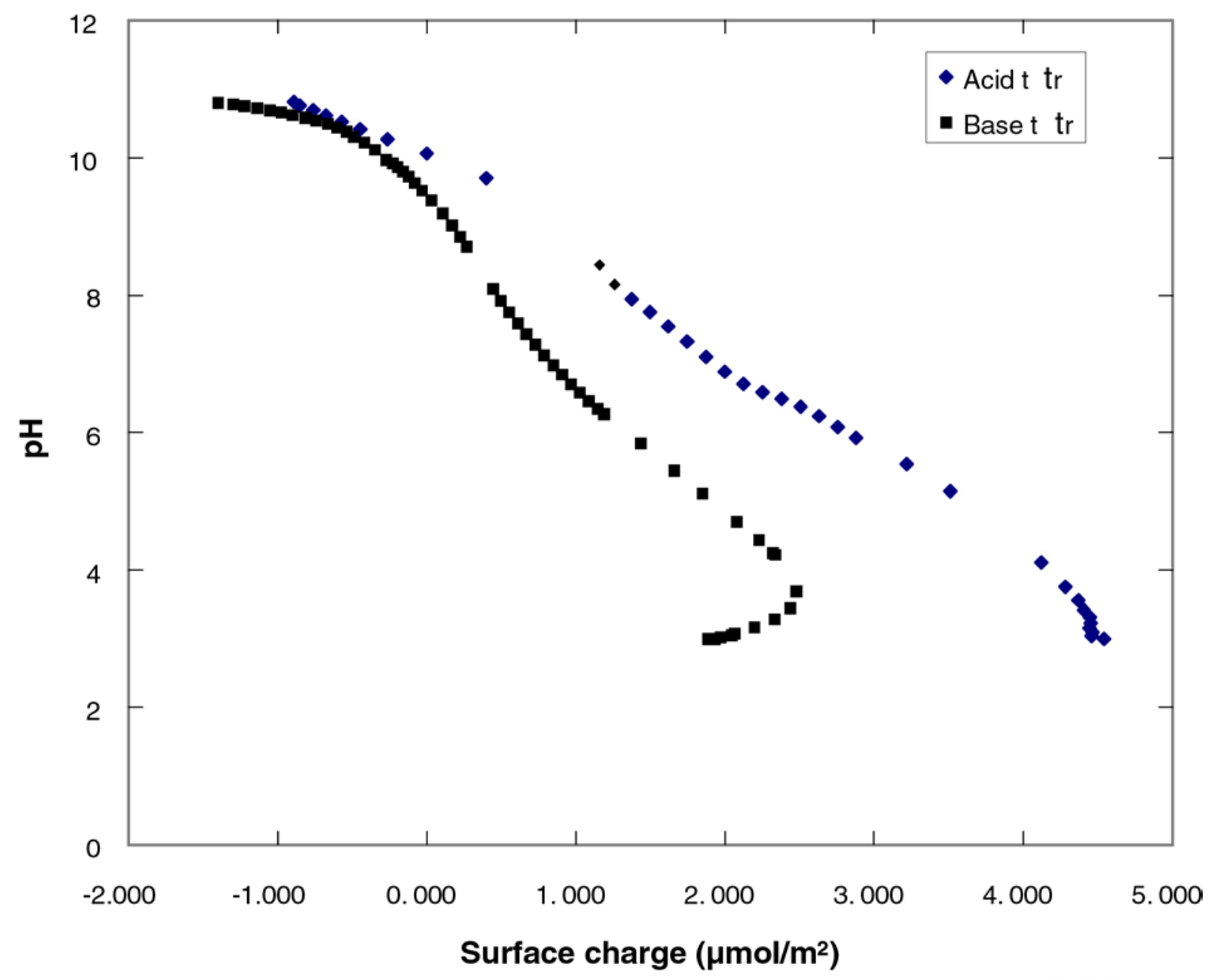

GJ99 0158

Figure 4. Proton stoichiometry titration of goethite. 
yield $24 \mu \mathrm{mol} / \mathrm{g} \mathrm{Fe}, 0.9 \mu \mathrm{mol} / \mathrm{g} \mathrm{Mn}, 4.1 \mu \mathrm{mol} / \mathrm{g} \mathrm{Al}$, and $13 \mu \mathrm{mol} / \mathrm{g} \mathrm{Si}$. Using the method of Kent et al 1998 and the results of the extractions surrogate reactive site concentrations of $2.8 \mu \mathrm{mol} / \mathrm{g}, 2.2 \mu \mathrm{mol} / \mathrm{g}$, $0.8 \mu \mathrm{mol} / \mathrm{g}$, and $1.0 \mu \mathrm{mol} / \mathrm{g}$ for $\mathrm{Fe}, \mathrm{Mn}, \mathrm{Al}$, and $\mathrm{Si}$ respectively. In addition to the reactive sites contributed by hydrous metal oxide coatings, INEEL soils contain clay minerals that have both fixed charged (cations exchange) and variably charged (Al-edge sites) reactive sites. The cation exchange capacity of the soil is being determined.

Scanning electron microscopy (SEM) was used to assess the abundance of crystalline and/or amorphous iron oxides in the soil. Crystalline iron oxide has a smaller surface area than amorphous iron oxide. Surface area is an important factor in the sorption process. Therefore, the correct crystalline structure of iron oxide present in the soil is necessary to predict and model the sorption of contaminants onto INEEL soil. Elemental analysis was also done on soil particles observed. Figure 5 is a SEM photomicrograph of the soil. The lighter areas in Figure 5 are iron-containing phases. Highly crystalline grains of iron minerals, such as magnetite, are not present in the soil. Iron minerals occur as small particles dotting the surfaces of mineral grains.

\section{Modeling $\mathrm{CO}_{2}$ production}

The development of methods for handling organic matter formation and decomposition has been a high priority during 1998-99 and significant progress can be reported. There is a vast body of literature on a decomposition of organic matter in soil and to a lesser extent on the respiration process. Almost all of the literature consists of experiments under limited environmental conditions with empirical models representing the data set. While these data sets may be useful for testing developed models, the published responses are not readily adaptable into the generalized process based model. Using MetLab, the coupled differential equations representing carbon substrate consumption and biomass production (Motz 1986) have been studied extensively. The equations have appeared in numerous scientific papers since they were initially published, and seem to be a good starting point for development of a predictive model. Their use has been primarily in description of microbial response to substrate addition and application to short term individual experiments and not in a generalized ecosystem model. We have determined that these equations yield unstable numerical solutions, stable oscillatory solutions, and damped oscillatory solutions for various sets of parameters. Through analysis of the results of these simulations we have obtained relationships among parameters that can ensure a stable solution. However, further analysis or the problem suggested that these equations probably do not provide sufficient process representation to be

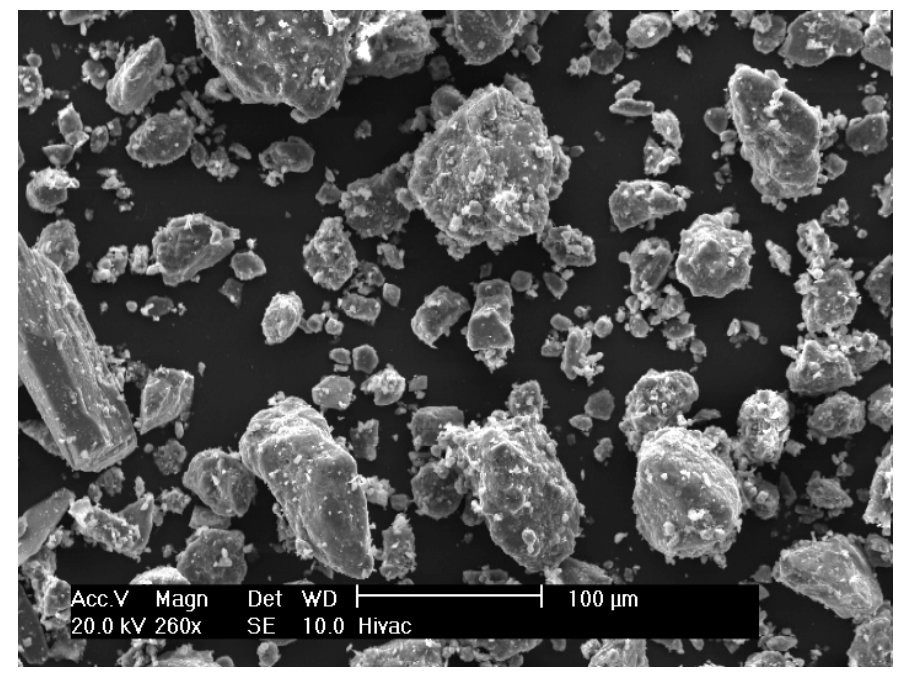

Figure 5. A picture of Spreading Area B soil taken by the scanning electron microscope (SEM). 
generally applicable to organic matter production and decomposition in soils. For example, this model treats only a single microbial population.

More recent models of the dynamics of microbial populations include the effects of nutrients, predators, and dormancy (Blagodatsky et al. 1998, Bosatta and Agren 1994). A minimal model of microbial dynamics should address the factors responsible for growth, cycling of the population between dormant and active states, and death of microbes. The response of these processes to temperature, hydraulic pressure (water content), gas phase composition, and nutrient availability can be represented by a set of first-order differential equations. These sets of equations were successfully programmed using MatLab m-files. In UNSATCHEM the substrate availability will be determined from a soil organic matter module, which in turn will receive input from a plant production model. Fresh biomass and its $\mathrm{C} / \mathrm{N}$ ratio is an essential component of a detailed soil respiration/gas phase predictive model. The success of this preliminary modeling encouraged us to develop a Fortran version utilizing a high-speed solver. This code was incorporated into UNSATCHEM to run as an alternative $\mathrm{CO}_{2}$ production module to the original module based on a reduction-function approach. The code has been tested and gives reasonable results but further work along this line will necessarily be parameterization of this model for specific applications.

Plants are a very significant factor for water flow near the soil surface. Removal of water by root water uptake may be important in determining the rate of recharge, especially in areas where the transpiration rate is a significant fraction of the infiltration rate. Water flow controls chemical transport by advection so plants are also an important factor in subsurface chemical transport. For this reason we have devoted significant effort to improving the representation of plant processes, particularly transpiration.

A generic plant growth model was developed that simulates plant growth including biomass production related to incident solar radiation, existing biomass and leaf area and water and salinity stress. Biomass is partitioned between roots and shoots, with calculation of leaf area index, green biomass, total shoot biomass and root biomass. The root biomass distribution with depth develops as a function of growth considering maximum rooting depth. Root length density development and distribution with depth is simulated with time in response to environmental factors (water availability and salinity). This submodel requires relatively modest input requirements of some plant specific response factors and solar radiation, air temperature, and reference evapotranspiration $\left(\mathrm{ET}_{0}\right)$ with time. The advantage of this approach is that for annual vegetation, detailed site-specific crop coefficients are not required. Calculation of actual ET will be significantly lower than the potential ET, especially in the early portion of the growing season. The approach also allows for the detailed organic matter distribution function with depth and time, essential for a detailed respiration and redox model. An accurate calculation of transpiration is prerequisite for accurate representation of water flow in the root zone.

\section{Coupled Reactive Transport}

Development of a new method for solving the aqueous speciation problem is proceeding using the MatLab (1998) software that was purchased at the start of the research. As a test case, equations representing aqueous speciation from major dissolved ions and carbon dioxide dynamics (26 equations and 26 unknowns) have been programmed in the MatLab native language. The set on nonlinear algebraic equations has been solved utilizing the Gauss-Newton and Levenberg-Marquardt methods, but convergence was slow and, in some cases, results were not found to be physically realistic. These tests were made to fully evaluate the capability of the solvers and included the universal practice of initial estimation of the variables. Additional work consisted of reducing the equation set to make them more amenable to numerical solution. This has resulted in a stable and rapid solver for this set of equations. Development of a stand-alone executable code (without need for user to purchase MatLab) is possible 
with additional effort. We are utilizing this software for development of the matrix solvers capability of other approaches, such as that used in MICROQL (Westall, 1979). The MICROQL approach involves generation of a matrix solution for each separate problem with problem definition, including equilibrium constants, fully specified by the user. UNSATCHEM does not require this degree of expertise on the part of the user. Instead, the equilibrium constants and other reaction-specific data are stored within the program. Our analysis of MICROQL indicates that a dynamic reconfiguration of the concentration matrix will be necessary at each time step to assure that the matrix solution will work. Various approaches to this technical problem are currently being investigated.

Future research will include a sensitivity study of the dependence of the numerical solutions on initial concentration estimates in order to evaluate the robustness of these algorithms in this application. Upon completion of this evaluation, variables (trace elements, exchange cations and constant capacitance modeled adsorption of anions) will be added and the algorithm reexamined.

The other process for removing water near the soil surface is soil evaporation. The original UNSATCHEM model required that the user should either specify soil evaporation rate and transpiration rate separately of specify evapotranspiration, in which case the model split this number into evaporation and transpiration components. Because the plant model now requires solar radiation as input data this number is available and could be used to calculate the soil evaporation separately. Therefore we developed an energy balance module that determines soil surface temperature implicitly and calculates the soil evaporation (van Bavel and Hillel 1976). The energy balance includes net solar radiation, sensible and latent heat fluxes, and soil heat flow due to both conduction and convection during water flow. The soil evaporation is calculated by dividing the latent heat flux by the latent heat of water.

\section{Enhanced Computational Methods}

\section{R. W. Johnson and M. D. Landon}

The characterization of the transport of contaminants in the variably saturated porous vadose zone is typically embodied in differential transport equations that involve mass and momentum fluxes, head, level of saturation, sinks and sources, and transport properties such as permeability. Traditionally, these equations are solved by first dividing the domain of interest into finite volumes and then discretizing the equations to render algebraic equations for each volume. Because each volume is connected to its neighboring volumes, the algebraic system of equations must be solved in large matrices of equations. The solution of the matrix equation may be straight forward, but still involves considerable compute time. Inevitably, the transport properties of the porous media are not known in a locally average sense that is required by the nature of the system of discrete equations. This situation is typically addressed by varying the numerical values of the transport properties as a function of space and attempting to predict some experimental data such as that produced by injecting a tracer into a well and measuring the time taken to exit at other wells. In order to obtain the best distribution of transport properties in the computational domain, many realizations that require solving the matrix of equations are made. This becomes very resource intensive and time consuming if each realization takes very much time. Hence, one of the goals of the numerical simulation of fluid flow in porous media or regular space is to reduce the compute time for each realization.

The overall objective of the present task is to examine the feasibility and features of a new numerical method for solving the differential equations that represent fluid flow. The method is based upon the implementation of well known functions that are commonly used in the fields of computer aided design (CAD) and computer aided graphic design (CAGD). The method can also be classified as a member of the family of finite element methods. In CAD and CAGD, curves and surfaces are typically represented by the use of Bezier curves, B-spline curves and NURBS (nonuniform rational B-splines) 
curves and surfaces (Farin 1997, Hoschek and Lasser 1993). These versatile functions were originally developed independently by two automotive engineers in France in the late 1950s and early 1960s (Farin 1997). With the advent of numerical machining tools, mathematical descriptions of the arbitrary curves and surfaces designed into auto body shapes were required. While the details of the construction of these geometric functions were initially proprietary, they were later made public. Additionally, the mathematical field of differential geometry has added much to the theory and usefulness of these functions.

B-spline surfaces are commonly used in computer-aided graphical design to represent arbitrary complex surfaces. Recently, a technique has been reported that employs a partial differential equation (PDE) to represent a desired surface. That is, the solution to the PDE represents a desired design surface. Parameters within the PDE may be varied to change the surface shape. Brown et al. (1990) and Brown et al (1998) describe this technique, called the PDE technique. The Galerkin finite element method using B-splines as basis functions is used to solve the PDE. Bloor and Wilson (1990) actually use the collocation method with B-splines to solve the PDE that represents the surface of interest. This approach is basically the same as the one examined herein except that here we are interested in solving the PDE to obtain its solution, whereas in (1990) they are interested in finding the PDE to solve to represent a surface of interest.

In the computational fluid dynamics (CFD) community, B-splines have been used with Galerkin methods in meshes near walls to resolve the near wall eddies by Shariff and Moser (1998) and Kravchenko, Moin, and Moser (1996) for large eddy simulations/direct numerical simulations of turbulence. They give the attractive features of B-splines as the arbitrary order of accuracy and high resolution available, the drastic reduction in required numbers of grid points, and the automatic $\mathrm{C}^{\mathrm{n}-1}$ continuity possible with n-degree B-splines. Ganesh and Sloan (1999) discuss the optimal order of spline methods for solving differential and integro-differential methods. They find that using higher degree splines reduces the number of required unknown variables by almost half over orthogonal collocation. The use of B-splines to represent the solutions to differential equations is also discussed and shown by de Boor (1978). B-splines were investigated as long ago as the nineteenth century by Lobachevshy, see Farin (1997).

\section{Bezier and B-Spline curves}

The solutions of differential equations can be seen as curves for one-dimensional problems, surfaces for two-dimensional problems and volumes for 3D problems. These curves and surfaces can be fairly complex and arbitrary, much as auto body shapes. The curves and surfaces are defined at every point on the curve or surface, much as an analytical solution. A Bezier curve can be of arbitrary degree. A linear Bezier is simply defined by two points in space. The curve is constructed parametrically. Consider a parameter ' $t$ ' that represents the fraction of distance from the first point to the second. As ' $t$ ' varies from 0 to 1, a straight line segment is traced. A 2nd degree (quadratic) Bezier curve is defined by three points, and so on. For a quadratic Bezier curve, the parameter ' $t$ ' is the fraction of the distance from the first to the second points and also the fraction of distance from the second to the third point, in straight lines. Two points are defined by ' $t$ ' on these two lines. Another line can be drawn between these two points, with a point on this third line also having a point a distance ' $t$ ' from the first to the second point. As ' $t$ ' varies from 0 to 1 , all three points move with the third point tracing out the quadratic Bezier curve. In order to represent a complex curve, either the degree of the Bezier can be increased, or a spline curve can be formed by connecting individual curves together. This latter is called a Bezier spline curve.

It is better to construct a Bezier spline curve to represent complex curves because if a Bezier point is changed for a particular curve, it has only local effect. Also, higher degree Bezier curves can exhibit undesirable wiggles. An important consideration, one that sets the new method apart from other 
traditional methods, is that a high level of continuity can be maintained from one individual curve to the next. $\mathrm{C}^{0}$ continuity is maintained if one curve begins at the same point where the last one ends. These junctions are called knots. If the spline curve has a continuous first parametric derivative at the knot, then we have $\mathrm{C}^{1}$ continuity, and so on. Because most equations associated with fluid mechanics include 2 nd order derivatives, we desire to maintain $\mathrm{C}^{2}$ continuity. This means that we need at least 3 rd degree Bezier curves. A convenient way to ensure that $\mathrm{C}^{2}$ continuity is preserved is to employ B-spline (Basis spline) curves. The same curve can be represented by either Bezier curves or B-splines. In fact, B-splines curves can be thought of as generalizations of Bezier spline curves. B-splines can be converted into Bezier spline curves following Boehm, see Farin (1997). The B-spline control points, in general, are not the same as the Bezier control points.

B-spline curves are defined by a vector of knots and associated control (or de Boor) points. These control points are, in general, not the same as Bezier points, although the latter can be derived from them. A cubic B-spline curve is automatically $\mathrm{C}^{2}$ continuous. Exact derivatives can simply be evaluated using well known formulae at any point on the curve. To obtain a B-spline surface, a tensor product of two Bspline curves can be formed. The B-spline surface and derivatives in any parametric direction can then be evaluated at any point. This means that the several terms in a differential equation can be easily evaluated from the B-spline surface and summed to determine if the equation holds. If not, the B-spline control points can be adjusted until the equation holds to some tolerance. If the tolerance is not met, additional spline segments can be added to increase the ability of the B-splines to represent the curve or surface in question. In fact, these geometric functions can be seen to be close approximations to the analytical solution of the differential equation in question. Because the B-spline curve or surface can be evaluated at any point, one must choose which points are to be used for the evaluations. These are termed collocation points and the method is said to belong to the class of collocation methods. The method can be considered a finite element collocation method where the test function used to form the variational statement is the Dirac delta function. Using the Dirac delta function in an integration has the effect of returning the original differential equation with no integration having been performed (Carey and Oden 1983, Lapidus and Pinder 1982).

The primary advantage of using B-spline curves/surfaces rather than traditional finite differences or volumes or elements is the fact that a curve or surface can be defined using far less information than for a standard finite difference/volume/element method. This fact means that the problem matrices that must be solved to find the numerical solution can be greatly reduced in size, leading to much faster solution times. Finally, there is no requirement to create a mesh in the traditional sense because there is no integration required or is there a requirement to provide schemes to approximate derivatives.

Figure 6 illustrates a cubic B-spline curve along with its B-spline control (de Boor) points and the data that the B-spline curve is interpolating. We use the definition of B-splines as given by Farin (1997); other authors define them slightly differently. The interpolated data come from a finite element solution (Fidap) of the axial velocity in a rectangular channel, at 1.25 channel heights downstream of the inlet. The Reynolds number for the channel flow is 500 based on channel width $(=1)$; the inlet velocity is uniform. This location was chosen because the finite element data (bilinear elements were used) first become well behaved at this location; that is, the transverse spatial oscillations become negligible for the finite element mesh used. The finite element solution data require 37 nodes in the transverse direction to resolve adequately the axial velocity at this location. The cubic B-spline requires the specification of values for seven de Boor points ( 2 being on the channel walls) along with a knot vector of $(0 ., 0 ., 0 ., 0.25,0.5,0.75,1 ., 1 ., 1$.$) . The multiple knots at the endpoints are required to ensure that the$ $\mathrm{B}$-spline curve begins at 0 and ends at 1 . Hence, the bilinear finite element solution requires the determination of 35 interior node values; only five B-spline control points are required. 
Because $n$-degree $B$-splines have $\mathrm{C}^{\mathrm{n}-1}$ continuity at the knots, their derivatives are defined to degree ' $\mathrm{n}-1$ ' everywhere. The fact that the Navier-Stokes, continuity, and energy conservation equations are at most 2 nd order in their derivatives, suggests the use of cubic B-spline curves. Formulae for the parametric derivatives of B-spline curves and surfaces are given in (Farin 1997, Hoschek and Lasser 1993); the Cartesian derivatives can be formed from the parametric derivatives using the chain rule. The B-spline in Figure 6 has an implicit form, meaning that the parametric coordinate is the same as the Cartesian coordinate. Figure 7 illustrates the 1st and 2nd derivatives of the B-spline curve of Figure 6 with respect to the transverse Cartesian coordinate. The B-spline curve is first converted to a Bezier spline curve before the derivatives are computed. As can be seen, the 2 nd derivative is continuous, although not smooth, as expected.

Figure 8 shows the transverse velocity at the same axial location of 1.25 channel widths. Here 14 knots or 12 de Boor points are needed to adequately resolve the velocity component profile. (The de Boor points are, in general, not located at the same points as the knots.) Of the 12 control points, two are on the wall, so that 10 data must be found to obtain the solution for the transverse velocity component. The fact that more control points are required for the transverse velocity relative to the axial velocity indicates that the fineness of the mesh used to solve the standard finite element problem is controlled by the velocity component with the sharpest curvatures. Using B-splines, however, the number of de Boor points used can be different for the different velocity components.

Because the present method does not require a mesh in the sense of finite differences or finite volumes/elements, savings of computational time can be realized from being able to use only the finest knot mesh required for each dependent variable.

In summary, we can say that the proposed numerical method has the following attractive features:

1. Any level of accuracy can be achieved by using B-splines of sufficient degree with sufficient numbers of spline segments.

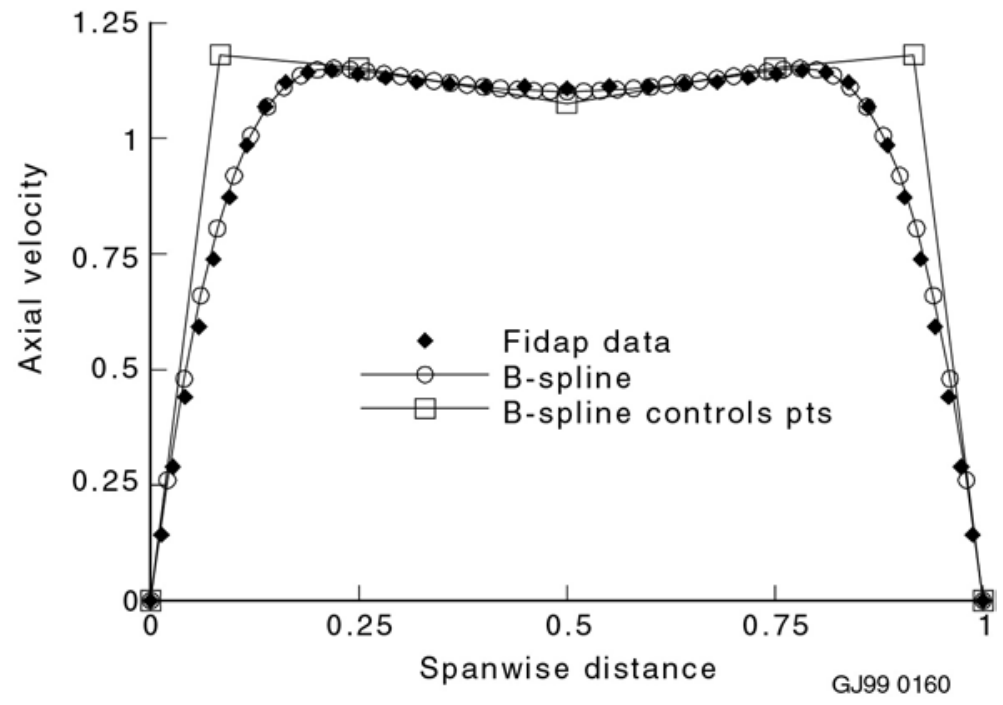

Figure 6. B-spline for the axial velocity of a channel flow at $\mathrm{x} / \mathrm{L}=1.25$ using 5 interior control points compared to finite element data (Fidap) using 35 interior grid points. 


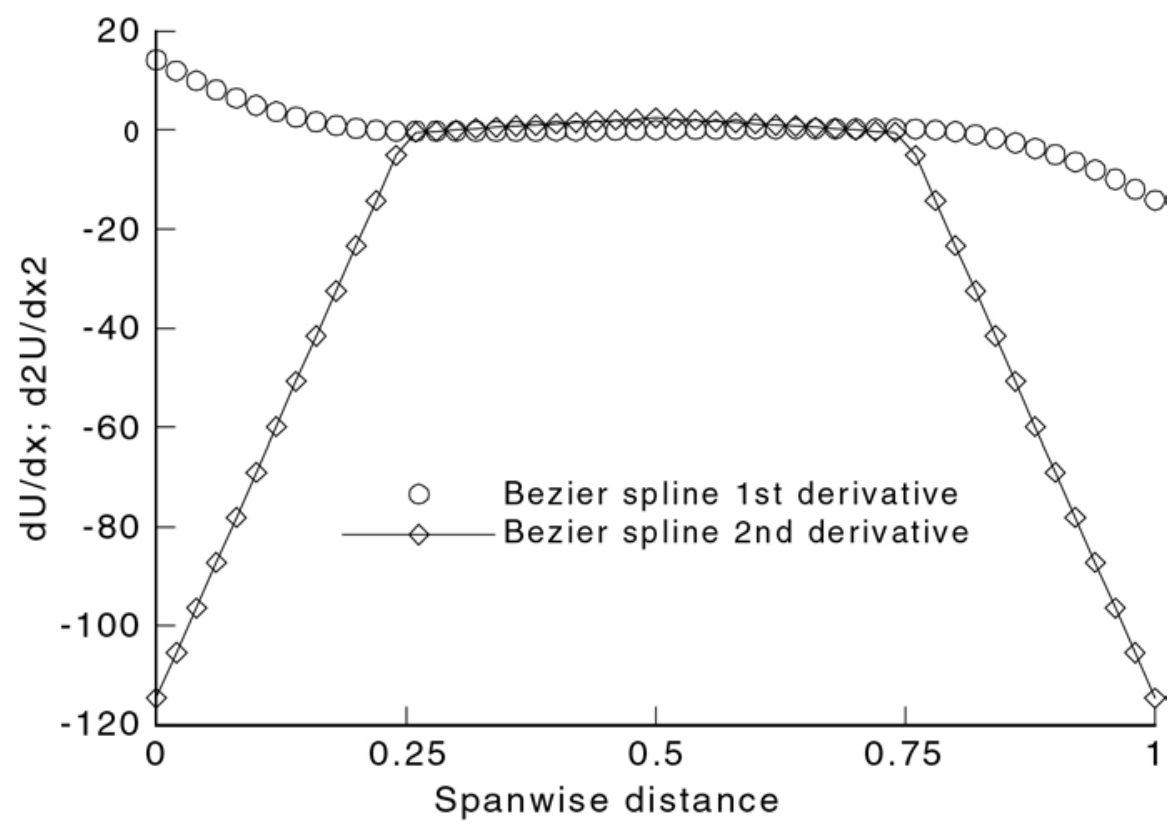

Figure 7. First and second derivatives of the axial velocity in the spanwise direction for the B-spline of Figure 6.

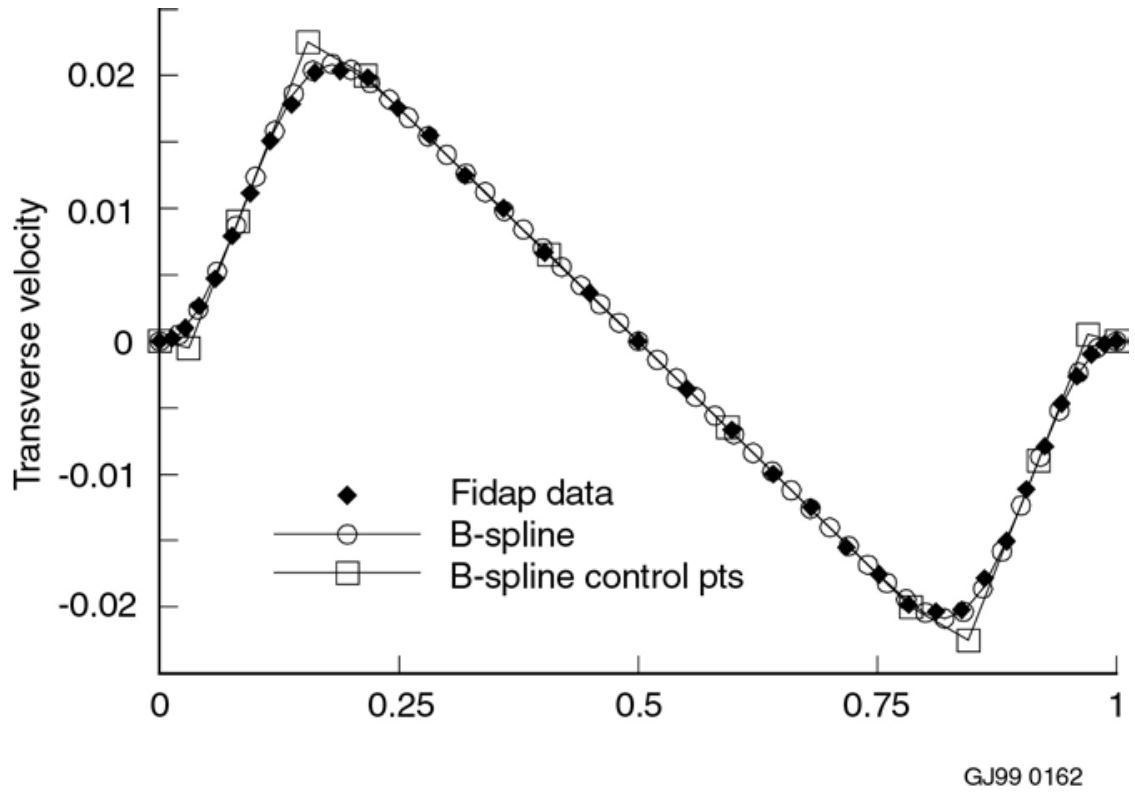

Figure 8. B-spline for transverse velocity at $\mathrm{x} / \mathrm{L}=1.25$ using 10 interior control points compared to the finite element data (Fidap) using 35 interior grid points. 
2. Large potential savings in solution time can be realized because fewer data are required to define solution curves and surfaces than for standard methods and each dependent variable can use the fewest data required for its solution.

3. A computational mesh in the standard sense is not required; the knot meshes that are required are expected to be generated during the solution procedure.

4. No truncation errors are associated with the method; the errors occur in how closely the Bspline curves can represent a solution variable.

5. No approximate schemes are needed to compute derivatives or perform quadrature.

\section{Example Problem}

An experimental code has been written that defines a B-spline surface using the tensor product of B-spline curves in a rectangular domain. The code converts the B-spline surface to a two-dimensional "patch-work" quilt of Bezier surface patches, then evaluates the surface and its 1st and 2nd derivatives in the $\mathrm{x}$ and $\mathrm{y}$ Cartesian coordinate directions. It is less computational work to convert the B-spline surface to Bezier surface patches when it is required to compute derivatives.

The B-spline code has been programmed for use in the solution of an incompressible, twodimensional, laminar, developing flow in a straight channel at Reynolds number 500 (based on channel width). The channel flow is the same one used earlier to demonstrate the use of B-splines to interpolate cross-stream data profiles. In order to avoid the region at the entrance to the channel where the finite element solution is not adequately resolved, we use a computational domain that begins at $\mathrm{x} / \mathrm{L}=1.25, \mathrm{~L}$ being the channel width. The domain ends at $\mathrm{x} / \mathrm{L}=13.0$. Inlet and exit profiles for the axial and transverse velocities for the B-spline computation are obtained from the finite element solution. Also, for simplicity, we use a B-spline surface interpolated to the finite element solution for the pressure for the present problem. We expect to generalize our procedure for future articles.

The B-spline surfaces for the axial (u) and transverse (v) velocity components are each defined by knot vectors in each of the two Cartesian coordinate directions and by the z-components of the de Boor (control) points. The z-components represent the dependent variables. The knot vectors are chosen to be able to interpolate the inlet data at $\mathrm{x} / \mathrm{L}=1.25$. Figures 6 and 8 actually represent the inlet conditions for $\mathrm{u}$ and $\mathrm{v}$. The solution is found by obtaining values for the z-components of the de Boor points. For our test problem here, this was performed by employing a commercial optimization code, OptdesX (1996). The two momentum equations and continuity equation that describe the straight channel flow are evaluated on a $(47 \times 50)$ matrix of points in the computational domain using the values and derivatives obtained by evaluating the B-spline surfaces. Initial values are set to zero except for the boundary conditions. Residuals are then computed for each equation and sent to the optimizer. The optimizer attempts to minimize the residuals by changing the control points using an optimization algorithm called the hybrid SQP-GRG (sequential quadratic programming — generalized reduced gradient) algorithm, see Parkinson and Wilson (1998). The solution procedure iterates until the residuals cannot be reduced further for a given B-spline surface. Obviously, a further reduction in the residuals could be achieved by inserting more knots, hence, more de Boor points until the desired level of accuracy is achieved.

Figures 9 and 10 illustrate (partial) B-spline solution surfaces for the axial and transverse velocities. The superimposed meshes on the surfaces show where the surfaces are evaluated for residual computation and plotting purposes; the surfaces are defined everywhere and can be evaluated anywhere. Hence, the solution surfaces actually represent bivariate geometric functions that approximate the true solutions. 


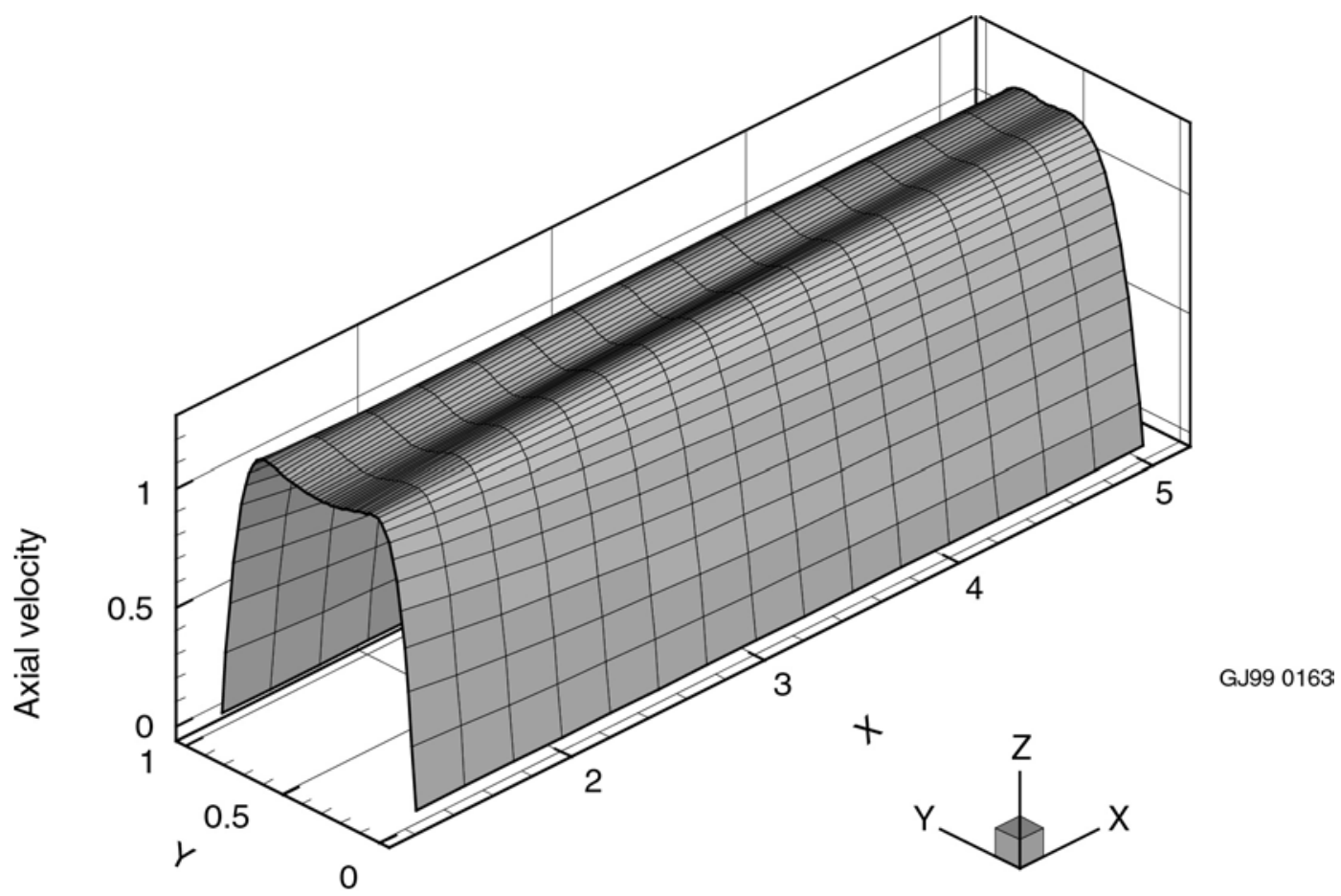

Figure 9. B-spline surface solution for the axial velocity for the 2-D channel flow.

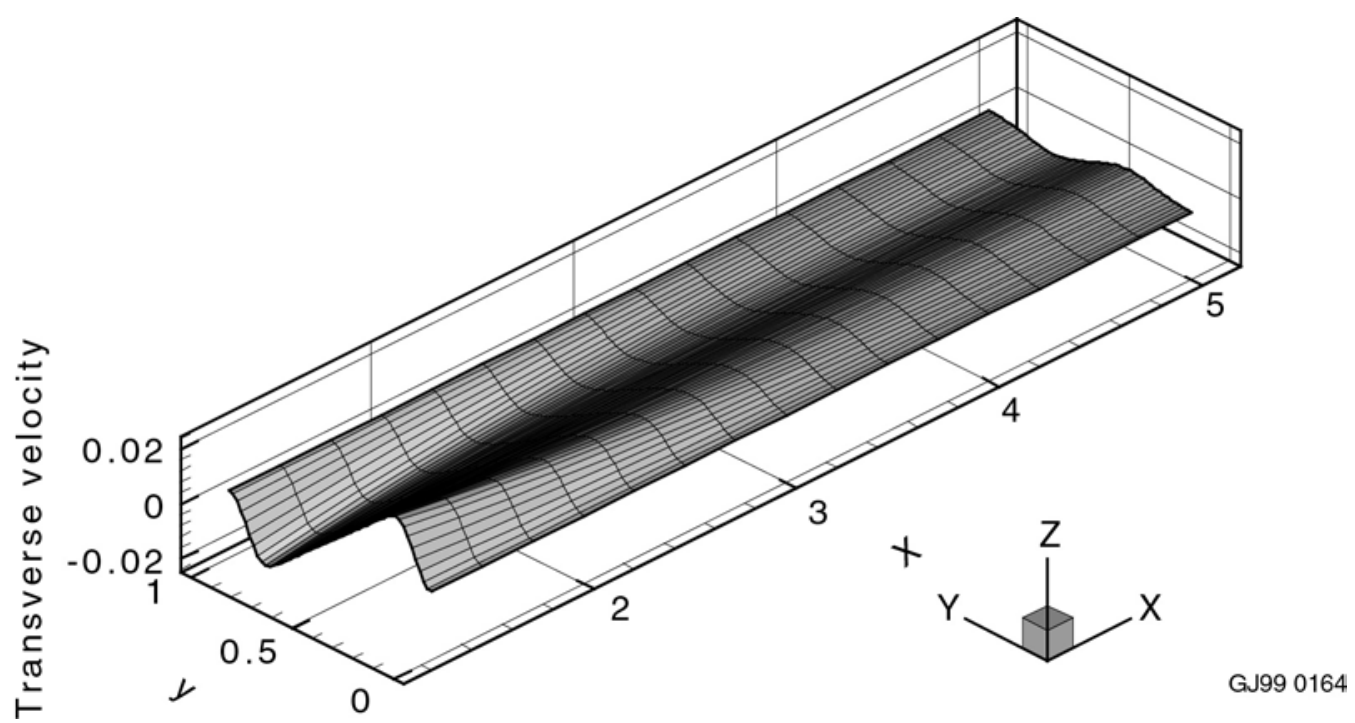

Figure 10. B-spline surface solution for the transverse velocity for the 2-D channel flow. Note that the y-direction scale is reversed from Figure 8.

\section{Further Progress}

The above example problem, reported in Johnson and Landon (1999), demonstrated the feasibility of using a B-spline collocation method to represent solutions to the equations of fluid dynamics.

However, the time required to find the solution using the optimization code was excessive. It is expected that a built-in solution algorithm will decrease the time required to find the solution. Newton's method has been employed to set up the problem matrix and iterate to a solution. Newton's method is used to 
linearize the problem or predict a solution assuming that the problem is linear. Because it is not, the method is repeated until the true nonlinear solution is approached.

In order to use Newton's method, a problem matrix must be set up. The matrix is actually the Jacobian of the system, that is, a matrix of sensitivity coefficients. A number of collocation points are chosen, the same number for which there are unknown control points. For each of these collocation points, the neighboring control points are perturbed to determine sensitivity. These values are the elements of the Jacobian. The matrix equation to be solved states that the Jacobian matrix multiplying the update vector of values that should be added to each control point is equal to the negative of the residual of the fluid equations. The residual is the value that the differential equations(s) that are being solved are different from what they should be at a given collocation point. This procedure is iterated until the residuals cannot be driven any closer to zero. Then it must be decided if this is close enough.

Several research issues must be addressed to obtain the best solution possible for a given B-spline surface. These include the following: (a) where should the collocation points be located?, (b) how many elements should be included in the Jacobian? (the fewer the faster, the more, the more accurate), (c) how far should the step be taken in the search direction found by solving the matrix equation? (the full step is only appropriate for a linear problem), (d) how well behaved is the solution algorithm as it navigates the solution space? (is there a unique solution?), (e) how can additional splines be added to further reduce the residual?, (f) what is the best matrix solver to use? Some of these issues have been addressed or are being addressed. Initially, the collocation points were located at the knots. This led, however, to kinks in the solution profiles, probably because of the finite precision of the computer. Initially, only five elements for each collocation point were included in the Jacobian matrix. Later all that had an influence were included, giving a better answer. However, it may be the case that fewer elements can be used early in the solution process when the solution is further away to save compute time. It seems best to calculate the step size based on what will yield the smallest possible residual, although this is more time consuming. A more optimal approach needs to be found. Sometimes, it seems that the solution search process works its way into a corner where it cannot escape, even though it is known that another solution is better. It is not clear whether this is because there are multiple solutions or because of a weakness in the search process. Currently, a simple lower-upper direct matrix solver is being used to solve the matrix equation. This will not be optimal for large matrices. We have not yet tried to add additional splines to the B-spline surface to better resolve the solution. There is work to be done to resolve these issues, and the resolutions may be problem size dependent.

\section{Vadose Zone Moisture Movement}

Soil water movement in the vadose zone is of vital importance to biological and geochemical processes. These processes strongly affect the fate and transport of chemical contaminants. In an effort to better predict the movement and distribution of the soil water in the vadose zone, accurate values of the transport parameters controlling the processes are necessary. However, hydrologic tools capable of providing estimates of the parameters needed by vadose zone transport models are lacking.

In order to estimate the spatial distribution of vadose zone transport parameters, two measurement tools have been developed. The first tool, the Exfiltrometer, measures the hydraulic conductivity and water potential as a function of water content. The hydraulic conductivity and water potential are highly nonlinear functions of water content and are typically measured by extraction of a soil core with subsequent lengthy laboratory analysis. The data collection from the Exfiltrometer is typically completed in a few days and has the advantage of measuring the soil transport properties in situ on undisturbed soil.

The second tool under development, the Vadose Zone Monitoring System, consists of a set of instruments that monitor the three state variables (water content, water potential, and solute concentration) of the vadose zone. These instruments are placed in a borehole and monitor the changes of the state variables as 
a function of time. Relationships between the state variables can be developed if the vadose zone system undergoes significant water flux changes.

\section{The Exfiltrometer}

Unsaturated hydraulic properties are needed in most environmental studies where the rate of soil water movement is a concern. The Exfiltrometer was and continues to be developed at the INEEL to provide unsaturated hydraulic properties under field conditions.

The Exfiltrometer relies on a dry subsurface beneath the instrument to remove soil water from a fixed volume within the instrument while recording the water content and water potentials. Using this method, the soil water retention curve (i.e., the relationship between the moisture content and the water potential) is obtained directly. Estimation of the soil water flux requires monitoring the change in soil water content over time. Changes of soil water content are related to water leaving the measurement zone through the bottom of the Exfiltrometer. The hydraulic conductivity is then calculated using Darcy's Law and the measured potential gradients using tensiometers.

Over the course of FY-99, the Exfiltrometer has undergone four revisions to make it more effective. To be effective it must provide for a rapid setup that is independent of the user, provide for deployment at depths to at least $20 \mathrm{~m}$ below land surface and provide a high level of repeatability. The latest version of the Exfiltrometer is shown on Figure 11 and consists of a metal tube, a water content sensor and two tensiometers. The circular outer casing was chosen to facilitate future borehole

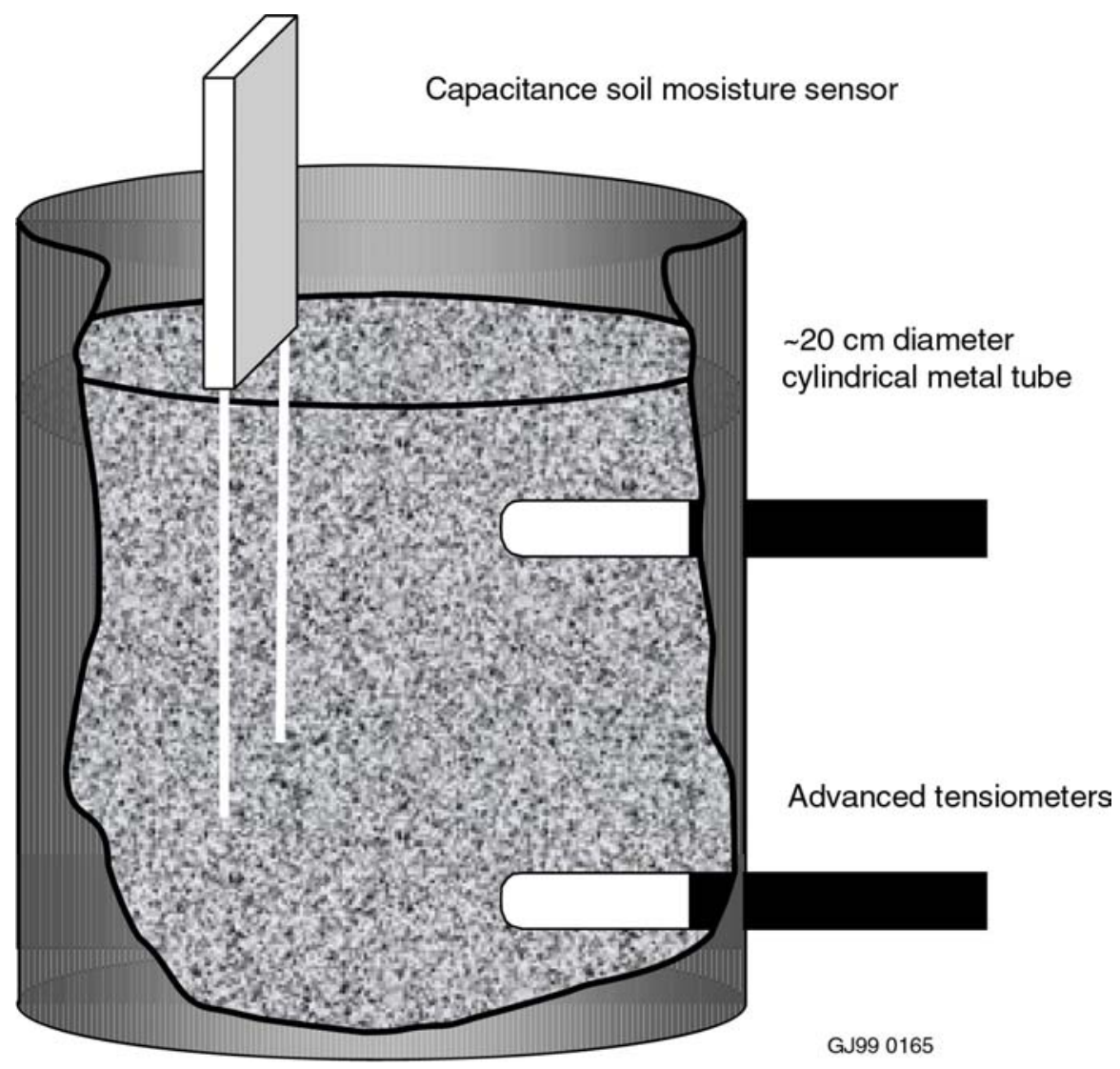

Figure 11. The latest Exfiltrometer. 
installation. The amount of soil water is monitored using a modified Campbell Scientific 505 capacitance probe. The water potential gradients are calculated from the difference in the measured water potentials using tensiometers spaced at a distance of approximately $5 \mathrm{~cm}$.

To evaluate the Exfiltrometer repeatability, a test was conducted in which the Exfiltrometer was operated sequentially without removing the metal tube or the monitoring instrumentation. For this test, the Exfiltrometer was installed in a sandy loam porous material and the soil was initially wetted to near saturation and allowed to drain for one day. This was necessary to achieve similar initial and boundary conditions between the two repeatability tests. The first repeatability test was conducted by adding water to the soil surface in the Exfiltrometer and monitoring the depth of water infiltration using the capacitance sensor and the advanced tensiometers. When the infiltration front reached the lower tensiometer, excess water was removed from the soil surface and data collection started. As the excess water drained from the Exfiltrometer, measurements of the soil water content and the water potential was monitored and recorded by a Campbell Scientific $23 \mathrm{X}$ data logger. After a period of 1.2 days, the test was terminated and the second repeatability test was conducted following the same procedures. The second test lasted 2.8 days.

The water retention curve data obtained during the repeatability tests are presented on Figure 12. Water contents range from $24 \%$ to approximately $12 \%$ and water potentials range from 0 to approximately $-280 \mathrm{~cm}$. This data represents the most dynamic portion of the water retention characteristic curve and it is encouraging that the two data sets generally fall upon one another. A water retention curve can be easily extended into drier regions with a single laboratory measurement on the same soil.

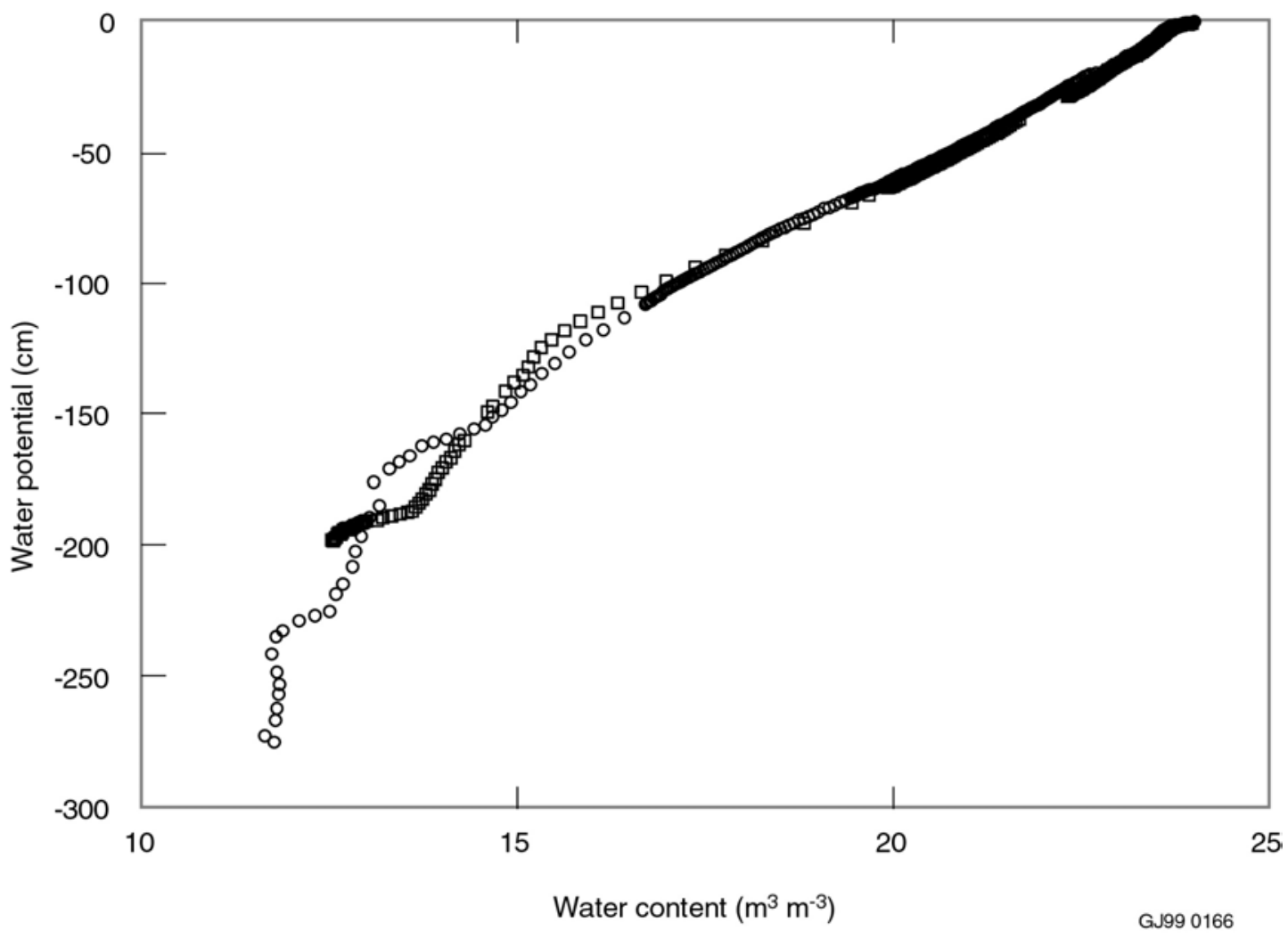

Figure 12. Water retention data. 
The unsaturated hydraulic properties derived from these data sets are given on Figure 13 in the form of the hydraulic conductivity water content relationship. Hydraulic conductivity spans approximately three orders of magnitude change over the change of water content measured during the Exfiltrometer test. As with the water retention curves, the hydraulic conductivity curves are also reproducible with the exception of a few outliers. These results are somewhat a surprise since the field temperatures during the repeatability experiments varied more than $20 \mathrm{C}$. These results are encouraging since hydraulic conductivity relationships are difficult to obtain under carefully controlled laboratory conditions.

The Exfiltrometer will be further tested over a small area to: (1) Develop correlations between the 7 van Genuchten parameters. The correlations will be incorporated into geostatistical models used to describe water and contaminant transport through the vadose zone. (2) Develop methods for using the Exfiltrometer to generate data sets that can be used to model hysteretic effects over the range important in field investigations. (3) Incorporate the unsaturated hydraulic properties into the microbiological studies being done to estimate microbial activity under unsaturated conditions.

\section{The Vadose Zone Monitoring System}

The Vadose Zone Monitoring System (VZMS) was designed to monitor the three state variables (water content, water potential, and solute concentration) at any depth below land surface. Existing monitoring tools were limited to operating near the ground surface to depths of a few meters. The VZMS has the advantage of examining these state variables as a function of depth allowing development of the spatial distribution parameters for geostatistical analyses.

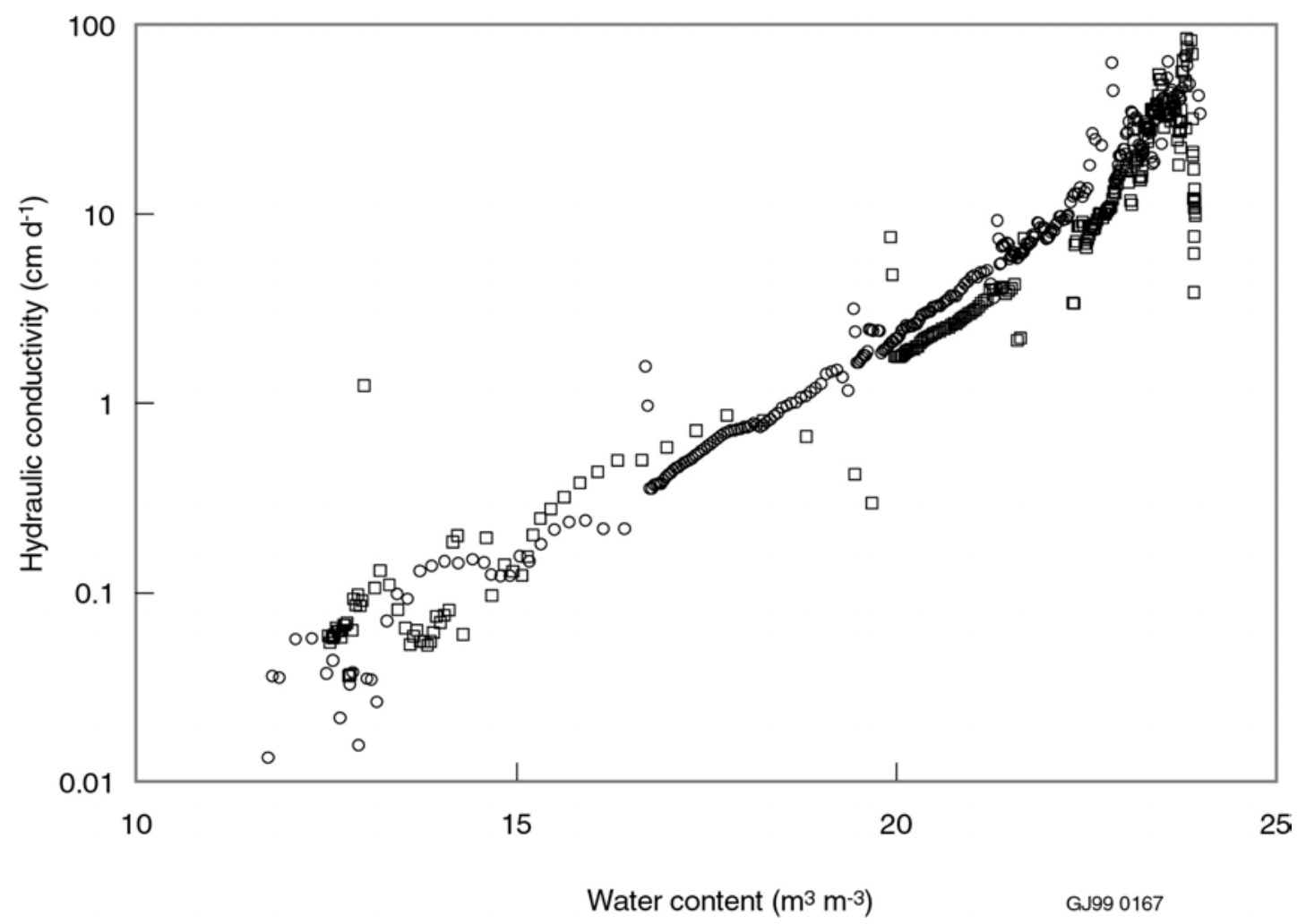

Figure 13. Unsaturated hydraulic properties derived from data. 
The VZMS consists of a water content sensor that relies on changes in dielectric properties, an Advanced Tensiometer for soil water potential and a commercially available vacuum lysimeter for extracting samples for solute, Figure 14. The suction lysimeter is directly attached to the advanced tensiometer where as the modified capacitance sensor is attached to the tensiometer with a lever arm. The lever arm allows a simple method to establish a direct contact of the capacitance sensor guides to the borehole wall. The VZMS is installed by drilling a borehole, lowering the unit to the desired depth, releasing the capacitance sensor, and applying a slight upward tension on the tensiometer to provide a force on the lever arm and secure the water content sensor against the borehole. The borehole is backfilled with silica flour around the instrument package and topped with either a bentonite seal or native soil backfill as with conventional tensiometers. Approximately six VZMS packages can be installed within a single borehole creating multiple monitoring levels.

Three boreholes were equipped with VZMS packages to a depth of 50 feet in the E-Area at Savannah River Site and have been operational since April 1999. Preliminary analysis of the data indicates the VZMS packages are operating better than expected. Moisture contents are stable with variations of less than $0.5 \%$ moisture. The suction lysimeters are able to withdraw soil water for chemical analyses.

The water potentials are stable $( \pm 5 \mathrm{~cm})$ and indicate two phenomena-barometric and earth tide effects - that are not normally observed with soil physic's monitoring tools. Figure 15 illustrates a portion of the water potential data from VZMS well SRS6 for a period of 10 days. Also plotted on this figure are the barometric pressure and the calculated earth tide. The relationship between water potential and the diurnal barometric pressure changes is fairly obvious and the semidiurnal behavior of water potential could be due to earth tides.

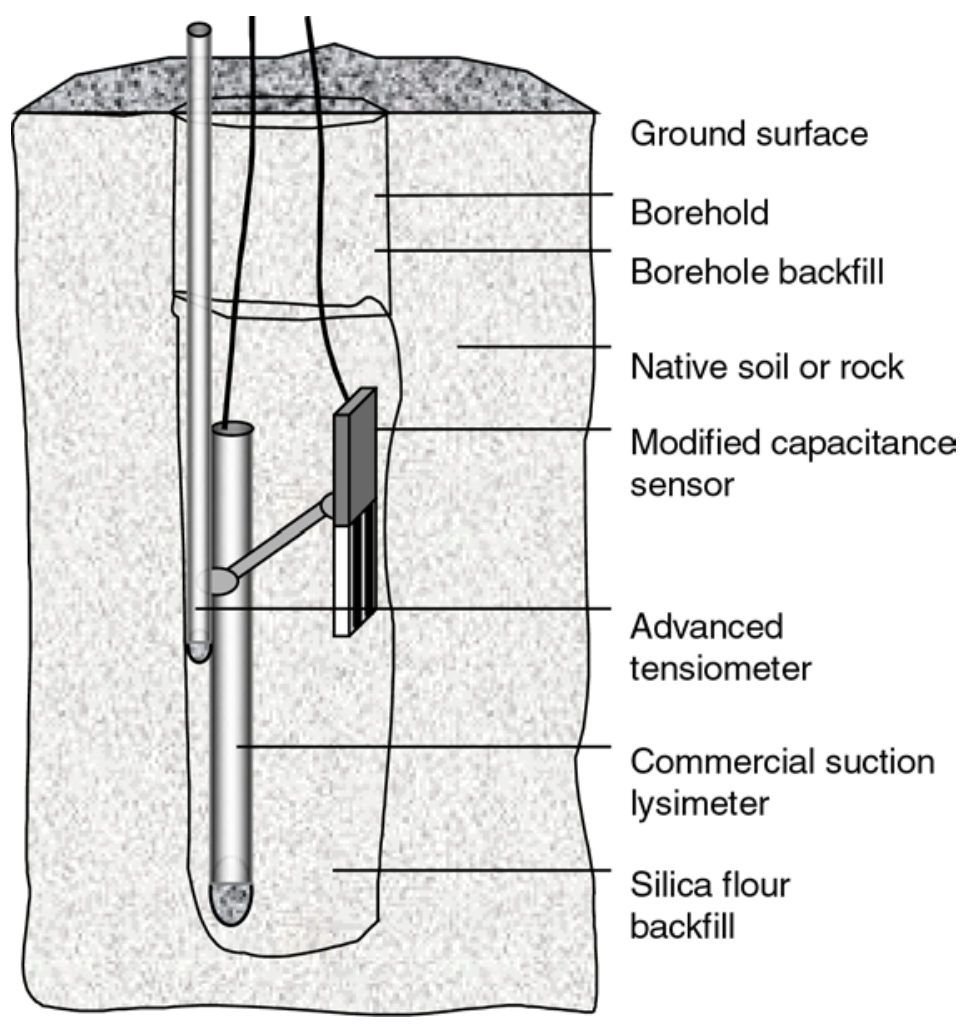

Figure 14. The Vadose Zone Monitoring System. 


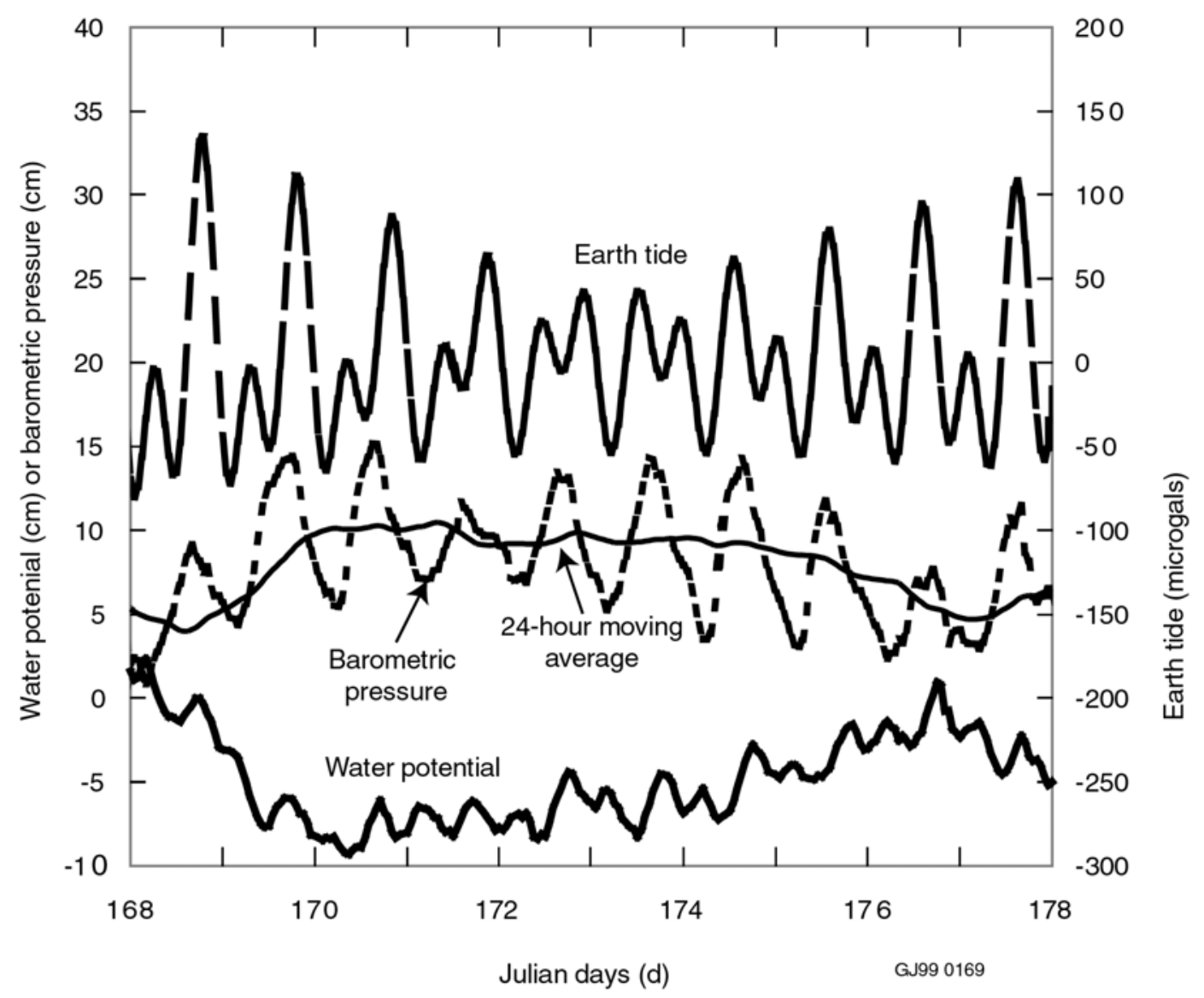

Figure 15. Water potential data from VZMS well SR6, barometric pressure, and calculated earth tide for a 10 day period

Future work includes presenting a manuscript on the Savannah River data with emphasis on the barometric and tidal effects. Additional VZMSs are being planned at SRS in FY-00. SRS and the Subsurface Contamination Program will directly fund the installation and monitoring of the VZMS.

The VZMS and the Exfiltrometer will also be deployed at the Jefferson Channel Research Site for monitoring the injection of water and tracers. This research is being conducted in collaboration with the University of Idaho and will include:

- Using subsurface radar to obtain initial water content distribution

- $\quad$ Correlating the initial water content distribution to unsaturated hydraulic properties

- Developing a stochastic model of subsurface properties

- $\quad$ Evaluating the inverse technique for obtaining hydraulic properties

- Modifying the Exfiltrometer to obtain unsaturated hydraulic properties in deep boreholes

- $\quad$ Modeling chemical transport in porous media. 
The Exfiltrometer and the VZMS tools will allow the INEEL to better understand the transport processes and parameter estimation within the vadose zone. However, the general problems in vadose zone transport will require additional innovations in the areas of data collection and interpretation. One need identified will require a monitoring system capable of seeing between boreholes with a high degree of spatial resolution. At present, no single system appears capable of adequately performing this task and it is expected that geophysical techniques combined with INEEL vadose zone instrumentation will be necessary.

\section{Relationships between Moisture Content, Tension, and Biogeochemical Activity in the Vadose Zone}

One of the most significant difficulties facing in situ bioremediation today is the biodegradability of the contaminant. The remediation takes place in the vadose zone, where water content can vary significantly within a small volume. While volume-averaged water content measurements can sometimes adequately predict flow patterns and mobility of contaminants, they have been less successful at predicting the remediation potential because of inconsistent results regarding microbial activity. The activity of microorganisms in the subsurface is controlled in part by the availability of water and water borne nutrients. The availability of water and its mobility in soil is in turn controlled to varying degrees by the activity of soil microorganisms and their production of $\mathrm{CO}_{2}$. For example, hydraulic conductivity (a measure of water mobility in soil) is strongly influenced by the concentration of $\mathrm{Ca}^{2+}$ (through precipitation and dissolution of calcium carbonate) and the $\mathrm{CO}_{2}$ level influences the $\mathrm{Ca}^{2+}$ concentration. The influences of $\mathrm{Ca}^{2+}$ and $\mathrm{CO}_{2}$ levels on the water retention curve are also unknown, and many microorganisms produce biofilms that may modify flow path. Finally, the production and migration of $\mathrm{CO}_{2}$ significantly influences the migration of radionuclides in the vadose zone. The relationship between water content and microbial activity is expected to be complex. Laboratory and field-scale unsaturated experiments will be conducted to test hypotheses and to develop an understanding of the relationship between microbial activity and moisture characteristics. This may include various soil types ranging from sandy to clay soils, but will be generally limited to water potentials in the range 0.1 to 6 bar. Adequate correlation of water content with biodegradability will be important in assessing remediation potential at most DOE sites, as well as other contaminated sites. This task supports ongoing work to develop vadose zone instrumentation including the Exfiltrometer and the dielectric soil water content sensors.

In porous media, microbial activity has been shown to decrease to zero at both very high and very low water contents (Figure 16). This has generally been attributed to higher osmotic pressures at low water contents and to oxygen limitations at high water contents. We hypothesize that this behavior also depends on the amount of water that is available to the microbes. In effect, at very low water contents, water in unsaturated soil becomes unavailable for microbial use because the bulk of the water is held in pores smaller than or similar in size to the bacteria. Thus, not all of the water in unsaturated soils is available, and the amount available would depend directly on the pore size distribution of the soil. This would shift the maximum activity range seen in Figure 16 to higher or lower water contents depending on how much water is available to the microbes. This effect would be expected to be more pronounced in sandy soils than in clays, since sand contains many more micropores $(<1 \mu \mathrm{m})$ than do clays. The addition of organic matter would also increase the relative amount of micropores in the soil.

This project is divided into four subtasks, including: (1) soil characterization, (2) aerobic microbial activity, (3) anaerobic microbial activity, and (4) estimation of diffusion path lengths and osmotic pressures. In Subtask 1, several properties of the model soil, which will be obtained from the USDA Sheep Experiment Station near Mud Lake, ID, will be measured to gain an understanding of the distribution of the water in the soil at the various water contents. These include surface area, interstitial volume, pore volume, and pore size distribution. In Subtask 2, soil will be adjusted to the desired water 


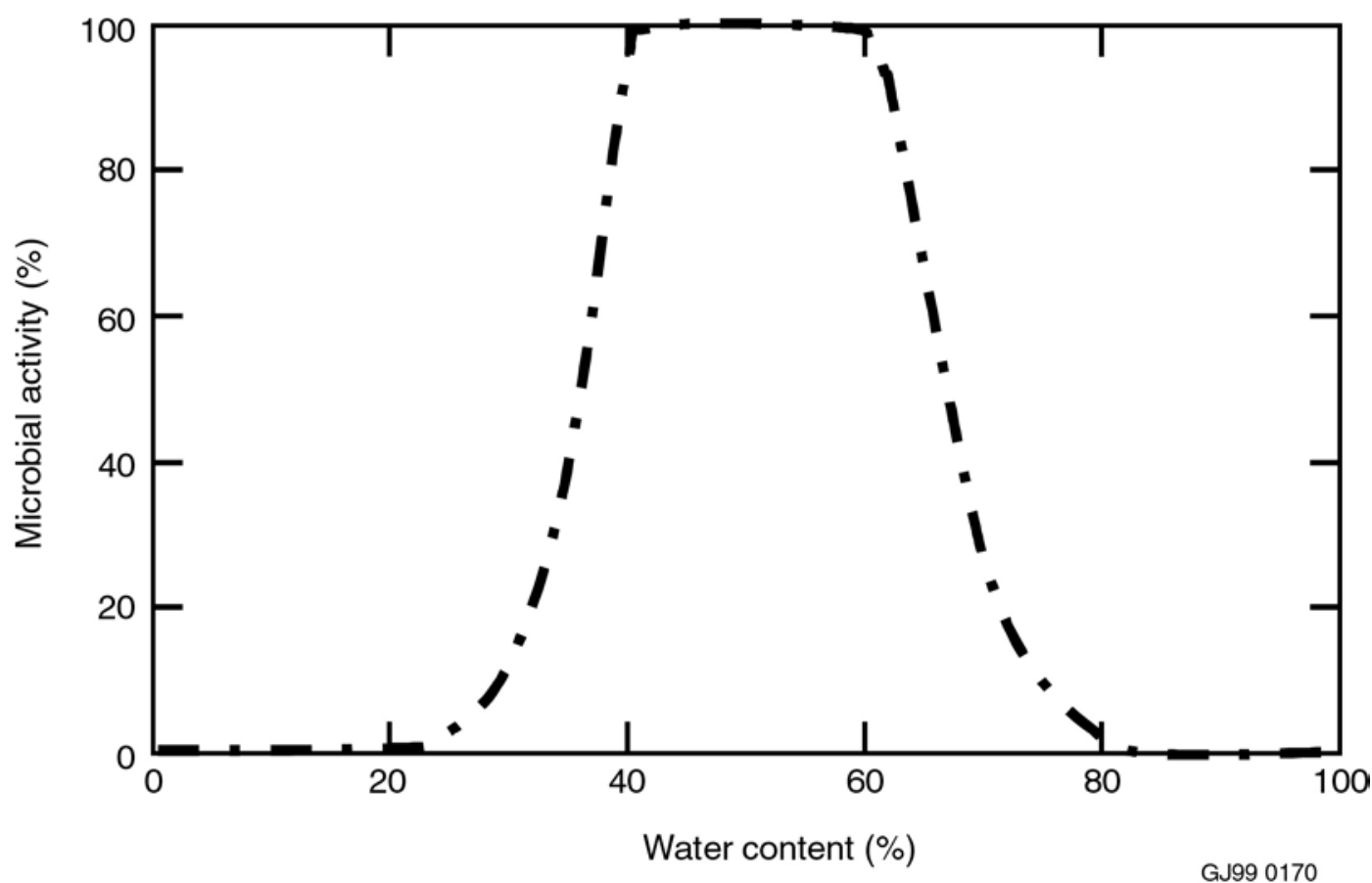

Figure 16. Microbial activity depends strongly on water content, decreasing to zero at both very low and very high water contents.

content using the Unsaturated Flow Apparatus (UFA ${ }^{\mathrm{TM}}$, see below). The solution used to adjust the water content will be a glucose solution spiked with ${ }^{14} \mathrm{C}$-UL-glucose. Microbial activity in the soil will then be measured by ${ }^{14} \mathrm{CO}_{2}$ evolution under aerobic conditions. These experiments will be done at water potentials ranging from 0.1-0.6 bar, and will be done in sealed batch systems. Small enough amounts of substrate will be added to ensure that the cells remain in maintenance metabolism. Subtask 3 will be similar to Subtask 2, except that the substrate will be acetate spiked with ${ }^{14} \mathrm{C}$-UL-acetate, and the sealed bottles will be maintained under anaerobic conditions. In Subtask 4, the diffusion path length will be measured for oxygen at various water contents. This value is important because it increases with water content, and limits the rate at which oxygen is supplied to the microbes. In batch or continuous experiments, an $\mathrm{O}_{2}$ microelectrode will be used (in a system that initially contains no oxygen) to measure decreases in gas phase concentration of oxygen with time from a known initial level of added $\mathrm{O}_{2}$. This data will be used to estimate an effective diffusion coefficient, which will be used with the actual oxygen diffusion coefficient to estimated the diffusion path length. Initial salt concentrations will also be estimated for the soil at its original water content, and used with the final water content to calculate the osmotic pressure at each water content.

Soil water content will be adjusted using a UFA ${ }^{\mathrm{TM}}$ rotor (see Figure 17). This step will also homogeneously distribute the substrate solution throughout the soil samples. The UFA ${ }^{\mathrm{TM}}$ is manufactured by UFA Ventures, Inc., Richland, WA. The UFA ${ }^{\mathrm{TM}}$ is an innovative hydrogeochemical instrument for rapidly, directly, and accurately determining the fluid transport properties of almost any porous media, such as soil, rock, cement, grout, ceramic or sludge, with respect to almost any fluid, including water, waste effluent, oils and organic solvents. The most important transport properties that require measurement are the hydraulic conductivity of liquids and gases (or the intrinsic permeability of the porous media), diffusion coefficients, the retardation factor or chemical behavior of migrating contaminants, and the composition of the pore fluids. Traditionally, these are very difficult to measure in relatively impermeable materials or under unsaturated conditions. But the UFA ${ }^{\mathrm{TM}}$ directly measures these in hours to days. 


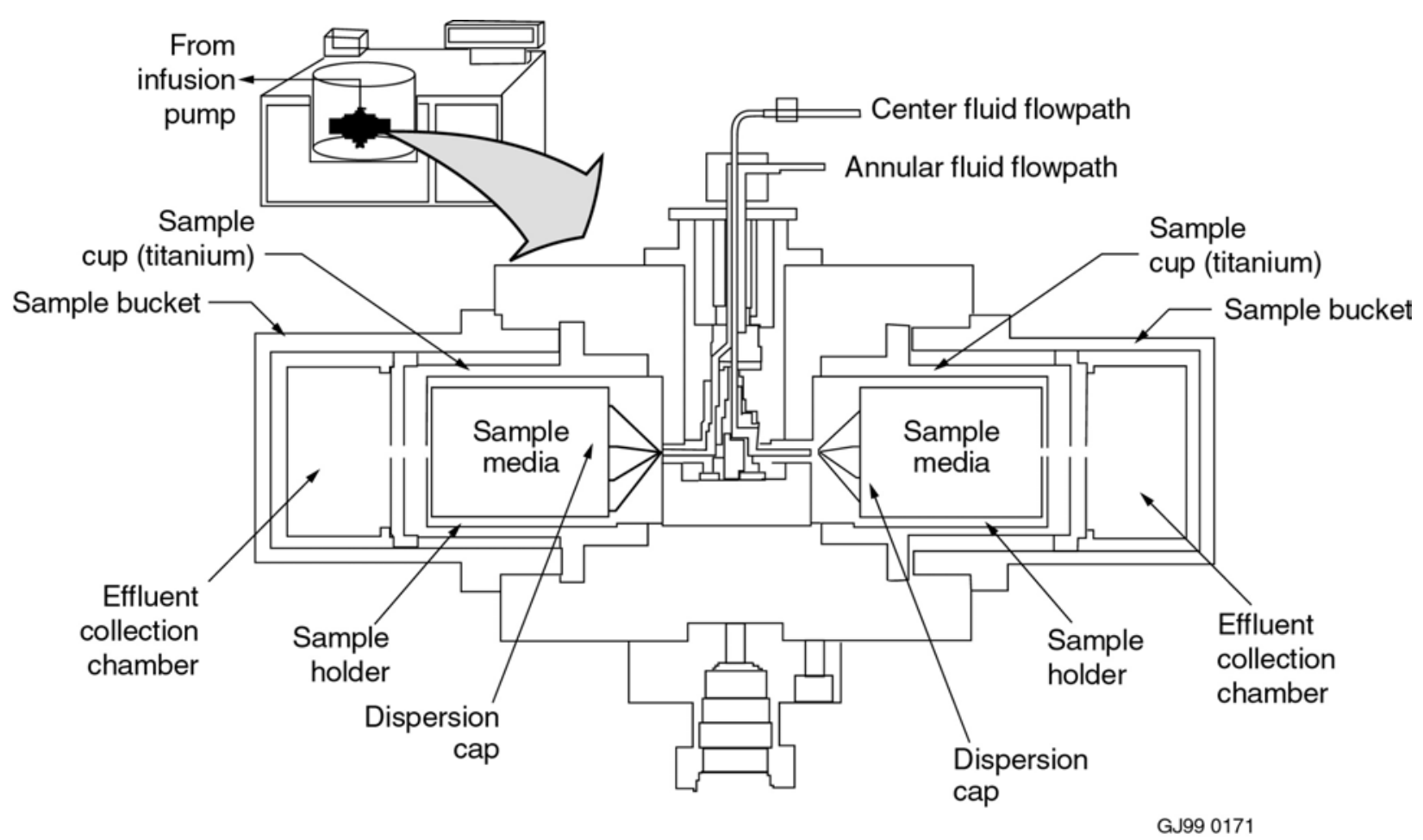

Figure 17. Diagram of the centrifuge rotor used in the Unsaturated Flow Apparatus (UFA) made by UFA Ventures, Inc. Richland, WA. Diagram reproduced from UFA Ventures, Inc. (1999).

The UFA ${ }^{\mathrm{TM}}$ subjects the sample to large fluid driving forces up to $10,000 \times g$ in an open-flow centrifugation device. A rotating seal assembly allows an ultralow flow pump to deliver fluid to the sample surface during rotation. Because of the large driving forces, steady state is reached within hours even at hydraulic conductivities as low as $10^{-10} \mathrm{~cm} / \mathrm{s}$, removing the traditional time barrier to these types of experiments. With rapid, direct, accurate measurements of transport properties possible for any porous media, the UFA ${ }^{\mathrm{TM}}$ can address issues facing expedited response actions, screening tests of formulations and material properties, and other problems needing immediate answers.

Knowledge of how soil physical properties limit microbial activity will be useful in estimating the potential microbial activity in a soil over a wide range of water contents. For example, soil physical properties including pore volume, pore size distribution, percentage sand/clay, etc., could be measured, and the relationships developed in this project used to predict the range of microbial activities that would be seen at different water contents based on the physical distribution of the water in the soil and its availability to the microbes. Thus, the process of in situ biodegradation can be better understood, and a method that is based on soil physical properties and local water contents can be devised to predict biodegradability. This result would directly benefit bioremediation efforts.

\section{ACCOMPLISHMENTS}

Specific scientific accomplishments for each task have been discussed in the preceding sections. Project accomplishments are summarized below.

- Developed an interfacial processes laboratory capabilities and applied capabilities to synthetic minerals and natural soils. 
- Demonstrated new numerical approach for the solution of transport equations

- $\quad$ Prepared, and had accepted for publication, two manuscripts.

- $\quad$ Received a US Patent (No. 5,915,476).

- $\quad$ Delivered two presentations at scientific meetings during FY-99 and had four others accepted for presentation in the fall of 1999.

- $\quad$ Deployed vadose zone monitoring system at the Savanna River and Hanford Sites.

- Initiated the development of Joint Use Field-Scale Experimental Facility where the hydrologic characterization, stochastic modeling, and geophysical methods are to be evaluated at a physical scale comparable to real buried waste sites.

- $\quad$ Prepared three science proposals for DOE based on concept developed in part in this project. Two proposal were funded for three years each with a total INEEL value of $\$ 1.2$ million.

- $\quad$ Hired two new vadose zone scientists to enhance INEEL capabilities.

\section{REFERENCES}

B. Baeyens and M. H. Bradbury, 1997, "A Mechanistic Description of Ni and Zn Sorption on NaMontmorillonite Part I: Titration and Sorption Measurements," Jour. of Cont. Hydrology, V 27, pp. 199-222.

S. A. Blagodatsky and O. Richter, 1998, "Microbial growth in soil and nitrogen turnover: A theoretical model considering the activity state of microorganisms," Soil Biol. Biochem, 30:1743-1755

M. I. G. Bloor and M. J. Wilson, "Representing PDE surface in terms of B-splines," Computer-Aided Design, 22, 6, 1990, 324-330.

E. Bosatta and G. Agren, 1994, "Theoretical analysis of microbial biomass dynamics in soils," S oil Biol. Biochem, 26:143-148

J. M. Brown, M. I. G. Bloor, S. Bloor, and M. J. Wilson, "Generation and Modification of B-Spline Surface Approximations to PDE Surfaces Using the Finite Element Method," in B. Ravani, ed., Advances in Design Automation, Computer Aided and Computational Design, ASME, 1990, $265-272$.

J. M. Brown, M. I. G. Bloor, S. Bloor, and M. J. Wilson, "The accuracy of B-spline finite element approximations to PDE surfaces," Comput. Methods Appl. Mech. Engrg., 158, 1998, 221-234.

S. Brunauer, P. H. Emmett, and E. Teller, "Adsorption of gases in multimolecular layers," Journal of American Chemical Society, 1938, 60: 309-319.

G. F. Carey and J. T. Oden, Finite Elements, A Second Course, vol. 2, Prentice Hall Inc., Englewood Cliffs, New Jersey, 1983, 167-182.

J. L. Concaand and J. Wright, "Diffusion coefficients in gravel under unsaturated conditions," 1990, Water Resources Res. 26(5):1055-1066. 
C. A. De Boor, A Practical Guide to Splines, Springer-Verlag, New York, 1978, 277-284.

D. A. Dzombak and F. M. M. Morel, Surface Complexation Modeling: Hydrous Ferric Oxide, Wiley Interscience, New York, 1990, p. 393.

G. Farin, Curves and Surfaces for Computer-Aided Geometric Design, A Practical Guide, 4th edition, Academic Press, San Diego, 1997.

M. Ganesh and I. H. Sloan, "Optimal order spline methods for nonlinear differential and integrodifferential equations," Appl. Num. Math., 29, 1999, 445-478.

R. F. Harris, "The effect of water potential on microbial growth and activity," Water Potential Relations in Soil Microbiology, Parr, J. F. (ed.), Soil Science Society of America Special Publication No. 9, Chapter 2, 1981.

J. Hoschek and D. Lasser, Fundamentals of Computer Aided Geometric Design, translated by L. L. Schumaker, A. K. Peters, Wellesley, Massachusetts, 1993.

E. A. Jenne, "Adsorption of Metals by Geomedia: Data Analysis, Modeling, Controlling Factors, and Related Issues," in Adsorption of Metals by Geomedia, edited E. A. Jenne, Academic Press, San Diego, 1998, pp. 1-73.

R. W. Johnson and M. D. Landon, “A B-Spline Based Collocation Method to Approximate the Solutions to the Equations of Fluid Dynamics," Paper FEDSM99-7160, Proceedings of the: 3rd ASME/JSME Joint Fluids Engineering Conference, July 18-23, 1999, San Francisco, California

D. B. Kent, V. S. Tripathi, N. B. Ball, J. O. Leckie, and M. D. Siegel, Surface-complexation modeling of radionuclide adsorption in subsurface environments, Division of High-Level Waste Management, Office of Nuclear Material Safety and Safeguards, U.S. Nuclear Regulatory Commission, NRC FIN A1756, 1988.

T. L. Kieft, S. A. Penny, F. J. Brockman, J. K. Fredrickson, B. N. Bjornstad, and L. L. Rosacker, "Microbial abundance and activities in relation to water potential in the vadose zones of arid and semiarid sites," Microb. Ecol, . 26(1):59-78, 1993.

A. Klute, Methods of soil analysis, Part 1 physical and mineralogical methods. Second edition, Soil Science Society of America, Inc., Madison, Wisconsin, 1986.

A. G. Kravechenko, P. Moin, and R. Moser, "Zonal Embedded Grids for Numerical Simulations of WallBounded Turbulent Flows," J. Comput. Phys., 127, 412-423, 1996.

L. Kroeckel and H. Stolp, "Influence of soil water potential on respiration and nitrogen fixation of Azotobacter vinelandii,” Plant Soil, 79(1):37-50, 1984.

L. Lapidus and G. F. Pinder, Numerical Solution of Partial Differential Equations, Equations in Science and Engineering, John Wiley \& Sons, New York, 1982, 49-60.

MATLAB 5.2, MATLAB, the language of technical computing The Math Works Inc., Natick, MA, 1998.

Molz, F. J. 1986. Simulation of microbial growth dynamics coupled to nutrient and oxygen transport in porous media. Water Resources Res. 22:1207-1216 
OptdesX, 1996, “A Software System for Optimal Engineering Design,” Design Synthesis, Inc., Provo, Utah.

D. L. Parkhurst, D. C. Thorstenson, and L. N. Plummer, PHREEQE-A computer program for geochemical calculations, Water Resources Investigations 80-96, 1980,U.S. Geol. Survey, Washington, D. C.

A. Parkinson and M. Wilson, "Development of a Hybrid SQP-GRG Algorithm for Constrained Nonlinear Programming," J. Mechan., Trans., \& Automat. In Design, Trans. of the ASME, 110, 308-315, 1980.

T. D. Payne, G. R. Lumpkin, and T. D. Waite, "Uranium (VI) adsorption on model minerals - controlling factors and surface complexation modeling," in Adsorption of Metals by Geomedia edited by Everett A. Jenne, 1998, Academic Press, San Diego, California, 75-98.

G. D. Redden, Jinhe Li, and James Leckie, “Adsorption of U(VI) and citric acid on goethite, gibbsite, and kaolinite," in Adsorption of Metals by Geomedia edited by Everett A. Jenne, 1998, Academic Press, San Diego, California, 291-315.

K. Shariff and R. D. Moser, "Two-Dimensional Mesh Embedding for B-spline Methods," J. Comput. Phys., 145, 471-488, 1998.

R. W. Smith and A. L. Schafer, "Effective Reactive Surface Area: An Anisotropic Property of Physically and Chemically Heterogeneous Media (ads.)," Scientific Basis for Nuclear Waste Management XXII, Materials Research Society 1998 Fall Meeting, Boston, MA, 1998.

R. W. Smith and E. A. Jenne, "Recalculation, Evaluation, and Prediction of Surface Complexation Constants for Metal Adsorption on Iron and Manganese Oxides," Environ. Sci. Technol, 25, 525-531, 1991.

R. W. Smith, T. L. McLing, A. L. Schafer, J. L. Wilson, and D. P. J. Swift, "Relationship Between Geochemical and Physical Heterogeneities," EOS 78, S158, 1997.

R. W. Smith, A. L. Schafer, and A. F. B. Tompson, "Theoretical Relationships between Reactivity and Permeability for Monomineralic Porous Media," in Scientific Basis for Nuclear Waste Management XIX, edited by W. M. Murphy and D. A. Knecht, Mat. Res. Soc. Symp. Proc., 412, Pittsburgh, PA, 1996, pp. 693-699.

J. M. Stark and M. K. Firestone, "Mechanisms for soil moisture effects on activity of nitrifying bacteria," Appl. Environ. Microbiol, 61(1):218-221, 1995.

D. L. Suarez and J. Simunek, "Solute Transport Modeling Under Variably Saturated Water Flow Conditions," Reactive Transport in Porous Media, Reviews in Mineralogy, Vol 34, pp. 229-264, 1996.

D. L. Suarez and J. Simunek, J., "UNSATCHEM: Unsaturated Water and Solute Transport Model with Equilibrium and Kinetic Chemistry," Soil Science Society of America Journal, Vol. 61, No. 6, pp. $1633-1646$.

A. F. B. Tompson, A. L. Schafer, and R. W. Smith, "Impact of Physical and Chemical Heterogeneity on Co-Contaminant Transport in a Sandy Porous Medium," Water Resour. Res., 32, 801-818, 1996. 
UFA Ventures, Inc. 1999. Richland, Washington. http://www.UFAVENTURES.COM/ index.html.

C. H. M. Van Bavel and D. I. Hillel, "Calculating potential and actual evaporation from a bare soil surface by simulation of concurrent flow of water and heat," Agricultural Meteorology, 17:453-476, 1976.

J. Westall, FITEQL - A Computer Program for Determination of Chemical Equilibrium Constants for Experimental Data, Report 82-02, Department of Chemistry, Oregon State University, Corvallis, Oregon.

J. Westall, MICROQL:I. A Chemical Equilibrium Program in BASIC. II. Computation of adsorption equilibria in BASIC, Swiss Federal Institute of Technology, Zurich, 1979.

G. Yeh and K. M. Salvage, HYDROGEOCHEM 2.0: A Coupled Model of HYDROlogic Transport and Mixed GEOCHEMical Kinetic/Equilibrium Reaction in Saturated-Unsaturated Media, Pennsylvania State University, University Park, PA, 1997.

\section{APPENDIX}

\section{Peer Reviewed Publications}

J. M. Hubbell and J. B. Sisson, June 29, 1999, Patent Number 5,915,476, US Patent and Trademark Office, "Monitoring Well," Assignee: Lockheed Martin Idaho Technologies Company, Idaho Falls, Idaho, 20 claims, 4 drawing sheets.

R. W. Johnson and M. D. Landon, M. D., "A B-Spline Based Collocation Method to Approximate the Solutions to the Equations of Fluid Dynamics," Paper FEDSM99-7160, Proceedings of the: 3rd ASME/JSME Joint Fluids Engineering Conference, July 18-23, 1999, San Francisco, California

R. W. Smith and A. L. Schafer, "Effective Reactive Surface Area: An Anisotropic Property of Physically and Chemically Heterogeneous Media," Materials Research Society Symposium. Proceedings 556:in press, 1999.

\section{Presentations}

R. W. Johnson and M. D. Landon, "A B-Spline Based Collocation Method to Approximate the Solutions to the Equations of Fluid Dynamics," 3rd ASME/JSME Joint Fluids Engineering Conference, July 18-23, 1999, San Francisco, California

R. W. Smith and A. L. Schafer, "Effective Reactive Surface Area: An Anisotropic Property of Physically and Chemically Heterogeneous Media (ads.)," Scientific Basis for Nuclear Waste Management XXII, Materials Research Society 1998 Fall Meeting, Boston, MA (1998). 


\title{
Transport Phenomena in Porous Media
}

\author{
G. A. Bala, J. M. Hubbel, B. D. Lee, R. M. Lehman, and J. B. Sisson \\ SUMMARY
}

This program is a multiyear program that investigates relationships between bulk-scale $(\mathrm{g}-\mathrm{Kg}$ or $\mathrm{ml}-\mathrm{L}$ ) biological, chemical, and physical heterogeneities in the subsurface to allow development of predictive knowledge of contaminant fate and transport. Microorganisms are the primary agents of geochemical change in the subsurface and these changes mediate transport phenomena. This program integrates microbial transport and partitioning with the effects of metabolic transformation of contaminant compounds and the movement of moisture (a primary means of microbial and contaminant physical transport) through the vadose zone and to underlying aquifers. We report here three research tasks that are driven by the identification of critical deficiencies in the existing knowledge base that limit in situ remedial applications. The program complements projects that investigate macro and microscale heterogeneity issues. Specifically, this program: (1) determines the segregation and partitioning of microbial populations and functions as groundwater interacts with geologic media; (2) investigates the coupling of microbial processes and geochemical reactions that affect actinide migration in subsurface environments, and (3) evaluates vertical and temporal distribution of soil water potential in deep vadose zones in semiarid and humid environments that affect chemical and biological transport phenomena. Endpoint applications of this work include remediation of contaminated geologic media at Department of Energy (DOE) sites and facilities nationwide and industrial sites throughout the world, as well as mining, resource recovery (energy), aquifer contamination, and waste disposal.

\section{Microbial Partitioning and Transport in Porous Media}

The ability to predict the location of microbial biomass and functions in saturated subsurface environments should improve the effectiveness of in situ manipulations for targeted applications. Current understanding is evolving with respect to the geochemical and physical controls on general spatial location of microbes in saturated media. However, ambiguous findings have been reported regarding the relative partitioning of microbes and their functions between the mobile and immobile phases and the conditions that may predictably alter this distribution. This project is dedicated to the empirical determination of the segregation of bacterial populations and functions as mixed groundwater communities interact with geologic media so that in situ biotransformations can be effectively modeled and implemented. The work relies on laboratory core floods and packed columns constructed with Snake River Plain Aquifer (SRPA) basalt, which are perfused with SRPA groundwater and destructively sampled so that both planktonic and sessile bacterial communities may be examined. Bacterial communities in the mobile phase and those harvested from the immobile phase are assayed by multiple culture-dependent and culture-independent methods. Initial studies have described differences in microbial community structure and function based on the microbial ecology of the system, while later studies have focused on populations responsible for desired processes, e.g., co-metabolic degradation of trichloroethylene (TCE) by methanotrophs. The effect of hydrogeology of the system as represented by fracture flow and porous flow on community partitioning has been investigated in replicated experiments with core floods constructed of basalt core with natural fractures and packed columns of the same material crushed. Incorporation of nutrients, contaminants, and manipulations to enhance degradation in the model system of these contaminants represent stepwise progressions in the complexity of the research. A critical limitation in the methods for examining community structure and function on heterogeneous surfaces is the loss of spatial information when the communities are harvested for analyses. A large part of the experimental effort in FY-99 was dedicated to developing methods of analyzing the structure and function of communities in situ (on the basalt surface). Two methods are being explored and optimized for in situ analyses: (1) confocal laser scanning microscopy (CLSM) and spectrometric analyses of these 
digital images and (2) synchrotron radiation-based Fourier transform spectromicroscopy. Transfer of scientific questions and methods of analyses from laboratory simulations to field conditions is being accomplished by examination of core and groundwater communities of samples retrieved from a shallow, sandy aquifer at the National Center for Integrated Bioremediation Research and Development (NCIBRD) at Wurtsmith Air Force Base, Michigan. This location is the site of an intentional release of labeled hydrocarbons into the aquifer. The ultimate goal of the basic research embodied in field and laboratory investigations is to develop the most economical methods to characterize microbial heterogeneity at a particular site so that in situ manipulations may be efficiently conducted with results predictable by the appropriate numerical model.

\section{Core Flooding}

Core floods were performed using three fractured basalt cores and one column packed with crushed basalt that were perfused with groundwater from USGS Well \#103 (as done previously for Replicated Hydrogeology [RH] experiment). After 10 days of continual flow, the columns were sacrificed and samples from the interior of fractured halves were taken from each core. For the packed columns, three samples of the packing material (crushed basalt) were taken from a longitudinal transect and duplicate samples of this crushed basalt were finely ground for comparison. In the RH experiment performed previously, only the fracture surfaces and exterior of the crushed basalt were examined. The interior of the fractured pieces and crushed pieces were examined for total bacterial cells and culturable cells. In the packed columns, the difference between the crushed basalt used for the packing column and finely ground crushed basalt represent the biomass inside of the packing material.

The expected values for the finely ground crushed basalt were expected to be equal to or higher than the crushed basalt (but not ground); however, the opposite was found: Ground, Crushed Basalt $=$ $1.47 \times 10^{5} \mathrm{CFU} / \mathrm{g}$ (average, $\mathrm{n}=3$ ); Crushed Basalt $=6.57 \times 10^{6} \mathrm{CFU} / \mathrm{g}$ (average, $\mathrm{n}=3$ ). This finding did not allow for a determination of the biomass inside of the crushed particles. The direct counts of cell numbers yielded similar findings. The presence of basalt particles in the preparation viewed microscopically seemed to obscure some of the cells making the data somewhat unreliable. It is speculated from these findings that the fine grinding of the crushed basalt and drying during this procedure resulted in a die-off of organisms. At this time, it seems unlikely to resolve the contribution of organisms on the outside of the crushed basalt from those on the inside of the crushed pieces. Surprisingly, measurable numbers of culturable heterotrophs were found in the samples from the interior of the fracture core $\left(\mathrm{ca} .1 \times 10^{4} \mathrm{CFU} / \mathrm{g}\right)$. Total cell counts were barely measurable $\left(\mathrm{ca} .1 \times 10^{5} \mathrm{cells} / \mathrm{g}\right.$ ) and again basalt particles obscured the viewing of cells. Since its seems unlikely that the interior of the basalt with permeabilities (ca. millidarcies) many orders of magnitude lower than the fracture itself (ca. megadarcies) would conduct much groundwater, the source of this biomass remains unknown.

The findings of this experiment to account for biomass inside of the core flood matrix are ambiguous and warrant repeated experimentation. On the other hand, harvesting or extracting cells from the solid matrix is not entirely satisfactory. Thus, methods for visualizing, in situ, cells on surfaces at concentrations seen in the environment need to be developed to address the central hypotheses regarding partitioning of attached and unattached bacterial populations in porous and fractured media.

Data from the replicated hydrogeology experiment were presented to Fractured Rock Science Team (FRST) and Department of Energy-Headquarters (DOE-HQ), Department of Energy Idaho Operations Office (DOE-ID), and Lockheed Martin Idaho Technologies Company (LMITCO) program managers associated with DOE's EMSP and FRST at the Idaho Falls Shilo Inn on October 20, 1998, in the report "Partitioning of Aquifer Bacterial Populations between Geologic Media and Groundwater," by M Lehman. 
A manuscript describing the results of the RH experiment is in preparation for submission to Applied and Environmental Microbiology.

\section{Sediment and Groundwater Sampling}

In collaboration with the University of Michigan National Center for Integrated Bioremediation Research and Development (NCIBRD), sediment and groundwater samples from the Michigan Integrated Remediation Technology Laboratory (MIRTL) field experiments for Community-Level Physiological Profiles (CLPP) were analyzed to examine response of attached and unattached communities during bioremediation.

In November of 1998, the first sets of sediments and groundwater that were from a postinjection (of labeled contaminants) timepoint from the MIRTL2: A Natural Gradient Reactive Fate and Transport Experiment at NCIBRD were received and processed for CLPP. During FY-99, 345 groundwater and 94 sediment samples were processed from MIRTL2 for CLPP. The groundwater samples were taken with a peristaltic pump from three depth intervals from the middle three (of nine) multiwell arrays in each transect, placed in sterile containers, shipped on ice, and analyzed in the laboratory within $24 \mathrm{~h}$ of sampling. Sediments from 18-22 ft below land surface (BLS) were taken from the saturated zone (water table ca. $16 \mathrm{ft}$ BLS) by geoprobe coring, capped in the lexan liner taken from the split coring barrel, placed on ice for shipment, and analyzed within $24 \mathrm{~h}$ of sampling. The cores were aseptically subcored in the laboratory for CLPP analyses by published methods. ${ }^{1}$ Representative data for sediments and groundwater sampled in May 1999 is graphed in Figure 1. This figure shows the CLPP response of groundwater and sediment communities along a transect through the longitudinal axis of the injected organic contaminants. Data from other months show a similar result, although the magnitude in reduction of carbon source utilization (i.e., CLPP) for a given transect position varies according to the time since injection and the corresponding relationship with the artificial plume. The data demonstrate two points: (1) there is a marked difference in the response of the attached (sediment) and unattached (groundwater) communities to the introduction of contaminants into the aquifer and the subsequent transport and

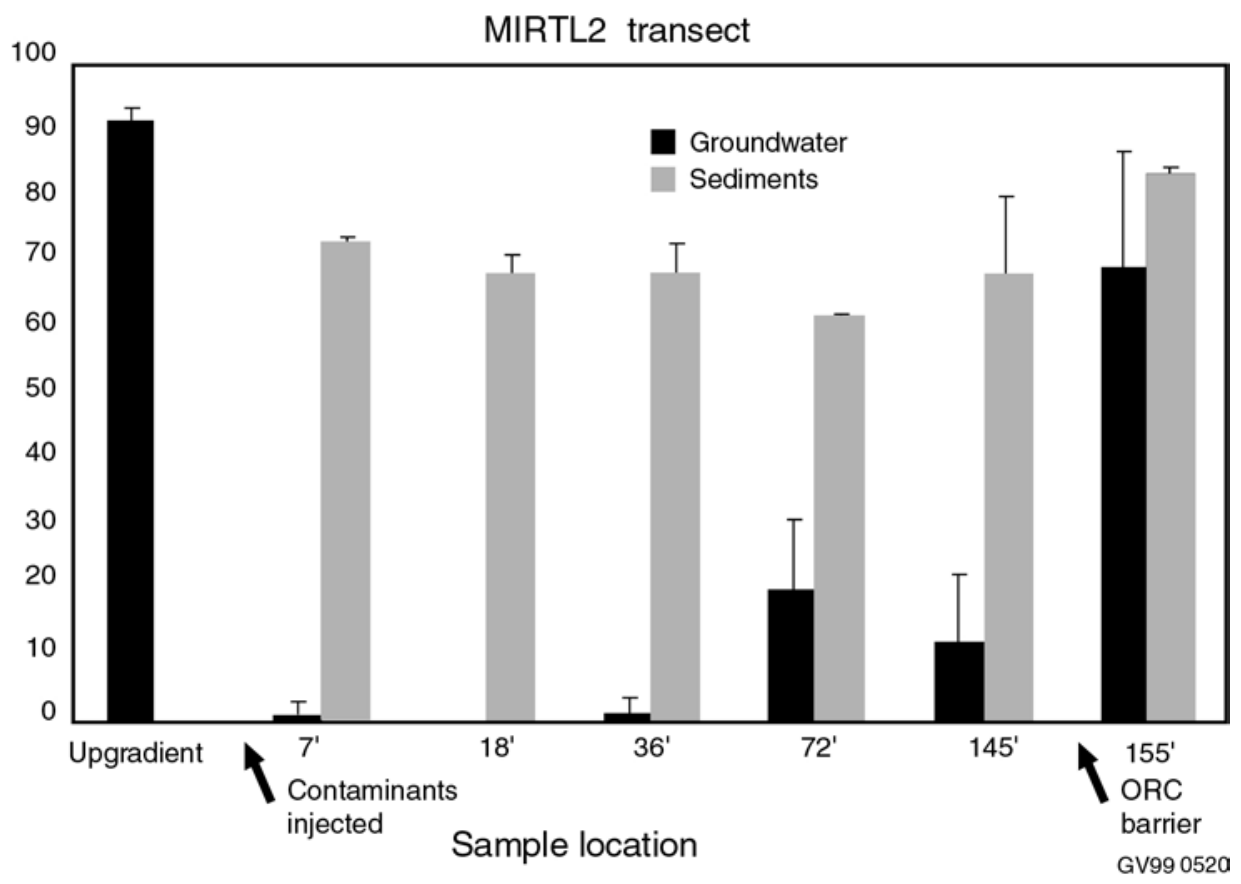

Figure 1. Change in carbon source utilization of groundwater and sediment communities along MIRTL2 transect. 
elimination of these contaminants and (2) the spatial relationship of the microbial communities with respect to the contaminant plume at this particular sampling date. Samples are being collected, shipped, and analyzed on a regular basis - weekly, then biweekly, now monthly as the plume disperses and is remediated. Dr. White (U. Tennessee) is conducting ester-linked phospholipid fatty acid (PLFA) analyses on whole communities on splits of these samples. The goal of this work is to document the effect of contaminants on microbial aquifer communities using complementary measures of microbial structure (PLFA) and function (CLPP). Besides examining the differences in response of groundwater and sediment communities, the hypothesis "Communities will return to pre-injection conditions" (and therefore be indicative of ecosystem status) is being tested in a controlled field setting that is optimal for this type of study. The expectation is a joint publication with the external collaborators detailing the findings at this site.

As part of this collaborative effort with Dr. Mike Barcelona (U. Michigan) and Dr. David White (U. Tennessee), M. Lehman traveled to the National Center for Integrated Bioremediation Research and Development (NCIBRD) at Wurtsmith Air Force Base, Oscoda, Michigan, in November 1998 and spent a week assisting a field team installing new multilevel well arrays and collecting groundwater samples for MIRTL2. In March 1999, a second trip to NCIBRD was made by M. Lehman who spent 1 week assisting with the collection of groundwater and sediment (by coring) samples. The contribution of in-kind resources (i.e., labor) to this multidisciplinary, collaborative study are key to continuing relations with collaborators. Plans have been made for a third site visit in November 1999 to spend a week coring to obtain background sediments outside of MIRTL2 test area and sediments from the transect that will be suitable for denaturing gradient gel electrophoresis (DGGE) profiling of microbial communities. Data produced so far indicate that microbial communities (attached and unattached) will continue to be affected by injected plume for about another year before we can determine whether a statisticallymeasurable recovery to pre-perturbation conditions occurs. Because the original objectives of MIRTL2 established by the host laboratory (U. Michigan) have wound to a conclusion this past summer, funds have been allocated to support field sampling of groundwater and sediment core from NCIBRD's MIRTL2 site beyond the intended duration of the reactive transport experiment.

\section{Count Specific Segments of the Microbial Community In Situ}

In collaboration with the Biochemical and Geochemical Reactions on Environmental Surfaces Project, spectroscopic and microscopic methods were developed to count specific segments of the microbial community (e.g., phylogenetic groups) in situ.

Defensible answers to questions regarding the partitioning of microorganisms and their activities between the groundwater and geologic media require not only controlled experimentation and validation of laboratory results in the field, but also improved methods for in situ examination. Counting and examining microbes in situ (subtasks \#3 and \#4) form an increasingly large percentage of this project as the limitations of traditional methods force advanced methods to answer more sophisticated questions with a higher degree of certainty in the conclusions. The essence of our work is to provide methods that allow in situ visualization of microbial identity and function when microbes are present at environmental concentrations and under physiological conditions similar to in situ.

CLSM and spectroscopic methods are being developed to examine bacterial cells that have been rendered fluorescent by whole-cell hybridization with 16S rRNA-directed oligonucleotide probes and are present on geologic surfaces. The ultimate objective is to quantify relative population abundance (based on phylogenetic groupings) by simultaneously measuring absorbances at three or more different wavelengths. Once reduced to practice, other microbial indices may be measured, e.g., enzyme activity in situ. The use of CLSM with its laser excitation confers several advantages to overcome the deficiencies in detection of environmental microorganisms by use of 16S rRNA-directed oligonucleotide probes, i.e., lower number of ribosomes. ${ }^{2,3}$ The specific advantages of CLSM are that laser excitation results in a 
stronger emission signal than epifluorescent illumination, digital imaging allows amplification and image analysis to improve the signal to noise ratio, and the ability to scan vertically allows integration of the signal from multiple cross sections of a stained cell. Counting microbes in situ involves several complementary efforts, which are detailed below.

Bacterial populations can be tagged with $16 \mathrm{~S}$ rRNA-directed oligonucleotide probes that are conjugated with different fluors; this procedure is termed whole cell fluorescent in situ hybridization (FISH) and allows distinction of different microbial phylotypes. The attractiveness of the technique is twofold: (1) cells are not cultured and (2) cells are not removed from their natural substrata. A variety of protocols have been published in the literature, although a single protocol has not emerged as being reliable by a consensus of practitioners. The application of FISH to environmental samples with low cell numbers and low activities per cell has been particularly problematic, ${ }^{2,3}$ although the potential return of in situ culture-independent identification of microorganisms provides substantial stimulus to pursue optimization of FISH protocols. The objective of optimizing FISH protocols in our laboratory is to provide the capability of determining the relative abundance of different phylogenetic groups in situ on geologic materials. Fifteen separate experiments were conducted to verify proper FISH labeling of different populations using the following probes, which correspond to phylogenetic groups of different levels: Eubacterial (Eub338), high G+C gram positives (GmPos1901), alpha Proteobacteria (Alpha19), beta Proteobacteria (Bet42A), and gamma Proteobacteria (Gamma42A). These phylogenetic groups include representatives that are commonly found in subsurface environments. ${ }^{7}$ All experiments included appropriate controls such as probe alone, cells with no probe, cross-hybridization controls, and hybridizations with a "nonsense probe" (Non338). Experiments were largely conducted on "type" strains of bacteria and some environmental samples were stained for comparison. Since the data that resulted from these labor-intensive experiments are qualitative, i.e., the cells stained or they did not, no data are included in this section of the report. The completed development of a lengthy protocol for these hybridizations is the product of this series of experiments. The cumulative result of these 15 experiments indicate that hybridizations with the Eubacterial (Eub338) and alpha Proteobacteria (Alpha19) can be reliably accomplished in our laboratory, while gram positives (GmPos1901) have provided a specific challenge. The nature of their cell wall precludes easy entry by the probes and necessitates alternative fixation approaches to prevent crosslinking of the peptidoglycan cell wall layer. ${ }^{5}$ These alternative fixations have been performed; however, the results remain unreliable at present. The probes for the beta (Beta42A) and gamma (Gamma42A) divisions of the Proteobacteria differ by only one base pair necessitating the use of "competitive probes" during hybridization to avoid cross-hybridization. ${ }^{3}$ Simultaneous application of competitive probes with different fluorescent labels (e.g., fluorescein, Cy-3, and $\mathrm{Cy}-5$ ) has been tested on type strains with mixed results. Two new filter sets for viewing Cy-3 and Cy-5 fluorescent labels (cyano dyes) with epifluorescent illumination were ordered, received, installed, and verified with new probes. A custom filter holder was purchased from a machine shop specializing in optical equipment to allow our Zeiss standard microscope to accept these filter sets. While probes conjugated with $\mathrm{Cy}-3$ have shown outstanding results, the use of $\mathrm{Cy} 5$ as a probe-conjugated fluor that fits in the long wavelength end of the spectrum has been problematic to date.

Work was initiated on developing spectral and hyperspectral methods for determining multiple microbial phylotypes in a single sample spectrum or spectral image after labeling with FISH probes. Each of the FISH probes are labeled with a different fluorophore with spectral characteristics different than any of the other probes in the assay. Ideally, each probe is completely resolved from the other probes and single excitation or emission wavelengths can be used for each probe. However, excitation and emission spectra for the available probes are very broad and overlap can be a severe problem, particularly when 10-30 $\mathrm{nm}$ bandpass filters are used. This spectral overlap problem can be overcome mathematically by collecting, at a minimum, images (i.e., spectral data points) at $\mathrm{n}$ excitation or emission wavelengths for $\mathrm{n}$ FISH probes and solving for the contributions of each of the probes. Ideally, the images at the $n$ excitation or emission wavelengths will be recorded simultaneously to limit translocation 
problems. Images at multiple wavelengths can be collected using the CLSM or a microspectroscopy system equipped with an appropriate CCD camera to collect spatially resolved spectra of cells along the entrance slit.

Initially, a laser fluorescence spectroscopy system was built using the $\mathrm{Ar}^{+}$ion and $\mathrm{HeNe}(543 \mathrm{~nm})$ lasers from the CLSM as excitation sources. The output of these lasers was focussed onto a 100- $\mu \mathrm{m}$ optical fiber to transfer the light to a standard 1-cm fluorescence cell. Emitted light was collected and focused on a 500- $\mu \mathrm{m}$ optical fiber and transferred to a $0.3-\mathrm{m}$ SpectraPro 300i polychrometer (Acton Research Corporation) equipped with a Princeton Instruments TEA1152 CCD detector. This system is being used with the neat probes and suspensions of FISH labeled cells to design the optical detection schemes for using the CLSM. The PARISS microspectrometer system mounted on a Nikon E600 microscope was upgraded by adding a more sensitive Dage DC-330 CCD camera to the spectrometer. The CLSM (Nikon PCM2000) has also been upgraded to allow use of laser excitation wavelengths of $488 \mathrm{~nm}(\mathrm{Ar}+), 514 \mathrm{~nm}(\mathrm{Ar}+), 543(\mathrm{HeNe})$, and $633 \mathrm{~nm}(\mathrm{HeNe})$ and simultaneous detection on three channels. With the upgraded CLSM system, it is now possible to spatially and spectrally resolve three probes with each image (i.e., one excitation wavelength and three emission wavelengths), even if they are not completely spectrally resolved. The hardware and software for the image analysis system have also been upgraded to provide capability commensurate with upgrades to the image acquisition software.

A limited number of experiments have been performed using probes labeled with fluorescein, Cy-3, and Cy-5. Fluorescein emission overlaps significantly with Cy-3 when exited at 488 or $514 \mathrm{~nm}$ as the fluorescein emission spectrum tails out to beyond $600 \mathrm{~nm}$. Cell suspensions of Pseudomonas stutzeri and Alcaligenes xylosidans were prepared from cultures grown overnight in nutrient broth. Cells were harvested by centrifugation, washed with phosphate buffered saline (PBS), and fixed by resuspending them in PBS with 3.7\% formalin for 30 minutes at room temperature. The cells were again centrifuged, washed twice with deionized water, resuspended in $200 \mu \mathrm{L}$ of hybridization buffer containing $5 \mathrm{ng} / \mu \mathrm{L}$ of the probe, and incubated for 2 hours at $46^{\circ} \mathrm{C}$ to "anneal" the probe. After centrifugation, excess probe was removed by washing twice with wash buffer. The cells were then resuspended in wash buffer and incubated for an additional 15 minutes at $48^{\circ} \mathrm{C}$, centrifuged, and finally resuspended in ice-cold DI water. In the initial experiment using the laser fluorescence spectrometer, no emission from cells with either the Cy-3 or fluorescein probes was noted. At the time, a 100- $\mu \mathrm{m}$ fiber was being used for collecting the emission from the cell. It is not known whether the cells did not accumulate the probe or if the light collection efficiency was too low to record a reasonable spectrum. In a second experiment, the cells were collected on the polycarbonate filters and examined using the PARISS microspectromer system. Pseudomonas stutzeri did not appear to be hybridized with the fluorescein labeled Eubacterial DNA probe, but the Alcaligenes xylosidans cells hybridized with the Cy-3 labeled beta Proteobacterial probe, and spatially resolved spectra were recorded. Future work will continue to refine the instrumental configurations and the preparation of the FISH labeling of cells. Once confident that pure cultures of cells can be consistently labeled and detected, we will move to mixed cultures and labeling with multiple FISH probes. Probes will be selected for maximum spectral differences while minimizing the excitation wavelengths that must be used on the CLSM. For example, using the probes fluorescein, Cy-3, and HEX (5-hexachloro-fluorescein), the 488-nm excitation line, and emission filters at 515, 540, and $580 \mathrm{~nm}$, three phylotypes can be resolved from a single image.

Ultimately, biofilms on actual mineral surfaces will be studied. Because the surfaces will be irregular and 3-D information will be desired, a technique is required to allow this 3-D examination. Such a procedure has been developed at Montana State University using cryosampling and cryosectioning techniques. ${ }^{6}$ This technique was evaluated using a biofilm established on a stainless steel coupon obtained from a biocorrosion project at the Idaho National Engineering and Environmental Laboratory (INEEL). The film was stained with acridine orange, air dried, coated with tissue embedding medium, and rapidly frozen to $-30^{\circ} \mathrm{C}$ with dry ice. The metal coupon was then removed from the frozen medium 
leaving the biofilm on the medium. The exposed base of the biofilm was coated with more tissue embedding medium and rapidly frozen again. The sandwiched biofilm was sectioned at $-25^{\circ} \mathrm{C}$ using a cryostat at Idaho State University. The thin sections were mounted onto glass slides and viewed by CLSM. Images from these sections appear similar to the images obtained from the surface films directly; however, some sections indicated a microbial contamination. The possible contamination structures present were not detected on the surface of the biofilm or in the biocorrosion liquid. At this point there is insufficient data to determine if the structures represent a microbial component of the biofilm or an artifact introduced during cryosampling. Future work will use biofilms of known cell populations to avoid this difficulty.

\section{Examine Microbial Populations and their Activities In Situ}

Using the Advanced Light Source at Lawrence Berkeley National Laboratory (LBNL), synchrotron radiation-based Fourier transform spectromicroscopy was applied to examine microbial populations and their activities in situ.

This project subtask was initiated in midyear FY-99 to evaluate a promising method for nondestructive assessment of microbial presence and activities in situ. ${ }^{4}$ An Associated Western Universities (AWU) subcontract was initiated to Mary Kauffman (interdisciplinary Microbiology and Geology Master's degree candidate at ISU) who is studying the degradation kinetics of aromatic and chlorinated hydrocarbons by attached and unattached organisms. Part of this work includes the study of the surface associations of organisms, degradative substrate, and mineral type on basalt by infrared spectromicroscopy using the particle accelerator at LBNL. A collaboration has been established and a proposal ("Investigation into the Spatial Distribution and Synchrotron FTIR Absorption Microspectra of Burkholderia cepacia G4 Attached to Basalt and Exposed to Toluene, Phenol, Trichloroethylene, or 3Hydroxyphenylacetylene") for beam time at Advanced Light Source (ALS) at LBNL was submitted and approved by the administrators of the ALS for 3 weeks of instrument beamtime. An experimental work plan for this project has been generated to direct this investigation. We are using surface-enhanced infrared absorption-reflectance (SEIRA) microspectroscopy to examine relations between Burkholderia cepacia, its biodegradation activities, and specific mineral phases as B. cepacia attaches to basalt. The work is in conjunction with Dr. Ho-Ying Holman (LBNL) on her study with SEIRA at LBNL for 3 weeks in July and August. Dr. Ken Tobin (U. Southern Illinois) is collaborating on the topic of the association of bacteria with specific basalt mineral phases using radiolabeled substrate uptake and visualization by phosphor-imaging. Tobin's interests are extremely similar; however, the major differences in the methods is the resolution-ca. 80 microns for phosphor-imaging and ca. 10 microns for SEIRA. The resolution is a key element in examining bacterial associations with specific mineral phases as an average bacterial cell is 1 micron in length and mineral phases occur over transects measured in tens to hundreds of microns. The essential results of our work with SEIRA using LNBL's ALS are detailed below.

Synchrotron radiation-based (SR) Fourier transform infrared (FTIR) spectromicroscopy has been used to study bacterial attachment and localization on individual mineral surfaces and on geologic materials. The purpose of this study was to evaluate the application of this technique to investigate potential biochemical differences between free-living and attached cells of a pure culture of Burkholderia cepacia G4. Additionally, we wanted to evaluate the applicability of this technique to investigate if a pure culture of Burkholderia cepacia $\mathrm{G} 4$ would exhibit preferential attachment to specific mineral phases within a heterogeneous geologic substratum. The bacterial-mineral interface spectra were collected using the 1.4.3 Beamline experimental endstation at LBNL's ALS. FTIR absorption spectra were recorded in the 4,000-650 cm $\mathrm{cm}^{-1}$ infrared region at a spectral resolution of $4 \mathrm{~cm}^{-1}$ and microscope-focused experimental spot size of 10 microns. Aluminum slides were spectrally characterized after exposure to a bacterial growth culture for 15 hours (attached cells) and compared to aluminum slides with freshly deposited (free-living) bacterial cells. Basalt specimens were spectrally characterized before, and after, inoculation with a pure culture of Burkholderia cepacia G4. Spectra of the distinct mineral phases in the basalt were 
compared to spectra obtained for individual mineral standards. The spectral characterization of the aluminum slides and basalt specimens after inoculation revealed absorption peaks near 1,740, 1,650, and $1,550 \mathrm{~cm}^{-1}$, which correspond to the phospholipids in the outer membrane of Gram-negative bacteria, protein Amide I, and protein Amide II, respectively. These absorption bands, indicative of bacterial cell walls, were consistent with previously published literature on infrared spectroscopy of biomolecules. Additionally, the aluminum slides with attached bacterial cells showed signs of corrosion evidenced by spectral differences in the $1,200-900 \mathrm{~cm}^{-1}$ region. Preliminary results indicate that SR FTIR spectromicroscopy can be a valuable tool in evaluating the contribution of distinct mineral phases within heterogeneous geologic substrata to subsurface microbiological environments. A sample spectra from the work with SEIRA appears in Figure 2.

\section{Microbially Mediated Actinide Transport in the Subsurface}

The primary objective of the proposed research is to better understand the coupling of microbial processes and surface geochemical reactions that affect the migration of actinides such as plutonium and uranium through saturated zones in the subsurface. To this end, laboratory research was performed to understand the effect of anaerobic enrichments on the mobility of uranium in basaltic aquifers. Uranium adsorption/desorption experiments were designed to maximize interaction of uranium and hydrous iron oxide moieties on the mineral oxide surfaces. This was accomplished using iron oxide coated quartz from Oyster, Virginia. Preliminary results indicated that anaerobic enrichments do not significantly affect adsorption of uranium to iron oxide coated quartz, but the enrichment in the absence of sulfate causes the concentration of uranium in solution to increase during the initial 12 days of enrichment. U(VI) species filtered through a $0.2-\mu \mathrm{m}$ filter were responsible for the increased uranium in solution. While U(VI) species in filtered samples decreased over time, the U(VI) concentration remained stable and increased, indicating that rather than readsorbing to the sand, that the $\mathrm{U}(\mathrm{VI})$ was forming colloids filterable by the $0.2-\mu \mathrm{m}$ filters. This type of colloid formation after microbially mediated desorption of U(VI) may lead to

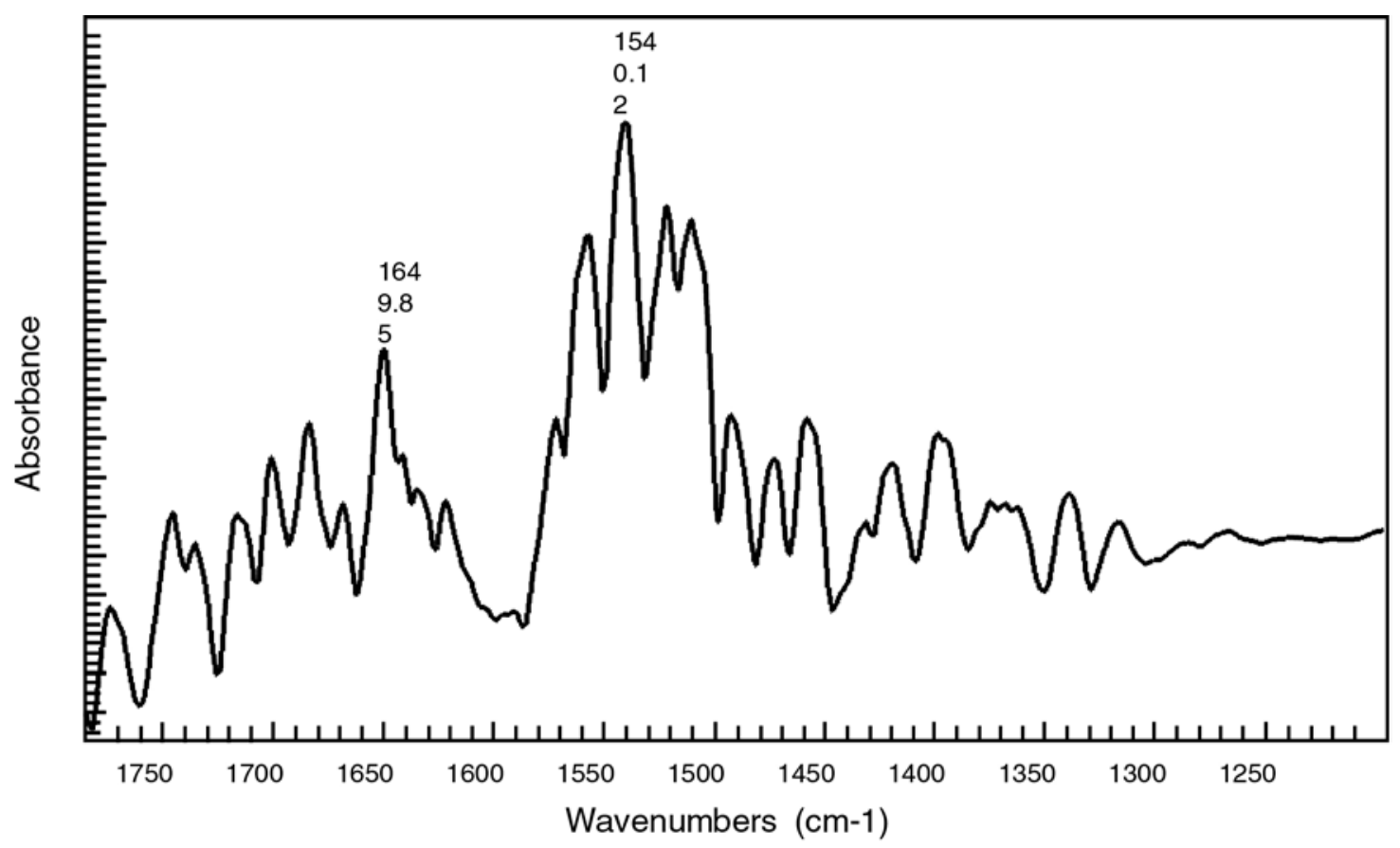

GV990521

Figure 2. FTIR spectra of Burkholderia cepacia G4 cells deposited on an aluminum slide after 21 minutes contact time. The adsorption peaks in the $1,695-1,620 \mathrm{~cm}^{-1}$ and $1,570-1,510 \mathrm{~cm}^{-1}$ regions are due to the $\mathrm{C}=\mathrm{O}$ amide vibrations of the peptide linkage in the bacterial protein Amide I and II systems. 
increased mobility of the actinide and potential migration to downflow receptors. Further research using batch and continuous flow experiments is planned to verify these results.

\section{Background}

Weapons production and energy research activities conducted at DOE facilities included: uranium enrichment, production of nuclear materials, spent-fuel reprocessing, development, testing, and fabrication of nuclear and nonnuclear weapons, construction and testing of nuclear reactors and management of various radioactive wastes and spent fuels. These activities have resulted in widespread contamination of subsurface sediments and groundwater with chlorinated organics, metals, and radionuclides. More than 5,700 known DOE contaminant plumes have contaminated over 600 billion gallons of water and 50 million cubic meters of soils and sediments. Some of the more prevalent contaminants that have been co-disposed at DOE sites are chlorinated solvents (e.g., perchloroethylene [PCE] and TCE), metals (e.g., lead, chromium, and mercury) and radionuclides (e.g., uranium, cesium and strontium). ${ }^{8}$ These contaminants, especially the metals and radionuclides, can persist in the environment for extremely long periods. Migration of these plumes threatens local and regional water sources, and in some cases has already adversely impacted offsite receptors. ${ }^{9}$

While a large amount of fundamental and applied knowledge has been gained regarding reductive dechlorination of chlorinated organic contaminants, ${ }^{10,11}$ the effect of these changes to the subsurface chemistry on mobility of actinides that have complexed to mineral surfaces has received limited attention. Microorganisms may affect the mobility of the actinide directly by changing the speciation, or indirectly by transforming the mineral matrix to which the metal has complexed. Once the uranyl ion has been released, it is available for complexation to counter ions in solution, allowing for mobilization of the actinide. During the reduction of the iron oxides and liberation of soluble ferrous iron, potential sites for adsorption are also lost.

The primary objective of the research during FY-99 was to perform bench-scale experiments to gain a basic scientific understanding of how enrichment of anaerobic microbial populations stimulated to biotransform chlorinated contaminants affect mobilization of actinides that have become chemically sorbed to basalt. Knowledge gained from the project will be directly applicable to the contaminant plume at Test Area North (TAN) and the ongoing Record of Decision (ROD) in place to remediate the organic fraction of the plume.

The bench-scale laboratory research will attempt to address a largely ignored issue associated with in situ remediation, which is the potential enhancement of mobility of co-disposed radionuclides resulting from remediation of organic groundwater contaminants. When microbial growth conditions are established in the subsurface for chlorinated solvent biotransformation, changes in chemistry will affect the fate of the uranium that has become chemically complexed to the geologic media. Anaerobic microorganisms require certain environmental factors to be favorable to maintain their baseline physiology, as well as dechlorination activity. One important factor is the redox potential (Eh) of the growth medium. A change in Eh facilitated by microorganisms has a strong possibility of affecting other chemistries in the area of changed Eh (i.e., metal mobility and speciation). ${ }^{12}$ The hypothesized mechanism of uranium release from the U-mineral oxide complex is similar for enrichments for sulfate reducers and sulfate free enrichments. In this mechanism, uranyl ions sorb to mineral oxides such as iron oxide, ferric oxyhydroxides or manganese oxides. Figure 3 shows potential mechanisms for microbially mediated transport of actinides such as uranium in a basaltic aquifer. For the purpose of the research, iron oxide minerals will be of primary interest. Once accelerated bioremediation is initiated, the required redox for microbial growth and supplementary electron transfer occurring in the system leads to the reduction and loss of the iron oxide and uranyl ions. The uranyl ions will complex with carbonate forming dioxouranium (VI) carbonate complexes. The iron is lost as ferrous iron, which may form 


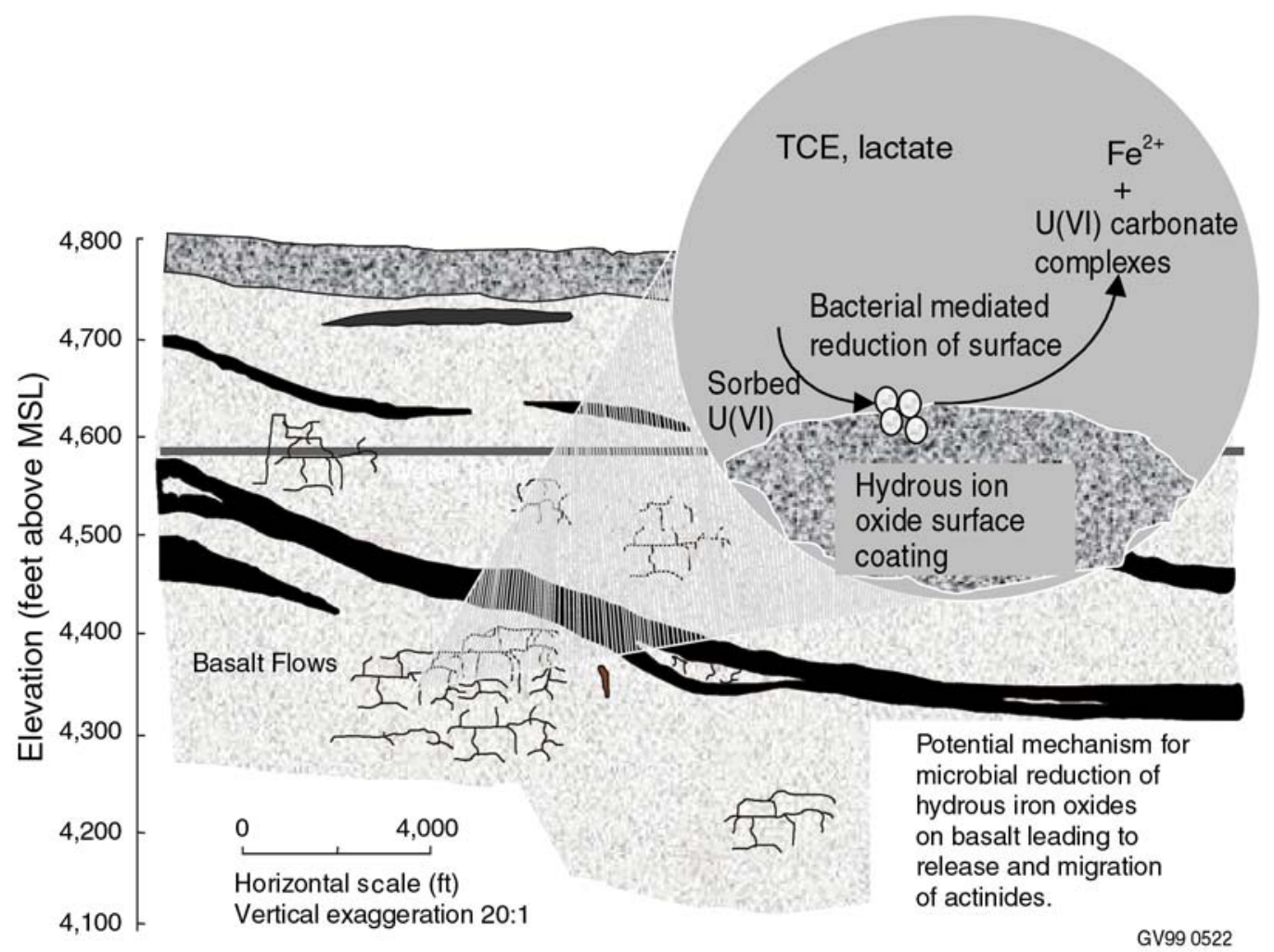

Figure 3. Schematic of potential mechanisms for microbially mediated mobilization of uranium in basaltic aquifers.

carbonate complexes. Under conditions of enrichment without sulfate, the uranium carbonate will stay in solution, depending on the microbial activity occurring or will be precipitated as solid uranous oxide. Uranium solubility will be affected under conditions of sulfate reduction due to the formation of metal sulfides, specifically iron sulfide. Two potential effects of the iron sulfide would be abiotic reduction of the U(VI) and reprecipitation onto the mineral matrix, or adsorption to iron sulfide particles followed by precipitation. The effect of oxidative conditions established after accelerated anaerobic remediation has been completed and the groundwater returns to oxygenated conditions on uranium mobility will also be determined.

The thrust of this phase of the project will be to study the effect of changes in the oxidationreduction potential of the groundwater on the mobility of geologically complexed actinides in saturated subsurface media, specifically basalt. The specific goals of the proposed research are:

- To demonstrate a link between anaerobic bioremediation of chlorinated contaminants and the mobilization/immobilization of uranium from basalt and iron oxide minerals

- To understand the mechanisms responsible for the release of uranium from specific mineral components (e.g., mineral oxide compounds) during anaerobic bioremediation

- $\quad$ To understand and develop adsorption/desorption kinetics for uranium mobilized from basalt and iron oxides under sulfate reducing conditions

- To understand and develop adsorption/desorption kinetics for uranium mobilized from basalt and iron oxides under methanogenic conditions 
- $\quad$ To understand the mechanisms for the release of uranium from mineral oxide compounds during reestablishment of oxidative conditions in groundwater.

The effect of remediating trichloroethylene using enrichments with and without sulfate on actinide mobility was determined. Previous research at the INEEL has shown that sulfate reducing and methanogenic enrichments are able to transfer electrons to organometallic compounds affecting the valence state of transition metal involved. The initial research task was to determine the interaction of redox conditions on uranium using simulated materials to mimic the mineral matrices found on the surface of basalt. Batch testing was performed during this phase of research. When culture conditions are established in the subsurface, changes in chemistry will affect the fate of uranium that has become specifically sorbed to basalt. After remediation of the plume is complete, the aquifer in the area will naturally return to oxidative conditions. The effect of the positive oxidation reduction potential on the chemically sorbed uranium will be determined.

The element of research was primarily focused on determining the effect of anaerobic microbial enrichments on the adsorption and desorption of uranium from hydrous iron oxide coated sand.

Results from the research indicate that anaerobic microbial enrichments with and without sulfate have no significant effect on uranium adsorption to iron oxide coated sand. Conversely, and probably the most interesting results from the research to date, enrichments without sulfate did appear to have an effect on desorption of uranium from iron oxide coated sand after the sand had been exposed to uranium for 5 months. After 12 days of incubation, the U(VI) concentration in the liquid increased by approximately $45 \mathrm{ppb}$ uranium in samples filtered through $0.2-\mu \mathrm{m}$ filters, as well as in unfiltered samples. U(VI) concentrations in the filtered samples steadily decreased over time, while U(VI) in unfiltered samples decreased slightly and then began to increase when microbial activity continued. Since there was not a substantial decrease in U(VI) concentration in the unfiltered samples, it would appear that the uranium was forming particles with a diameter greater than $0.2 \mu \mathrm{m}$ or was sorbing to the microbes, but was not readsorbing to the iron oxide coated sand. Formation of insoluble particles is important because this would allow for migration of $\mathrm{U}(\mathrm{VI})$ from the contaminated zone to downflow receptors. Additional research will be required to determine whether adsorptive sites on the iron oxide coated sand were chemically reduced by the cultures removing some of the sorptive capacity of the sand.

Assay results also indicated that there is substantial variability for the adsorptive capacity between various batches of sand. This factor may have contributed to the variability between results noted between the various replicates and sampling points and between the various assays. Future batch testing will be attempted using sequential sampling from the same vial. In this case, larger vials equipped with Mininert valves will be used.

One other interesting result was that the enrichment culture taken from TCE degradation kinetics experiments was not able to reduce TCE in any of the adsorption/desorption assays performed. This may be due to the fact that the culture has changed over the extended culture period. The TAN culture was not grown in the presence of TCE before inoculation of the enrichments, which may have decreased activity toward TCE. To alleviate this problem, a sample of water will be requested from the current remediation operation being performed at TAN, where TCE reduction is occurring.

Once some of these questions have been resolved, the column experiments will be initiated.

\section{Analysis}

Vials used to assay uranium adsorption/desorption contained mineral salts, lactate, TCE, uranium, and sulfate depending on the enrichment specified. Lactate was monitored using high-pressure liquid chromatography. Trichloroethylene and associated reduction products were analyzed using gas 
chromatography (GC) using solid-phase microextraction (SPME) for sampling, while uranium was analyzed using a kinetic phosphorescence analyzer (KPA).

\section{Inoculum}

A microbial consortium enriched from groundwater from TAN at the INEEL was used as the inoculum for the experiments. The microbial culture was initially used to determine TCE biotransformation kinetics in an unrelated project. The culture has been maintained under anaerobic conditions with biweekly additions of fresh growth medium and lactate as the carbon source.

\section{Uranium Adsorption/Desorption Experiments}

Serum vials were loaded with iron oxide coated quartz to determine the effect of anaerobic enrichments with and without sulfate on uranium immobilization and mobilization from U-mineral oxide complexes on the surface of the quartz. Iron oxide coated quartz was used to simulate mineral surfaces on basalt, while enhancing the amount of uranium adsorption occurring due to the excess of reactive surface. This research task will help to determine the fate of soluble uranium during an active anaerobic bioremediation project. TCE was studied as the co-contaminant for the experiment. During the past year, four separate assays were run to determine the effect of anaerobic enrichments on sorption and desorption of uranium from the iron oxide coated sand. The assays are as follows:

- $\quad$ Assay 1 was used to determine the effect of anaerobic enrichments on adsorption of uranium to iron oxide coated sand

- Assay 2 was used to determine the effect of anaerobic enrichments on desorption of uranium from iron oxide coated sand after 2.5 months of adsorption

- Assay 3 was used to determine the effect of anaerobic enrichments on desorption of uranium from iron oxide coated sand after 5 months of adsorption

- $\quad$ Assay 4 was used to determine the effect of uranium on the microbial population in the absence of the iron oxide coated sand.

Assay 1. Serum vials were used to monitor U (VI), TCE and associated products of reduction, and volatile fatty acids using the analytical instruments described above. Destructive sampling was used for analysis and samples were taken every 3 weeks.

Uranium (1 ppm) was added to growth medium containing lactate, phosphate, and nitrate. A starting lactate concentration of $500 \mathrm{ppm}$ was used for testing. The liquid was sparged with nitrogen to drive off excess oxygen and the vials were sealed. Inoculum was added just before sealing the vials. TCE was added to the vials to initiate the experiment and a time zero sample was taken. TCE was added to a target concentration of $10 \mathrm{ppm}$. A $10 \%$ inoculum was used for testing. The vials were incubated statically at room temperature, in the dark. Five separate treatments were set up to monitor the effect of various test conditions on uranium adsorption. Each test consisted of enrichments with and without sulfate, a killed control containing TCE, a blank with TCE, and a blank with no TCE. Blank in this instance is defined as the growth medium containing no inoculum. The killed control was prepared by autoclaving an aliquot of inoculum and was used to determine whether biomass from the inoculum affected uranium concentrations in the liquid, while the blank was used to monitor uranium adsorption/desorption in the absence of cells or TCE. The TCE containing blank was used to determine whether the TCE had any co-contaminant effects during the adsorption/desorption process.

Figure 4 shows the hexavalent uranium $(\mathrm{U}(\mathrm{VI}))$ concentration over time for the various treatments tested. Symbols annotated with the cross indicate treatments that contained biomass, while solid symbols 


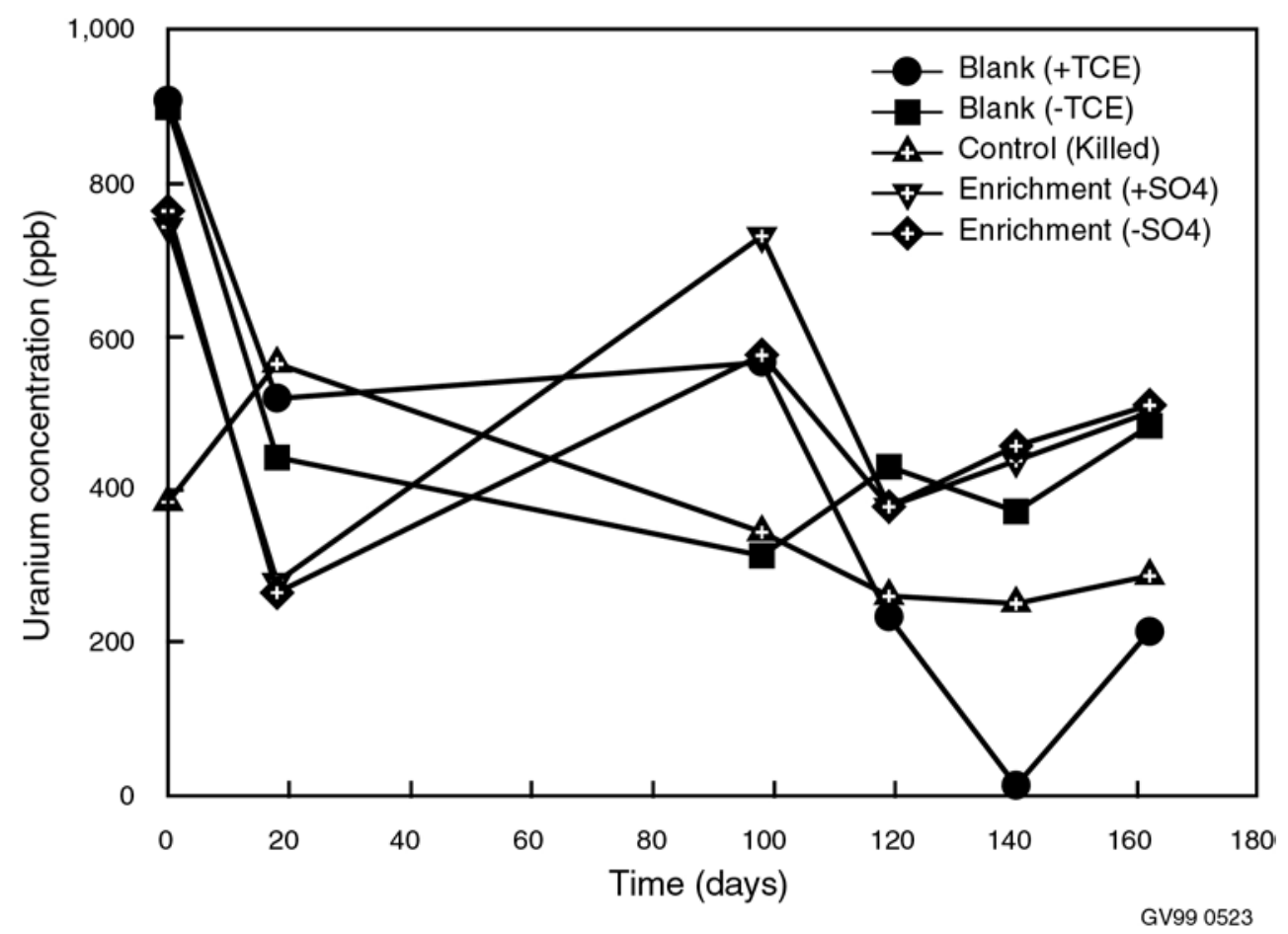

Figure 4. U(VI) concentrations from an experiment to determine the effect of anaerobic microbial enrichments on uranium adsorption to iron oxide coated quartz. Symbols with crosses indicate samples that contained biomass.

represent cell free blanks. The initial U(VI) concentration for unfiltered samples from the enrichments was approximately $100 \mathrm{ppb}$ below the level demonstrated for the blanks. After 18 days of incubation the $\mathrm{U}(\mathrm{VI})$ concentration in the enrichment vials decreased to below $300 \mathrm{ppb}$ while $\mathrm{U}(\mathrm{VI})$ concentration in the killed control and the blanks ranged between 440 and $570 \mathrm{ppb}$. The next sampling period, which was performed after 100 days of incubation, indicated high U(VI) concentrations for the enrichment vials, although values are questionable due to decreased lifetime of the phosphorescence decay of the samples during analysis. After 119 days of incubation, the U(VI) concentration appeared to stabilize for most of the treatments. Adsorption of U(VI) in the blank with TCE and the killed control continued to decrease over the duration of the assay indicating that uranium may have some effect on adsorption in the absence of microbial activity. Similar microbial activity was demonstrated between the enrichment with sulfate and the enrichment without sulfate as indicated by the biotransformation of lactate (Figure 5). Lactate was completely removed after 100 days of incubation with acetate and propionate appearing in the growth medium. Methane production by microbes in both enrichments was minimal and there was no reduction of TCE noted in any of the samples.

The mass of uranium in the sand compared to the liquid after 119 days of incubation can be seen in Figure 6. Results indicate less uranium on the sand from the enrichments compared to the blanks and controls, while the amount of U(VI) in solution was similar for most of the treatments with the exception of the blank containing TCE. The mass of uranium on the sand from the killed control and the blanks was similar.

Over the long term of the assay, U(VI) concentrations in the liquid phase from this assay did not indicate a significant effect of the anaerobic enrichments compared to the killed control and blanks. Mass of uranium on the sand may indicate that the adsorption of uranium to the sand is hindered by the enrichments. The differences in uranium levels on the sand may be due to reduced species of uranium bound in surface complexes compared to treatments with no microbial activity. 

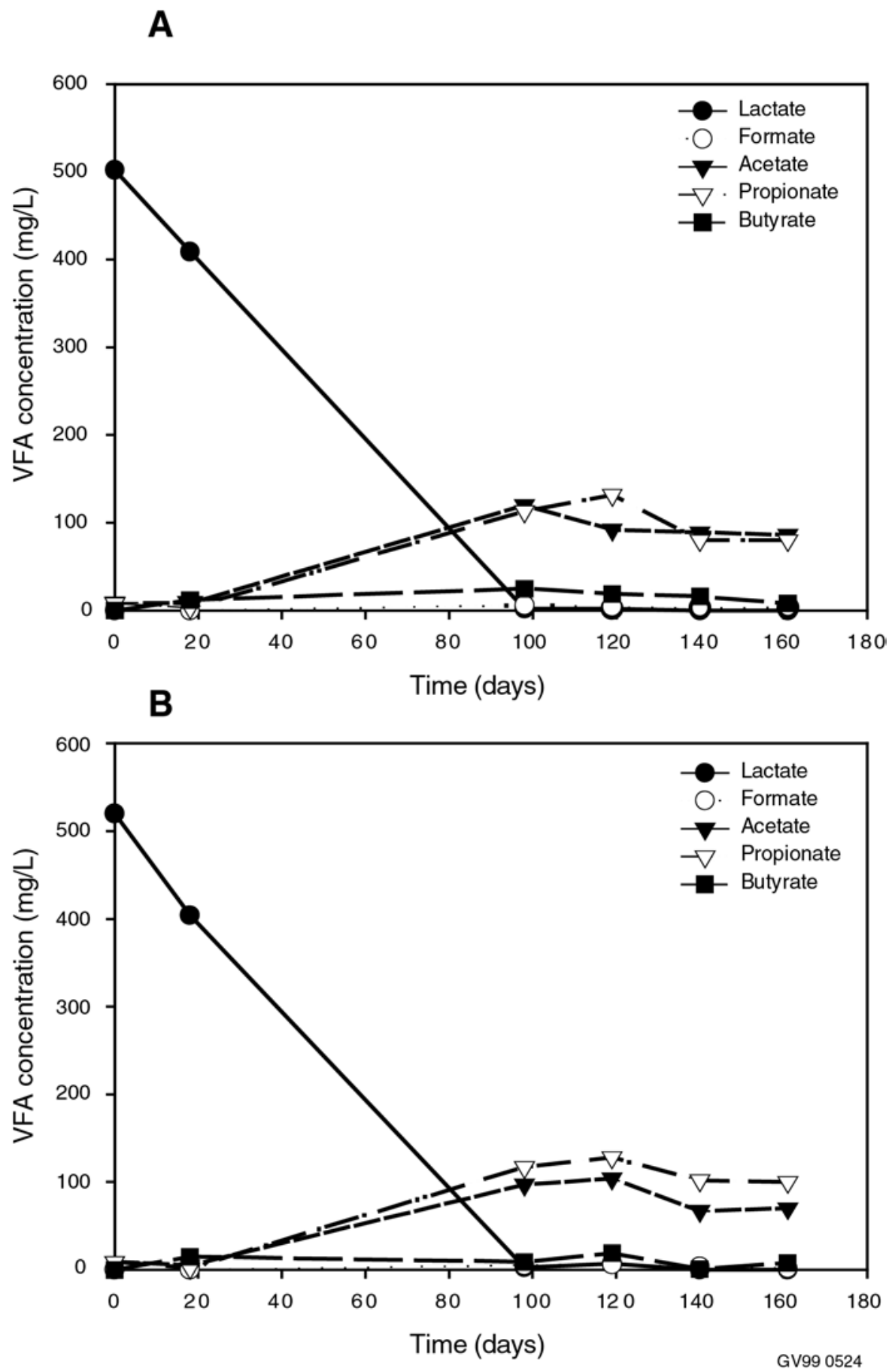

Figure 5. Lactate biotransformation results from an experiment to determine the effect of anaerobic microbial enrichments on uranium adsorption to iron oxide coated sand. Graph A is from an enrichment containing sulfate. Graph B represents data from an enrichment without sulfate. 


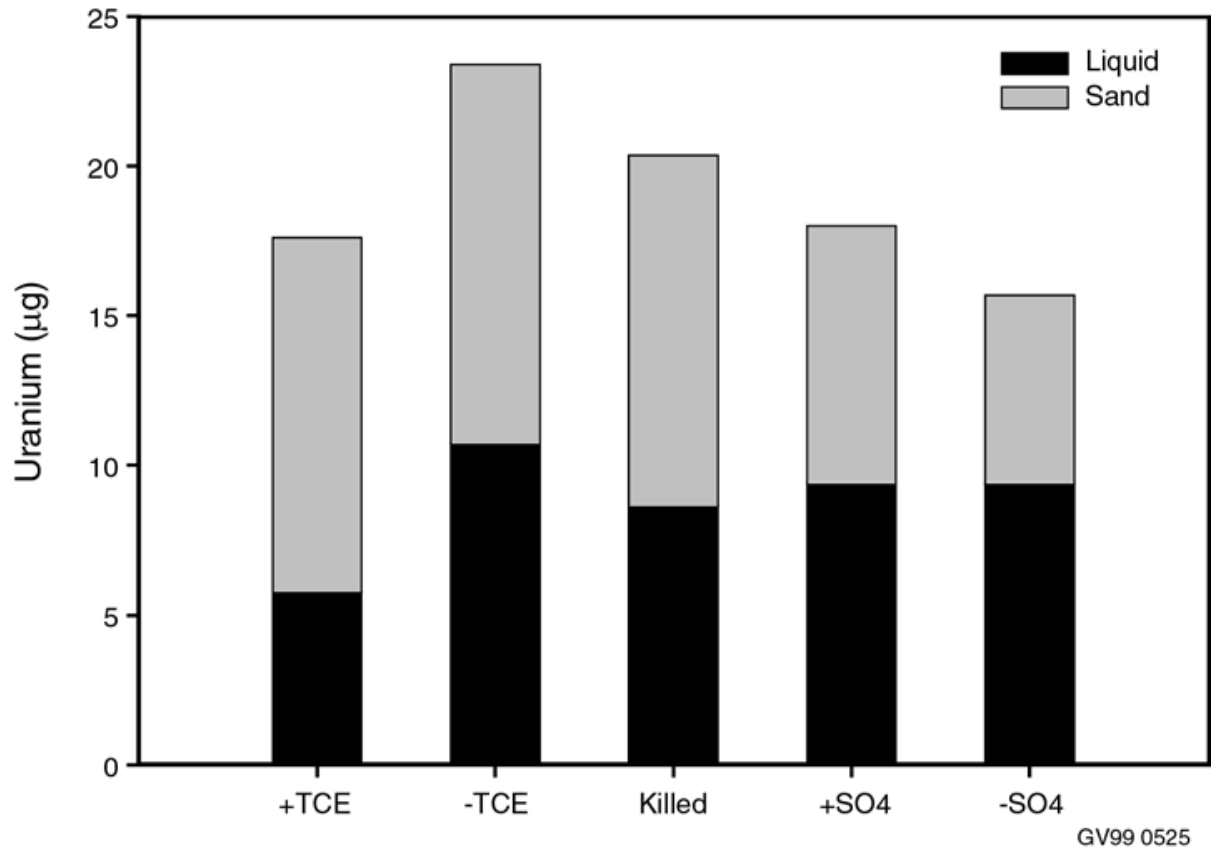

Figure 6. Mass of $\mathrm{U}(\mathrm{VI})$ in liquid and sand fractions from an experiment to determine the effect of anaerobic microbial enrichments on uranium adsorption to iron oxide coated sand.

Assay 2. A second set of vials was set up to determine the effect of microbial enrichments on desorption of uranium from iron oxide coated sand after 2.5 months of adsorption. Vials were set up containing $0.5 \mathrm{~g}$ of sand and then $25 \mathrm{~mL}$ of nitrogen phosphorus growth medium was added. A stock uranium solution was then added to a target uranium concentration of $1 \mathrm{ppm}$. Vials were then sealed and incubated in the dark for 2.5 months. Following incubation, the adsorption medium was aspirated from the vials and then the various treatments were set up as indicated in Assay 1.

As seen in Figure 7, uranium adsorption was still occurring after the desorption experiment had been initiated. Adsorption appeared to stabilize after Day 21 of sampling in unfiltered samples. Very low concentrations of $U(V I)$ seen in the enrichment containing sulfate is thought to be the product of precipitation through the formation of metal sulfides. The U(VI) concentration in the enrichment containing sulfate increased from near zero to $10 \mathrm{ppb}$ of uranium after 42 days of incubation. Concentrations of U(VI) were similar in the blanks, killed control, and the sulfate free enrichment. Similar analytical results (data not shown) for U(VI) were noted in samples analyzed after filtering through a $0.2-\mu \mathrm{m}$ filter. Microbial cultures used during the test were shown to be active as indicated by their ability to remove lactate. Both enrichments removed all of the lactate in the growth medium within 60 days (Figure 8). Lactate was converted to primarily acetate and propionate. The killed control showed little lactate removal over the duration of the test. Methane production in all vials sampled was minimal. As with the previous assay, no TCE biotransformation was noted in any of the treatments.

The mass of U(VI) adsorbed to the sand was determined at sample intervals similar to those used for the liquid samples. Mass of uranium on the sand from the various treatments can be seen in Figure 9. The amount of uranium seen on the sand was similar to levels shown during the adsorption experiment outlined in the discussion of Assay 1, with the exception of the killed control, which showed less uranium on the sand then even the enrichments. 


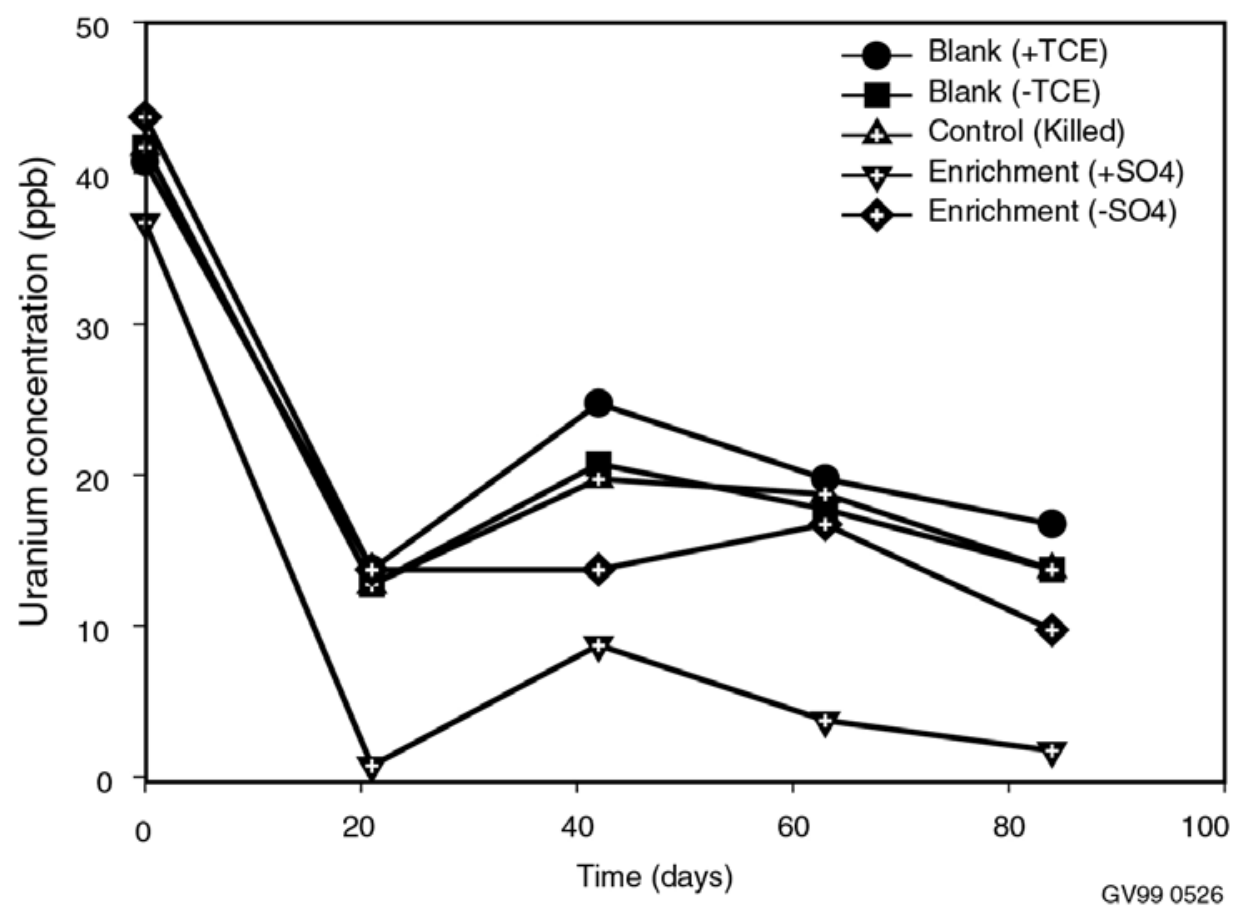

Figure 7. U(VI) concentrations from an experiment to determine the effect of anaerobic microbial enrichments on uranium desorption from iron oxide coated quartz. Sand had been exposed to a $1 \mathrm{ppm}$ uranium solution for 2.5 months before initiation of enrichments. Symbols with crosses indicate samples that contained biomass.

Figure 10 shows a micrograph from an environmental scanning electron microscope (ESEM) scan of a sand sample from the enrichment without sulfate. Black rod shaped patterns can be seen across the surface of the sand, indicating attachment of the enrichment culture on the surface of the sand. ESEM analysis of sand samples that had not been exposed to uranium or microbes showed no microbes, while analysis of sand from the enrichments with sulfate indicated very few bacteria (data not shown). Sulfatefree conditions were initially set up to enrich for methanogenic microorganisms. The lack of methane production in the samples, along with the attachment of microbes to the ferric iron rich surface of the sand, may be an indication that sulfate-free enrichments are selecting from iron reducing bacteria instead.

Assay 3. A third set of vials was prepared to determine the effect of microbial enrichments on desorption of uranium from iron oxide coated sand after exposure to a uranium adsorption medium for 5 months. Vials were loaded with $0.5 \mathrm{~g}$ of the iron oxide coated sand and $25 \mathrm{~mL}$ of nitrogen phosphorus growth medium. A stock uranium solution was then added to a target uranium concentration of $1 \mathrm{ppm}$. Vials were then sealed and incubated statically in the dark for 5 months. Following incubation, the adsorption medium was removed from the vials through aspiration and the various treatments used for the first two assays were prepared.

Following adsorption, a few vials were sacrificed to determine the amount of uranium that had adsorbed to the iron oxide coated sand. KPA analysis indicated masses of U(VI) varied between 9.89 and $20.55 \mu \mathrm{g}$. It should be noted that these amounts are twice to four times the amounts noted on time zero samples taken during Assay 2. As would be expected, the amount of U(VI) remaining in the adsorption solution varied inversely compared to the amount of U(VI) found on the sand (i.e., low uranium in solution when uranium on sand was high). These significant differences in adsorption may have affected the results obtained in all adsorption and desorption assays run to date. 

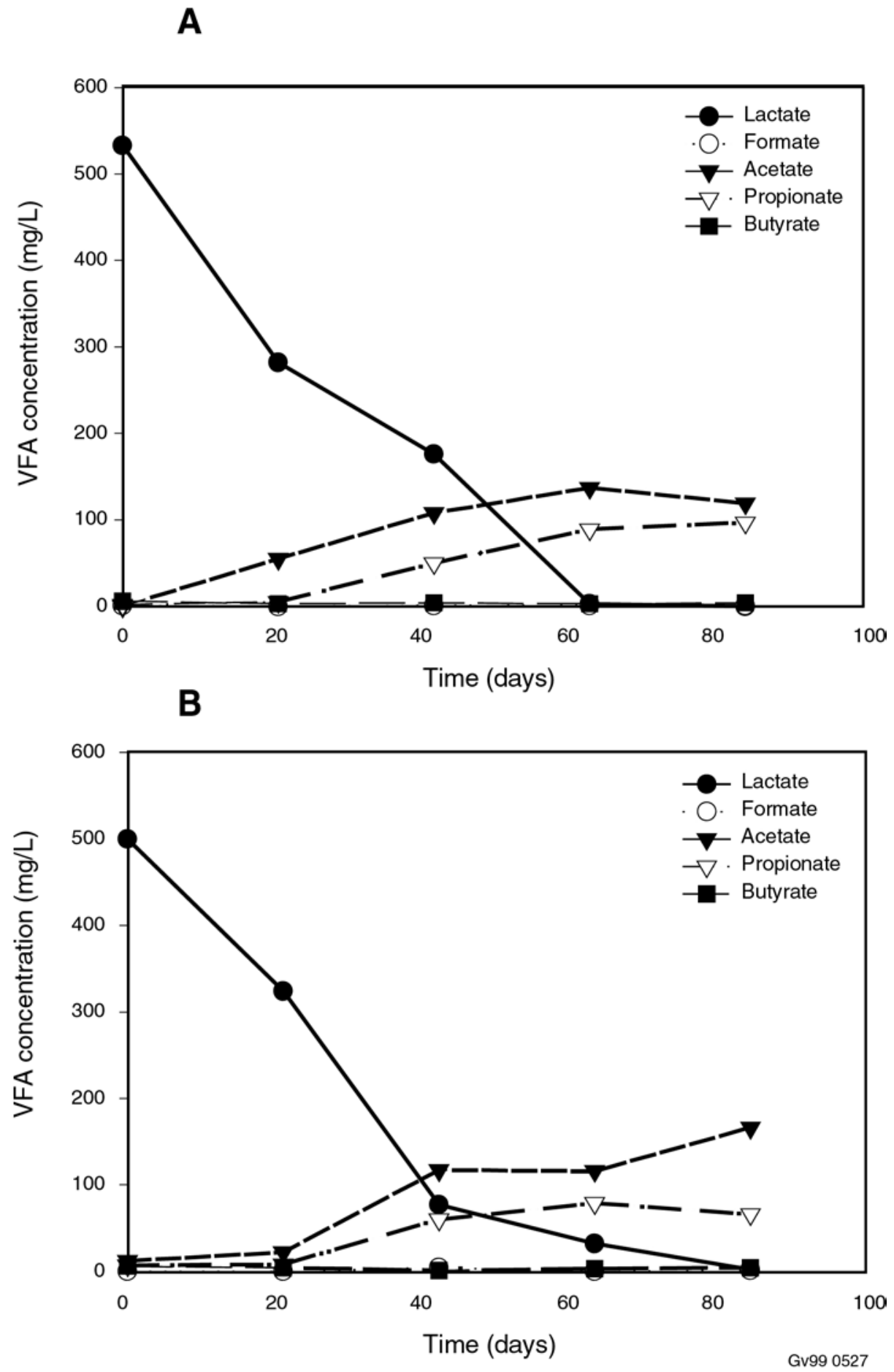

Figure 8. Lactate biotransformation results from an experiment to determine the effect of anaerobic microbial enrichments on uranium desorption from iron oxide coated sand. Sand had been exposed to a $1 \mathrm{ppm}$ uranium solution for 2.5 months before initiation of enrichments. Graph A is from an enrichment containing sulfate. Graph B represents data from an enrichment without sulfate. 


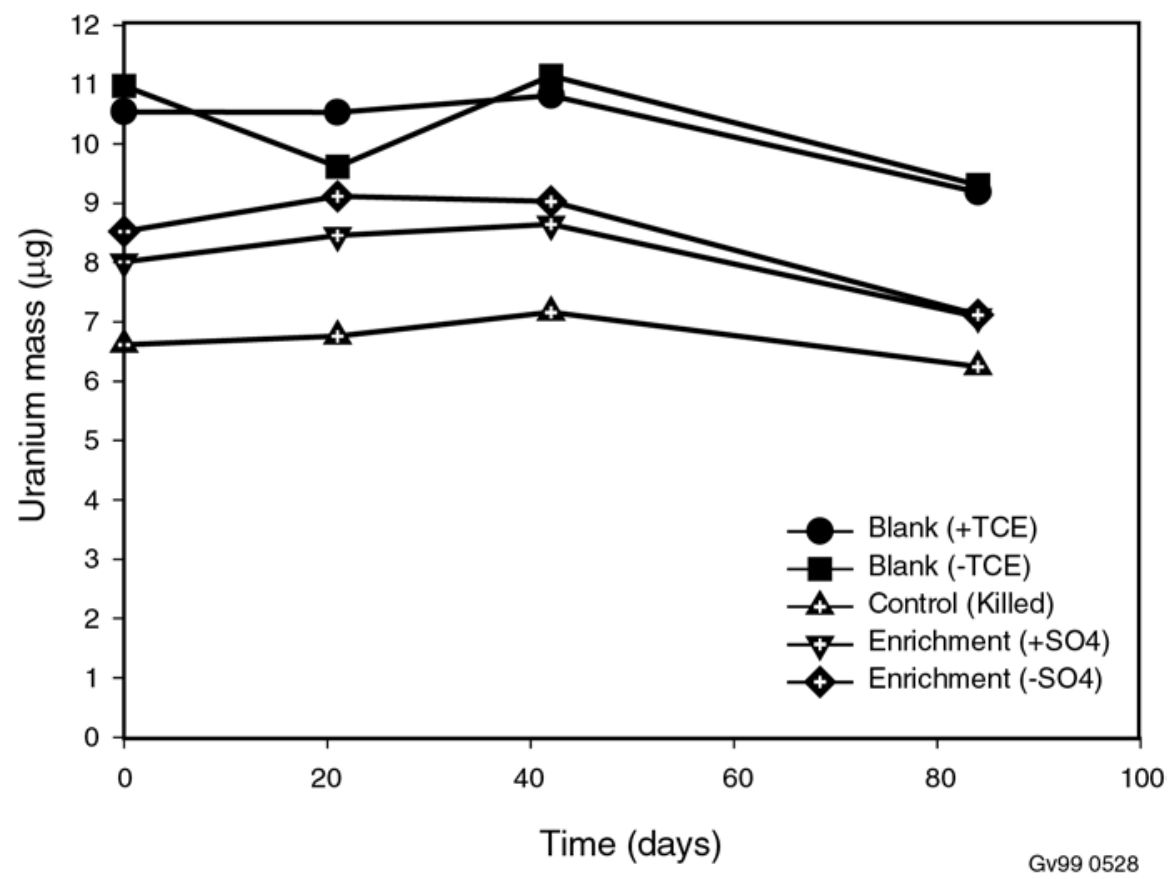

Figure 9. Mass of U(VI) on sand fractions from an experiment to determine the effect of anaerobic microbial enrichments on uranium desorption from iron oxide coated sand. Sand had been exposed to a 1 ppm uranium solution for 2.5 months before initiation of enrichments. Symbols with crosses indicate samples that contained biomass.

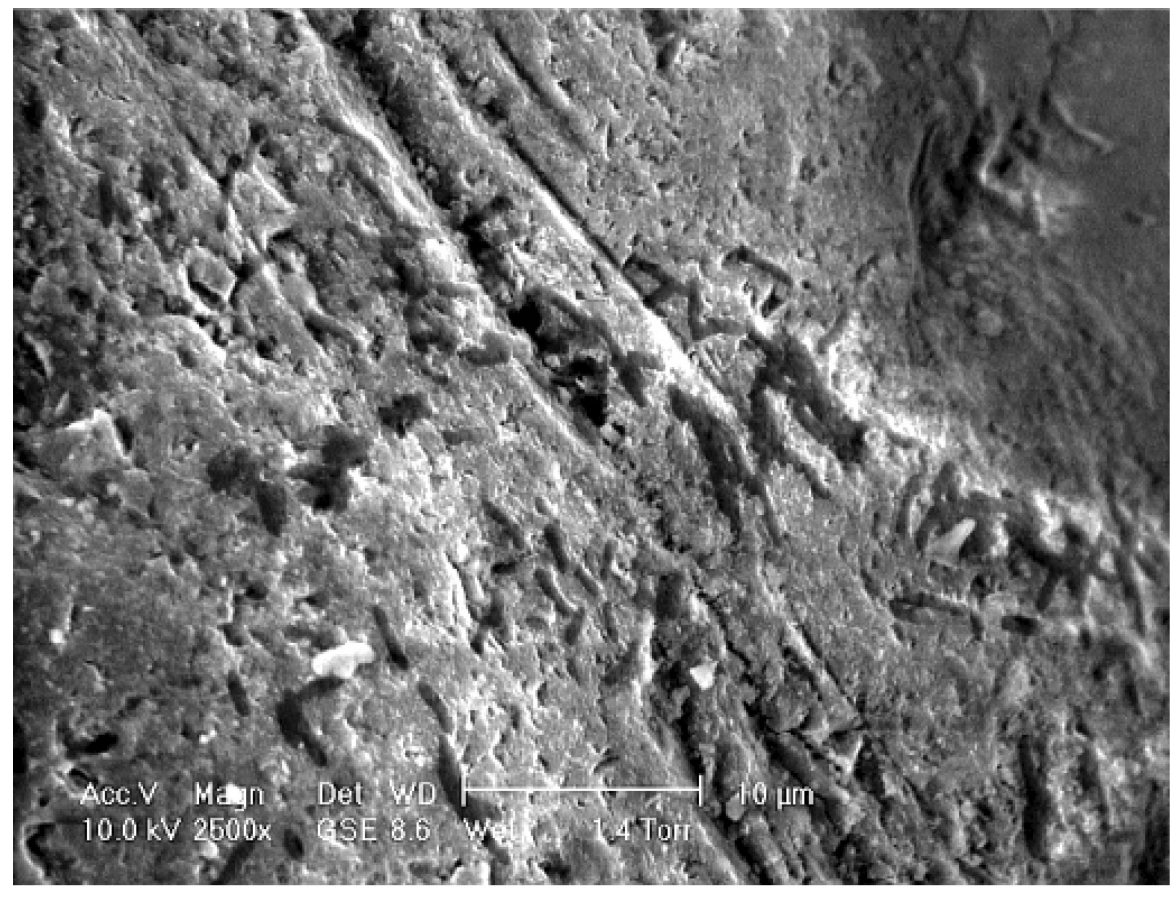

GV99 0529

Figure 10. ESEM micrograph of sand particle surface from an experiment to determine the effect of anaerobic microbial enrichments without sulfate on uranium desorption from iron oxide coated sand. Sand had been exposed to a $1 \mathrm{ppm}$ uranium solution for 2.5 months before initiation of enrichments. Black rod shaped structures represent bacteria growing on surface of sand. 
U(VI) concentrations for the various treatments in Assay 3 did not decrease upon initial exposure as was the case for sand exposed to uranium for only 2.5 months (Figure 11). These results may be an indication that uranium adsorption in Assay 2 was not completed and continued after the desorption experiment was initiated. After 12 days of incubation, the U(VI) in solution for unfiltered samples increased to near $110 \mathrm{ppb}$ in the enrichments without sulfate. No significant differences were noted in the other treatments at this sampling point. After this time, the U(VI) levels in these unfiltered samples began to decrease slightly until after Day 42, when the concentration again increased. Lactate removal from this enrichment can be seen in Figure 12. In an attempt to maintain optimum microbial activity for TCE dechlorination, vials were spiked with lactate after Day 12, increasing the concentration in solution to over $800 \mathrm{mg}$ of lactate/L. Little lactate removal activity was noted between Days 12 and 42, which is the same period in which the $\mathrm{U}(\mathrm{VI})$ concentration began to increase. When the enrichment began to remove the lactate, the uranium concentration again began to increase. U(VI) concentrations in the enrichments with sulfate increased slightly after 12 days and then gradually decreased over the duration of the test, eventually falling below $20 \mathrm{ppb}$ U(VI). As with the sulfate-free enrichment, the enrichment containing sulfate was spiked with lactate, which appeared to slightly inhibit the culture as indicated by slow conversion of lactate (Figure 13). Lactate removal in the control was minimal (data not shown). The cause of the gradual increase in the U(VI) concentration in the blank without TCE and the killed control is not known.

U(VI) concentrations from filtered samples from the same assay can be seen in Figure 14. These results represent $\mathrm{U}(\mathrm{VI})$ present in solution after being filtered through a $0.2-\mu \mathrm{m}$ filter before acidification and analysis. An increase in the amount of U(VI) in both enrichments was noted by Day 12, which was the first analysis after time zero. A slight increase in the U(VI) concentration in the killed control was also noted. $U(V I)$ concentrations in filtered blanks remained stable for the duration of the experiment. Increases in U(VI) noted in the enrichments without sulfate indicate that most of the increase in uranium concentration in solution was in a soluble form, at least in size fractions smaller than $0.2 \mu \mathrm{m}$. After Day 12, the U(VI) concentration in all of the treatments with cells began to steadily decrease with time

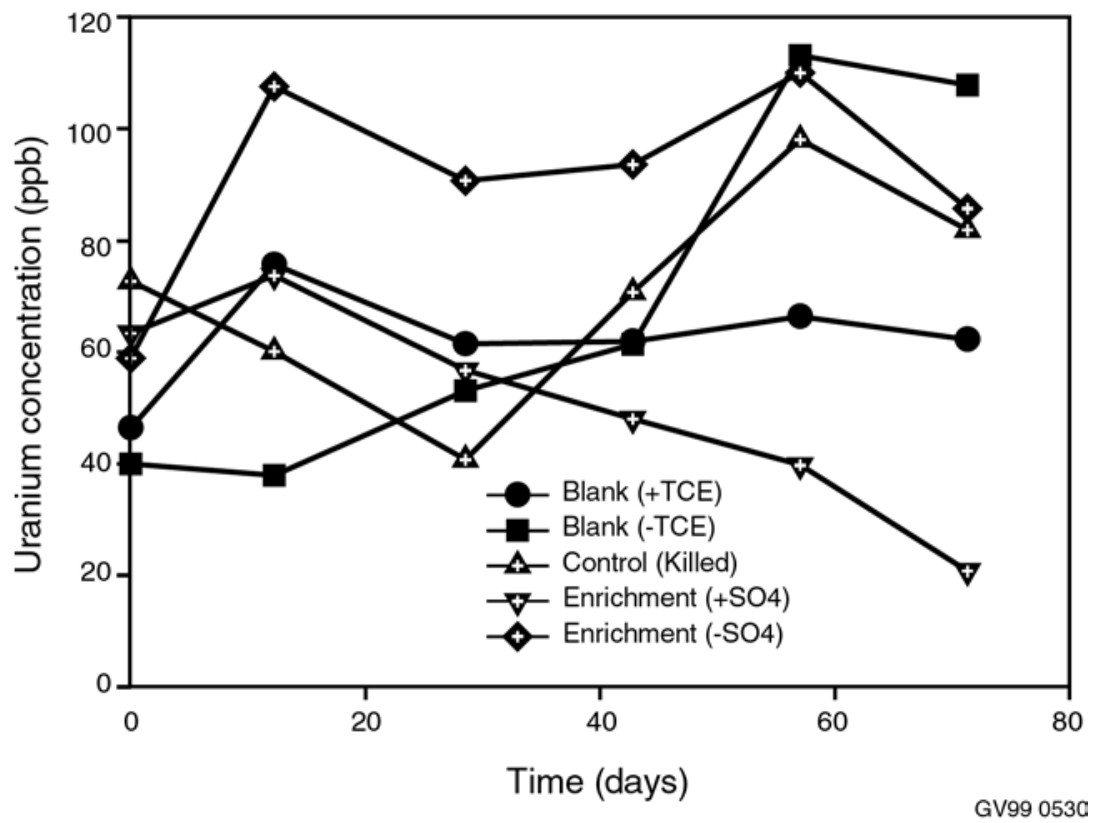

Figure 11. U(VI) concentrations in unfiltered samples from an experiment to determine the effect of anaerobic microbial enrichments on uranium desorption from iron oxide coated quartz. Sand had been exposed to a $1 \mathrm{ppm}$ uranium solution for 5 months before initiation of enrichments. Symbols with crosses indicate samples that contained biomass. 


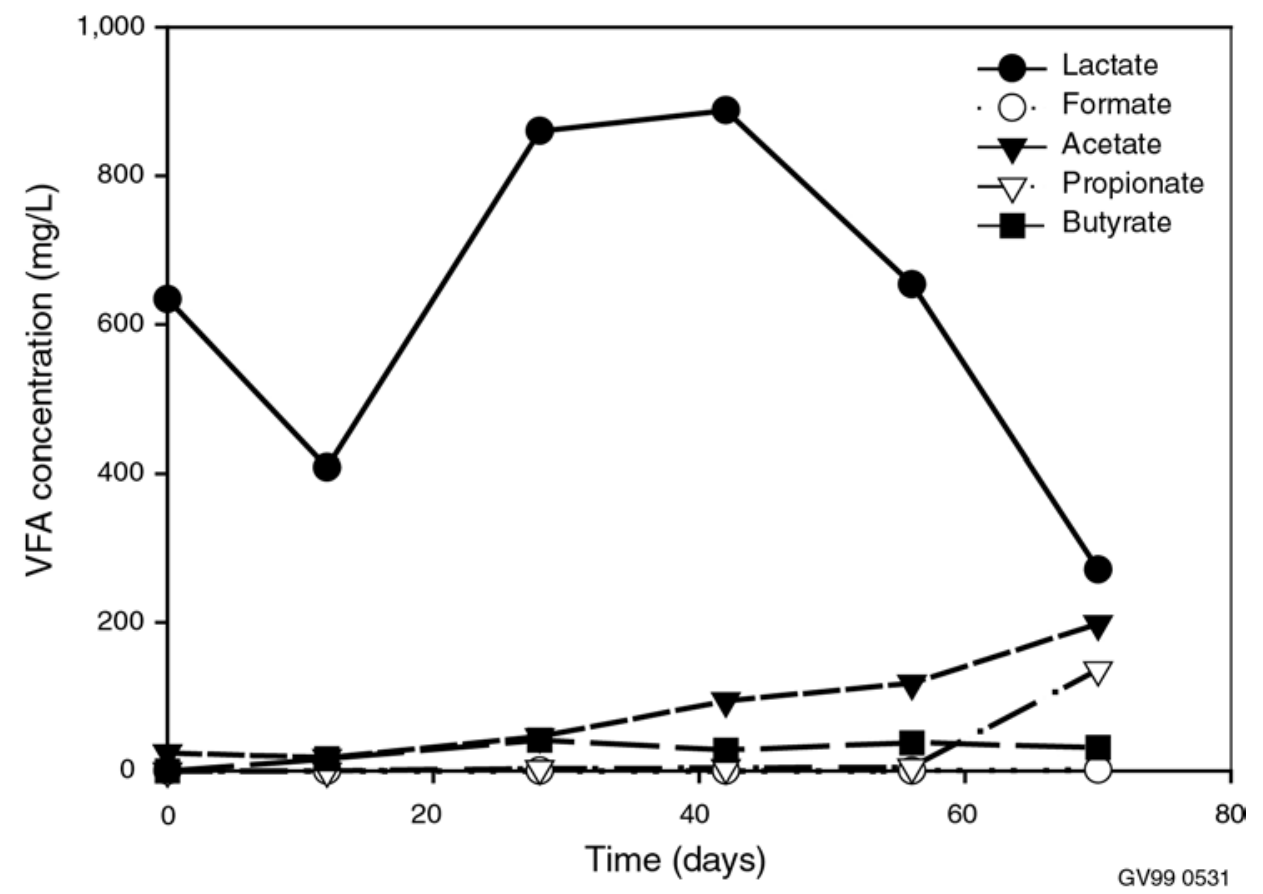

Figure 12. Lactate biotransformation results from an experiment to determine the effect of sulfate-free, anaerobic microbial enrichments on uranium desorption from iron oxide coated sand. Sand had been exposed to a $1 \mathrm{ppm}$ uranium solution for 5 months before initiation of enrichments.

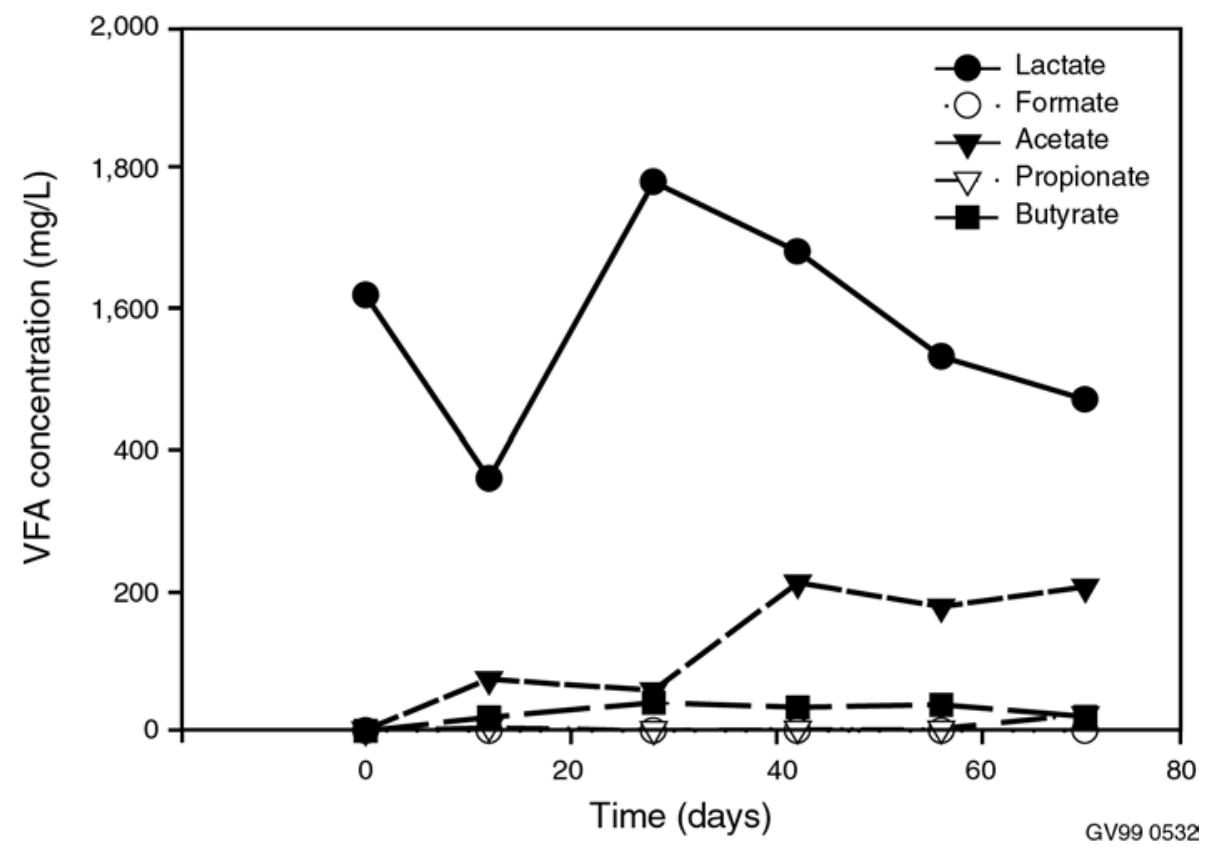

Figure 13. Lactate biotransformation results from an experiment to determine the effect of anaerobic microbial enrichments with sulfate provided as an electron acceptor on uranium desorption from iron oxide coated sand. Sand had been exposed to a $1 \mathrm{ppm}$ uranium solution for 5 months before initiation of enrichments. 


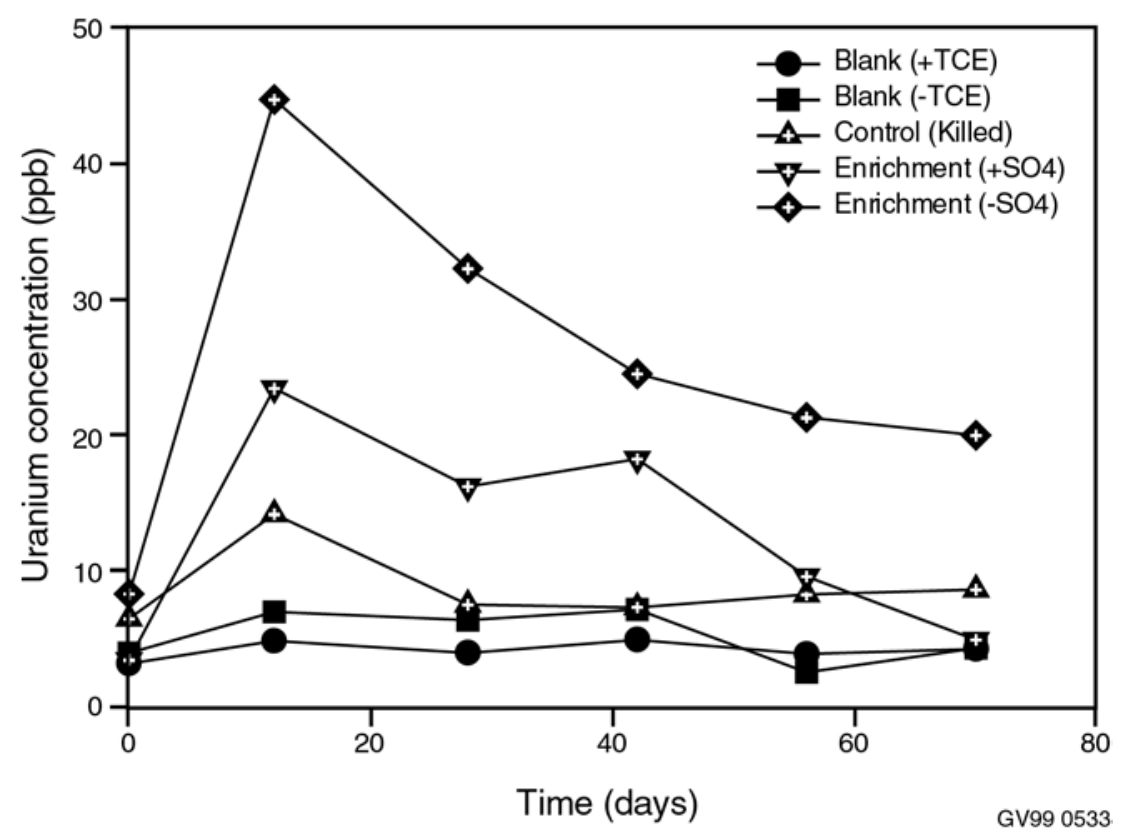

Figure 14. U(VI) concentrations in filtered samples from an experiment to determine the effect of anaerobic microbial enrichments on uranium desorption from iron oxide coated quartz. Sand had been exposed to a $1 \mathrm{ppm}$ uranium solution for 5 months before initiation of enrichments. Symbols with crosses indicate samples that contained biomass.

for the remainder of the experiment. Results from Figures 11 and 14 for the sulfate free enrichment indicate that while soluble uranium was decreasing gradually over time, that U(VI) in the unfiltered sample increased slightly. These results show that the uranium was not readsorbing, but was forming some sort of particulate filterable with a $0.2-\mu \mathrm{m}$ filter. It has not yet been determined whether the uranium being removed by filtration was associated with the microbes or chemical colloid. Potential explanations for the phenomenon being seen is that after initial adsorption the microbes, especially in the sulfate free enrichments, are causing mobilization and potentially solubilization of U(VI) on the iron oxide surface. Following mobilization of the actinide, rather than readsorption to the iron oxide surface, the $\mathrm{U}(\mathrm{VI})$ was being complexed in solution to form a particulate greater than $0.2 \mu \mathrm{m}$ in diameter. It has not been determined whether the observed reaction is chemically or microbially mediated. Formation of $\mathrm{U}(\mathrm{VI})$ containing colloids once the U(VI) is desorbed may increase the possibility of migration to downflow receptors. Laboratory and field evidence indicates that colloidal material, whether organic or inorganic in nature, can lead to the transport of radionuclides in groundwater. ${ }^{13}$ As with the two previous experiments, no methane production or TCE removal was noted in any of the enrichments, blanks or control.

Assay 4. A final enrichment assay was set up during FY-99 to determine the effect of enrichments on uranium when no iron oxide was present. The five treatments used during the previous three assays were set up, but no iron oxide coated quartz was added to the vials. An additional set of vials was set up to determine whether uranium was inhibitory to the microbes used for the enrichments. Enrichments were set up with and without sulfate; no uranium or iron oxide coated sand was added.

Experiments to determine whether uranium was inhibitory to the culture used for testing showed that enrichments with sulfate were inhibited, while enrichments without sulfate appeared to transform lactate at a similar rate whether uranium was present or not. Figure 15 shows lactate removal for the enrichments with sulfate in the presence of uranium (Graph A) and without uranium (Graph B). When uranium was absent from the vials, lactate was completely removed within 20 days, while in the presence 

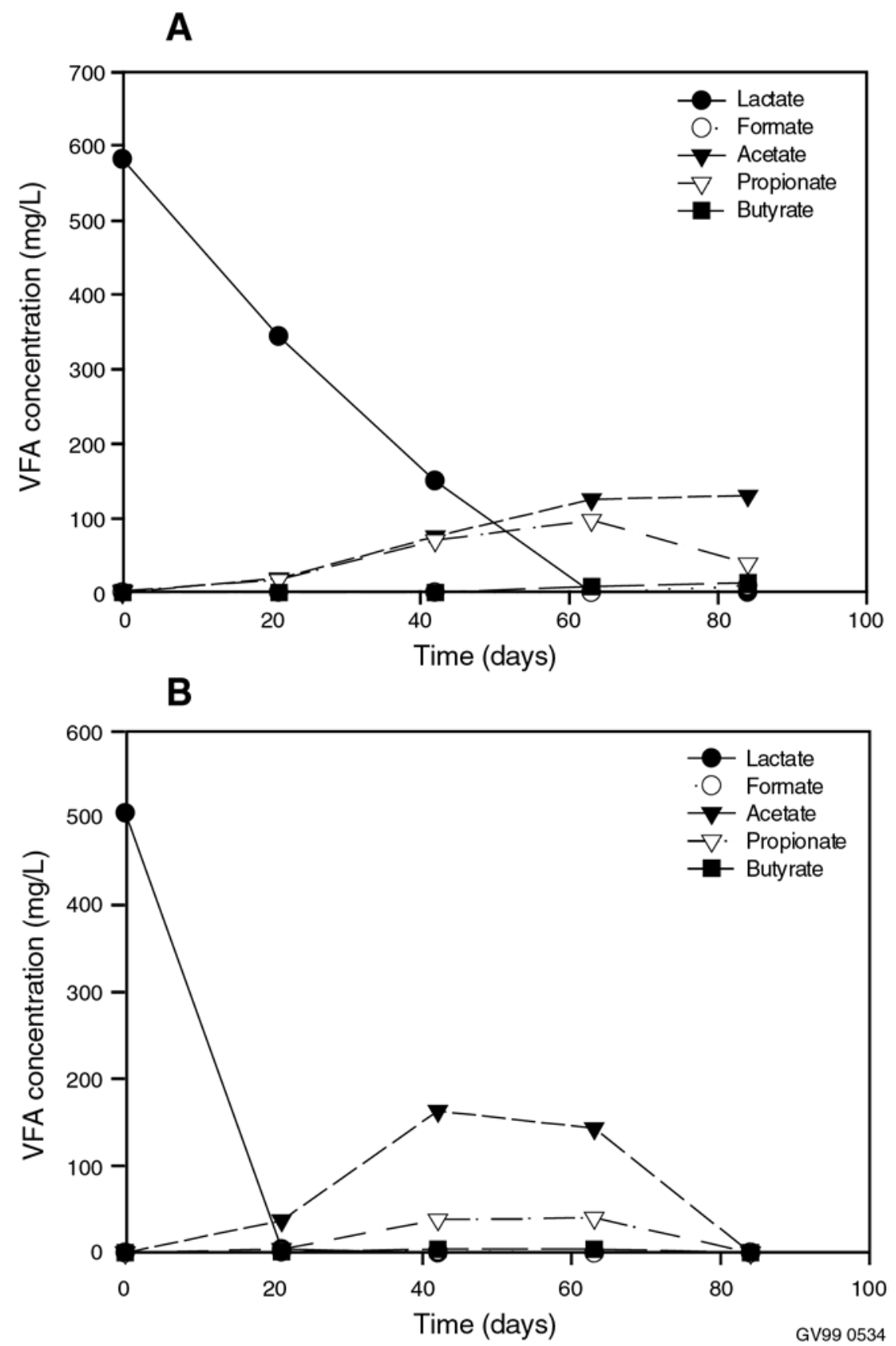

Figure 15. Lactate biotransformation results from an experiment to determine the effect uranium on the physiology of enrichments used for adsorption/desorption testing. Graph A represents lactate biotransformation by an enrichment with sulfate in liquid media containing uranium but no iron oxide coated sand. Graph B represents lactate biotransformation by an enrichment with sulfate in liquid medium without uranium and without iron oxide coated sand. 
of uranium complete removal of lactate occurred after 60 days, which is an indication that uranium may have been inhibitory to these microbes during the adsorption/desorption testing. Uranium mediated inhibition of respiration by soil microbes has been previously reported ${ }^{14}$ Sulfate-free enrichments grown with or without uranium removed lactate at similar rates (complete removal by Day 60), indicating no inhibition by uranium.

\section{Using Deep Tensiometers to Interrogate and Validate Numeric Models and Existing Field Data}

The subsurface movement of moisture is the primary means by which contaminants are transported through the vadose zone and to underlying aquifers. Field data, obtained by instrumentation, are required to monitor these migration phenomena and provide data for predictions of contaminant transport and model calibration. In this task, advanced tensiometers, which provide field data not previously attainable, were used to characterize soil water potential distributions over time in different geologic and climatic environments.

The subsurface movement of moisture is the primary means by which contaminants are transported through the vadose zone and to the aquifer. Field data are required to monitor these migration phenomena and provide data for model calibration. The advanced tensiometer provides data not previously attainable that is critical for understanding transport through deep vadose zones. These data are needed to monitor and evaluate contaminant plume movement and verify landfill and hazardous waste site conditions. Advanced tensiometers are one of the vadose zone instruments that can be used to: (a) determine the potential for moisture movement, (b) determine initial moisture conditions, and (c) obtain characterization data for input to risk assessment modeling, and as part of preoperational, operational, and postclosure monitoring systems to ensure disposal and remedial actions are effective.

The objectives of this project are to: (a) characterize the distribution of soil water potential in deep vadose zones using the advanced tensiometer, (b) collect deep water potential over time periods relevant to transport processes, (c) determine variability of water potential over short distances (tens of meters), (d) acquire soil water potential in different geologic environments, (e) acquire soil water potential at sites with different climates, and (f) perform technical transfer activities to disseminate information on water potential measurements in the deep vadose zones.

Advanced tensiometers were maintained and monitored at depths ranging from 2.4 to $31.5 \mathrm{~m}$ in a variety of geologic and climatic settings. The well sites represent both arid and humid settings in geologically diverse materials, including sediments (gravel, sands, clay, and silts) and porous rock (basalt). The well sites are located in a variety of locations: in Idaho Falls (INEEL Research Center [IRC]), at the INEEL west of Idaho Falls (Radioactive Waste Management Complex [RWMC], Idaho Nuclear Technology and Engineering Center [INTEC], and south of TAN), Oak Ridge, Tennessee (Bear Creek Site) and Savannah River Site. The following section presents results and conclusions from data obtained from several wells at the INEEL representative of sites located in fractured basalt and sedimentary interbeds.

\section{Background}

Recharge through deep vadose zone profiles in arid and semiarid climites is difficult to quantify and detect. Indirect physical monitoring methods include water balance, zero-flux plane, and use of Richards' equation to estimate the recharge flux. These methods are limited by uncertainty of the input data need to determine the recharge flux. The use of direct methods, such as weighing lysimeters, are also limited due to expense, and nonrepresentativeness of disturbed repacked soil within the lysimeter. 
Two of the indirect physical methods, the zero-flux plane and Richards' equation, rely on accurate determination of the water potential within the soil profile to calculate the recharge flux. Water potential measurements in dry soil, such as by thermocouple psychrometers or heat dissipation sensors, exhibit larger measurement errors than that measured by tensiometers. However, conventional tensiometers are only applicable to near surface soils due to the hanging column of water and are limited to measured water potentials that are greater than the vapor pressure of water.

The advanced tensiometer ${ }^{15}$ represents a significant improvement in determining the water potential within the soil profile. Their design removes the hanging water column limitation of the conventional tensiometer by placing a seal at the porous ceramic cup. This tensiometer design offers two advantages. First, the net water potential that can be measured is the entire tensiometric range since the hydrostatic pressure head effect of the water column has been removed from the potential measurement. Secondly, since the water column and the porous cup are disconnected, water potential measurements can be obtained at depth. Direct measurement of the water potential is preferable to other monitoring methods (e.g., moisture content) since it allows direct calculation of the hydraulic gradient.

Recharge through fractured vadose zones is more difficult to quantify than that through porous media vadose zones. Water can move through either the fractures or the material matrix. Most researchers agree that saturated recharge through a fractured vadose zone occurs through the fractures and not through the medium. ${ }^{16,17}$ Field and modeling results from a large-scale infiltration test conducted at the INEEL suggests that infiltration was through the fractures and the matrix had little effect on the advance of the wetting front. ${ }^{18}$ Methods to quantify of these fluxes through fractured media are the subject of numerous studies.

Long-term advanced tensiometer measurement of water potentials in fractured basalt in southeast Idaho was examined. These measurements were analyzed to document episodic and steady-state recharge events. Although the water potential measurements were recorded in a fractured matrix, the applications of the advance tensiometers are also applicable in porous media at hazardous waste sites, landfills, and other areas where estimates of infiltration are desired.

Advanced tensiometers were installed in a fractured basalt matrix and sedimentary interbeds between 2 to $30 \mathrm{~m}$ to evaluate water potentials in deep vadose zones. Although the climate generally receives less than $300 \mathrm{~mm}$ of precipitation, water potential readings were in the tensiometric range.

Water potential measurements indicated a pseudosteady-state gradient for most of the year interrupted by episodic infiltration events. Spring snowmelt creates episodic recharge that moves rapidly through the fractured basalt. During high magnitude recharge events, preferential saturated channeling of the recharge occurs, bypassing zones within the vadose zone. Milder episodic recharge events appear to infiltrate more uniformly through the fractured basalt as indicated by the tensiometric readings. Sedimentary interbeds substantially reduce the recharge percolation, acting as a barrier to flow. Unit gradient analysis of the steady-state recharge flux indicates that unsaturated fracture flow is the major recharge mechanism through the fractured basalt during much of the year.

The results from this long-term water potential study indicate that the advanced tensiometers can be used to monitor infiltration events and estimate steady-state recharge flux. The stability of the water potential readings allows documentation of recharge events throughout the year. The measurement of water potential is advantageous in the allowing of the hydraulic gradient. Such monitoring is essential at waste disposal sites. 
Specific accomplishments are as follows:

- Instruments at the IRC (W03 and W05), Oak Ridge (Bear Creek site), RWMC (77-2, 78-1, and 76-5), Site 14, Savannah River Site, and INTEC are currently maintained and monitored on an hourly basis. These sites represent arid and humid sites in geologically diverse materials, including sediments (gravel, sands, clay, and silts) and porous rock (basalt). Data were downloaded from all the sites, stored in a database, and plotted to evaluate the data.

- Water potentials were analyzed from a deep vadose zone at a semiarid site in southeast Idaho to examine long-term water potential over several years. Advanced tensiometers were installed in near surface alluvial sediments and an underlying fractured Snake River Plain basalt profile. Intermittent sedimentary interbeds of approximately $2-\mathrm{m}$ thickness were also instrumented with tensiometers when present. Water potential measurements were collected from 2.4 to $31.4 \mathrm{~m} \mathrm{BLS}$, for time periods of $11 / 2$ to $31 / 2$ years. Despite the semiarid climate, water potential measurements were within the tensiometric range, from approximately +100 to $-250 \mathrm{~cm}$ of water. For most of the year, water potentials within the fractured basalt exhibited a standard deviation of less than $10 \mathrm{~cm}$ and exhibited a pseudosteady-state unit-gradient downward flux. Tensiometers located near the surface (i.e., less than $4 \mathrm{~m} \mathrm{BLS}$ ) exhibited water potential standard deviations of greater than $25 \mathrm{~cm}$, likely due to evapotransporation and drainage. Episodic snowmelt infiltration events within the basalt and sedimentary interbeds were recorded, indicating recharge pulses occurring at depth. Water potential during episodic infiltration events varied both temporally and spatially, indicating preferential flow pathways through the fractured basalt.

- Installed vadose zone monitoring instruments at the E-slit trench area at Savannah River Site in cooperation with their Accelerated Site Technology Deployment (ASTD) program. These data will be used to complement our vadose zone monitoring. This site represents a deep vadose zone composed of fine-grained sedimentary material in a humid climate.

Advanced tensiometers were installed at the Hanford Buried Waste Test Facility (BWTF) that serves as an analog for the Hanford Tank Farm. Six advanced tensiometers were installed at the BWTF in a $7.6 \mathrm{~m}$ deep lysimeter to evaluate the use of advanced tensiometers in sediments similar to those found at the tank farm. Advanced tensiometers were placed adjacent to existing instruments (water content sensors, neutron access tube, and conventional tensiometers installed using an instrument caisson) at depths $0.9,1.53,2.07$, 4.30, 5.55, and $7.16 \mathrm{~m}$ to allow comparison of data. A seminar on vadose zone instrumentation and current monitoring concepts was presented at the Pacific Northwest National Laboratory (PNNL) to technical staff and DOE representatives.

- Met with a representative from Applied Research Associates (ARA) to explore the feasibility and practicability of incorporating the advanced tensiometers into a cone penetrometer for waste management and agricultural applications. The discussion indicated that there should be a good fit between their apparatus and the advanced tensiometers. A Teaming Agreement was prepared and signed for the INEEL to work with ARA. This work will initially entail preparation of a submittal of a United States Department of Agriculture (USDA) Phase I Small Business Innovation Research (SBIR) for incorporating the advanced tensiometer into a soil moisture monitoring system. A prototype instrument would be built and tested in the laboratory under a variety of temperature, moisture, and soil water potential conditions. Successful completion of these tasks will follow with preparation of a Phase II SBIR proposal. A draft of the Phase I proposal was written, edited, and submitted to the USDA. 
- $\quad$ The patent for the advanced tensiometers was granted by the U.S. Patent and Trademark Office, June, 29, 1999, \# 5,915,476.

- $\quad$ Fourteen new invention disclosures records were prepared and submitted to the Technology Transfer Office on vadose zone and groundwater monitoring devices.

- Several discussions have been held on design and installation of advanced tensiometers for supporting the Environmental Restoration programs at INTEC and RWMC. They are currently considering installing several dozen advanced tensiometers at these sites. Plans were drawn up for specially designed advanced tensiometers that could be installed to depths of about $380 \mathrm{ft}$.

Water potential measurements collected from the tensiometers in two boreholes are illustrated in Figures 16 and 17. In general, the data ranges from -300 to $+50 \mathrm{~cm} \mathrm{H}_{2} \mathrm{O}$ for the monitored period. Small fluctuations in water potential data were observed in the tensiometer data are attributed to changes in barometric pressure. ${ }^{15}$

Borehole 76-5 was monitored for nearly 3 years from July 1996 through May 1999 (Figure 16). The data set can be view as three distinct sections. During the first section that lasted approximately 2 months, water potentials were initially in the range of -150 to $-375 \mathrm{~cm}$ of water and increased to pseudosteady-state values of approximately -50 to $-250 \mathrm{~cm}$ of water. The silt loam material surrounding the porous cup was installed in a dry condition. It is suggested that during this initial 2-month period, the backfill material was equilibrating with the surrounding bulk matrix as indicated by the rise in the water

Well 76-5

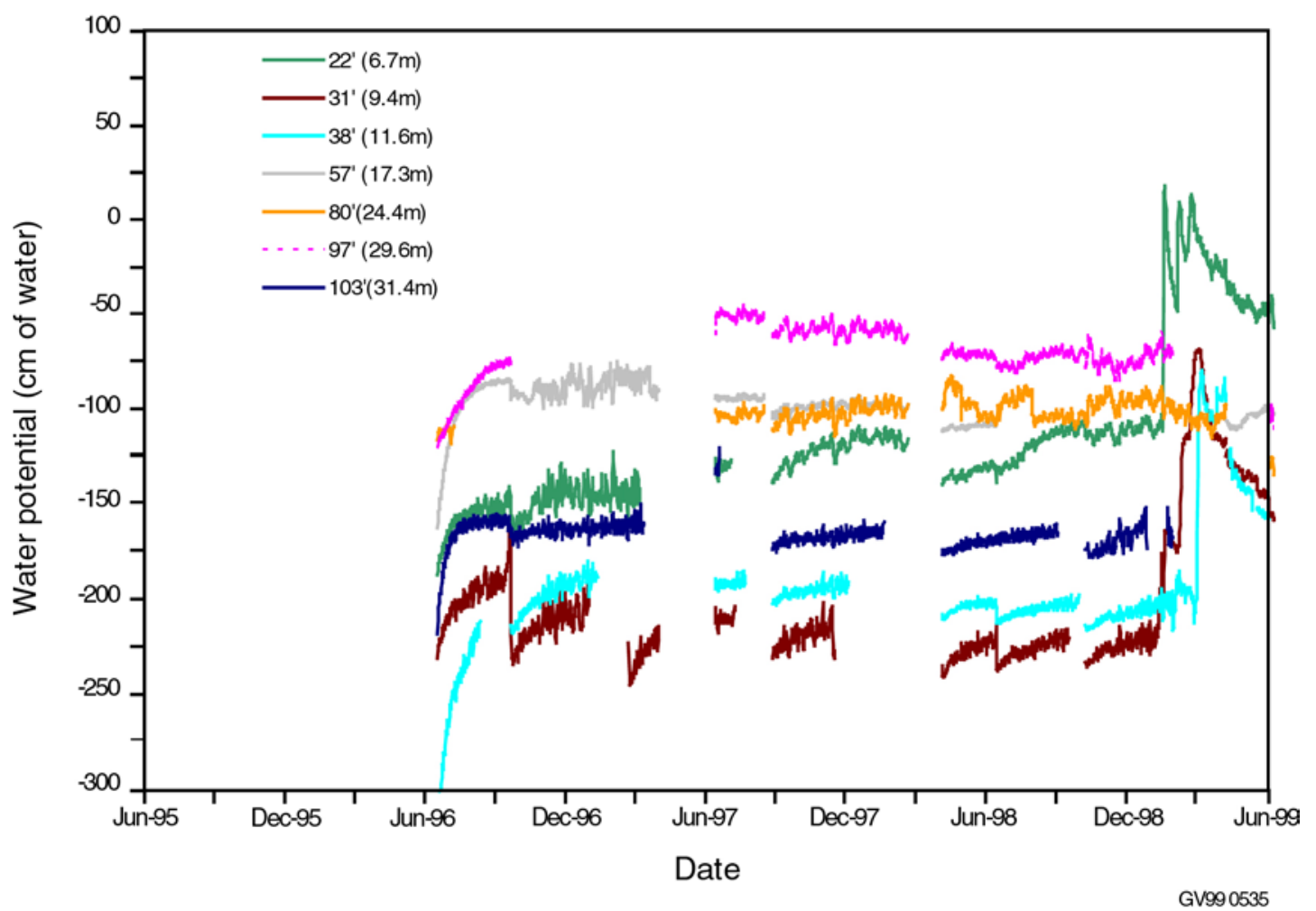

Figure 16. Monitoring of Borehole 76-5. The borehole was monitored for nearly 3 years from July 1996 through May 1999. 


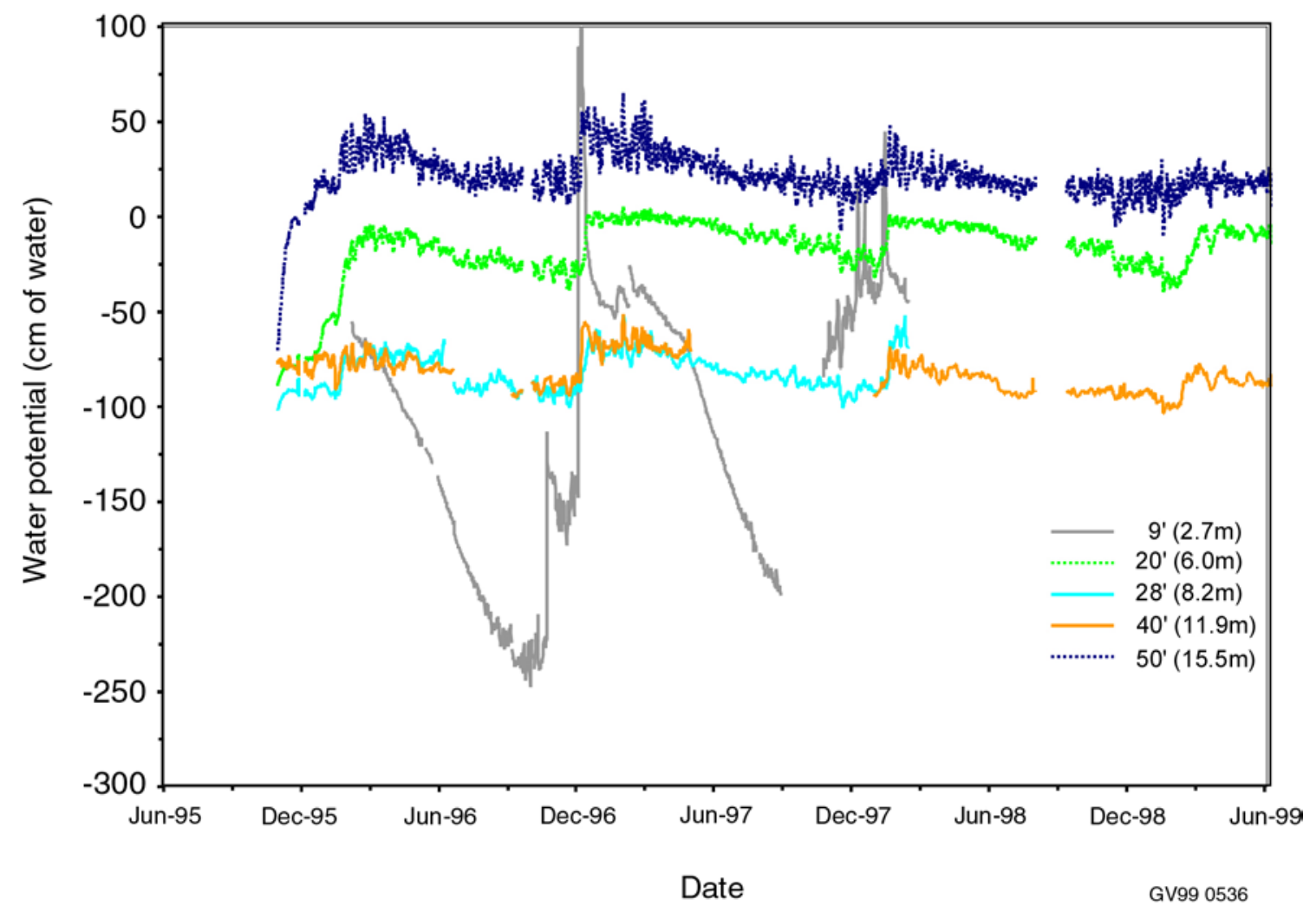

Figure 17. Monitoring of Borehole IRC-03. The borehole was monitored for a $31 / 2$-year period, from November 1995 through May 1999.

potential values. The second section of the data set illustrates fairly stable water potential values for a period of $21 / 2$ years until January 1999. The last section of the data set indicates a large infiltration event occurred as illustrated by the increase in water potential measured by the shallower tensiometers.

Borehole IRC-03 was monitored for a 3 1/2-year period, from November 1995 through May 1999 (Figure 17). Measured water potentials illustrate an initial equilibrium period, and long-term stable periods (10 to 12-month periods) interrupted by near annual infiltration events in the winter/spring. Water potentials are generally higher than those measured in Borehole 76-5. The deepest instrumented depth, $15.5 \mathrm{~m}$, showed near saturated to saturated conditions for the majority of the monitored period.

Both episodic and steady-state infiltration were recorded using the advanced tensiometers in the fractured basalt at the INEEL and IRC sites. Episodic infiltration was documented through examination of temporal changes in the tensiometric readings. Steady-state infiltration was inferred through the determination of a unit hydraulic gradient potential for the bulk of the year.

\section{Episodic Flux}

Episodic infiltration events interrupt the near steady-state conditions on a near-annual basis for the IRC site (Figure 17), while only one episodic infiltration event was noted during the 3 years of monitoring at the INEEL 76-5 site (Figure 16). Recharge at arid sites is highly variable and is dependent on both local topography and plant cover. When a surface alluvial cover overlies a fractured matrix, recharge is further complicated by the depth of the overlying alluvium. Stothoff ${ }^{19}$ numerically examined 
the effects of alluvial cover depth on the amount of infiltration into an underlying fractured matrix. Stothoff concluded that little to no alluvial cover resulted in maximum infiltration due to minimum available storage within the overlying alluvium. Intermediate alluvial cover exhibited the least recharge due to increased alluvial storage combined with the alluvium-fractured interface acting as a capillary barrier maintaining water within the alluvium for subsequent transpiration. Deep alluvial depths effectively isolate the alluvium-fracture interface from evaporation/transpiration implying that the interface has no effect on the infiltration rate.

It is suggested that the 1997 and 1998 spring snowmelt infiltration events at Borehole 76-5 never reached the alluvium-fractured basalt layer. An episodic infiltration event through the fracture basalt was noted during the spring 1999 timeframe. The temporal variability of the episodic infiltration at this site could be due to the deeper overlying sediment, surface topography, ground freezing, and/or amount of potential infiltration available at Borehole 76-5 compared to the IRC site.

Infiltration events are noted yearly for the IRC wells in contrast to only a single infiltration event for the 76-5 Borehole. Table 1 lists the major infiltration events and the date of the wetting front arrival, as indicated by a significant change in water potential, for each measuring depth.

Episodic infiltration events tend to move rapidly through the basalt. One of the most pronounced and easily traced wetting events occurred as a result of a snow melt event in late December 1996, and was monitored in Borehole IRC-03 (Figure 17). The wetting front passed from the 2.7 to the $15.5-\mathrm{m}$ depth in 3 days. All of the tensiometers in this borehole responded to this infiltration event. However, the sequence of first arrival indicated preferential flow paths within the basalt. Following formation of perched water at $2.7 \mathrm{~m}$ at the basalt/sediment interface, the water potential responded first at the 11.9 and $15.5-\mathrm{m}$ depths and then a few days later at the $6 \mathrm{~m}$ and finally the $8.2-\mathrm{m}$ depth. The velocity of the water pressure pulse ranged from 1 to $3 \mathrm{~m} \mathrm{~d}^{-1}$ through the basalt. ${ }^{15}$ This velocity range is close to the $5 \mathrm{~m} \mathrm{~d}^{-1}$ averaged vertical wetting front velocity through basalt during a large-scale infiltration test at the INEEL ${ }^{20}$ near Borehole 76-5.

Table 1. First arrival of water of infiltration to specified depth.

\begin{tabular}{|c|c|c|c|c|c|c|}
\hline \multicolumn{2}{|r|}{$76-5$} & \multicolumn{5}{|c|}{ IRC-03 } \\
\hline $\begin{array}{c}\text { Depth } \\
\text { (m) }\end{array}$ & Spring 1999 & Depth & Spring 1996 & Winter 96-97 & Spring 1998 & Spring 1999 \\
\hline$-^{\mathrm{a}}$ & $-^{\mathrm{a}}$ & $2.7 \mathrm{~m}$ & No data & $12-29-96$ & $2-5-98$ & No data \\
\hline$-^{\mathrm{a}}$ & $-^{\mathrm{a}}$ & $-^{\mathrm{a}}$ & $-^{\mathrm{a}}$ & $-^{\mathrm{a}}$ & $-^{\mathrm{a}}$ & $-^{\mathrm{a}}$ \\
\hline 6.7 & $2-11-99$ & $6.0 \mathrm{~m}$ & $2-15-96$ & $1-6-97$ & $2-10-98$ & $3-6-99$ \\
\hline 9.4 & $3-3-99$ & $8.2 \mathrm{~m}$ & $2-18-96$ & $1-1-97$ & $2-13-98$ & No data \\
\hline 11.6 & $3-26-99$ & $11.9 \mathrm{~m}$ & $2-17-96$ & $1-1-97$ & $2-13-98$ & $3-6-99$ \\
\hline$-^{\mathrm{a}}$ & $-^{\mathrm{a}}$ & $15.5 \mathrm{~m}$ & $2-17-96$ & $1-1-97$ & No infiltration & No infiltration \\
\hline $17.3 \mathrm{~m}$ & No data to date & $-^{\mathrm{a}}$ & $-^{\mathrm{a}}$ & $-^{\mathrm{a}}$ & $-^{\mathrm{a}}$ & $-^{\mathrm{a}}$ \\
\hline $24.4 \mathrm{~m}$ & No infiltration & $-^{\mathrm{a}}$ & $-^{\mathrm{a}}$ & $-^{\mathrm{a}}$ & $-^{\mathrm{a}}$ & $-^{\mathrm{a}}$ \\
\hline $29.9 \mathrm{~m}$ & No data to date & $-^{\mathrm{a}}$ & $-^{\mathrm{a}}$ & $-^{\mathrm{a}}$ & $-^{\mathrm{a}}$ & $-^{\mathrm{a}}$ \\
\hline $31.4 \mathrm{~m}$ & No data to date & $-^{\mathrm{a}}$ & $-^{\mathrm{a}}$ & $-^{\mathrm{a}}$ & $-^{\mathrm{a}}$ & $-^{\mathrm{a}}$ \\
\hline
\end{tabular}


Both saturated and unsaturated flow conditions likely existed in the basalt fractures during infiltration events. For the December 1996 infiltration event at IRC-03, water potentials increased approximately 30 to $40 \mathrm{~cm}$ throughout the basalt profile. The intermediate depth water potential measurements $(8.2$ and $11.9 \mathrm{~m})$ indicated continuous unsaturated conditions in contrast to the saturated conditions at $15.5-\mathrm{m}$ depth. During the infiltration event, the 6-m tensiometric measurement increased from approximately $-25 \mathrm{~cm}$ to positive values indicating a change from unsaturated to saturated conditions within the basalt fractures. The 6-m water potential value maintained a value of approximately zero (saturated) for a few months, then became increasingly more negative (unsaturated) until the following spring infiltration event.

It appears that the episodic infiltration through the basalt fractures is time spatial dependent. Arrival dates listed in Table 1 for Borehole IRC-03 suggest that the sequence of the infiltration front arrival varies according to the infiltration event. For example, at depth $6.0 \mathrm{~m}$, the arrival of the infiltration front is recorded of having the first arrival in the spring of 1996 and 1998, but shows the infiltration front arriving last during the winter 1996-1997 event. We suggest that the magnitude of the infiltration flux is responsible for variable preferential infiltration pathways. As illustrated by the $2.7-\mathrm{m}$ water potential measurements in Figure 17, the magnitude of the winter 1996-1997infiltration event was the largest infiltration event recorded during the 4-year monitoring period. It is suggested that significant saturated channel flow was present during this infiltration event creating preferential flow channels. During subsequent recharge events, the magnitude of the infiltration event were less than the winter 1996-1997 event, and the infiltration preferential flow pattern appears to have changed to a more sequential travel time with respect to depth.

The presence of a sedimentary interbed appears to be the controlling profile structure slowing the infiltration process. The infiltration front moves through the fractured basalt profile at the IRC borehole at rates of meters per day. Figure 17 illustrates steady-state background conditions interrupted in the spring of 1999 by an infiltration event. A sharp increase in water potential as a result of the infiltration event was measured down to $11.9 \mathrm{~m}$ bls at Borehole IRC-03. The wetting front moved from 6 to $11.9 \mathrm{~m}$ within 2 days $\left(2.95 \mathrm{~m} \mathrm{~d}^{-1}\right)$. In contrast, infiltration through Borehole $76-5$ (Figure 16) that contains two sedimentary interbeds at 9.4 and $11.6 \mathrm{~m}$ bls, occurred more slowly. The wetting front at Borehole 76-5 infiltrated from a depth of 6.7 to $11.6 \mathrm{~m}$ in 43 days $\left(0.11 \mathrm{~m} \mathrm{~d}^{-1}\right)$. The sedimentary interbed may have behaved as a capillary barrier retarding the advance of the infiltration front through the profile. This hypothesis is supported by the higher $(\sim-100 \mathrm{~cm})$ water potential readings above the sedimentary interbed compared to the $\sim-200-\mathrm{cm}$ water potential readings in the sedimentary interbeds.

\section{Steady-State Flux}

Water potentials measured over the long term in each of these boreholes indicated pseudosteadystate background conditions generally existed at depths below $4 \mathrm{~m}$ for most of the year. Average water potentials were calculated for the pseudosteady-state period from March 1998 to February 1999 for each tensiometer (Figures 16 and 17).

Average water potential values in Borehole 76-5 are all within the tensiometric range and exhibit a relatively small standard deviation. During the pseudosteady-state portion of the year, a macroscale near unit-gradient exists through the basalt profile. At Borehole IRC-03, variation in average water potential with depth is less than $1.1 \mathrm{~m}$ over the $15-\mathrm{m}$ profile. At Borehole $76-5$, the maximum variation is $1.6 \mathrm{~m}$ over the 31.4-m profile.

In Borehole 76-5, large changes in averaged water potential do occur between the basalt and sedimentary interbeds. The hydrologic characteristics of the sedimentary interbeds are markedly different from the basalt. Hydraulic conductivity characteristic curves were determined for typical interbed material and basalt matrix. The curves for the sedimentary interbed were developed from a composite of 
three soil samples taken from the 10-m interbed ${ }^{21}$ collected in the general area of Borehole 76-5

(Reference 22). The basalt curve was obtained from hydrologic characterization of a near surface basalt block. $^{23}$

The tensiometric data suggest significant unsaturated water flux is transported through the fractures during most of the year. If a unit hydraulic gradient is assumed through both the basalt and the interbed layers of Borehole 76-5, the water flux is equal to the hydraulic conductivity. At approximate water potentials of $-100 \mathrm{~cm}$ in the basalt and $-200 \mathrm{~cm}$ in the interbed, implies an estimated flux of $10^{-9} \mathrm{~cm} \mathrm{~s}^{-1}$ through the basalt matrix and $10^{-6} \mathrm{~cm} \mathrm{~s}^{-1}$ in the interbed. Continuity of water flux must exist at the basaltinterbed under steady-state conditions. It is suggested that unsaturated film flow exists along the fracture walls at a flux equal to the interbed. No measurements of unsaturated flow of INEEL basalt has been made to verify whether the unsaturated flow is dominated by matrix or fractured phenomena at these field tensions.

\section{ACCOMPLISHMENTS}

\section{Core Flooding}

Perform supplementary core floods to address possible presence of organisms in fractured cores and crushed basalt. Core floods were performed using three fractured basalt cores and one column packed with crushed basalt. After 10 days of flow, the columns were sacrificed and samples were taken. For the packed columns, three samples were taken and duplicate samples of this crushed basalt were finely ground for comparison. In previous experiments, only the fracture surfaces and exterior of the crushed basalt were examined. The interior of the fractured pieces and crushed pieces were examined for total bacterial cells and culturable cells. In the packed columns, the difference between the crushed basalt used for packing column and finely ground crushed basalt represent the biomass inside of the packing material.

The expected values for the finely ground crushed basalt were expected to be equal to or higher than the crushed basalt (but not ground); however, the opposite was found. This finding did not allow determination of the biomass inside of the crushed particles. The direct counts of cell numbers yielded similar findings. It is speculated from these findings that the fine grinding of the crushed basalt and drying during this procedure resulted in a die-off of organisms. Presently, it seems unlikely to resolve the contribution of organisms on the outside of the crushed basalt from those on the inside of the crushed pieces. Surprisingly, measurable numbers of culturable heterotrophs were found in the samples from the interior of the fracture core. Total cell counts were barely measurable. Since its seems unlikely that the interior of the basalt with permeabilities (ca. millidarcies) many orders of magnitude lower than the fracture itself (ca. megadarcies) would conduct much groundwater, the source of this biomass remains unknown.

Data from this experiment were presented to FRST and DOE-HQ, DOE-ID, and LMITCO program managers associated with DOE's EMSP and FRST at the Idaho Falls Shilo Inn on October 20, 1998, in the report "Partitioning of Aquifer Bacterial Populations between Geologic Media and Groundwater," by M Lehman.

A manuscript describing the results of the RH experiment is in preparation for submission to Applied and Environmental Microbiology. 


\section{Sediment and Groundwater Sampling}

In collaboration with the University of Michigan National Center for Integrated Bioremediation Research and Development (NCIBRD), analyze sediment and groundwater samples from the Michigan Integrated Remediation Technology Laboratory (MIRTL) field experiments for Community-Level Physiological Profiles (CLPP) to examine response of attached and unattached communities during bioremediation.

Sediments and groundwater from a postinjection (of labeled contaminants) from the MIRTL2: A Natural Gradient Reactive Fate and Transport Experiment at NCIBRD were received and processed for CLPP. During FY-99, 345 groundwater and 94 sediment samples were processed from MIRTL2 for CLPP. Sediments from 18-22 ft BLS were taken from the saturated zone (water table ca. $16 \mathrm{ft} \mathrm{BLS).}$ The cores were aseptically subcored in the laboratory for CLPP analyses. The data demonstrate two points: (1) there is a marked difference in the response of the attached (sediment) and unattached (groundwater) communities to the introduction of contaminants into the aquifer and the subsequent transport and elimination of these contaminants; and, (2) the spatial relationship of the microbial communities with respect to the contaminant plume at this particular sampling date. The goal of this work is to document the effect of contaminants on microbial aquifer communities using complementary measures of microbial structure (PLFA) and function (CLPP).

\section{Count Specific Segments of the Microbial Community In Situ}

In collaboration with work package 3YW432001, develop spectroscopic and microscopic methods to count specific segments of the microbial community (e.g., phylogenetic groups) in situ.

Initially, a laser fluorescence spectroscopy system was built using the $\mathrm{Ar}^{+}$ion and $\mathrm{HeNe}(543 \mathrm{~nm})$ lasers from the CLSM as excitation sources. This system is being used with probes and suspensions of FISH labeled cells to design the optical detection schemes for using the CLSM. The PARISS microspectrometer system mounted on a Nikon E600 microscope was upgraded by adding a more sensitive camera to the spectrometer. The CLSM has also been upgraded to allow use of laser excitation wavelengths of $488 \mathrm{~nm}(\mathrm{Ar}+), 514 \mathrm{~nm}(\mathrm{Ar}+), 543(\mathrm{HeNe})$, and $633 \mathrm{~nm}(\mathrm{HeNe})$ and simultaneous detection on three channels. With the upgraded CLSM system, it is now possible to spatially and spectrally resolve three probes with each image (i.e., one excitation wavelength and three emission wavelengths), even if they are not completely spectrally resolved. The hardware and software for the image analysis system have also been upgraded to provide capability commensurate with upgrades to the image acquisition software.

A limited number of experiments have been performed using probes labeled with fluorescein, Cy-3, and Cy-5. Fluorescein emission overlaps significantly with Cy-3 when exited at 488 or $514 \mathrm{~nm}$ as the fluorescein emission spectrum tails out to beyond $600 \mathrm{~nm}$. In an initial experiment using the laser fluorescence spectrometer, no emission from cells (Pseudomonas stutzeri, Alcaligenes xylosidans) with either the Cy-3 or fluorescein probes was noted. It is not known whether the cells did not accumulate the probe or if the light collection efficiency was too low to record a reasonable spectrum. In a second experiment, the cells examined using the PARISS microspectromer system. Pseudomonas stutzeri did not appear to be hybridized with fluorescein labeled Eubacterial DNA probe, but the Alcaligenes xylosidans cells hybridized with the Cy-3 labeled beta Proteobacterial probe, and spatially resolved spectra were recorded. 


\section{Examine Microbial Populations and their Activities In situ}

Using the Advanced Light Source at Lawrence Berkeley National Laboratory (LBNL), apply synchrotron radiation-based Fourier transform spectromicroscopy to examine microbial populations and their activities in situ.

This project subtask was initiated to evaluate a promising method for non-destructive assessment of microbial presence and activities in situ (4). Part of this work includes study of the surface associations of organisms, degradative substrate, and mineral type on basalt by infrared spectromicroscopy using the particle accelerator at Lawrence Berkeley National Laboratory (LBNL). A collaboration has been established and a proposal was submitted and approved by the administrators of the ALS. We are using surface-enhanced infrared absorption-reflectance (SEIRA) microspectroscopy to examine relations between Burkholderia cepacia, its biodegradation activities, and specific mineral phases as B. cepacia attaches to basalt.

Synchrotron radiation-based (SR) Fourier transform infrared (FTIR) spectromicroscopy has been used to study bacterial attachment and localization on individual mineral surfaces and on geologic materials. The purpose of this study was to evaluate the application of this technique to investigate potential biochemical differences between free-living and attached cells. Additionally, we wanted to evaluate the applicability of this technique to investigate if it would exhibit preferential attachment to specific mineral phases within a heterogeneous geologic substratum. Preliminary results indicate that SR FTIR spectromicroscopy can be a valuable tool in evaluating the contribution of distinct mineral phases within heterogeneous geologic substrata to subsurface microbiological environments. A sample spectra from the work with SEIRA appears in Figure 2.

\section{Microbially Mediated Actinide Transport in the Subsurface}

Results from the research indicate that anaerobic microbial enrichments with and without sulfate have no significant effect on uranium adsorption to iron oxide coated sand. Conversely, enrichments without sulfate did appear to have an effect on desorption of uranium from iron oxide coated sand after the sand had been exposed to uranium for 5 months. After 12 days of incubation, the U(VI) concentration in the liquid increased by approximately $45 \mathrm{ppb}$ uranium in samples filtered through $0.2-\mu \mathrm{m}$ filters, as well as in unfiltered samples. U(VI) concentrations in the filtered samples steadily decreased over time, while U(VI) in unfiltered samples decreased slightly and then began to increase when microbial activity continued. Since there was not a substantial decrease in U(VI) concentration in the unfiltered samples, it would appear that the uranium was forming particles with a diameter greater than $0.2 \mu \mathrm{m}$ or was sorbing to the microbes, but was not readsorbing to the iron oxide coated sand. Formation of insoluble particles is important because this would allow for migration of U(VI) from the contaminated zone to downflow receptors. Additional research will be required to determine whether adsorptive sites on the iron oxide coated sand were chemically reduced by the cultures removing some of the sorptive capacity of the sand.

Assay results also indicated that there is substantial variability for the adsorptive capacity between various batches of sand. This factor may have contributed to the variability between results noted between the various replicates and sampling points and between the various assays. Future batch testing will be attempted using sequential sampling form the same vial.

One other interesting result was that the enrichment culture taken from TCE degradation kinetics experiments was not able to reduce TCE in any of the adsorption/desorption assays performed. This may be due to the fact that the culture has changed over the extended culture period. The culture was not grown in the presence of TCE before inoculation of the enrichments, which may have decreased activity toward TCE. 


\section{Using Deep Tensiometers to Interrogate and Validate Numeric Models and Existing Field Data}

The results from this long-term water potential study indicate that the advanced tensiometers can be used to monitor infiltration events and estimate steady-state recharge flux. The stability of the water potential readings allows documentation of recharge events throughout the year. The measurement of water potential is advantageous in the allowing of the hydraulic gradient. Such monitoring is essential at waste disposal sites.

Water potential measurements collected from the tensiometers in two boreholes are illustrated in Figures 16 and 17. In general, the data ranges from -300 to $+50 \mathrm{~cm} \mathrm{H}_{2} \mathrm{O}$ for the monitored period. Small fluctuations in water potential data were observed in the tensiometer data are attributed to changes in barometric pressure (Hubbell and Sisson 1998).

Both episodic and steady-state infiltration were recorded using the advanced tensiometers in the fractured basalt at the INEEL and IRC sites. Episodic infiltration was documented through examination of temporal changes in the tensiometric readings. Steady-state infiltration was inferred through the determination of a unit hydraulic gradient potential for the bulk of the year.

\section{REFERENCES}

1. R. M. Lehman, F. S. Colwell, D. B. Ringelberg, and D. C. White, "Combined Microbial Community-Level Analyses for Quality Assurance of Terrestrial Subsurface Cores," Journal of Microbiological Methods, 1995, 22:263-281.

2. R. Amann, W. Ludwig, and K. Schleifer, "Phylogentic Identification and In Situ Detection of Individual Microbial Cells without Cultivation,” Microbiological Reviews, 1995, 59(1):143-169.

3. W. Manz, R. Amann, W. Lugwig, M. Wagner, and K. Schleifer, Phylogenetic Oligodeoxynucleotide Probes for the Major Subclasses of Proteobacteria: Problems and Solutions. System. Appl. Microbiol, 1992, 15:593-600.

4. H.-Y. N. Holman, , D. L. Perry, and J. C. Hunter-Cevera, "Surface-Enhanced Infrared AbsorptionReflectance (SEIRA) Microspectroscopy for Bacterial Localization on Geologic Material Surfaces," Journal of Microbiological Methods, 1998, 34:59-71.

5. C. Roller, M. Wagner, R. Amann, W. Ludwing, and K. Schleifer, "In Situ Probing of GramPositive Bacteria with High DNA G+C Content using 23S rRNA-Targeted Oligonucleotides," Microbiology, 1994, 140:2849-2858.

6. J. A. Flood, N. J. Ashbolt, and P. J. Beatson, Simultaneous Morphological and Population Analyses of Environmental Biofilms, In H. C. Flemming (ed.), Investigation of Biofilms, 1999 (in press).

7. J. Fredrickson, D. Balkwill, J. Zachara, S. Li, F. Brockman, and M. Simmons, "Physiological Diversity and Distributions of Heterotrophic Bacteria in Deep Cretaceous Sediments of the Atlantic Coastal Plain,” Applied and Environmental Microbiology, 1991, 57:402-411.

8. Riley, R. G., J. M. Zachara, and F. J. Wobber, Chemical Contaminants on DOE Lands and Selection of Contaminant Mixtures for Subsurface Science Research, DOE/ER-0547T, April 1992. 
9. U.S. Department of Energy, Subsurface Contaminants Focus Area Summary Report, Washington, DC, August 1996.

10. P. Adriaens and T. M. Vogel, Biological Treatment of Organics, In Microbial Transformation and Degradation of Toxic Organic Chemicals, L. Young and C. Cerniglia, eds. John Wiley \& Sons, Inc. New York, 1995, pp. 435-486.

11. S. Fetzner, "Bacterial Dehalogenation," Appl. Microbiol. Biotechnol. 1998, 50: pp. 633-657.

12. K. W. Tsang. P. R. Dugan, and R. M. Pfister, "Mobilization of Bi, Cd, Pb, Th, and U Ions from Contaminated Soil and the Influence of Bacteria on the Process," In Emerging Technologies in Hazardous Waste Management IV (D. Tedder and F. Poland, eds.) American Chemical Society, Washington, DC, 1994, pp. 78-93.

13. I. Beak and W. W. Pitt, Jr., "Colloid-Facilitated Radionuclide Transport in Fractured Porous Rock," Waste Management, 1996, 16(4), pp. 313-325.

14. M. C. Meyer, M. W. Paschke, T. McLendon, and D. Price, "Decreases in Soil Microbial Function and Functional Diversity in Response to Depleted Uranium," J. Environ. Qual., 1998, 27, pp. 1306-1311.

15. J. M. Hubbell and J. B. Sisson, "Advanced Tensiometer for Shallow or Deep Soil-Water Potential Measurements,” Soil Science, April 1998, 163 (4), pp. 271-277.

16. R. Nativ, E. Adar, O. Dahan, and M. Geyh, "Water Recharge and Solute Transport through the Vadose Zone of Fractured Chalk under Desert Conditions," Water Resources Research, 31(2), 1995, pp. 253-261.

17 F. M. Dunnivant, M. E. Newman, C. W. Bishop, D. Burgess, J. R. Giles, B. D. Higgs, J. M. Hubbell, E. Neher, G. T. Norrell, M. C. Pfiefer, I. Porro, R. S. Starr, and A. H. Wylie, "Water and Radioactive Tracer Flow in a Heterogeneous Field-Scale System," Groundwater, 36(6), 1998, pp. 949-958.

18. S. M. Magnuson, Inverse Modeling for Field-Scale Hydrologic and Transport Parameters of Fractured Basalt, Report No. INEEL-95/0637, 1995, Idaho Falls, Idaho: U.S. Department of Energy, Idaho Operations Office.

19. S. A. Stothoff, "Sensitivity of Long-Term Bare Soil Infiltration Simulations of Hydraulic Properties in an Arid Environment," Water Resources Research, 33(4), 1997, pp. 547-558.

20. I. Porro and C. W. Bishop, Large-Scale Infiltration Test CPN Data Analysis, Report No. INEEL-95/059, 1995, Idaho Falls, Idaho: Idaho National Engineering Laboratory.

21. S. O. Magnuson and D. L. McElroy, Estimation of Infiltration from In Situ Moisture Contents and Representative Moisture Characteristic Curves for the 30', 110', and 240' Interbeds, Report No. RWM-93-001, EG\&G Idaho, Inc., 1993, Idaho Falls, Idaho.

22. D. L. McElroy and J. M. Hubbell, Hydrologic and Physical Properties of Sediments at the Radioactive Waste Management Complex, Report No. EGG-BG-9147, EG\&G Idaho, Inc., 1990, Idaho Falls, Idaho. 
23. C. W. Bishop, HydraulicProperties of Vesicular Basalt. M. S. Thesis, Dept. of Hydrology and Water Resources, University of Arizona, Tucson, Arizona, 1991.

\section{APPENDIX \\ Microbial partitioning and Transport in Porous Media}

\section{Journals}

Garland, J. L., and R. M. Lehman, 1999, Dilution/Extinction of Community Phenotypic Characters to Estimate Relative Structural Diversity in Mixed Communities, FEMS Microbiol. Ecol., 30(4): 333-343.

Genter, R. B., and R. M. Lehman, 1999, Metal Toxicity Ascertained by Algal Population Density, Heterotrophic Substrate Utilization, and Fatty Acid Profile in a Small Stream, Environmental Toxicology and Chemistry (In press).

O'Connell, S. P., Lawson D., Watwood M. E., and R. M. Lehman, "A BASIC Program for Reduction of Data from Community-Level Physiological Profiling Using Biolog Microplates," (submitted to Journal of Microbiological Methods following rejection from Applied and Environmental Microbiology).

\section{Presentations}

Kauffman, M. E., H-Y Holman, R. M. Lehman, M. C. Martin, and W. R. McKinney, 1999, Application of Synchrotron Radiation-Based Fourier Transform Infrared Spectromicroscopy to the Investigation of Bacterial Attachment to Aluminum and to Mineralogically-Heterogeneous Geologic Substrata. Abstract, Advanced Light Source Users Conference, Berkeley, CA, October 1999.

Kauffman, M. E., H-Y Holman, R. M. Lehman, M. C. Martin, and W. R. McKinney, 1999, Investigation of Bacterial Attachment to Distinct Mineral Phases within Heterogeneous Geologic Substratum using Synchrotron Radiation-Based Fourier Transform Spectromicroscopy, Abstract, Fall Meeting of the American Geophysical Union, San Francisco, CA, December 1999.

Garland, J. L., and R. M. Lehman, 1999, Dilution/Extinction of Community Phenotypic Characters to Estimate Relative Bacterial Species Diversity in Mixed Communities, Abstract, ASM Conference on Microbial Biodiversity, Chicago, IL, August 1999.

O’Connell, S. P., F. S. Colwell, R. M. Lehman, and M. E. Watwood, Groundwater Microbial Species Diversity as Affected by Trichloroethylene in Microcosm Experiments, Abstract, ASM Conference on Microbial Biodiversity, Chicago, IL, August 1999.

Wilson, M. S., R. M. Lehman, and F. S. Colwell, 1999, Comparison of Culture and Nonculture-Based Methods for Measuring Abundance of Methanotrophic Bacteria in a Deep Basalt Aquifer, Abstract, International Symposium on Subsurface Microbiology - 1999, Vail, CO, August 1999.

Ingram, J. C., M. M. Cortez, G. S. Groenwold, W. F. Bauer, R. M. Lehman, and S. P. O'Connell, 1999, Static SIMS Detection of Cell Membrane Biomolecules," Abstract, 47th American Society for Mass Spectrometry Conference on Mass Spectrometry, Dallas, Texas June 13-17, 1999. 


\section{Using Deep Tensiometers to Interrogate and Validate Numeric Models and Existing Field Data}

\section{Book}

IAEA (International Atomic Energy Agency), Monitoring and Surveillance in Near Surface Disposal Facilities, Vienna, Austria, summer 2000.

U.S. Salinity Laboratory, Proceedings of the International Workshop on Characterization and Measurement of Hydraulic Properties of Unsaturated Porous Media, "Water Potential to Depths of $30 \mathrm{~m}$ in Fractured Basalt and Sedimentary Interbeds," University of California, Riverside CA, USA, U.S. Salinity Laboratory, edited by M. Th. van Genuchten, F. J. Liij, and L. Wu, 1999, pp. 855-865.

\section{Presentations}

J. M. Hubbell, Vadose Zone Monitoring Using Advanced Tensiometers, University of Central Florida, Orlando, FL, December 9-12, 1998, TechnoVentions'98.

J. M. Hubbell, Field Observations of Soil Water Potential in Deep Vadose Zones Using advanced tensiometers, Henderson, NV, January 4-6, 1999, Western Regional Soil Physics Society W-188.

\section{Patent}

J. M. Hubbell and James B. Sisson, June 29, 1999, Patent Number 5,915,476, U.S. Patent and Trademark Office, "Monitoring Well," Assignee: Lockheed Martin Idaho Technologies Company, Idaho Falls, ID, 20 claims, 4 drawing sheets. 


\section{Transport Aspects of Selective Mass Transport Agents}

The ability to separate radioactive and hazardous wastes is a critical need in the efforts to costeffectively treat and process the wide variety of wastes for which DOE-EM is responsible. Liquid separations are today's and the future's most urgently needed type of purification technology. Polymer and solid phase sorbent chemistries are tools by which liquid separations may be performed. In spite of the need for liquid separations, very few programs are focused on polymer-based separation processes for harsh environments. This program recognizes that need and focuses upon developing polymeric and solid state materials with enhanced liquid separation performances in harsh environments.

Both industry and the government need separations materials that function in harsh environments. Research conducted at the INEEL has produced a variety of materials and techniques that have been demonstrated for specific harsh environmental separations. Applications for this technology include separations of acid gases from air and liquids, organic vapors from air, and organic liquids from water and each other. This program is focused on the two critical issues relative to effective separation methods targeted for waste treatment and disposal, pollution prevention, and waste characterization and monitoring. The two critical issues are: 1) the physical properties of the separation media-specifically, what chemistry is required to provide the needed stability to survive and perform in harsh conditions?, and 2) the inter- and intra- molecular interactions involved in the transport/retention mechanisms-in particular, how does one chemically tune the material to be specific for given target compounds? Understanding these two key areas will provide the basis for development of new separation tools for DOE-EM use.

The program consists of three research tasks:

1. Selective Mass Transport in Polymers. Thin polymer film membranes can be formed as either selective chemical passing materials or barrier materials but have not been developed for extremely hazardous environments as encountered in many industrial and government processes. This project will determine the key fundamental parameters that lead to the development of membranes for the separation of hazardous and radioactive DOE legacy wastes.

2. Pore Size and Morphology Control for Solid and Polymer Matrices. Thin organic polymer films have proven to be effective separations membranes, but they often lack the requisite stability for cost-effective application to harsh environments. This project is investigating the effects of harsh environments on polymer morphology and will develop stabilized hybrid organic/inorganic materials for use in the separation of hazardous and radioactive DOE legacy wastes.

3. Adsorption and Absorption Materials for Molecular Separations. Development of novel porous and high-surface-area molecular absorbents/adsorbents-such as the copper sulfides-is being pursued. Mesostructure in candidate materials is controlled via the use of sacrificial surfactants that alter precipitation kinetics. This project will develop stabilized inorganic materials with broad applications in the separation of hazardous and radioactive DOE legacy wastes. 


\title{
Selective Mass Transport in Polymers
}

\author{
F. F. Stewart, T. A. Luther, R. P. Lash, C. J. Orme \\ SUMMARY
}

The U.S. Department of Energy is replete with demanding separations needs. Due to the Cold War legacy, there are numerous stockpiles of aqueous wastes that are both highly radioactive and highly caustic. The need to minimize this volume is extreme. Work performed in this project proposes that membranes may be able to play a role in the solution of the DOE waste problem through selective mass transport through a polymeric matrix and the formation of a barrier to non-selected chemical species. The key to expanding the state-of-the-art in membrane technology is to understand how membrane transport occurs such that it can be predicted. During this reporting period, a series of polymeric materials were synthesized that embodied significantly different solubility behavior. A balance of hydrophilic versus hydrophobic character to the materials was employed and it was accomplished using polyphosphazenes as a model material with the goal of studying water selective transport. This polymer system was chosen because it embodies the requirements for separations in harsh environments: thermal, chemical, and radiation stability far superior to more common organic polymers. Polymers were synthesized from a common backbone precursor in an attempt to limit the number of morphology differences between the polymers. The series spanned from hydrophobic to hydrophilic. The materials were characterized using NMR, GPC, and thermal analysis followed by membrane casting, pure gas and water pervaporation testing. To accurately describe the solubility characteristics of these new materials, solubility characteristics of the polymers were determined from comparison to various solvents. The net result of these experiments was that Hanson expanded solubility parameters appear to be able to describe pervaporation membrane transport phenomena, thus membrane transport can be qualitatively predicted.

\section{PROJECT DESCRIPTION}

Solution-diffusion theory ${ }^{1}$ of transport through a polymer matrix can be thought of as a process with two distinct parameters-solubility and diffusivity. Diffusivity is the mechanism of transport that describes molecular transport through void volume or defects in the polymer matrix. This quantity has been well investigated through membranes formed from glassy polymers and ceramics. ${ }^{2}$ The operative mechanism employed is size exclusion, where a small molecule can move through the polymer matrix but a larger molecule is retained.

A significant aspect of membrane transport that has been investigated to a lesser degree is the solubility component to transport. Solubility can be defined as the intermolecular attractions that either encourage or discourage intimate molecular interactions between a permeate molecule and a polymer substrate. To achieve efficient molecular transport with high selectivities, polymers would be designed to maximize molecular interaction with the selected permeate while minimizing these interactions with the desired rejectate. Experimentally, this entails matching certain chemical characteristics of transported molecules with the polymer substrate. The advantage of this type of analysis is a separation of chemical species based upon their intrinsic chemical affinities regardless of molecular size, thus versatility of the separation can be directly expanded through selection of appropriate polymeric media.

Effective application of membrane technology to separation science requires a mechanistic understanding of the separation process and how to control it. When the mechanistic picture is completed, it allows for the prediction of chemical transport and separation behavior through determination of the key parameters that constitute the solubility aspect of membrane transport. To identify parameters that may control solubility in a polymer and thus membrane transport, this project was 
designed to use a model polymer, polyphosphazenes, which embodied all of the necessary features that a polymer must have to be able to be employed in separations relevant to DOE. Necessary properties include the ability to form defect-free films, to be thermally and chemically stable, and to be able to maintain physical integrity in high radiation environments. Additionally, polymers can be formed with near infinite chemical and physical variety.

A mechanistic understanding of the molecular aspects of selective mass transport through a host matrix is the key to the development of new materials for challenging environmental separations. Intermolecular and intramolecular interactions of selected permeates and macromolecular solid matrices are the focus for this work. A plan is outlined here that identifies strategies and tasks for developing and probing selective mass transport matrices that draws on materials synthesis, testing, evaluation, characterization, and modeling capabilities to build a fundamental understanding of membrane transport and how it can be described and predicted. Beyond determination of the nature of the significant molecular interactions that occur in separations materials, general chemoselective characteristics must be chosen and the effect of these choices on the macroscopic properties of the materials must be understood. Macroscopic physical properties to be understood and controlled are ruggedness, inherent stability (chemical, thermal, and radiation), and film-forming ability. Likewise, design of a material for an exact physical property can have a deleterious effect on the desired chemoselectivity, thus the two factors of permeate affinity and macroscopic physical properties can be linked together and clearly have an effect on each other.

In this work, we seek to further the understanding of separations material design by determining and ultimately predicting clear structure-function relationships in polymeric matrices for factors such as backbone morphology, crosslinking, and chemical content. Outlined below are four tasks that investigate inter- and intramolecular interactions between small permeates and host matrices. The first task will entail the synthesis of materials to be characterized for their gross physical properties and molecular inclusion phenomena, with the first consisting of amorphous rubbery polymers and the second involved with the synthesis of novel inorganic materials. The second task will develop materials formed in the materials synthesis task into thin film membranes including crosslinking studies. The third task will provide characterization data necessary to form structure-function relationships. This task will include gas permeability testing using a pure gas test cell. Finally, the fourth task concerns the prediction and characterization of molecular permeability within polymeric matrices. Additionally, work will continue on the development of a computational computer-based modeling capability to predict molecular solubility and thus membrane performance.

\section{Material Synthesis}

\section{Linear Polymer Synthesis}

Linear polymers based on polyphosphazenes were synthesized so that their solubility behavior could be controlled. Solubility is defined as the affinity of a polymer to swell or dissolve in a particular solvent.

Phosphazene polymers embody an inorganic backbone of alternating phosphorus and nitrogen that contain two pendant groups on phosphorus. These pendant groups are typically organic ${ }^{3}$ and are attached through nucleophilic substitution processes; thus these polymers are an organic-inorganic hybrid. These polymers are formed through a unique two-step process where the backbone is synthesized with highly labile chlorine substituents on phosphorus. Poly[dichlorophosphazene] is unstable to water, however this instability can be eliminated through the attachment of pendant groups. ${ }^{4}$ The choice of pendant group is generally thought to control the solubility behavior of the polymer. Hydrophilic polymers have been readily synthesized ${ }^{5}$ by attaching hydrophilic organic pendant groups to the polymer backbone. An 
example of this is poly[bis(2-(2-methoxyethoxy)ethoxy)phosphazene] (MEEP), a water soluble polymer with 2-(2-methoxyethoxy)ethanol (MEE) pendant groups. Likewise, substitution with hydrophobic groups such as phenol and $p$-methoxyphenol (MEOAr) has yielded hydrophobic polymers. ${ }^{6}$

In an earlier work, ${ }^{7}$ phosphazene terpolymers have been synthesized with varying ratios of hydrophilic MEE and hydrophobic MEOAr. The term terpolymer is employed because when two differing pendant groups are attached, three differing monomers are possible: $\mathrm{PN}(\mathrm{MEE})_{2}$, $\mathrm{PN}(\mathrm{MEE})(\mathrm{MEOAr})$, and PN(MEOAr) ${ }_{2}$. The advantage of this synthetic strategy is that polymers were formed with a controllable affinity for a chemical species, in this case water. With polymers containing higher levels of MEE, thought to be appropriate to enhance selectivity for water, tests in a water-dye pervaporation apparatus were performed with poor results. The data showed decreased fluxes with time suggesting poor mechanical stability of the polymer.

Crosslinking of the polymers after formation of membranes was identified as the method to provide mechanical durability. To maximize the crosslink density, an additional pendant group was added with a moiety that allows for facile crosslinking, $o$-allylphenol. This pendant group was attached through the same processes as the MEE and MEOAr. The allylic group provides a relatively low energy pathway to achieve crosslinks through free radical processes. Methods that will effectively crosslink polymers through the use of allylic moieties include electron beam irradiation and thermal processes that effect free radical generation through the decomposition of an initiator.

To explore solubility, it was envisioned that five separate polymers could be synthesized with a consistent content of $o$-allylphenol and varying amounts of MEE and MEOAr from one lot of poly[dichlorophosphazene] yielding an array of five controlled solubility polymers. The benefit of this type of synthesis is the potential for eliminating morphological and molecular weight deviations inherent to the use of multiple lots of poly[dichlorophosphazene]. Additionally, the $o$-allylphenol could be added to the polymer in a uniform manner such that all of the separate polymers contained the same amount. The reactions were conducted and the ratios of each pendant group were measured by integration of proton nuclear magnetic resonance (NMR) spectra, see Table 1. An analysis of the data yields a strong deviation in the percent of the o-allylphenol on the polymers with the highest percentages of MEE. It is proposed that this is due to fractionalization of the polymer during purification, fractions of the polymer were removed during the numerous precipitations that were employed to purify the polymers.

Additionally, there was some degree of deviation between the sought and measured amount of MEE on the backbone. This type of behavior is common in these syntheses due to the requirement of an excess amount of pendant group during synthesis that is required to ensure that all sites are substituted.

Table 1. Pendant group distribution of the controlled solubility polymers.

\begin{tabular}{ccccccc}
\hline & $\begin{array}{c}\text { Pendant Group } \\
\text { Distribution } \\
\text { Sought } \\
\text { MEE(\%) }\end{array}$ & $\begin{array}{c}\text { MEOAr } \\
(\%)\end{array}$ & $\begin{array}{c}\text { O-allylphenol } \\
(\%)\end{array}$ & $\begin{array}{c}\text { Pendant Group } \\
\text { Distribution } \\
\text { Measured MEE } \\
(\%)\end{array}$ & $\begin{array}{c}\text { MEOAr } \\
(\%)\end{array}$ & $\begin{array}{c}\text { o-allylphenol } \\
(\%)\end{array}$ \\
\hline Molymer & 5 & 80 & 15 & 6 & 75 & 19 \\
M2B & 25 & 60 & 15 & 38 & 49 & 13 \\
M2C & 42.5 & 42.5 & 15 & 41 & 45 & 14 \\
M2D & 60 & 25 & 15 & 48 & 48 & 4 \\
M2E & 80 & 5 & 15 & 74 & 24 & 2 \\
\hline
\end{tabular}


Remaining amounts of chlorine on the backbone represent sites where hydrolytic degradation of the backbone can occur. Full characterization of these materials was accomplished through the use of DSC, NMR, and dilute solution characterization.

\section{Phosphazene Cyclomatrix Materials}

The other portion of the materials synthesis task was focussed on cyclomatrix phosphazene polymers. Cyclomatrix polymers consist of organic links between six-membered phosphazene rings. Previous work identified a synthetic route that provided soluble polymeric materials in good yield through the use of an ester linkage methodology. Further work concentrated in two general directions. First, new cyclomatrix precursors were developed that offered different routes of chemical crosslinking were synthesized. Second, electron beam irradiation was employed to form cyclomatrix polymers with the potential to be used as separation materials.

Three potential cyclomatrix precursors were synthesized employing specific pendant groups on a cyclotriphosphazene, see Figure 1. Structure B, hexa(methylhydroquinone)cyclotriphosphazene was readily synthesized using a previously reported procedure. This synthesis was significant for two reasons.

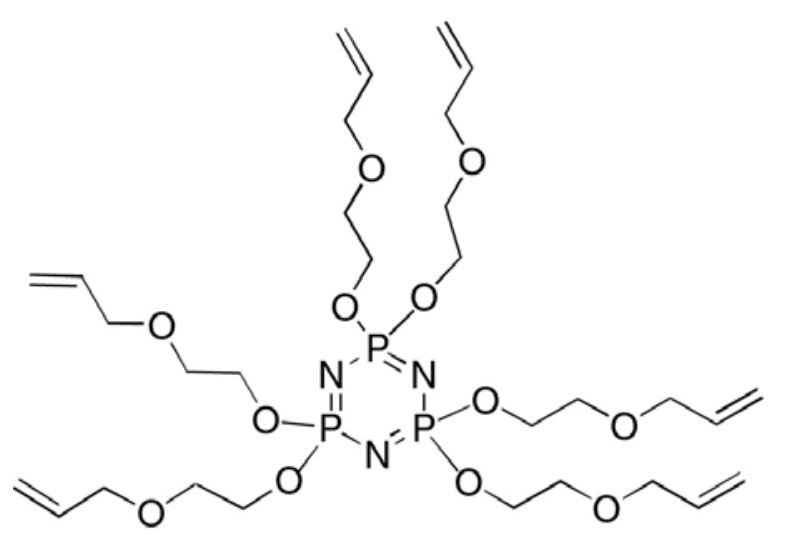

$\underline{A}$<smiles>Cc1cc(OP2(Oc3ccc(O)c(C)c3)=NP(Oc3ccc(O)c(C)c3)(Oc3ccc(O)cc3C)=NP(Oc3ccc(O)cc3C)(Oc3ccc(O)cc3C)=N2)ccc1O</smiles><smiles>COCCOCCOP1(OCCOCCOC)=NP(OCCOCCOC)(OCCOCCOC)=NP(OCCOCCOC)(OCCOCCOC)=N1</smiles>

GV99 0441

Figure 1. Three cyclomatrix phosphazene precursors. A. Hexa(2-allyloxyethoxy)cyclotriphosphazene.

B. Hexa(methylhydroquinone)cyclotriphosphazene.

C. Hexa(2-(2-methoxyethoxy)ethoxy)cyclotriphosphazene. 
First, the methyl groups represent a local of potential functionalization chemistry to accommodate polymerization. Second, the regiochemistry of the methyl group attachment favored the meta position with respect to phosphazene ring, but was not complete as was seen for the $t$-butyl analog. ${ }^{8}$ The two possible orientations, ortho and meta, were both clearly observable by proton and ${ }^{13} \mathrm{C} N \mathrm{NR}$, favoring the meta position (90\%) over the ortho position (10\%). Additionally, this effect on the spectra was reflected by the ${ }^{31} \mathrm{P}$ NMR spectra.

Hexa(2-allyloxyethoxy)cyclotriphosphazene, structure A, was synthesized by the straightforward route of the reaction of hexachlorocyclotriphosphazene with sodium 2-allyloxyethoxide. The structure, with allylic moieties included in the structure, offers a readily available chemical pathway for the synthesis of cyclomatrix polymers. This material was crosslinked using electron beam irradiation at a dose level of 20 and 40 Mrad to yield a semi-solid material that was not found to be suitable for membrane formation. This conclusion is drawn from the fact that the material lacked the necessary physical integrity and film forming ability. Crosslinking of this cyclotriphosphazene is proposed to occur through the allylic groups. This conclusion is supported by electron beam crosslinking of hexa(2-(2-methoxyethoxy)ethoxy)cyclotriphosphazene, structure C. This fluid material, similar to structure A but without the allylic moiety, showed no significant physical manifestations of crosslinking with doses as high as $140 \mathrm{Mrad}$.

\section{Materials Processing and Testing}

The focus of this task was to study the controlled solubility linear polymer series for optimum membrane performance in a liquid pervaporation separation. These materials were designed for certain solubility characteristics without regard to physical properties. For example, polymer M2A is a hydrophobic amorphous rubber with excellent film forming characteristics. Thermal crosslinking of this polymer was accomplished through doping of a polymer-THF casting solution with a free radical initiator such as benzoyl peroxide. Membranes were solution cast onto a glass or ceramic substrate and the THF was allowed to evaporate. Once the THF was removed, the membranes were cured in an oven at $130^{\circ} \mathrm{C}$ for at least 10 minutes. Previous work suggests that this method is the optimum for these types of phosphazenes. ${ }^{9}$

Effective membranes for pervaporation were formed as composites consisting of a thin film $(15-60 \mu \mathrm{m})$ cast on to a controlled pore size ceramic with $0.2 \mu \mathrm{m}$ pores. Curing of these membranes provided sufficient crosslink density within the polymer to prevent penetration of polymeric materials into the pores. Penetration or creep of the polymer into the pores would have a clogging effect upon the ceramic, thus lowering flux rates.

Electron beam crosslinking of these polymers was conducted to study the effect of radiation induced crosslinking upon the controlled solubility polymer series. Moderate dose levels, 10-30 Mrad, were found to crosslink all five polymers such that they were no longer soluble. Membrane performance testing of these electron beam crosslinked membranes, however, was not accomplished due to the mechanical properties of the polymers. With substantial amounts of MEE within the polymer matrix, the membranes tend to become brittle making physical manipulation impractical.

\section{Characterization of Polymeric Membranes}

Appropriate physical and chemical characterization experiments were performed on the controlled solubility polymer series. NMR, DSC, and TGA proved to be the most revealing. The polymers were studied (see Table 2) in the solution state by ${ }^{31} \mathrm{P}$ NMR where they were observed to give three resonances, which is consistent with previous reports. Additionally, GPC analysis was employed to study molecular weight with all data in the $10^{6}$ range, as expected. 
Table 2. Phosphorus-31 NMR data, glass transition temperature $\left(\mathrm{T}_{\mathrm{g}}\right)$, and decomposition temperature $\left(\mathrm{T}_{\mathrm{d}}\right)$ data for the controlled solubility polymer series.

\begin{tabular}{|c|c|c|c|}
\hline Polymer & $\begin{array}{c}{ }^{31} \text { P NMR Data } \\
(\mathrm{ppm})\end{array}$ & $\begin{array}{c}\mathrm{T}_{\mathrm{g}} \\
\left({ }^{\circ} \mathrm{C}\right)\end{array}$ & $\begin{array}{c}\mathrm{T}_{\mathrm{d}} \\
\left({ }^{\circ} \mathrm{C}\right)\end{array}$ \\
\hline M2A & $-8,-16.9,-17.2,-17.6$ & 4.0 & 322 \\
\hline M2B & $-7.2,-12.0,-17.0$ & -18 & 312 \\
\hline $\mathrm{M} 2 \mathrm{C}$ & $-7.2,-12.0,-13.0,-17.0,-17.5$ & -49 & 263 \\
\hline M2D & $-7.2,-12.0,-13.0,-17.0,-17.5$ & -43 & 287 \\
\hline M2E & $-7.5,-12.0,-13.0$ & -63 & 270 \\
\hline
\end{tabular}

The data show a clear trend with increasing amounts of MEE on the backbone giving both lower glass transition $\left(\mathrm{T}_{\mathrm{g}}\right)$ temperatures and lower decomposition $\left(\mathrm{T}_{\mathrm{d}}\right)$ temperatures. This is a reflection of the increased level of polymer chain flexibility and relative thermal instability induced by MEE. A pure MEE polymer, MEEP, shows its $\mathrm{T}_{\mathrm{g}}$ at $-84^{\circ} \mathrm{C}$ and $\mathrm{T}_{\mathrm{d}}$ at $260^{\circ} \mathrm{C}$ suggesting that the aromatic constituents do impart greater thermal stability. Physically, the increased amount of molecular motion imparted by the

MEE is a disadvantage due to polymer creep, the aromatic component gives the polymers a robust quality that increases with the amount of aromatic component. This must be balanced by the MEE component, which gives the polymers water permeability.

\section{Pure Gas Transport Experiments}

Gas permeability experiments were performed on a variety of polymers including a well characterized phosphazene (PPXP) and polydimethylsiloxane (PDMS). PDMS is a common polymer whose gas transport characteristics are well understood and the data obtained are shown in Table 3 . In this table, data is provided for permeability, which is a product of the solubility terms and the diffusive terms of transport. The small monatomic and diatomic gases yield very similar data while a larger gas such as carbon dioxide exhibits a much larger solubility component, which reflects this gases' ability to solubilize into the polymer and plasticize it.

The benchmark phosphazene polymer, PPXP, is a commercial formulation containing sec-butylphenol, MEOAr, and $o$-allylphenol as pendant groups. PPXP polymer is hydrophobic, has good film-forming characteristics, and is crosslinked in a similar manner as other phosphazenes discussed above. Samples of this polymer were delivered to a collaborator (Dr. Donald H. Weinkauf, New Mexico Institute of Mining and Technology) where measurements were taken using helium, nitrogen, oxygen, and carbon dioxide, see Table 4. A comparison of data collected at INEEL and New Mexico Tech gives good agreement suggesting an adequate calibration of the instruments.

Pure gas transport experiments were also conducted to study crosslinking in MEE containing polymers. The phosphazene (lot \# 098) employed in this study was a polymer with $23 \% \mathrm{MEE}, 72 \%$ MEOAr, and 5\% o-allylphenol as pendant groups. The material was tested as thin films without crosslinking, with thermally initiated free radical crosslinking, and with electron beam irradiation at three different dose levels. Helium, argon, and $\mathrm{CO}_{2}$ were used as test gases, see Table 5. The data suggest that the method and the degree of crosslinking have little effect upon the gas transport characteristics of the polymer. This is a significant observation because it allows for the decoupling of membrane physical characteristics and transport characteristics. Thus, a polymer may be designed with specific solubility 
Table 3. Pure gas transport through PDMS films.

\begin{tabular}{|c|c|c|c|c|c|c|}
\hline Gas & $\mathrm{He}$ & $\mathrm{H}_{2}$ & $\mathrm{~N}_{2}$ & $\mathrm{O}_{2}$ & Methane & $\mathrm{CO}_{2}$ \\
\hline $\begin{array}{l}\text { Permeability } \\
\text { (Barrers) }\end{array}$ & 310 & 574 & 242 & 488 & 736 & 2341 \\
\hline Diffusivity & $6.3 \times 10^{-5}$ & $6.3 \times 10^{-5}$ & $1.5 \times 10^{-5}$ & $1.6 \times 10^{-5}$ & $1.2 \times 10^{-5}$ & $1.13 \times 10^{-5}$ \\
\hline$\underline{\text { Solubility }}$ & 0.037 & 0.08 & 0.112 & 0.23 & 0.46 & 1.6 \\
\hline
\end{tabular}

Table 4. Pure gas transport through PPXP films.

\begin{tabular}{ccccc} 
Gas & $\begin{array}{c}\text { INEEL } \\
\text { Permeability } \\
\text { (Barrers) }\end{array}$ & $\begin{array}{c}\text { NM Tech } \\
\text { Permeability } \\
\text { (Barrers) }\end{array}$ & $\begin{array}{c}\text { INEEL } \\
\text { Diffusivity }\end{array}$ & $\begin{array}{c}\text { NM Tech } \\
\text { Diffusivity }\end{array}$ \\
\hline $\mathrm{He}$ & $10-20$ & 14.9 & $4 \times 10^{-6}$ & Not determined \\
$\mathrm{N}_{2}$ & $2-3$ & 1 & $1.12 \times 10^{-6}$ & $2.5 \times 10^{-7}$ \\
$\mathrm{O}_{2}$ & $3-5$ & 3.9 & $1.4 \times 10^{-7}$ & $3.8 \times 10^{-7}$ \\
$\mathrm{CO}_{2}$ & $20-30$ & 22.4 & $1.8 \times 10^{-7}$ & $2.6 \times 10^{-7}$ \\
\hline
\end{tabular}

Table 5. Permeability (Barrers) of gases through Polymer 098.

\begin{tabular}{cccccc}
\hline Gas & No Crosslinking & Thermal & $10 \mathrm{Mrad}$ & $15 \mathrm{Mrad}$ & $20 \mathrm{Mrad}$ \\
\hline $\mathrm{He}$ & 8.2 & 7.8 & 8.1 & 7.7 & 12.5 \\
$\mathrm{Ar}$ & 2.8 & 2.2 & 2.7 & 2.3 & 3.5 \\
$\mathrm{CO}_{2}$ & 39 & 29 & 33 & 30 & 45 \\
\hline
\end{tabular}

characteristics and subsequently may be treated to obtain appropriate physical properties without loss of transport behavior.

Pure gas characterization for permeability was performed on the controlled solubility series of polymers, see Table 6 . The general trend suggests that with increasing amounts of MEE on the backbone, permeability of all gases increases as compared to the lowest MEE containing polymer, M2A. This effect is accentuated for $\mathrm{CO}_{2}$, consistent with data reported above.

The ability to transport a specific gas is significant, however, separation of one gas from another must be considered. It is generally accepted that as a membrane transports increasing amounts of a certain gas, the potential separation factor is reduced. A benchmark separation ${ }^{10}$ is oxygen from nitrogen. In an earlier work, ratios of $\mathrm{O}_{2} / \mathrm{N}_{2}$ separation factors were plotted versus the permeability of $\mathrm{O}_{2}$. The data yielded an upper bound region that represented a maximum in the separation factor/ $\mathrm{O}_{2}$ permeability ratio. The data for $\mathrm{O}_{2}$ and $\mathrm{N}_{2}$ from Table 6 were plotted to reveal strong correlation between pendant group concentration of separation factor. Polymers with higher percentages of MEE on the backbone were observed to give lower separation factors than materials with lower amounts. In fact, a general trend can be drawn that is perpendicular to the upper bound suggesting that at lower amounts of MEE, membranes are more effective at separating nitrogen from oxygen, see Figure 2. PDMS is included in the plot as an example of a polymer with high rates of transport but low separation factor. 
Table 6. Permeability (Barrers) of gases through the controlled solubility polymers.

\begin{tabular}{lccccc}
\hline Gas & M2A & M2B & M2C & M2D & M2E \\
\hline $\mathrm{H}_{2}$ & 4.2 & 34.7 & 4.9 & 23.7 & 28.8 \\
$\mathrm{He}$ & 4.7 & 25.3 & 4.6 & 16.6 & 21.9 \\
$\mathrm{~N}_{2}$ & 0.3 & 7.3 & 2.4 & 8.3 & 10.2 \\
$\mathrm{O}_{2}$ & 1.7 & 13.8 & 3 & 14.8 & 18 \\
$\mathrm{CH}_{4}$ & 1.4 & 13 & 3 & 16 & 19.2 \\
$\mathrm{CO}_{2}$ & 9.3 & 117.2 & 28.5 & 115.9 & 226.7 \\
\hline
\end{tabular}

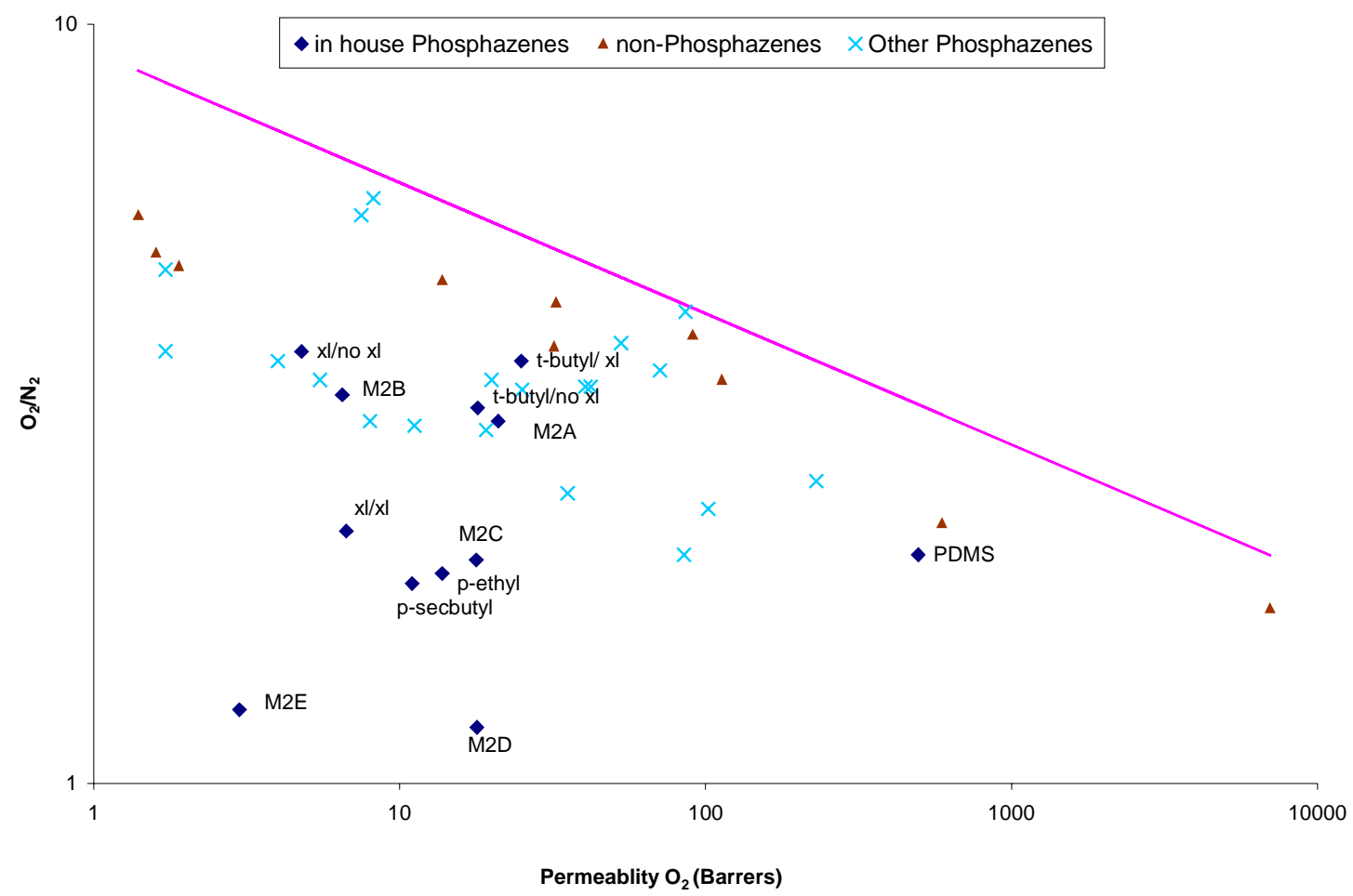

Figure 2. Plot of separation factor $\left(\mathrm{O}_{2} / \mathrm{N}_{2}\right)$ versus permeability of oxygen.

\section{Solubility Parameter Determinations}

Essential to generating a predictive model, quantification of an individual solvent interaction with a specific polymer must be performed. Hildebrand solubility parameters ${ }^{11}$ describe in a single term a product of the polar, hydrogen bonding, and weak forces of a particular solvent. To determine the Hildebrand solubility parameter for a polymer, lightly crosslinked portions of the polymer are immersed in various solvents of known parameter. The solvent that causes the highest degree of swelling measured gravimetrically has the closest Hildebrand parameter to that of the polymer. As illustrated in Figure 3, one difficulty that often arises is a bimodal distribution of parameters, instead of one there are two or more maxima. Maximum solubility is observed for all polymers at approximately 9.3 , which corresponds to methylene chloride. A second maximum is observed at approximately 14.5 (methanol) that appears to vary based upon polymer substitution. A way to deconvolute this situation is through the use of Hanson expanded solubility parameters ${ }^{9}$ where three independent terms are employed to describe the solubility 


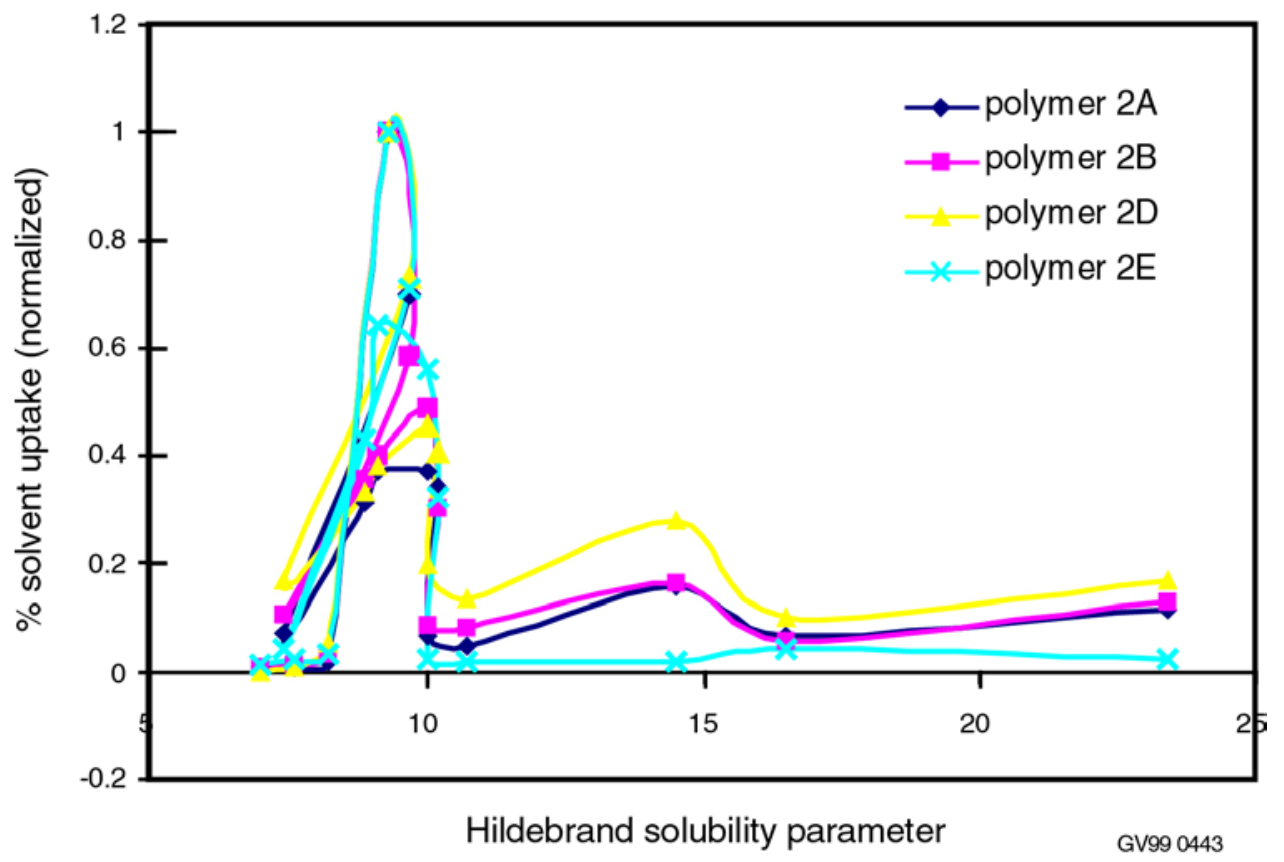

Figure 3. Hildebrand solubility parameter plot for the controlled solubility polymer series.

behavior of a polymer. These expanded parameters are termed $\delta_{\mathrm{d}}, \delta_{\mathrm{h}}$, and $\delta_{\mathrm{p}}$ where $\delta_{\mathrm{d}}$ is the dispersive term that accounts for all of the weaker intermolecular forces such as London forces and Van der Waals. $\delta_{\mathrm{h}}$ describes the hydrogen bonding component, generally a strong force and $\delta_{\mathrm{p}}$ describes the polar component which is attributable to molecular dipoles.

Lightly crosslinked portions of each polymer were immersed in a variety of solvents for several weeks to ensure an equilibrium swelling condition. The masses of the polymer portions were measured before and after immersion and the difference was expressed as a percent. From this data, a judgement as to the "goodness" of each solvent was made. The expanded Hanson parameters for the solvents used in this study are shown in Table 7. Additionally, for each polymer, the good, weak, and non-solvents are indicated. Polymer M2A shows the clearest behavior with regions of good solvent and non-solvent behavior only. Addition of MEE into the polymer matrix yields three different solubility behaviors, good, weak, and non-solvent behavior. Solvents that give weak solvent-polymer interactions are highlighted in Table 7. It should be noted that water is included in this matrix. Initially, it was expected that the addition of MEE to the polymer matrix would fundamentally change their solubility behaviors and that solvents that solubilize, for example, M2A, would not swell M2E, and vice versa. The data actually suggest that solubility in certain solvents like those highlighted, increases with the addition of MEE. However, the strongest solubility behavior remains with solvents such as toluene and methylene chloride. The enhancement for water, the goal of the project, appears to be minor.

The above result was unexpected. As an attempt to justify the observation that the addition of MEE to the polymer matrix represents a slight deviation in solubility behavior, group contribution theory ${ }^{11}$ was explored. Group contribution theory states that the solubility of any molecule is a product of each of the contributions of the atoms within a molecule. Using group contributions, the Hanson solubility parameters for polymeric units may be predicted. Calculated values for monomers representing the two major constituents of the controlled solubility polymer series are presented in Table 8 . The most significant feature to these calculations is the similarity between all three parameters for the two polymers. Also included in Table 8 are the Hanson parameters for water. In comparing these three data sets, the hydrogen bonding term difference between the two polymeric species and water is most glaring. 
Table 7. Hanson solubility parameters for solvents and degree of solvent behavior for controlled solubility polymers ( $\mathrm{Y}=$ good solvent, $\mathrm{W}=$ weak solvent, $\mathrm{N}=$ nonsolvent).

\begin{tabular}{|c|c|c|c|c|c|c|c|}
\hline Solvent & $\delta_{\mathrm{d}}$ & $\delta_{\mathrm{p}}$ & $\delta_{\mathrm{h}}$ & $\mathrm{M} 2 \mathrm{~A}$ & M2C & M2D & M2E \\
\hline Pentane & 14.5 & 0 & 0 & $\mathrm{~N}$ & $\mathrm{~N}$ & $\mathrm{~N}$ & $\mathrm{~N}$ \\
\hline Octane & 15.5 & 0 & 0 & $\mathrm{~N}$ & $\mathrm{~N}$ & $\mathrm{~N}$ & $\mathrm{~N}$ \\
\hline Cyclohexane & 16.8 & 0 & 0.2 & $\mathrm{~N}$ & $\mathrm{~N}$ & $\mathrm{~N}$ & $\mathrm{~N}$ \\
\hline Diethyl ether & 14.5 & 2.9 & 5.1 & $\mathrm{~N}$ & $\mathbf{W}$ & $\mathbf{W}$ & $\mathbf{W}$ \\
\hline 2-Propanol & 15.8 & 6.1 & 16.4 & $\mathrm{~N}$ & $\mathbf{W}$ & $\mathbf{W}$ & $\mathbf{W}$ \\
\hline Ethylene glycol & 17 & 11 & 26 & $\mathrm{~N}$ & $\mathbf{W}$ & $\mathbf{W}$ & $\mathbf{N}$ \\
\hline Methanol & 15.1 & 12.3 & 22.3 & $\mathrm{~N}$ & $\mathbf{Y}$ & $\mathbf{Y}$ & $\mathbf{W}$ \\
\hline Tetraethylene glycol & 16 & 11 & 17 & $\mathrm{~N}$ & $\mathbf{W}$ & $\mathbf{W}$ & $\mathbf{N}$ \\
\hline Water & 12 & 31 & 34 & $\mathrm{~N}$ & $\mathbf{W}$ & $\mathbf{W}$ & $\mathbf{W}$ \\
\hline Chloroform & 17.8 & 3.1 & 5.7 & $\mathrm{Y}$ & $\mathrm{Y}$ & $\mathrm{Y}$ & $\mathrm{Y}$ \\
\hline Methylene chloride & 18.2 & 6.3 & 6.1 & $\mathrm{Y}$ & $\mathrm{Y}$ & $\mathrm{Y}$ & $\mathrm{Y}$ \\
\hline Toluene & 18 & 4.1 & 2 & $\mathrm{Y}$ & Y & $\mathrm{Y}$ & $\mathrm{Y}$ \\
\hline THF & 16.8 & 5.7 & 8 & $\mathrm{Y}$ & $\mathrm{Y}$ & $\mathrm{Y}$ & $\mathrm{Y}$ \\
\hline 1,4-Dioxane & 19 & 1.8 & 7.4 & $\mathrm{Y}$ & $\mathrm{Y}$ & $\mathrm{Y}$ & $\mathrm{Y}$ \\
\hline $\begin{array}{l}\text { Diethylene glycol } \\
\text { monomethyl-ether }\end{array}$ & 16.2 & 9.2 & 12.3 & Y & Y & Y & Y \\
\hline
\end{tabular}

Table 8. Calculated Hanson solubility parameters from group contributions.

\begin{tabular}{|c|c|c|c|}
\hline Parameters calculated for: & $\delta_{\mathrm{d}}$ & $\delta_{\mathrm{p}}$ & $\delta_{\mathrm{h}}$ \\
\hline $\mathrm{PN}(\mathrm{MEE})_{2}$ & 17 & 5.5 & 8.3 \\
\hline $\mathrm{PN}(\mathrm{MEOAr})_{2}$ & 19.3 & 5.5 & 7.2 \\
\hline Water & 12 & 31 & 34 \\
\hline
\end{tabular}

This observation suggests that the use of MEE as the hydrophilic component does not match well with the solubility characteristics of water, the desired permeate.

\section{Liquid Pervaporation}

Pervaporation experiments were conducted on thermally initiated crosslinked polymer thin films formed from the controlled solubility polymer series. These polymers were designed to embody clear solubility characteristics without regard to their dimensional stability and this is evidenced by the data in Table 9. Polymers M2A and M2D are excellent film formers and have given good pervaporation data. Membranes cast from Polymer M2B exhibited extremely high fluxes however this data appears to be too high. More work needs to be done to properly characterize this polymer. Polymer M2C exhibited an extreme amount of fluid-like creep behavior that made pervaporation membrane formation impossible. Polymer M2E also is a good film-former; however in the course of five membranes, all have failed 
Table 9. Pervaporation of water from a water-dye feed at $50^{\circ} \mathrm{C}$.

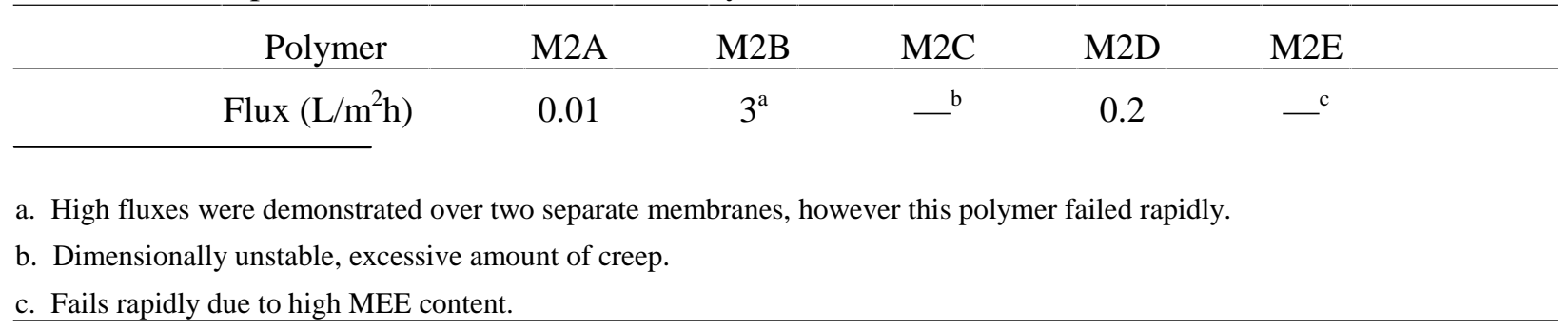

suggesting that this polymer, with a high level of 2-(2-methoxyethoxy)ethanol as a pendant group, exhibit poor physical stability which was not unexpected.

Polymer M2D was tested for an isopropanol-water separation. This experiment consisted of a feed of 50\%/50\% (v/v) water and isopropanol. Fluxes were an order of magnitude higher than comparable experiments with water alone and the separation favored isopropanol to a degree, see Figure 4. All experiments were conducted at $50^{\circ} \mathrm{C}$ where the separation factor favored isopropanol increased as the concentration of isopropanol decreased. Furthermore, the fluxes of permeate through the membrane also declined, however, these fluxes are generally very high compared to other pervaporation processes. Generally, the separation factors are low and this would be suggested from the solubility parameter experiments, which shows that both water and isopropanol are weak solvents for polymer M2D.

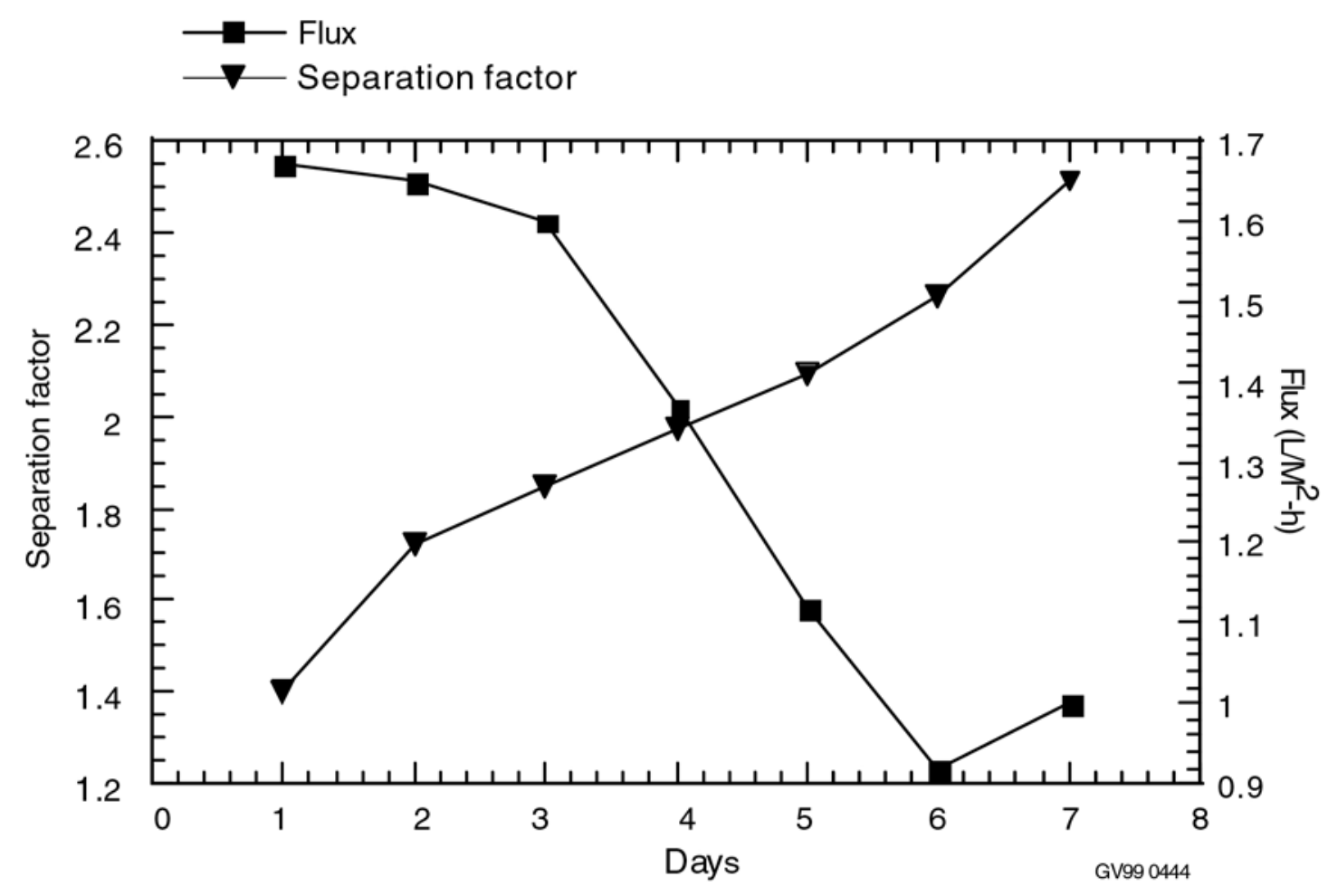

Figure 4. Flux and separation factor measurements for a pervaporation of isopropanol from water using a membrane formed from Polymer M2D. 


\section{Prediction of Polymer Properties and Membrane Efficacy}

The theoretical work conducted at New Mexico Tech., which was related to INEEL's Phosphazene research effort, consisted of three facets. First, the solubility of gases in rubbery polymers was continued by Joanne Budzien. Both traditional Flory-Huggins theory and more modern PRISM integral equation theory were pursued with both yielding reasonable results.

The PRISM work suggested a Flory-Huggins-like form ${ }^{12}$ that we hope will be more accurate than Flory-Huggins theory and simpler in application than PRISM theory. Under a new student, Morgan Pileggi, we have been comparing this approach to experimental measurements; however, this has been difficult due to differences in experimental technique and in sample preparation (degree of crystallinity, etc.). Recently, we have focused upon comparisons with simulation for the initial analysis of the theory. We plan to revisit the experimental results at a later date.

\section{ACCOMPLISHMENTS}

\section{Material Synthesis}

- $\quad$ Synthesized five polymers with varying solubility characteristics with the goal of matching these solubility characteristics to selected permeates.

- $\quad$ Synthesized three new phosphazene cyclomatrix precursors. A manuscript describing this work is in preparation.

- Crosslinked linear polymers and cyclomatrix polymers to gain durable materials that can remain stable in harsh chemical environments. Materials were studied using thermal free radical processing and electron beam processing.

\section{Materials Processing and Testing}

- $\quad$ Formed thermally crosslinked membranes for testing in our laboratories. These materials were defect-free films supported by a microporous ceramic.

\section{Characterization of Polymeric Membranes}

- Structurally characterized the five new polymers using Nuclear Magnetic Resonance spectrometry, thermal methods (DSC, TGA), and by dilute solution characterization (GPC, LLS).

- Determined the permeability of helium, hydrogen, nitrogen, oxygen, methane, and carbon dioxide through the five new polymers. The nitrogen and oxygen experiments allow for the comparison of the polymers to others reported in the literature, oxygen and nitrogen are benchmark tests. A manuscript describing this work is in preparation.

- Determined Hanson solubility parameters for the new polymer series that will allow for the prediction of membrane transport.

- $\quad$ Performed characteristic water/dye separations using pervaporation and the five new polymers. Several failed to give satisfactory data due to poor mechanical stability. 
- $\quad$ On one of the polymers with better mechanical stability, an isopropanol-water separation was performed with good results. Fluxes (mass of transported material) were very high with a good separation factor favoring isopropanol.

\section{Prediction of Polymer Properties and Membrane Efficacy}

- Continued study into the application of Flory-Huggins theory to prediction of membrane transport. We have focussed the study onto the amorphous polymers synthesized for this work. A manuscript describing this work is in preparation.

\section{REFERENCES}

1. G. S. Park, In Synthetic Membranes: Science and Engineering Applications; P. M. Bungay, H. K. Lonsdale, M. N. DePinho, Eds., NATO A.S.I. Series, Dordrecht: D. Reidel Publishing, 1983, pp. 57-108.

2. J. Stastna, D. DeKee, Transport Properties in Polymers, Lancaster, PA: Technomic Publishing Co., 1995.

3. H. R. Allcock, "Recent Advances in Phosphazene Chemistry," Chemistry Reviews, Vol. 72, No. 4, 1972, p. 305.

4. J. E. Mark, H. R. Allcock, R. West, Inorganic Polymers; Englewood Cliffs, NJ: Prentice-Hall, 1992, pp. 61-140.

5. H. R. Allcock, P. E. Austin, T. X. Neenan, J. T. Sisko, P. M. Blonsky and D. F. Shriver, "Polyphosphazenes with Etheric Side Groups: Prospective Biomedical and Solid Electrolyte Polymers," Macromolecules, Vol 19, 1986, pp. 1,508-1,512.

6. R. E. Singler, G. L. Hagnauer, N. S. Schneider, B. R. Lalierte, R. E. Sacher, R. W. Matton, "Synthesis and Characterization of Polyaryloxyphosphazenes," J. Poly. Sci.: Polymer Chem. Ed. , Vol.12, 1974, pp. 433-444.

7. F. F. Stewart, E. S. Peterson, M. L. Stone, and R. E. Singler, "Synthesis and Characterization of Polyphosphazene Copolymers Using Phosphorus-31 NMR Spectroscopy," Amer. Chem. Soc., Polymer Preprints Vol. 38, No. 1, 1997, pp. 836-837.

8. F. F. Stewart and M. K. Harrup, "Phosphazene Monomers from the Regiospecific Reaction of tert-Butylhydroquinone with Hexachlorocyclotriphosphazene: A New Composite Material Precursor," J. Appl. Poly. Sci., 1999 72(8), p. 1,085.

9. F. F. Stewart, R. E. Singler, M. K. Harrup, E. S. Peterson, and R. P. Lash, "Electron Beam Crosslinking of Fluoroalkoxy, Methoxyethoxyethoxy and Substituted Phenoxy Polyphosphazenes: Physical and Chemical Characterization and Comparison to a Thermally Induced Free Radical Process and Ionic Complexation," J. Appl. Poly. Sci., In Press.

10. L. M. Robeson, "Correlation of Separation Factor versus Permeability for Polymeric Membranes," J. Mem. Sci., Vol. 62, 1991, pp. 165-185.

11. A. F. M. Barton, CRC Handbook of Solubility Parameters and Other Cohesion Parameters; Boca Raton, FL: CRC Press, 1983. 
12. Joanne Budzien, M.S. Thesis, New Mexico Tech., December 1998; "Prediction of the Solubility of Simple Gases in Rubbery Polymers."

\section{APPENDIX}

\section{Peer Reviewed Publications}

1. F. F. Stewart and M. K. Harrup, "Phosphazene Monomers from the Regiospecific Reaction of tert-Butylhydroquinone with Hexachlorocyclotriphosphazene: A New Composite Material Precursor," J. Appl. Poly. Sci., 1999 72(8), p. 1,085.

2. F. F. Stewart, R. E. Singler, M. K. Harrup, E. S. Peterson, and R. P. Lash, "Electron Beam Crosslinking of Fluoroalkoxy, Methoxyethoxyethoxy and Substituted Phenoxy Polyphosphazenes: Physical and Chemical Characterization and Comparison to a Thermally Induced Free Radical Process and Ionic Complexation," J. Appl. Poly. Sci., In Press.

3. F. F. Stewart, M. K. Harrup, R. P. Lash, and M. N. Tsang, "Synthesis, Characterization and Thermal Stability of Phosphazene Terpolymers with 2-(2-Methoxyethoxy)ethoxy and Diacetone D-Glucofuranosyl Pendant Groups," Polymer International, In Press.

4. F. F. Stewart, M. K. Harrup, T. A. Luther, C. J. Orme, and R. P. Lash, "Formation of Pervaporation Membranes from Polyphosphazenes Having Hydrophilic and Hydrophobic Pendant Groups: Synthesis and Characterization," Submitted to Chemistry of Materials.

\section{Presentations}

5. F. F. Stewart, E. S. Peterson, M. L. Stone, M. K. Harrup, L. A. Polson, and C. J. Orme, "Membrane Separations Using New Polymers,” Filtration ‘98, Atlantic City, NJ, December 1998.

6. J. L. Budzien, J. D. McCoy, J. G. Curro, R. A. Laviolette, "A Simple Relation for the Solubility of Gases in Polymers," poster at the American Physical Society Conference, Atlanta, GA, March, 1999.

7. T. A. Luther, F. F. Stewart, and M. K. Harrup, "Preparation of Hexa(Methyl Hydroquinone) Cyclotriphosphazene and Substitution Reactions with Alkyl Halides," American Chemical Society Northwest Regional Meeting, Portland, Oregon, June 1999, Paper \# 364.

8. F. F. Stewart, M. K. Harrup, E. S. Peterson, M. L. Stone, C. J. Orme, and L. A. Polson, "Chemically Tailored Phosphazene Polymers for Water Separations Applications: Synthesis, Characterization, and Membrane Formation." Presented to the Macromolecular Secretariat, Symposium on Polymeric Materials in Separations, $218^{\text {th }}$ National Meeting of the American Chemical Society, New Orleans, LA, August 1999, MACR 19. 


\title{
Pore Size and Morphology Control for Solid and Polymer Matrices
}

\author{
Mason K. Harrup and Alan K. Wertsching \\ SUMMARY
}

The separation needs of the Department of Energy's Office of Environmental Management are extremely demanding on separation materials due to the highly aggressive thermal and chemical environments and also due to the presence of high levels of radiation. Typical organic membrane materials employed for this purpose do not maintain structural integrity in these types of environments. In order to address this pressing need, this project has focused upon two aspects of the transport of small molecules through solid polymer and composite materials that exhibit great promise for solving these problems. The first aspect of this project extended the investigation of novel hybrid organic/inorganic materials that offer greatly enhanced durabilities, namely molecular composites. Composites of this kind were first synthesized at the INEEL during FY-98 under this program. During FY-99, factors that control structure property relationships and other pertinent synthetic aspects of these composites were more fully explored. It was found that the nature of the catalyst, be it acid, base, or simple ionic species, employed to form these materials was critical to the final material properties. Through the course of this research, a tremendous amount of understanding of how these composites form-bonding schemes and ceramic/polymer interactions-was gained as well as an enhanced ability to apply this understanding to form composites with predictable, desired properties. Not only do these composites exhibit superior physical properties to their polymer-only counterparts, they have been shown to possess superior radiation stability - greater than a four-fold increase. This greater stability in exactly the types of harsh environments that the DOE is interested in remediating, coupled with our enhanced understanding of the fundamental science behind the formation of these materials, extends the high promise for these materials as a solution to some of the department's toughest separations problems.

The second focus of this project involved the investigation of the effects of polymer morphology upon pure polymer membrane performance. Polymer morphology is a little studied phenomenon that could significantly impact membrane lifetimes and transport rates. A series of new polymers, designed with EM-relevant separations in acidic environments in mind, was synthesized and evaluated to assess the importance of polymer morphology during water transport. It was found that ligand-based morphological differences in polymers that possess similar pendant groups contribute do indeed have a significant impact on the properties and the overall performance and useful lifetime of separations membranes of this type. Further work in this area is needed to fully understand the effects observed and to quantify the magnitude of the advantage gained by this selective stabilization strategy.

\section{PROJECT DESCRIPTION}

\section{Objectives}

One of the biggest challenges in making membrane separations technologies viable for EM applications is increasing the rate of permeation through the membrane while maintaining high selectivity. This must be achieved with a durable material, capable of withstanding harsh environments, without making the material prohibitively expensive. Membrane materials that allow for a high transmission rate generally swell to a great degree and lose their physical integrity leading to rapid failure of the membrane. To combat this problem, all manners of crosslinking strategies have been explored in the open and patent literature. While this type of strategy does increase membrane durability, it has met 
with limited success for several reasons. First, crosslinked polymers generally have lower transmission rates than their uncrosslinked counterparts. Second, inhomogeneity in crosslink density results in batchto-batch variability and does not completely alleviate the problem of early membrane failure.

One recent development that offers promise to address these problems is the formation of molecular composites. ${ }^{1,2}$ These composites combine polymers with ceramic components in a novel manner, retaining the beneficial aspects of both. The resulting material is more physically and chemically robust than the polymer alone, yet the material loses none of the desired qualities of the parent polymer. Recent research in the area of molecular composites has yielded new composite materials that exhibit promise for a variety of EM applications.

In this work, we seek a better fundamental understanding of the mechanisms of transport into and through polymer and composite matrices. This enhanced understanding will facilitate rational design improvements upon existing materials used for challenging environmental separations. The scope of this project has two main focuses:

1. Molecular composites for enhanced selective mass transport:
a. Controlled pore size materials
b. Enhanced selective permeation
c. Better durability/longer useful lifetimes.

2. Morphology effects on selective mass transport and durability
a. Ligand-based morphology
b. Backbone-based morphology effects.

\section{Strategy}

The strategy is to examine the areas delineated above in detail and draw conclusions about molecular interactions/transport from the combined results of these efforts. Each of the parts of this work will be executed separately initially, with crossovers anticipated to occur as data is collected and the work progresses. Four tasks in the two main areas are outlined below.

\section{Molecular Composites}

The expertise that exists here in the separations group at the INEEL will be leveraged to provide both the synthetic background and the testing capabilities necessary to execute this project. By investigating these composite materials at the molecular level, a greater understanding of the specific interactions between the polymer component and the condensed ceramic component will be realized. This knowledge will hopefully lead to two improvements over current all-polymer or all-ceramic membranes. First, direct condensation of the ceramic component in the presence of organic small molecules $^{3}$ or a micellular matrix ${ }^{4}$ is known to render at least some control over the resulting pore size of the final ceramic material. By condensing the ceramic within a polymer matrix, enhanced control over not only pore size but pore shape and distribution may be realized. Second, having a rigid ceramic structure within the polymer matrix will control the swelling of the polymer in the presence of a permeating solvent. This should result in enhanced chemical selectivity of membranes made from these materials by preventing large solvent filled regions within the matrix. 
In the first task, well characterized polyphosphazenes known to possess very high water transmission rates, but very short lifetimes before failure will be synthesized. These will be fully characterized and their mechanical and solubility/solvent swelling properties examined.

Molecular composites will subsequently be formed with these same polymers and the exact formulation varied to produce many related new materials. These new materials will be fully characterized using the new NMR solids imaging capability, an analytical technique closely related to MRI commonly used by health professionals. This new instrumentation was acquired during FY 1998, and significantly adds to the core capabilities of this lab. Additional studies will be performed using thermal analysis methods, using DSC, TGA, and DMA instrumentation.

After this rigorous characterization, these composite materials will then be evaluated using the same test protocols as used for the parent polymers. The results will then be compared to the parent systems to determine the magnitude of the advantage gained with these materials. It is anticipated that these materials, possessing the added durability imparted by the ceramic component, will form more rugged membranes with much increased mechanical properties that will translate into greater useful lifetimes. Further development of this concept, and its application to a variety of EM-relevant separation challenges is anticipated based upon positive initial results.

In the second task, the bonding between the ceramic and polymer component of these composites will be investigated. There are two possible ways in which the components of molecular composites can interact - via direct covalent bonding or by the formation of an interpenetrating network (IPN). A nonpolymeric model compound that simulates the polymers being used in the first task will be synthesized to aid in the elucidation of the extent and exact nature of this bonding. This model will be condensed with ceramic precursors and studied by solid state NMR and elemental analysis techniques. Solvent swelling and washing studies will be performed to attempt to gain direct quantitative evidence for the presence of covalent linkages between the ceramic superstructure and the model compound.

\section{Polymer Morphology}

Batch-to-batch reproducibility is a pressing problem in working with linear polyphosphazenes as separation materials. Since the substitution of organic ligands for chlorine on the polymer skeleton is readily controllable and highly reproducible, it must be some other factor-polymer morphology-that accounts for the differences in the observed physical and chemical properties. There are two major sites for morphological variation within polyphosphazene membranes-backbone branching sites and ligand crosslinking sites. Each could have a profound effect upon macroscopic transport properties through the polymeric matrix, or the effect could be minimal due to averaging of sites throughout a practical separations membrane that is extremely thick compared to the molecular scale.

In the third task, polymer backbone morphology will be investigated to determine the effects upon macro-scale transport through the polymer matrix. Until now, little attention has been given to the morphology of the polymer skeleton in polyphosphazenes. Materials made via the Allcock method ${ }^{5}$ are assumed to be completely linear. This however seems unlikely in the strictest sense, since it is known that under polymerization conditions $\left(250^{\circ} \mathrm{C}\right)$ the linear dichloropolymer readily undergoes thermal crosslinking, eventually becoming completely insoluble inorganic rubber. ${ }^{6}$

Thermal crosslinking or branching of the polymer increases in frequency as the reaction progresses and the supply of starting trimer is consumed. ${ }^{7}$ It is reasonable therefore to assume that at early reaction times (5-15\% trimer consumed) the polymer strands are the closest to truly linear structures, while having a high enough molecular number to be true high polymers. Terminating the polymerization reaction at this stage and separating the high polymer material from the unreacted trimers and low molecular weight 
oligiomers will afford a benchmark material that is as close to a purely linear material as is practically achievable. Alternatively, a new process for generating the parent polymer $\left(\mathrm{NPCl}_{2}\right)_{\mathrm{n}}$, recently developed with the aid of the INEEL, could be employed to render the linear benchmark materials. ${ }^{8}$ A complete analysis of this material by NMR, viscometry, laser light scattering, and GPC will render benchmark values for a polyphosphazene that possesses a minimum of branchpoints.

This linear benchmark material can then be thermally crosslinked in a vacuum/Argon oven to various degrees, either lightly to produce a branched material or heavily to produce a hyperbranched material. Utilizing this strategy, backbone branching occurs during a stage in the synthesis when all of the ligands are identical, namely chlorine. It is reasonable to assume that branching will occur in a very homogeneous manner as there are no participating electronic, steric, or associative effects to differentiate one segment of the polymer strand from another. This should offer a significant improvement over more typically employed ligand-based crosslinking strategies that suffer from uneven crosslink densities, the need for extra process steps, and other problems. NMR, viscometry, laser light scattering, and GPC will test these materials and their values will be tabulated along with those of the linear benchmark. This series of increasingly branched polymers forms a database of materials, which can be thought of as "calibration standards" for degree of polymer morphology. Additionally, materials made normally (via the complete Allcock method) will be subjected to the same battery of tests and the data compared to that obtained from the calibrated materials to assess the degree of branching inherent in typical polymers.

A sample representing the full spread of the parent polymer materials produced above will then be substituted with the appropriate ligands to produce well studied materials for dewatering applications. These polymers (aryloxy-substituted) are known to undergo scission along the phosphazene backbone under the same conditions that induce branching in the parent polymer. Using this methodology, various distinctive backbone morphologies can be generated. This also affords a viable synthetic route to star polymers, which are receiving considerable attention ${ }^{9}$ at this time but are so far unknown for polyphosphazenes.

With this strategy, the more highly branched polymer samples can be reduced in molecular weight to achieve two goals. The first is to render the materials more tractable to testing and membrane formation. The second is to produce materials of identical composition and similar molecular number that differ only in backbone morphology. The full battery of analytical testing delineated above will be performed to adequately characterize these materials. Membranes will then be cast from these materials and permeation tests (liquids and/or gasses) performed to evaluate the difference in performance observed. These differences can then be appropriately correlated to backbone morphology to give an enhanced understanding of the fundamental manner in which backbone morphology affects membrane performance.

In the fourth task, the effect of ligand-based morphological differences will be investigated. The alternate strategy to backbone-based crosslinking is pendant group-based crosslinking. Typically, hydrophobic crosslining groups are employed to perform the crosslinking. This may lead to preferentially high crosslink density in hydrophobic polymer regions and concomitantly low crosslink density in hydrophilic polymer regions. This inhomogeneity in crosslink density may in turn lead to shorter useful membrane lifetimes. Pendant groups identical to those employed in Task 3 will be used to form waterpassing polymers, with the sole difference being that a hydrophilic group, such as 2-ethoxy allyl ether, will be employed as the active crosslinking group. Several polymers will be synthesized that emulate

a. Similar ligands tend to associate in the reaction solution during the substitution process. This leads to heterogeneous substitution patterns among individual polymer strands. 
ones prepared previously ${ }^{10}$ using hydrophobic crosslinking groups. Membranes will then be cast from these new materials and permeation tests (liquids and/or gasses) performed to evaluate any differences observed between the performance of these materials and the performance of the hydrophobically crosslinked materials.

The theoretical basis behind this approach relies upon the selective stabilization of the polymer matrix where such additional stability is most warranted. It is well known that crosslinking the polymers is a necessary process step to ensure the physical stability of the polymer membranes and greatly extend membrane lifetime before failure. In mixed substituent polymer systems with components of greatly differing solubility properties, as is the case in these systems, regions will exist within the polymer matrix that are more dense in one component than another through preferential association during the evaporative film formation process. Previous efforts have used hydrophobic pendant groups to afford the needed crosslinking sites. However, it is not the hydrophobic component of the polymer matrix that is responsible for water transport, therefore it is logical to assume that additional stabilization in this region is not a fully effective strategy. Instead, crosslinking in the region of the matrix that is the least physically stable and the most heavily involved in the active transport of water, namely the hydrophilic region, could reasonably be expected to have a greater benefit in stabilizing the membrane. Using this more effective selective stabilization strategy, one could be able to incorporate a higher percentage of hydrophilic components in the polymer formulation, resulting in higher rates of water transport and thus, a superior membrane for waste dewatering applications.

The caveat here, though, is as follows: selective membrane stabilization is a molecular scale phenomenon. Dewatering a waste stream using a selective membrane is a macroscopic scale application. A practical membrane is tens to hundreds of microns thick in order to be able to withstand the actual physical forces present in such a membrane system. This scale is very large compared to a single polymer strand or individual crosslinking sites. An averaging effect throughout the thickness of the membrane may prevent molecular scale fine-tuning, such as this selective stabilization approach, from having a significant impact on the observed performance of dewatering membranes.

\section{RESULTS AND DISCUSSION}

\section{Molecular Composites}

\section{Bonding in Molecular Composites}

One of the primary goals of research in this area was to gain a better understanding of the bonding between the polymer component and the ceramic component of these molecular composites. This interaction is very difficult to probe using high molecular weight polymeric species. Therefore, model compounds had to be synthesized that accurately simulated the pendant groups on the benchmark methoxyethoxyethoxyphosphazene (MEEP) polymer yet possessed a discrete, lower molecular weight. The compound that was chosen was HexaMEEP-T, a trimeric phosphazene that incorporates the same pendant groups as MEEP (see Figure 1). A series of molecular composites was formed with this model compound and characterized using solid-state NMR and elemental analysis to provide evidence that the nonceramic component was indeed within the composite matrix. The material was subsequently shattered into small pieces and subjected to a repeated washing procedure designed to remove all of the trimer material that was not covalently bound to the insoluble ceramic superstructure. The resulting ceramic material was subjected to the same analytical protocols as before and no directly observable traces of the model compound were detected. While this indicates that the amount of covalent bonding in materials such as this is low (detection limits for NMR are $\sim 1 \%$ ), it does not completely rule out the presence of such linkages. It does however, show that the dominant mode of interaction within these composites is an interpenetrating network (IPN). The nature of this IPN remains under investigation. 
A

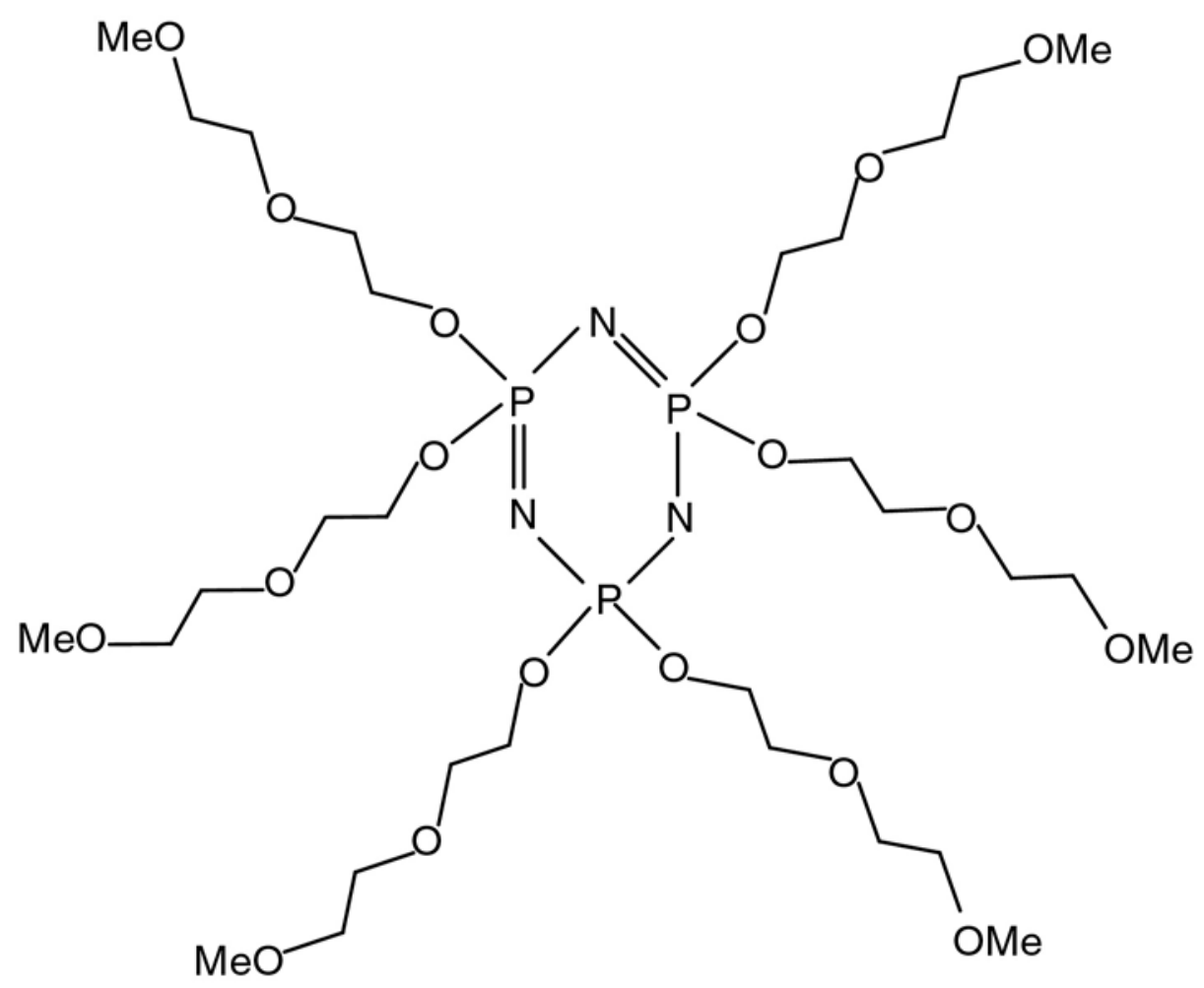

B<smiles>COCCOCCO[PH](N)(N)OCCOCCOC</smiles>

Figure 1. Structures of the nonceramic materials used in all molecular composite studies.

(a) Hexa-MEEP-T model compound used for the bonding studies, (b) a representation of one mer of the MEEP polymer used to formulate the polymeric molecular composites. 


\section{Catalysis of Silicate Networks}

In the ceramics community, use of acid, base or ionic neutral conditions to catalyze formation of silicate networks aging of solutions and sintering temperatures are known to produce radically different glass and ceramic morphologies. ${ }^{11}$ Implementation of these approaches has been attempted for some organic polymer-based hybrid nanocomposites ${ }^{12}$ but not for polyphosphazene silicate composites. The desire to create unique and novel network morphologies became the impetus for establishing new catalysis protocols. The surprising inability to reproduce a well established benchmark composite material from the literature, ${ }^{13}$ was one principal driver that initiated this in-depth investigation into the roles that the nature and amount of catalysts play in the production and physical properties of these composites.

It quickly became apparent, in the course of experiments, that use of lower critical solubility temperature (LCST) purified MEEP, ${ }^{14}$ instead of dialysis purified MEEP, prevented our attempts to successfully reproduce the mechanical properties reported for MEEP composites using the Ferrar method (for elaboration, see below). ${ }^{13}$ Only after salt was incorporated as a co-catalyst in the MEEP (obtained without utilizing rigorous LCST purification), did the Ferrar method produce tractable composites in our hands. This result proved that in order for dilute acid (as $\mathrm{HCl}$ ) to catalyze the silicate formation an ionic salt co-catalyst was necessary. The opportunity to explore salt-only catalyzed composites became possible with the availability of rigorously pure MEEP. In addition, the ability to synthesize a series of acid or base catalyzed composites both with and without salt interference has provided us with the ability to decouple mixed catalyst effects on the properties of the composites.

Multiple series of composites were produced using a variety of reaction conditions in an attempt to create and optimize desirable physical properties. The synthetic variables initially identified as critical and subsequently explored include the type of the catalyst (acid, base, or ionic salt), concentration of catalyst, mixed catalyst schemes, and the effects of aging of solutions before casting. A pictorial representation of how the catalyst affects the nature of the derived ceramic is depicted in Figure 2. This view of the ceramic morphology is derived from the sol-gel pure ceramics literature, but evidence gathered in the course of our experiments shows that this generalized view is valid in our systems as well. A typical synthesis begins by dissolving a 40/60 ratio of MEEP/TEOS with THF/ethanol mixed solvent in a capped vial. The catalyst is introduced as an aqueous solution and the mixture is then sonicated. The solution can then be aged or immediately poured into a Teflon mould and covered. The composite selfassembles as volatile solvent slowly escapes and a composite film is created.

Mechanical Analysis. Each molecular composite was cast as a thin film and was cut to specific dimensions before analysis with TA Instruments Dynamic Mechanical Analyzer (DMA), equipped with a thin-film clamp accessory. The DMA can place specific load forces on composite films in order to obtain relevant mechanical data. The stress-strain (force ramp) experiment was the most common analytical experiment performed on these composite films. Ramped force experiments involve stretching a film sample under increasing force until failure. The resulting plot (Figure 3a) can reveal important mechanical characteristics of the composite. For example, the linear region as annotated is the force range in which a material can be deformed in a fully reversible manner, that is the material retains full memory. It becomes critical to remain within the linear range when removing films from casting moulds to obtain an unstressed, undeformed membrane. This can be particularly difficult when films exhibit a high degree of adhesiveness. The linear region also provides force limits for use in more extensive modulus experiments.

Compression tests involve placing films on a flat platform while a peg of known area is forced down through the sample. These compression or penetration tests (Figure $3 b$ ) provide valuable 

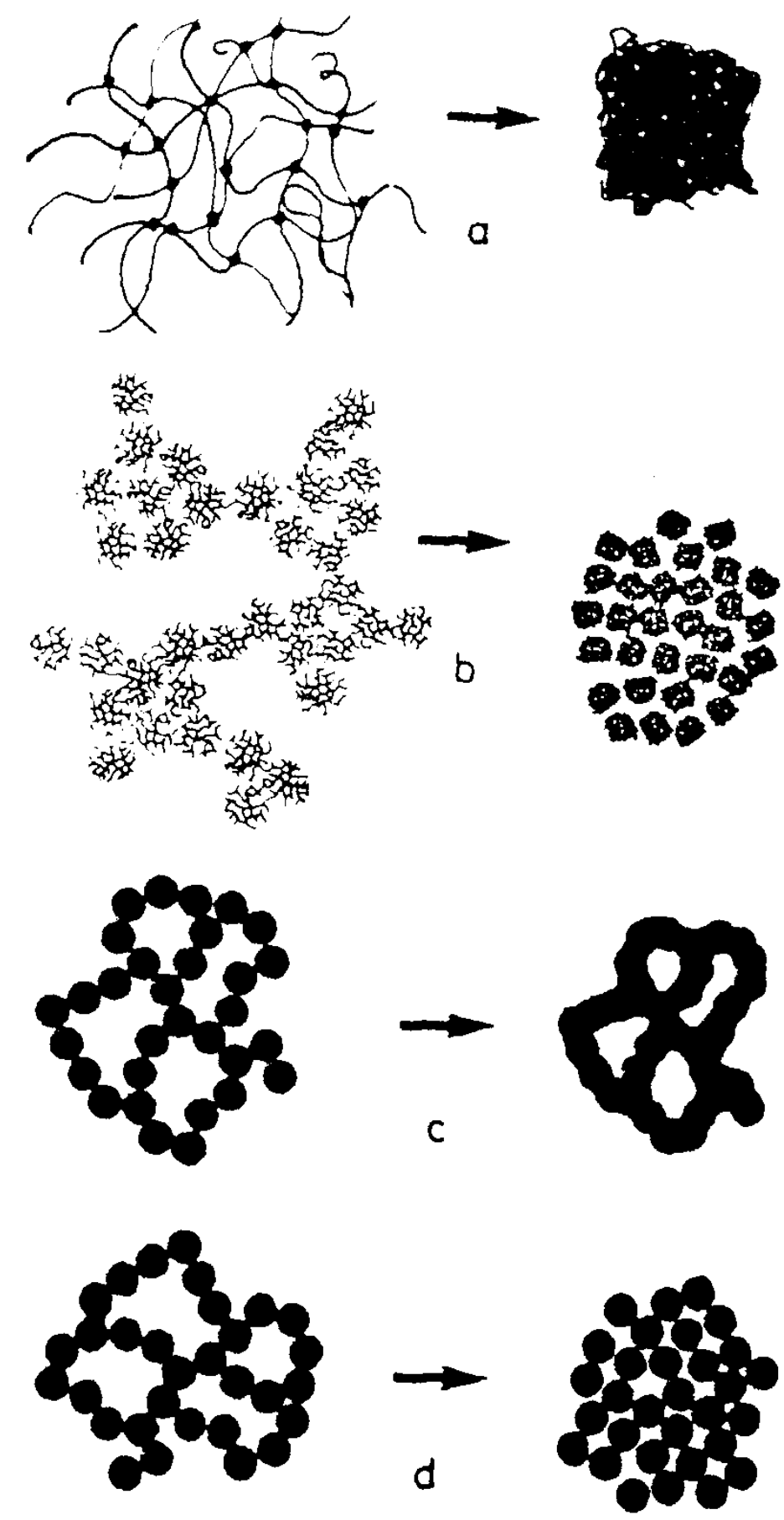

Figure 2. Schematic representation of ceramic gel formation and curing for: (a) acid-catalyzed gels, (b) base-catalyzed gels, (c) colloidal gels aged under high silica solubility conditions, (d) gels formed from existing weakly interacting particles. 

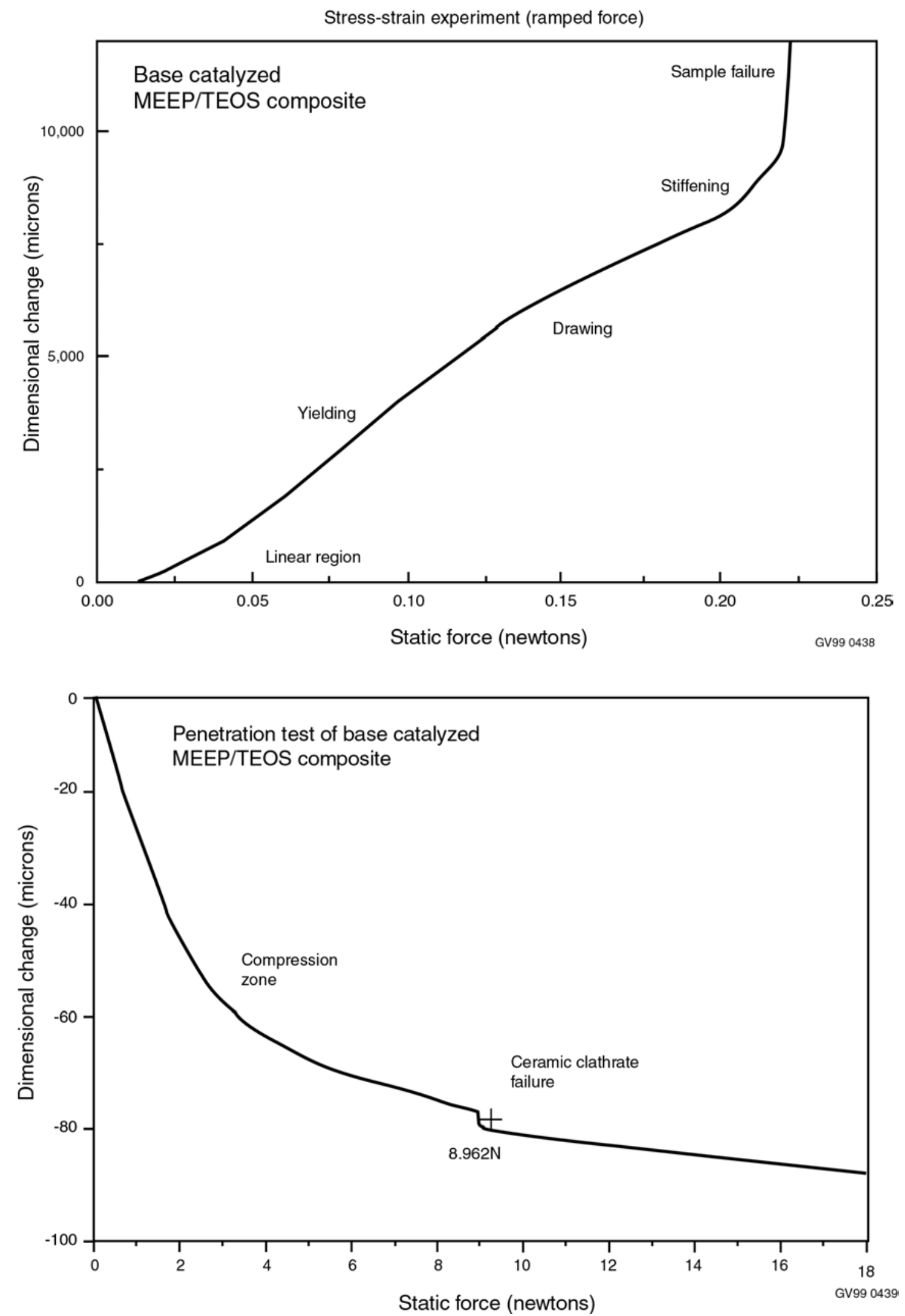

Figure 3. Typical data obtained from DMA experiments with a base catalyzed molecular composites. (a) force ramp test, (b) penetration test. Important features are labeled on the graphs. 
information concerning the silicate substrate within the composite. The point at which the silicate clathrate fails, as annotated, not only provides the force limit under compression but the minimum of composite thickness before failure while under compression. These compression values are particularly useful for battery applications for which these composite materials are already being developed.

Additional tests were performed to assess not just the mechanical properties of these composites themselves, but how they change upon contact with water. Swell testing of composite films is useful in evaluating their applicability as membrane films for water passing applications. How well a membrane swells in solution relates directly to solvent flux. In MEEP/silicate composites, the MEEP component is fully soluble in aqueous solutions while the silicate component is not. How the silicate matrix forms is critical for composite stability in solutions (refer to Figure 2). Using various catalysts and other factors in the silicate formation can stabilize these composite membranes against loss of MEEP or failure of the composite while in contact with aqueous feed streams. Swell deswell tests in conjunction with DMA analysis can determine whether or not hydrostatic pressure exceeds the linear force region of the composite and from this, the useful lifetime of the composite in actual water passing usage can be estimated.

Since multiple catalysts had been identified as effective in forming these composites, and differences in the nature and amount of the catalyst known to be critical to the final material properties, a discussion of the results from several representative composite series is warranted.

Salts such as lithium fluoride had not previously been used as catalysts in the formation of silicate polyphosphazene composites. Data concerning catalyst LiF loading were tabulated in Table 1 . One notable trend in Table 1 is increasing composite strength with increasing concentration of LiF catalyst. Another is the relatively large displacement, above $6 \mathrm{~mm}$, for each LiF composite before failure. Large displacements are good indicators of improved swelling characteristics for these types of composites. All the LiF composites swell exceedingly well under aqueous conditions, unfortunately this occurs with the loss of some MEEP from the ceramic superstructure.

Although correlation between strength and base concentration in Table 2 is more ambiguous, the general trend is similar to $\mathrm{LiF}$ composites. One advantage the $\mathrm{KOH}$ composites display is the large displacements, above $9 \mathrm{~mm}$, in ramp force experiments. As expected, $\mathrm{KOH}$ composites have excellent swell/deswell characteristics. Another advantage is that MEEP does not appear to leak out of the silica matrix upon repeated swelling. This suggests a more intimately woven silicate polyphosphazene matrix as compared to composites catalyzed by LiF.

Table 1. Lithium fluoride composite stress strain results. Catalyst loading study of MEEP/TEOS 60/40 composites with variable aqueous LiF concentrations, all at $150 \mu \mathrm{L}$ loading level.

\begin{tabular}{cccc}
\hline $\begin{array}{c}\text { LiF Loading } \\
(\mathrm{M})\end{array}$ & $\begin{array}{c}\text { Maximum. Force } \\
(\mathrm{N})\end{array}$ & $\begin{array}{c}\text { Displacement } \\
(\mu \mathrm{m})\end{array}$ & $\begin{array}{c}\text { Thickness } \\
(\mu \mathrm{m})\end{array}$ \\
\hline 0.016 & 0.0583 & $6,248.9$ & 90 \\
0.033 & 0.0961 & 7,513 & 80 \\
0.050 & 0.1457 & $6,962.6$ & 130 \\
0.067 & 0.5764 & $6,685.9$ & 130 \\
0.080 & 0.6523 & 11,683 & 115 \\
\hline
\end{tabular}


Table 2. Potassium hydroxide composite Stress strain results. Catalyst loading study of MEEP/TEOS $60 / 40$ composites with variable $\mathrm{KOH}(\underline{\mathrm{M}})$ solutions, all at $150 \mu \mathrm{L}$ loading level.

\begin{tabular}{llcl}
\hline $\begin{array}{c}\text { KOH Loadings } \\
(\underline{\mathrm{M}})\end{array}$ & $\begin{array}{c}\text { Failure Force } \\
(\mathrm{N})\end{array}$ & $\begin{array}{c}\text { Displacement } \\
(\mu \mathrm{m})\end{array}$ & $\begin{array}{c}\text { Thickness } \\
(\mu \mathrm{m})\end{array}$ \\
\hline 0.16 (intractable) & $\mathrm{NA}$ & $\mathrm{NA}$ & $\mathrm{NA}$ \\
0.33 & 0.3545 & 9,727 & 120 \\
0.50 & 0.5146 & 10,610 & 85 \\
0.67 & 0.4265 & 10,929 & 100 \\
0.80 & 0.8064 & 17,012 & 105 \\
\hline
\end{tabular}

Before aging protocols were specifically investigated, immediate casting of acid-catalyzed composite solutions after mixing was the standard procedure for all MEEP/TEOS composites. Aging of solutions, before casting, was not recognized as a significant factor until after base catalysis $(\mathrm{KOH})$ was investigated. Without aging $\mathrm{KOH}$ solutions, composites could never harden enough to remove from Teflon casting moulds. Aging studies were then deemed relevant and the most surprising result is observed in Table 3. A maximum of one day of solution aging is enough to dramatically improve cast films and any additional time appears to reduce maximum recoverable force on each film. Rapid silanol formation associated with hydroxide catalysts are known to create star-like silicate structure in solution. ${ }^{15}$ Data from Table 3 would indicate formations of discrete structures of specific size are also highly timedependent. Also inferred is that there is an optimal size for these star-like structures that promotes a maximum efficiency for the crosslinking of these structures.

Whereas $\mathrm{LiF}$ and $\mathrm{KOH}$ composites typically form rubbery and highly adhesive materials, $\mathrm{HCl} / \mathrm{NaCl}$ composites are less ductile, more glassy composites. High concentrations of halides in sol-gel cures are known by ceramists to encourage heavy cross-linking, which explains this radical difference in morphologies. Stress-strain curves observed for these materials are very similar to those reported for the Ferrar composites. ${ }^{13}$ This observation leads us to believe that the authors' previous works were unaware of salt contamination in their source of MEEP and their conclusions may also be tainted. Both composites have short displacements before failure and similar maximum force limits (see Table 4). As the small displacements would indicate swelling in aqueous solution is poor and under hydrostatic pressure these composites fracture and fragment. Loss of MEEP from the composite occurs readily as the silicate matrix breaks up. An interest caveat here is that increased cross-linking does not appear to significantly improve maximum force limits, as compared to the other composites.

Table 3. Aging solution study. Aging study of MEEP/TEOS 60/40 solutions (THF/ethanol) before casting with $150 \mu \mathrm{L}$ of $0.33 \underline{\mathrm{M}} \mathrm{KOH}$ catalyst.

\begin{tabular}{clcc}
\hline Solution Storage & \multicolumn{1}{c}{$\begin{array}{c}\text { Maximum Force } \\
(\mathrm{N})\end{array}$} & $\begin{array}{c}\text { Displacement } \\
(\mu \mathrm{m})\end{array}$ & $\begin{array}{c}\text { Thickness } \\
(\mu \mathrm{m})\end{array}$ \\
\hline No storage & NA (intractable) & NA & NA \\
1 day & 0.4054 & 13,659 & 100 \\
3 days & 0.3585 & 12,924 & 100 \\
5 days & 0.2885 & 11,910 & 150 \\
\hline
\end{tabular}


Table 4. Acid with variable salt loading as catalysts. Salt study of MEEP/TEOS 60/40 solutions

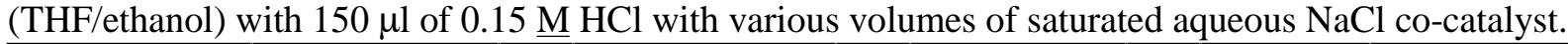

\begin{tabular}{clll}
$\begin{array}{c}\text { NaCl Saturation } \\
(\mu \mathrm{L})\end{array}$ & \multicolumn{1}{c}{$\begin{array}{c}\text { Maximum Force } \\
(\mathrm{N})\end{array}$} & $\begin{array}{c}\text { Displacement } \\
(\mu \mathrm{m})\end{array}$ & $\begin{array}{c}\text { Thickness } \\
(\mu \mathrm{m})\end{array}$ \\
\hline 0.0 & NA (intractable $)$ & NA & NA \\
10 & 0.6285 & 322 & 100 \\
20 & 0.6180 & 985 & 60 \\
30 & 0.9562 & 1,041 & 120 \\
\hline
\end{tabular}

\section{Polymer Morphology}

Polymer morphology plays an important role in any polymer membrane. Studies of phosphazene membranes both at this laboratory and in the open literature have focused upon the nature of the substituent pendant groups attached to the polymer backbone as a means of controlling permeation and selectivity behaviour. Little attention has been given to the potential effects of polymer morphology on such systems. Since there is a wealth of experimental data on the dewatering performance of phosphazene membranes that possess hydrophobic crosslinkable pendant groups, ${ }^{10}$ it was decided to leverage this information to assess the effects of ligand-based polymer morphology. The system chosen as this benchmark was the well studied ternary system that incorporates the hydrophilic pendant group di(ethyleneglycol) methyl ether (DEG) and the hydrophobic pendant group 4-methoxy phenol (MeOP) with 2-allyl phenol as a hydrophobic crosslinkable functionality (see Figure 4). These constituents are incorporated into the polymer formulations at various levels to tune the performance of the polymers. The DEG component is primarily responsible for water transport, while the MeOP component is responsible for dimensional stability and good film-forming characteristics. For this study, the 2-allyl phenol used to stabilize the polymer was to be replaced with another functionality that was capable of effecting polymer crosslinking yet was also hydrophilic so it would preferentially associate with more hydrophilic regions of the polymer matrix. By comparing the performance of these membranes to those that possessed similar DEG/MeOP ratios, the effects of ligand-based polymer morphology could be accurately assessed.

To accomplish these goals, the first necessary task was to discover a water-soluble pendant group that would mimic the crosslinking properties of the benchmark functionality, 2-allyl phenol.

Additionally, this ligand would need to be compatible with the synthesis of polyphosphazenes. The initial group investigated, 2-hydroxyethyl acrylate, was chosen because acrylates were known to be active freeradical crosslinkable moieties, and also because this particular acrylate was commercially available and readily water-soluble. Model substitution reactions were carried out on phosphazene trimer molecules to assess compatibility with typical phosphazene chemistry—namely nucleophilic substitution. Initial reactions were unsuccessful due to the competing, undesired side-reaction of acrylate self-polymerization. A second series of attempts to complete this substitution reaction was made with the addition of Schlenk techniques to exclude atmospheric oxygen, known to catalyze the autopolymerization of acrylates. While this new technique met with limited success, it was evident that polymers that contained this pendant group would not enjoy appreciable long-term stability upon exposure to air. Based on these results, this moiety was abandoned in favor of one that would not undergo free radical self-polymerization as readily and thus represent a much more practical system for study.

The next crosslinkable pendant group chosen, and the one ultimately used for this study, was one that contained the more stable allyl ether group. 2-Hydroxyethyl allyl ether (shown in Figure 4) was first 


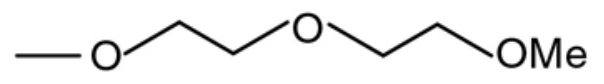

DEG

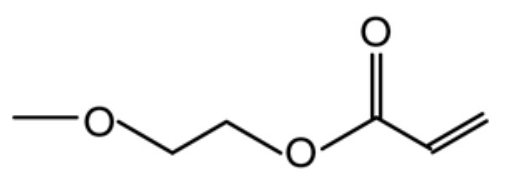

2-Hydroxyethyl acrylate

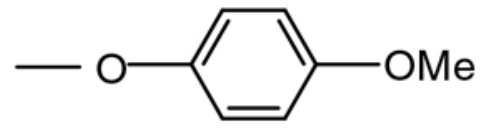

$\mathrm{MeOP}$

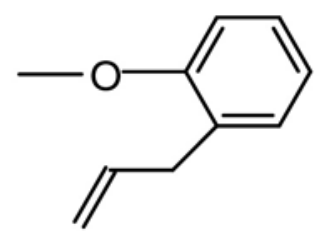

2-Allyl phenol

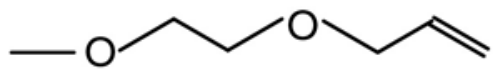

2-Hydroxyethyl ally ether

GV99 0436

Figure 4. Pendant groups used in the ligand-based polymer morphology study.

employed in model reactions to ensure that the synthesis would proceed to completion without problems similar to those encountered with the acrylate pendant group. After these reactions were completed successfully, an entire series of polymers that varied only the DEG to MeOP ratio while having a constant level of the hydrophilic crosslinkable group was designed and slated for synthesis. This series consisted of five polymers (designated WPHX-\#) and after synthesis, these polymers were fully characterized by solution multinuclear NMR, with pendant group composition determined by integratable ${ }^{1} \mathrm{H}$ NMR measurements. A summary of the composition of each of the polymers is delineated in Table 5. Work up of these polymers proved to be somewhat problematic, and several of the polymers crosslinked spontaneously during the purification and drying procedures. However, the five polymers used for the remainder of this study were well behaved and no spontaneous crosslinking was observed.

From the table above, it is easily seen that the hydrophobic component of the polymers was systematically varied from $0 \%$ to $71 \%$ in increments of approximately $25 \%$. This was done to obtain a representative spread of solubility properties, from WPHX-6, which is fully water-soluble, to WPHX-9, which is quite hydrophobic. It is known from previous research with these types of polymers ${ }^{10}$ that below approximately $75 \%$ hydrophobic component, the membranes swell to such a great extent that they tend to fail rapidly in an aqueous separation. Characterization of the behavior of this series of polymers was accomplished by performing swelling studies (with TGA analysis) and pervaporation studies to determine if, through hydrophilic crosslinking, this limit could be extended to include even less hydrophobic component, thus increasing water transport rates. Swelling in water was extensive for each of these 
Table 5. Composition of the constituent polymers of the WPHX series.

\begin{tabular}{cccc}
\hline Polymer & $\begin{array}{c}\text { DEG } \\
(\%)\end{array}$ & $\begin{array}{c}\text { MeOP } \\
(\%)\end{array}$ & $\begin{array}{c}\text { 2-hydroxyethyl } \\
\text { allyl ether XL } \\
(\%)\end{array}$ \\
\hline WPHX-6 & 97.1 & 0.0 & 2.9 \\
WPHX-7 & 94.9 & 0.0 & 5.1 \\
WPHX-5 & 71.2 & 24.7 & 4.1 \\
WPHX-3 & 44.1 & 49.2 & 6.7 \\
WPHX-9 & 20.8 & 71.0 & 8.2 \\
\hline
\end{tabular}

polymers, as expected. Unfortunately, after crosslinking through the hydrophilic component, membrane lifetimes in pervaporation experiments were very long, with low total fluxes of water through the membranes. Testing of these membranes has therefore taken much longer than anticipated, but the results are potentially exciting. From the evidence gathered to-date, it is apparent that molecular level ligandbased morphological changes within phosphazene polymers do exert a significant influence upon the macroscopic performance of the membranes. Further detailed study of these types of morphological effects is needed to fully understand the effects observed and to quantify the magnitude of the advantage gained by this selective stabilization strategy.

\section{ACCOMPLISHMENTS}

\section{Molecular Composites}

- $\quad$ Experiments designed to reveal the nature of bonding in these composites were successfully completed. These studies showed that covalent bonding (in polyether-based systems) was a minor component of the interaction between the ceramic and the polymer component.

- $\quad$ Developed and refined synthetic protocols for formation of molecular composites. These included not only synthetic conditions, but also thin film formation protocols that provide a convenient pathway for adaptation of these materials as membranes for challenging EM-relevant separations.

- Identified and explored several synthetic variables critical to the properties of the compositesthese included the polymer/ceramic ratio, the nature of the catalyst employed, catalyst loading levels, water loading levels, and precursor solution aging time. Each of these critical variables was explored singularly and the data obtained was correlated with the observed physical properties to form the basis for understanding the structure-property relationships evident in these materials.

- Investigated co-catalyst schemes, to further expand and validate understanding of the structureproperty relationships. This fundamental understanding has afforded us the ability to formulate composite materials with a broad range of desirable properties.

- $\quad$ Extensive dynamic mechanical analysis of these composites was performed. This has provided information crucial to accurately assessing the potential usefulness and limitations of these materials. 
- Durability studies were performed, and it was observed that by forming composite materials, a four-fold increase in radiation stability over the polymer component alone was achieved. It is believed that this tolerance to high-energy radiation can be broadened to include both a greater degree of radiation resistance and to include other (all organic) polymer systems.

\section{Polymer Morphology}

- A series of five new polymers that possess hydrophilic crosslinkable ligands, specifically designed to address ligand-based morphological differences and the corresponding effects upon membrane performance, was successfully synthesized.

- $\quad$ The above mentioned series of polymers were all fully characterized by multinuclear NMR $\left({ }^{31} \mathrm{P}\right.$,

${ }^{13} \mathrm{C}$, and ${ }^{1} \mathrm{H}$ ), multiple thermal analysis techniques (DSC, TGA and DMA), and by laser light scattering molecular weight determination experiments.

- $\quad$ Swelling studies with water were conducted to assess solvent/polymer interactions.

- $\quad$ Both thermal and e-beam crosslinking procedures were performed upon all of these polymers followed by further materials characterization (predominantly thermal analyses).

- Water purification trials were conducted using these polymers, both crosslinked and uncrosslinked, as pervaporation membranes. The results of these pervaporation experiments are being compared to existing benchmark data for similar polymers that possess hydrophobic crosslinkable ligands. From these comparisons, the true effects of ligand-based morphology upon polymer membrane performance can be determined.

\section{REFERENCES}

1. B. K. Coltrain, W. T. Ferrar, C. J. Landry, T. R. Molaire, and N. Zumbultadis, "Polyphosphazene Molecular Composites 1. In Situ Polymerization of Tetraethoxysilane," Chem. Mater. 4, 1992, 358.

2. Fifth International Symposium on Polymer Electrolytes, Nanocomposites Division, April 1998.

3. A. F. M. Leenaars, K. Keizer, and A. J. Burggraaf, "The Preparation and Characterization of Alumina Membranes with Ultra-fine Pores,” J. Mater. Sci. 19, 1984, 1,077.

4. S. A. Bagshaw, E. Prouzet, and T. J. Pinnavaia, "Templating of Mesoporous Molecular Seives by Nonionic Polyethylene Oxide Surfactants," Science 269, 1995, 1,242.

5. H. R. Allcock and R. L. Kugel, "Synthesis of High Polymeric Alkoxy- and Aryloxyphosphonitriles," J. Am. Chem. Soc., 87, 1965, 4, 216.

6. $\quad$ H. N. Stokes, "On the Chloronitrides of Phosphorous (II)," Amer. Chem. J., 19, 1897, 782.

7. H. R. Allcock, R. L. Kugel, and K. J. Valen, "Phosphonitrilic Compounds. 06. High Molecular Weight Poly(alkoxy- and aryloxyphosphazenes)," Inorg.Chem., 5, 1966, 1,709.

8. C. A. Allen, and E. S. Peterson, U. S. Patent, Filed October, 1998.

9. Focus category_PMSE Division, ACS National Meeting, Las Vegas, NV, 1997. 
10. F. F. Stewart, M. K. Harrup, T. A. Luther, C. J. Orme, and R. P. Lash, "Formation of Pervaporation Membranes from Polyphosphazenes Having Hydrophilic and Hydrophobic Pendant Groups: Synthesis and Characterization," Chem. Mater., Submitted.

11. J. A. Pope and J. D. Mackenzie, "Sol-Gel Processing of Silica," Journal of Non-Crystalline Solids, 87, No. 3, 1986, 185-198.

12. B. M. Novak, "Hybrid Nanocomposite Materials-Between Inorganic Glasses and Organic Polymers," Advanced Materials, Vol. 5, No. 6, 1993, 422-433.

13. B. K. Coltrain, W. T. Ferrar, C. J. T. Landry, T. R. Molaire, D. E. Schildkraut, and V. K. Smith, "Polyphosphazene Molecular Composites. II.," Polymer Preprints (ACS) 1993, 34 (1), 266-267.

14. M. K. Harrup and F. F. Stewart, "Improved Method for the Isolation and Purification of WaterSoluble Polyphosphazenes," J. Appl. Poly. Sci., Submitted.

15. G. W. Scherer, “Aging and Drying of Gels," Journal of Non-Crystalline Solids, Vol. 100, No. 1, 1988, 77-92. 


\title{
Adsorption and Absorption Materials of Molecular Separations
}

\author{
Glenn A. Moore, Phil Martellaro, and Eric Peterson \\ SUMMARY
}

\begin{abstract}
We report the development of novel molecular absorbents/adsorbents "sorbant" materials for removing/separating target environmental contaminants from waste and/or process streams. Synthesis of metal sulfides for solid-phase mercury-vapor capture-agents was the primary focus of our FY-99 effort. These materials are being developed in support of DOE's Environmental Management (EM) program needs in the area of waste treatment and disposal. Namely, to provide enabling technology for cost effective remediation of mercury containing solid and liquid waste forms. ${ }^{1,2,3}$ Presently, as stated in DOE's EM Research and Development Program Plan: "Mercury mixed waste cannot be commercially recycled. Current treatment and stabilization technologies cannot meet Land Disposal Restrictions." ${ }^{4}$ Toxicity of mercury is greatest when it is in an organic form, produced by biotransformation of ionic mercury to methylmercury. ${ }^{5}$ This transformation is driven by bacterial action on the sediment-bound mercury. Methylmercury dissolves in water and is easily absorbed by organisms. This toxicant is persistent because it is not easily removed from the body, and as a result mercury can remain in the body for up to 20 to 30 years. Toxicity from inorganic mercury is primarily an occupational disease resulting in central nervous system damage and kidney failure. Given the established environment and human health hazards of mercury, the development of cost-effective mercury control technologies is of increased government and industry priority. Metal sulfides that react with mercury to form the insoluble mineral cinnabar, $\mathrm{HgS}$, is our area of interest. ${ }^{6,7,8,9,10,11,12,13,14}$
\end{abstract}

Metal sulfide powders can be prepared using solid state fusion, spray pyrolysis, or precipitation techniques. ${ }^{15,16,17,18,19}$ When precipitation techniques are employed, particle size and morphology can be controlled via synthesis conditions and the use of particulate stabilizing agents. ${ }^{20}$ Surfactant assemblies, i.e., micelles, vesicles, microemulsions, and monolayers, can serve as nucleation sites for nanoparticulates or templates for ordered hybrid mesoporous materials. ${ }^{21,22,23,24,25}$ Likewise, surfactant-monolayer assembly attached to nanoparticle surfaces can mediate particle growth and facilitate formation of colloidal suspensions. Surfactant molecules can be removed from organic/inorganic hybrid structures using solvent extraction or pyrolysis. ${ }^{26}$ When pyrolysis (calcining) is employed, organized hybrid-structures are may be destroyed; however, nanocrystalline morphology can generally be maintained. Two primary synthesis approaches were investigated, (1) aqueous based precipitation utilizing a surfactant for particlesize-mediation, and (2) formation of metal-thiourea complexes. Using these techniques, powders, films, and infiltrated porous ceramics were produced. This report summarizes the preparation of a series of nanocrystalline metal sulfides (MSs) and the results of reactive-capture experiments involving vapor- and aqueous-phase mercury species.

\section{PROJECT DESCRIPTION}

\section{Processing of Metal Sulfides}

\section{Particle-Size-Mediated Synthesis of Copper Sulfide using CTAB and $\mathrm{Na}_{2} \mathrm{~S}$}

The formation of 3D lamellar biphase copper sulfide nanoparticles was accomplished using aqueous solutions of dissolved $\mathrm{Cu}\left(\mathrm{NO}_{3}\right)_{2} 3 \mathrm{H}_{2} \mathrm{O}$ and cetyltrimethylammonium bromide $\mathrm{C}_{19} \mathrm{H}_{42} \mathrm{NBR}$ (CTAB), Sigma chemical Company, a surfactant acting as a particle-size-mediating agent. The general 
procedure for the aqueous synthesis is similar to that reported by Anderson and Newcomer. ${ }^{21}$ Commercially available copper nitrate from Alpha Products was used as received. Precipitation was initiated by addition of a sodium sulfide, $\mathrm{Na}_{2} \mathrm{~S} 9 \mathrm{H}_{2} \mathrm{O}$, solution. The precipitate was filtered, rinsed, and subjected to a pyrolysis treatment for removal of residual CTAB, $250-350^{\circ} \mathrm{C}$ in $4 \% \mathrm{H}_{2} \mathrm{~S} / \mathrm{Argon}$ (Ar) gas.

Transmission electron microscopy (TEM) of precalcined powder, Figure 1, shows the layered structure characteristic of lamellar ordering. Powder X-ray diffraction (XRD) analysis was performed using a Phillips PW1710 diffractometer to confirm the phase identity of synthesized and calcined samples. Quantitative crystalline phase analysis "Rietveld Analysis" was performed on several precalcined precipitate and mixed phase copper sulfide samples. ${ }^{27}$ Crystal structure models, i.e., $\mathrm{CuS}$, $\mathrm{Cu}_{2} \mathrm{~S}, \mathrm{Cu}_{7.2} \mathrm{~S}_{4}$, were constructed using MSI's Cerius2 Crystal Builder. The structure models and our experimental X-ray diffraction data were used in conjunction with the quantitative phase analysis routine, DBWS-Rietveld. The Rietveld analysis provided a means of isolating diffraction peaks associated with the novel surfactant/sulfide mesophase. Once isolated, the unidentified peak-set was processed using the powder indexing program DICVOL91. Powder indexing results were consistent with literature reported mesophase structures incorporating CTAB. ${ }^{23}$ Our pre-calcined materials exhibited a series of low angle diffraction peaks that could be indexed on an orthorhombic unit cell having $a=5.96 \AA, b=8.66 \AA$, and $c=20.65 \AA$.

Calcining at $250^{\circ} \mathrm{C}$ for $18 \mathrm{~min}$ in $4 \% \mathrm{H}_{2} \mathrm{~S} / \mathrm{Ar}$ produced hexagonal $\mathrm{CuS}$, (covellite) while samples calcined in $100 \%$ argon converted to tetragonal $\mathrm{Cu}_{2} \mathrm{~S}$ (chalcocite). Figure 2 is a TEM micrograph of

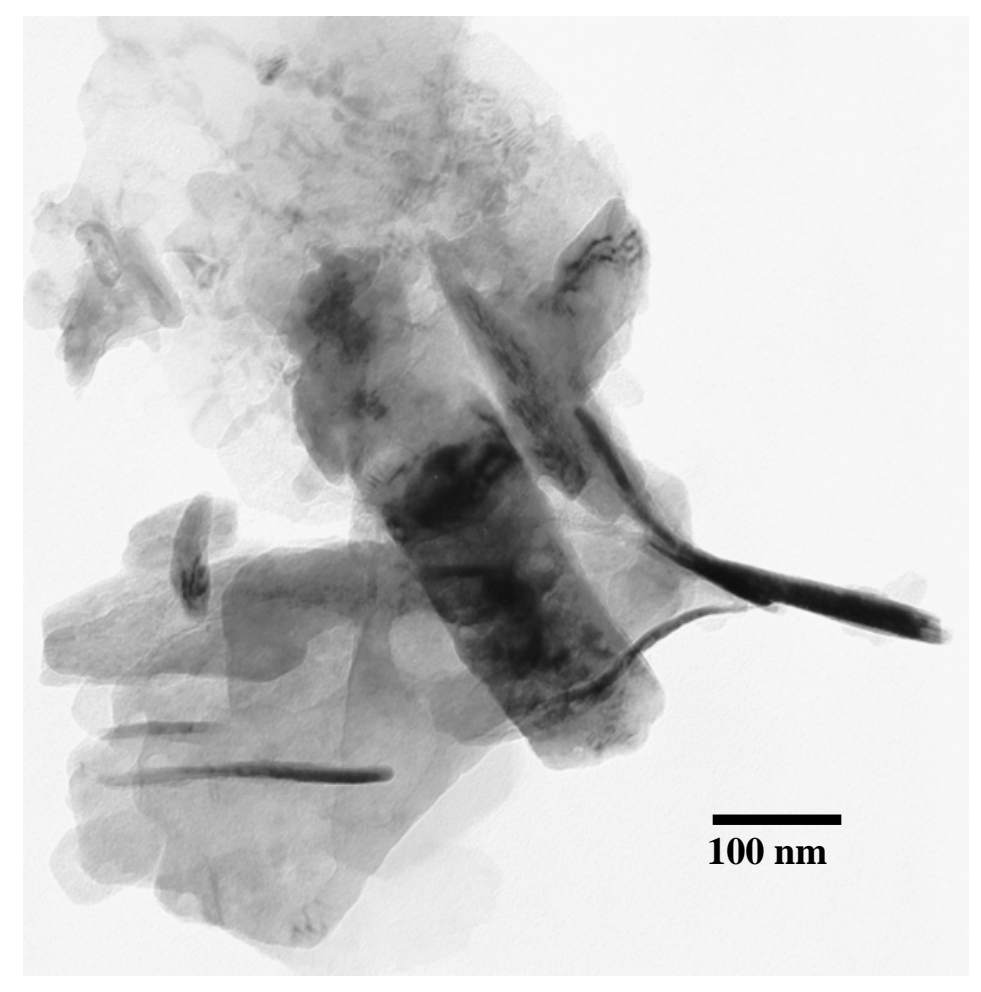

Figure 1. TEM micrograph of as precipitated lamellar CTAB/sulfide mesophase particles or TEM micrograph of precalcined $\mathrm{CuS}$ particles exhibiting lamellar order. 


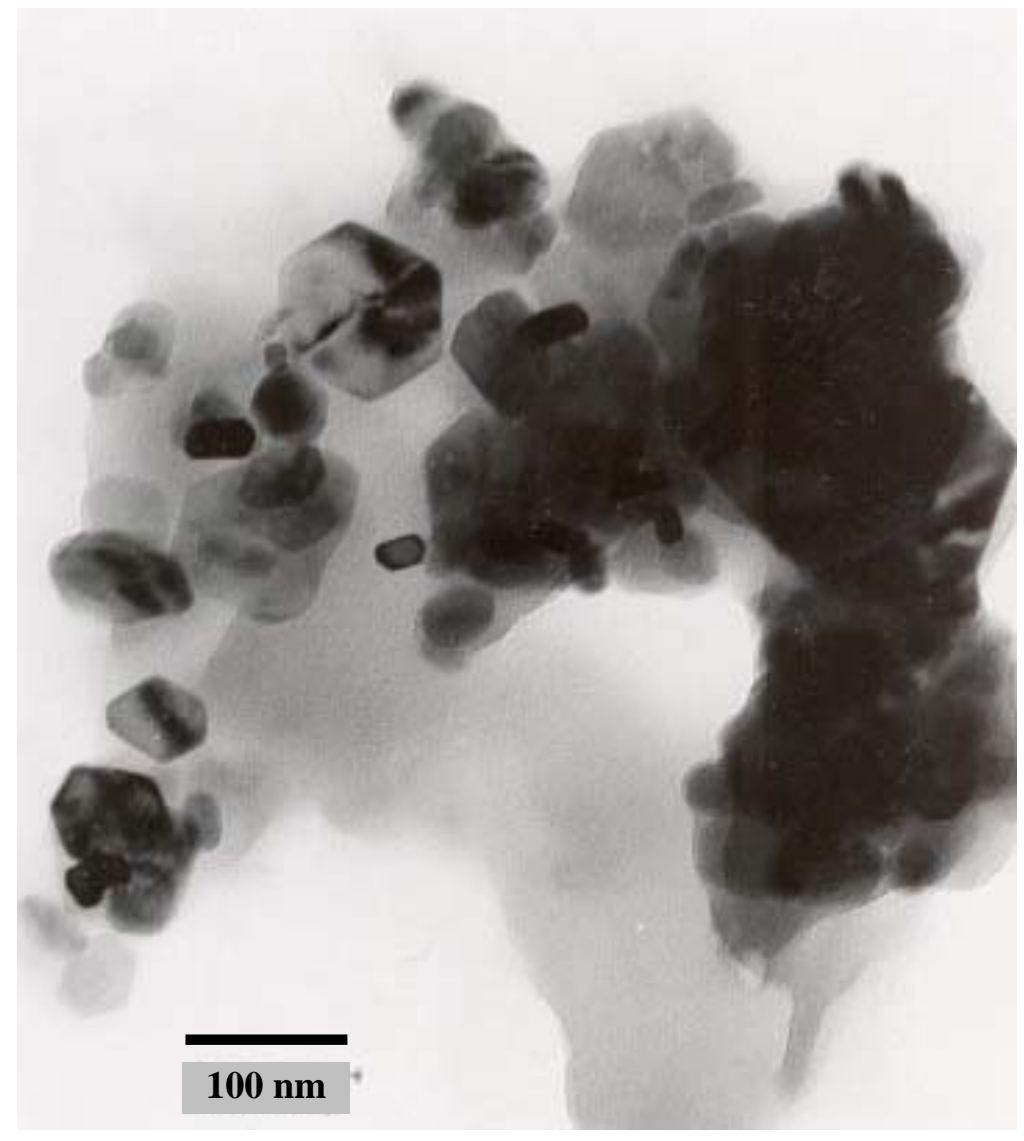

Figure 2. TEM micrograph of $\mathrm{CuS}$ nanplatelets.

covellite powder, showing the presence of well-defined hexagonal platelets $\sim 10-200 \mathrm{~nm}$ in diameter, 125-2500X smaller than those observed in unmediated precipitate samples. The low angle diffraction peaks of the lamellar mesophase were not present after calcining. Thus, indicating the elimination of the organic/inorganic hybrid structure.

\section{Particle-Size-Mediated Synthesis of Copper Sulfide using CTAB and $\mathrm{H}_{2} \mathrm{~S}$}

As an alternative to the use of sodium sulfide in the preparation of metal sulfide powders, we investigated the substitution of hydrogen sulfide gas. For these experiments, a solution of $\mathrm{Cu}\left(\mathrm{NO}_{3}\right)_{2} \cdot 3 \mathrm{H}_{2} \mathrm{O}(2.5 \mathrm{wt} \%) / \mathrm{CTAB}(4.5 \mathrm{wt} \%)$ was exposed to $4 \% \mathrm{H}_{2} \mathrm{~S} /$ argon using an immersed gas bubbling tube. The gas mixture in the presence of the surfactant created froth above the cupric nitrate/CTAB solution where the formation of metal sulfide precipitate was observed. The foam was expelled from the reaction flask and collected in a secondary container. Figure 3 is a TEM micrograph of the as-precipitated copper sulfide, $\sim 5-20 \mathrm{~nm}$ in diameter, particles. The nanoparticles appear uniformly dense and non-faceted in contrast to the layered hybrid structure observed for material processed using $\mathrm{CTAB}$ and $\mathrm{Na}_{2} \mathrm{~S}$. X-ray diffraction analysis of a $200^{\circ} \mathrm{C}$ calcined sample revealed the presence of chalcocite $\left(\mathrm{Cu}_{2} \mathrm{~S}\right)$ and digenite $\left(\mathrm{Cu}_{1.8} \mathrm{~S}\right)$. 


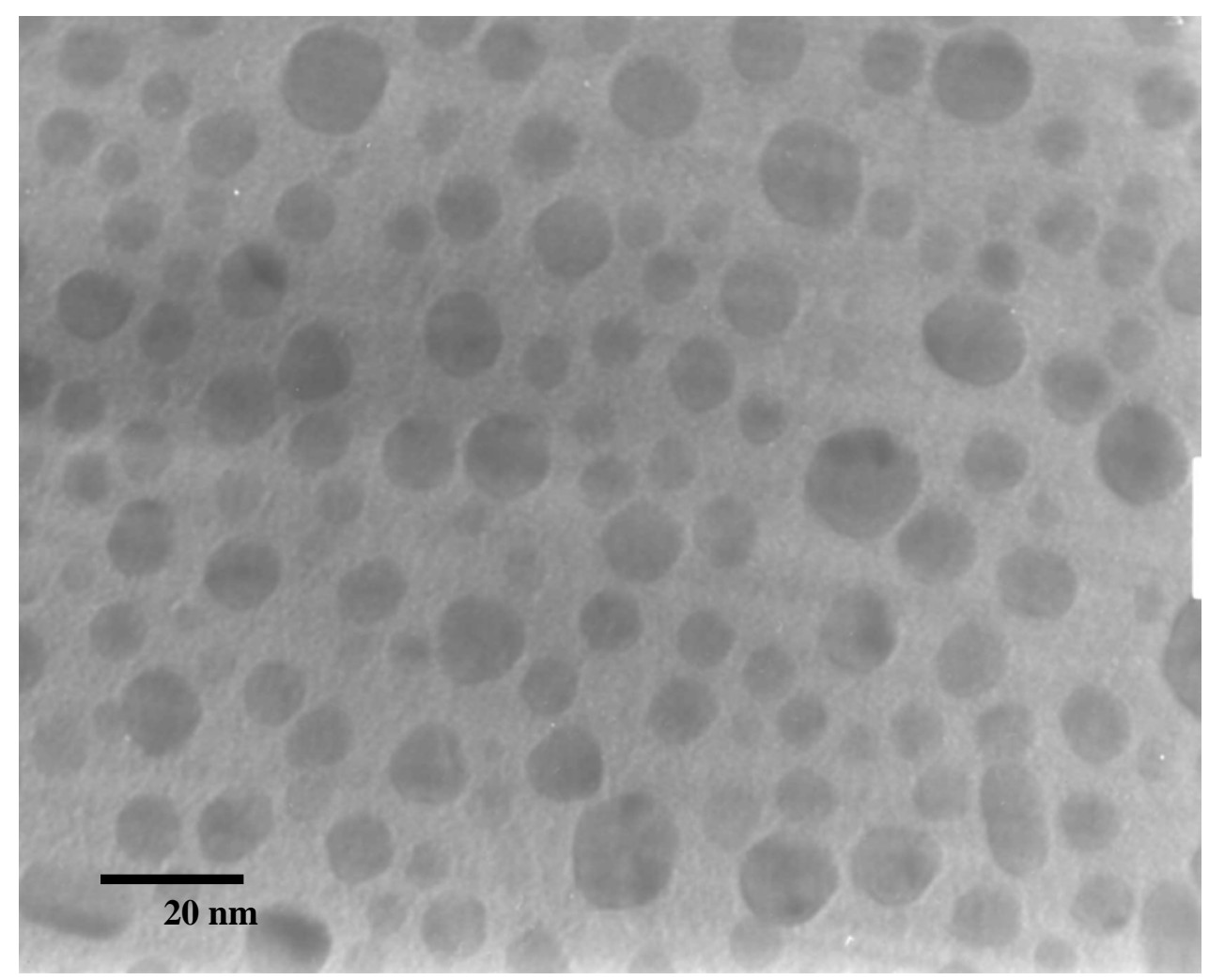

Figure 3. TEM micrograph of copper sulfide particles formed, using hydrogen sulfide gas reactant.

\section{Particle-Size-Mediated Synthesis of Copper Sulfide using Beta-Cyclodextrin and $\mathrm{Na}_{2} \mathrm{~S}$}

The third synthesis route investigated involved the use of Beta-cyclodextrin $(\beta-\mathrm{CD})$ mediated precipitation of copper sulfide. Cyclodextrins are a family of starch derivatives having toroidal-shaped cage structures conducive to the formation of guest-host inclusion complexes. ${ }^{28}$ These molecules are water-soluble and can form hydrated gel structures. Our objective for these experiments was to determine if particle-size-mediation or molecular templating could be achieved as was the case for the CuS-CTAB system.

For these experiments, an aqueous solution for cupric nitrate was added to an aqueous solution of sodium sulfide and dissolved $\beta$-CD (CTD, Inc., Trappsol TBCD, MW=1135.0 Aq. Sol.=1.8\%) resulting in the formation of a fine precipitate. X-ray diffraction of the as-produced precipitate revealed the presence of submicron digenite " $\mathrm{Cu}_{9} \mathrm{~S}_{5}$ " and a small low angle hump indicative of a mesophase, i.e., a molecular composite between the digenite and the $\beta$-CD. Precipitate powder, calcined in argon at $250^{\circ} \mathrm{C}$, consisted primarily of digenite and Copper Sulfate $\mathrm{CuSO}_{4}$. It is suspected that the thermal decomposition products of $\beta$-CD oxidized the copper sulfide. XRD and TEM analysis of a calcined sample did not reveal indications of any retained mesoporous structure.

\section{Processing of Copper-Thiourea Complexes}

Processing of metal sulfide coatings and submicron powders via spray pyrolysis of dissolved metal salt/thiourea solutions has been reported in the literature. ${ }^{17,18,20}$ We investigated the use of copper-thiourea solutions for coating and infiltrating ceramic membranes and reticulated ceramic foam structures. The 
objective being to fabricate thin coatings on the pore walls of permeable substrates, thus affording a viable means of selectively extracting and immobilizing mercury vapor and/or mercury dissolved in water.

For these experiments, thiourea powder or an aqueous thiourea solution was added to a solution of copper nitrate to form a greenish-white precipitate; with heating to $\sim 100^{\circ} \mathrm{C}$, a slightly viscous "clear" solution was obtained. This solution was subsequently dried and the crystalline product ground and calcined at $300^{\circ} \mathrm{C}$ in $4 \% \mathrm{H}_{2} \mathrm{~S} / \mathrm{Ar}$ to form single phase $\mathrm{CuS}$ having a surface area of $\sim 4.2 \mathrm{~m}^{2} / \mathrm{g}$. $\mathrm{Cu}$-thiourea complex crystal formation was observed upon slow cooling a 1:1 stoichiometric ratio of cupric nitrate and thiourea solution, Figure 4. Larger $\mathrm{Cu}$-thiourea crystals, Figure 5, were formed by aging an aqueous solution for a period of approximately two months.

When $50 \mathrm{wt} \%$ acetone was added to the aqueous $\mathrm{Cu}$-thiourea solution, prior to or after the heating step, the solution stability was greatly increased; with only minor amounts of complex precipitate observed after two weeks at room temperature. Coating experiments with this $\mathrm{Cu}$-thiourea formulation produced good quality, i.e., crack-free and adherent, thin films via evaporation of $\mathrm{Cu}$-thiourea/water/acetone solution on glass substrates. The precalcined coatings were determined via XRD to be amorphous. Figure 6, shows an optical micrograph of the resulting covellite film calcined at $300^{\circ} \mathrm{C}$ for $10 \mathrm{~min}$. in $4 \% \mathrm{H}_{2} \mathrm{~S} / \mathrm{Ar}$.

We also investigated the influence of CTAB within this processing scheme. When dissolved $\mathrm{CTAB}$ was present in the initial $\mathrm{Cu}\left(\mathrm{NO}_{3}\right)_{2}$ solution, titration with a thiourea solution resulted in the formation of a milky-white product that could not be solublized with further heating $\left(\sim 100^{\circ} \mathrm{C}\right)$. This product was dried, forming a plastic-like substance, and calcined at $300^{\circ} \mathrm{C}$ in $4 \% \mathrm{H}_{2} \mathrm{~S} / \mathrm{Ar}$ to produce single phase $\mathrm{CuS}$.

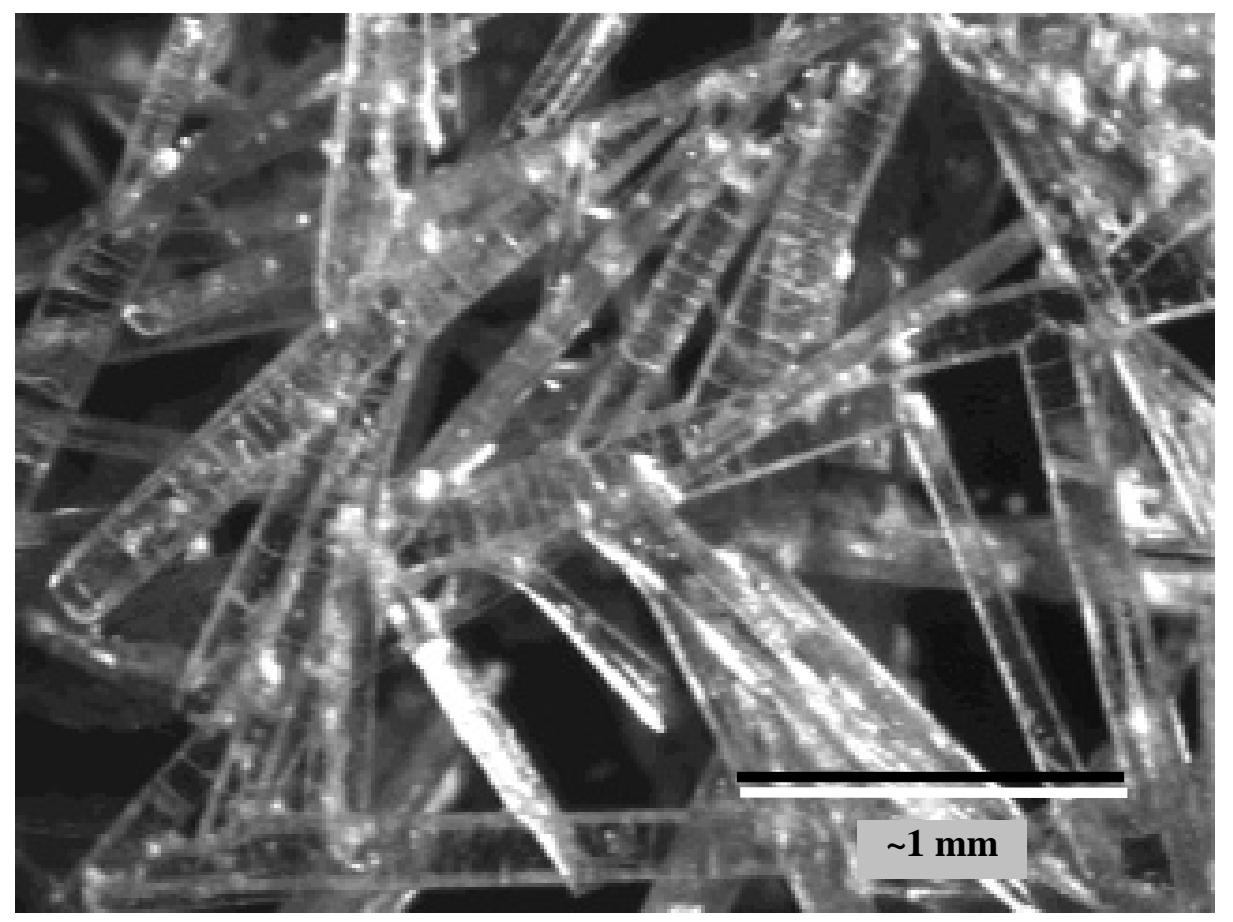

Figure 4. Transparent crystallites formed in an aqueous solution of $\mathrm{Cu}$ nitrate/thiourea. 


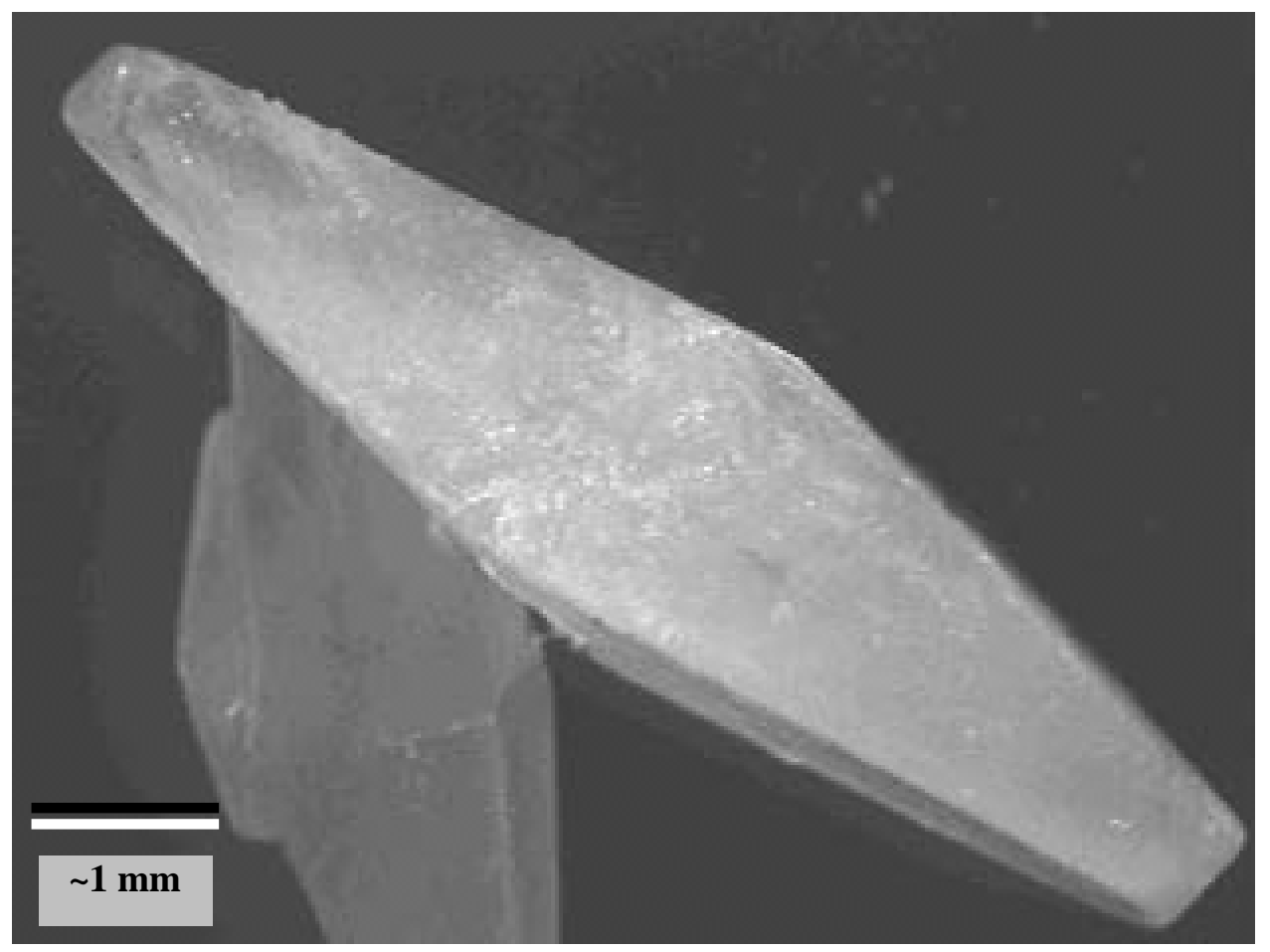

Figure 5. Cu-thiourea crystals formed with aging of saturated cupric nitrate/thiourea.

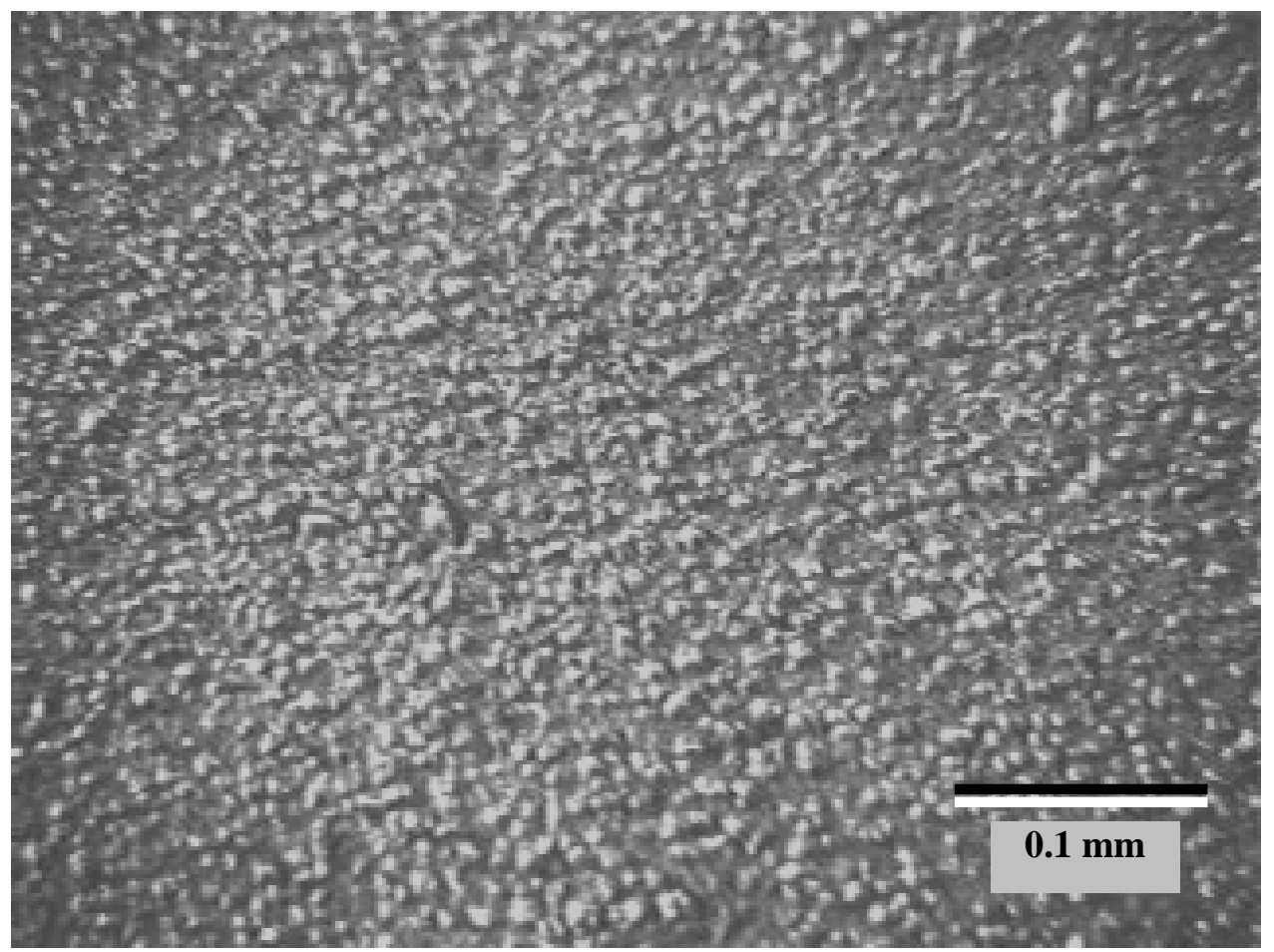

Figure 6. Corvellite film formed on glass slide via a $\mathrm{Cu}$-thiourea/water/acetone solution. 
Solutions/suspension of the $\mathrm{Cu}$-thiourea complexes (with and without CTAB) were prepared and infiltrated into porous ceramic disks (Osmonics, mullite \#46401-10-13 $\mu \mathrm{m}$ ) and alumina membranes (Whatman, Anodisc $0.2 \mu \mathrm{m}$ ). Addition of $1 \mathrm{wt} \% \mathrm{CTAB}$ surfactant to the $\mathrm{Cu}$-thiourea solution resulted in the formation of submicron particulate, as opposed to high-aspect-ratio transparent crystallites. Figure 7 shows SEM micrographs of alumina membranes infiltrated with $\mathrm{Cu}$-thiourea complexes "with and without CTAB". The calcined membrane infiltrated with the CTAB-containing Cu-thiourea suspension exhibits discrete $\mathrm{CuS}$ particles dispersed throughout the membranes' cross section. The calcined membrane infiltrated with the Cu-thiourea solution "no CTAB" shows a more continuous $\mathrm{CuS}$ deposit.

Porous ceramic substrates were infiltrated with $\mathrm{Cu}$-thiourea solutions/sols with and without CTAB. Solutions without $\mathrm{CTAB}$, were infiltrated at $80-100^{\circ} \mathrm{C}$ and subsequently pyrolyzed at $300^{\circ} \mathrm{C}$ in $4 \%$ $\mathrm{H}_{2} \mathrm{~S} / \mathrm{Ar}$. Figure 8 show a SEM micrograph of the resulting nodule-like CuS particles in the pores of the porous substrate. The $\mathrm{Cu}$-thiourea sol containing $\mathrm{CTAB}$ was processed in a like manner and resulting in the formation of a sintered coating structure composed of submicron CuS particles, Figure 9.

\section{Synthesis of MnS, Ga2S3, FeS, NiS, ZnS, Ag2S, In2S3 and CuFeS2}

Reagent grade metal nitrate was utilized for each synthesis except for iron where the dichloride was used. Each of the metal nitrates was commercially available and used as received. The $\mathrm{Na}_{2} \mathrm{~S} \cdot 9 \mathrm{H}_{2} \mathrm{O}$ was obtained from Fisher Scientific and was used as received. The ethanol was reagent grade and used as received. Table 1 shows the metal sulfide targeted composition and the actual metal sulfide phase obtained before and after calcining. The commercially purchased metal sulfides included -325 mesh $\mathrm{Cu}_{2} \mathrm{~S},-100$ mesh CuS 99+\%, $\mathrm{Au}_{2} \mathrm{~S}_{3} 99.9 \%, \mathrm{Au}_{2} \mathrm{~S} 99.9 \%$ and $\mathrm{Ag}_{2} \mathrm{~S} 99.9 \%$ which were all obtained from Aldrich Chemical Company and used as received.

Synthesis of $\mathrm{MnS}, \mathrm{Ga}_{2} \mathrm{~S}_{3}, \mathrm{FeS}, \mathrm{NiS}, \mathrm{ZnS}, \mathrm{Ag}_{2} \mathrm{~S}$, and $\mathrm{In}_{2} \mathrm{~S}_{3}$ : CTAB (0.02moles) and $\mathrm{Na}_{2} \mathrm{~S} 9 \mathrm{H}_{2} \mathrm{O}$ (0.02moles) were dissolved in a $250 \mathrm{~mL}$ beaker containing a mixture of $25 \mathrm{~mL}$ of ethanol and $25 \mathrm{~mL}$ of water. The beaker was slightly warmed to $50^{\circ} \mathrm{C}$ to facilitate dissolution of the solids. In a separate vessel $\mathrm{M}\left(\mathrm{NO}_{3}\right)_{\mathrm{x}} \mathrm{xH}_{2} \mathrm{O}(0.02 \mathrm{Moles})\left(\mathrm{FeCl}_{2} \cdot 4 \mathrm{H}_{2} \mathrm{O}\right.$ in the case of $\left.\mathrm{FeS}\right)$ was dissolved in $25 \mathrm{~mL}$ of water. For MnS, $\mathrm{Ga}_{2} \mathrm{~S}_{3}$ and $\mathrm{FeS}$ synthesis the $7 \mathrm{M} \mathrm{HCl}$ was added dropwise to the $\mathrm{CTAB} / \mathrm{Na}_{2} \mathrm{~S}$ solutions to lower the $\mathrm{pH}$ to 8 before combining with the metal containing solution. The two solutions are then combined and an immediate dark colored precipitate was observed. The solution $\mathrm{pH}$ was monitored and adjusted to about 7.5 in the case of $\mathrm{MnS}, \mathrm{Ga}_{2} \mathrm{~S}_{3}$ and $\mathrm{FeS}$. No pH adjustment is necessary for the other metal sulfides. The
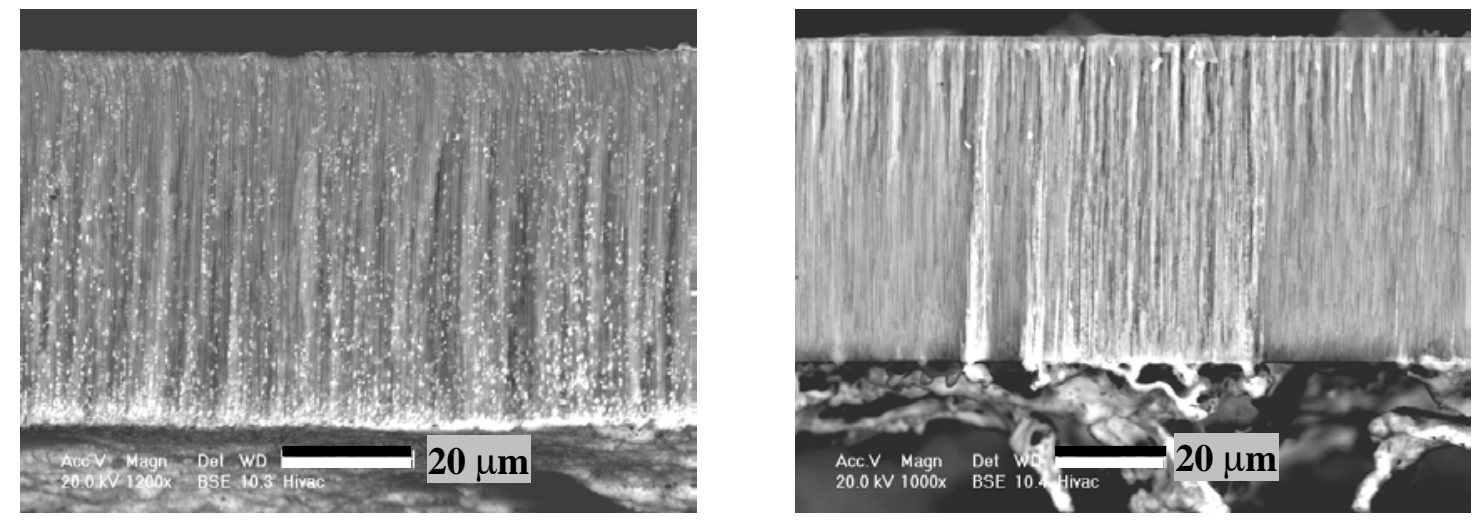

Figure 7. SEM images of calcined alumina membranes; infiltrated with Cu-thiourea complexes. In "a," discrete particle of $\mathrm{CuS}$ "white dots" formed from a $\mathrm{Cu}$-thiourea-CTAB suspension, while in "b" limited infiltration of a $\mathrm{Cu}$-thiourea solution resulted in more continuous $\mathrm{CuS}$ deposit "white streaks." 


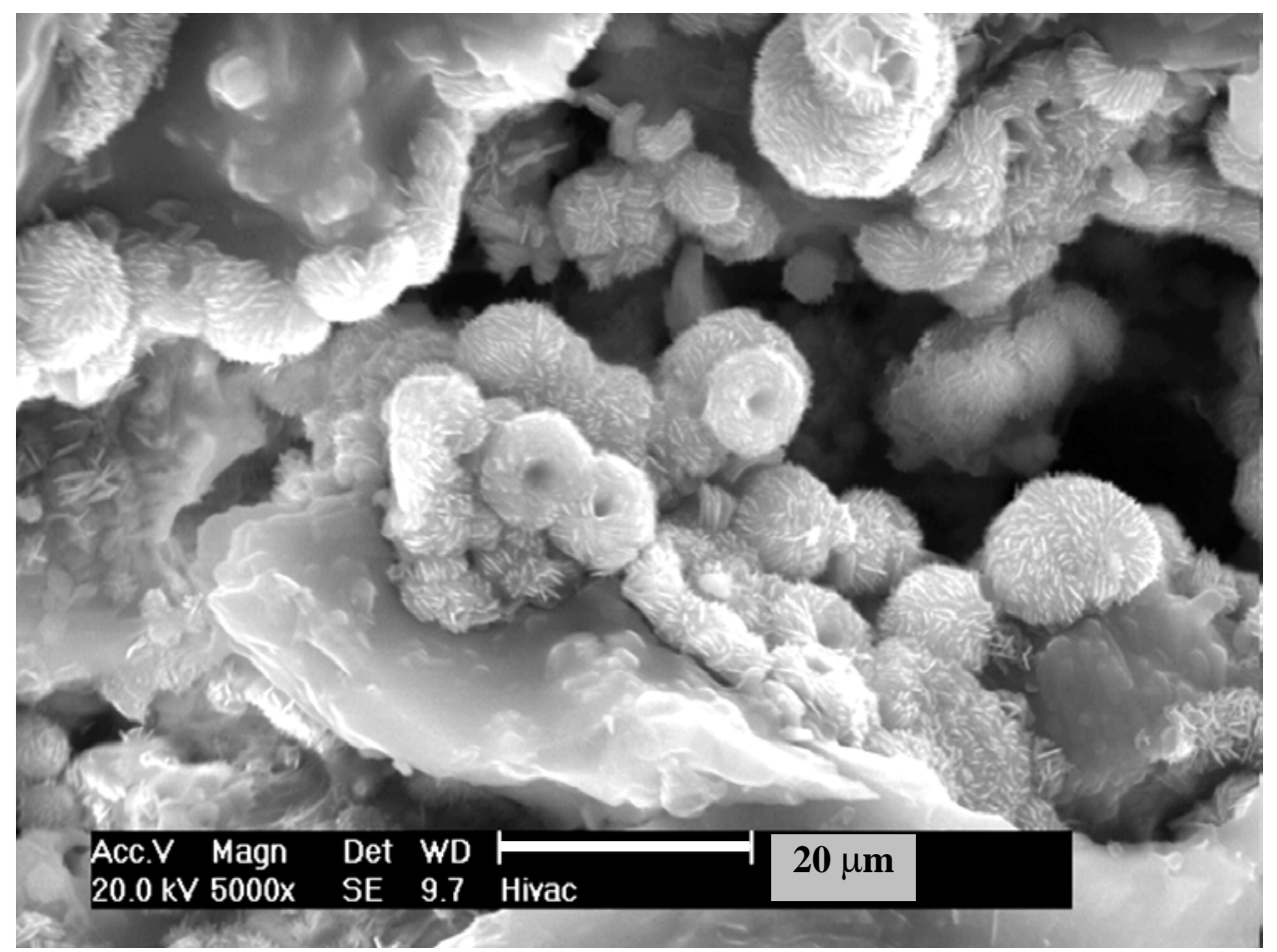

Figure 8. Copper sulfide particles formed in the pores of a mullite disk via pyrolysis of infiltrated $\mathrm{Cu}$-thiourea solution without CTAB.

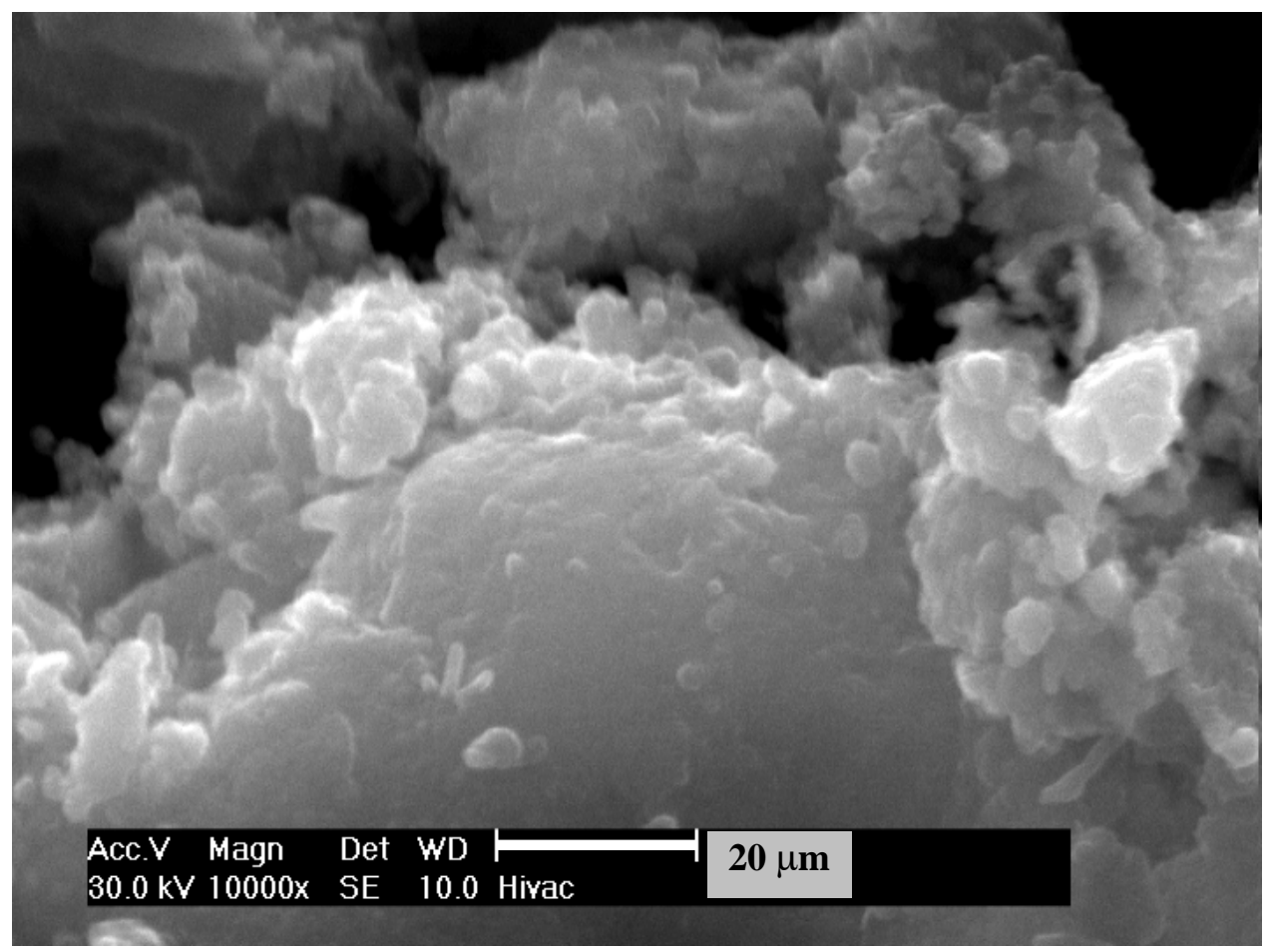

Figure 9. Copper sulfide particles formed in the pores of a mullite disk via pyrolysis of infiltrated $\mathrm{Cu}$ thiourea sol with CTAB. 
Table 1. Summary of metal sulfide syntheses and XRD characterization of pre-calcined and calcined materials.

\begin{tabular}{|c|c|c|}
\hline Target Compound & Pre-Calcine Phases & Calcined Phases \\
\hline $\mathrm{MnS}$ & $\mathrm{MnO}, \mathrm{MnS}$ & $\mathrm{MnS}$ \\
\hline $\mathrm{MnS}(\mathrm{CTAB})$ & $\mathrm{MnO}, \mathrm{MnS}$ & $\mathrm{MnS}$ \\
\hline $\mathrm{FeS}$ & $-^{\mathrm{a}}$ & $?$ \\
\hline $\mathrm{FeS}(\mathrm{CTAB})$ & $\mathrm{FeO}, \mathrm{FeS}$ & $\mathrm{FeS}$ \\
\hline $\mathrm{NiS}$ & $\mathrm{NiS}$ & $\mathrm{NiS}$ \\
\hline $\mathrm{NiS}(\mathrm{CTAB})$ & $\mathrm{NiS}$ & $\mathrm{NiS}$ \\
\hline $\mathrm{ZnS}$ & $\mathrm{ZnS}$ & $\mathrm{ZnS}$ \\
\hline $\mathrm{ZnS}(\mathrm{CTAB})$ & $\mathrm{ZnS}$ & $\mathrm{ZnS}$ \\
\hline $\mathrm{Ga}_{2} \mathrm{~S}_{3}$ & $-^{a}$ & $\mathrm{Ga}_{2} \mathrm{~S}_{3}$ \\
\hline $\mathrm{Ga}_{2} \mathrm{~S}_{3}(\mathrm{CTAB})$ & $-^{a}$ & $\mathrm{Ga}_{2} \mathrm{~S}_{3}$ \\
\hline $\mathrm{In}_{2} \mathrm{~S}_{3}$ & $-^{a}$ & $\operatorname{In}_{2} S_{3}$ \\
\hline $\mathrm{In}_{2} \mathrm{~S}_{3}(\mathrm{CTAB})$ & $-^{a}$ & $\operatorname{In}_{2} S_{3}$ \\
\hline $\mathrm{CuS}(\mathrm{CTAB})$ & $\mathrm{Cu}_{2} \mathrm{~S} / \mathrm{CuS}$ & $\mathrm{Cu}_{2} \mathrm{~S} / \mathrm{CuS}$ \\
\hline $\mathrm{Ag}_{2} \mathrm{~S}$ & $\mathrm{Ag}_{2} \mathrm{~S}$ & $\mathrm{Ag}_{2} \mathrm{~S}$ \\
\hline $\mathrm{Ag}_{2} \mathrm{~S}(\mathrm{CTAB})$ & $\mathrm{Ag}_{2} \mathrm{~S}$ & $\mathrm{Ag}_{2} \mathrm{~S}$ \\
\hline
\end{tabular}

solutions were then allowed to stir for 10 minutes and filtered. The resulting solid was dried and recombined with $50 \mathrm{~mL}$ of water, placed in a sonication bath (Fisher Scientific FS-14) for 10 minutes, followed by a second filtration. The solid was collected and the sonication/filtration process repeated two more times to remove the excess CTAB. The solid product was thoroughly air dried and calcined under a $4 \% \mathrm{H}_{2} \mathrm{~S} / \mathrm{Ar}$ mixture. Calcining was typically performed at $300^{\circ} \mathrm{C}$.

Synthesis of CuFeS 2 : $3.65 \mathrm{~g} \mathrm{CTAB}\left(0.01\right.$ moles) and $2.4 \mathrm{~g}$ of $\mathrm{Na}_{2} \mathrm{~S} 9 \mathrm{H}_{2} \mathrm{O}(0.01$ moles $)$ were dissolved in $25 \mathrm{~mL}$ of ethanol and $25 \mathrm{~mL}$ of water. In separate vessels $(0.01$ moles $)$ each of $\mathrm{Cu}\left(\mathrm{NO}_{3}\right)_{2} 3 \mathrm{H}_{2} \mathrm{O}$ and $\mathrm{Fe}\left(\mathrm{NO}_{3}\right)_{3} \cdot 3 \mathrm{H}_{2} \mathrm{O}$ were dissolved in $25 \mathrm{~mL}$ water. The three solutions were combined and the resulting black precipitate washed three times and calcined at $350^{\circ} \mathrm{C}$ for 2 hours under a $\mathrm{H}_{2} \mathrm{~S} / \mathrm{Ar}$ atmosphere.

\section{Mercury Sorption Experiments}

A series of mercury vapors and ionic $\mathrm{Hg}^{2+}$ reactivity experiments were performed using the synthesized and commercial metal sulfides. Initial studies were conducted to determine the efficiency and rate of $\operatorname{Hg}(0)$ sorption in the metal sulfide samples. Some samples were treated with a saturated aqueous solution of oxalic acid; we refer to this process as "activation". Ukrainian researchers investigating mineral sulfides as mercury vapor capture materials have found that oxalic acid treatment enhances mercury vapor reactivity. ${ }^{12,13}$ It is known that organic chelation of dissolved metals enhance the solubility of various minerals and that adsorption of organic anions on mineral surfaces catalyzes the dissolution and precipitation of minerals. ${ }^{29}$ To our knowledge, the exact mechanism for the observed 
$\mathrm{Hg}(0)$ reaction rate increase in "activated" metal sulfides has not been established. To date, our research efforts have not attempted to address this specific question. None the less, we incorporated the use oxalic acid treated samples in our test matrixes. When the activation process was performed, a metal sulfide/oxalic acid slurry was prepared and subsequently filtered, collected, and dried prior to exposure to $\mathrm{Hg}(0)$ vapor. The products of the metal sulfide $\mathrm{Hg}(0)$ vapor reactions were determined using XRD in order to gain a better understanding reaction mechanisms. The experimental goals for $\mathrm{Hg}^{2+}$ sorption in aqueous solutions were similar as those for gas phase $\mathrm{Hg}(0)$ sorption.

\section{Exposure of Metal Sulfides to $\mathrm{Hg}(0)$ Vapors}

For these experiments, metal sulfide powder samples were weighed and placed into a small recrystallization dish. The dish was then placed into a closed container (desiccator) containing elemental $\mathrm{Hg}$. The desiccator was placed in a laboratory oven at 70 or $90^{\circ} \mathrm{C}$ for up to 80 days. The concentration of $\mathrm{Hg}(0)$ in the desiccator was calculated to be $\sim 1500 \mathrm{ppm} @ 70^{\circ} \mathrm{C}$ and $\sim 2500 \mathrm{ppm} @ 90^{\circ} \mathrm{C}$. The samples were periodically removed from the desiccator and weighed. When a sample's reaction with $\mathrm{Hg}(0)$ was complete, i.e., constant weight between measurements, phase composition analysis was performed using powder XRD.

The observed reactions of $\operatorname{Hg}(0)$ with the copper, silver and gold sulfides are listed below (Reactions 1-4). In the case of copper and gold sulfides the reaction with elemental mercury appears to be entirely a redox process. These reactions produce cinnabar and a reduced form of the leading metal, i.e., $\mathrm{Cu}(\mathrm{I}), \mathrm{Au}^{0}$. In Reaction 1 , the chalcocite $\left(\mathrm{Cu}_{2} \mathrm{~S}\right)$ is produced whereas in Reactions 3 and 4 elemental gold is formed. Acanthite $\left(\mathrm{Ag}_{2} \mathrm{~S}\right)$ reacted in a one-to-one molar ratio with $\mathrm{Hg}(0)$ to form a mercury silver amalgam mercurian (Ag:0.69, $\mathrm{Hg}: 0.32)$, imiterite $\left(\mathrm{Ag}_{2} \mathrm{HgS}_{2}\right)$, and a trace amount of cinnabar( $\left.\mathrm{HgS}\right)$, Reaction 2. Reaction 2 was derived with the aid of Rietveld analysis, applied to the $A g_{2} \mathrm{~S} / \mathrm{Hg}(0)$ reaction product's XRD powder pattern. For this analysis, the experimentally observed 2:1 Ag: $\mathrm{Hg}$ reaction ratio was used as a mass balance constant and the stoichiometry of the $\mathrm{Ag}-\mathrm{Hg}$ amalgam adjusted appropriately. Our estimated $\mathrm{Ag}-\mathrm{Hg}$ amalgam composition $(\mathrm{Ag}: 0.69, \mathrm{Hg}: 0.32)$ is within $1.5 \%$ of that reported by Grundmann, et al., PDF\# 43-1465. ${ }^{30}$ Unaccounted for in the Rietveld analysis is $\sim 65 \%$ of originally present sulfur . It is believed that free sulfur is a product of the reaction, however, the primarydiffraction-line for sulfur was outside the XRD scan-range. Additional XRD scans and chemical analyses are needed before the fate of the sulfur can be definitively established. At this time, we can conclude that the Ag-Hg amalgam formed is "typical" and $~ 13$ times more prevalent than the silver mercury sulfide product "imiterite".

In all four cases, it appears that $\mathrm{Hg}(0)$ uptake occurs by chemical reaction with the starting metal sulfide and not via physisorption processes such as intercalation..$^{31,32}$

$$
\begin{aligned}
& 2 \mathrm{CuS}+\mathrm{Hg}(0)) \rightarrow \mathrm{HgS}+\mathrm{Cu} 2 \mathrm{~S} \\
& \mathrm{Ag} 2 \mathrm{~S}+\mathrm{Hg}(0)) \rightarrow 0.18 \mathrm{Ag} 2 \mathrm{HgS} 2+2.4(\mathrm{Ag}: 0.7, \mathrm{Hg}: 0.3)+0.02 \mathrm{HgS}+0.65 \mathrm{~S} \\
& \mathrm{Au} 2 \mathrm{~S}+\mathrm{Hg}(0)) \rightarrow \mathrm{HgS}+2 \mathrm{Au} 0 \\
& \mathrm{Au} 2 \mathrm{~S} 3+3 \mathrm{Hg}(0) \rightarrow 3 \mathrm{HgS}+2 \mathrm{Au} 0
\end{aligned}
$$

$\mathrm{CuS}$ and $\mathrm{Ag}_{2} \mathrm{~S}$ were the only INEEL synthesized samples that removed $\mathrm{Hg}(0)$ vapors from air. The commercial $\mathrm{CuS}, \mathrm{Ag}_{2} \mathrm{~S}, \mathrm{Au}_{2} \mathrm{~S}$, and $\mathrm{Au}_{2} \mathrm{~S}_{3}$ also adsorbed $\mathrm{Hg}(0)$. Our initial studies using commercial grade covellite revealed the redox properties associated with $\mathrm{Hg}(0)$ removal. The redox potentials of the constituent metals are listed in Table $2 .{ }^{33}$ Although the standard potentials listed are for the metals in acidic solutions, the values correlate with the observed $\mathrm{Hg}(0)$-sorption results. That is, only those metal 
Table 2. Standard potentials for the reduction of the metal ions in acidic solution.

\begin{tabular}{cc}
\hline Reduction Couple & $\mathrm{EMF}^{0}$ \\
\hline $\mathrm{Mn}^{2+} / \mathrm{Mn}^{0}$ & -1.05 \\
$\mathrm{Fe}^{2+} / \mathrm{Fe}^{0}$ & -0.44 \\
$\mathrm{Zn}^{2+} / \mathrm{Zn}^{0}$ & -0.25 \\
$\mathrm{Ga}^{3+} / \mathrm{Ga}^{0}$ & -0.52 \\
$\mathrm{Cu}^{2+} / \mathrm{Cu}^{1+}$ & 0.17 \\
$\mathrm{Ag}^{1+} / \mathrm{Ag}^{0}$ & 0.79 \\
$\mathrm{Au}^{1+} / \mathrm{Au}^{0}$ & 1.68 \\
$\mathrm{Au}^{3+} / \mathrm{Au}^{0}$ & 4.26 \\
\hline
\end{tabular}

sulfide powders containing metals with a positive reduction potential were effective, i.e., $\mathrm{Cu}^{+2}, \mathrm{Ag}^{+1}$, $\mathrm{Au}^{+1}, \mathrm{Au}^{+3}$.

After 80 days at $90^{\circ} \mathrm{C}$ in the presence of $\sim 2500 \mathrm{ppm} \mathrm{Hg}(0)$, the "pure" commercial covellite sample was determined to be fully reacted; i.e., consisting solely of cinnabar and chalcocite and being $51 \mathrm{wt} \%$ $\mathrm{Hg}$. Figure 10 shows $\mathrm{Hg}(0)$ uptake with time; the complete covellite-to-cinnabar reaction of commercial and activated $\mathrm{CuS}$ samples is evident. The INEEL-synthesized mediated covellite sample gained $80 \%$ of weight needed for full $\mathrm{Hg}(0)$ saturation of a pure covellite. Rietveld analysis performed on INEEL-synthesized mediated covellite sample established that the sample was not pure covellite, but $\sim 80$ mole $\%$ covellite and $\sim 20$ mole $\%$ copper(I) sulfide "chalcocite". Thus, our phase composition estimate is consistent with the observed $\mathrm{Hg}(0)$ capacity. Namely, $100 \%$ of the covellite present reacts according to Reaction 1.

Table 3 shows $\mathrm{Hg}(0)$ capacity and sorption rate for $\mathrm{CuS}, \mathrm{Ag}_{2} \mathrm{~S}, \mathrm{Au}_{2} \mathrm{~S}$ and $\mathrm{Au}_{2} \mathrm{~S}_{3}$. Rates given are for the initial three days of $\mathrm{Hg}(0)$ exposure. For oxalic acid treated commercial covellite "activated", the reactive adsorption rate increased three fold 0.2 to $0.6 \mathrm{moles}(\mathrm{Hg}) /(\operatorname{mole}(\mathrm{MS}) *$ day $)$. The increased reactivity resulting from the oxalic acid activation of commercial covellite was shown to be reversible. Namely, when activated covellite samples were washed with water and dried, the $\operatorname{Hg}(0)$ reaction rate and final weight gain (wt \%) was found to be identical to those of inactivated covellite samples. For INEEL synthesized covellite, $\operatorname{Hg}(0)$ uptake was five times more rapid than observed for the commercial covellite sample. Considering the unreactive chalcocite component, $\sim 20 \%$, it should be possible to adsorb $\sim 0.15$ moles $\mathrm{Hg}(0) /(\operatorname{mole}(\mathrm{CuS}) *$ day $)$ at $90^{\circ} \mathrm{C}$ using single phase INEEL synthesized covellite. Since these experiments were performed, it has been established that calcining CTAB mediated precipitate at $350^{\circ} \mathrm{C}$ in $4 \% \mathrm{H}_{2} \mathrm{~S} / \mathrm{Ar}$ yields single-phase covellite. Reactive adsorption results for this material has not yet been obtained.

Samples of commercial $\mathrm{Ag}_{2} \mathrm{~S}$ reacted with $\mathrm{Hg}(0)$ in a 1:1 molar ratio. The time dependent adsorption data is shown in Figure 11. The CTAB mediated sample exhibited the most rapid reactive adsorption rate, 0.11 moles $\mathrm{Hg}(0) /\left(\operatorname{mole}\left(\mathrm{Ag}_{2} \mathrm{~S}\right) *\right.$ day $)$ at $70^{\circ} \mathrm{C}$, Table 3 . This uptake rate is $9 \mathrm{X}$ and $5 \mathrm{X}$ greater that observed for commercial $\mathrm{Ag} 2 \mathrm{~S}$ and No-CTAB INEEL synthesized $\mathrm{Ag}_{2} \mathrm{~S}$, respectively. All three samples showed nearly identical saturation levels, $\sim 98.3 \%$, after 80 days of $\sim 1500 \mathrm{ppm} \mathrm{Hg}(0)$ exposure. 


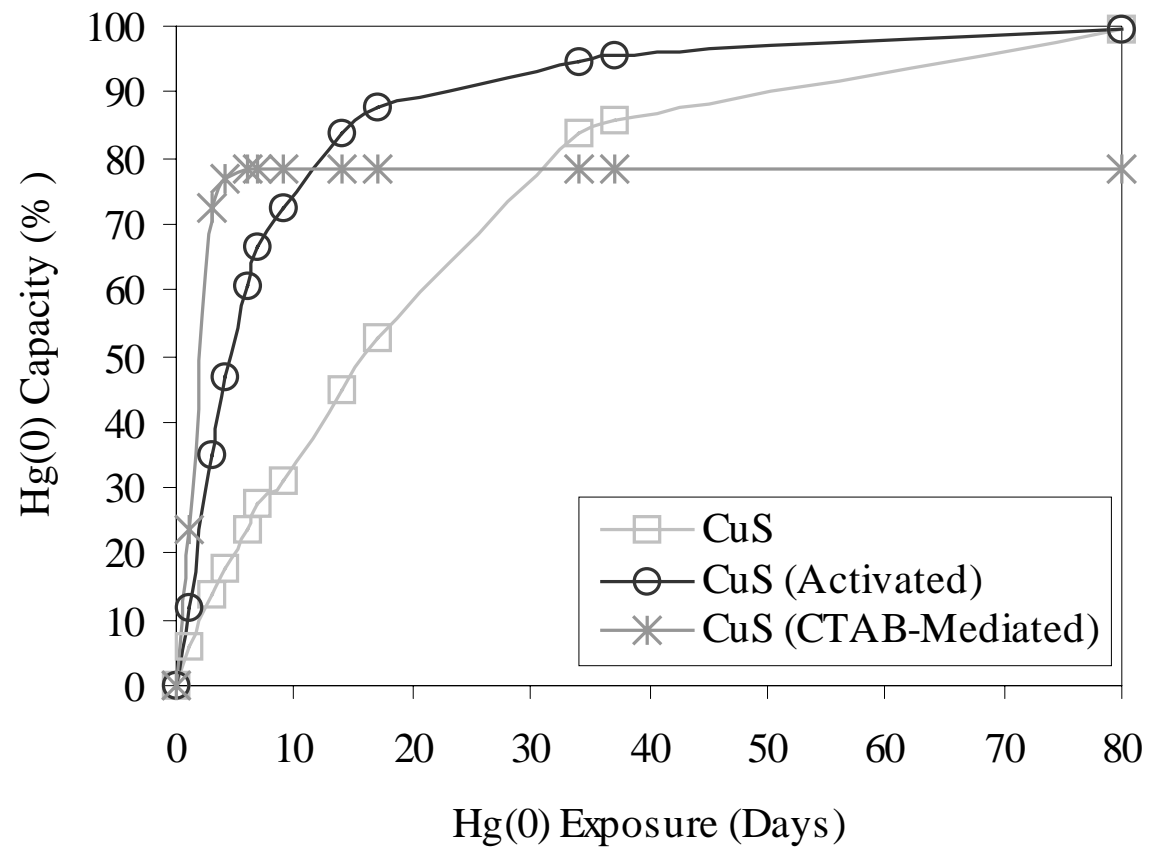

Figure 10. Percent of $\operatorname{Hg}(0)$ capacity with time for copper sulfide samples. $\mathrm{T}=90^{\circ} \mathrm{C}$.

Table 3. Reactive adsorption data for $\mathrm{CuS}, \mathrm{Ag}_{2} \mathrm{~S}, \mathrm{Au}_{2} \mathrm{~S}$ and $\mathrm{Au}_{2} \mathrm{~S}_{3}$ samples exposed to $\mathrm{Hg}(0)$. T $=70^{\circ} \mathrm{C}$ for $\mathrm{Ag}$ and $\mathrm{Au} ; \mathrm{T}=90^{\circ} \mathrm{C}$ for $\mathrm{Cu}$.

\begin{tabular}{|c|c|c|c|}
\hline Metal Sulfide & $\begin{array}{c}\mathrm{Hg}(0) \text { Capacity } \\
\text { moles }(\mathrm{Hg}) \\
\text { mole }(\mathrm{MS}) \\
\end{array}$ & $\begin{array}{c}\mathrm{Hg}(0) \text { Sorption Rate } \\
\text { moles }(\mathrm{Hg}) \\
\text { mole }(\mathrm{MS})^{*} \text { Day }\end{array}$ & $\begin{array}{l}\mathrm{Hg}(0) \text { Uptake } \\
\text { (\% Capacity) } \\
\text { "Days@Temp" }\end{array}$ \\
\hline $\mathrm{CuS}(\mathrm{CTAB})$ & 0.5 & 0.121 & 80 “80@90 C” \\
\hline $\begin{array}{l}\text { CuS(Commercial,) } \\
\text { Activated }\end{array}$ & 0.5 & 0.059 & 100 “80@90C” \\
\hline $\mathrm{CuS}($ Commercial $)$ & 0.5 & 0.023 & 100 “80@90 C” \\
\hline $\mathrm{Ag}_{2} \mathrm{~S}(\mathrm{CTAB})$ & 1.0 & 0.086 & 98.2 "80@70C" \\
\hline $\mathrm{Ag}_{2} \mathrm{~S}$ (No-CTAB) & 1.0 & 0.017 & 98.4 “80@70C" \\
\hline $\mathrm{Ag}_{2} \mathrm{~S}($ Commercial $)$ & 1.0 & 0.009 & 98.4 “80@70C” \\
\hline $\mathrm{Au}_{2} \mathrm{~S}($ Commercial $)$ & 1.0 & 0.15 & 100 “12 @ 70C" \\
\hline $\mathrm{Ag}_{2} \mathrm{~S}_{3}($ Commercial $)$ & 3.0 & 0.46 & 89.5 “10@70C" \\
\hline
\end{tabular}




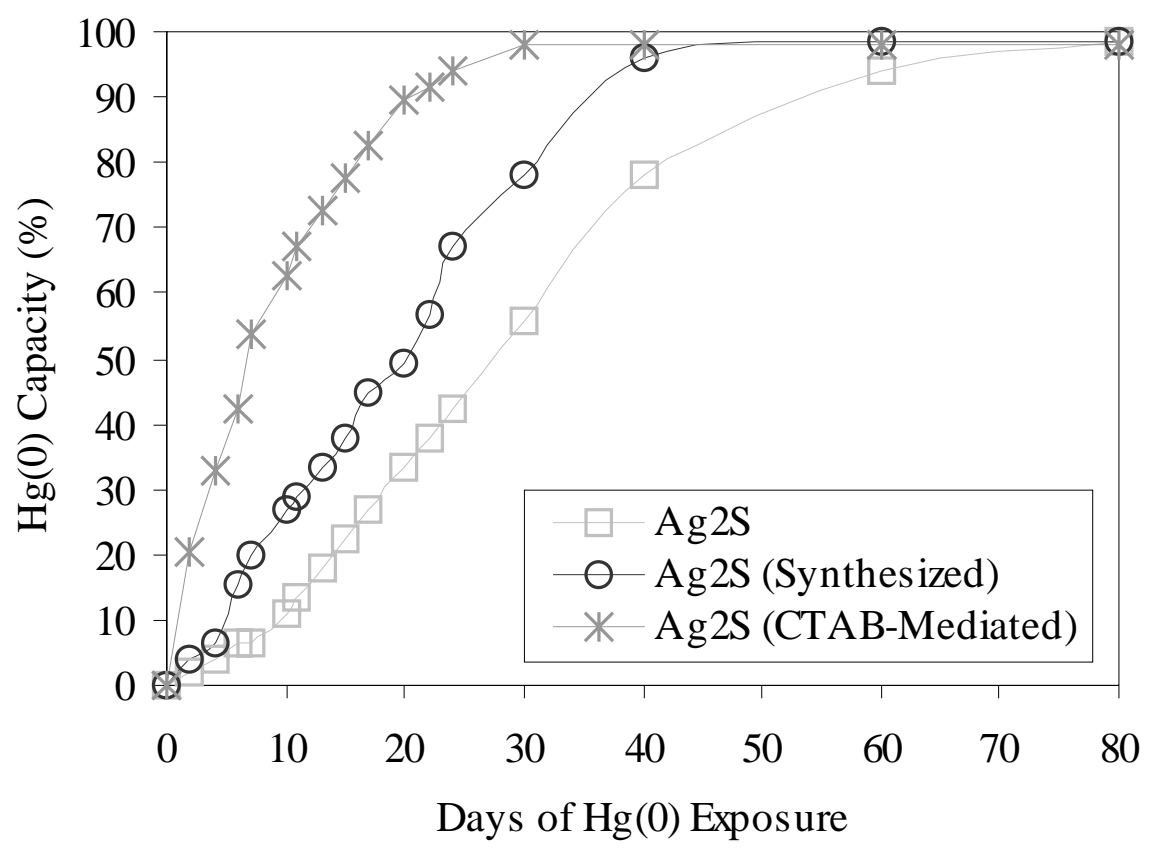

Figure 11. Percent $\operatorname{Hg}(0)$ capacity with time for silver sulfide samples. $\mathrm{T}=70^{\circ} \mathrm{C}$.

Figure 12 compares the rates of $\operatorname{Hg}(0)$ reactive adsorption rate for each of the commercial grade metal sulfides at $70^{\circ} \mathrm{C}$. The rates of $\mathrm{Hg}(0)$ adsorption correlate with the Electro Motive Force (EMF) values reported in Table 2 . The rate of $\mathrm{Hg}(0)$ adsorption increase proportionately with the positive reduction potential for each metal sulfide studied; even though silver sulfide does react with $\mathrm{Hg}(0)$ solely by a redox process, i.e., a portion of the $\mathrm{Ag}^{+1}$ is retained in the imiterite formed.

\section{Capture of $\mathrm{Hg} 2+$ from Aqueous Solutions}

For this series of experiments, a five-fold excess of each binary metal sulfide was stirred in an $\mathrm{HNO}_{3}$ acidified 20ppm $\mathrm{Hg}^{2+}$ "standard" solution. In the case of the ternary chalcopyrite, a 35ppm $\mathrm{Hg}^{2+}$ "standard" solution was used. All of the metal sulfides were stirred in their respective standard solutions for 2 hours, the particulate product removed via filtration, and the $\mathrm{Hg}^{2+}$ depleted standard solution analyzed using atomic absorption spectroscopy (AAS). The results of the $\mathrm{Hg}^{2+}$ sorption study are shown in Table 4. No difference between mediated and non-mediated metal sulfides was observed, thus, Table 4 shows only the non-mediated sample data. Additionally, oxalic acid activation did not alter the $\mathrm{Hg}^{2+}$ reactivity.

The most effective metal sulfide evaluated was chalcocite $\left(\mathrm{Cu}_{2} \mathrm{~S}\right)$; removing $\mathrm{Hg}^{2+}$ from solution to $<1 \mathrm{ppm}$, below the detection limit of the AAS instrument. Additional $\mathrm{Hg}^{2+}$ adsorption experiments were performed with the $\mathrm{Cu}_{2} \mathrm{~S}$ because of its observed ability to remove $\mathrm{Hg}^{2+}$ from solutions as compared with the other metal sulfides. Studies on the rate and efficiency of $\mathrm{Hg}^{2+}$ removal showed that a $2 \mathrm{X}$-mole excess of $\mathrm{Cu}_{2} \mathrm{~S}$ would remove nearly all of the $\mathrm{Hg}^{2+}$ after stirring in the solution for only 1.5 hours. The powder XRD pattern of the resulting solid was found to be amorphous. Investigation of the reaction $\mathrm{Cu}_{2} \mathrm{~S} / \mathrm{Hg}^{2+}$ mechanism and characterization of the reaction product is planned for $\mathrm{FY}-00$. 


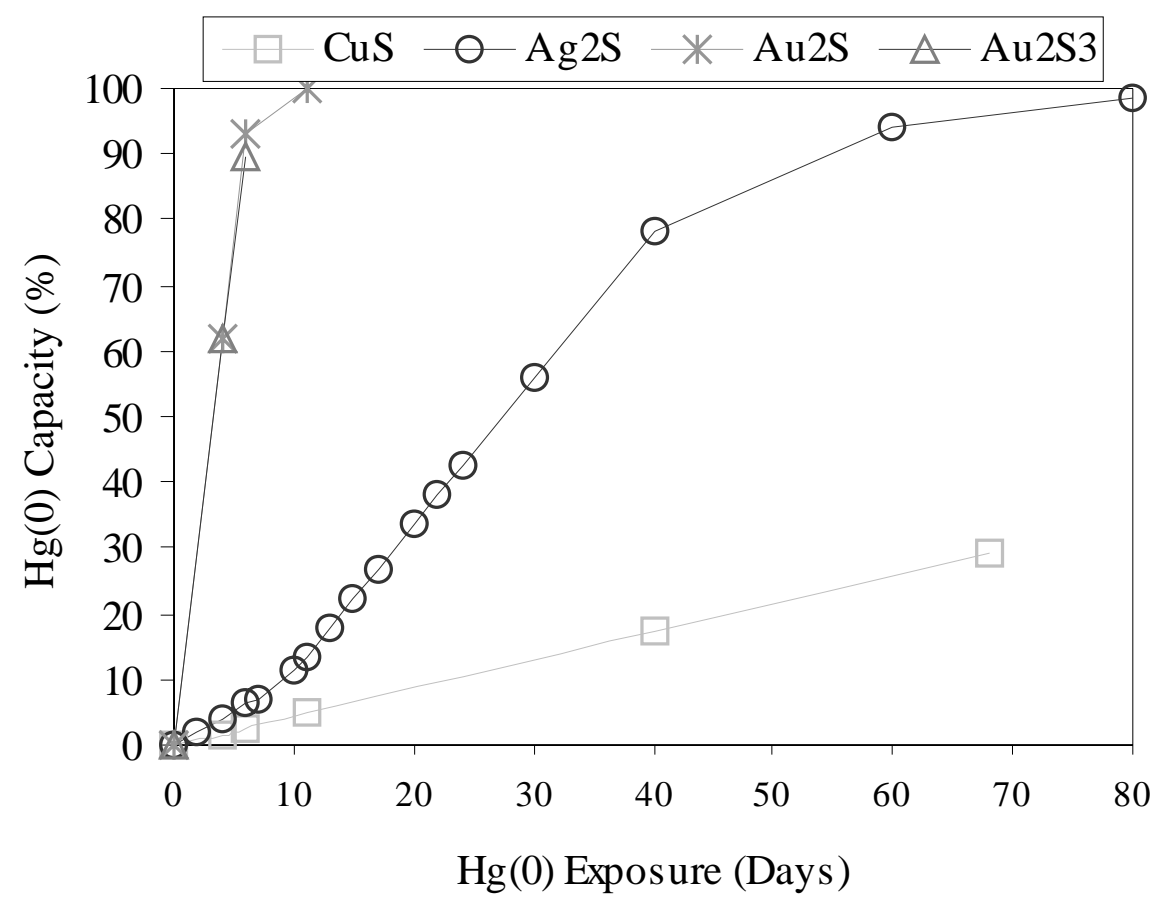

Figure 12. Percent $\operatorname{Hg}(0)$ capacity with time for commercial metal sulfides. $\mathrm{T}=70^{\circ} \mathrm{C}$.

Table 4. $\mathrm{Hg}^{2+}$ removal studies using mediated metal sulfides.

\begin{tabular}{|c|c|c|}
\hline Metal Sulfide & $\begin{array}{c}\text { Initial } \mathrm{Hg}^{2+} \mathrm{Conc} \\
(\mathrm{ppm})\end{array}$ & $\begin{array}{c}\text { Final } \mathrm{Hg}^{2+} \mathrm{Conc} \\
(\mathrm{ppm})\end{array}$ \\
\hline $\mathrm{MnS}$ & 20 & 13 \\
\hline $\mathrm{FeS}$ & 20 & 9 \\
\hline $\mathrm{NiS}$ & 20 & 20 \\
\hline $\mathrm{CuS}$ & 20 & 14 \\
\hline $\mathrm{ZnS}$ & 20 & 18 \\
\hline $\mathrm{Ga}_{2} \mathrm{~S}_{3}$ & 20 & 18 \\
\hline $\mathrm{Ag}_{2} \mathrm{~S}$ & 20 & 18 \\
\hline $\mathrm{In}_{2} \mathrm{~S}_{3}$ & 20 & 5 \\
\hline $\mathrm{CuFeS}_{2}$ & 35 & 9 \\
\hline $\mathrm{Cu}_{2} \mathrm{~S}$ & 20 & $<1$ \\
\hline
\end{tabular}




\section{TEM Analysis Tool}

Research Systems Inc. Software package IDL was used to create a graphical user interface (GUI) for performing analysis of scanned images of TEM negatives. A Microtek ScanMaker 5 transparency scanner having 1000 dots per inch (dpi) optical resolution was used to create 8 or 16 bit grayscale TIF files from TEM negatives. The TIF files were subsequently imported to the IDL TEM routine that we call TEM- Image Analysis Tool (TEM-IAT). A screen capture of the GUI is shown in Figure 13. TEMIAT allows a user to determine the d-spacing's for a TEM ring or spot type diffraction pattern, measure feature' dimensions of bright-field or dark-field images, and place a micron bar on the image. The routine was found to be much better than the traditional methods of using calipers or a ruler to index a diffraction pattern. Namely, the use of a zoom window and a profile graph allowed the point or radius of greatest intensity "exposure" to be precisely determined and marked.

\section{Multi-Phase Reactive Flow CFD Model Development}

Spray coating/drying techniques are commonly used in the materials processing field to fabricate particulate, polymeric, and ceramic-precursor coatings as well as spherical particles. In order for our nanocrystalline metal sulfide powders to be utilized in flowing air-streams and liquid feed-streams, we must make porous bulk and/or coated media that, once reacted with mercury, is fully recoverable. The spray coating and drying processes is well suited to this task. Critical however, is our ability to establish a sturdy porous matrix that maximizes reactivity with the target analyte, i.e. $\mathrm{Hg}(0) \mathrm{or}_{\mathrm{Hg}}{ }^{+2}$.

Coating/particle morphology is primarily controlled via the evaporation rate of a carrier solvent from a consolidating product phase. In order to obtain a homogeneous product having controlled porosity, one should first be able to generate a near monosize-droplet spray distribution, create a uniform thermal environment within the coating chamber, and establish/maintain a uniform flow-field for droplet entrainment. This chore is not easily accomplished in an experimental fashion and thus, we have chosen to develop a process-modeling tool to aid us. Namely, a multi-phase fluid dynamics model that allows process variables, i.e. temperature, gas flow rate, droplet size and distribution, spray volume, vessel
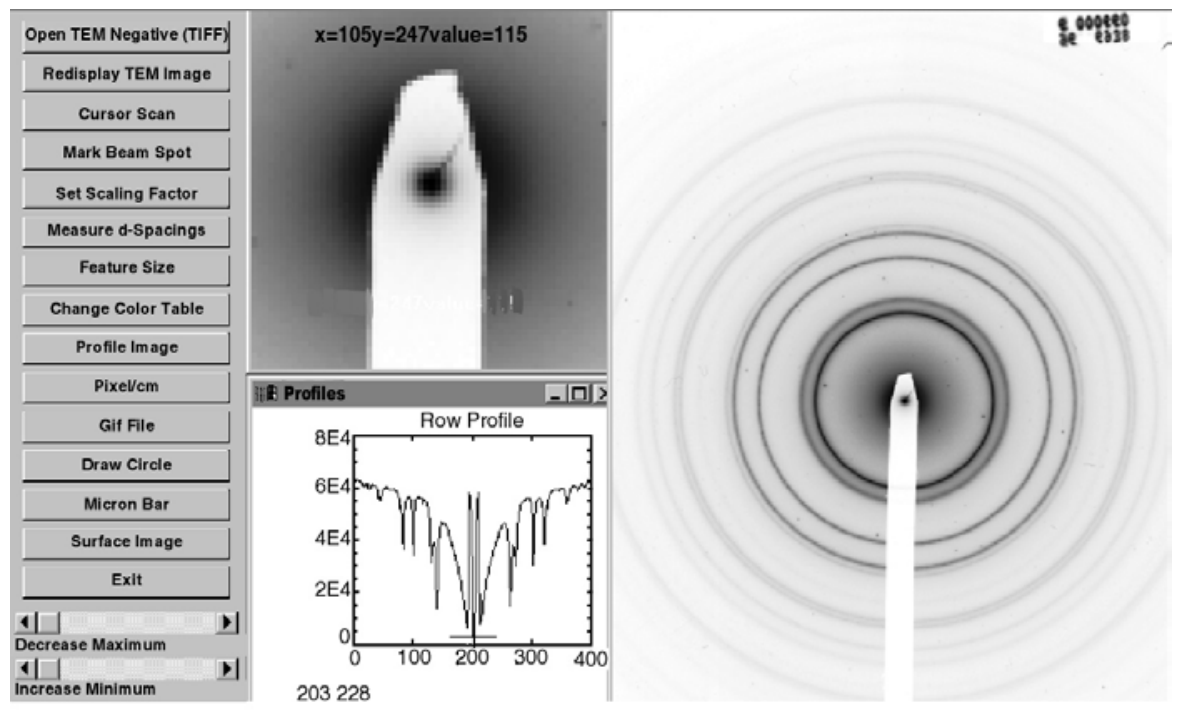

GV99 0575

Figure 13. Screen-capture of the IDL routine TEM-IAT used for indexing and image analysis of scanned TEM negatives. 
geometry, etc., to be parametrically studied. This approach should facilitate the development of robust porous media sorbents composed of nanocrystalline metal sulfides.

The Computational Fluid Dynamics (CFD) code under development will facilitate simulations of the ultrasonic nozzle spray generation process, the spray drying process, and fluidized bed coating schemes. The goal of this research task is the development an efficient, high-resolution, pressure based scheme for simulating multi-phase reactive flows on unstructured grids. Upon completion of this research effort, a semi-implicit pressure-based CFD code would be available for INEEL researchers. Having an in house code where modifications and additions can be performed has a distinct advantage over commercially available codes. All too often the physics needed to simulate the multi-phase processes we investigated are not implemented in commercial codes. Also, contracting costs for commercial code modifications can be prohibitive, with the rights to the modifications being retained by the code's owner. A state-of-the-art in house code could be strictly controlled as to modifications and additions (version control). Also, in house training would be considerably cheaper than commercial user group classes along with a proper user's manual and pre and post-processing tools.

The research centered on deriving a semi-implicit version of a two-step explicit scheme. The pressure, momentum, and density variables are treated in an implicit fashion, hence semi-implicit. The three implicit variables are coupled by substituting the momentum equation into the conservation of mass equation to eliminate momentum as an unknown. Density is then put in terms of pressure by employing the equation of state. These substitutions result in a single second-order differential equation in terms of pressure, hence pressure based scheme. This semi-implicit treatment has two advantages over explicit schemes. The first advantage is that the acoustic component from the time step size limit is removed. The spatial stiffness due to slow flows on small computational cells is then eliminated. The second advantage is that the pressure obtained with this semi-implicit treatment corrects the momentum to satisfy continuity requirements. This allows incompressible flows to be simulated with the compressible flow equations. This type of scheme can then be used to simulate any flow from incompressible to supersonic.

The pressure-based scheme proposed for this research is adapted from an explicit scheme developed at the Naval Research Laboratory (NRL) and the University of Wales. This explicit scheme, called Finite Element Method Flux-Corrected Transport (FEM-FCT), employs a two-step (predictorcorrector) time integration based upon the second-order Taylor-Galerkin temporal discretization. The semi-implicit pressure based treatment described above is applied to the second (corrector) step. Linear triangular elements are used for spatial discretization to provide second-order spatial accuracy and to be able to fit the computational domain to complex geometries. Flux-Corrected Transport (FCT) is a highresolution method, which limits the convective fluxes to avoid spurious oscillations in the solution near large changes in solution gradients. For example in high-speed flows where shocks are present, oscillations in the solution variables are generated by the second-order space discretization. FCT suppresses these oscillations and maintains the positivity of the conserved variables, density and total energy. Large changes in density can also occur in multi-phase simulations. Flows with liquid droplets entrained in a gas phase typically have density variations on the order of three magnitudes, even though the entire flow field may be nearly incompressible (slow flows).

There is an important novel aspect to this research. The typical semi-implicit pressure based scheme requires an iterative solution to maintain second order temporal accuracy and continuity requirements. For stability reasons, the typical scheme does not solve the total energy equation until the second-order differential pressure equation is solved. However, for a compressible gas, pressure is a function of the density and internal energy. The typical scheme uses an iterative process between the differential pressure equation and the total energy equation until pressure is converged. The new pressure based scheme is able to accurately convect total energy in an explicit manner before the differential pressure equation is solved. The energy effects on the equation of state can then be accounted for in the 
differential pressure equation. The iterative process is then not required. This aspect is highly advantageous for computational simulations involving highly compressible systems having high-energy deposition rates, reactions, or phase change. This gain in efficiency is also apparent when marching the solution to steady state with quicker convergence.

In the Second Quarter of FY-99, one problem identified was what appeared to be a boundary condition problem. Namely, a de-coupling between the pressure and density at the boundaries was creating changes in the outward normal direction (curved surfaces). The actual origin of the de-coupling has since been established and resolved. Namely, the de-coupling was found to be a consequence of an inconsistent calculation of domain boundary normals, defined at boundary cell faces, and boundary nodes. This inconsistency can only be found in schemes that incorporate weak formulations (integral) based on cell-vertex (nodes) discretizations (i.e., the Finite Element Method and the integral form of the Finite Volume Method). Most pressure-based schemes employ a cell centered differencing where the solution variables are defined at the centroid of the cell. Therefore, the solution is never actually computed on the domain boundary, only adjacent to the boundary. The pressure-based scheme being researched here incorporates a nodal discretization where the solution variables are computed at the cell vertices; some of which coincide with the domain boundary. There are certain advantages (and disadvantages) to using a nodal scheme that will not be discussed here.

To solve the problem, the face-length averaging procedure, used to compute the boundary node normals, was replaced by an integral-form consistent with that used to compute the boundary face normals. The correct integral form is obtained by integrating over all the elements common to a specific boundary node. This procedure gave slightly different boundary node normals compared to those obtained from the face-length-averaging scheme, yet, the change had a significant impact on the solution at curved boundaries. Thus, at this time, the "consistent " normal calculation equations are being integrated comprehensively throughout the semi-implicit pressure-based CFD code. When integration and validation are accomplished, modeling of the ultrasonic nozzle spray process can commence.

\section{ACCOMPLISHMENTS}

Nanocrystalline metal sulfide powders, thin films, and infiltrated porous ceramics were successfully prepared using aqueous base precipitation and thiourea complexation techniques. The surfactant CTAB was employed as a particle-size-mediating agent. Lamellar organic/inorganic hybrid structures were observed with the CTAB/copper sulfide system.

Experiments were performed to determine the reactive adsorption capabilities of the metal sulfides to vapor- and aqueous-phase mercury. The reaction of covellite with $\mathrm{Hg}(0)$ to form cinnabar was shown to proceed to completion in 80 days at $90^{\circ} \mathrm{C}$. The rate of mercury sorption was found to increase three fold when an oxalic acid pretreatment "activation" was applied to commercial CuS. The nanocrystalline $\mathrm{CuS}$, prepared using $\mathrm{CTAB}$ exhibited a five fold $\mathrm{Hg}(0)$ uptake rate increase when compared to commercial "unactivated" covellite. The copper and gold sulfides adsorb mercury by a redox process, resulting in cinnabar. Silver sulfide gives both redox and non-redox products.

Metal sulfides were also tested for the removal of $\mathrm{Hg}^{2+}$ from aqueous solutions. Most of the metal sulfides tested removed some $\mathrm{Hg}^{2+}$ from solution. Chalcocite, $\mathrm{Cu}_{2} \mathrm{~S}$, was the most effective, with a five fold excess removing $>95 \%$ of $\mathrm{Hg}^{2+}$ from a standard $20 \mathrm{ppm}$ solution. Formation of cinnabar was not observed in any of the post-reaction XRD powder patterns; acquired for all metal sulfides tested. The solid reaction products were found to be primarily amorphous in nature. Thus, an complete understanding of the metal sulfide $/ \mathrm{Hg}^{2+}$ reaction chemistry has not been established at this time. 
Research Systems Inc. Software package IDL was used to create a graphical user interface (GUI) for performing analysis of scanned images of TEM negatives. The TEM- Image Analysis Tool (TEMIAT) provides a computer based means of determining the d-spacings for a TEM ring or spot type diffraction pattern, measure feature' dimensions of bright-field or dark-field images, and allows micron bar annotation.

Development of a CFD code having a pressure based scheme for simulating multi-phase reactive flows on unstructured grids was explored in FY-99. The code, when implemented, will be used for multiphase simulations of the ultrasonic nozzle spray generation process, the spray drying process, and fluidized bed coating schemes.

\section{REFERENCES}

1. Final Standards for Hazardous Air Pollutants for Hazardous Waste Combustors, U.S. EPA, RIN 2050-AEO1, 1999.

2. E. J. Granite, H. W. Pennline and R. A. Hargis, Sorbents for Mercury Removal from Flue Gas, DOE/FETC/TR-98-01, U.S. DOE, Federal Energy Technology Center, Pittsburgh, PA, (1998).

3. C. D. Livengood, H. S. Huang, M. H. Mendelsohn and J. M. Wu, Development of Mercury Control Enhancements for Flue-Gas Cleanup Systems, Proc. EPRI/DOE International Conference of Managing Hazardous and Particulate Air Pollutants, Toronto, Ontario, Canada, (1995).

4 DOE Environmental Management Research and Development Program Plan, November 1998

5. P. Kruus, Chemicals in the environment, Chapter VII, Metal Pollution, Polyscience Publications, (1991).

6. L. N. Oji, Mercury Disposal via Sulfur Reactions, Jour. Env. Eng. Vol 124 No. 10 (1998) 945.

7. A. Sugier and F. Villa, Process for Removing Mercury from a Gas or a Liquid by absorption on a Copper Sulfide containing Mass, U.S. Patent 4,094,777, (1978).

8. H. Toulhoat, et al., Process for Preparing and Regenerating a Copper Containing Mercury Collecting Mass, U.S. Patent 4,902,662, (1990).

9. C. Cameron, et al., Method of Preparing a Solid Mass for Mercury Recovery, U.S. Patent 5,350,728, (1994).

10. T. Y. Yan, A Novel Process of Hg Removal from Gases, Ind. Eng. Chem. Res., 33 (1994) 3010-3014.

11. T. Y. Yan, Method for removing Mercury from Hydrocarbon Oil by High Temperature Reactive Adsorption, U.S. Patent 4,909,926, (1990).

12. A. E. Gorenbain, A. Rebitser, D. Paul and Y. F. Linov, The Method of Producing the Sorbents for Scrubbing the Mercury From Gases, Russian Federation Patent 930445418/26, (1993).

13. A. E. Gorenbain, A. Rebitser, D. Paul, Y. F. Linov, The Method of Scrubbing the Mercury from Gases, Russian Federation Patent 930445416/26, (1993). 
14. C. J. Cameron and Y. Barthel, Proc. Mercury Removal from Wet Natural Gas, Seventy-Second GPA Annual Convention, New Orleans, Louisiana, (1994) 256.

15. C. Nascu, I. Pop, V. Ionescu, E. Indrea and I. Bratu, Spray pyrolysis deposition of CuS thin films, Materials Letters, 32 (1997) 73-77.

16. T. Sugimoto, S. Chen and A. Muramatsu, Synthesis of uniform particles of CdS, ZnS, PbS and CuS from concentrated solutions of the metal chelates, Colloids Surfaces A: Physicochem. Eng. Aspects, 135 (1998) 207.

17. I. Wuled Lenggoro, Y. C. Kang, T. Komiya, K. Okuyama and N. Tohge, Formation of Submicron Copper Sulfide Particles Using Spray Pyrolysis Method, Jpn. J. Appl. Phys., 37 (1998) L288.

18. K.Okutama, I. Wuled Lenggoro and N. Tagami, Preparation of $\mathrm{ZnS}$ and CdS fine particles with different particles sizes by a spray-pyrolysis method, J. Mat. Sci., 32 (1997) 1229-1237.

19. I. Grozdanov, C. K. Barlingay and S. K. Dey, Novel applications of chemically deposited CuxS thin films, Materials Letters, 23 (1995) 181-185.

20. S. G. Dixit, A. R. Mahadeshwar and S. K. Haram, Some aspects of the role of surfactants in the formation of nanoparticles, Colloids Surfaces A: Physicochem. Eng. Aspects, 133 (1998) 69-75.

21. M. T. Anderson and P. Newcomer, Molecular Approach to Mesoporous Metal Sulfides, Mat. Res. Soc. Symp. Proc., 371 (1995) 117.

22. C. T. Kresge, M. E. Leonowicz, W. J. Rothe, J. C. Vartuli and J. S. Beck, Orderd mesoporous molecular sieves synthesized by a liquid-crystal template mechanism, Nature, 359 (1992) 710-712.

23. G. D. Stucky, et al., Organization of Organic Molecules with Inorganic Molecular Species into Nanocomposite Biphase Arrays, Chem. Mater., 6 (1994) 1176-1191.

24. P. Yang, D. Zhao, D. I. Margolese, D. R. Chmelka and G. D. Stucky, Generalized syntheses of large-pore mesoporous metal oxides with semicrystalline frameworks, Nature, 396 (1998) $152-155$.

25. J. Liu, et al., Hybrid Mesoporous Materials with Functionalized Monolayers, Adv. Mater., 10 No. 2 (1998) 161-165.

26. P. V. Braun, P. Osenar and S. I. Stupp, Semiconducting superlattices templated by molecular assemblies, Nature, 380 (1997) 325-328.

27. R. A. Young, The Rietveld Method, Oxford University Press, ISBN 019 855912 7, (1993).

28. L. DeQuan, J. Shi, K Springer and B. I. Swanson, Cyclosdextrin-Based Surface Acoustic Wave Chemical Microsensors, Proc. Nuclear and Hazadous Waste Management Spectrun '96, American Nuclear Society, IL, (1996).

29. J. Ganor and A. C. Lasaga, The effects of oxalic acid on kaolinite dissolution rate, Mineralogical Magazine, 58A (1994) 315. 
30. Powder Diffraction File, Release 1998 Data Sets 1-48 pus 70-85, International Centre for Diffraction Data, Newton Square, PA.

31. E. Gash, A. L Spain, L. M. Dysleski, C. J. Flaschenreim, A. Kalaveshi, P. K. Dorhout and S. H. Strauss, Environ. Sci. Technol., 32 (1998) 1007.

32. P. J. Martellaro, G. A. Moore, A. Gorenbein, E. H. Abbott and E. S. Peterson, Environmental Application of Mineral Sulfides for Removal of Gas-Phase $\mathrm{Hg}(0)$ and Aqueous $\mathrm{Hg} 2+$, Sep. Sci. and Tech., (1999), Submitted.

33. W. M. Latimer and J. H. Hildebrand, Refer. Book of Inor. Chem., 3rd Ed., The Macmillian Co. (1959) Appendix II. 


\section{Computational Simulation of Mechanical and Chemical Systems}

For any simulation problem, the ability to predict the outcome of a proposed solution is an essential ingredient in making an informed decision concerning future research for or deployment of that solution. Recent advances in computation, both in terms of hardware and software, place reliable predictions for environmentally relevant problems within reach, often for much less effort than required for a full physical demonstration. Therefore, the two-fold objective of this task is the delivery of a computational infrastructure, including hardware, software, and networking, to INEEL scientists, and the trustworthy prediction of the outcome of the wide variety of technologies, processes, or strategies that have been or soon will be proposed for the solution of EM needs. The following two projects constitute this task:

1. Computational Infrastructure Project. Acquire or develop, then implement and maintain, state-of-the-art hardware, software, and networking infrastructure for computational simulation.

2. Computational Simulation Project. Employ state-of-the-art computational tools to predict and evaluate the outcome of a wide variety of mechanical or chemical systems, specifically for the following problems: (1) chemical and molecular transport processes in liquids and solids, (2) macroscopic dynamics of complex fluids and solids, (3) fracture propagation in solids, and (4) determinism in time series. 


\title{
Computational Simulation Project
}

\author{
Randall LaViolette (PI), Ray Berry, Eric Greenwade, Eric Steffler, and Charles Tolle \\ SUMMARY
}

The primary objective of the Computational Simulation Project is the prediction of the outcome of a wide variety of proposed technologies, processes, or strategies that might be employed in environmental management. Secondarily, we ourselves may discover improved solutions to environmental problems. This objective was chosen in order to contribute to the larger goal of providing informed choices from among the bewildering variety of strategies and technologies that might be applied to the resolution of a wide variety of environmental problems.

This year (FY-99) we focused upon chemical or transport behavior of materials under conditions that might be employed in environmental, especially subsurface, applications. Within this large focus, we selected topics that were both rich in application to EM problems at the INEEL and other DOE sites, and sufficiently compact for investigation within the scope of the ESR program. These topics, which have become tasks for this project, are indicated in Table 1 below, along with two-letter codes for reference:

Accomplishments included five manuscripts submitted for publication in peer-reviewed journals, two published so far, two invited presentations at universities, and three contributed presentations at national conferences. Two more manuscripts are in preparation as of September 20, 1999.

\section{PROJECT DESCRIPTION}

This year (FY-99) we focused upon chemical or transport behavior of materials under conditions that would be encountered in environmental, especially subsurface, applications. Within this large focus, we selected topics that were both rich in application to EM problems and sufficiently compact for investigation within the scope of the ESR program. These topics are indicated in Table 1, with the leaders and team members indicated below in Table 2.

All of these tasks are strongly connected with EM Science Needs (see also http://www.em.doe.gov/info/scitech.html). Table 3 lists in alphabetical order those (20 out of 107) Science Needs that specifically called for modeling like that employed in or developed under the tasks in this project. Additional Science Needs that clearly could have been employed in at least one of the kinds of modeling that we pursue, but did not specifically call for them, were excluded from this list. Task BW also is not mentioned in Table 3 because it applies to all tasks that employ parallel computing.

Table 1. Tasks in the Computational Simulation Project.

$\mathrm{CH}$ Chemical and transport processes in liquids and interfaces: Molecular description of transport and chemistry in separation membranes and solvation in aqueous and ionic solutions.

FD Complex fluid and solid dynamics: Particle-based methods for continuum fluids with complicated initial or boundary conditions, reactive multi-phase flow, and nucleation in a flow.

FP Dynamic fracture propagation in solids: Development of new approaches to predict fractures in solids and validation with the experiment.

TS Determinism in time series: Detection and exploitation of determinism in noisy time series.

BW Squadron: Construction and upgrade of a beowulf-class computer cluster. 
Table 2. Task leaders and team members for Computational Simulation Project.

\begin{tabular}{|c|c|}
\hline \multirow{5}{*}{$\begin{array}{l}\mathrm{CH} \\
\text { Team }\end{array}$} & r. Randall LaViolette, Advisory Scientist, Chemical Materials \& Processes, INEEL yaq@inel.gov \\
\hline & W. Alan Propp, Advisory Scientist, Chemical \& Biological Sciences, INEEL wp1@inel.gov \\
\hline & Joanne Budzien, Associated Western Universities Graduate Fellow, New Mexico Tech., and INEEL \\
\hline & $\begin{array}{l}\text { Dr. John McCoy, Assoc. Prof., Materials Engineering, New Mexico Tech. } \\
\text { http://www.nmt.edu/ mtls/new_mccoy.html }\end{array}$ \\
\hline & Dr. Lawrence Pratt, ${ }^{a}$ Technical Staff Member, Theory, LANL http://tdivweb.lanl.gov:80/ 1rp/ \\
\hline FD & Dr. Ray Berry, Advisory Engineer, Metals \& Ceramics, INEEL $\underline{\text { rxb@inel.gov }}$ \\
\hline Team & Dr. Bryan Kashiwa, ${ }^{a}$ Technical Staff Member, Theory, LANL http://gnarly.lanl.gov:80/Cfdlib/Cfdlib.html \\
\hline & Dr. Robert McGraw, ${ }^{a}$ Scientist, Environmental Chemistry, BNL http://www.das.bnl.gov/Cv_eed.053.htm\#mcgraw \\
\hline FP & Dr. Eric Steffler, Principal Engineer, Metals \& Ceramics, INEEL stefed@inel.gov \\
\hline \multirow[t]{3}{*}{ Team } & Dr. Richard Williamson, Consulting Engineer, Metals \& Ceramics, INEEL $\underline{\text { rlw@inel.gov }}$ \\
\hline & $\begin{array}{l}\text { Dr. Mark Rashid, Assoc. Prof., Civil \& Environmental Engineering, U.C. Davis } \\
\text { http://cee.engr.ucdavis.edu/faculty/rashid }\end{array}$ \\
\hline & Tonya Emerson, Research Assistant, Civil \& Environmental Engineering, U.C. Davis \\
\hline TS & Dr. Charles Tolle, Principal Engineer, Applied Materials \& Technology, INEEL tollcr@inel.gov \\
\hline BW & L. Eric Greenwade, Consulting Engineer, Software \& Electronics, INEEL http://vizlab.inel.gov/ \\
\hline Team & Jed Haile, Senior Engineer, Software \& Electronics, INEEL http://vizlab.inel.gov/ \\
\hline
\end{tabular}

a. Indicates a collaborator who received no funds from the ESR in FY-99.

Table 3. Selected EM Science Needs and Computational Simulation Tasks.

\begin{tabular}{|c|c|}
\hline Selected EM Science Needs (alphabetical order) & CS Task(s) \\
\hline Chemical Sensor Principles & $\mathrm{CH}$ \\
\hline Chemistry of Problem Constituents for High Level Waste (HLW) Vitrification & $\mathrm{CH}$ \\
\hline Contaminant Mobility Beneath Tank Farms & $\mathrm{CH}, \mathrm{FD}$ \\
\hline Contaminant Transport in a Fractured Rock Vadose Zone & $\mathrm{CH}, \mathrm{FD}$ \\
\hline Decision Support Assistance for Remediation and Assessment Design & $\mathrm{CH}, \mathrm{FD}, \mathrm{TS}$ \\
\hline Differences Between Saturated and Unsaturated Systems & FD \\
\hline Effect of Subsurface Heterogeneities on Chemical Reaction and Transport & $\mathrm{CH}, \mathrm{FD}$ \\
\hline Fracture Toughness Properties for Carbon Steel Utilized for Nuclear Waste Containment Vessels & FP \\
\hline Integration of Reactive Chemistry into Field-Scale Transport & $\mathrm{CH}, \mathrm{FD}$ \\
\hline Issue of Scale in Flow Prediction and Contaminant Remediation in Porous Media & $\mathrm{CH}, \mathrm{FD}, \mathrm{TS}$ \\
\hline Low-Frequency Stress-Wave Stimulation for Enhanced Transport of NAPL Groundwater Contaminants & $\mathrm{CH}, \mathrm{FD}$ \\
\hline Mathematical Formulations of Chemical Reaction/Material Transport & $\mathrm{CH}, \mathrm{FD}$ \\
\hline Mechanisms of Line Plugging & FD \\
\hline Moisture Flow and Contaminant Transport in Arid Conditions & FD \\
\hline Physics of Fracture Flow and Transport in the Vadose Zone & FD, TS \\
\hline Quantifying Uncertainty in Predictions of Remediation Performance & $\mathrm{CH}, \mathrm{FD}, \mathrm{TS}$ \\
\hline Transport of Contaminants & $\mathrm{CH}, \mathrm{FD}$ \\
\hline Transport of HE and Metals in Fractured Rock and Surface Alluvial Systems & $\mathrm{CH}, \mathrm{FD}$ \\
\hline Vadose Zone Flow Simulation Tool Under Arid Conditions & $\mathrm{CH}, \mathrm{FD}, \mathrm{TS}$ \\
\hline Water Fluxes and Solute Transport in Arid and Semiarid Environments & $\mathrm{CH}, \mathrm{FD}$ \\
\hline
\end{tabular}




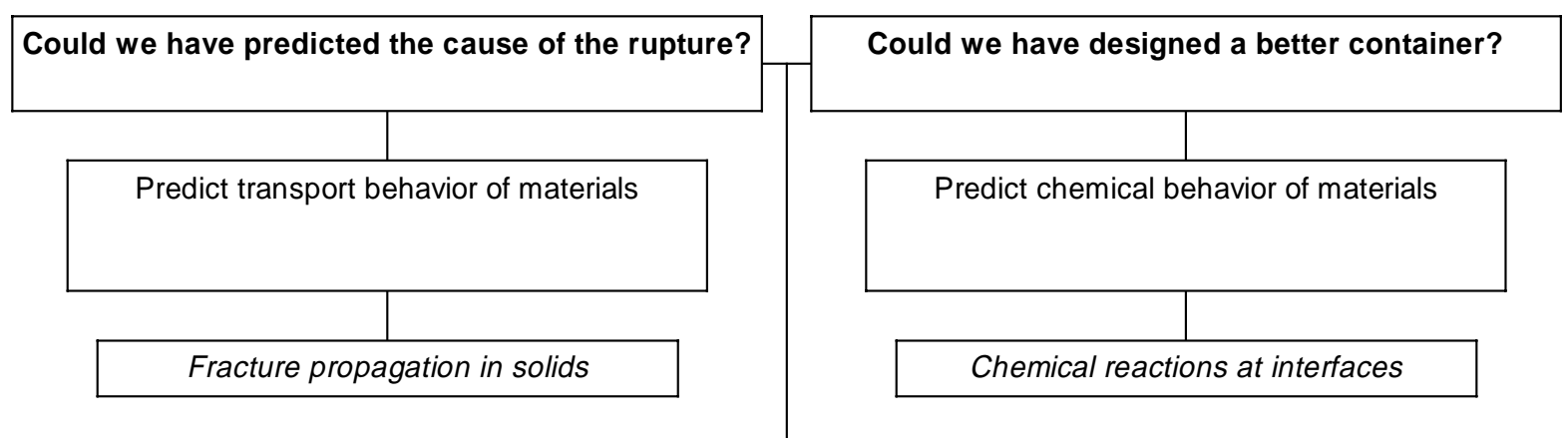

A container of contaminated solution ruptures. Contaminated solution spills into the ground.

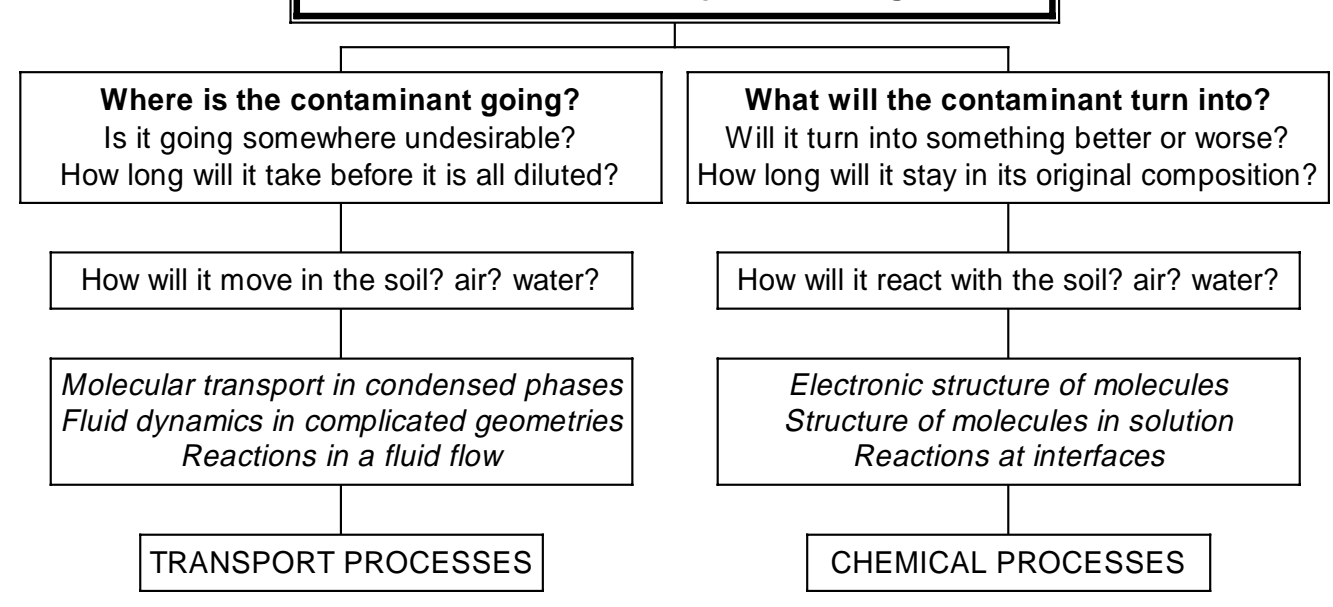

Figure 1. How environmental concerns become Computational Science tasks.

The relationship between Computational Science tasks and environmental concerns is also illustrated with Figure 1, where we consider the problem of storing a solution of hazardous waste in a faulty container, as just one example.

The remainder of this section is devoted to descriptions of the individual tasks.

\section{Chemical and Transport Processes in Liquids and Interfaces (CH)}

This task had two main sub-tasks: the development of additional capability in computational chemistry, and the pursuit of applications. In both cases the focus was on the chemical and transport processes in solutions at the molecular level, since these processes are prominent in the study of the contamination and remediation of soils and the subsurface.

Our capabilities in computational chemistry are enhanced in four ways in this project:

1. Development of theories in collaboration with other laboratories

2. Development (writing, testing) of codes here at the INEEL

3. Acquisition of codes from vendors or other laboratories

4. Training of staff in the use of computational chemistry codes. 


\section{Capabilities}

In the first instance, we have an ongoing collaboration with Dr. Lawrence Pratt of Los Alamos National Laboratory (LANL), to develop a compact theory for solvation in aqueous solutions. The idea here is that the interaction of hydrogen-bonding molecules in certain small clusters, identified by the theory, contain most of the information about solvation in the liquid; the rest can be treated in a meanfield-like theory that is straightforward to implement. This approach reduces the study of solvation in aqueous solutions to a few molecules that, in turn, can be calculated ab initio with commonly available electronic structure codes (GAUSSIAN, DMOL, GAMESS, etc.). Apart from this approach, there has been no consistent way to link $a b$ initio cluster studies in a vacuum to properties of a liquid. From the other side, studies of solvation in liquids have been for the most part controlled by empirical, nontransferable, few body interactions that have always been suspect, but are still employed in the absence of anything better. Eventually, with continued increases in computing power, the very slow many body $a b$ initio techniques now under development elsewhere (Car-Parinello, VASP, CASTEP, etc.) will be able to provide a comprehensive picture without resorting to theory, besides Schrodinger's equation, but these are still many years from practical use. The theory provides a direct way to use the tools that are available to deliver an accurate picture of solvation in aqueous solutions. ${ }^{1}$ We have begun to test the theory by comparing the predictions with molecular dynamics simulations of neat methanol; although the results are encouraging, the work is still in process. In the second instance, we have been writing and developing a wide variety of codes. These include a new random number generator (used for MonteCarlo and other stochastic simulation techniques), a new molecular dynamics code optimized for polymers, and several utility codes that provide interfaces between existing codes. In the third instance, we continue to acquire modules to the Cerius 2 suite of computational chemistry codes (Molecular Simulations Inc., www.msi.com), the GAMESS electronic structure code (Iowa State University, www.msg.ameslab.gov/gamess/gamess.html), and upgrades to the NWCHEM suite (PNNL, www.emsl.pnl.gov:2080/docs/nwchem). We have also begun collaboration with Prof. Tom Beck (University of Cincinnati, http://bessie.che.uc.edu/tlb/beck.html) for the development of a fast multi-grid electronic structure code; interest and support from MSI has also been solicited. Finally, we have sent staff to Molecular Simulations, Inc. (MSI) headquarters for training in the use of the Cerius2 suite of codes.

\section{Applications}

The applications pursued this year have been primarily ionic transport in polymer membranes, and secondarily, the structure of ionic liquids, and mercury transport in copper sulfides. The ion transport study was pursued in support of the chemical separations studies under ESR project, Selective Mass Transport in Polymers. Polyphosphazenes have long been recognized for their use in highly selective chemical separation membranes, and are likely to have a large role in future remediation and decontamination technologies. However, the fundamentals of ion transport and reaction in polyphosphazene has only begun to be elucidated. One significant problem that must be addressed in order to design optimal polyphosphazene membranes is identifying the predominant paths of ion transport. Even in relatively simple systems, the transport mechanism has become controversial. For example, the transport of the lithium ion ( $\mathrm{Li}+$ ) in methoxy-ethoxy-ethoxy-poly-phosphazene (MEEP) has been recently reported to occur primarily along the farthest extent of the ethoxy side chains, far from the phosphazene backbone. Yet new and unpublished nuclear magnetic resonance (NMR) data obtained at the INEEL indicate a substantial interaction between the ion and nitrogen atoms, suggesting that at least some transport occurs along the backbone. Modeling the ion transport in MEEP faces formidable obstacles. First, equilibrium MEEP configurations are difficult to obtain because the structural relaxation times are very long on the scale of computer simulation. Second, the very slow transport of the ion itself precludes a direct simulation of the transport. The first problem is tackled with a combination of brute force and annealing, in which the temperature is frequently raised, then lowered, in order to speed the 
sampling of low energy configurations separated by high energy barriers. The second problem requires much more care. A strategy was developed in which the transport was divided into two parts: ion motion within accessible but disconnected cavities in the polymer and in the motion of the cavities themselves, as they separate and re-connect with other cavities. This study occupied the majority of the $\mathrm{CH}$ task in both effort and elapsed time. A manuscript is in preparation summarizing the results; the main result is that not only is ion transport along the phosphazene backbone possible, it is preferred.

The structure of ionic liquids, both melts and solutions, has until recently been an area of intense study, but has become strangely neglected in this decade. In particular, no one has elucidated what Stillinger has called the inherent structure ${ }^{2}$ of an ionic liquid. As an adjunct to our studies of ionic transport, we decided to calculate the inherent structure of a primitive ionic melt, zinc (II) bromide. The zinc bromide has the advantage of being simple enough to calculate quickly, but complicated enough to be representative. One of the "holy grails" of the study of inherent structures is the discovery of systems for which the inherent structure is substantially the same as the equilibrium liquid. Liquid water is the best known example of a system like that. ${ }^{3}$ We can now add that the liquid zinc bromide is partly like that for the zinc-bromide interactions, but not for the other interactions. These and other results are summarized in a manuscript now in preparation. We also incorporated some of these results into another manuscript (submitted to Physica D) in which we showed that the inherent structure obeys "Benford's Law," a test of homogeneity, which apparently has never before been explored in the context of molecular dynamics simulations. This last work also was the first direct collaboration between tasks (CH and TS) in this instance of this project.

The study of mercury transport in copper sulfides was carried out in support of the ESR project, Adsorption and Absorption Materials for Molecular Separations. A variety of copper sulfide minerals have been employed by the French and the Russians for the removal of mercury from gas or liquid flows, and show promise as an inexpensive technology for treating mercury-contaminated sites. We were asked to follow up on a suggestion, given in a Russian report, that mercury intercalates chalcopyrite or covellite. Our simulations and thermodynamics calculations showed that intercalation of mercury in these minerals is impossible at ordinary pressures. Independently and almost simultaneously, $\mathrm{x}$-ray measurements obtained at Montana State and analyzed at the INEEL provided another confirmation of our conclusion. The chemical reaction and transport of mercury in copper sulfides appears now to be entirely interfacial. However, many puzzles remain, and there is room for a substantial contribution for computational chemistry to provide insight into the mechanism of mercury transport and reaction in copper sulfides. It is especially important to determine the charge distributions in these compounds, which apparently has never been carried out with recent electronic structure techniques.

\section{Complex Fluid and Solid Dynamics (FD)}

This task began with the construction and demonstration of a class of modeling tools to enable more realistic simulations of complex dynamics in continuum systems. Emphasis was placed on building tools permitting the exploration of problems in complex material dynamics, in particular on multi-component, multiphase, and multi-material mixtures and other complex interactive flow processes.

Most engineering bodies, materials, and fluids are mixtures. Often times one of the constituents is preponderant and the other constituents insignificant so the body is assumed to be of the predominant single constituent. There are many situations, however, where none of the constituents present in the body can be ignored with respect to the others; e.g., slurries and suspensions, sediment transport, porous rocks and soil infused with water or oil, plasmas and gaseous mixtures, boiling coolant flows, and corpuscle motion in the blood plasma of mammals. Frequently, a material moves relative to another; this interpenetration is sometimes useful, as in the promotion of mixing, and sometimes destructive, as in the erosion of pipe walls of pneumatic conveying equipment. Often times, the constituents in a mixture 
undergo phase change, resulting in the interconversion between one phase and another, or they undergo chemical reactions, resulting in an interconversion between constituents and the formation of new compounds. While the importance of the study of mixtures was recognized long ago, and mathematical models were developed to describe the flow of mixtures, these early models frequently did not provide adequate agreement in many physical situations. The theory of mixtures has evolved in recent years to overcome these inadequacies. The theory of mixtures (or mixture theory) provides a rational framework for studying problems involving mixtures. The basic premise of the theory is that the space occupied by a mixture can be considered as a continuum in its own right. Thus, at each point in the space occupied by the mixture, there will be a particle belonging to each of the constituents. This requires that the constituents are dense enough that they can be homogenized over the region of the mixture as a continuum in a time-, volume-, or ensemble-averaged sense. Each of these homogenized continua moves relative to the others, due to the application of forces and interacts with each other. For each constituent, we can define its motion, mass density, partial stress tensor, internal energy, temperature, entropy, and other relevant physical quantities, as we do for a single constituent continuum. The theory of mixtures allows for the interconversion of mass and exchange of momentum and energy between the various constituents. Balance laws for mass, linear and angular momentum, and energy take into account contributions of any of these quantities with regard to a particular constituent due to the influence of the other constituents. An appropriate thermo-mechanical setting can also be provided within which to study mixtures that takes into account the basic laws of thermodynamics.

Several variants of the theory of mixtures have been proposed in order to study problems of diffusion, phase change, and the flow of coexistent phases. A multiphase mixture (e.g., sand particles suspended in a gas flow) is characterized by well-defined interfacial areas between the constituents of the mixture and steep gradients of properties close to the interface. The interface between the phases may be only a few molecular diameters thick, across which mass, momentum, and energy transfer processes take place. A multiphase flow is, then, the interpenetrating motion of dissimilar materials. A multi-component mixture (e.g., a mixture of different gases) differs from a multiphase mixture in that no steep gradients of the properties between the constituents of the mixture can be macroscopically discerned. Sometimes, the averaged properties resulting from mixture theory are inadequate. Sometimes we need to examine the interactions of dissimilar materials with such resolution that a detailed analysis of the dynamics of each constituent is necessary. The solution of flows with large deformation and multiple materials remains a major area of research and development. A characteristic feature of all solutions of these problems is their immense complexity, which necessitates using numerical solution methods on modern computers. Such methods collectively represent a very specialized area of computational fluid dynamics (CFD) or computational mechanics (CM).

With the resources available to pursue this enormous problem, this project was narrowly aimed at beginning the construction of a class of modeling tools to enable more realistic simulations of complex dynamics in continuum systems. Emphasis was placed on building tools permitting the exploration of problems in complex material dynamics, in particular on multi-component, multiphase, and multimaterial mixtures and other complex interactive flow processes. This year, this task was extended along three main directions:

1. The extension of current research on gridless, particle-based methods

2. The continuation of our work with LANL on the extension of the CFDLIB advanced research code to INEEL and EM needs

3. Collaboration with BNL on development of an advanced simulation capability for nucleation and growth of nanocrystalline metals. 
Traditional modeling of deformable continuous media employs grid-based numerical methods such as finite element, finite volume, or finite difference methods. Although grid-based methods are very powerful, they are unsuited to handle many complex fluid flows, such as mixing of streams and other highly dynamical material motions. In recent years, several classes of numerical methods have been proposed which require no active grid for their solution. These grid-free methods, collectively referred to (here) as particle methods, have several appealing features. While several of these were researched under this task, the main focus this year has been on smoothed-particle hydrodynamics (SPH) methods. ${ }^{4}$

\section{Particle Methods}

An efficient SPH particle-based code was constructed, wherein realistic constitutive equations could be employed to demonstrate the macroscopic energy effects of compression and heat conduction in a highly dynamical setting. The code uses an efficient neighbor locator algorithm developed recently at SNL. ${ }^{5}$ An explicit time integration of the SPH equations is used along with conservative smoothing. ${ }^{6}$ Compressibility effects are allowed in a liquid phase by using a barotropic equation of state. Heat conduction and subsequent phase change (freezing or melting) are allowed in both liquid and solid phases. Density, pressure, internal energy, temperature, and solid volume fraction are tracked as dependent variables for each computational particle. The SPH code is demonstrated here by applying it to the collision of two aluminum droplets. The top droplet is molten at $1100 \mathrm{~K}$ and is initially traveling downward at $100 \mathrm{~m} / \mathrm{s}$. The bottom droplet (ball) is a rigid solid with thermal properties of aluminum at $300 \mathrm{~K}$ and is initially traveling upward at $100 \mathrm{~m} / \mathrm{s}$. This bottom ball represents a thermally active, moving boundary.

Figure 1 shows a time sequence of the collision at $4.17,45.87,90.35$, and $139.0 \mu$ s. The deformations, temperatures, and solid volume fractions can be readily seen. When the two droplets/balls are in contact, the cold solid ball begins to warm up while the hot molten ball begins to cool. The molten aluminum begins to solidify at $932 \mathrm{~K}$. At that point the solid volume fraction begins to change from its initial value of 0.0 (all liquid) to its final value of 1.0 (all solid). During this transition (solidification), heat of fusion is given up, keeping the temperature of the droplet at $932 \mathrm{~K}$. As the initially molten material is finally solidified totally, its temperature again begins to drop below $932 \mathrm{~K}$. This simulation is stopped at this time because once the initially molten material fully solidifies, it is not given the properties of a deformable solid; this level of modeling sophistication has yet to be developed. The SPH method shows an impressive capability for handling such highly dynamical events. Closer examination of these results identified the need for improvements, some of which will be address during the next fiscal year.

\section{Multiphase Mixtures}

CFDLIB (http://gnarly.lanl.gov:80/Cfdlib/Cfdlib.html) is a library of computer codes, currently under development by LANL and other researchers, capable of solving a wide variety of advanced CFD problems in two- and three-dimensions. ${ }^{7,8}$ The codes use a finite-volume computational scheme, a multi-block data structure, and an arbitrary, Lagrangian-Eulerian (ALE) split computational cycle and are constructed to run efficiently in a parallel computing environment. The codes allow simulation of flows of all speeds from fully incompressible to hypersonic. Modules have been constructed that enable multifluid and multiphase computations with several fluid/solid fields. Realistic simulations are created by pulling together the physical models used to represent the effects of chemical species conversion, physical kinetics of phase change, granular flow, multiphase turbulence, and other complex interactive flow processes. Recently, the FLIP scheme, ${ }^{9}$ a particle method with extremely low numerical dissipation, was incorporated within the CFDLIB structure. This method allows interaction with finite volume treatment, giving distinct advantage for highly deforming material flows, chemically reacting flows, and multiphase flows. The material point method ${ }^{10}$ is used in the solid mechanics version of FLIP which can, for some problems, eliminate the need for mixture theory by evaluating equation of state and strength models 

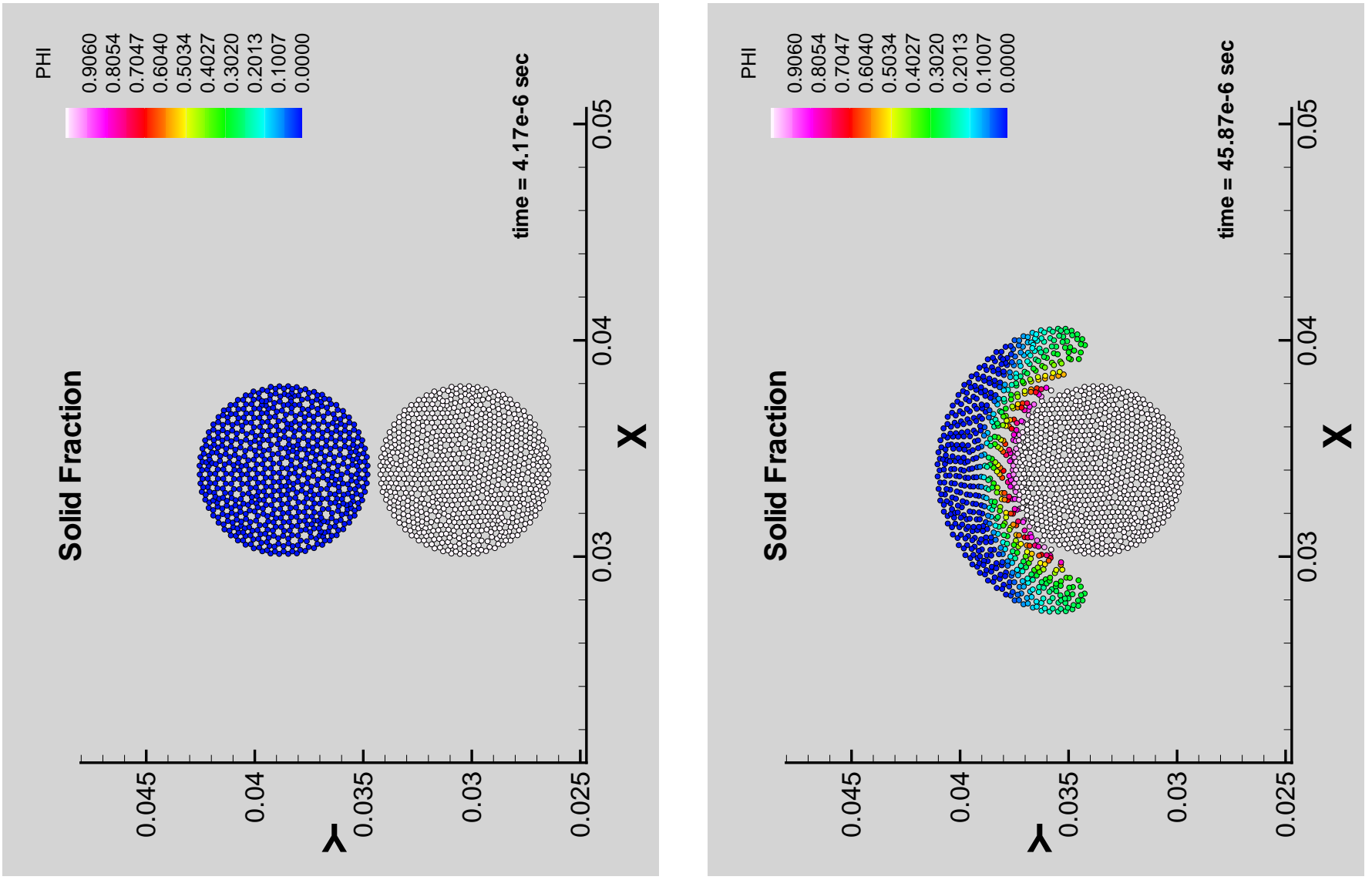

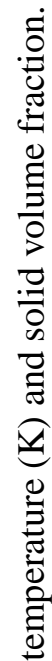
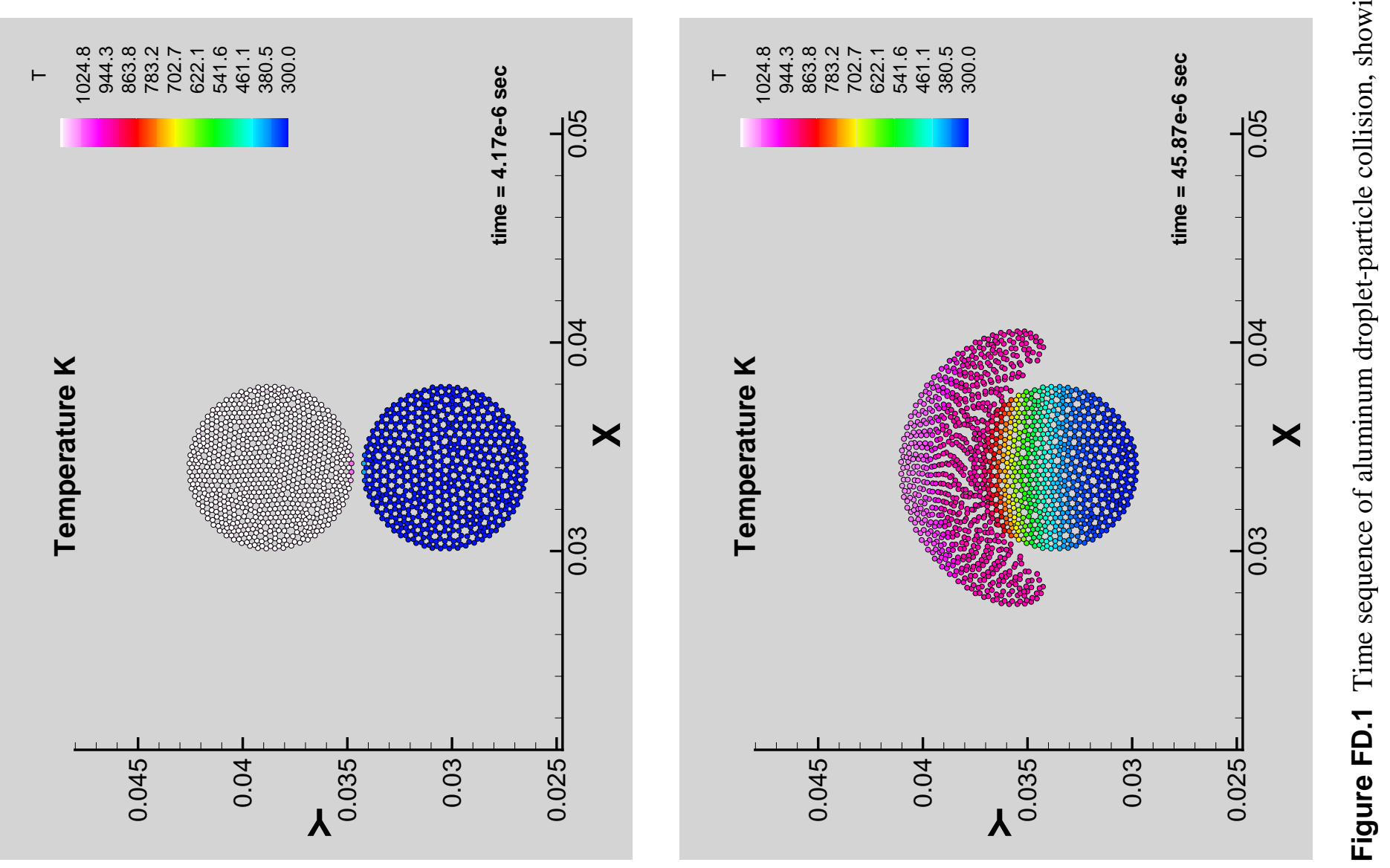

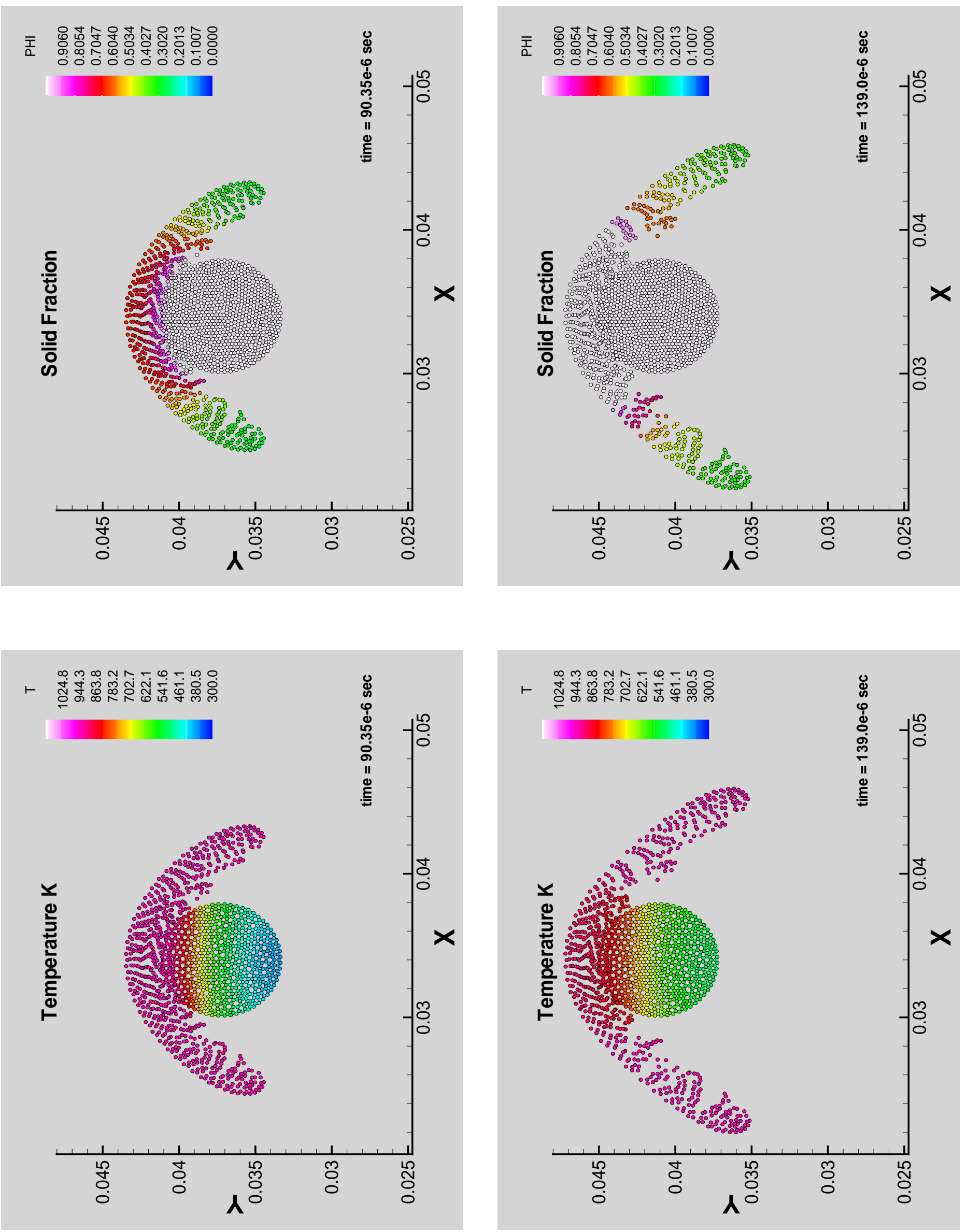

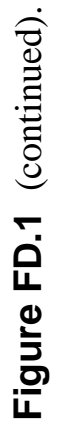


particle by particle. We are using the codes to test the validity of various theories and to provide important guidance to the design and operation of modern equipment and processes. In short, CFDLIB serves as a vehicle for the exploration of problems in complex material dynamics.

We strongly emphasize that the code volumes of CFDLIB are being developed with the sophisticated user in mind; the experienced computationalist can set up and solve a large variety of problems, but must do so with little instruction from code manuals and internal documentation. Since CFDLIB is a state-of-the-art research tool, most useful to the computational physicist interested in exploring highly specialized effects in complex fluid/solid dynamics, we spend a considerable effort learning, and remaining current with, the evolving capabilities of this valuable tool. This year our efforts have been focused on implementation of CFDLIB within the parallel and sequential computing environment of the INEEL and demonstration of its capability.

The latest versions of CFDLIB were implemented on a PC, a serial workstation, and on both the SGI Origin2000 and Squadron (the beowulf cluster) in parallel at the INEEL. In fact we successfully ran a test problem in parallel using some processors from the SGI Origin2000 and some processors from Squadron. To demonstrate the power of CFDLIB, a practical multiphase problem is simulated wherein it is postulated that some energetic (explosive) materials, with similar burn characteristics to gunpowder, were buried long ago and may now detonate if drilled into as part of a waste remediation project. Of course, for such a problem, one would be interested in whether the explosion would breech through to the surface releasing potential contaminants and whether anything could be placed over the surface to mitigate such a breech. One liter of this energetic material is assumed to be buried $100 \mathrm{~cm}$ below the surface in a sand, air, and water mixture medium (volume fractions: sand 0.6 , air 0.3 , water 0.1 ). The energetic material is assumed to burn rapidly to form a gas at a pressure of $38 \mathrm{kbar}$. Air is assumed to be compressible and to obey a BKW equation of state, while the sand and water are treated as incompressible. The air and water phases are treated on the ALE grid while the sand is treated by the FLIP method.

Figure 2 shows a time sequence of the underground gas expansion and of the deformation of the surrounding sand, air, and water mixture at $0.0,20.0$, and $100.0 \mathrm{~ms}$. If the simulation is studied more carefully, it is seen that the expansion has slowed at $100 \mathrm{~ms}$ and that the pressure in the cavity is actually less than atmospheric. Even though the simulation is here stopped before a breech is shown, it is easily surmised that since sand has negligible strength, gravity will pull the bridge of sand back into the cavity, causing a large breech. CFDLIB is clearly seen to be capable of handling such highly dynamic multiphase events. While CFDLIB already has some significant equation of state capability included, much more needs to be included in order to broaden its capabilities for application to pertinent engineering problems at the INEEL.

\section{Nucleation}

In many multiphase flows of interest, the initial transition from single phase to two or more phases is of key importance, both to basic understanding and the effective design of pertinent processes. This initial transition event, known as nucleation, has been examined previously with development of simplified, one-way coupling along steamlines (steady flow) for simulating nucleation and growth of nanocrystalline metals from a gas flow field. ${ }^{11,12}$ As a collaborative effort with researchers at BNL, our main focus this year was to begin the extension of our previously demonstrated method to unsteady, volumetrically-coupled flow fields.

Because of some unavoidable delays with our research partners at BNL, we did not progress to the extent anticipated with the development of an unsteady, volumetrically coupled model for nucleation from a flow field. Our BNL research partners supplied us with references for the transport algorithm to 

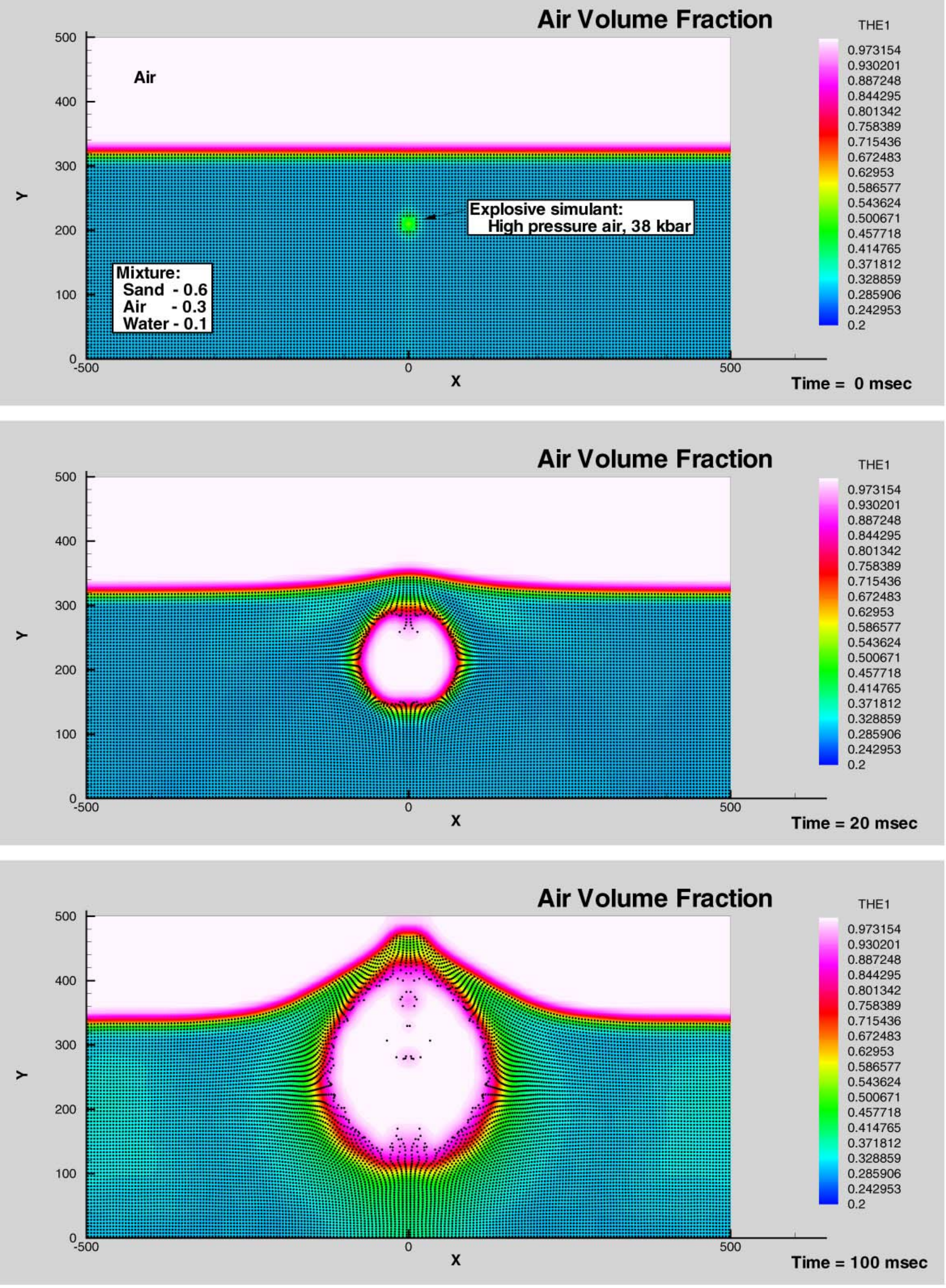

Figure FD.2 Time sequence of explosive expansion in a sand/air/water mixture, showing air volume fraction. 
which chemistry is coupled in BNL's 3D Eulerian hemispheric-scale model. While the method they use may be conservative, monotonic, and positive definite for scalar variables, it may not possess the compatibility property. ${ }^{13}$ Specifically, coupled transport equations of the form

$$
\frac{\partial}{\partial \mathrm{t}}\left(\begin{array}{l}
\rho \\
\mathrm{A}
\end{array}\right)+\nabla \cdot\left(\begin{array}{l}
\rho \\
\mathrm{A}
\end{array}\right) \mathrm{u}=0
$$

can produce artificial extrema in the ratio $T \equiv A / \rho$, even though $T$ satisfies a simple advection equation and is expected to preserve monotonicity. Here $\mathbf{u}$ is velocity, $\rho$ is mass density, and $A$ is a conserved quantity such as momentum density, energy density, or chemical species density, so $T$ is a mass-specific transport quantity such as velocity, energy per unit mass, temperature, or species mass fraction. The compatibility property ensures that the average $T$ in a given cell is bounded by the average previous time $T$ from that cell and its neighbors. This is a nice property to have in any numerical advection scheme, but this property could become critically necessary for systems having large density gradients, where overshoots and/or undershoots could be a problem. We know of only a few advection algorithms that possess the compatibility property ${ }^{13,14}$ A coupled advection algorithm that possessed the compatibility property was written and tested. The coupling of our nucleation model with flow transport will continue during the next fiscal year.

\section{Dynamic Fracture Propagation in Solids (FP)}

The usefulness, lifetime, or safety of solid systems or components (e.g., waste containers and encapsulation matrices) is largely determined by fractures in solids. The ability to simulate and predict the behavior of fractures is therefore crucial to decisions about the choice of materials for these components or estimates of the lifetimes of existing structures. The ability to predict the behavior of propagating cracks is important for an assessment of the behavior of solid components for long times, as might be required by waste container specifications, for example. Current remediation techniques used to remove contaminated concrete can be improved by examining alternate, more efficient material breakup technologies based on a comprehensive understanding of fracture processes and numerical simulations. The task will continue to support ESR projects in the Materials Dynamics area; i.e., Coatings for Environmental Applications and Corrosion and Aging of Solids. Secondly, this technique will be extended and verified via experimental comparison to elastic and elastic-plastic materials. Ultimately, with this tool we will analyze portions of full scale structures or containers to assess the propensity for crack growth and estimate the time and conditions that would lead to catastrophic system failure.

This task is devoted to the development of computational capability and expertise to simulate (dynamic) propagating fractures in both elastic and elastic-plastic materials. Fracture modeling is one of the more challenging aspects of solid mechanics computations because of the large stress and strain gradients and microscopic spatial scales involved. Since many INEEL tasks have included the need for fracture modeling, the necessary capability and expertise to simulate static (non-propagating) fractures has been well established. Such simulations are clearly limited, however, since fractures naturally propagate; i.e., they evolve in time and space. The task has involved the use of the two-dimensional Arbitrary Local Mesh Replacement (ALMR2D) method to compare numerically predicted fracture paths to published linear-elastic material fracture experimental results to validate and test the algorithm range of applicability. The current modeling capability, unique to the INEEL, is a direct result of FY-98/FY-99 accomplishments and cannot be done with commercial software. The subsequent sections give an overview of tasks completed in FY-99 concerning the ALMR2D algorithm and related efforts. 


\section{Staff Training}

The latest release of ALMR2D was installed on a workstation for INEEL staff access along the distribution of the latest ALMR algorithm user's manual. A comprehensive tutorial was also included which afforded interested personnel the opportunity to learn the programming conventions as needs were identified. The inherent capabilities of the algorithm to model and predict a crack trajectory without knowing the crack path is a powerful tool that only exists here at the INEEL within the fracture group.

\section{Experimental Work}

Fracture test results of A710 structural steel were conducted in an effort to quantify constraint effects on crack extension that is being used as algorithm validation. Tonya Emerson, a graduate student from the University of California at Davis, was at the INEEL for the past summer to strengthen the collaborative efforts with the fracture group. The experimental work conducted during that time was used as benchmark information to refine/validate the ongoing development of the elastic-plastic algorithm.

A710 steel was used to fabricate three specimens with dimensions $12.0 \times 2.5$ inches with three thicknesses of 0.50 inches, 0.90 inches and 1.25 inches, respectively. These dimensions were chosen to determine the constraint effects of this material and to use all of the recorded engineering data to validate the algorithm. A schematic of the geometry test is shown in Figure 1.

After test completion, the specimen was cooled using $\mathrm{LN}_{2}$ and the remaining ligament was fractured. This exposed the crack surface and enabled identification of the extent of fatigue crack profile, final crack front geometry, and the type of crack growth that occurred during corresponding events in the load and displacement measurements. Figure 2 and Figure 3 are digital images two different specimen fracture surfaces.

Next, we consider two-dimensional applications.

\section{Concrete Test}

An extended abstract written by Winkler, Hofstetter and Niederwange ${ }^{15}$ conducted three fracture experiments in an effort to verify modified constitutive relations in a concrete mix. The authors employed a simple L shaped beam arrangement to compare experimental results to finite element model results. While they compared the experimental results to their numerical models, the process seemed to be controlled more by geometry than their simulations suggested. Figure 4 shows the test geometry and resulting fracture paths. Figure 5a (magnified displacement) and $5 \mathrm{~b}$ (element failure) show the resulting

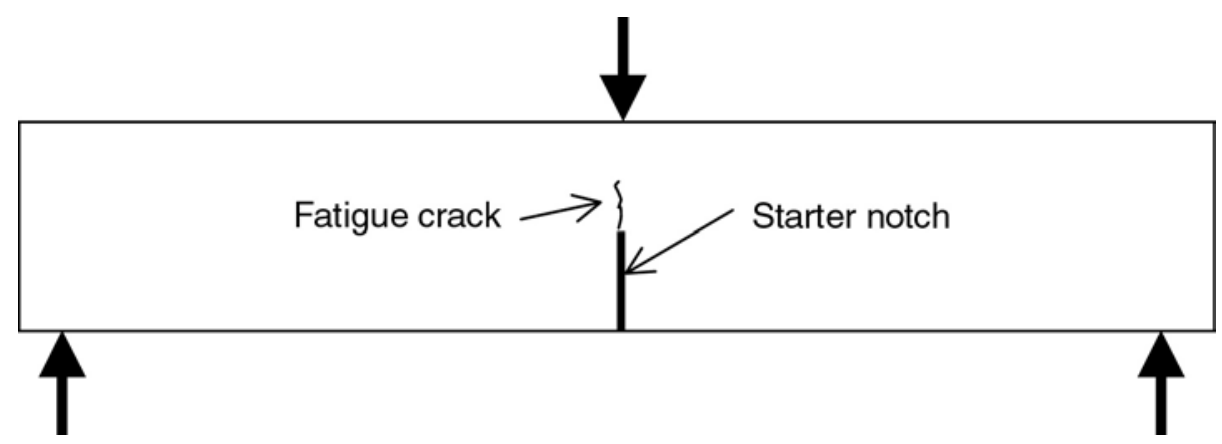

Figure FP.1. 3 pt. bend bar geometry used for experimental work. 

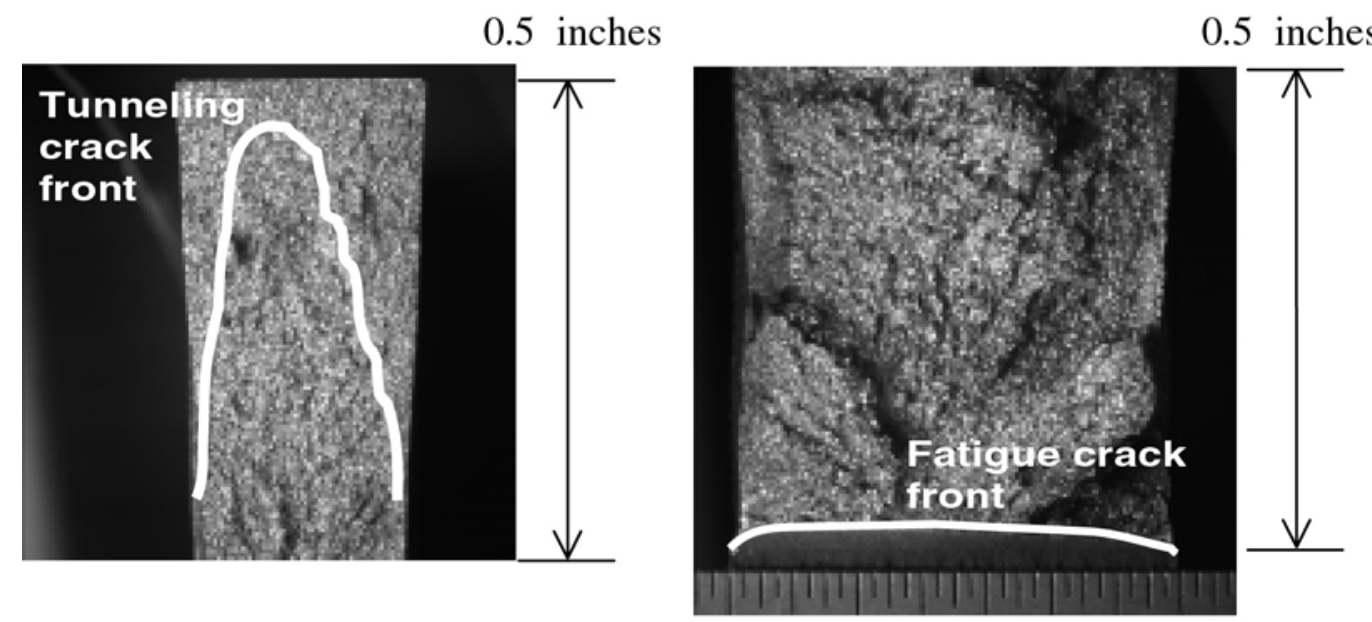

GV99 0473

Figure FP.2 Final crack profile showing substantial tunneling at final load.
Figure FP.3 Fatigue crack profile and large features on fracture surface.

finite element analysis conducted by Winkler et al. with the approximated resulting fracture path prediction superimposed for reference.

The interest within the fracture modeling group at the INEEL was the resulting crack trajectories reported. The same geometry was modeled using the working fracture propagation code supplied by Dr. Mark Rashid for linear elastic bodies. A comparison of their reported fracture paths are compared to simulations using the INEEL research code FEFRAC. Figures 6a-d illustrate the magnified crack trajectories predicted by FEFRAC. A small existing flaw was assumed at 4 different angles, 0, 45, 60 and -45 degrees relative to the horizontal plane with the algorithm using a standard maximum opening mode fracture stress criterion to calculate the crack trajectory. Figures 7a-d illustrate the FEFRAC fracture traces with the approximated element failure path of Winkler et al. superimposed in the dashed line. Figures 8a-d show the same FEFRAC traces with the experimental fracture paths overlaid, illustrating improved agreement for two of the tests with the 45 degree initial notch orientation (best viewed magnified or printed).

\section{Brittle Coating}

Protective coatings are often applied to metal materials, for example, using thermal spraying or naturally grown oxide scales. Since these coating processes are typically done at high temperatures, and the substrate and coating possess significantly different thermal expansion coefficients, large and often detrimental thermal stresses are developed during cooling. At locations of significant geometry changes, such as corners, the stresses can be concentrated, resulting in increased probability of fractures and thus undesirable failure of the brittle coating. At the INEEL in the DOE-BES program, Mechanically Reliable Surface Oxide, significant modeling efforts have investigated the quantitative effects of these stress risers and the resulting fracture paths. In recent modeling efforts, a multiple crack simulation has demonstrated the ability to predict crack extension in the brittle coating with initiation and extension in various locations. After a primary crack has extended and arrested, the redistribution of stress results in crack extension at other flaws. The primary drawback to this approach is that the crack paths need to be predefined. This is a significant limitation when complex stress fields and geometries are encountered, since the probable crack path of an actual system cannot be known beforehand. An example of this type of model with multiple flaws is shown in Figure 9 and was produced using the commercial package ABAQUS (http://www.abaqus.com/). 


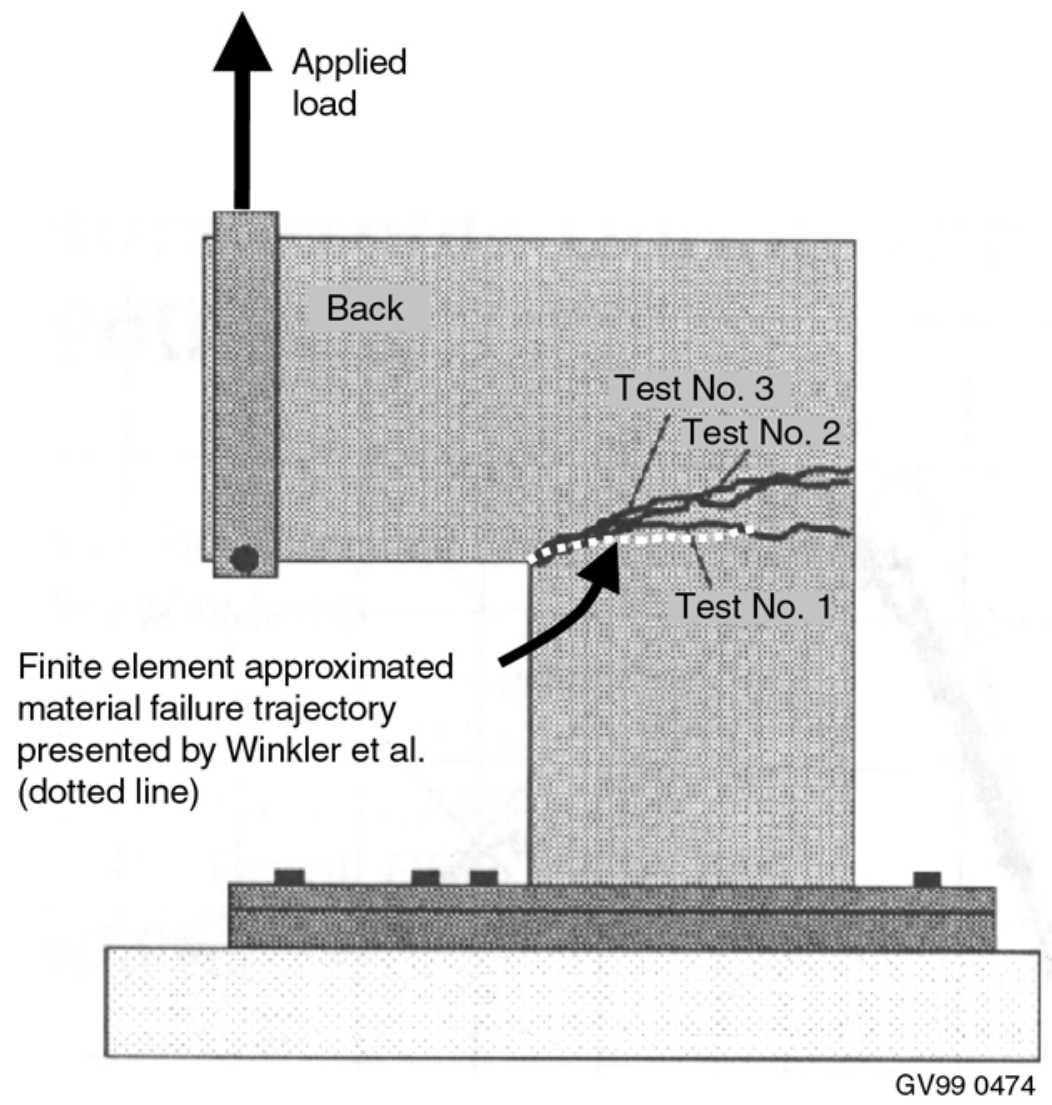

Figure FP.4 Experimental geometry used to test concrete L-shaped specimens with fixed base and tensile applied vertical load. Cracks initiated at the corner and traveled to the free surface.

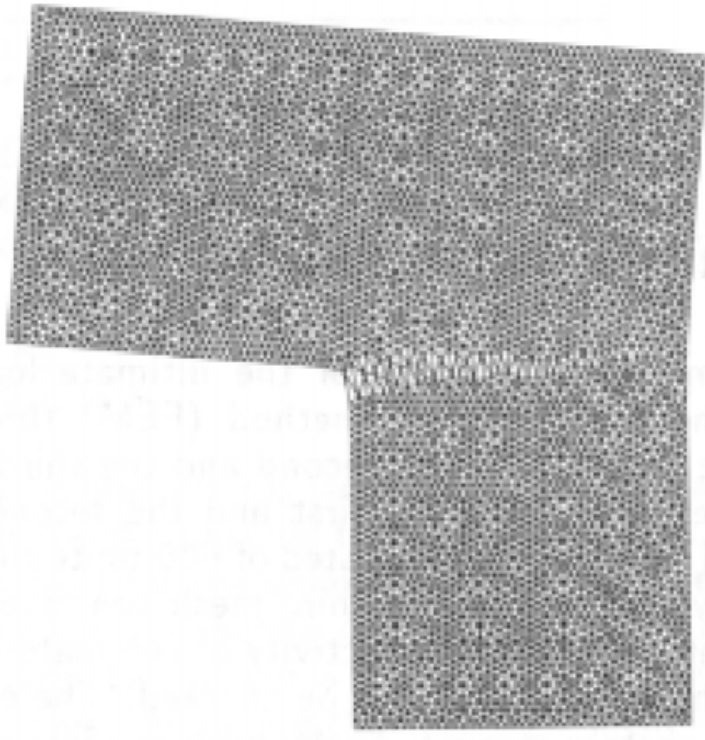

a)

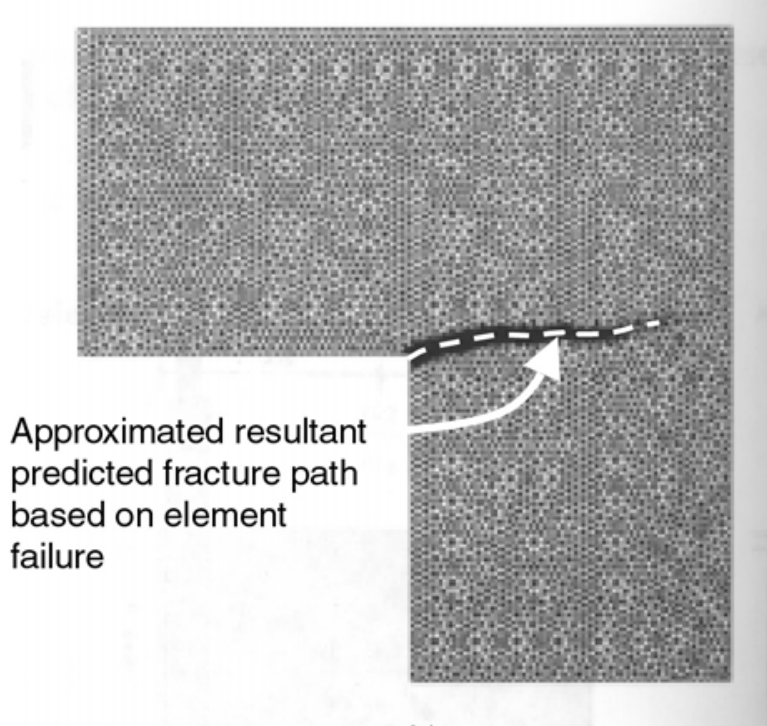

b)

GV99 0475

Figure FP.5 Finite element mesh and element failure path used to predict failure path. a) deformed mesh and b) mesh with approximate resultant failure path. 


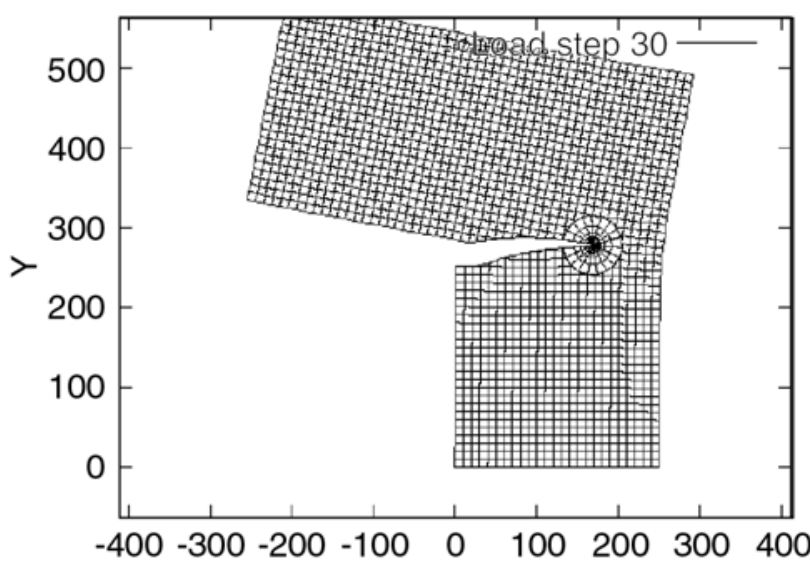

a)

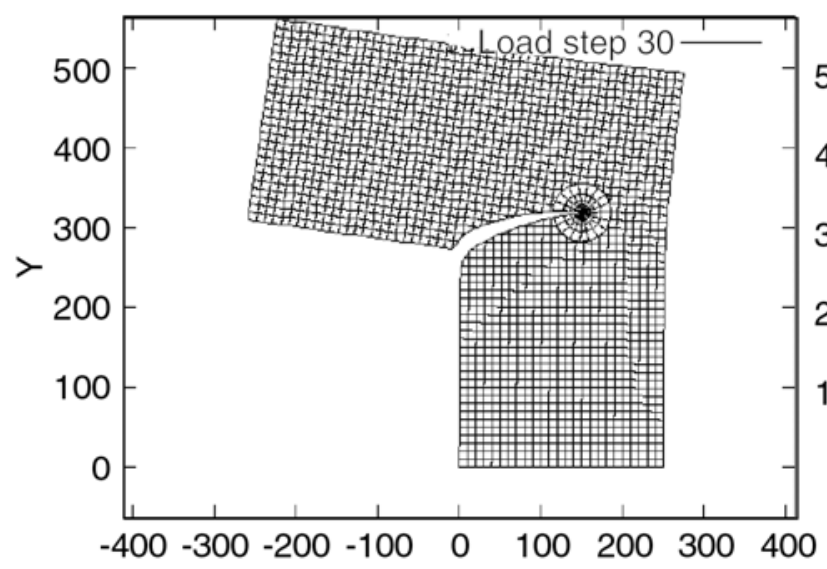

c)

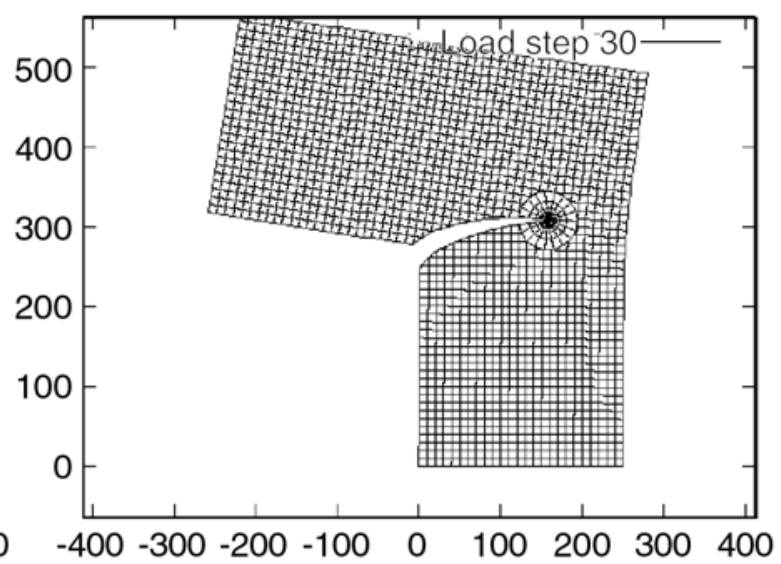

b)

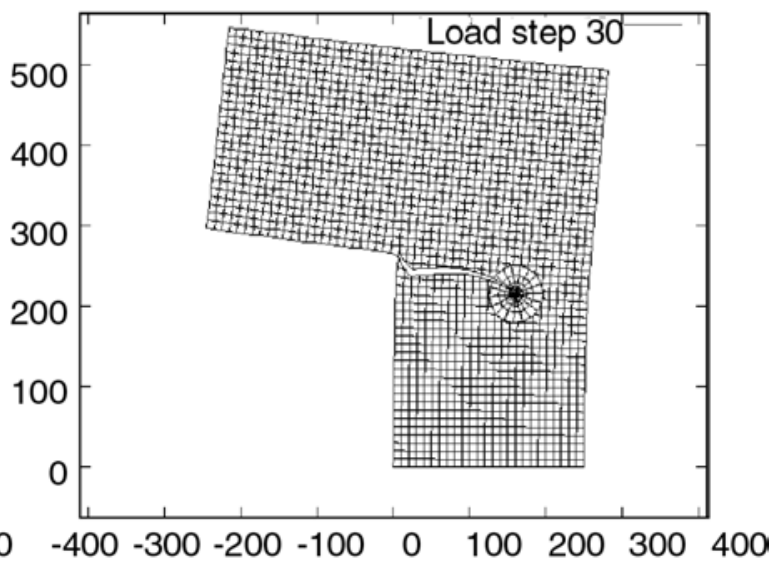

d)

GV99 0476

Figure FP.6 Four different displacement-magnified FEFRAC simulations with starting notch angles of a) 0 , b) 45 , c) 60 and d) -45 degrees. 


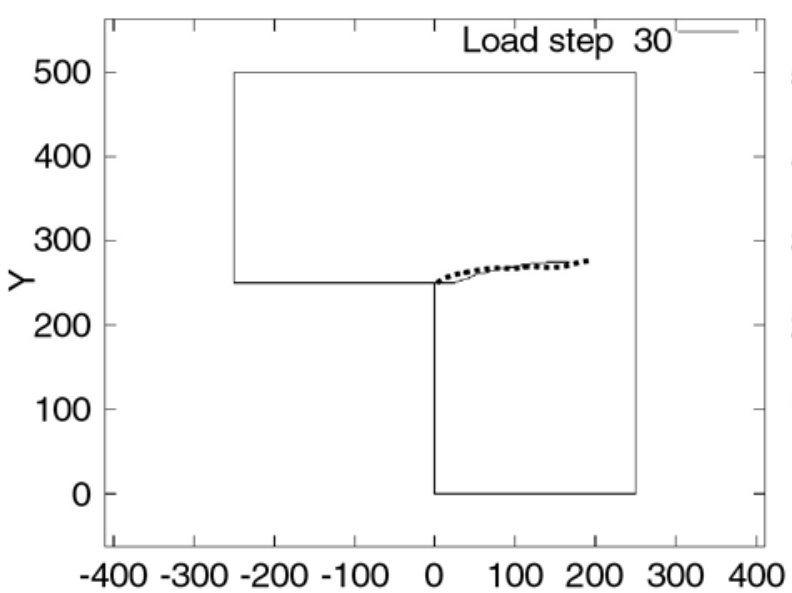

a)

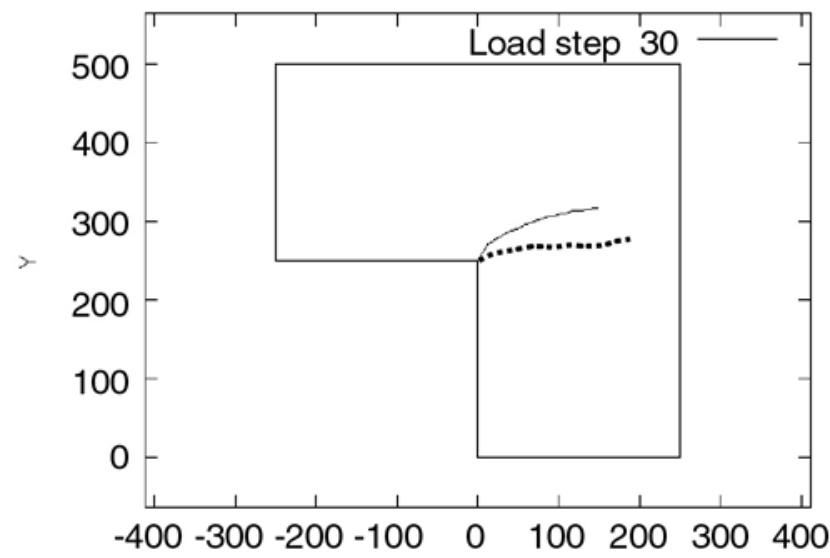

c)

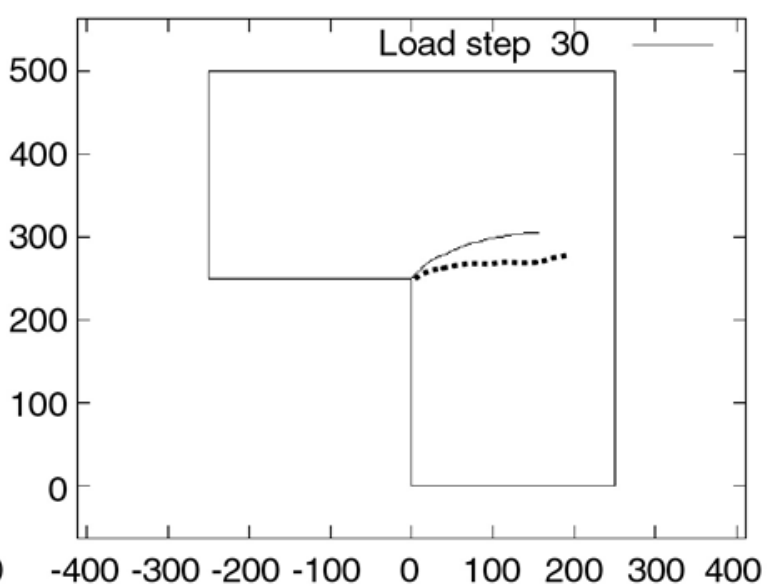

b)

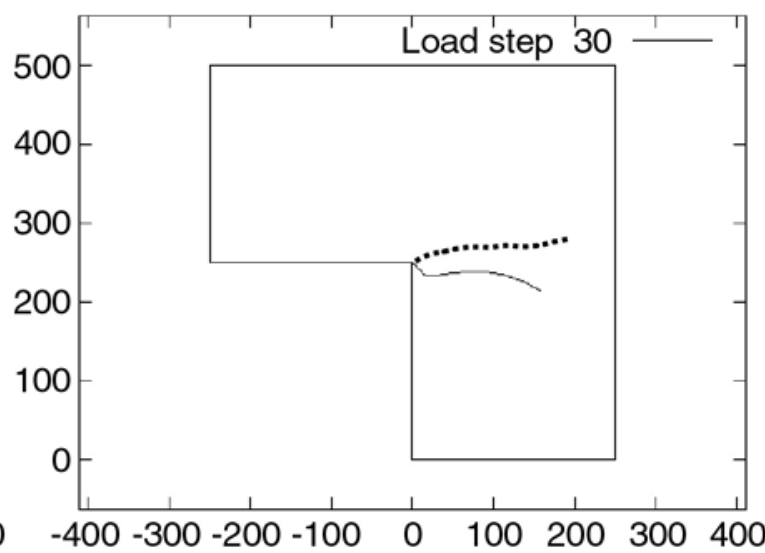

d)

GV99 0477

Figure FP.7 Four different fracture traces using FEFRAC simulations with starting notch angles of a) 0 , b) 45 , c) 60 and d) -45 degrees. Dotted line is approximated fracture path predicted by Winkler et al. 


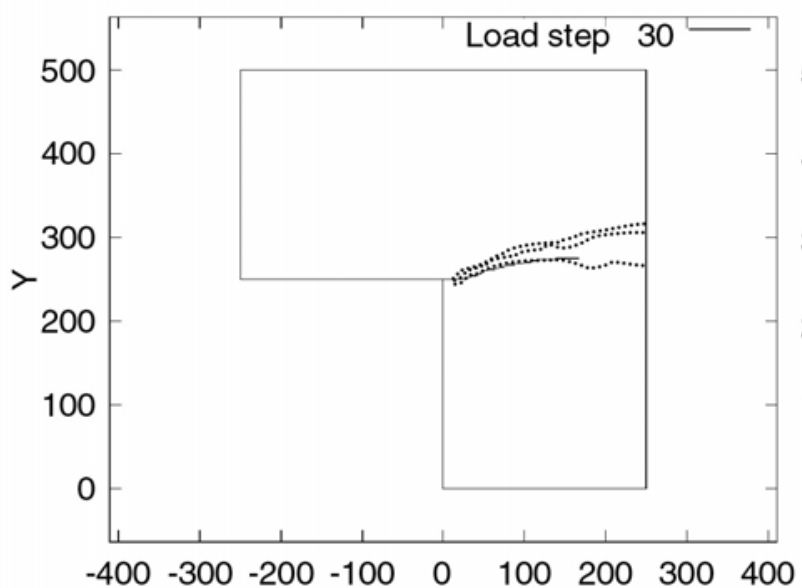

a)

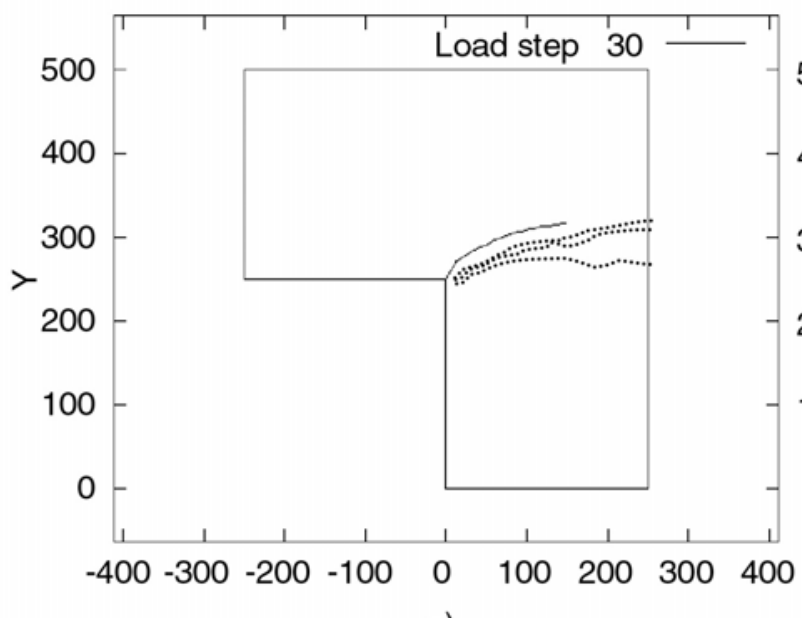

c)

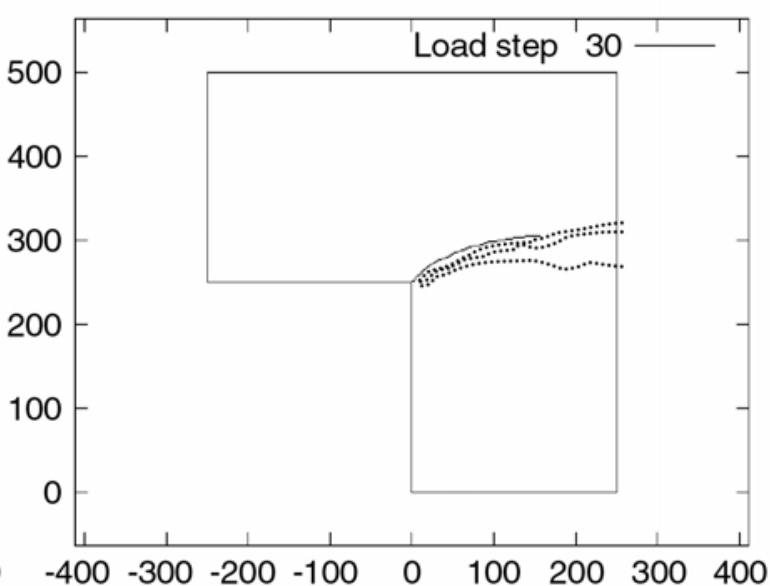

b)

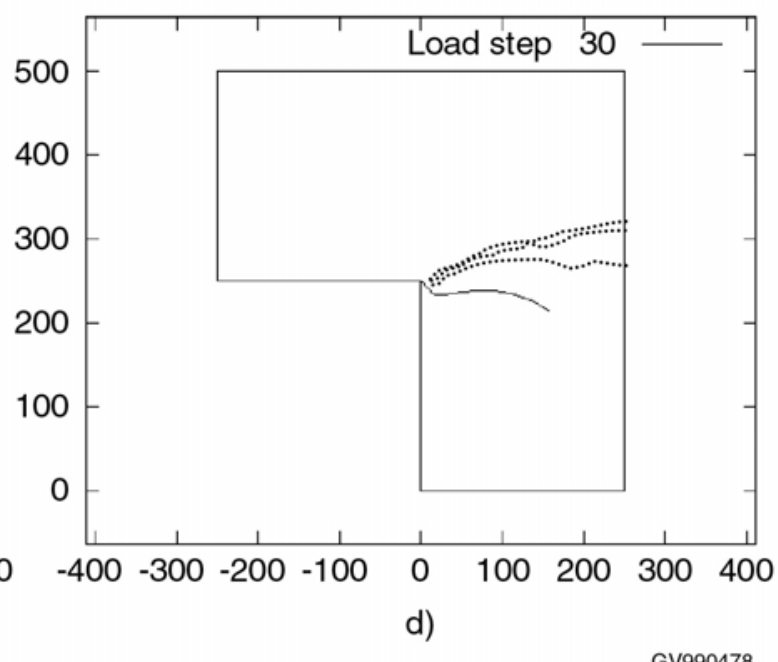

Figure FP.8 Four different fracture traces using FEFRAC simulations with starting notch angles of a) 0, b) 45 , c) 60 and d) -45 degrees. Dotted lines are experimental fracture paths reported by Winkler et al.

FEFRAC was used to calculate similar crack geometries. A thermo-mechanical ABAQUS model was used to calculate the displacements along a coating to substrate interface. Since FEFRAC is currently only available for linear elastic materials, the ABAQUS calculated boundary displacements along the coating portion of the model were extracted and used as boundary condition input for FEFRAC. This two step process enabled the modeling of crack growth without guessing the probable fracture path. The geometry used by ABAQUS with the metal substrate and brittle coating is shown in Figure 10. Note the region of interest is at the corner of the model. Symmetry was employed and only $1 / 4$ of the original component was modeled. Figure 11 shows a magnified image with the first flaw calculation introduced at nearly 45 degrees through the corner. 


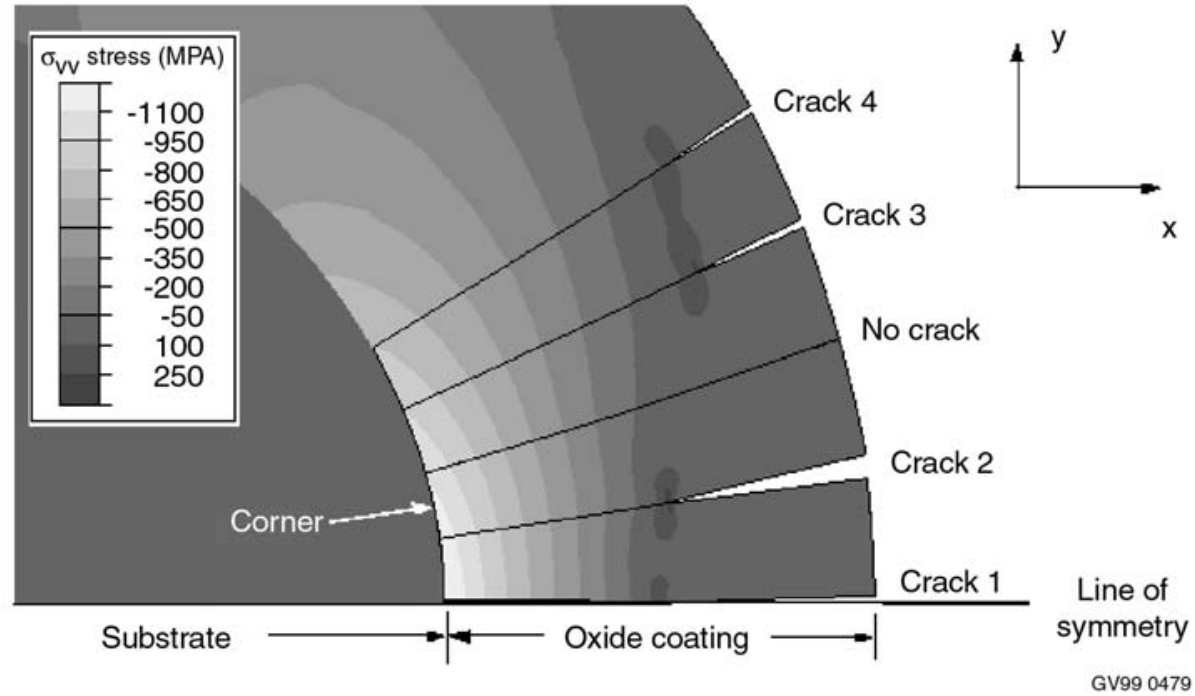

Figure FP.9 Multiple pre-defined crack ABAQUS model.

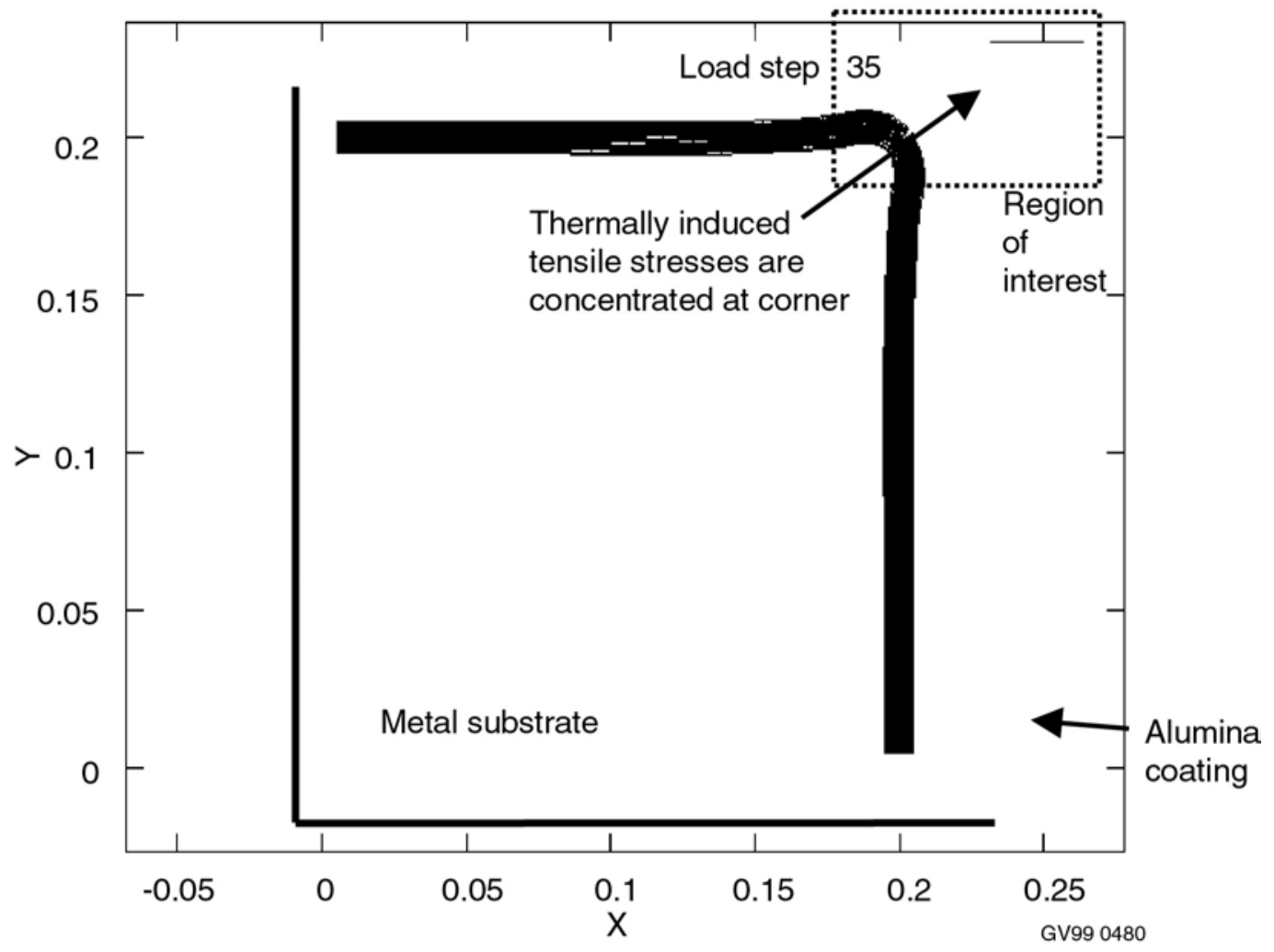

Figure FP.10 Overall model used in analysis showing the 1/4 geometry with brittle coating and metal substrate. 


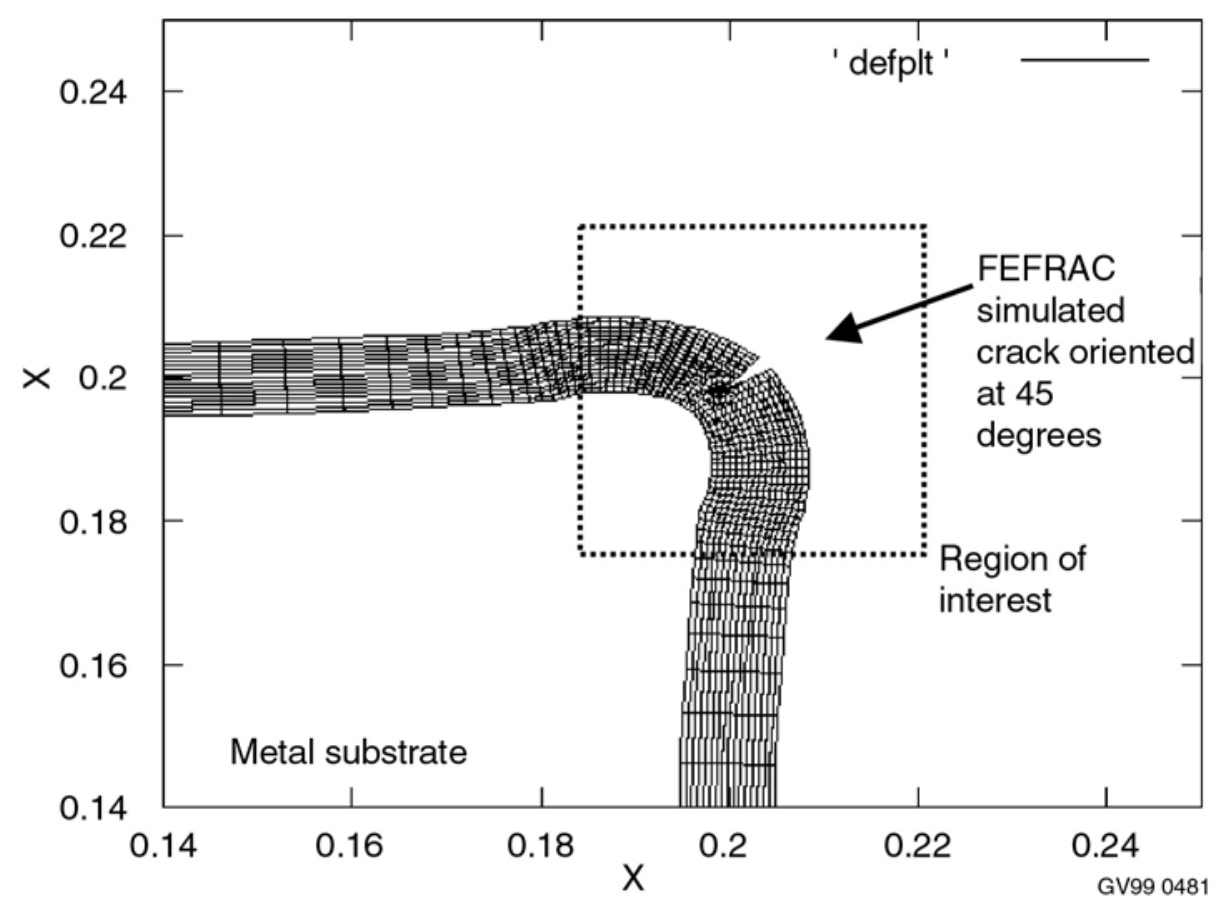

Figure FP.11. Magnified image of corner model showing initial 45-degree flaw introduction and resulting path.

The same boundary conditions were used with flaws introduced at 55 and 65 degrees. Figures 12, 13, and 14 show large magnifications of the corner region of interest with extended flaw paths. Displacements are magnified for illustrative purposes. Note the significant crack path changes predicted by the model. The FEFRAC model used here is a course approximation of anticipated crack paths. Significant model refinement is necessary to increase confidence in predicted paths. Ultimately, experimental verification at larger laboratory scale simulations is necessary to compare the sensitivity of the model to specific geometric influences.

In the ABAQUS model illustrated in Figure 9 the secondary flaws grow and arrest at shorter flaw lengths as the distance from the maximum stress region is increased. This spatially makes physical sense, except that there is a slight asymmetry in the stress contours surrounding the shorter flaws. If the stress intensity at the tips of these secondary flaws is both mode I and mode II, the maximum tensile stress direction may not be coincident with the required ABAQUS predefinition. This is evidenced in the initial numerical analysis of the same system using FEFRAC where the maximum stress surrounding the crack tip defines the direction of extension. If this can be confirmed with refined models, it will help explain why spallation of coating material consistently occurs near geometric features on actual components. With this knowledge, the geometric designs can be optimized to reduce and or eliminate this type of coating failure resulting in improved component integrity and cost savings.

\section{Elastic Plastic Code Development}

Elastic-plastic version of the code experienced developmental delays. While Dr. Mark Rashid at U.C. Davis intended to deliver the updated algorithm, unexpected developmental delays have pushed delivery of the algorithm into some time in the coming FY. The latest version will enable the fracture 


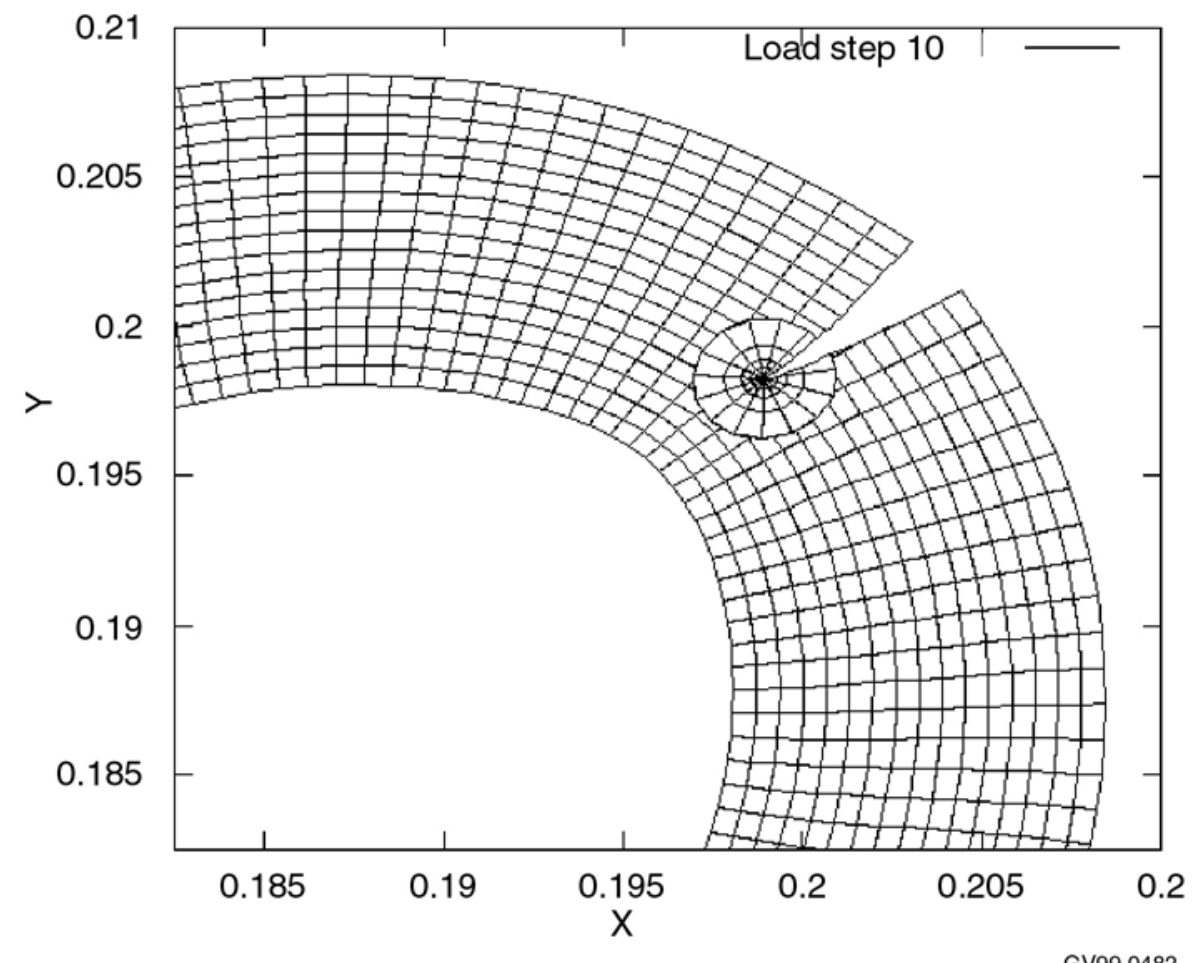

Figure FP.12. FEFRAC predicted crack trajectory with initial flaw at 45 degrees.

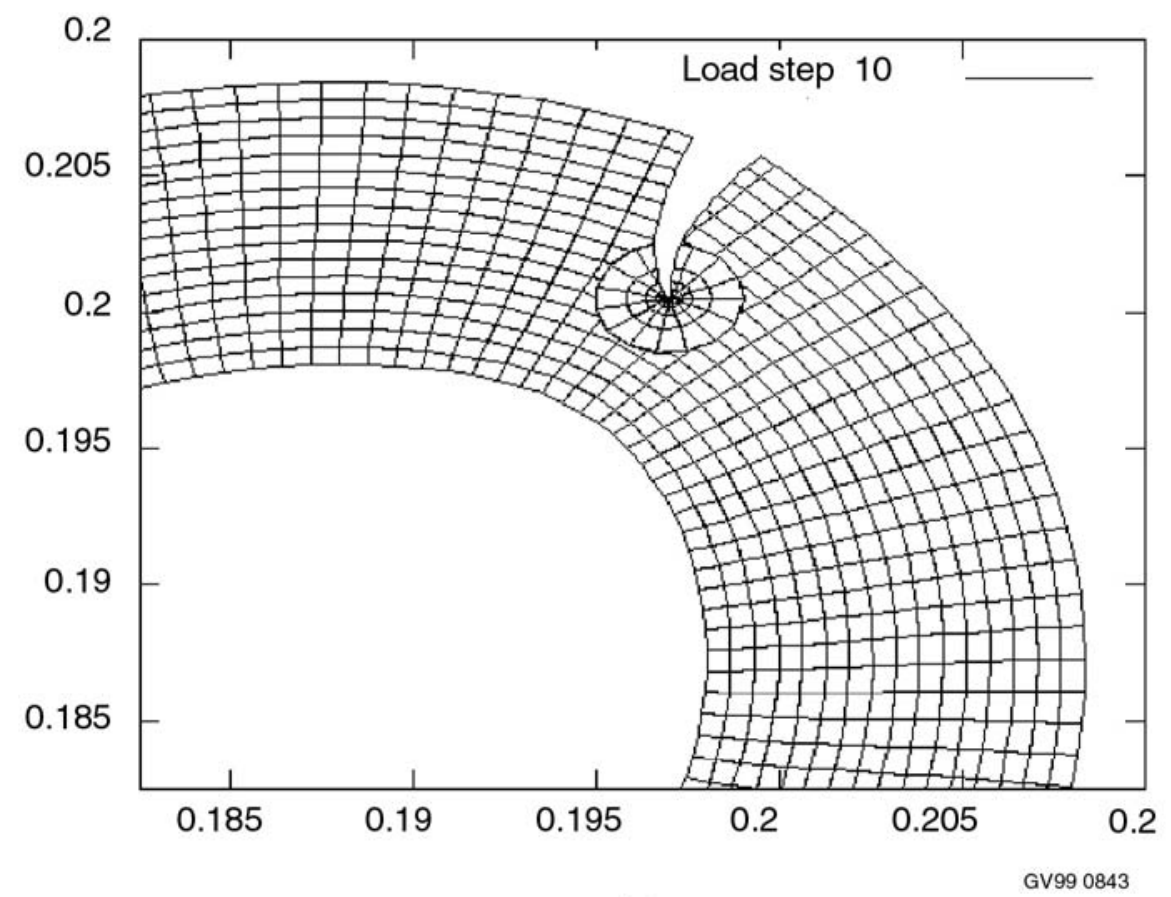

Figure FP.13. FEFRAC predicted crack trajectory with initial flaw at 55 degrees. 


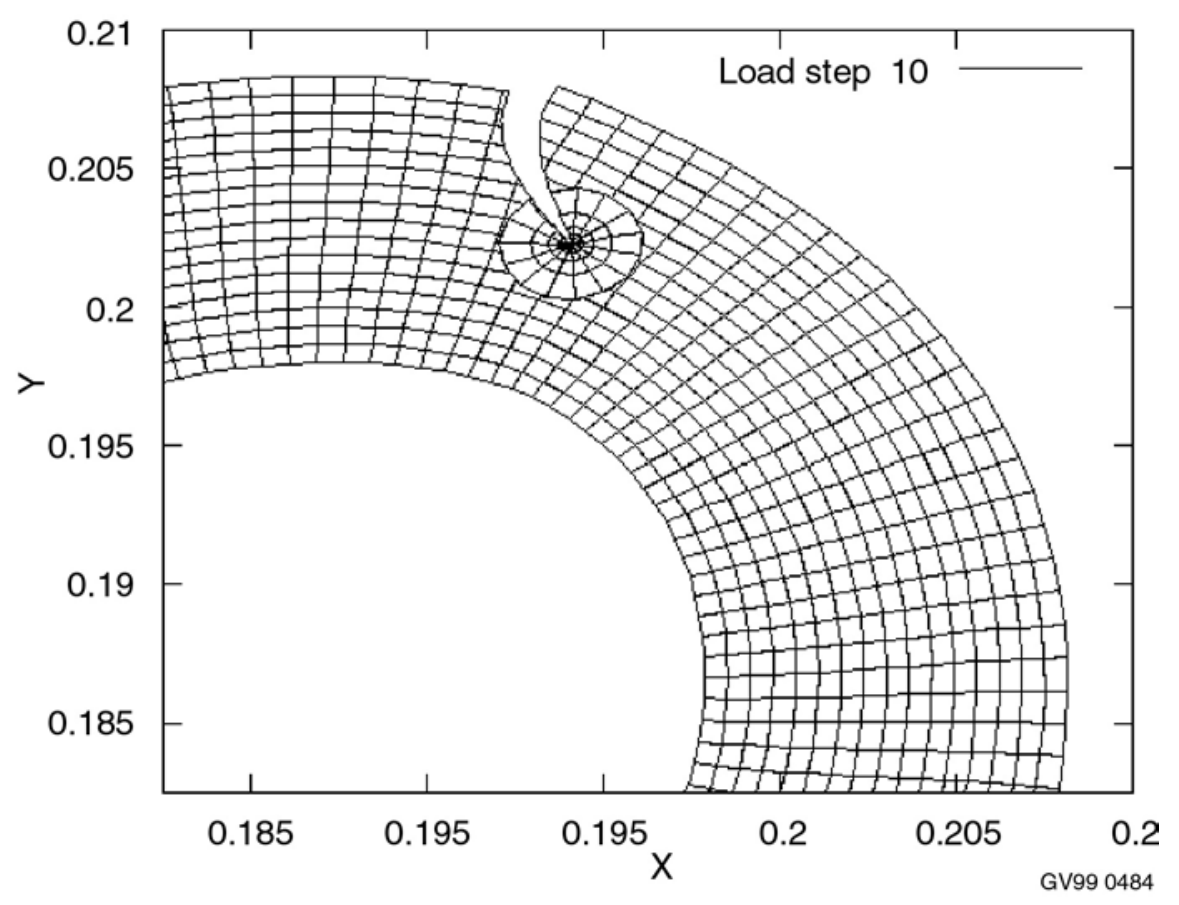

Figure FP.14. FEFRAC predicted crack trajectory with initial flaw at 65 degrees.

modeling group to compute the predicted fracture path in elastic-plastic materials. This latest technology, which will be delivered to the INEEL, is a direct result of the ESR program.

\section{Summary}

The unique research code FEFRAC enables the fracture analysis group to model propagating cracks without the confines of crack path predetermination. The algorithm developed by Dr. Mark Rashid was supplied to the INEEL at no cost, as a result of ongoing collaborative research. Dr. Rashid visited the INEEL and assisted in experiments that are currently being used to verify the latest elasticplastic versions of the algorithm that will also be supplied to the INEEL at no cost. The working linear elastic version running at the INEEL has been used for comparison to published experimental fracture test results. The algorithm has proven to be an improvement over traditional modeling methods in predicting crack path and has been used to supplement ongoing research in Coatings for Environmental Applications. Numerical results indicate that FEFRAC predicted probable fracture paths that could be obtained in no other conventional finite element analysis packages. Ongoing and anticipated extensions of this work will extend the unique capabilities for fracture modeling at the INEEL.

\section{Determinism in Time Series (TS)}

Many experiments, from laboratory to field studies, can be expressed as the measurement of a time series. We extended the state-of-the-art in the detection of determinism (e.g., periodicity) in complicated time series by extending the recent advances in time-based clustering (TBC), iterated function systems maps (IFSM), and hypothesis testing by doubling the number of symbols used within this detection method. Determinism in otherwise overwhelmingly complex signals permits one to represent the underlying dynamics with a few constants in simple models. Besides the economy achieved with a compact representation, the model obtained often provides insight into the mechanism that generates the signal, and may even identify a route for the control of the underlying dynamics. The techniques 
developed here are general and have wide application in physics, chemistry, biology, ecology, and control systems.

This task had two main objectives: upgrading and porting an existing TBC application from the Macintosh to an independent platform core that can run on Windows NT, Unix, and MacOS, and extending the two-dimensional IFSM determinism technique to a 3D IFSM technique. By extending the determinism detection technique from the two-dimensional procedure to the more complex threedimensional procedure, more complex signals can be analyzed. This is important for next year's tasks, which include analyzing real world complex signals for underlining determinism. In addition to code development, this project also investigated the implications of Benford's law (a test of homogeneity) for dynamical systems such as Lorenz, Hénon, and Rössler.

\section{Squadron (BW)}

The task here was simply to acquire and connect the components for a beowulf-class parallel processor (Squadron). The beowulf concept includes using only commodity hardware and software to construct the system; there is no attempt to integrate all the processors onto a single backplane, for example. Applications are anticipated to support all other computational science tasks. Squadron is patterned after the LOKI and AVALON systems at LANL (http://cnls.lanl.gov/Frames/avalon-a.html). Squadron consists of three main components, as follows:

- $\quad$ Head end system (root)

- $\quad 20 "$ monitor

- $\quad$ X windows compatible frame buffer

- $\quad$ Dec Alpha 21164 processors at 500MHz

- $\quad$ 512MB DRAM

- $\quad$ 4B SCSI disk

- $\quad$ Red Hat Linux operating system

- $\quad$ EGCS compilers-Version 2.90.29

- $\quad$ MPICH-Version 1.1.1

- $\quad$ Portable Batch System

- $\quad$ Back end system (16 nodes)

- $\quad$ Dec Alpha 21164 processors at $500 \mathrm{MHz}$

- $\quad$ 512MB DRAM

- $\quad$ 4GB SCSI disk

- $\quad$ Red Hat Linux operating system 
- $\quad$ EGCS compilers-Version 2.90.29

- $\quad$ MPICH-Version 1.1.1

- $\quad$ Networking

- $\quad$ Cisco 4000 using 100Mbps full duplex switched ethernet

- $\quad$ Cyclades serial interface for admin and troubleshooting.

Ten more Dec Alpha 21164 processors are on order as of September 20, 1999. The target number of processors for Squadron is 48; any more would require an additional switch and a modified networking scheme. Finally, the work of construction, testing, debugging, profiling, and maintenance of Squadron, was carried out under the Computational Infrastructure Project.

\section{ACCOMPLISHMENTS}

- $\quad$ Manuscripts and Presentations

- $\quad$ Five manuscripts were submitted to peer-reviewed journals or peer-reviewed proceedings for publication this fiscal year; two have been published already, and the other three are still in review.

- $\quad$ Two more manuscripts have been in preparation since the end of Summer of 1999, and are expected to be submitted for publication sometime in the Fall of 1999.

- $\quad$ Presentations of aspects of our work were made this year at two invited seminars, and at three national conferences. One of us also attended the semi-annual Statistical Mechanics Meeting at Rutgers University in December 1998.

- We sponsored two seminars here, with speakers from LANL and the University of Cincinnati, (Dr. Christopher Jarzynsky and Prof. Tom Beck, respectively), as part of our effort to develop or maintain collaborations.

- $\quad$ Capabilities Added

- $\quad$ A beowulf class parallel computing cluster (Squadron) has been built with 17 DEC Alpha processors. Squadron now both external and internal source code in parallel.

- A new high performance molecular dynamics simulation code was written that has been optimized for polymers; the code runs on a parallel computer cluster.

- $\quad$ A new random number generator code was written that passes every hypothesis test that we have subjected it to, and seems to be free of the correlations recently detected in other, previously trusted generators.

- $\quad$ An new efficient smoothed-particle hydrodynamics code was written and tested that handles energy effects of compression and heat conduction.

- $\quad$ The latest versions of CFDLIB from LANL have been implemented on both the SGI Origin 2000 and Squadron, our beowulf-class cluster. 
- $\quad$ A new dynamic fracture propagation code, FEFRAC, has been written, tested, and used here as part of our collaboration with U. C. Davis.

- $\quad$ The basic features of the original Macintosh time-based clustering and iterated function system map routines were ported to the platform independent software package that currently is implemented on Windows NT.

- $\quad$ A three-dimension iterated function system map and its related Markoff chain hypothesis test were implemented within the new platform independent software package.

- A new staff member was trained in the Cerius 2 computational chemistry suite, bringing to four the number of INEEL staff who have been trained by MSI in the use of their software.

- $\quad$ Three staff members have been or are being trained to use the new FEFRAC code.

- $\quad$ Spinoffs (projects that received no ESR funding but employed capabilities developed here)

- Molecular dynamics simulation of the structure of methane in water under seafloor conditions for Methane Hydrates URC.

- Molecular dynamics simulation of hydrogen-intercalated graphite, for Hydrogen Technologies Leadership Forum.

$\mathrm{CH})$

\section{REFERENCES}

1. $\quad$ L. R. Pratt and R. A. LaViolette, "Quasi-Chemical Theories of Associated Liquids," Mol. Phys. 94 (1998) 909.

2. F. H. Stillinger and R. A. LaViolette, "Sensitivity of Liquid State Inherent Structure to Details of Inter-Molecular Forces," J. Chem. Phys. 83 (1985), 6413 (Inherent structures were first described in an obscure and now out-of-print journal: F. H. Stillinger and T. A. Weber, Kinam 3A (1981) 159)

3. F. H. Stillinger and T. A. Weber, "Inherent Structure in Water," J. Phys. Chem. 87 (1983) 2833; A. Pohorille, L. R. Pratt, R. A. LaViolette, M. A. Wilson, and R. D. MacElroy, "Comparison of the Structure of Harmonic Gasses and Liquid Water", J. Chem. Phys. 87 (1987) 6070

FD)

4. J. J. Monaghan, "Smoothed Particle Hydrodynamics," Annu. Rev. Astron. Astrophys., 30, 1992, 543-574.

5. B. A. Kashiwa and R. M. Rauenzahn, "A Multimaterial Formalism," ASME Symposium on Numerical Methods for Multiphase Flows, Lake Tahoe, Nevada, June 19-23, 1994.

6. B. A. Kashiwa, N. T. Padial, R. M. Rauenzahn, W. B. VanderHeyden, "A Cell-Centered ICE Method for Multiphase Flow Simulations," ASME Symposium on Numerical Methods for Multiphase Flows, Lake Tahoe, Nevada, June 19-23, 1994. 
7. J. U. Brackbill, D. B. Kothe, and H. M. Ruppel, "FLIP: A Low-Dissipation, Particle-in-Cell Method for Fluid Flow," Comput. Phys. Comm., 48, 1988, 25-38.

8. D. Sulsky, S.-J. Zhou, and H. L. Schreyer, "Application of a Particle-in-Cell Method to Solid Mechanics," Comput. Phys. Comm., 87, 1995, 236-252. R. A. Berry, R. A. LaViolette, and R. McGraw, "Numerical Simulation of Titanium Production in the Plasma Quench Reactor," in Synthesis/Processing of Lightweight Metallic Materials, edited by F. H. Froes, C. Suryanarayana, and C. M. Ward-Close, TMS, 1995, 141-150.

9. R. A. LaViolette, R. A. Berry, and R. McGraw, "Homogeneous Nucleation of Metals in a PlasmaQuench Reactor," Plasma Chemistry and Plasma Processing, 16, 2, 1996, $249-264$.

10. R. A. Berry, R. A. LaViolette, and R. McGraw, "Numerical Simulation of Titanium Production in the Plasma Quench Reactor," in Synthesis/Processing of Lightweight Metallic Materials, edited by F. H. Froes, C. Suryanarayana, and C. M. Ward-Close, TMS, 1995, 141-150.

11. J. W. Swegle, S. W. Attaway, M. W. Heinstein, F. J. Mello, and D. L. Hicks, "An Analysis of Smoothed Particle Hydrodynamics," SAND93-2513 (unpublished), 1994.

12. P. W. Randles and L. D. Libersky, "Smoothed Particle Hydrodynamics: Some Recent Improvements and Applications," Comput. Methods Appl. Mech. Engrg., 139, 1996, 375-408.

13. C. Schar and P. K. Smolarkiewicz, "A Synchronous and Iterative Flux-Correction Formalism for Coupled Transport Equations," J. Comput. Phys., 128, 1996, 101-120.

14. W. B. VanderHeyden and B. A. Kashiwa, "Compatible Fluxes for van Leer Advection," J. Comput. Phys., 146, 1998, 1-28.

FP)

15. B. Winkler, G. Hofstetter, G. Niederwanger, "Experimental Verifications of a Constitutive Model for Concrete Cracking", Proceedings of Society of Experimental Mechanics Annual Conference on Theoretical, Experimental and Computational Mechanics, Cincinnati, Ohio, June 7-9, 1999.

\section{APPENDIX}

\section{Peer Reviewed Publications}

- $\quad$ C. R. Tolle, J. L. Budzien, and R. A. LaViolette, "Do Dynamical Systems Follow Benford's Law?" Physica D, (submitted and in review, 1999).

- $\quad$ D. H. Dahl and R. A. LaViolette, "RAWKRAB: A Random-Walk Random-Byte Generator," Appl. Math. Mod. (submitted and in review, 1998)

- $\quad$ C. R. Tolle and L. Allred, "Time-Based Clustering Symbol Train Creation," NASA Tech Briefs, (submitted and in review, 1998).

- $\quad$ R. A. LaViolette, M. E. Watwood, T. R. Ginn, and D. L. Stoner, "Spatial Disorder and Degradation Kinetics in Intrinsic Biodegradation Schemes," J. Phys. Chem., Vol. 103, 1999, p. 4480. 
- $\quad$ C. R. Tolle, and R. W. Gundersen, "Searching for Determinism in Erratic Signals: Fuzzy TimeBased Clustering of Delay Vectors," Intelligent Engineering Systems Through Artificial Neural Networks 8, Edited by C. H. Dagli, New York, NY: ASME Press, 1998.

- $\quad$ L. R. Pratt and R. A. LaViolette, "Quasi-Chemical Theories of Associated Liquids," Mol. Phys., Vol. 94, 1998, p. 909.

- J. L. Budzien, J. D. McCoy, J. G. Curro, R. A. LaViolette, and E. S. Peterson, "The Solubility of Gases in Polyethylene: Integral Equation Study of Standard Molecular Models," Macromolecules Vol. 31, 1998, p. 6669; erratum p. 8653.

- J. L. Budzien, J. D. McCoy, D. H. Weinkauf, R. A. LaViolette, and E. S. Peterson, "Solubility of Gases in Amorphous Polyethylene," Macromolecules, Vol. 31, 1998, p. 3368.

\section{Presentations}

- $\quad$ M. M. Rashid and R. Roy, "Experimental Comparison and Model Assessment for a Novel Approach to Inelastic Fracture," Fifth U. S. National Congress on Computational Mechanics, U. of Colorado, Boulder, Colorado, August 4-6, 1999 (contributed).

- J. L. Budzien, J. D. McCoy, J. G. Curro, and R. A. LaViolette, "A Simple Relation for the Solubility of Gases in Polymers," Centennial Meeting of the American Physical Society, Atlanta, Georgia, March 20-26, 1999 (contributed).

- $\quad$ R. A. LaViolette, "Spatial Disorder and Degradation Kinetics in Soil Restoration," Physical Chemistry Seminar, University of Oregon, Eugene, Oregon, February 15, 1999 (invited).

- $\quad$ R. A. LaViolette, "Spatial Disorder and Degradation Kinetics in Soil Restoration," Physical Chemistry Seminar, University of Cincinnati, Cincinnati, Ohio, January 21, 1999 (invited).

- $\quad$ C. R. Tolle, and R. W. Gundersen, "Searching for Determinism in Erratic Signals: Fuzzy TimeBased Clustering of Delay Vectors," ANNIE '98, St. Louis, Missouri, November 1-4, 1998 (contributed). 


\title{
Computational Infrastructure
}

\author{
L. E. Greenwade(PI), M. J. Cebull, J. T. Haile, S. R. Woolsey \\ SUMMARY
}

The Computational Infrastructure (CI) task of the Environmental Systems Research Program has the charter to produce a robust, effective, and modern set of computing technologies to support modeling and simulation of computational components of environment problems. The charter includes support for all elements of computational work including physical and process simulation, data analysis, and archiving along with appropriate visualization and communication tools. The principal tasks for FY 1999 were infrastructure development and numerical optimization and data formats conversions.

FY-99 has seen substantial progress made on each of these principal tasks. Working on infrastructure development, team members have made improvements in the environment, hardware, networking, and software with the goal of building a state-of-the-art computing environment. Team members working on the numerical optimization have worked to decrease the time required to run scientific applications. Runtimes have been decreased by optimizing scalar codes for the users and providing users with information and tools to optimize their own code. Team members have assisted scientists in developing, compiling, and executing parallel applications on the Suns, SGIs, Beowulf cluster, and combinations of these platforms by developing new tools, communication models, and web page documentation of instructions. One goal of the data formats conversion part of the second task is to move to a standard data storage and retrieval format to allow for greater reuse of existing visualization and data analysis capabilities. A standard data storage and retrieval format was selected that is already in use throughout the DOE Laboratory complex, and some of the scientist's data has been converted to this format. The 3-D graphics task provides the scientist with a 3-D view of the data allowing easier analysis of the data. Several 3-D graphic plots and animations were created to aid the scientists in their data analysis and presentations.

\section{PROJECT DESCRIPTION}

\section{Infrastructure}

Infrastructure development is necessary to maintain a usable, reliable, high performance computing (HPC) environment. The Network Simulation Laboratory (NSL) team works to provide stable userfriendly computing environment with as little downtime as possible on the computer platforms.

The NSL provides a number of different platforms for users. These include PC class machines running either Linux or Windows NT, workstation class systems running Solaris and Irix, and large-scale multiprocessor systems running Linux or Irix. This provides the NSL team and users a wide variety of application and problem solving capabilities. This heterogeneous environment also creates difficulties in maintaining user accounts, consistent user environments, access to data, and resources. The NSL team has used NIS+, Samba, and Network File Systems to provide easy access to user accounts and data from any NSL computer system.

NIS+ is a network system information database. Through NIS+ a central location of user accounts, home directories, host names, group info, and other information is made available to all Unix operating systems. This makes for easy password maintenance, central user administration, and streamlines many system administration tasks. The only drawback is that Windows NT will not use NIS+ as a directory service. 
File sharing is accomplished for all platforms through Network File Systems (NFS) and Samba. All user directories and data repositories are available from any NSL system. On the Unix systems this is handled seamlessly by NFS and automount. When a directory or file is requested, the system gets the resources from wherever they reside and makes them available. On the Windows systems these resources are available as network drives which may be mapped by a user whenever needed.

These services create a large amount of network traffic. To handle the demand with a minimal impact on performance, all NSL systems have been migrated from the $10 \mathrm{megabit} / \mathrm{sec}$ shared ethernet available in the EROB to dedicated high performance Cisco switches located in the lab. All NSL systems are on a minimum of 100 megabit/sec switched ethernet, with the high-end systems also having 1,000 megabit/sec (gigabit) ethernet. Performance testing indicates that this has gained as much as a 40X improvement in network performance. Major systems are configured with multiple network interfaces to allow for better load balancing and improved availability. This migration also improved reliability; our networking has remained viable at times when the networking in the rest of EROB has failed.

Mira (sun enterprise 3500), the main file server, mail server, web server, and application server has been improved to provide large amounts of fault tolerance. The system runs with multiple power supplies, system boards, and disk controllers to allow for hardware failures. Mira's file systems have been built to span multiple disks with an emphasis on performance and redundancy. This paid off when Mira suffered a disk controller failure. Another disk was available as a mirror that took over the failed disk's functionality until the failed component could be replaced. Mira delivered better than $99.9 \%$ uptime for FY-99.

A Beowulf cluster was built during FY-99. This was a first time effort for the INEEL. A Beowulf cluster is a group of workstation class systems grouped together with dedicated networking to create a supercomputer class parallel computing system. The NSL cluster is composed of $17500 \mathrm{MHz}$ DEC Alpha workstations with $512 \mathrm{mb}$ RAM and a 4 gigabyte hard drive running the Linux operating system. Each of the workstations is considered to be a node of the cluster. A Beowulf cluster's main advantage is cost. The NSL cluster was built for a small fraction of the cost of Orion, a 24 CPU Origin 2000, but it provides about the same level of performance. The major disadvantage of a cluster is complexity. The NSL team overcame many technical challenges building this cluster. A Beowulf cluster is difficult to build, maintain, and use. During this fiscal year lots of work has been done to make the Beowulf cluster more user friendly.

Some of the challenges which were encountered while building the Beowulf cluster included user management, network performance, fault recovery, file management, operating system issues, boot initialization, and compiler issues. A number of Beowulf clusters have been built across the DOE Lab complex and across the country, but they all vary in architecture, hardware, operating system levels, and purpose. Due to this and the home brew nature of these systems, few documented tools are available. The NSL team built most of the tools required to create a useable system.

One difficulty in running an application on the Beowulf cluster involves the setup required in executing a multiple processor application. The Beowulf cluster connects 17 different machines. In many ways the different machines still act as individual machines, and that makes operating the cluster more difficult for the user. For example the user must have the executable file(s) available on each of the machines in the cluster that will be used to run the application. To run an application on all 17 machines, the user was required to copy the file to each of the machines. The copy command would be entered 16 times changing the name of the machine for each copy. A parallel copy tool was developed to copy a file from the master machine to each of the slave machines. Several other parallel tools have been developed. The pfind tool is a parallel find routine. The pkill tool allows an application to be killed on all of the different machines where it is running instead of killing the application on each machine. The pps tool 
provides a parallel report process status. These are just a few examples of tools that have been developed this year. Tool development has been documented in man pages on the cluster, and it has been placed on the Intranet under the NSL's user support area.

Another difficulty that users encountered when working on the Beowulf cluster was keeping track of which node on the cluster they were actually logged in to. When working on the cluster, it is very easy to hop from one node to the next via rlogin, making it very easy to get confused. Changes were made to the user's login shells and working environments to make it possible to determine which node is being used at a glance.

On Orion the user tells the machine how many processors to use, and Orion continually looks for unused processors and schedules the work. The Beowulf cluster did not have a scheduling routine. The user was required to see which processors were open and schedule their work. If another user scheduled work on the same processor, the Beowulf cluster was not able to detect this and place the work on a different processor. The Portable Batch System (PBS) was installed on the Beowulf cluster to address some of these difficulties. PBS, a batch job and computer systems resource management package, was developed at NASA Ames Research Center and is freely available from the Internet. PBS can be installed to run jobs on a single system or several systems grouped together. PBS was also installed on the SGI and Sun machines to allow jobs to run over multiple platforms.

\section{Numerical Optimization and Data Formats}

INEEL users are running dozens of numerical simulation codes on the NSL hardware. Many of these are modeling extremely complex and tightly coupled phenomena involving diverse environmental concerns at the INEEL. In many cases these calculations take prohibitively long and hence limit the user's ability to perform their analysis at the level of detail required by the problem. Therefore a number of efficiency improvements are being pursued to enable more effective utilization of existing computational resources. These include scalar and parallel code optimization and a standard data storage format.

Scalar optimization is the process of reviewing single processor code and determining changes that decrease the runtime. The INEEL has many scientific applications that require large amounts of computing time to complete. Reducing the runtime of these applications on a single processor is the first step in code optimization. This provides an immediate benefit to the code users by reducing the time required in performing a particular simulation. It also allows for longer, more complex simulations to be run and for a greater number of users to gain access to these capabilities.

An example of scientific code that could benefit from a decreased runtime is a 3-D the subsurface flow and transport code (TETRAD). The code is most often run on Orion, the SGI Origin2000 machine located in the NSL. The WorkShop Debugger tool available for the SGI machines has several performance analyzer tools. These tools provide useful options such as determining bottlenecks, getting ideal times, tracing I/O activities, tracing page faults, finding memory leaks, and many more. Once the performance analyzer has located trouble spots in the code the analyst can attempt to correct them with the appropriate optimization techniques.

The scalar optimization of TETRAD met with limited success. Several of the tools mentioned above were used to try to find the problem areas in the TETRAD code. The code exhibited a number of problems that relate to the lack of structure utilized in its implementation. This is very common with such 'dusty deck' Fortran programs. Work on the scalar optimization of Tetrad was stopped until a nondisclosure agreement (NDA) signed between the code's owner and Silicon Graphics, Inc. (SGI). SGI's support was required to read the optimized Tetrad file, but SGI could not help unless they were 
given the relevant source code. SGI had signed a nondisclosure agreement, but the code owner would not provide the needed signature. The test that had been run and problem areas that were found were documented for future use when the nondisclosure agreement problems were resolved.

Parallel computing is an increasingly important core capability due to the ever-lengthening runtimes caused by the greater complexity of today's scientific applications. Allowing these applications to run on multiple processors decreases their total runtime and increases users' productivity. Providing scientists with the knowledge to develop, compile, and run applications on multiple processors and multiple platforms at the NSL is an important task.

In many cases the scientist had an existing multiple processor code, and an NSL team member provided the information to compile and execute the application on the multiple processors. The latest releases of Argonne National Laboratory (ANL) Message Passing Interface (MPICH) were installed and tested on all the NSL parallel computing hardware. The tests were successfully completed on SGI (24 processors), Sun (16 processors) and the Beowulf cluster (17 processors). A parallel graphics environment that allows multiple processors to write to a single graphics window was also tested. The parallel test codes were run on any of the three parallel hardware platforms by typing a single command that will build the appropriate executable for the computer on which execution is desired. The work performed in setting up and using MPICH for user level applications has been documented and presented in the form of a set of web pages (http://mira/nsl/mpich/Mpich_main.html). There were many specific procedures that had to be followed in order the make use of the parallel computing environment under MPICH. The issues were resolved and the results consolidated in a format that will assist future users and accelerate their work in developing parallel code under MPICH. NSL team members have provided support by installing the application for users on the multiple platforms and providing tools for the scientist. An internal INEEL user who has limited time and does not need to understand the details of installing applications on multiple platforms may prefer to have the NSL team do the installation.

The NSL team supports the internal INEEL users by providing information that can be used to evaluate the runtimes of their parallel applications. A standard set of benchmark results of the parallel hardware within the NSL was produced. It serves as a way to better quantify the INEEL capability in this area as well as provide local experience with the industry standard benchmarks. The Numerical Aerospace Simulations Facility (NAS) parallel benchmarks were developed at NASA Ames to provide a way to evaluate the performance of parallel computers. The seven NAS Parallel Benchmarks (NPB) benchmark procedures are commonly used in scientific application. INEEL internal users can compare the runtime of their applications to similar benchmarks to evaluate whether the application is running as expected. Most of the benchmarks were run on both the SGI Origin 2000 and the Beowulf cluster. A web page (http://mira/nsl/benchmarks/benchmark.html) has been developed to display the results of these runs for internal INEEL users. The benchmarks are a valuable tool in evaluating the times that should be expected by users on the different parallel computers. The tests compiled with both the compiler defaults and the more aggressive $\mathrm{O} 3$ optimization that is frequently used by application developers to increase performance. Several of the benchmarks would not run on the Beowulf cluster. This will continue to be worked so that a complete set of comparative results will exist, a better understanding of the difficulties of running on the cluster will be obtained, and additional experience will be acquired in porting applications to the cluster environment.

Another tool provided for the user to baseline application performance was the model of communication in parallel applications. In most parallel applications, a master processor sends data and allocates work to slave processors. The slave processors do the work and send back the resulting data. An application was written to model an arbitrary parallel application. The model allows the user to input the several parameters that represent a variety of computational and communication scenarios. The number of individual tasks that will be completed by the slave processors is input as a parameter to the 
model. The tasks can be completed in a synchronous time schedule or an asynchronous time schedule. If a synchronous time schedule is selected, the time interval for each task to complete is a required input. If an asynchronous time schedule is selected, information about the task scheduling must be input. The distributions implemented for the asynchronous time schedule are a normal distribution requiring the mean and standard deviation as inputs and a lognormal distribution requiring mean and standard error as inputs. The last input required by the user is the size of the data to be communicated to each task.

After the application receives the input, the master processor sends arbitrary data equaling the size input and the time interval input or calculated to each processor. Each slave processor receives the data and time interval. The slave process waits for the indicated time interval and then sends the data back to the master processor. The master processor receives the data back from each slave processor and allocates a new task to the slave processor until all of the tasks have been allocated.

The model was run on the primary NSL data and file server, Mira (Sun Enterprise 3500). This executes process 0 (master process) and utilizes processors from the Beowulf cluster as the client (slave) processors. This was done in order to maximize available hardware resources. The Sun server is used as the IO device since it is the only system in production with a true parallel file system. The Cisco switch can aggregate the network traffic from up to 10 Beowulf nodes to the Sun simultaneously. Hence each component is used optimally - the Sun for network and disk IO, the Cisco for its packet throughput, and the Beowulf cluster for its numerical computing capability.

The model was run with synchronous and asynchronous time scheduling, different time intervals, different data sizes, and on different numbers of processors. The model results were analyzed, plotted, and can be used as a resource for users developing parallel applications. A user would characterize their application with respect to the parameters used within this model and then review the model results and decide what to expect from a parallel version of the code. Hence a user will be able to determine potential performance bottleneck a priori and make the appropriate modifications to their application or expectations.

Another key to efficiently using computing and personnel resources is moving to a standard data storage and retrieval format. Such a format will allow for greater reuse of existing visualization and data analysis capabilities and remove one more burden from the application scientist. The ability to share scientific data without having to worry about platform dependencies is an important capability for the INEEL to develop and maintain. It is an added benefit that such a format is in wide use through the DOE Laboratory complex, the nation, and world. The Hierarchical Data Format (HDF) is a library and a platform-independent data format for storing and exchanging scientific data. HDF is developed and supported by the National Center for Supercomputing Applications (NCSA) and is available free of charge from the Internet. HDF is the selected data storage format for the Accelerated Strategic Computing Initiative (ASCI) project, a combined effort between Los Alamos, Sandia, and Lawrence Livermore National Laboratories.

The INEEL has used the TETRAD code for many years in the simulation of ground water. However, this code has some disadvantages that can be overcome by converting the output of the simulations into the standard HDF files. In the simulations are set up on an unstructured grid with refined areas of the grid where more detailed analysis is required. The refinement areas are stored as separate files from the TETRAD output, and each of the files must be viewed individually making it difficult to determine how and where the refinements fit into the unrefined data. It can become confusing and time consuming to keep switching between data regions, while trying to determine where the refinements fit together in the overall data. The TETRAD data storage also makes extremely large data files when areas with many refinements are run. The HDF file can store the results of the entire TETRAD simulation in one file removing confusion and decreasing the amount of disk storage. In processing TETRAD test files, 
it was found that on average 50 kilobytes $(\mathrm{KB})$ of disk space were saved on a single TETRAD file without refinements on a small original area. The space savings increased to $125 \mathrm{~KB}$ with the addition of a refinement area file, and the space reduction was $350 \mathrm{~KB}$ with three additional refinement areas. The disk space reduction increases with larger sample areas and more refinement areas. In HDF Version 5, the original structure can be defined and maintained in a hierarchical form to ensure no loss of information which is essential for accurate analysis of the results from a simulation run.

The application used to translate the TETRAD output to the HDF files was written to allow the users maximum flexibility. The application can be used with a GUI interface allowing the user to interact with the conversion. In the GUI version of the translator the user can select files that will be translated from a list and view updates of the translation process. If interaction is not desired, the translation system can be run automated from command-line scripts without requiring user attention and the resulting messages can be saved in a log file. In either case the result of the translation is the one HDF file.

The HDF file can then be processed into a visualization system known as AVS to allow the user see a 3-D model of the simulation results. The 3-D model allows the user to quickly analyze the output of the groundwater flow simulation. The model can be viewed from any angle and displayed showing crosssectional planes shaded with colors representative of the amounts of the variable of interest found in the area. The refinement areas are automatically shown as part of model, and a zoom feature may be used to view the refinement areas in more detail. The model allows the user to view all of the data at once, without the need to try to superimpose the model of one refinement area on to the total area.. The user can change quickly between variables, instantly seeing the relationships between variables and areas of interest in the simulated region. The model may also be run over the different time steps showing the user the changes in the variable of interest in the ground water flow over time.

\section{ACCOMPLISHMENTS}

\section{Infrastructure}

The NSL computers maintained a better than $99 \%$ uptime for the year. During FY-99 the number of users increased by $500 \%$ and system utilization remained very high, typically between $70 \%$ and $90 \%$. Quite a few new software packages were added for both existing and new users. The network information services were dramatically improved proving greater flexibility to users while decreasing the administrative costs for user account management. This allows users to log into any NSL machine a see a consistent file system structure that is machine-independent. Physical infrastructure was maintained at its present level through monitoring and preventative maintenance. Great strides were made in the computer network infrastructure providing an order-of-magnitude improvement over the previous system. A Beowulf class supercomputing cluster was designed, acquired and build by the NSL staff. This system was brought up with all the necessary operating and user software and run through numerous test and evaluation procedures. This system was transferred to the end user who will begin using it in FY 2000.

\section{Numerical Optimization and Data Formats}

Several numerical simulation codes in use at the INEEL were analyzed for computational efficiency. These included codes developed within the INEEL as well as those from outside. These codes were profiled so as to identify areas that may benefit from improvements. The primary code used for contaminant migration in groundwater was shown to operate at an efficiency of only $4 \%$. Many specific areas of improvement were identified and where communicated to the INEEL users. Others were optimized for a single processor and showed improvements of 50-1,000\%. Several libraries for developing parallel codes were acquired and installed on the NSL parallel computing hardware. These 
were used by multiple users in the development and porting of simulation codes that were then run on the NSL hardware. The TETRAD groundwater modeling code was integrated with the Accelerated Strategic Computing Initiative's parallel library and data formats software. This was integrated with the AVS visualization toolkit to provide the first, interactive, machine-independent data exploration tool for this application. This increased the overall efficiency of the application and provided a much more effective analysis environment. The overall result is that INEEL researchers can conduct simulations of larger size and complexity in shorter time than ever before. 


\section{Materials Dynamics}

Reliable intermediate and long-term storage of hazardous materials depends critically on understanding the mechanisms that lead to protective behavior or degradation in engineered barriers and the fundamental processes by which corrosive fluids are transported to storage systems and contamination is transported to the environment. This topical area is focused on three different facets of these issues: mechanisms of biologically assisted degradation of cladding materials in spent nuclear fuel, fabrication of dense impermeable ceramic coatings for waste processing equipment and engineered barriers, and transport of contaminated fluids in porous or fractured media. The technical approach in each of these subject areas has been to develop a fundamental understanding of underlying mechanisms of materials behavior so that defensible prediction of materials behavior over very long time scales are possible, as well as rational strategies for control and mitigation of materials degradation. Detailed descriptions of the technical progress in each of the topical areas are presented in the following sections. 


\title{
Coatings for Environmental Applications
}

\author{
R. N. Wright, J. K. Wright, W. D. Swank and R. A. Gavalya \\ SUMMARY
}

Coatings have been identified as potential solutions for several critical environmental management needs. For example, coatings are needed to improve resistance to elevated temperature degradation to extend the lifetime of process equipment for vitrification and to provide aqueous corrosion protection of casks for long term storage of spent nuclear fuel. While coatings are widely used in engineering applications, there is little scientific understanding of the relationship of processing to microstructure and properties. As a result, it is difficult to predict the performance of coatings in service. The objective of this project is to develop and experimentally validate models that allow one to predict the influence of process variables on the microstructure, bonding, and mechanical integrity of ceramic coatings applied to metallic substrates, using a variety of thermal spray processes. Simulations were validated by postmortem characterization of microstructure, cracking, and bond strength of a model $\mathrm{MgAl}_{2} \mathrm{O}_{4}$ spinel coating on Type 304 stainless steel substrates. A unique residual stress measurement capability, developed in the previous year of this project, has been used to determine the magnitude of both components (quenching and thermal expansion mismatch) of the residual stress in the coating-substrate system. Methods have been developed to consistently prepare and quantitatively characterize substrate and coating microstructures. It has been determined that surface preparation by grit blasting introduces substantial cold work into the stainless steel that is subsequently annealed by coating deposition. Alteration of the near-surface region of the substrate is significant for corrosion properties. Application of a Ni-Al bond coat instead of grit blasting greatly improves adhesion of the ceramic coating, but also introduces significant residual stress. The quenching contribution of the residual stress dominates the thermal expansion portion of the stress. The quenching contribution is much greater in metallic bond coat compared to the ceramic, apparently because the ceramic has mechanisms, such as cracking and debonding, available to shed residual stress. Thermal cycling of the coating/substrate system results in permanent relief of residual stress in the ceramic coating as a result of cracking. The Ni-Al bond coat material exhibited considerably less hysteresis during thermal cycling.

\section{PROJECT DESCRIPTION}

\section{Introduction}

Residual stresses arise during thermal spraying and are a major driving force for failure and debonding of coatings. The stresses are comprised of a quenching contribution, which is caused as the hot splats adhere to the cold substrate and contract as they cool from the temperature at impact to some local equilibrium temperature achieved by the coating and adjacent substrate material. The contraction is constrained by the underlying substrate, and a tensile stress necessarily develops in the deposit. In addition to the quenching contribution, there is a mismatch contribution resulting from the difference in thermal expansion coefficients between the coating and the substrate materials as they cool from some elevated temperature obtained at the end of the spray process back to room temperature. When the coating is ceramic and the substrate is metallic, this contribution typically results in a compressive stress in the coating. The net residual stress depends on the sum of the quenching and mismatch contributions.

One of the most common ways to determine the residual stresses experimentally is by measuring the curvature of a thin sample. Figure 1 shows a schematic of the curvature resulting from the quenching and mismatch contributions for the case of a ceramic coating applied to a metallic substrate. The direction of curvature in the sample is an indication of the relative dominance of these two contributions. 
a. Quenching contribution
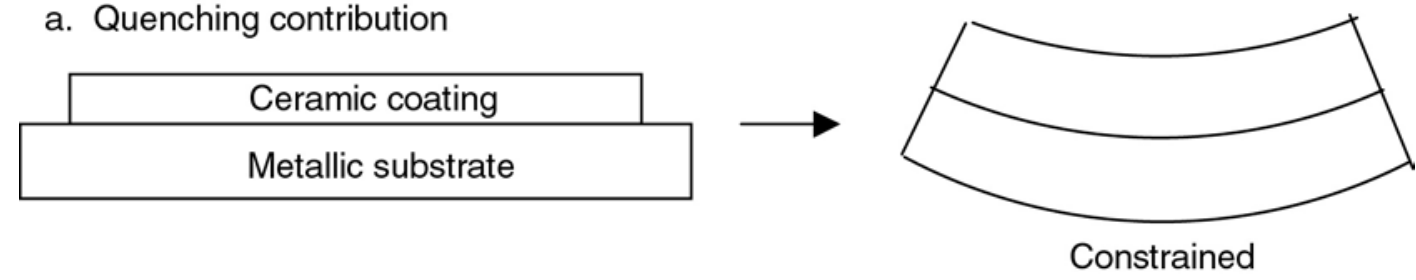

b. Mismatch contribution

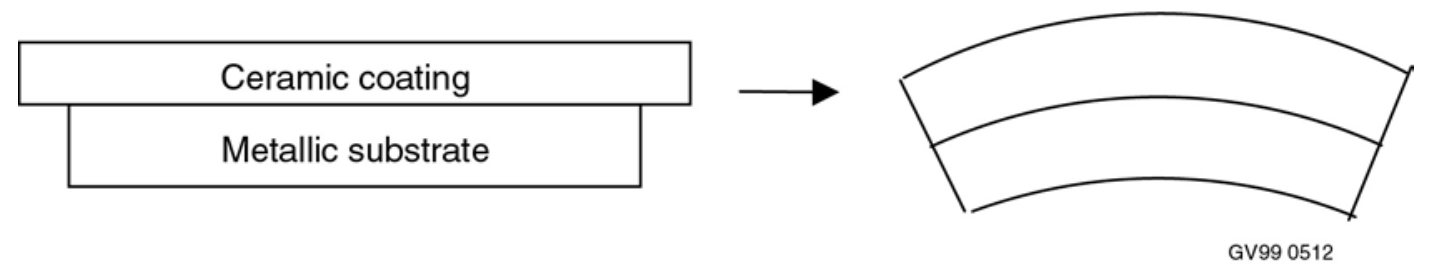

Figure 1. Schematic of curvature resulting from quenching and thermal expansion mismatch.

The curvature is dependent on both the change in temperature, $\Delta \mathrm{T}$, and the difference in thermal expansion coefficients, $\Delta \alpha$. The $\Delta \mathrm{T}$ can be separated into a quenching portion, $\mathrm{T}_{\text {splat }}-\mathrm{T}_{\text {entire sample during }}$ deposition, which occurs during deposition, and a mismatch portion, $\mathrm{T}_{\text {entire sample during deposition }}-\mathrm{T}_{\text {room }}$ which occurs during final cooling. The $\Delta \alpha$ applies only during final cooling as well.

The residual stress can be directly calculated from the curvature of the sample if certain assumptions are valid. Among these are perfect bonding of the coating, elastic deformation of the metal, and uniform curvature of the coupon. In reality it is likely that debonding, localized plasticity, creep, and/or micro-cracking occur. Some of these features, for example plasticity and creep, can be accounted for by use of more complex finite element method (FEM) calculations. Others remain difficult to quantify.

Ceramic coatings with $\mathrm{MgAl}_{2} \mathrm{O}_{4}$ spinel composition applied to Type 304 stainless steel substrates were used as a model system for this work. The spinel was selected as the model ceramic because of expressed interest as a candidate coating material for waste canisters for long term storage of spent fuel and because it is free of phase transformations upon cooling from the deposition temperature. Type 304 stainless steel was used as a substrate material because this alloy has well-characterized mechanical properties, and resists oxidation. In most cases a $\mathrm{Ni}$-Al bond coat that is typical of commercial practice was applied between the stainless steel substrate and the spinel.

\section{Experimental Details and Results}

\section{Method of Stress Determination}

A multi-beam optical sensor (MOS) was used to acquire laser-based curvature measurements. The measurements are accomplished with an InGaAIP laser diode, etalon, charged-coupled device (CCD), frame grabber, and MOS software. A single laser beam enters a highly reflective etalon and exits as multiple parallel beams. The parallel beams strike the sample's polished surface and are then reflected to the CCD where the image, which appears as spots, is captured by a frame grabber.

Prior to starting the software program, the user is prompted to choose the number of spots that will be monitored. Sets of "windows" corresponding to the number of spots that will be monitored are generated and are positioned to enclose each spot. When the MOS program starts running, each window 
automatically locates the centroid of its individual spot and tracks the spot and its centroid during data acquisition. The distances between neighboring spots/centroids are then calculated and either stored as a reference or compared to a reference. In order to determine the substrate curvature, $\kappa$, the spacing between each adjacent spot is measured and compared to a "flat" reference that was taken previously. In our case, a two-inch optical mirror is assumed to be a flat surface and spot spacing images reflected from the mirror are gathered to establish a reference.

An analytic solution was used to convert measured spot spacing into curvature, $\kappa$, from the following equation, ${ }^{1}$

$$
\frac{\Delta \mathrm{D}}{\mathrm{D}_{0}}=\frac{2 \mathrm{~L} \kappa}{\cos \alpha}
$$

where $D_{0}=$ reference spot spacing, $\Delta D=D(t)-D_{0}, L$ is the length from sample to CCD array, $\kappa$ is the curvature, and $\alpha$ is the angle of incidence on the sample ( $\alpha=0^{0}$ for normal incidence). The spacing between the spot centroids of the coupon, $D(t)$, are then compared to the spot centroid spacing of a 50mm optical mirror, $D_{0}$, to determine $\Delta D$.

The MOS system is coupled with a furnace capable of heating to $\sim 1000^{\circ} \mathrm{C}$ and a vacuum chamber capable of $\sim 10^{-7}$ Torr to enable elevated temperature measurements without oxidation. Note that because of the large curvature imparted by grit blasting described in detail below, none of the coupons for residual stress measurement were prepared by this method. Coatings were applied directly after application of a bond coat. Spot spacing was measured after initial coupon preparation, after bond coat application, and after spinel coat application. In most cases, additional measurements were taken during subsequent heating and cooling of the coated coupon. Thermocouples were laser-welded directly towards one end of the back of the coupon to monitor sample temperature during sample heating. Since the laser welding caused local distortion, spot spacing measurements at the opposite end of the coupon were used to determine the curvature during these experiments.

Residual stresses were subsequently calculated ${ }^{2,3,4}$ from experimentally determined curvatures and coating thickness, and bulk tabulated values for elastic modulus. Biaxial modulus was obtained by dividing elastic modulus by $1-v$, where $v$ is Poisson's ratio and was assumed to be 0.3 for all materials.

\section{General Sample Preparation}

The substrates used in this work were $1.5 \mathrm{~mm}$, thick Type 304 stainless steel, cut into $12 \times 75 \mathrm{~mm}$ strips. Thin samples curve more and thus are sensitive to changes in residual stress. Electrical discharge machining (EDM) was used to cut samples to size after preliminary experiments revealed that shearing and water jet cutting caused non-uniform twisted or bent coupons. The backside of the coupon was then polished to a mirror finish to achieve adequate reflection of the laser spots so the spacing could be measured. The other side was prepared for the thermal spray coating by application of a Ni-Al bond coat, or in some cases grit blasting.

Both bond coat and spinel coatings were applied using a conventional Metco 9MB plasma spray torch in laboratory air. The torch was operated using $40 \mathrm{slm}$ of argon and $10 \mathrm{slm}$ hydrogen as the process gases. A standoff distance of $100 \mathrm{~mm}$ was maintained from the torch to the substrate for all of the experiments. The torch was fixed, while the substrate was rastered through the plume to apply the coating. The traverse speed was varied from 70 to $200 \mathrm{~mm} / \mathrm{s}$ to vary the amount of material deposited in each pass so that spinel coatings of nominally constant thickness were deposited either in 10 or 24 layers. 
The Ni-Al bond coat was deposited using four passes. All coupons were cooled with an air jet from the back.

\section{Metallography}

Consistent metallographic sample preparation techniques and image analysis are critical in coating development. A procedure was developed for automated preparation of spinel coatings on stainless steel coupons that retained edge features and minimized pullout of unmelted particles imbedded in the coating. The procedure was validated by repeated polishing and re-examining of an identical area on several coupons and determining that the nature of the structure and quantitative measures of porosity and substrate grain size did not vary within experimental accuracy. The automated image analysis system purchased in the first year of the program was used extensively to characterize spinel coatings. Subroutines were written for the program to automatically measure porosity, pore size distribution, the lineal extent of cracking and the orientation of cracks with respect to the spray direction.

It was determined that there was a consistent difference in the apparent volume fraction of porosity between images obtained using the optical microscope compared to backscattered electron images obtained using the scanning electron microscope. Optical images consistently indicated a lower volume fraction of porosity and apparently revealed a second phase within the coating. Backscattered electron images showed that what appeared to be a second phase in the optical images was unmelted ceramic particles imbedded in the coating. These unmelted particles were badly fragmented and contained porosity that was below the resolution of optical microscopy. The final procedure for quantitative metallography was to examine the coatings optically during preparation, followed by capture of electronic images using backscattered electron imaging and automated analysis using preset parameters in custom subroutines. A representative image of a coating made up by spraying ten relatively thick layers is shown in Figure 2. In the example shown, cracking is evident between every other layer of the coating, i.e., there are ten layers and five cracks are observed. Coatings deposited by application of twice as many thin layers exhibited less cracking than those formed by application of thick layers.

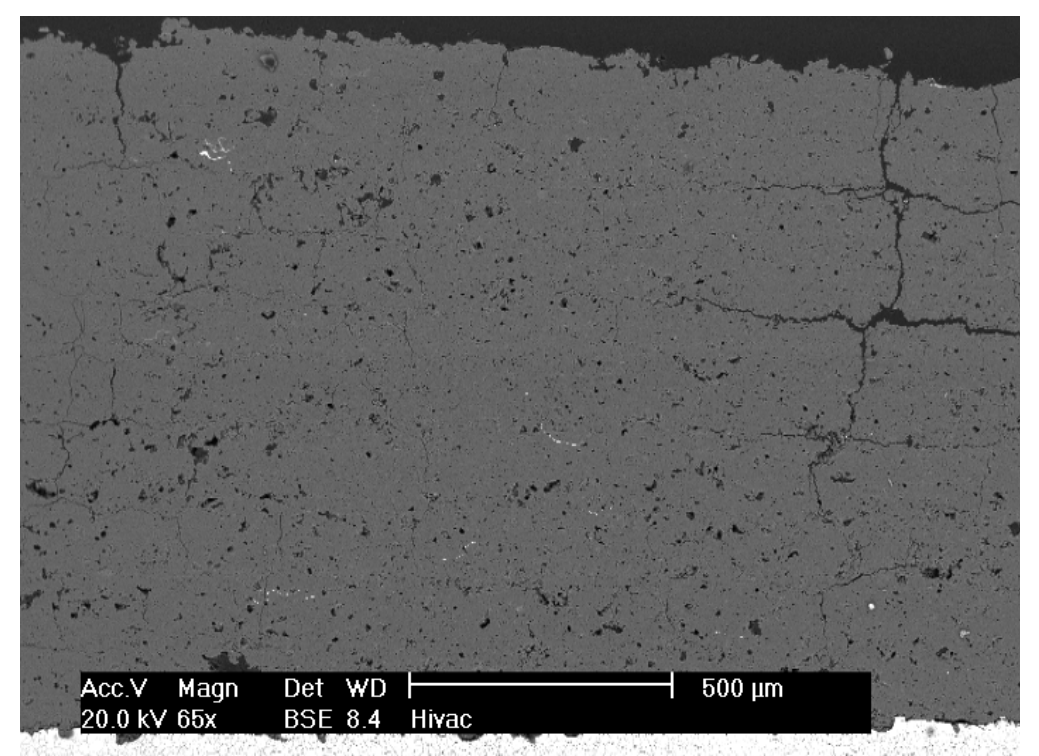

Figure 2. Backscattered scanning electron micrograph of a $\mathrm{MgAl}_{2} \mathrm{O}_{4}$ ceramic coating on a Type 304 stainless steel substrate. 


\section{Grit Blasting Study}

Metallic substrates for coating are typically prepared by abrading the surface with high velocity ceramic grit, occasionally followed by application of a metallic bond coat. The purpose of this surface treatment is twofold; first it helps remove loose oxide or contamination. Second, it is believed that much of the bonding between a ceramic coating and the substrate is mechanical interlocking with a rough surface. Grit blasting the $1.5 \mathrm{~mm}$ thick stainless steel coupons was found to cause greater curvature than application of the coating. The radius of curvature after grit blasting was on the order of $0.7 \mathrm{~m}$ compared to a typical value of $5 \mathrm{~m}$ for bond and spinel coating (where a small radius corresponds to large deflection). It is possible to calculate the elastic stress required to cause the observed curvature. The elastic residual stress calculated in this way is unreasonably large, comparable to the modulus. Clearly, the curvature results from significant plastic deformation. Extensive plasticity in the substrate is a potential problem for long term performance since deformation can lead to enhanced corrosion rates.

The nature and extent of plasticity after grit blasting, and the annealing effects of coating deposition (e.g., recovery or recrystallization) were examined in a series of experiments using optical and scanning and transmission electron microscopy (TEM). The variation in surface condition and degree of induced plasticity was examined as a function of grit size, velocity (over the practical measured range from approximately 100 to $200 \mathrm{~m} / \mathrm{s}$ ) at a constant stand-off distance, and exposure time (i.e., a surrogate for total particle impacts). Two specific particle velocities, 140 and $180 \mathrm{~m} / \mathrm{s}$, were examined in detail. In

addition, a series of samples grit blasted with a particle velocity of $180 \mathrm{~m} / \mathrm{s}$ were subsequently coated with bond coat or ceramic alone, or a bond coat with ceramic applied also.

TEM examination of grit-blasted samples showed that there was substantial cold work in the near surface region indicated by a very high density of defects. The cold work was not uniform; there were grains that were essentially undeformed. At the midline of the specimen, approximately $750 \mu \mathrm{m}$ below the surface, the material remained in the annealed condition. TEM examination showed that there was substantial annealing of defects resulting from application of a coating. Representative photographs from the TEM study are shown in Figures $3 \mathrm{a}-\mathrm{c}$. There were no qualitative differences in the amount of annealing resulting from thick or thin ceramic coatings, or a thin bond coat. There was no evidence of either recrystallization or grain growth visible using optical microscopy. There was little evidence of dissolution of grain boundary carbides, although subtle differences were apparent in the way electropolishing affected grain boundaries during TEM sample preparation of coated materials. In the coated materials TEM sample preparation caused preferential thinning at the grain boundaries, suggesting that some change in the grain boundary chemistry had occurred.

Dissolution of grain boundary carbides and recrystallization are higher temperature processes than recovery of the defect structure. Carbide dissolution, for example, is thought to occur only for temperatures above $950^{\circ} \mathrm{C}$. Depending on the amount of cold work, recrystallization in Type 304 stainless steel typically does not occur below about 500 to $600^{\circ} \mathrm{C}$. ${ }^{5,6}$ The observation that recovery occurs, but high temperature processes do not, even in close proximity to the substrate/coating interface places an upper limit on the substrate temperature of a few hundred degrees. These observations are consistent with measured maximum temperature measured at the rear surface of the sample of $250^{\circ} \mathrm{C}$ and the sluggishness of grain growth of stainless steel.

\section{Examination of the Influence of Surface Preparation on Bond Strength}

In addition to being convenient for residual stress measurement experiments, bond coating was found to yield better adhesion between the coating and substrate compared to grit blasting. Bond strength results measured using standard ASTM pull tab methods are shown in Table 1 for specimens prepared with the Ni-Al bond coat, and by grit blasting at 140 and $180 \mathrm{~m} / \mathrm{s}$ particle velocities. For each surface 


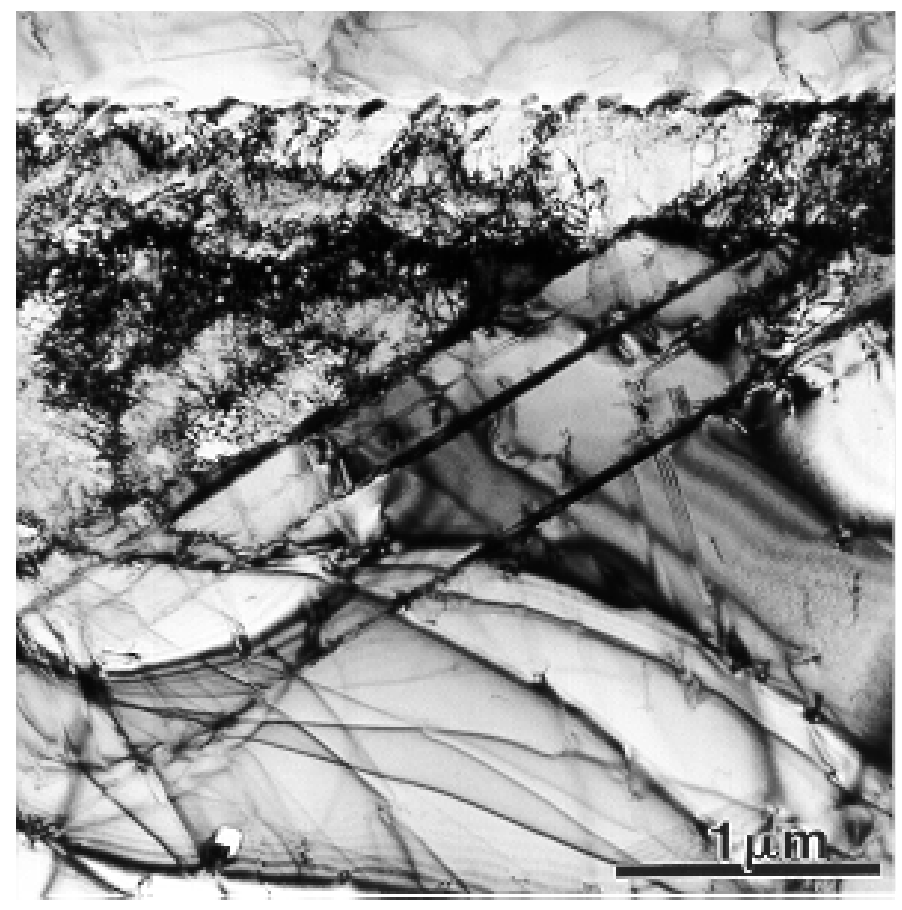

(a)

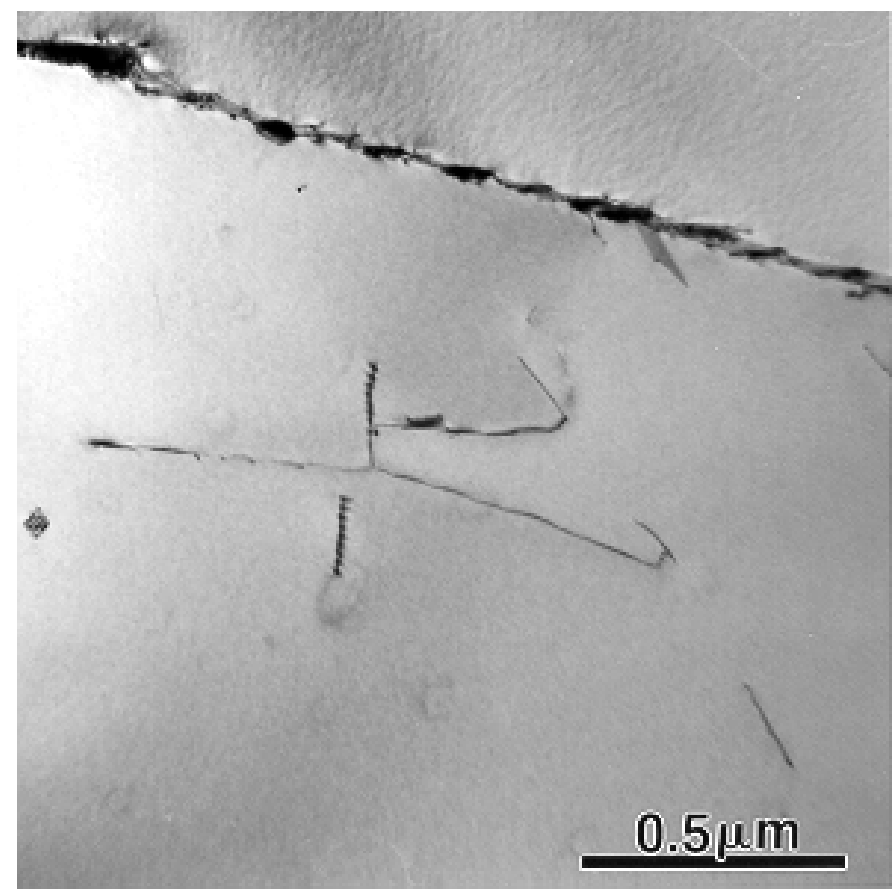

(b)

Figure 3. Transmission electron micrographs of the defect structure in Type 304 stainless steel after grit blasting with particles at a velocity of $180 \mathrm{~m} / \mathrm{s}$ (a) in the near surface region, (b) $750 \mu \mathrm{m}$ below the surface, and (c) after subsequent deposition of a spinel coating. 


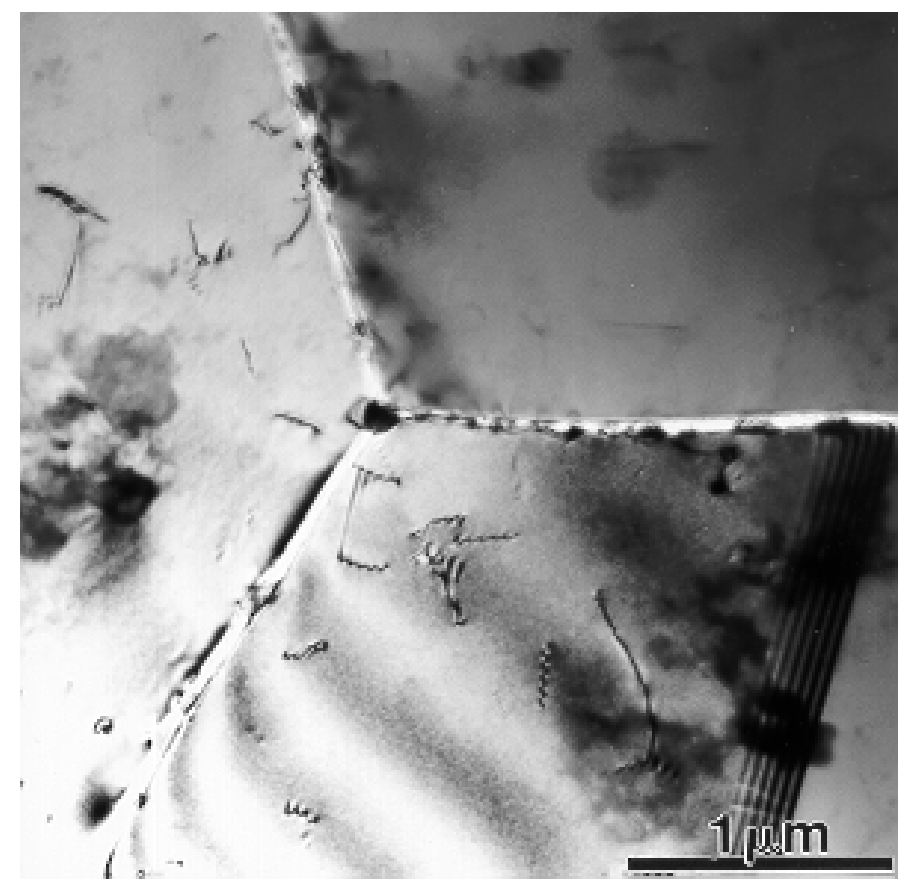

(c)

Figure 3. (continued).

Table 1. Bond strength values and location of failure for spinel coatings as a function of substrate surface preparation and number of layers in the coating.

\begin{tabular}{lccccc}
\hline $\begin{array}{c}\text { Specimen Surface } \\
\text { Preparation }\end{array}$ & $\begin{array}{c}\text { Strength } \\
(\mathrm{Mpa})\end{array}$ & $\begin{array}{c}\text { Number of } \\
\text { Coating Layers }\end{array}$ & $\begin{array}{c}\text { Bond Failure } \\
(\%)\end{array}$ & $\begin{array}{c}\text { Coating Failure } \\
(\%)\end{array}$ & $\begin{array}{c}\text { Epoxy Failure } \\
(\%)\end{array}$ \\
\hline Ni-Al bond coat & 16.7 & 24 & 40 & 40 & 10 \\
Ni-Al bond coat & 13.8 & 12 & 10 & 75 & 15 \\
Grit blasted, $140 \mathrm{~m} / \mathrm{s}$ & 9.5 & 24 & 100 & 0 & 0 \\
Grit blasted, $140 \mathrm{~m} / \mathrm{s}$ & 8.6 & 12 & 100 & 0 & 0 \\
Grit blasted, $180 \mathrm{~m} / \mathrm{s}$ & 9.2 & 24 & 100 & 0 & 0 \\
Grit blasted, $180 \mathrm{~m} / \mathrm{s}$ & 8.6 & 12 & 100 & 0 & 0 \\
\hline
\end{tabular}

preparation method coatings of nominally identical thickness were applied using 12 or 24 passes.

Estimates of the fraction of the fracture surface where failure occurred are indicated. The notation "bond failure" corresponds to separation of the coating from the substrate, "coating failure" indicates fracture within the coating material, and "epoxy failure" refers to failure of the adhesive used to attach the coated sample to the fixture used to apply tensile loading. The results clearly indicate that the strength of the coating exceeds the bond strength for any of the samples prepared by grit blasting. Generally the samples prepared using a bond coat have better bond strength compared to those that were grit blasted. There is also clear evidence in the bond coat samples that depositing the coating in many thin layers improves the properties of the coating. From the relative proportion of failure in the coating and at the substrate/coating interface, it appears that the strength of the coating is related to the propensity toward microcracking shown for a sample deposited in thick layers in Figure 2. 


\section{Coating Residual Stress Study}

Good repeatability was observed among curvature measurements of samples processed similarly, as seen in Table 2, which presents some example data collected after each stage in processing for three samples. Most curvature comes after the bond coat has been applied. Adding the spinel decreases the curvature, while heating the sample in the MOS furnace increases the curvature. The direction of the curvature indicates that the samples are dominated by the quenching rather than the mismatch effect (refer to Figure 1), as expected, since the quenching $\Delta \mathrm{T}$ is large while $\Delta \alpha$ between the metallic bond coat and the metallic substrate, as well as the mismatch $\Delta \mathrm{T}$ are relatively small. At this stage, the bond coat is in tension.

After the spinel has been applied, the sample still curves in the same direction, but less severely. The curvature resulting from the spinel appears to be dominated by mismatch. In this case, $\Delta \alpha$ between the ceramic coating and the metallic substrate (and bond coat) is large. Also, the mismatch $\Delta \mathrm{T}$ is slightly more than that obtained during bond coat application $\left(\sim 130^{\circ} \mathrm{C}\right.$ vs. $\left.70^{\circ} \mathrm{C}\right)$ because the much thicker spinel coating imparts more heat to the sample. As the sample cools to room temperature, the ceramic contracts less than the metal and the curvature decreases. If a spinel could be applied directly to a substrate (i.e., without a bond coat), curvature in the opposite direction might be observed, as illustrated in Figure 1. Although the quenching component of $\Delta \mathrm{T}$ is large, the quench stresses are not as large as expected, perhaps because of microcracking (Figure 2) or debonding of the coating. A similar result has been observed previously, ${ }^{2}$ with an $\mathrm{Al}_{2} \mathrm{O}_{3}$ coating dominated by mismatch and a Ni-based coating dominated by quenching.

Since the sample is bent, stress varies as a function of position through the thickness of the sample. While the bond coat is thin, and stress variations are minimal, the spinel coatings in this study are nearly as thick as the substrates, and the residual stress varies dramatically with thickness. Residual stresses in the spinel must be further defined as total, if the uncoated coupon curvature is considered as the starting condition, or net, if the curvature after bond coat application is considered as the starting condition. The total average stress in the coating (i.e., the residual stress at the midplane of the coating) is tensile, while the interface stress is more tensile and the surface of the coating is compressive. Conversely, the net average stress in the coating is compressive, while the interface stress is more compressive and the surface of the coating is tensile. In all cases room temperature residual stress magnitudes in the spinel are less than $100 \mathrm{MPa}$.

\section{Thermal Cycling}

As the fully coated sample is heated in the MOS furnace, the ceramic expands less than the metal and the curvature increases. As a result, the total stress magnitudes at all locations increase. Net residual stresses actually switch sign (i.e., change from compressive to tensile or vice versa) upon heating. In theory, the net residual stress determined upon heating the sample to the temperature achieved by the

Table 2. Radius of curvature of three samples, in meters, measured at various processing stages.

\begin{tabular}{lccc}
\hline \multicolumn{1}{c}{ Condition } & 16EDM21 & 16EDM22 & 16EDM23 \\
\hline Before thermal spraying & 404.7 & 252.9 & 69.8 \\
After bond coat & 4.8 & 3.7 & 4.2 \\
After spinel coat & 4.9 & 4.5 & 5.7 \\
Spinel coat, heated to $150^{\circ} \mathrm{C}$ & 2.4 & 2.2 & 2.4 \\
\hline
\end{tabular}


entire sample during spraying $\left(\sim 150^{\circ} \mathrm{C}\right.$, as measured at the sample back surface) should be equivalent to the quench stress component, provided that perfect bonding is maintained. Accordingly, an average value of about $14 \mathrm{MPa}$, tension, was calculated as the quench stress for the samples listed in Table 1.

Measuring the curvature as a function of temperature also permits calculation of the thermal expansion coefficient of the spinel coating directly, rather than relying on tabulated values for bulk spinel. ${ }^{2}$ Thermal expansion coefficients calculated by this method for the thermally sprayed spinel ranged from $7.7 \times 10^{-6}$ at $100^{\circ} \mathrm{C}$ to $8.7 \times 10^{-6}$ at $250^{\circ} \mathrm{C}$, while those tabulated for bulk spinel range from $7.5 \times 10^{-6}$ at $100^{\circ} \mathrm{C}$ to $8.4 \times 10^{-6}$ at $300^{\circ} \mathrm{C}$. However, this calculation requires the elastic modulus as input, and to date, only the tabulated modulus for bulk spinel is available.

Hysteresis-type effects are observed in the coupons when they are heated and cooled in the furnace. After being heated and allowed to cool, the curvature is less than the curvature recorded prior to heating. As the coupon heats, the stainless steel substrate expands more than the attached coating, due to the difference in thermal expansion coefficients. As a result, tensile stresses develop in the coating at the interface and debonding between the coating/substrate interface and fracturing within the coating occurs. This relieves part of the residual stress within the coupon and allows it to partially "relax" with a subsequent decrease in curvature. Hysteresis is shown in Figure 4 for thermal cycling of coupons with $\mathrm{Ni}-\mathrm{Al}$ bond coat and spinel coating.

Coupons coated only with $\mathrm{Ni}-\mathrm{Al}$ were observed to decrease slightly in curvature when thermally cycled, whereas spinel coated coupons displayed a more pronounced decrease in curvature. Thermal expansion coefficients between the stainless steel substrate and the Ni-Al bond coat are similar in value. Consequently, when the coupon is heated, the substrate and coating expand almost equally, and less tensile stress is placed on the $\mathrm{Ni}-\mathrm{Al}$ coating. In contrast, thermal expansion coefficient differences are larger between the spinel and stainless steel substrate, as compared to $\mathrm{Ni}-\mathrm{Al}$ and the substrate. In addition to a smaller $\Delta \alpha$ driving force during thermal cycling, the bond coat may deform plastically rather than microcrack as the spinel would.

\section{Furnace Thermal Gradient Study}

In order to obtain curvature measurements on samples at elevated temperature, samples were slowly heated and spot spacing was collected as the sample reached the temperature of interest. Although the heating rate is relatively slow, it is conceivable that a temperature gradient exists through the thickness of the substrate due to differences in the reflectivity of the stainless steel and spinel surfaces. This potential temperature gradient could cause curvature that is not related to the residual stress.

A substrate, polished on one side for the purpose of curvature measurements, was prepared with a thermocouple attached to each side. The bond coat and spinel were then applied to the unpolished side of the sample as usual, causing one of the thermocouples to be located at the substrate/coating interface. The coated sample was heated in the curvature-measuring apparatus at the same rate used during previous measurements. The temperature history of the thermocouples indicated a difference in temperature between the two substrate surfaces. The difference occurs because of the difference in radiation properties between the polished and highly reflective stainless steel surface and the more absorptive spinel surface. A maximum difference of about $12^{\circ} \mathrm{C}$ was observed in the vicinity of $120^{\circ} \mathrm{C}$. Heat transfer calculations, considering the thickness and thermal properties of the two layers, indicated that if the polished surface of the steel was $120^{\circ} \mathrm{C}$, and the interface was $132^{\circ} \mathrm{C}$, then the spinel surface would be about $143^{\circ} \mathrm{C}$.

Using these temperatures as input in a finite element model (FEM), the radius of curvature, resulting from this through-thickness temperature gradient, was estimated to be about $26 \mathrm{~m}$. Although this 


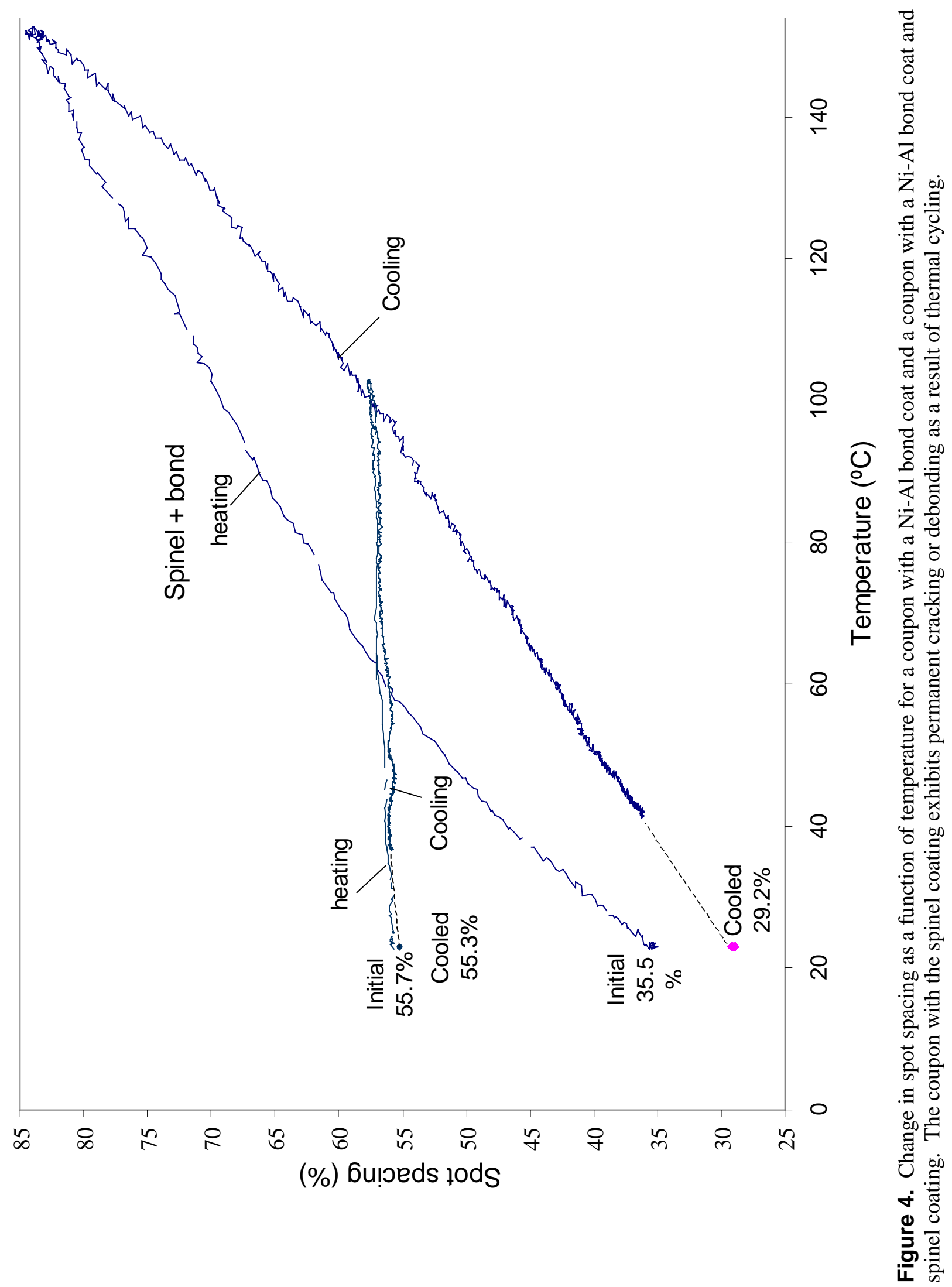


is an order of magnitude less than curvatures resulting from coatings, it was determined that all further measurements at elevated temperature would incorporate a 15-minute hold at temperature to bring the sample to steady state before curvature was determined.

\section{Finite Element Modeling}

\section{Approach}

The long, narrow strip geometry selected for this study was used in the hope that residual stresses could be accurately calculated using straight-forward analytic solutions available from the literature ${ }^{2,7,8,9}$ or two dimensional finite element models of the $x-y$ plane in Figure 5. It was necessary to validate these simplified approaches before proceeding with more complex FEM analyses.

Several types of finite elements are available for this type of analysis, each with an inherent set of assumptions. Two dimensional plane strain requires $\varepsilon_{z}=0$, a condition appropriate if the $\mathrm{z}$ dimension is large. However, $\varepsilon_{\mathrm{z}}$ is the total strain, including the thermal strains. Since thermal expansion is being modeled, this assumption results in unrealistically large $\sigma_{z}$, so plane strain is inappropriate for this case. In contrast, generalized plane strain allows the xy planes (i.e., the sample edges perpendicular to $\mathrm{z}$, shown shaded in Figure 5) to expand or rotate with temperature, but they must remain planar. Two dimensional plane stress requires $\sigma_{\mathrm{z}}=0$, an appropriate condition if the $\mathrm{z}$ dimension is very small. The most accurate but computationally intensive elements are solid three-dimensional elements, which permit the exact geometry to be modeled. Calculations were run with each type of element, except plane strain, and compared with analytic solutions for a plate subjected to biaxial conditions $\sigma_{\mathrm{x}}=\sigma_{\mathrm{z}}, \sigma_{\mathrm{y}}=0$.

To date, generalized 2D plane strain elements had been used exclusively; however, the xy (shaded) edges had been required to remain parallel during thermal contraction. Three-dimensional modeling showed that although most of the curvature that results from residual stress occurs along the $\mathrm{x}$ axis, some does occur along the $\mathrm{z}$ axis. Therefore, the $\mathrm{xy}$ planes must be allowed to rotate with respect to each other. The element definitions were modified accordingly. Once done, generalized plane strain gave the same results, within $2 \%$, as the centerline (x-axis) of the 3D model. At the edges of the sample, conditions differ from those in the bulk sample, but the effect is very localized. In essence, the stress state is constant along the $\mathrm{x}$ and $\mathrm{z}$ axis until very near the sample edges. Plane stress elements give results similar to those along the xy edges of the 3D model.

The 3D model verified that most of the sample is under a biaxial stress condition, and stresses can be calculated accurately using analytic solutions, provided materials remain linearly elastic. If non-linearity such as plasticity or temperature dependence exist, stresses can be calculated using 2D generalized plane strain elements.

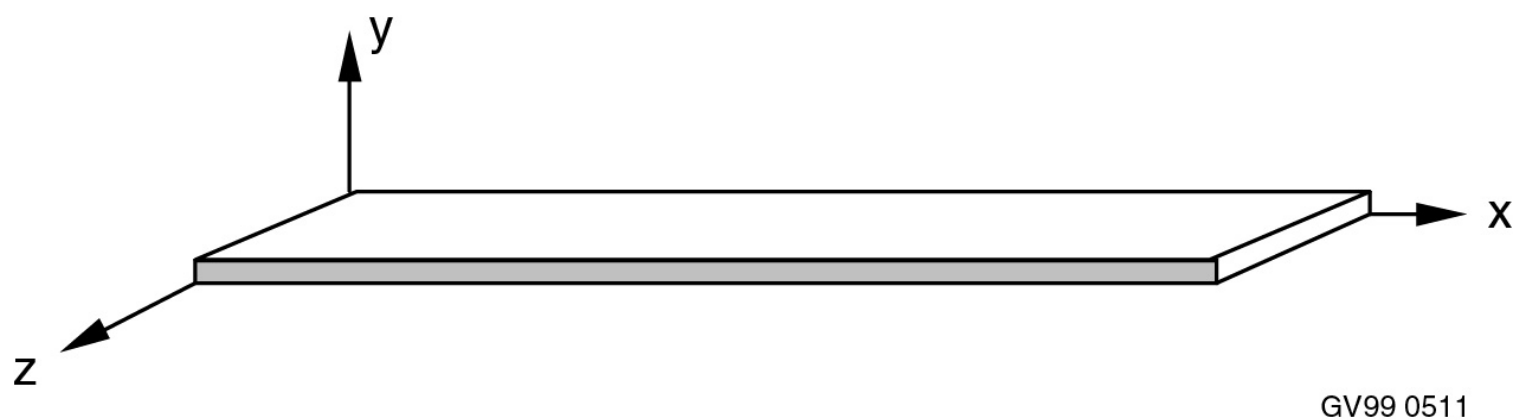

Figure 5. Sample schematic. 


\section{Bond Coat Results}

A finite element model of the thin $(130 \mu \mathrm{m})$ bond coat applied to the stainless steel substrate was developed. The bond coat material is primarily $\mathrm{Ni}$ with about $5 \% \mathrm{Al}$, so bulk tabulated properties for pure $\mathrm{Ni}$ were used. It was assumed that the $\mathrm{Ni}$ was applied as a single layer that was well bonded to the substrate. The calculation involves two stages: obtaining thermal equilibrium between the hot Ni layer as it comes in contact with the cool stainless steel substrate during the thermal spray process and cooling the entire sample from this steady state temperature to room temperature. The first stage results in quenching stresses, while the latter causes thermal expansion mismatch stresses.

Although the temperature of splats upon impact with the substrate is probably in the vicinity of $2000^{\circ} \mathrm{C}$, the melting temperature of $\mathrm{Ni}, 1445^{\circ} \mathrm{C}$, was used as the initial deposit temperature. Since molten metal can support no load, no stress is generated during cooling from the actual deposition temperature to solidification, and that portion need not be modeled. The substrate was assumed to be at $20^{\circ} \mathrm{C}$. The steady state temperature calculated by the model was $120^{\circ} \mathrm{C}$. This value is in good agreement with $90^{\circ} \mathrm{C}$, the temperature obtained from a thermocouple attached to the back of a substrate during spraying. The actual specimen does not reach a steady state temperature during the thermal spray process, so a temperature measured on the back surface is expected to be lower.

The radius of curvature calculated by the FEM model is $5.29 \mathrm{~m}$ after deposition and $7.06 \mathrm{~m}$ after cooling to room temperature, with the sample cupping toward the bond coat. These values are in good agreement with measured value of $4.4 \mathrm{~m}$. Most of the curvature occurs during the quenching stage, which is then somewhat relaxed during the mismatch stage because the thermal expansion coefficients used for $\mathrm{Ni}$ are less than those for stainless steel. Possible sources for the discrepancy between final modeled and measured curvatures include inaccurate material properties, such as expansion coefficients and plasticity (especially at high temperatures), incorrect bond coat melting temperature, insufficient FEM mesh resolution, inaccurate layer thickness, and the assumptions that perfect bonding is maintained and that the sample reaches an intermediate steady state temperature.

These calculations indicate that thermal spray processes can be modeled for metal coatings with FEM by using the melting point of the coating layer as the deposition temperature. Plastic strains of several percent were calculated in the bond coat; therefore, simple analytic solutions, which generally assume elasticity or at best, temperature independent plasticity, would not be adequate for calculating residual stress states in these samples.

Modeling ceramic coatings results in an excessive quenching contribution, because the particles remain solid to such a high temperature that $\Delta \mathrm{T}$ during the initial stage is very large. In reality, micro-cracking and debonding relieve much of this stress, but these mechanisms have not been successfully incorporated into a model to date. Improved results may be possible by using either an artificially low deposition temperature or by modifying the ceramic coating or interface properties to account for the coating imperfections.

\section{Future Work}

The results of the current work suggest several important areas for future research. Numerical simulations of coatings typically use properties for the coating materials that are derived from tabulated values for bulk materials. It is clear that porosity, texture, and microcracking of the coating materials may result in elastic properties and coefficient of thermal expansion that vary significantly from the bulk values. More accurate finite element simulations to account for these microstructural differences require the ability to measure the directional properties of materials in the form of coatings. Understanding the 
relationship of coating microstructures to observed properties is also important in developing processing strategies for mitigating residual stress during fabrication of coatings.

In order to predict the service behavior of coatings, it will also be necessary to more completely understand the transport properties. For aqueous corrosion protection in long term storage, for example, it is important to characterize and eventually control transport of water and water vapor. The thermal conductivity of coatings is also an important property for predicting thermal barrier properties of coatings for elevated temperature applications.

\section{ACCOMPLISHMENTS}

Automated methods to consistently prepare and examine cross-sections of ceramic coatings on metallic substrates have been developed. Software routines have been developed and validated to capture electronic images and quantify the major microstructure features. The features that are critical for predicting coating performance include porosity and cracking, both parallel and normal to the deposition direction.

The influence of substrate preparation on the substrate material characteristics has been determined. Typical grit blasting parameters result in particle velocities in the range of $100-200 \mathrm{~m} / \mathrm{s}$. For Type 304 stainless steel substrates, grit blasting results in deformation that is consistent with elastic deformations of the order of the elastic modulus. These results indicate that plasticity is a significant contribution to measured residual deformation. Transmission electron microscopy confirms that grit blasting results in severe cold work in a region of the substrate that is $100 \mu \mathrm{m}$ deep. Deposition of a coating anneals this damage to a large extent. Under the conditions examined here for optimum coating deposition there is little carbide dissolution in the stainless steel and no measurable grain growth. These experimental results are consistent with a temperature at the coating substrate interface of less than $500^{\circ} \mathrm{C}$ during coating deposition.

Application of a Ni-Al bond coat was investigated as an alternative to grit blasting. Even a relatively thin bond coat results in significant residual stress in the Type 304 stainless steel substrates. Much less damage is introduced compared to grit blasting. Using the Ni-Al bond coat results in greater coating adhesion compared to grit balsting. The residual stress imposed by the bond coat is significantly greater than that caused by ceramic coating. It has been concluded that the bond coat has less effective mechanisms available to relieve stress compared to the ceramic, which shows considerable fracture apparently as a result of the stress caused by quenching and cooling from the deposition temperature.

A specimen preparation method and geometry have been developed so that reproducible residual stress determinations can be made from total curvature measurements. The stress contribution from quenching is significantly larger than the thermal expansion mismatch stress contribution. Thermal cycling the spinel coating results in hysteriesis in the residual stress even at temperatures as low as $150^{\circ} \mathrm{C}$. It appears that some irreversible process, like cracking, occurs in the ceramic to relieve stress. In the metallic bond coat, little change in the residual stress is observed for thermal cycles as high as $350^{\circ} \mathrm{C}$. These results are consistent with the observation that the total residual stress from the metallic bond coat is significantly greater than that from a much thicker ceramic coating. These results suggest that the stress relief mechanisms available to the metallic material, i.e., plasticity or creep, are not as effective as cracking and debonding in the ceramic. 


\section{REFERENCES}

1. L. Gavard, F. Montheillet and J. Le Coze, "Recrystallization and Grain Growth in High Purity Austenitic Stainless Steels," Scripta Materialia, Vol. 39, No. 8, 1998, pp. 1095-1099.

2. R. N. Wright, G. E. Korth and J. E. Flinn, "Particle Bonding, Annealing Response, and Mechanical Properties of Dynamically Consolidated Type 304 Stainless Steel Powders," Metallurgical Transactions A, Vol. 20A, 1989, pp. 2449-2457.

3. J. A. Floro, E. Chason and S. R. Lee, "Real Time Measurement of Epilayer Strain Using a Simplified Wafer Curvature Technique," Materials Research Society Symposium Proceedings Vol. 406, 1996, pp. 491-496.

4. O. Kesler, J. Matejicek, S. Sampath, S. Suresh, T. Gnaeupel-Herold, P. C. Brand, H .J. Prask, "Measurement of Residual Stress in Plasma-sprayed Metallic, Ceramic and Composite Coatings," Materials Science and Engineering, Vol. A257, 1998, pp. 215-224.

5. O. Kesler, M. Finot, S. Suresh and S. Sampath, "Determination of Processing-induced Stresses and Properties of Layered and Graded Coatings: Experimental Method and Results for Plasma-sprayed Ni-Al ${ }_{2} \mathrm{O}_{3}, "$ Acta Materialia, Vol. 42, No. 2, 1997, pp. 3123-3134.

6. C. H. Hsueh and A. G. Evans, "Residual Stresses in Metal/Ceramic Bonded Strips" Journal of the American Ceramic Society, Vol. 68, No. 5, 1985, pp. 241-248.

7. Y. C. Tsui and T. W. Clyne, "An Analytical Model for the Generation of Residual Stresses in Sprayed Coatings Deposited Progressively onto Planar Substrates," Thermal Spray: A United Forum for Scientific and Technological Advances, C. C. Berndt (Ed.), Materials Park, Ohio: ASM International, 1997, pp. 813-822.

8. Chin-Chen Chiu, "Residual Stresses in Ceramic Coatings as Determined from the Curvature of a Coated Strip," Materials Science and Engineering, Vol. A150, 1992, pp. 139-148.

9. Chin-Chen Chiu, "Elastic Modulus Determination of Coating Layers as Applied to Layered Ceramic Composites," Materials Science and Engineering, Vol. A132, 1991, pp. 39-47.

\section{APPENDIX}

\section{Presentations}

1. Microstructure Effects on Stainless Steel Substrates from Deposition of Plasma Spray Ceramic Coatings, R. N. Wright, W. D. Swank, J. R. Fincke, to be presented at the International Thermal Spray conference, 8-11 May 2000, Montreal, Canada.

2. Residual Stress Determination from a Laser-Based Curvature Measurement, W. D. Swank, R. A. Gavalya, J. K. Wright, R. N. Wright, to be presented at the International Thermal Spray

Conference, 8-11 May 2000, Montreal, Canada. 


\title{
Corrosion and Aging
}

\author{
Patrick J. Pinhero and Richard N. Wright \\ SUMMARY
}

Microbial-influenced corrosion (MIC) is a potential problem of significance at the Idaho National Engineering and Environmental Laboratory (INEEL) related to the storage of spent nuclear fuel (SNF) at the Idaho Nuclear Technology and Engineering Center (INTEC). Understanding and mitigating MIC in SNF storage is the primary goal of this task, and several basic questions have been answered in FY 1999. Many microbes originating from SNF storage pools at INTEC-603 and -666 have been isolated, grown, and positively identified by $16 \mathrm{~S}$ rRNA and Biolog ${ }^{\circledR}$. Additionally, through collaboration with the Idaho State University Linear Accelerator Center, we have demonstrated that $60 \%$ of these bacteria tolerated $60,000 \mathrm{rad} \gamma$-irradiation doses. By approximately doubling the $\gamma$-ray exposure, the number of surviving species that survived was only reduced by $10 \%$. Studies examining the electrochemical effect of microbes upon corrosion were continued in FY 1999. A systematic study of the sterility problems associated with using a coupled-electrochemical cell apparatus identified probable contamination sources and furnished corrections that eliminate them. In the arena of the MIC community, this is a significant accomplishment. Results using coupled-cell apparati provided preliminary evidence for enhanced corrosion of Al 6061 through inoculation with Pseudomonas aeruginosa. Scanning Reference Electrode Technique (SRET) has been implemented to examine the dynamics of localized corrosion. Results from experiments using SRET indicate an ennoblement of Al 6061 in the presence of microorganisms with respect to polarization at or near the breakdown potential $\left(\mathrm{E}_{\mathrm{bd}}\right)$. Images obtained with the SRET instrumentation show evidence of localized electrochemical activity in Al 6061. Extremophillic research has developed methods to isolate, identify, and culture bacteria from a variety of environments. Focus on bacteria that most probably exist in harsh environments typical of nuclear facilities and in long term geological storage environments was emphasized. Extreme environments that have been considered include high and low temperatures, low $\mathrm{pH}$, and high pressure. Novel bioreactors have been developed to culture bacteria at high pressure and elevated temperatures. Sulfur reducing bacteria that are thought to be significant for corrosion in spent fuel storage were extensively studied.

\section{PROJECT DESCRIPTION}

\section{Task 1. Biocorrosion}

The Department of Energy is custodian of several thousand metric tons of spent nuclear reactor fuel (SNF) which were a primary result of experimental nuclear reactor development and Naval reactor activities during the period of the Cold War. Also included in this stockpile is fuel from energy research and naval reactors. The long-term containment performance of the fuel under storage and disposal conditions is uncertain. Uncertainties have a direct bearing on the DOE's ability to license disposal methods. The EM Office emphasizes a fundamental need for identifying mechanisms that may adversely affect the performance of the fuel package during storage. Deleterious effects recognized as being incompletely characterized include: corrosion and degradation rates for the fuel matrices, mechanisms that may lead to accelerated degradation of containers; and effects of microbes on fuel packages.

Microbial-influenced corrosion (MIC) is a known problem in various industries. For example, severe biofouling occurs in transport piping used in the petroleum industry. Sulfate-reducing bacteria (SRB) are of particular importance because they are responsible for souring oil and for MIC. Water content of just $1 \%$ is adequate to support substantial development of SRBs within a few weeks. At the 
INEEL, large "swimming pools" of water are used to temporarily store SNF as it cools prior to processing, transport, and final storage. During transition to final storage, the SNF is dried and then placed in containers for transport to the repository for final deposition. Obvious questions arise given this scenario. (1) Can microbes survive in temporary storage pools where SNF is resides? (2) What effect do the microbes, if present, have on the surface of the cladding? Do they initiate corrosion? Do they accelerate or exacerbate corrosion processes? Or, do they inhibit degradation of SNF cladding? (3) If MIC is present, is it the result of one type of microbe, or is it a complex interaction induced by several classes of microbes? (4) Does the chemistry change when the SNF is transitioned from wet to dry storage? This is by no means an exhaustive list of questions. Its purpose is to give some baseline for scientific inquiry.

\section{Radiation Tolerance of Bacteria Study}

Reports of the observation of viable microbes in wet and dry SNF storage facilities is not novel, although it has not yet been unambiguously demonstrated that microbes can exist on SNF itself. Scientists in Canada have established a long-term study that has been concerned with microbes introduced during construction of the Underground Research Laboratory. ${ }^{1,2,3,4}$ Recently, a group of our colleagues at the INEEL published an account detailing the existence of organisms on coupons placed within two of the storage pools at Idaho Nuclear Technology and Engineering Center (INTEC). ${ }^{5}$ Though these research groups have observed microbes in storage facilities, nothing has been published thus far about the identity or radiation tolerance of the organisms. Resistance to radiation is significant because it implies that biofilms could survive on SNF.

It is well documented that some microbes such as Deinococcus radiodurans (D. radiodurans) survive in a radiation-rich environment. ${ }^{6}$ The tolerance of this organism to radiation may be related to its well-documented DNA repair mechanism, its peculiar cell wall/membrane structure, its high concentration of antioxidants, and its multiple copies of genetic information. ${ }^{7}$ As a first step in examining possible MIC damage at INTEC, we have set out to isolate microbes from the SNF storage pools and determine their respective sensitivities to radiation.

Specimens were collected at two SNF storage pools in INTEC-603 and INTEC-666. The primary differences in these facilities are the age of the pool and the quality of the water within the pool. INTEC-603 is the older of the two, having been constructed in 1951-2. It is an unlined concrete basin with its water re-circulated through a closed-loop cooling system equipped with both sand and UV filtration. INTEC-666 is a stainless-steel-lined concrete basin that was constructed in 1980-2. The water in INTEC-666 is re-circulated through ion-exchange resins, UV irradiation, and treated by reverse osmosis in order to retain high purity. Samples were collected from low radiation locations within each pool. Microorganisms isolated from these samples were identified using either the Biolog technique ${ }^{8}$ or a $16 S$ rRNA technique. ${ }^{9}$ Table 1 includes the identities of the bacteria with respect to INTEC location, growth conditions, and gram staining.

Each of the isolated bacteria grew from a single colony at least 24 hours prior to irradiation. Some isolates were slow growers and thus required longer incubation periods. Cell counts were determined microscopically before centrifuging the cultures (10,000 rpm for 10 minutes). Cell pellets of each different culture were re-suspended in water and stored on ice until they were irradiated. Each isolate was prepared in triplicate for irradiation.

In the course of the experiments, the microbes were irradiated for total exposures of approximately $5 \times 10^{3} \mathrm{Rad}, 6 \times 10^{4} \mathrm{Rad}$, and $1.1 \times 10^{5} \mathrm{Rad}$. The time of dose ranged from 7 minutes to 75 minutes, depending on the desired level of exposure. Following irradiation, each of the exposed samples was spread on agar-solidified growth material and the colonies were counted after 24 hours and up to 10 days. 
Table 1. Identity, sampling origin, growth medium, and result of gram staining for each of the irradiated microbes.

\begin{tabular}{llll}
\hline \multicolumn{1}{c}{ Microbe Identity } & \multicolumn{1}{c}{ Origin } & \multicolumn{1}{c}{ Growth Medium } & Gram Stain \\
\hline Escherichia Coli & Laboratory control & Luria Bertani Broth & - \\
Deinococcus radiodurans & Laboratory control & Nutrient broth w/ 1\% dextrose & + \\
Pseudomonas aeruginosa & Laboratory addition & $5 \%$ PTYG & - \\
Pseudomonas putida & INTEC-666 & $5 \%$ PTYG & - \\
Comamonas acidovorans & INTEC-666 & $5 \%$ PTYG & - \\
Corynebacterium aquaticum & INTEC-666 & $5 \%$ PTYG & + \\
Gluconobacter cerinus & INTEC-666 & $5 \%$ PTYG & - \\
Sulfate Reducing Bacteria A & INTEC-603 & ATCC 1249 SRB media & - \\
Sulfate Reducing Bacteria B & INTEC-603 & ATCC 1249 SRB media & - \\
Micrococcus diversus & INTEC-603 & Nutrient broth w/ 1\% dextrose & + \\
Rhodococcus rhodochrous & INTEC-603 & $5 \%$ PTYG & + \\
Micrococcus luteus & Laboratory addition & $5 \%$ PTYG & + \\
\hline
\end{tabular}

Two controls were maintained throughout the irradiation study. E. coli without the recA gene was selected as our radiation-sensitive control for the set of experiments.

The lack of the recA gene prevents $E$. coli from engaging its SOS regulatory system, a complex cellular survival mechanism that repairs DNA. ${ }^{7}$ D. radiodurans served as the radiation resistant control because of its well-documented radiation tolerance. In addition, we included $P$. aeruginosa in the irradiation study because it is used in other associated MIC research.

The radiation dose was delivered using a 6-MeV LINAC in the Idaho State University LINAC Facility. Electrons within the LINAC were accelerated to a kinetic energy of 6-MeV and projected onto a tungsten target to produce the continuous bremsstrahlung and characteristic x-ray spectrum. A $0.64 \mathrm{~cm}$ thick aluminum plate was placed between the tungsten target and the specimen to both harden the photon beam and remove any high energy electrons that may have passed through the tungsten target. The best control of this instrument was achieved when attempts were made at controlling the absorbed dose (total exposure) delivered to a specimen. The dose rate was, at best, coarsely controlled due to the operational parameters that were adjusted manually by the operator during the course of an exposure. The radiation dose received by the specimen was determined using a combination of radiochromic dosimeters ${ }^{\mathrm{a}}$ and thimble-ionization chambers. ${ }^{b}$ The response of the thimble-ionization chambers as a function of the magnitude of incident field strength was linear over a broad range of exposure rates. Calibration of these chambers was made versus absorbed doses in the $2 \times 10^{5}$ rad range, as measured with the radiochromic dosimeters. This type of calibration correlates the positionally-dependent detector's response in units of Roentgen or Roentgen/minute to units of absorbed dose (i.e., $\mathrm{rad}$ or $\mathrm{rad} / \mathrm{minute}$ ) for a particular photon

\footnotetext{
a. Far West Technology, 30-D South Kellog, Goleta, CA 93117.

b. Radcal Corporation, Model 1515 Radiation Monitor. Initial experiments were performed using a 1.5-MeV build-up cap. Later experiments employed 6-MeV build-up cap.
} 
energy spectrum. This calibration was performed within a day before or after experiments where the bacteria were irradiated.

The results from the irradiation experiments are displayed in Table 2. In this table, mean populations are tabulated according to the amount of radiation absorbed. The second column represents the initial population prior to irradiation. We tracked an additional set of specimens over a six-hour period to account for natural changes to the cell populations, independent of irradiation. The value reported for each of the irradiated bacteria is corrected by a simple ratio calculation that accounts for the natural growth or death inherent to a specific microbial isolate. The data indicate that about half of the microbes tested survive irradiation. Upon closer inspection, it appears that of the four microbes originating from INTEC-666, only one, $C$. aquaticum, survived irradiation. Of the four microbes identified from INTEC-603, three survived irradiation. The overall trends are displayed in Figure 1. This plot shows the surviving fraction of the initial cell count as a function of the total amount of radiation absorbed by each microbial isolate. We observed two distinct trends: microbes that demonstrate a tolerance to large amounts of radiation and microbes whose populations are significantly reduced or totally killed off. The dashed diagonal line is provided to help show the separation between the two distinct sets of microbes. Points above the line represent organisms that display radiation resistance.

It is well documented that ionizing radiation does not kill cells directly, but acts indirectly by creating reactive free radicals. ${ }^{7}$ These free radicals can chemically alter sensitive macromolecules in the cell leading to their inactivation. Though most of the macromolecules in the cell can be influenced in one way or another by interaction with ionizing radiation, it is the interaction with a DNA macromolecule that is often implicated in cell death. This is because DNA is a unique molecule that often contains single copies of the genes that comprise it. Other macromolecules, such as proteins, may have several exact copies, so that inactivation of one will not lead to cell death. Proteins can also be re-synthesized if the genetic coding, DNA, remains intact. The role of oxygen is also significant in radiation processes.

Table 2. Data from the irradiation experiments. Each reported cell population is an average value computed from three sets of triplicates, i.e., $\mathrm{N}=9$. Statistical deviations are on the order of $\pm 10 \%$.

\begin{tabular}{lcccc}
\hline & \multicolumn{3}{c}{ Mean Microbe Population for Each Absorbed Dose } \\
\cline { 2 - 5 } Microbe Identity & Initial & $\sim 5 \times 10^{3} \mathrm{rad}$ & $\sim 6 \times 10^{4} \mathrm{rad}$ & $\sim 1.1 \times 10^{5} \mathrm{rad}$ \\
\hline E. Coli & $1.8 \times 10^{9}$ & $1.8 \times 10^{7}$ & 0 & 0 \\
D. Radiodurans & $9.5 \times 10^{6}$ & $7.1 \times 10^{6}$ & $3.8 \times 10^{6}$ & $9.9 \times 10^{6}$ \\
P. aeruginosa & $2.6 \times 10^{9}$ & $4.5 \times 10^{5}$ & 0 & 0 \\
P. putida & $8.0 \times 10^{8}$ & $1.5 \times 10^{7}$ & $2.7 \times 10^{2}$ & 0 \\
C. acidovorans & $5.4 \times 10^{8}$ & $2.1 \times 10^{6}$ & 0 & 0 \\
C. aquaticum & $2.7 \times 10^{9}$ & $9.9 \times 10^{8}$ & $7.4 \times 10^{5}$ & $8.1 \times 10^{4}$ \\
G. cerinus & $7.9 \times 10^{8}$ & $9.0 \times 10^{7}$ & $5.7 \times 10^{2}$ & $9.1 \times 10^{0}$ \\
SRB A & $4.8 \times 10^{8}$ & $1.4 \times 10^{8}$ & $1.4 \times 10^{6}$ & $1.9 \times 10^{7}$ \\
SRB B & $2.0 \times 10^{7}$ & $4.8 \times 10^{7}$ & $6.1 \times 10^{7}$ & $1.0 \times 10^{6}$ \\
M. diversus & $2.1 \times 10^{8}$ & $1.3 \times 10^{8}$ & $1.3 \times 10^{7}$ & $2.7 \times 10^{4}$ \\
R. rhodochrous & $1.5 \times 10^{8}$ & $1.8 \times 10^{8}$ & $2.1 \times 10^{5}$ & $7.7 \times 10^{0}$ \\
M. luteus & $9.7 \times 10^{6}$ & $3.4 \times 10^{7}$ & $3.6 \times 10^{6}$ & $2.2 \times 10^{6}$ \\
\hline
\end{tabular}




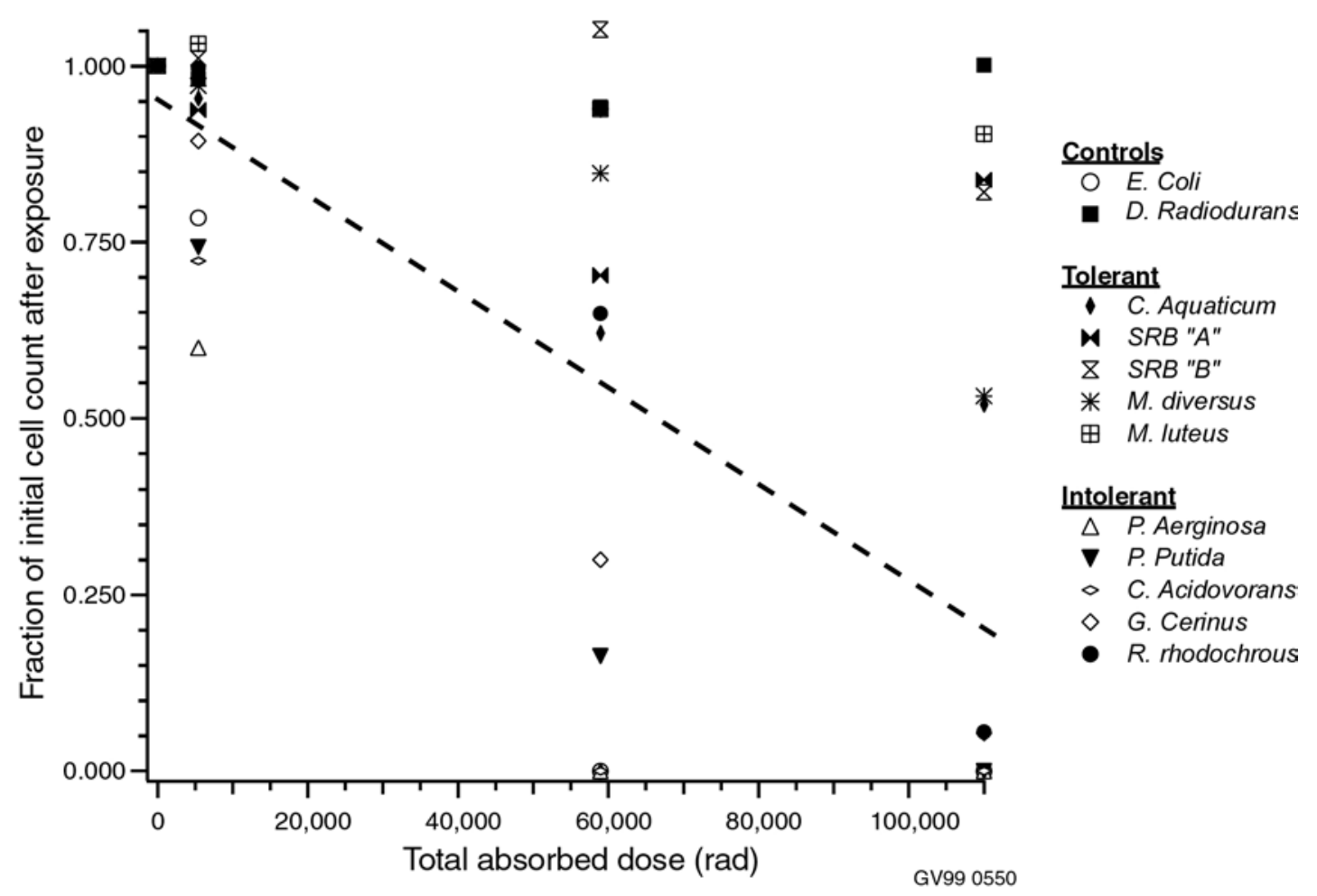

Figure 1. Plot of the fraction of surviving cells in each of the irradiated microbes versus the total absorbed dose in rad. Note the two groupings: microbial colonies that demonstrate resistance to the absorbed dose (above diagonal line), and those are killed off (below line).

Three times as much radiation damage is observed in aerobic environments as in anaerobic conditions. ${ }^{10}$ This is significant because our sulfate-reducing bacteria (SRB) were grown, transported, and irradiated under anaerobic conditions. Therefore, we believe that the radiation resistance demonstrated by the SRBs may not be a characteristic of the microbe, but a function of the low oxygen concentration.

The variation in the radiation sensitivity of the other microbes may be due to differences that exist between gram-positive and gram-negative bacteria. We observed that three of the four gram-positive species (D. radiodurans, $C$. aquaticum, $M$. diversus, and $M$. luteus) exhibit a lower sensitivity to ionizing radiation. The exception, $R$. rhodochrous, does show radiation resistance at $6 \times 10^{4} \mathrm{rad}$, but its population is dramatically reduced by absorption of the larger total dose. It should be noted that the dose rate at the $1.1 \times 10^{5} \mathrm{rad}$ level is almost twice as intense as the rates at the lower exposures. This may contribute to the observed deviation of the $R$. rhodochrous from the other three gram-positive organisms. In sporeproducing, gram-positive bacteria, it is known that the spores contain storage proteins that bind tightly to DNA, possibly protecting it from radiation damage. ${ }^{11,12}$ If all gram-positive organisms possess similar protection mechanisms that contribute to an intrinsic radiation resistance, it seems logical that larger numbers of gram-positive organisms versus gram-negative organisms have been isolated from areas like Chernobyl, where high levels of radioactive contamination are present. ${ }^{13}$

We note that the radiation dose supplied by the LINAC in these experiments is more intense than "hot" SNF in the INTEC pools. This is an important point, since the microbes determined to be radiationsensitive by the bounds of our experiment are robust enough to survive within the INTEC SNF storage pools. As a final point, it should be stated that the number of organisms we have isolated and examined 
here is likely to represent only a small fraction of the total number of unique organisms present in the INTEC facilities, based upon our limited sampling opportunities.

\section{Generalized Corrosion Studies Using Coupled-Electrochemical Cells}

Solving the Sterility Problem. In analytical studies, it is useful to make direct comparisons between the system of interest and a controlled system. The control contains everything that the system of interest contains, except for the components that make this system "interesting." Within the realm of biocorrosion studies, a direct coupling, between an electrochemical cell containing well-chosen microorganisms and a sterile electrochemical cell, would give the researcher an ability to measure differences between the two cells, while everything else remains equal. Brenda Little designed such a system which is built upon the use of a semi-permeable membrane. ${ }^{14}$ Figure 2 shows the layout of this apparatus. In September 1998, after several efforts at setting up the coupled-electrochemical cell system only to observe contamination in a couple of weeks, our research team made the decision to attempt a methodical study to pinpoint and solve the contamination problem. We came up with the following modes of contamination:

1. Leakage of microorganisms from biotic electrochemical cell to abiotic electrochemical cell through the membrane or around sides of the membrane

2. Leakage of microorganisms through the Vycor tips of the bridge tubes

3. Insufficient sterilization of the thermometer with ethanol

4. Insufficient sterilization of sample holder assembly with ethanol

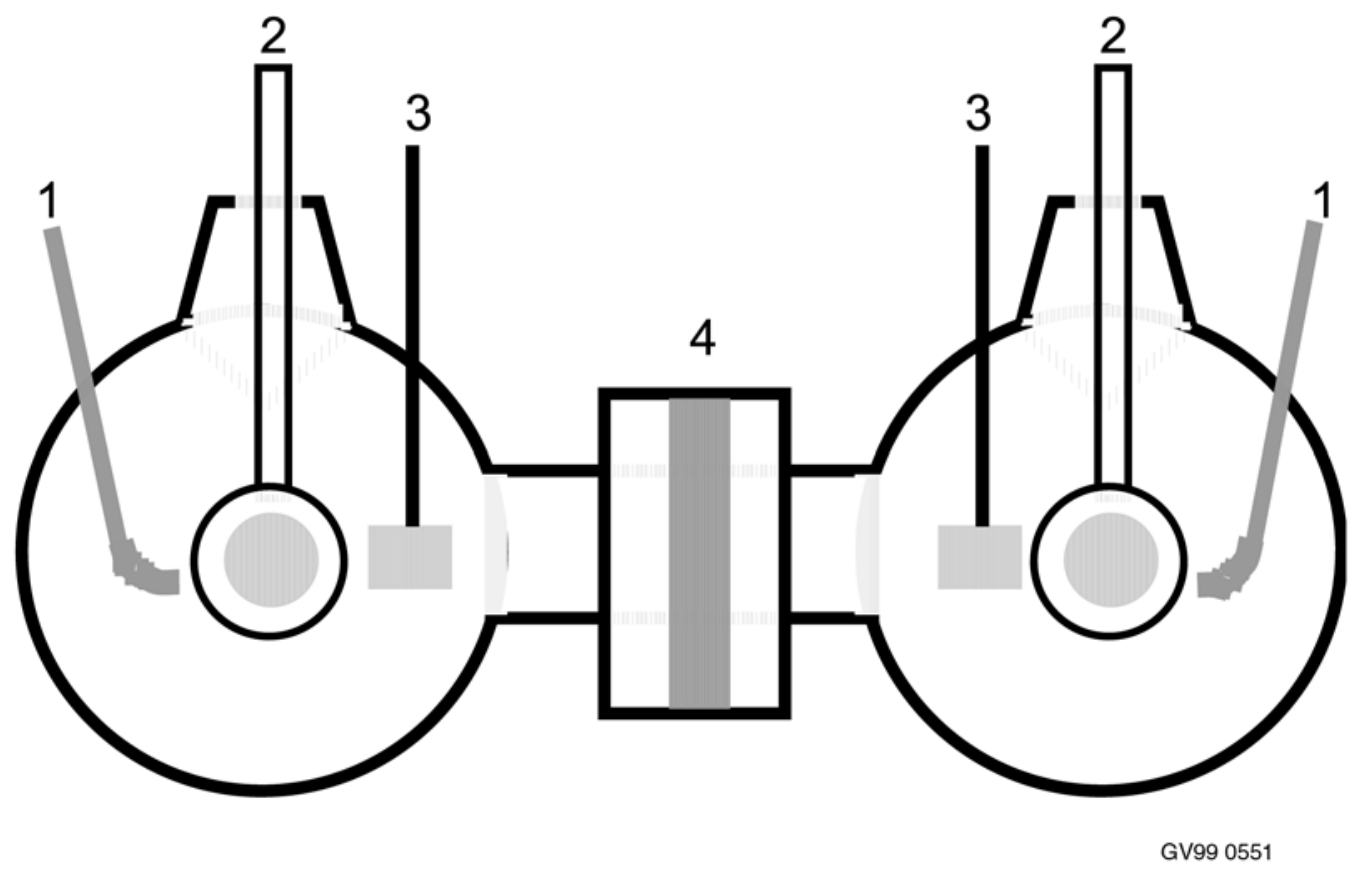

Figure 2. Diagram of the Coupled-Electrochemical Cell Apparatus. One side of the cell is kept sterile, while bacteria are inoculated into the other compartment. Many of the components in the individual cells are the same, e.g., part (1) is the SCE reference electrode, (2) is the working electrode, - Al 6061, and (3) is a Pt counter electrode. The important component is part (4), a porous membrane which allows equilibration of chemistry, but separation of microbiology. 


\section{Water (raw or polished), not filter-sterilized properly}

\section{Handling during assembly.}

To determine the presence of microorganisms in both the biotic and abiotic cells, we stained extracted working electrodes from each cell with acridine orange and counted cells with the fluorescent microscope. Environmental scanning electron microscopy (ESEM) was also used to examine the extent of contamination, i.e., determine whether a biofilm formed. Our results are presented with respect to each of the listed questions.

Addressing the first question, leakage from biotic cell to abiotic cell through the membrane or around the sides of the membrane, is a complicated issue. The individual electrochemical cells do not exactly match at the junction where they couple; therefore, the bacteria may be able migrate around the membrane edge to the other side. To fully answer the question of contamination associated with the transport of microorganisms through or around the membrane, we asked five related questions.

First, does contamination come from the membrane and/or the joint? The flasks, membrane, and filters were autoclaved for $20 \mathrm{~min}$ at $120^{\circ} \mathrm{C}$ and $20 \mathrm{psi}$ on a gravity (dry) load. We then set up the coupled system with the $0.2 \mu \mathrm{m}$ polycarbonate membrane and glass fiber filters for support, as in the original experiment. We wrapped the coupling with Teflon tape to seal it and clamped the two cells together. No other parts were added to the flasks. Using sterile techniques, we transferred 2 liters of $1 / 4 \mathrm{x}$ TSB to each flask and inoculated with Pseudomonas aeruginosa. After 2 days, we observed contamination of the abiotic flask.

Second, is the contamination coming through the membrane? We repeated the same experiment with a different membrane. The membrane in this case is Gelman Metricel polysulfone with a pore size of 0.2 micron. The membrane was cut down from its original $142 \mathrm{~mm}$ diameter to $110 \mathrm{~mm}$, then it was taped around the outside of the joint. This was to prevent bacteria from migrating around the membrane to the other side. The membrane on the flask was autoclaved with a gravity load. The two sides were secured with Teflon tape and clamped together. Media were added and allowed to equilibrate for 2 days without inoculation to determine if handling glassware leads to contamination of the electrochemical cells. No growth was observed due to handling. We then inoculated with P. aeruginosa. After 1 week the abiotic flask was contaminated. Our conclusion is that either the membrane is being damaged during autoclaving and/or handling, or bacteria are penetrating its 0.2 micron pores. The solution we propose is to try a different method of autoclaving and use different membranes.

Third, does autoclaving on gravity (dry) load rather than liquid load damage the microstructure of the membrane? We repeated the same experiment with the exception of autoclaving the Metricel® membrane on a liquid load in a petri dish of water. The liquid load brings the pressure down slower than the gravity load and the water may prevent damage to the membrane due to expansion. We then secured the membrane into the coupling with Teflon tape and clamped the two electrochemical cells together. This test remained sterile for 1 month. Our conclusion is that a liquid load is a necessary modification to the autoclaving procedure.

Fourth, is the contamination coming around the membrane? We repeated the same experiment using a dense film membrane (plastic wrap) cut to $47 \mathrm{~mm}$ (same as original experiment). We secured this membrane with Teflon tape and clamped the cells together. We then added a few drops of red food coloring to the biotic flask. After a few hours the red color was also in the abiotic flask. Our solution is to use gaskets to seal the edges around the membrane. 
Fifth, do the new design of Viton gaskets around the membrane and new clamps prevent contamination? We repeated the same experiment with a dense film membrane and 2 Viton gaskets sandwiching the membrane. We secured the membrane in place using a new aluminum clamp instead of the old clamps. We added red food coloring to the biotic side. No color was observed in the abiotic side after several days.

To further address the issue of contamination from around the membrane, a sample sheet of silicone was obtained. This sample, Poron Silicones product \#HT6240 Trans, batch \#D8572-1, 1/16-in., is extremely pliable and adheres well to itself and other materials. A set of gaskets was cut from this material, and the gaskets were used to make a "sandwich" with a $42 \mathrm{~mm}, 0.2 \mu \mathrm{M}$ polycarbonate membrane. This sandwich was placed between the necks of a Brenda Little setup, and a clamp was loosely applied to hold the apparatus together. The ground glass stoppers of two air vents and two bridge tubes were sleeved in wide Teflon tape before being inserted into the appropriate openings, and the entire assemblage was autoclaved for 33 minutes. After cooling, the clamp between the two cells was tightened. Inside the transfer hood, sterile 1/8x TSB was added to each cell and the completed setup was incubated on the benchtop in B-9. Although uninoculated, turbidity was observed on one side within 7 days. A second cell set was treated similarly, with the exception of having the biotic side inoculated with P. putida after 5 days. (No turbidity was detected before inoculation.) Within three days of inoculation, the abiotic cell was also visibly turbid.

To address contamination from membrane failure and inadequate initial sterilization, two sets of cells were tested. Set A was assembled with air vents and bridge tubes as described above, but all other openings were capped in aluminum foil. The separated cells were autoclaved for 65 minutes, allowed to cool, then assembled in the transfer hood with a separately sterilized gasket/membrane sandwich, as described above. Sterile 1/8x TSB was also added in the hood. Set B was completely assembled, including media, and autoclaved in its entirety for 90 minutes. Both sets were incubated on the bench top for 18 days with no observable contamination. On day 18, ampicillin and choramphenicol were added to each cell to final concentrations of $85 \mu \mathrm{g} / \mathrm{mL}$ and $35 \mu \mathrm{g} / \mathrm{mL}$, respectively. The biotic side of each cell was then inoculated with a culture of $E$. coli possessing resistance to these antibiotics. Set A showed breakthrough in 4 days, while set $\mathrm{B}$ showed breakthrough in 5 days.

A similar experiment was performed to test the integrity of the Vycor® tips on our bridge tubes. Three bridge tubes were autoclaved inside separate 500-mL flasks. After cooling, 1/8x TSB was added to the flasks and the inside of the bridge tubes in the transfer hood. Each bridge tube was then inoculated with one colony of $P$. putida and incubated static at room temperature. Each flask became contaminated within 10 days of inoculation. The analogous antibiotic experiment was performed with the bridge tubes. Each bridge tube/flask/ media combination was autoclaved for 20 minutes, then incubated static at room temperature for 18 days. Antibiotic was added, as for the coupled-electrochemical cells. Bridge tubes were carefully inoculated, using the transfer hood, and all replicates again were incubated static at room temperature. Contamination of replicate \#1 was noted at 10 days from inoculation, contamination of replicate \#2 was noted at 11 days from inoculation, and replicate \#3 remained sterile 13 days from inoculation.

We are currently working on a new filtration system for the modified Brenda Little cells. One idea is to use a filter with much more surface area than the filters we are currently using. We decided to try the Barnstead D3750 hollow fiber filter (the final filter on our Nanopure systems) because it is readily available. Implementation of this system has produced no visible contamination over a period of 5 months. Tests of ion mobility indicate that ions can be transported freely through the membrane, a necessary requirement in keeping both systems' electrolyte equivalent in both electrochemical cells, except for the microorganisms on the biotic side. A second idea is to use ceramic-coated stainless steel membranes with a pore size of 0.1 micron because of greater durability and smaller pore size. A $42 \mathrm{~mm}$ 
membrane was cut from a sheet sample of Ceramesh ( 0.1 micron pore size, ceramic-coated, stainless steel membrane). As before, this membrane was sandwiched between a set of Poron silicone gaskets, and the sandwich was clamped into place between two coupled cells. Dilute TSB was added to each side, and the entire apparatus autoclaved for 40 minutes. The next day, chloramphenicol was added to $35 \mu \mathrm{g} / \mathrm{ml}$ and ampicillin was added to $80 \mu \mathrm{g} / \mathrm{ml}$. Again, the biotic side was inoculated with the same resistant $E$. coli strain as previously described. No contamination was detected on the abiotic side as of 4 months postinoculation.

The last variable, involving inadequate sterilization of the metal sample, was tested using two methods: one was sonicating in acetone for 10 minutes followed by rinsing in pontificated ethanol, and the other was autoclaving for 30 minutes at $120^{\circ} \mathrm{C}$ and $20 \mathrm{psi}$. Before the sterilization treatments, each 120 grit aluminum sample was placed on 5\% PTYG plates to see how many colonies grew. After treatment, samples were placed on new 5\% PTYG plates and then transferred to 1/8X TSB. Each test was done in triplicate. After 2 weeks, all of the plates with acetone and flame-sterilized samples had several colonies growing, but the TSB did not have any growth at all. This probably can be explained due to outside contamination of the plates, either prior to plating or during plating. Of the 3 plates with autoclaved samples, one was contaminated, and the other two were not. All in TSB were not contaminated. Therefore, it appears that both methods adequately sterilize the aluminum samples. Since autoclaving the produces an oxide layer that will slow down the corrosion rate, we do not want to autoclave these samples. So the conclusion is to acetone sonicate for 10 minutes, followed by a rinse in pontificated ethanol.

Implementation of the Refined Coupled-Electrochemical Cell System. Once the contamination problems were reduced to the point that we could retain sterility over a period of several months, we set up coupled-electrochemical cells to examine the effect of $P$. aeruginosa on the corrosion potential and corrosion current of $\mathrm{Al}$ 6061. In order to determine the effects of "base" electrolyte purity, we constructed three conditions. Condition I consisted of a 1:1 ratio of reverse osmosis purified water to raw water from the Snake River Aquifer at the INEEL site. Condition II consisted wholly of reverse osmosis purified water. Condition III consists solely of $18 \mathrm{M} \Omega$ water from the Barnstead Nanopure filtration system. The top three rows, panels (a)-(f), of Figure 3 show a graphical representation of the data obtained in Condition I, Condition II, and Condition III, respectively. Each of the panels forming the left-hand column corresponds to corrosion potential measurements versus time, while the right-hand column shows time-dependent, corrosion current measurements. In each of the corrosion potential measurements, the open symbols indicate measurements associated with a biotic cell, while the closed (filled) symbols correspond to measurements on abiotic cells. Partner cells, abiotic and biotic, forming a coupled-electrochemical system, share the same symbol shape, i.e., an open circle and a closed circle that are members of the same coupled-electrochemical cell pair. The shape designation is retained in the graphs displaying the corrosion current data; thus, a circle represents a corrosion current measurement for the coupled-electrochemical cells in the left-hand column shown with circles.

In panel (a), corrosion potentials of Condition I cells, there is no observable trend that correlates the abiotic samples with being more corrosive (more negative corrosion potential) or more passive (more positive corrosion potential) than their corresponding biotic mate. Observation of contamination (turbidity) in the abiotic cells at days 23, 29, and 54 demonstrates the failure of the experiment. This visual observation is echoed in examining the data. Panel (a) shows that two of the three coupled-pairs have abiotic and biotic samples with the same corrosion potential. Panel (b) demonstrates the same, which means that the same environment exists in the individual cells comprising each coupled-pair (net cell resistances $\rightarrow \infty$ ). The remaining coupled-pair would probably gradually extrapolate to the same result if it was projected over a longer time period, but this is incongruent with the purpose of the study. 

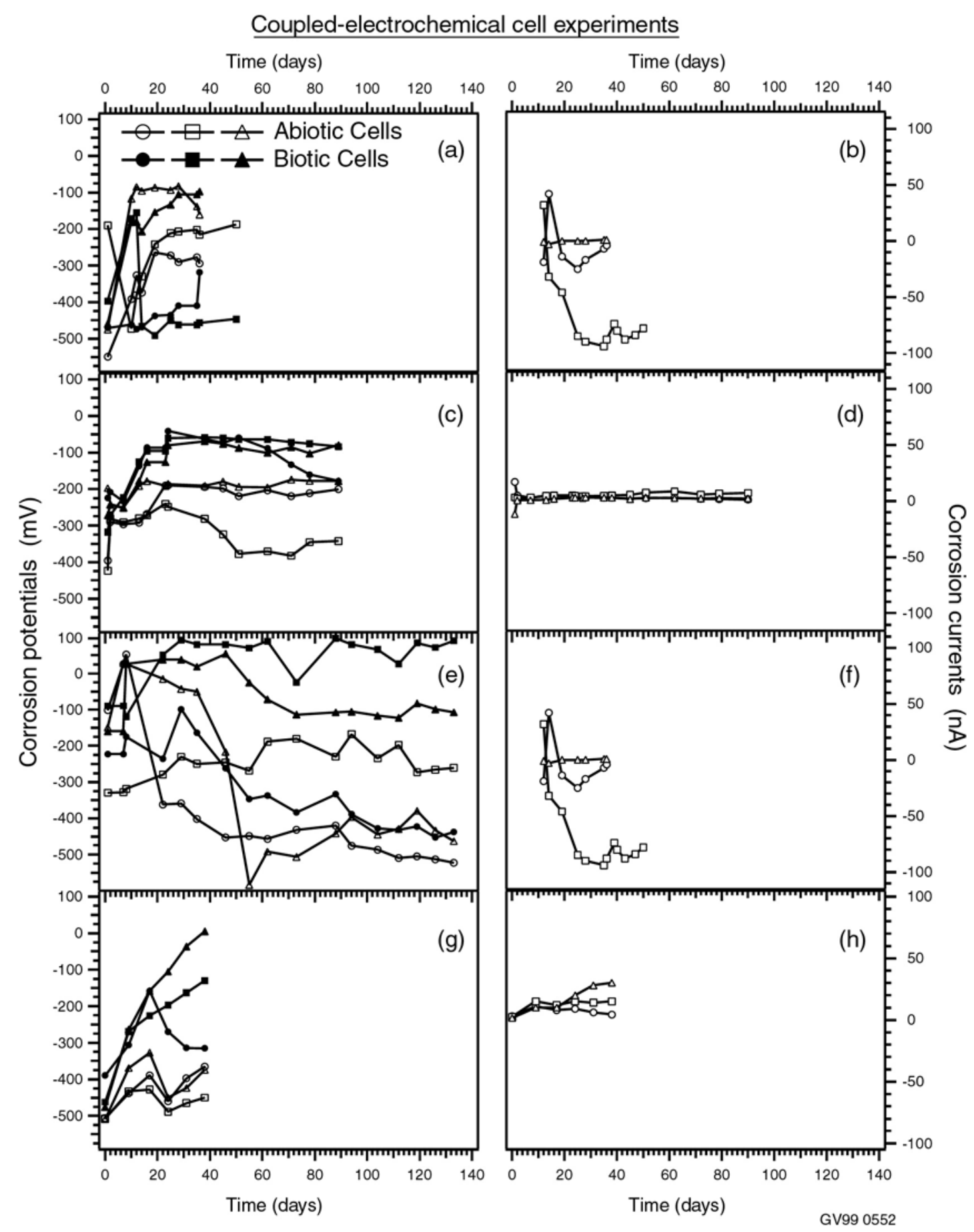

Figure 3. Plots of time-dependent corrosion potentials and time-dependent corrosion currents for the coupled electrochemical cells with $P$. aeruginosa as inoculant. Panel (a) is corrosion potential ( $\mathrm{E}_{\text {corr }}$ ) for 1:1 raw water and reverse osmosis purified water mixture, (b) corrosion current $\left(\mathrm{I}_{\text {corr }}\right)$ for same, (c) $\mathrm{E}_{\text {corr }}$ for R.O. only, (d) $\mathrm{I}_{\text {corr }}$ for R.O., (e) $\mathrm{E}_{\text {corr }}$ for $18 \mathrm{M}$ Nanopure water, (f) $\mathrm{I}_{\text {corr }}$ for same, (g) $\mathrm{E}_{\text {corr }}$ for cartridge filter membrane, and (h) $\mathrm{I}_{\text {corr }}$ for cartridge filter membrane. 
One coupled-pair comprising Condition II displayed contamination at day 64, while the other two became contaminated at day 91. Panel (c ) shows the measured time-dependent corrosion potential. In all coupled-pairs, the abiotic constituent was observed to be more passive than the biotic. The associated corrosion currents, panel (d), were all very small. The same observation of the abiotic cell being more passive than its corresponding biotic cell is again demonstrated in the corrosion potential measurements for Condition III, panel (e). All of the abiotic cells in Condition III remained sterile through 120 days.

Panels (g) and (h) correspond to a repeat of Condition II in a modified coupled-electrochemical cell apparatus using Barnstead D3750 hollow fiber filters rather than the regular membrane. Again, these coupled-pairs show that the abiotic member of each pair is more passive than the biotic. This set of experiments remains contaminant-free after 40+ days.

The general trend in the coupled-electrochemical cell experiments is that the introduction of $P$. aeruginosa to the "base" water electrolyte creates an environment that is more corrosive to $\mathrm{Al} 6061$ than $\mathrm{Al} 6061$ that is in the base water alone. It should be noted that measurements using the coupledelectrochemical cell apparatus are only valid if the electrodes are absolutely identical. ${ }^{15}$ For $\mathrm{Al} 6061$ this may be a valid assumption, but in the case of 300 series stainless steel, it may not be as straightforward.

\section{Localized Corrosion Studies Using the SRET}

Scanning reference electrode technique (SRET) is an imaging tool that allows examination of localized activity via measurement of minute changes in electrochemical potential. In our experiments, we coupled a commercial SRET instrument, EG\&G SVET 100, with an EG\&G PAR 275 Potentiostat (see Figure 4). This mating of instrumentation furnishes us with a method for examining the polarized

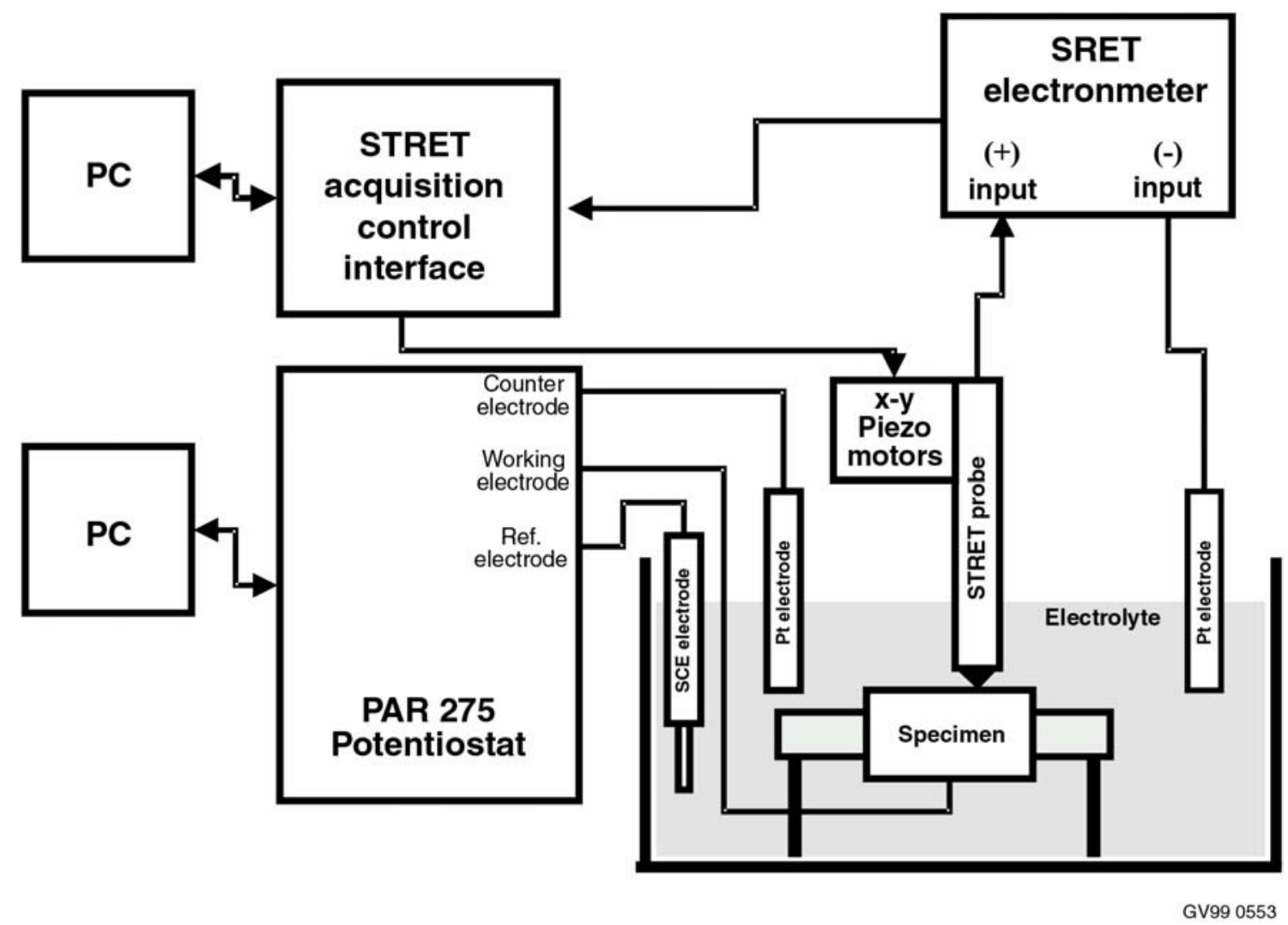

Figure 4. Block schematic of SRET instrumentation including integration of potentiostatic capability. 
local breakdown of metal surfaces in different electrolytes. Using this method we began looking at the corrosive local breakdown of $\mathrm{Al} 6061$ in electrolytes containing microbes. We have examined two different mixtures. The first is a mixture of $P$. aeruginosa, D. radiodurans, and SRBs. The second mixture is a "kitchen sink," multi-component electrolyte containing all bacteria in our possession. To date, three experiments have been completed, and one is in progress. Currently, there is an analogous experiment in progress using an SS 304 working electrode instead of $\mathrm{Al} 6061$.

Figure 5 displays an example set of SRET data acquired from an Al 6061 sample kept abiotic for 22 days, panel (a), and a sample exposed to a biotic environment for 23 days, panel (b). Each panel

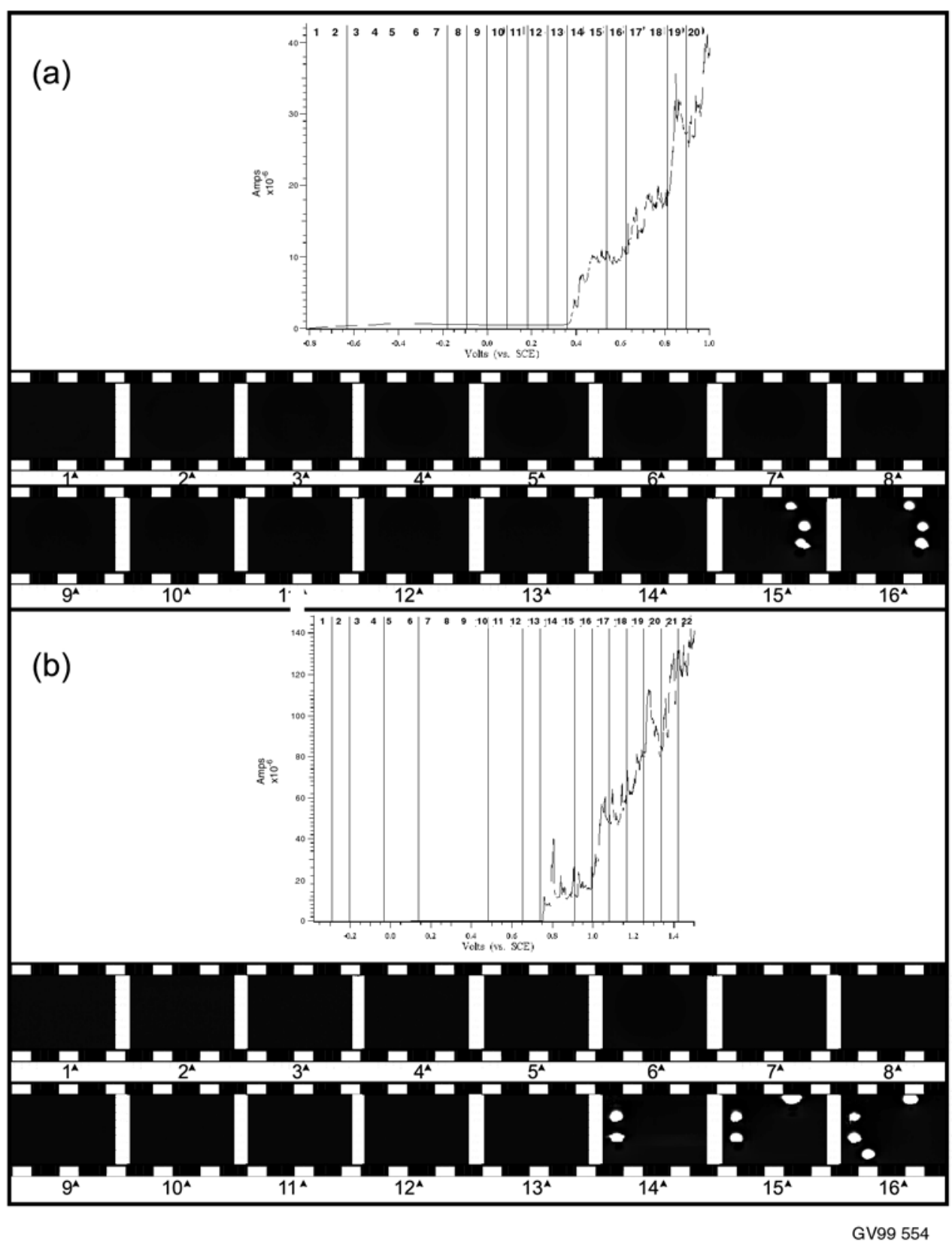

Figure 5. Data from SRET scans of (a) Al 6061 kept in a sterile environment for 22 days, and (b) Al 6061 exposed to a kitchen sink biotic environment for 23 days. 
contains a plot of a potentiodynamic scan and a strip of images that is associated with it. The number beneath each image corresponds with a section of the potentiodynamic scan that is defined by a range of applied potentials. The potentiodynamic scans in the panels show marked increases in detected current in Section 14. For the abiotic sample, panel (a), this onset of current occurs at $+0.367 \mathrm{~V}$ vs. a saturated Calomel electrode (SCE). The biotic sample in panel (b) displays an onset at $+0.750 \mathrm{~V}$ vs. SCE. For the abiotic sample, the image corresponding to this voltage region does not show a localized breakdown, but the following image, frame \#15, does. After forming three distinct local regions of electrochemical activity, there is little change in subsequent scans. The nobler biotic sample shows initial formation of two localized features. This grows by one additional feature in each of the next two frames. Open circuit measurements of the corrosion potentials prior to polarizing the samples corroborate the observation of microbial ennoblement. Figure 6 displays this in all cases, with the exception of open circuit measurements in an environment of raw water with a mixed culture of $P$. aeruginosa, D. radiodurans, and SRBs. Wild fluctuations in the measured breakdown potentials, $E_{b d}$, need to be addressed in future experiments. Thoughts that this can be attributed with microbial changes can be discounted, since variations are observed in both the biotic and abiotic measurements. Future integration of additional measures and advanced electrochemical techniques should shed light on this observation

It should be noted that these SRET studies are preliminary attempts. Pronounced effort currently is being applied to refining techniques to ensure that we are measuring overall corrosion effects and not mere attenuation of signal by a biofilm that carpets the surface. Additionally, we are in the process of refining our scans so that we can examine both breakdown and repassivation. This combined measurement will give us valuable information associated with the hysteresis formed by the breakdown and repassivation curves. Surface analytical techniques have recently been used to characterize chemical changes at the surface of the working electrode. Figure 7 displays X-ray photoelectron spectra (XPS) that demonstrates a phosphorous fingerprint present only in the samples that were exposed to mediumcontaining microorganisms.

\section{Task 2. Extremophiles}

Another task that is related to spent fuel and waste storage has been to develop capabilities to isolate, identify, and manipulate extremophilic microorganisms under different harsh conditions. Topics for investigation include applications to biotechnology, to conditions associated with nuclear industrial environments and other potentially hazardous industrial sites, and to assessment of human global climate and environmental effects. Extreme environmental conditions under consideration include high and low temperatures, low $\mathrm{pH}$, high pressure, high radiation, and high levels of contaminants, such as heavy metals.

\section{Bioreactor Development}

Methods being developed include both culture and non-culture (molecular) based methods. Culture-based methods require development and construction of novel bioreactors. Molecular methods are being applied innovatively to identify genetic types and communities and to select genetic capabilities of interest. Projects within the past 18 months have focused on developing novel high pressure/temperature bioreactors, field sampling and labor-intensive cultivation to develop cultures of interest, and obtaining molecular assessment of cultures. Most recently, work has been started to assess cold-adapted communities from glacial ice cores.

Large quantities of biomass from extremophiles, adapted to conditions of interest, are necessary for molecular and biotechnological studies. The project began with development of a high hydrostatic pressure, high temperature, continuous culture instrument (chemostat). The model organism used in these 


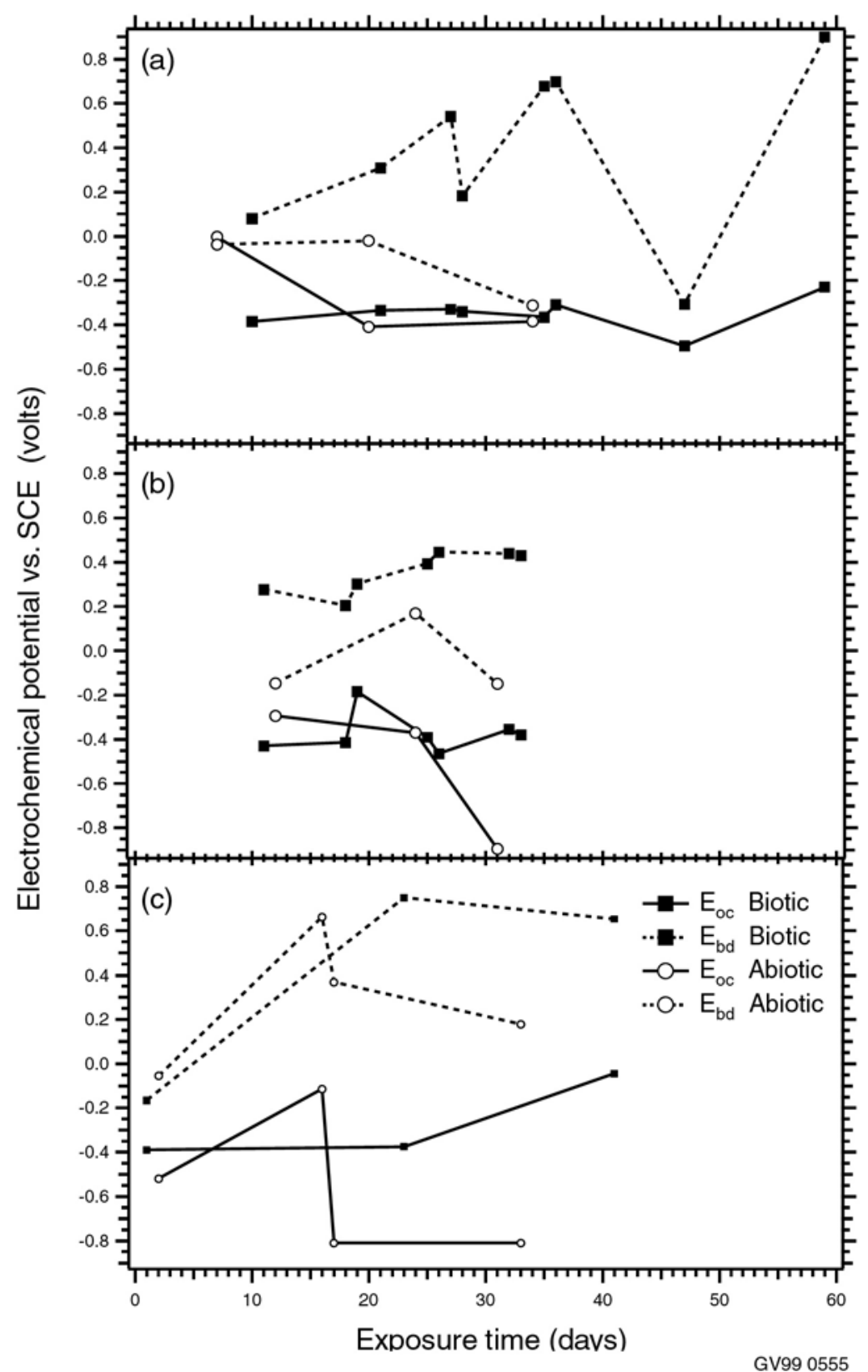

Figure 6. Plots of measured electrochemical potentials vs. SCE vs. exposure time for SRET experiments (a) of biotic and sterile Al 6061 involving raw water with P. aeruginosa, D. radiodurans, and SRBs, (b) the same except for $18 \mathrm{M} \Omega$ water, and (c) Al 6061 involving kitchen sink biotic mixture. The broken lines represent measurements of breakdown (or pitting) potentials, $\mathrm{E}_{\mathrm{bd}}$, and the solid lines indicate measurements of the of open circuit (or corrosion) potentials, $\mathrm{E}_{\mathrm{oc}}$. 


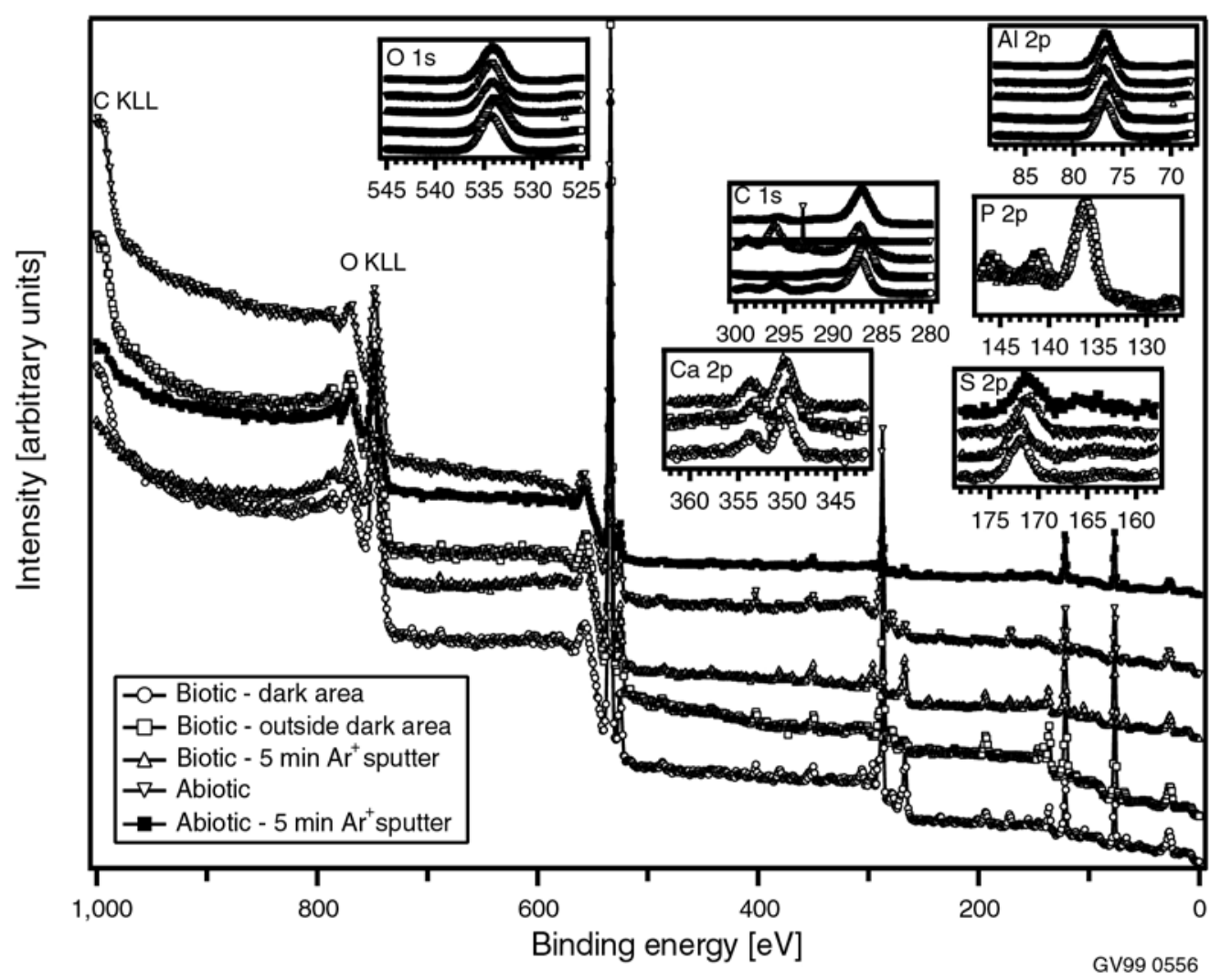

Figure 7. This is XPS survey spectra of biotic and abiotic samples after SRET scanning. Insets show high resolution regional scans of $\mathrm{Al}, \mathrm{C}, \mathrm{Ca}, \mathrm{O}, \mathrm{P}$, and $\mathrm{S}$. Please note the $\mathrm{P} 2 \mathrm{p}$ fingerprint for the biotic samples.

experiments was Thermotoga maritima, a marine thermal vent bacterium with unusual genetics transitional between the microbial groups Archaea and gram- positive bacteria. This setup was then shifted to continuous culture of the acidophilic thermophile, Sulfolobus acidocaldarius, that is in the Archaean group. This species is considered "sulfur dependent" and can grow aerobically using either complex carbon or sulfur for energy. When growing on sulfur, it is autotrophic and uses $\mathrm{CO}_{2}$ from the atmosphere as a carbon source.

A second instrument was developed for hyperbaric (gas pressurized) cultivation at elevated temperatures with control of either or both liquid and gas feeds. Again the model organism was S. acidocaldarius. Both physiological types were tested, including a strain that had been maintained in INEEL/Biotechnologies laboratories and an autotroph that was isolated from samples taken from Moose Pool at Yellowstone National Park.

In conjunction with the hyperbaric chemostat, a sampling sub-task was undertaken to isolate and culture three genera of Sulfolobus related organisms, all sulfur-dependent acidophiles but with differing physiologies and optimal growth conditions. The purpose for this sampling was to begin development of an extremophile culture collection and gene library for experimental and biotechnological applications. Sulfate-reducing bacteria have been identified in the spent fuel storage pool and are thought to contribute to corrosion. The nature of the environment being sampled in this task will allow development of hypotheses related to high radiation exposure and high background levels of heavy metal contaminants. To date, three species have been isolated and a portion of the genome from two has been sequenced. 
According to these sequences, the organisms appear to be closely related to but possibly genetically distinct from the target groups. This task will not be continued as part of the Corrosion and Aging Project after FY-99.

\section{ACCOMPLISHMENTS}

Several species of bacteria have been isolated and cultured from spent fuel storage pools at the INEEL. The bacteria were identified, using 16S rRNA analysis and Biolog® methods. Isolates included both gram positive and gram negative species and sulfate-reducing bacteria. From an ecological perspective, the bacteria found in the spent fuel pools are typical of soil communities.

The radiation resistance of bacteria isolated from the spent fuel storage pools was assessed, using high energy irradiation at the Idaho State University linear accelerator facility. Demonstration of radiation resistance is significant, since it helps demonstrate that bacteria could survive the environmental conditions expected on the surface of spent fuel plates. Several species of bacteria from the INEEL were found to be resistant to irradiation at doses as high as $1.1 \times 10^{5} \mathrm{Rad}$. It was demonstrated that the sulfatereducing bacteria tended to be resistant to irradiation. The radiation resistance of these bacteria is thought to be a result of growth and testing in an anaerobic environment. Reduced availability of oxygen presumably results in less radiation-induced formation of free radicals. Gram positive bacteria also exhibited greater resistance to irradiation compared to gram negative bacteria.

Improved methods have been developed to maintain sterility in control chambers for long periods of time in coupled electrochemical corrosion cells. Sterilization of the sample coupon has been achieved without autoclaving. In general, this is desirable for corrosion testing $\mathrm{Al} 6061$ alloy since autoclaving produces a surface oxide that is significantly different from that formed in normal atmospheric conditions. Better methods of sealing glass joints and effective filter membranes between coupled cells have been developed and demonstrated. Sterility in the control chamber has now been maintained routinely for periods of weeks. The general trend in coupled-electrochemical cells is that introduction of $P$. aeruginosa creates an environment that is more corrosive to $\mathrm{Al} 6061$ than the sterile control.

The SRET analysis has enabled imaging localized electrochemical activity on Al 6061 surfaces as a function of microbial environment. Results from experiments involving a three-component mixture ( $P$. aeruginosa, $D$. radiodurans, and SRBs) correlate with trends observed in the set of kitchen sink experiments. Both cases demonstrate ennoblement of the alloy surface in the microbial environment over time. Demonstration of SRET imaging for examining localized electrochemical activity in microbiological environments is a unique capability for the program.

Methods have been developed to isolate, identify, and culture extremophillic bacteria from a variety of environments. This task has focused on bacteria that might be expected to exist in harsh environments typical of nuclear facilities and in long term geological storage environments. Extreme environments that have been considered include high and low temperatures, low $\mathrm{pH}$, and high pressure. Novel bioreactors have been developed to culture bacteria at high pressure and elevated temperature. Sulfur-reducing bacteria that are thought to be significant for corrosion in spent fuel storage have been extensively studied.

\section{REFERENCES}

1. Stroes-Gascoyne, S. and J. M. West, “An Overview of Microbial Research Related to High Level Nuclear Waste Disposal with Emphasis on the Canadian Concept for the Disposal of Nuclear Fuel Waste.” Canadian Journal of Microbiology, Vol. 42, No.4, 1996, pp. 349-366. 
2. Stroes-Gascoyne, S. and J. M. West, Microbial Studies in the Canadian Nuclear Fuel Waste Management Program. Fems Microbiology Reviews, Vol. 20, No. 3-4, 1997, pp. 573-590.

3. Stroes-Gascoyne, S. and M. Gascoyne, "The Introduction of Microbial Nutrients into a Nuclear Waste Disposal Vault During Excavation and Operation," Environmental Science and Technology, Vol. 32, No. 3, 1998, pp. 317-26.

4. Stroes-Gascoyne, S. and F. P. Sargent, "The Canadian Approach to Microbial Studies in Nuclear Waste Management and Disposal.” Journal of Contaminant Hydrology, Vol. 35, No. 1-3, 1998, pp. 175-190.

5. Wolfram, J. H., R. E. Mizia, and W. J. Dirk. "Microbial Sampling of Aluminum-Clad Spent Nuclear Fuel," NACE International Corrosion 98, Houston, Texas, 1998.

6. Lin, C. L. and S. T. Tan, "Isolation and Characterization of a Novel Deinococcus Radiodurans Mutant Abnormally Susceptible to Mutation Induction by UV, Gamma-Ray, and Mitomycin C, ” International Journal of Radiation Biology, Vol. 69, 1996, pp. 493-502.

7. Brock, T. D. and M. T. Madigan, Biology of Microorganisms, 5 ed., Englewood Cliffs, NJ: Prentice Hall, 1988, pp. 198-9, 345, and 751.

8. Bochner, B. R., “Sleuthing out Bacterial Identities,” Nature, Vol. 339, 1989, pp. 157-8.

9. Woese, C. R., Bacterial Evolution. Microbiological Reviews, Vol. 51, No. 2, 1987, pp. 221-71.

10. Hall, E. J., Radiobiology for the Radiologist, 2 ed., New York: Harper and Row, 1978.

11. Neidhardt, F. C., J. L. Ingraham, and M. Schaechter, Physiology of the Bacterial Cell, Sunderland, MA: Sinauer, 1990.

12. Duwat, P., et al., "Characterization of Lactococcus Lactis UV-Sensitive Mmutants Obtained by ISS1 Transportation,” Journal of Bacteriology, Vol. 179, No. 14, 1997, pp. 4473-9.

13. Zavilgelsky, G. B., et al., "Isolation and Analysis of UV and Radio-Resistant Bacteria from Chernobyl," Journal of Photochemistry and Photobiology B-Biology, Vol. 43, No. 2, 1998, pp. $152-157$.

14 Little, B. J. and Wagner, P., "An Electrochemical Evaluation of Microbiologically Induced Corrosion by Two Iron-Oxidizing Bacteria," Paper 122, presented at Corrosion/86, National Association of Corrosion Engineers, Houston, TX, 1986.

15. Dexter, S. C. Duquette, D. J., Siebert, O. W., and Videla, H. A., "Use and Limitations of Electrochemical Techniques for Investigating Microbiological Corrosion," Corrosion, Vol. 47, No. 4, 1991, p. 308. 


\section{APPENDIX}

\section{Presentations}

1. D. F. Bruhn, C. R. Breckenridge, M. N. Tsang, C. S. Watkins, W. E. Windes, F. F. Roberto, R. N. Wright, R. R. Brey, and P. J. Pinhero, "Irradiation Of Microbes From Spent Nuclear Fuel Storage Pool Environments," Global '99, Jackson, WY, September 2, 1999.

2. P. J. Pinhero, C. S. Watkins, "Imaging Microbiologically-Influenced Pitting Corrosion of Spent Nuclear Fuel Cladding Material with a Scanning Reference Electrode Technique (SRET)," MRS1999, Symposium QQ18: Microbial Processes in Waste Management, Boston, Massachusetts, December 1, 1999. 


\title{
Transport in Solid and Liquid Media
}

\author{
Clinton Van Siclen \\ SUMMARY
}

This research project addresses the transport properties of heterogeneous materials, such as porous and fractured rock, that pertain to environmental issues. For example, fluid permeability and molecular diffusivity are relevant transport properties for contaminant migration in groundwater, while measurements of electrical conductivity (another transport property) may reveal structural characteristics of the porous rock containing the groundwater. An example specific to DOE is radioactive waste storage, where the thermal conductivity of the waste container and surrounding medium determines the dissipation of the decay heat.

The objective of the research is increased understanding of the relationship between the transport properties and the morphology of the material. In general, this will take the form of analytical expressions for the transport properties that are functions of the morphological (or microstructural) attributes of the material (e.g., pore-space tortuosity in porous rock, fractal dimension of rock fracture faces, and spatial distribution of phases in composites). Such equations follow from the development of realistic transport models and statistical representations of the material systems. While this research is basic science, the results are intended to be of use to hydrologists, geophysicists, etc.

A significant accomplishment in FY-99 was the demonstration of a critical length scale, the correlation length $\xi$, below which a material is heterogeneous and above which the material is effectively homogeneous with respect to the transport property of interest. The correlation length is thus a nontrivial parameter of a material system; furthermore, as it is a complicated function of the constituent properties as well as the microstructure morphology, it cannot be determined by visual inspection. This work was recently published in Journal of Physics A.

Considerable progress was made towards relating the transport properties (permeability and electrical conductivity) and geometry of single, fluid-saturated fractures. This work is preliminary to considering the transport properties of fracture networks.

\section{PROJECT DESCRIPTION}

Research during FY-99 included (1) a demonstration that heterogeneous materials possess distinct correlation lengths that are specific to transport properties, and (2) a study of the fluid permeability and electrical conductivity of a single fracture. Project 1 was completed and published in FY-99, while project 2 will be completed in FY 2000. An overview of this work is given in the two subsections that follow.

\section{Transport Property-Specific Correlation Lengths for Heterogeneous Materials}

The correlation length $\xi$ is that length scale above which a material property is independent of sample size or volume over which the property is measured. An immediate corollary of this definition is that accurate measurement of a transport property requires that the linear dimension of the sample exceed $\xi$. This requirement is not always realized. For example, megascopic systems such as oil reservoirs and aquifers cannot be studied in their entirety so subsystems (subvolumes), often of size less than $\xi$, are 
characterized instead. The measured physical property (e.g., fluid permeability) will then be subsystem size-dependent, and phenomenological equations of motion (e.g., Darcy's law) that require a homogeneous system may not be obeyed.

Another corollary is that the sample may not possess a characteristic length scale less than $\xi$. (To make this statement, the typical domain or pore size over which the system is homogeneous must be regarded as the "unit" of length.) From statistical physics it then follows that the corresponding transport property can be expressed as a power law function of $\xi$ with exponent that reflects the morphology of the sample. If the sample dimensions are less than $\xi$, the transport property is a power law function of the sample size instead, with no change in the exponent.

It is thus important to determine the relationships between the morphology of the material, the transport properties, and the correlation lengths corresponding to those transport properties.

This is achieved by use of the "walker diffusion method" (WDM). ${ }^{1}$ The WDM, which was developed by this project last year, is a stochastic method for calculating transport properties. In contrast to finite difference schemes that directly solve the transport equations and thus produce only numerical values for the transport properties, the WDM provides useful analytical and graphical results. This new method exploits the isomorphism between the transport equations and the diffusion equation for a collection of noninteracting walkers (hence the name).

The phase domains in a composite microstructure correspond to distinct populations of walkers, where the walker density of a population is given by the value of the transport coefficient of the corresponding phase domain. The principle of detailed balance ensures that the population densities are maintained and provides the following rule for walker diffusion over a digitized microstructure: a walker at site (or pixel) $i$ attempts a move to a randomly chosen adjacent site $j$ during the time interval $\tau=(4 d)^{-1}$, where $d$ is the Euclidean dimension of the space; this move is successful with probability $p_{i j}=\sigma_{j} /\left(\sigma_{i}+\right.$ $\sigma_{j}$ ), where $\sigma_{i}$ and $\sigma_{j}$ are the transport coefficients for the phases comprising sites $i$ and $j$, respectively. The path of a walker thus reflects the composition (population density) and morphology of the domains that are encountered, and may be described by a diffusion coefficient $D_{w}$ that is related to the macroscopic transport coefficient $\sigma$ by

$\sigma=\langle\sigma(\boldsymbol{r})\rangle \mathrm{D}_{\mathrm{w}}$

where $\langle\sigma(\mathrm{r})\rangle$ is the volume average of the constituent transport coefficients. The diffusion coefficient $D_{w}$ is calculated from the equation

$\mathrm{D}_{\mathrm{w}}=\left\langle R^{2}\right\rangle /(2 d t) \mathrm{F}$

where the set $\{R\}$ of walker displacements, each occurring over the time interval $t$, comprises a Gaussian distribution that must necessarily be centered well beyond $\xi$.

For displacements $R<\xi$, the walker diffusion is anomalous rather than Gaussian, due to the heterogeneity of the microstructure at length scales less than $\xi$ (note that the specimen or periodic length $L$ replaces $\xi$ here and in the work that follows when $L<\xi$ ). There is, however, an additional characteristic length $\xi_{0}<\xi$ below which the microstructure is again effectively homogeneous; this may correspond, for example, to the average phase domain size. A walker displacement of $\xi$ requiring a travel time $t_{\xi}=\xi^{2} /\left(2 d D_{w}\right)$ is then comprised of $\left(\xi / \xi_{0}\right)^{d_{w}}$ segments of length $\xi_{0}$, each requiring a travel time of $t_{\xi}=\xi_{0}{ }^{2} /\left(2 d D_{0}\right)$, where $D_{0}$ is the walker diffusion coefficient calculated from displacements $R<\xi_{0}$.

Setting 
$t_{\xi}=\left(\xi / \xi_{0}\right)^{d_{w}} t_{0}$

gives the relationship

$$
D_{w}=D_{0}\left(\xi / \xi_{0}\right)^{2-d_{w}}
$$

between the walker diffusion coefficient $D_{w}$ and the correlation length $\xi$. This equation has been derived previously in the context of percolation theory ${ }^{2}$ and for conducting pore-insulating matrix systems, 3,4 where $d_{w}$ is then the fractal dimension of the walker path on a percolating cluster near the percolation threshold and through a system of connected pores comprising a fractal structure, respectively.

Equations (1) and (4) together show that all transport properties of a heterogeneous material have a power law-dependence on the correlation length, reflecting the absence of a natural length scale between $\xi_{0}$ and $\xi$. Like $\xi$, the exponent $d_{w}$ is a complex function of the material composition and morphology.

Because $D_{w}$ is a functional of the ratios $\sigma_{i} / \sigma_{j}$ of the constituent phase property values, the dependence of $\xi$ and $d_{w}$ on the phase properties is expected to likewise take the form of ratios.

The dimension $d_{w}$ of a walker path that is fractal over length scales $\xi_{0}<R<\xi$ may be determined from the generalization of equation (3),

$$
t_{R}=\left(\left\langle R^{2}\right\rangle / \xi_{0}^{2}\right)^{d_{w} / 2} t_{0}
$$

Composites, however, do not in general give rise to walker trajectories that can be characterized by a single fractal dimension, whereupon $d_{w}$ in equation (5) must be replaced by the length scale-dependent dimension $d_{w}(R)$. In any case, $d_{w}=2$ for $R>\xi$, which allows an unambiguous graphical determination of the correlation length.

Composites are comprised of phase domains arranged in a distinct microstructure. A typical morphology is shown in Figure 1 $(a)$, where second-phase particles (phase $B$ ) are embedded in a host matrix (phase $A$ ). Figure $1(b)$ presents values for $\left\langle R(t)^{2}\right\rangle$ for this two-dimensional $(200 \times 200$-site periodic) composite with $\sigma_{B} / \sigma_{A}=10^{3}$. Each data point is the average of twelve values, where each of those twelve values is in turn the average $R(t)^{2}$ produced by $10^{5}$ walks, each of duration $t$. In every case the error bar of two standard deviations is obscured by the plotted point. The anomalous and Gaussian regimes are readily distinguished in Figure $1(b)$ : the correlation length $\xi$ and crossover time $t_{\xi}$ are identified by the intersection of the sequence of data points with the solid line fit to those points lying in the Gaussian regime. A straight line fit to the points lying in the anomalous regime would have slope $2 / d_{w}$; this was not done because $d_{w}$ is clearly not constant over length scales less than $\xi$, as discussed above. The dashed line with a slope of 1 and y-intercept equal to $\ln 4$ would be produced by a walker diffusing through a single-phase system $\left(D_{w}=1\right)$, and so gives the limiting behavior at $t \rightarrow 0$ for the particulate-matrix system. An anomalous diffusion regime terminating at the correlation length $\xi$ is therefore inevitable.

The labyrinthine two-phase morphology shown in Figure 2(a) is also typical of real microstructures. Figure $2(b)$ presents values for $\left\langle R(t)^{2}\right\rangle$ for this two-dimensional $(200 \times 200$-site periodic) composite with $\sigma_{B} / \sigma_{A}=10^{-3}$, obtained in the same manner as for the particulate-matrix system. Again, the correlation length $\xi$ and crossover time $t_{\xi}$ separating the anomalous and Gaussian regimes are easily identified. Calculated values for the correlation length $\xi$, the walker diffusion coefficient $D_{w}$, and the conductivity $\sigma$ for these particulate-matrix and labyrinthine systems are $\left(8,0.0080,0.0020 \sigma_{B}\right)$ and $(91$, 


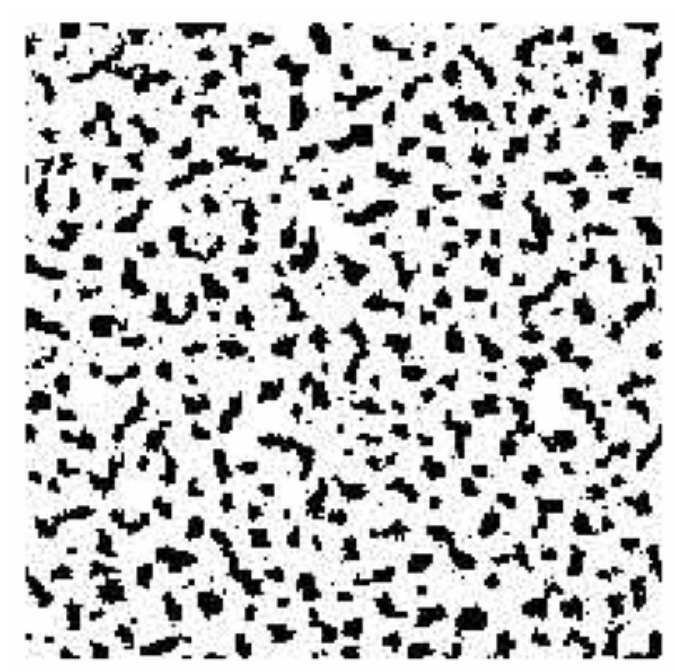

(a)

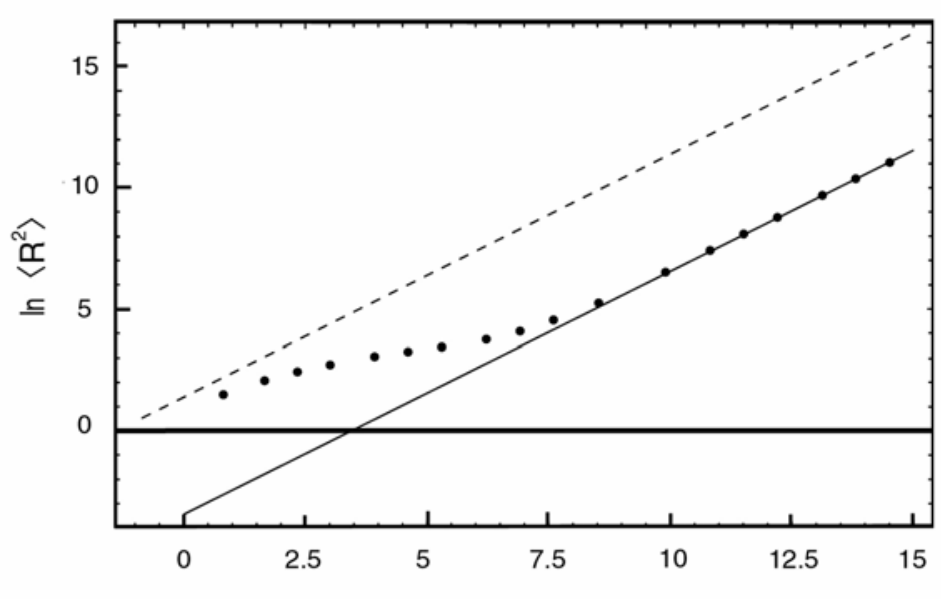

$\ln t$

(b)

GV99 0515

Figure 1. (a) Two-phase composite with a particulate-matrix microstructure. The black particulate phase $B$ has volume fraction 0.25 . (b) Average values for walker displacement-squared $R(t)^{2}$ for walks over the indicated time intervals $t$ through this composite with phase conductivity ratio $\sigma_{B} / \sigma_{A}=10^{3}$. The solid line is a fit to the data points in the Gaussian regime, while the dashed line indicates the walker behavior in a single-phase material.

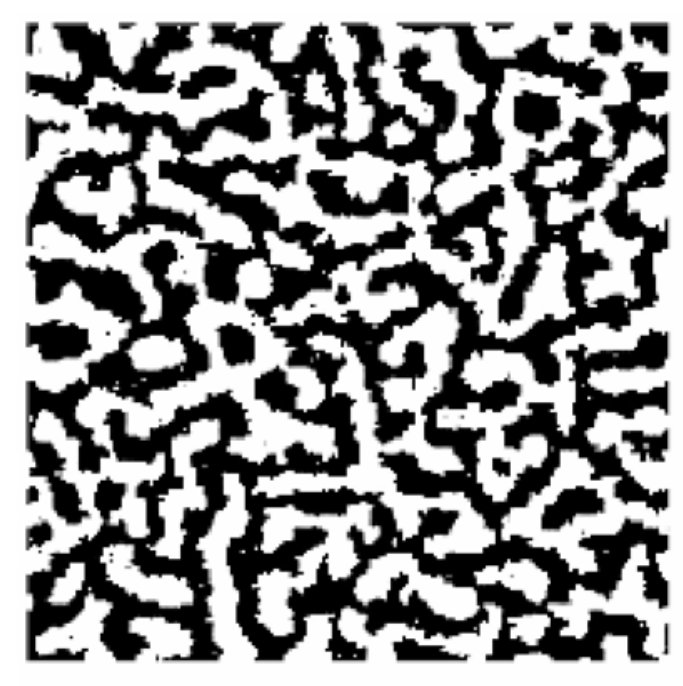

(a)

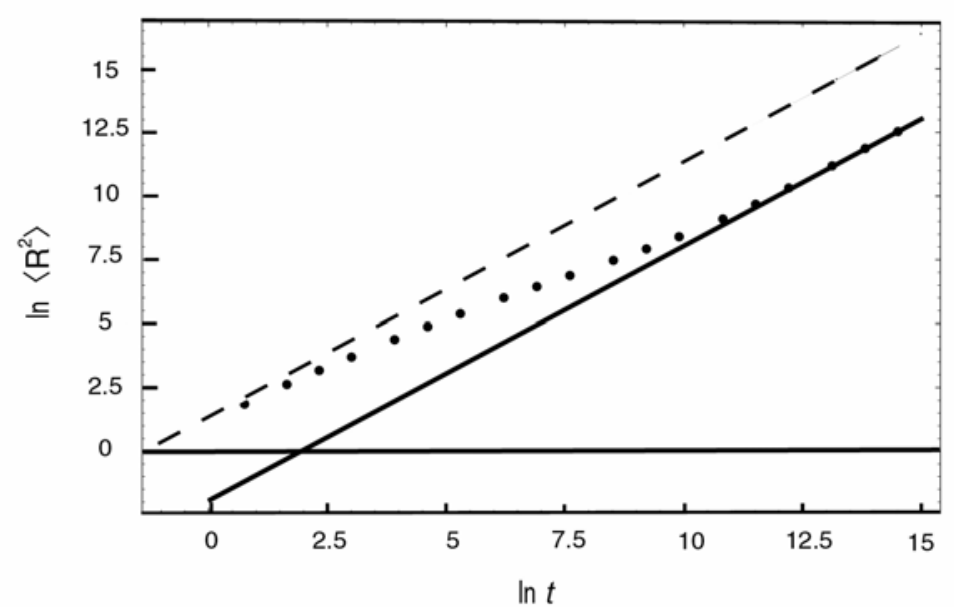

(b)

Figure 2. (a) Two-phase composite with a labyrinthine microstructure. Each phase has volume fraction 0.5. (b) Average values for walker displacement-squared $R(t)^{2}$ for walks over the indicated time intervals $t$ through this composite with phase conductivity ratio $\sigma_{B} / \sigma_{A}=10^{-3}$. The solid line is a fit to the data points in the Gaussian regime, while the dashed line indicates the walker behavior in a single-phase material. 
$0.0347,0.0174 \sigma_{A}$ ), respectively. The larger values in the latter case are due to the percolating domain structure of the higher conductivity phase.

These examples demonstrate that a distinct correlation length associated with a particular transport property exists even for non-fractal composite microstructures. This is because the heterogeneity of the microstructure (as expressed by the morphology, phase domain distribution, etc., as well as by the ratios $\left.\sigma_{i} / \sigma_{j}\right)$ is length scale-dependent at all length scales up to $\xi$.

A more detailed discussion of transport property-specific correlation lengths is provided in a recent publication, ${ }^{5}$ which also includes analytical and numerical results for two-phase disordered composites. These microstructures are of fundamental interest due to their connection with percolation theory, but are perhaps of less practical interest than heterogeneous microstructures such as those considered here.

\section{Hydraulic and Electrical Conductivity of Single Fractures}

The transport properties of rock may be substantially determined by an existent network of fractures, particularly in cases where the rock matrix is relatively impermeable. Such networks are difficult to model for purposes of studying transport properties because fracture densities and orientations tend to be highly anisotropic. In addition, the basic unit of a fracture network - a single fracture-has spatially variable aperture and orientation which affect the local transport properties.

Before addressing the properties of fracture networks, then, it is important to understand the relationship between the geometry of a single fracture and its transport properties. The fluid permeability and electrical conductivity may be straightforwardly obtained by solving the Navier-Stokes equations and the Laplace equation, respectively, over the interior of a three-dimensional, computer-generated fracture. ${ }^{6,7}$ However, this approach is computationally intensive, which severely limits the number of fractures (distinguished by aperture and surface roughness) that can be considered and the length scale over which surface roughness can be created. In addition, the purely numerical results of the calculations provide no basis for proposing a mathematical relationship between the two properties. Such a relationship would be very useful, as the electrical conductivity of a fracture network is much easier to measure experimentally and to calculate for a model system than the permeability.

The approach taken here is inspired by the "parallel plate" model for a fracture. For this model, where the fracture surfaces are smooth and parallel, the steady-state solution of the Navier-Stokes equations for laminar flow yields the "cubic law"; that is, the volume flow rate $\boldsymbol{q}$ is proportional to the cube of the aperture $d$. For the computer-generated fractures with rough surfaces, this cubic law is assumed to hold locally:

$\boldsymbol{q}(\boldsymbol{r})=-\delta \frac{d(\boldsymbol{r})^{3}}{12 \mu} \nabla p(\boldsymbol{r})$

where the vector $\boldsymbol{r}$ identifies the point $(x, y)$ (the fracture lies roughly parallel to the $x-y$ plane), $\boldsymbol{q}(\boldsymbol{r})$ is the volume of fluid discharged per unit time through the cross-sectional area $\delta d(\boldsymbol{r}), \mu$ is the fluid viscosity, and $p$ is the fluid pressure. The condition of fluid incompressibility, $\nabla \cdot \boldsymbol{q}(\boldsymbol{r})=0$, then leads to the Reynolds equation for fluid flow,

$$
\nabla \cdot\left(d^{3} \nabla p\right)=0
$$


Two observations may be made. First, equation (7) is simply a scalar diffusion equation with a spatially dependent diffusion coefficient and so can be solved by a variety of conventional methods. Second, equation (6) may be regarded as a microscopic counterpart to Darcy's Law,

$$
Q=-\frac{K}{\mu} \nabla P
$$

which expresses the linear relationship between the macroscopic volumetric flow rate $\boldsymbol{Q}$ through a system and the imposed pressure gradient $\nabla P$. The proportionality constant $K$ is the effective permeability of the system and is the quantity of interest in this study. Clearly, the value of $K$ will reflect the fracture geometry.

This model for fluid flow through a rough fracture was developed by Brown, ${ }^{8,9}$ who solved the Reynolds equation (7) for various computer-generated fractures by the finite-difference method. However, the model is ideally suited for application of the walker diffusion method (WDM), which can provide some useful analytical results in addition to numerical results.

For a given fracture, the WDM produces the quantity

$$
k \equiv\left\langle\frac{d(\boldsymbol{r})^{3}}{12}\right\rangle D_{h},
$$

where $D_{h}$ is the walker diffusion coefficient for a two-dimensional system with local transport coefficient $d(\boldsymbol{r})^{3}$, which satisfies the macroscopic relationship,

$\boldsymbol{Q}=-\frac{k}{\mu\langle d(\boldsymbol{r})\rangle} \nabla P$

As this relationship resembles Darcy's Law (8), the quantity $k /\langle d(\boldsymbol{r})\rangle$ is identified with the effective permeability $K$. Thus

$$
K=\frac{1}{12} \frac{\left\langle d(\boldsymbol{r})^{3}\right\rangle}{\langle d(\boldsymbol{r})\rangle} D_{h} .
$$

Note that, because $D_{h} \leq 1$, equation (11) gives an upper bound for $K$ when $D_{h}$ is set equal to one.

A similar approach is taken for electrical current flow through a fracture (the fracture is filled with a conducting fluid such as water, while the rock matrix is insulating). In this case, the effective conductivity $\sigma=\sigma_{0} D_{e}$, where $\sigma_{0}$ is the conductivity of the fluid and $D_{e}$ is the walker diffusion coefficient for a two-dimensional system with local transport coefficient $d(\boldsymbol{r})$. Interestingly, this expression for $\sigma$ is also obtained when the Laplace equation is solved for a three-dimensional fracture by the WDM, but the calculated value of $D_{e}$ is different. The difference between the two diffusion coefficients is thus a measure of the accuracy of the two-dimensional Reynolds model for electrical current flow considered here.

WDM calculations will be performed for realistic fractures with various apertures and degrees of surface roughness. The two faces of a real fracture are correlated over large length scales (that is, they "match up") and are uncorrelated over small length scales (reflecting the differential weathering 
subsequent to fracture formation). The faces are self-affine with roughness exponent $\zeta$ typically close to 0.8. ${ }^{10}$ While a number of methods $\mathrm{s}^{11}$ exist to generate self-affine surfaces, the successive random additions algorithm ${ }^{12}$ (where the "additions" are taken randomly from a Gaussian distribution with zero mean and variance $\sigma_{\mathrm{G}}{ }^{2}$ ) is particularly straightforward to formulate in a computer code and will be used here. After the two fracture faces are created, their separation produces the set $\{d(\mathbf{r})\}$ of local apertures appearing in the Reynolds equation (6). It can be shown that this set forms a Gaussian distribution with mean $A$ and variance

$\sigma_{A}^{2}=2 \sigma_{G}^{2} \frac{L^{-2 \varsigma}-M^{-2 \varsigma}}{2^{-2 \varsigma}-1}$

where $A$ is a chosen average aperture, $L^{2}$ is the system size, and $L / M$ is the shortest wavelength over which the two faces are correlated.

It may be noted that the variance $\sigma_{A}^{2}=0$ when $M=L$, that is, when the two faces are correlated over all wavelengths. In this case, the set $\{d(\mathbf{r})\}$ is comprised of identical values $A$, and the Reynolds model gives an effective permeability $K=A^{2} / 12$ and an effective electrical conductivity $\sigma=\sigma_{0}$. These are of course incorrect results (it can be shown, for example, that $\sigma \sim \sigma_{0} L^{-2(1-\zeta)}$ for a very narrow twodimensional fracture with perfectly correlated faces), which reveal a limitation of this model; namely, that the tortuosity of the fluid and electrical flow paths is not fully accounted for. Indeed, Mourzenko et al. ${ }^{13}$ have shown that the two-dimensional Reynolds equation produces fracture permeabilities larger than those found using the three-dimensional Stokes equation, while Volik et al. ${ }^{14}$ have shown that the Reynolds equation produces thermal conductivities larger than those found using the three-dimensional Laplace equation.

Thus only fractures with completely uncorrelated $(M=1)$ faces will be considered here. A variety of fractures with different roughness exponents will be created in order to discover relations between the transport properties and $\zeta$.

As the mean aperture $A$ is reduced, the two fracture surfaces come into contact. Where this occurs, the aperture $d(\mathbf{r})$ is set to zero. It can be shown that the fractional open area (i.e., the fraction of members of the set $\{d(\mathbf{r})\}$ that are greater than zero)

$$
S_{A}=\frac{1}{2} \operatorname{erfc}\left(-\frac{A}{\sqrt{2} \sigma_{A}}\right) .
$$

There are a number of interesting issues to explore with regard to partially closed fractures. For example: (1) When the two uncorrelated, self-affine surfaces first touch, their separation $d(\boldsymbol{r})$ is fractal with the same dimension 3- $\zeta$ as those surfaces. ${ }^{15}$ The set $\{d(\boldsymbol{r})\}$ may then be imagined as a self-affine surface with valleys that subsequently fill in as the mean fracture aperture $A$ is further reduced. The filling in of the valleys creates coastlines that are fractals with dimension $2-\zeta .{ }^{16}$ How are these fractal structures reflected in the correlation length $\xi$ associated with a transport property, and how are they reflected in the dimension $d_{w}$ of the walker path over and around these structures? (2) Mourzenko et al. ${ }^{7}$ have shown that fractures with uncorrelated, self-affine faces do not exhibit a unique percolation threshold with respect to the fractional open area $S_{A}$. Nonetheless it may be possible to determine a value $S_{A}{ }^{\prime}$ such that the transport properties exhibit critical behavior as $S_{A}$ approaches $S_{A}{ }^{\prime}$. How will those critical exponents (obtained for a partially correlated aperture) differ from the corresponding universal critical exponents for uncorrelated two-dimensional lattice systems? 
A justification for using the two-dimensional Reynolds equation to calculate transport and flow properties of fractures is that these properties can be straightforwardly related to one another. This is realized by expressing the walker diffusion coefficients $D_{h}$ and $D_{e}$ as power law functions of their respective correlation lengths, ${ }^{5}$

$$
D_{h} \propto \xi_{h}^{2-d_{h}} \text { and } D_{e} \propto \xi_{e}^{2-d_{e}}
$$

where $d_{h}$ and $d_{e}$ are the fractal dimensions of the walker paths over length scales less than the correlation length. It is reasonable to expect that $\xi_{h} \cong \xi_{e}$, so that

$$
D_{h}=D_{e}^{\left(2-d_{h}\right) /\left(2-d_{e}\right)}=F^{-\left(d_{h}-2\right) /\left(d_{e}-2\right)},
$$

where the formation factor $F \equiv \sigma_{0} / \sigma=D_{e}^{-1}$. Then combining equations (11) and (15) gives an analytic relation between the permeability $K$ and the formation factor $F$,

$$
K=\frac{1}{12} \frac{\left\langle d(\boldsymbol{r})^{3}\right\rangle}{\langle d(\boldsymbol{r})\rangle} F^{-\left(d_{h}-2\right) /\left(d_{e}-2\right)},
$$

for single fractures. Note that for a parallel plate fracture, $d_{h}=d_{e}=2$ so that $K \propto F^{-1}$ as expected. For rough fractures, the fluid flow is more sensitive than the electrical current to aperture variations since the local WDM transport coefficients in the two cases are $d(\boldsymbol{r})^{3}$ and $d(\boldsymbol{r})$, respectively; thus in general $d_{h}>d_{e}>2$. WDM calculations for a variety of rough fractures will provide a more quantitative understanding of the influence of fracture geometry (expressed by the mean $A$ and variance $\sigma_{A}{ }^{2}$ of the aperture, the surface roughness exponent $\zeta$, and the fractional open area $S_{A}$ ) on the walker path dimensions $d_{h}$ and $d_{e}$.

\section{ACCOMPLISHMENTS}

The transport of contaminants through materials is a primary concern of DOE-EM. In a typical study, computer codes are run that solve the transport equations for a large volume containing the (simulated) contamination source; those equations incorporate spatially varying transport coefficients (e.g., permeability and electrical conductivity) derived from a geostatistical analysis of well data. This ESR project, by attempting to relate the transport properties (i.e., transport coefficients) to the local morphology of the material, is intended, ultimately, to improve the analysis of the geophysical data.

To this end, a study was made of the relationship between the correlation length of a material and the material morphology. It was shown that a distinct correlation length $\xi$ exists for each transport property; this means that the material is effectively homogeneous with respect to that transport property over length scales greater than $\xi$, or equivalently, that measurements of that property on all samples of size greater than $\xi$ will produce the same value. Because $\xi$ distinguishes between the length scales over which a system is heterogeneous and homogeneous, it is a fundamental, albeit derived, quantity characterizing a material. A property (e.g., permeability) measured over a volume of size less than $\xi$ will give an anomalous result that should not be used uncritically.

Contaminant migration in rock may be greatly enhanced when transport occurs through a network of fractures. To predict the transport properties of fracture networks, it is first necessary to understand the relationship between the properties and the geometry of a single fracture. Significant progress has been made in this regard: analytic expressions for fluid permeability and electrical conductivity have been 
derived for single fractures parameterized by mean aperture, fractal dimension of the fracture face roughness, and degree of fracture closure. This work will be completed in FY-2000.

\section{REFERENCES}

1. C. DeW. Van Siclen, "Walker Diffusion Method for Calculation of Transport Properties of Composite Materials," Physical Review E, Vol. 59, No. 3, 1999, pp. 2804-2807.

2. Y. Gefen, A. Aharony, and S. Alexander, "Anomalous Diffusion on Percolating Clusters," Physical Review Letters, Vol. 50, No. 1, 1983, pp. 77-80.

3. A. J. Katz and A. H. Thompson, "Fractal Sandstone Pores: Implications for Conductivity and Pore Formation," Physical Review Letters, Vol. 54, No. 12, 1985, p. 1325-1328.

4. S. Roy and S. Tarafdar, "Archie's Law from a Fractal Model for Porous Rocks," Physical Review B, Vol. 55, No. 13, 1997, pp. 8038-8041.

5. C. DeW. Van Siclen, "Anomalous Walker Diffusion through Composite Systems," Journal of Physics A, Vol. 32, 1999, pp. 5763-5771.

6. X. Zhang, M. A. Knackstedt, and M. Sahimi, "Fluid Flow across Mass Fractals and Self-Affine Surfaces," Physica A, Vol. 233, 1996, pp. 835-847.

7. V. V. Mourzenko, J.-F. Thovert, and P. M. Adler, "Percolation and Conductivity of Self-Affine Fractures," Physical Review E, Vol. 59, No. 4, 1999, pp. 4265-4284.

8. S. R. Brown, "Fluid Flow through Rock Joints: the Effect of Surface Roughness," Journal of Geophysical Research, Vol. 92, No. B2, 1987, pp. 1337-1347.

9. S. R. Brown, "Transport of Fluid and Electric Current through a Single Fracture," Journal of Geophysical Research, Vol. 94, No. B7, 1989, pp. 9429-9438.

10. S. R. Brown and C. H. Scholz, "Broad Bandwidth Study of the Topography of Natural Rock Surfaces,” Journal of Geophysical Research, Vol. 90, 1985, pp. 12575-12582.

11. A. R. Mehrabi, H. Rassamdana, and M. Sahimi, "Characterization of Long-Range Correlations in Complex Distributions and Profiles,” Physical Review E, Vol. 56, No. 1, 1997, pp. 712-722.

12. J. Schmittbuhl, J.-P. Vilotte, and S. Roux, "Percolation through Self-Affine Surfaces," Journal of Physics A, Vol 26, 1993, pp. 6115-6133.

13. V. V. Mourzenko, J.-F. Thovert, and P. M. Adler, "Permeability of a Single Fracture; Validity of the Reynolds Equation," Journal of Physics II (France), Vol. 5, 1995, pp. 465-482.

14. S. Volik, V. V. Mourzenko, J.-F. Thovert, and P. M. Adler, "Thermal Conductivity of a Single Fracture," Transport in Porous Media, Vol. 27, 1997, pp. 305-326.

15. M. B. Isichenko, "Percolation, Statistical Topography, and Transport in Random Media," Reviews of Modern Physics, Vol. 64, No. 4, 1992, pp. 961-1043.

16. J. Feder, Fractals, New York: Plenum, 1988. 


\section{APPENDIX}

\section{Peer Reviewed Publications}

1. C. DeW. Van Siclen, "Walker Diffusion Method for Calculation of Transport Properties of Composite Materials," Physical Review E, Vol. 59, No. 3, 1999, pp. 2804-2807.

2. C. DeW. Van Siclen, "Anomalous Walker Diffusion through Composite Systems," Journal of Physics A, Vol. 32, 1999, pp. 5763-5771. 


\section{Characterization Science}

DOE's EM Program is responsible for managing newly generated and legacy waste in addition to cleaning up or monitoring contaminated sites; consequently it faces a wide range of characterization and monitoring issues. Waste must be characterized before and after processing. Contaminated sites must characterized, monitored during remediation, and often monitored after remediation as part of long-term stewardship activities. Characterization plays an important part in understanding the fate and transport of contaminants and is therefore key to understanding natural attenuation processes. Disposal of waste at WIPP requires accurate characterization of the TRU components of the waste.

This research area supports a variety of activities that include development of NDA instrumentation, nuclear spectroscopy and structure studies, sensor development, expert system development, and enhancement of existing instrumental techniques for environmental measurements. The NDA instrumentation, nuclear spectroscopy and structure studies, and expert system development will lead to an improved capability for required characterization of boxed and drummed waste prior to its shipment to WIPP. The sensor development and instrumental enhancement activities address remediation and long-term monitoring as well as the need for field portable characterization instruments. 


\title{
Adaptive Sensors
}

\author{
K. L. Telschow, R. S. Schley, N. Thantu, V. A. Deason, S. M. Watson
}

SUMMARY

Sensing is an important part of efforts to characterize, treat, and dispose hazardous materials as information on the physical and chemical state of the material must be known before, during, and after remediation. In situ measurements require monitoring that is noncontacting, nonintrusive, and capable of wide-area imaging.

This project is investigating the use of nonlinear optical methodologies that offer imaging solutions to environmental sensing problems. Previous results have shown that dynamic holography can produce sensors that are self-adaptive, self-processing, highly sensitive, and provide wide area imaging in realtime. A primary function of the present work is to investigate ways of implementing this full-field measurement technique for imaging chemical information through the atmosphere and at surfaces. A part of this research program uses materials that exhibit strong second order optical nonlinearities.

Continuous or quasi-continuous excitation methods and narrow band detection techniques were employed for high sensitivity and imaging. Theoretical and experimental characterization of a "containerless" photoacoustic spectroscopic measurement technology using photorefractive materials were successfully conducted to determine detection limits and measurement speed. Spectroscopic measurements were conducted in the visible region with existing tunable laser facilities. Laser sources in the infrared molecular "fingerprint" region of the absorption spectrum shall be used in future work. Advances toward understanding the photorefractive two-wave mixing process in anisotropic materials were made both theoretically and experimentally FY 2000.

A second task on this research program employs a time-domain approach using third and higher order nonlinearities that exist in most materials. This approach offers the potential for highly selective spectroscopy based on a particular materials nonlinear optical properties. A new researcher experienced in ultrafast laser techniques has joined this project and established an ultrafast laser facility with femtosecond time resolution.

\section{PROJECT DESCRIPTION}

\section{Containerless Photoacoustic Spectroscopy}

The first environmental sensing application under study is photoacoustic spectroscopy. The goal of this work is to determine the sensitivity and limitations for containerless photoacoustic spectroscopy using the dynamic holographic approach. At first, emphasis has been placed on determining the ability to measure the absorption cross-section for a known molecular species from the heat generated during the photoacoustic process along the measurement beam. This process uses a continuous laser for detection and a second tunable chopped laser for generation. Future work is repeating this measurement using a single laser that both provides tunable absorption and dynamic holographic detection. Detection at surfaces is also planned for investigation FY 2000.

\section{Experimental Measurement Setup}

During FY-99 the project has established the capability to perform containerless photoacoustic spectroscopy using a tunable Ti:Sapphire laser over the range of $700 \mathrm{~nm}$ to $1,000 \mathrm{~nm}$ and detection at $532 \mathrm{~nm}$ using a very stable solid state continuous Nd:YAG laser. The basic setup is shown in Figure 1. 


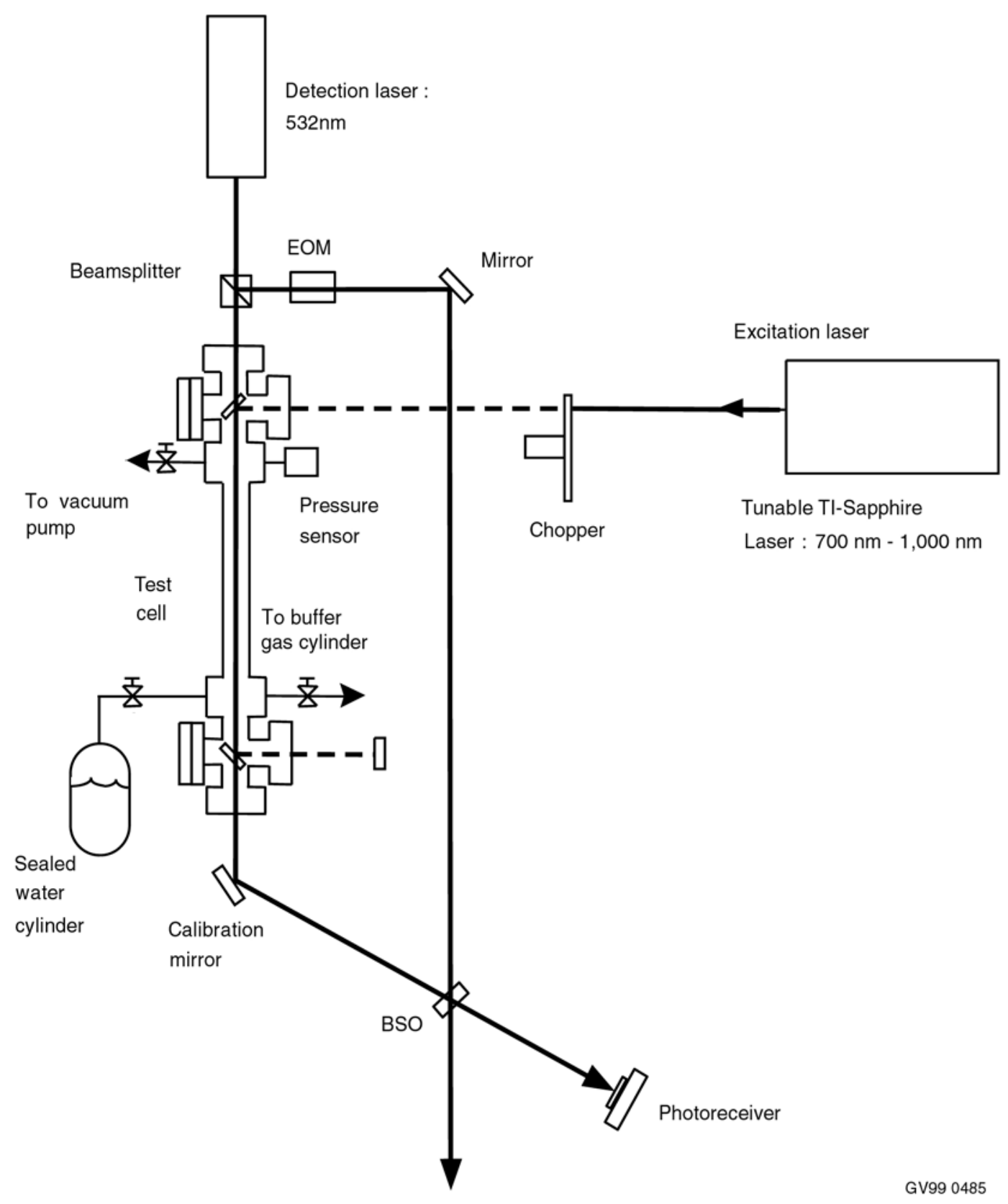

Figure 1. Experimental apparatus configured for the frequency-domain nonlinear optical and photoacoustic dynamic holographic measurements, showing the detection and generation laser beams and the gas containment enclosure.

The excitation laser is modulated by a chopper and sent through a cell that contains controllable amounts of trace and background gases. The Ti:Sapphire laser is tuned to an absorption line of the trace gas. The absorbed light energy is ultimately transferred to the background gas producing alternating local temperature and density modulations in and near the beam path. Acoustic waves are also launched into the surrounding gas region. The local heating in the gas produces a corresponding modulation in the index of refraction of the gas. A second detection laser beam passes through the absorption region and experiences a phase shift due to the temperature modulation. The nonlinear optical properties of the photorefractive material, bismuth silicon oxide (BSO) or gallium arsenide (GaAs), establish a grating that 
demodulates the small photoacoustic phase shift by interaction with a suitably modulated reference beam as indicated in the figure.

\section{Simulated Trace Gas Measurement Results}

Quantitative photoacoustic measurements were performed on water vapor and nitrogen mixtures at selected known absorption lines in the $800-900 \mathrm{~nm}$ range. Water vapor was used as it is easily obtained, benign and its spectroscopic properties quantitatively known. Several absorption lines of water were experimentally measured. The aim of this approach was to conduct photoacoustic optical absorption measurements in a geometry that does not require a closed volume cell for the detection process, as is normally the case in conventional photoacoustic spectroscopy employing acoustic detection with microphones. A closed cell was used here only for the purpose of controlling the concentrations of the gas mixtures investigated. The interferometrically measured photoacoustic signal was shown to be proportional to the water vapor concentration for several absorption lines and the peak magnitudes to be in approximate agreement with published values for these lines. In addition, the effect of adding a background gas, not participating in the optical absorption but contributing to the photoacoustic heating, was explored. Figure 2 shows the absorption line in water vapor at $12,226.1 \mathrm{~cm}^{-1}$ both without (top) and with (bottom) background nitrogen gas. The data without the background gas show that the photoacoustic signal is proportional to the water vapor density, as expected. Addition of a background gas ultimately reduces the photoacoustic signal due to collision broadening and addition of more heat capacity. The data in the bottom of the figure show this behavior as the background gas is added.

Modeling the Photoacoustic Detection Process:

A theoretical model of the containerless gas mixture photoacoustic process was developed in order to determine the minimum detectable trace gas concentration. The model results were shown to be in quantitative agreement with measurements for background gas pressures up to one atmosphere described as follows. Consider a temperature modulation produced by absorption of chopped laser radiation along a beam path, L. Neglecting thermal diffusion, the fundamental component of the average temperature modulation along the detection beam path, as measured by lock-in detection, is given by

$$
\Delta \mathrm{T}=\frac{\left(\mathrm{k}_{\mathrm{v} 0} \eta_{0}+\mathrm{k}_{\mathrm{v} 1} \eta_{1}\right) \mathrm{I}_{0}}{\left(\rho_{0} \mathrm{C}_{\mathrm{p} 0}+\rho_{1} \mathrm{C}_{\mathrm{p} 1}\right) \pi^{2} \mathrm{f}}
$$

where $k_{v 0,1}, \eta_{0,1}, \rho_{0,1}, C_{p 0,1}$ refer to the optical absorption cross section, molecular concentration, mass density, and heat capacity of the background and trace gases respectively. The optical absorption depends on the excitation laser wavelength and provides the selectivity for a particular molecular species. The factor $I_{0}$ describes the excitation laser intensity and the chopping, at the frequency $f$, allows the use of lock-in detection techniques for high noise immunity. When the excitation wavelength is chosen so that the background gas absorption coefficient is negligible, the above equation illustrates that the background gas primarily dampens the photoacoustic temperature fluctuation. This is a drawback to the photoacoustic approach as most measurement problems involve a background gas at one atmosphere.

The local heating along the beam path modulates the index of refraction of the gas through a small change in density. The net optical phase shift along the beam path is given by

$$
\Delta \Phi=\frac{2 \pi}{\lambda} \int\left(\mathrm{n}\left(\mathrm{x}, \mathrm{T}_{\mathrm{a}}+\Delta \mathrm{T}\right)-1\right) \mathrm{dx} \approx \frac{2 \pi \Delta \mathrm{nL}}{\lambda}
$$



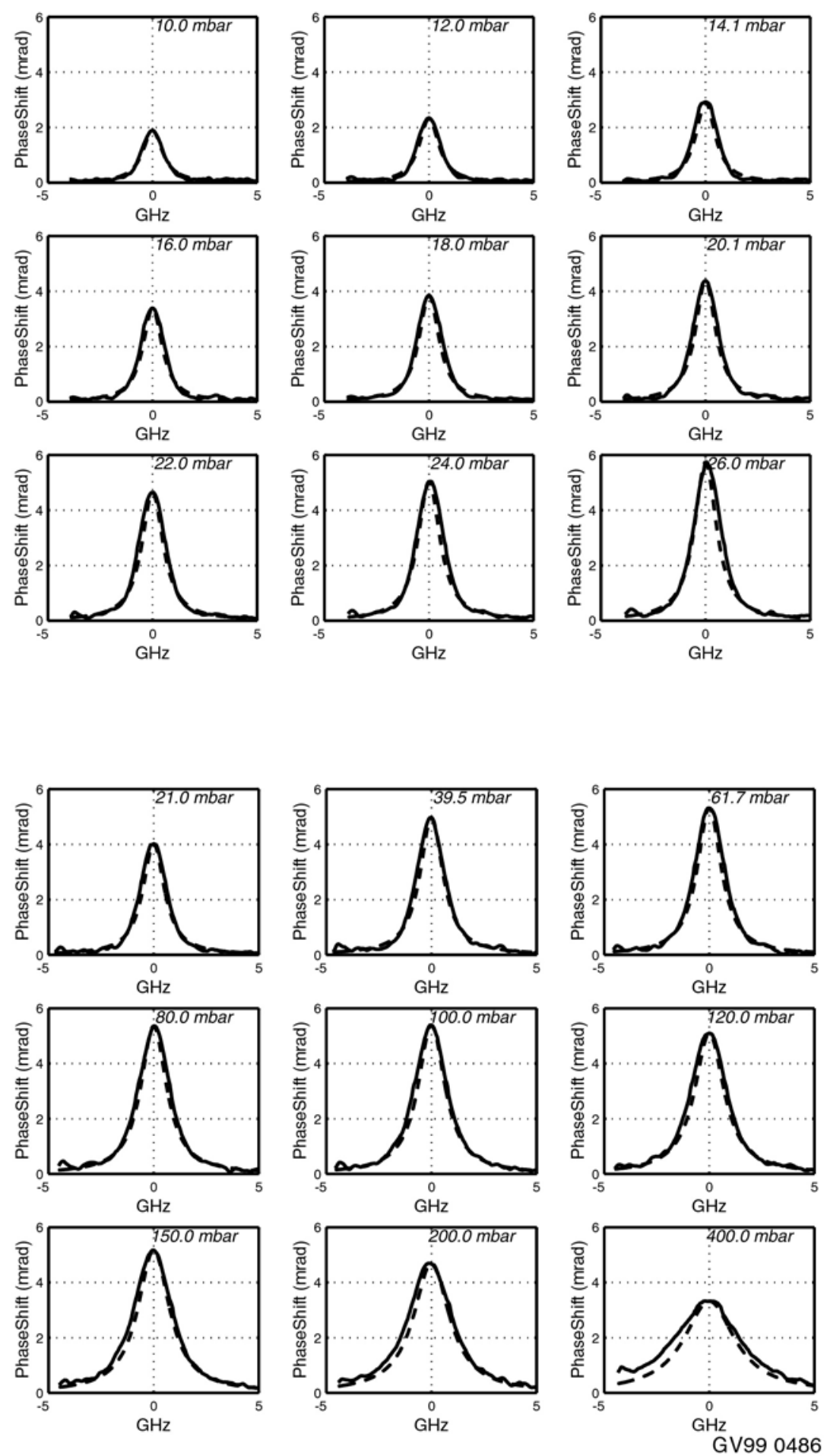

Figure 2. Phase shift measurement and Gaussian curve fits of the optical absorption spectra for various water vapor pressures (top) and for 21 mbars of water vapor in nitrogen (bottom) at the total pressure indicated. 
where $L$ is the beam path length, $\lambda$ is the optical wavelength, $n(x, T)$ is the local temperature dependent index of refraction and $T_{a}$ is the ambient temperature. Optical interferometric detection techniques were used to demodulate the small photoacoustic phase shift. For a two-component gas mixture, the index of refraction is given by the Claussius-Mossotti relation, $\frac{\mathrm{n}^{2}-1}{\mathrm{n}^{2}+2}=\frac{\mathrm{R}_{\mathrm{m} 0} \rho_{0}}{\mathrm{M}_{0}}+\frac{\mathrm{R}_{\mathrm{m} 1} \rho_{1}}{M_{1}}$, where $\mathrm{R}_{\mathrm{m} 0,1}\left(\frac{\mathrm{m}^{3}}{\mathrm{~mole}}\right)=\frac{\mathrm{N}_{\mathrm{A}} \alpha_{\mathrm{P} 0,1}}{3 \varepsilon_{0}}$ with $N_{A}$ as Avogadro's number, $\alpha_{\mathrm{P} 0,1}\left(\frac{\mathrm{Cm}^{2}}{\mathrm{~V}}\right)$ the atomic or molecular polarizability and $\mathrm{M}_{0,1}(\mathrm{~kg} / \mathrm{mole})$ the molecular weight. The rise in temperature at constant pressure causes a change in index of refraction through

$$
|\Delta \mathrm{n}| \approx \frac{1}{2 \varepsilon_{0}} \mid \alpha_{\mathrm{P} 0} \eta_{0}+\alpha_{\mathrm{P} 1} \eta_{1}\left(\frac{\Delta \mathrm{T}}{\mathrm{T}_{\mathrm{a}}}\right) .
$$

Combining Equations 1-3 produces the net photoacoustic phase shift as

$$
\Delta \Phi=\left(\frac{2 \pi \mathrm{L}}{\lambda}\right)\left(\frac{\mathrm{I}_{0}}{\pi^{2} \mathrm{fT}_{\mathrm{a}}}\right)\left(\frac{\alpha_{\mathrm{P} 0} \mathrm{~N}_{\mathrm{A}}}{2 \varepsilon_{0} \mathrm{M}_{0} \mathrm{C}_{\mathrm{p} 0}}\right) \frac{\left(\eta_{0}+\left(\frac{\alpha_{\mathrm{P} 1}}{\alpha_{\mathrm{P} 0}}\right) \eta_{1}\right)}{\left(\eta_{0}+\left(\frac{\mathrm{M}_{1} \mathrm{C}_{\mathrm{p} 1}}{\mathrm{M}_{0} \mathrm{C}_{\mathrm{p} 0}}\right) \eta_{1}\right)}\left(\mathrm{k}_{\mathrm{v} 1} \eta_{1}\right) .
$$

The optical absorption cross-section for a given molecular transition at resonance is given by $\mathrm{k}_{v}=\frac{\mathrm{S}_{v v^{\prime}}(\mathrm{T})}{\pi \gamma(\mathrm{p}, \mathrm{T})}\left(\mathrm{cm}^{2} /\right.$ molecule $)$, where $\mathrm{S}_{v v^{\prime}}(\mathrm{cm} /$ molecule $)$ is the line strength and $\gamma(\mathrm{p}, \mathrm{T})\left(\mathrm{cm}^{-1}\right)$ is the line width. Values for these quantities were obtained from the HITRAN96 database developed by the Air Force for prediction of atmospheric optical absorption conditions lists of spectral lines and absorption cross-sections. ${ }^{1}$ Figure 3 shows a comparison between the predictions of the measured phase shift from Equation 4 as a function of nitrogen (top) and helium (bottom) background pressures. Both cases normalize the predictions to the experimental measurements through a scale factor as shown. Good agreement between the model and experimental results were obtained as shown. The discrepancies (scale factors not equal to one) can probably be accounted for by incomplete beam overlap and other experimental measurement uncertainties and is under further study.

An analysis of the minimum detectable trace gas concentration was performed using the photoacoustic model and the experimentally obtained noise level. The results are shown in Figure 4 as a series of phase shifts versus trace gas concentration for different line strengths. The line strength for the particular absorption line of water measured was $4.2 \times 10^{-23}\left(\mathrm{~cm}^{-1} /\right.$ molecule) which lies on a line showing a minimum detectable concentration of about $1,000 \mathrm{ppm}$ with the current experimental noise level. Much improvement is possible as is shown by the location of the shot-noise limit detection that is possible with optical measurements leading to a theoretical limit of less than $1 \mathrm{ppm}$. Even smaller concentrations can be measured using stronger absorption lines for the trace gas molecule as indicated. Generally, stronger absorption lines are observed in the infra-red absorption region for wavelengths greater than 1 micron. The experimental noise level observed was determined to be due to phase noise in the lasers and from other vibrational and acoustic sources at low frequencies near the operating frequency of $100 \mathrm{~Hz}$. This low frequency was used due to the rapid fall off of the photoacoustic signal with frequency. Improvements are possible with the use of additional photorefractive components to compensate for the noise sources and are the subject of further study. 

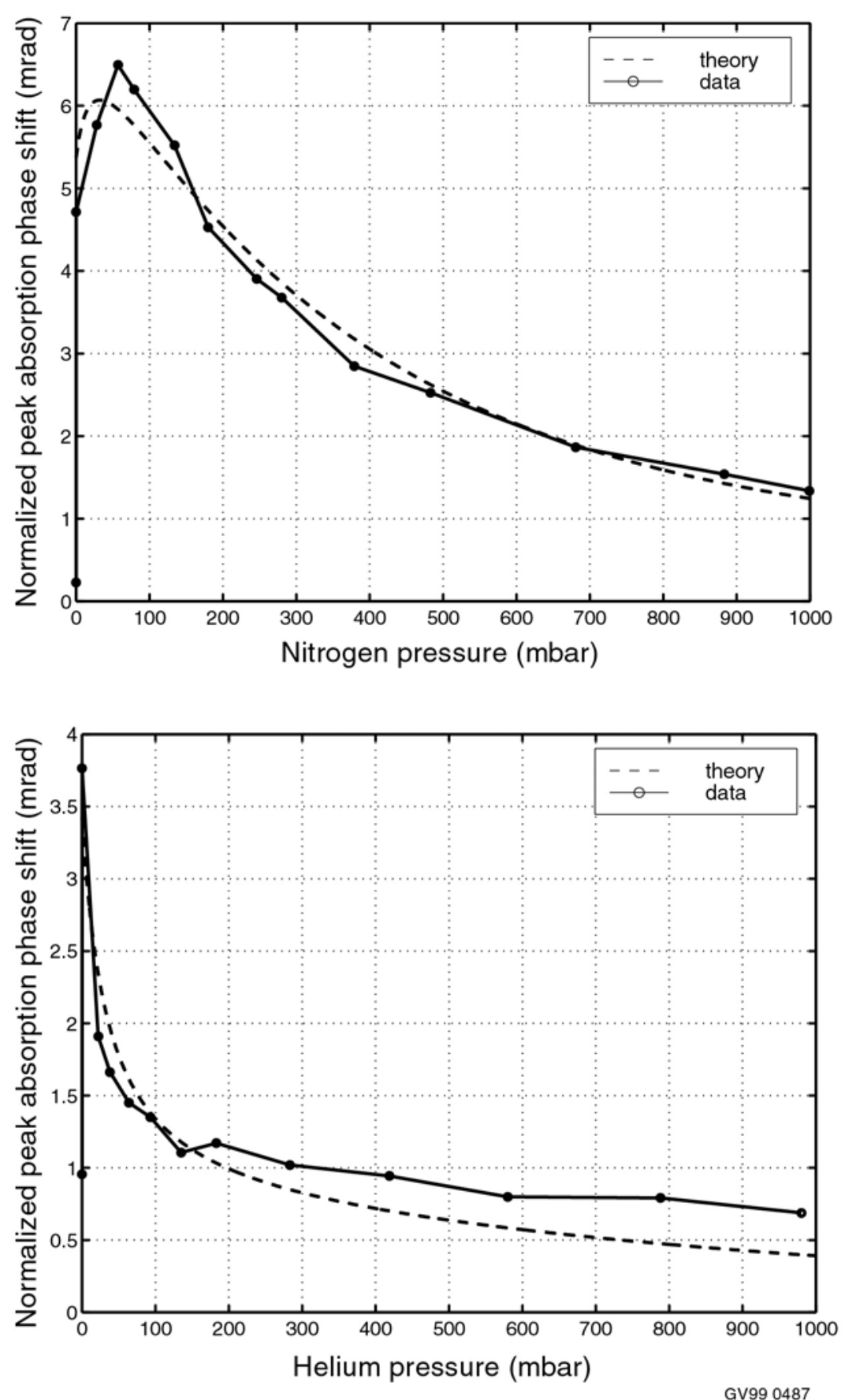

Figure 3. Theoretical and experimental normalized peak absorption phase shifts determined from the nitrogen (top) and helium (bottom) buffered water vapor measurements. 


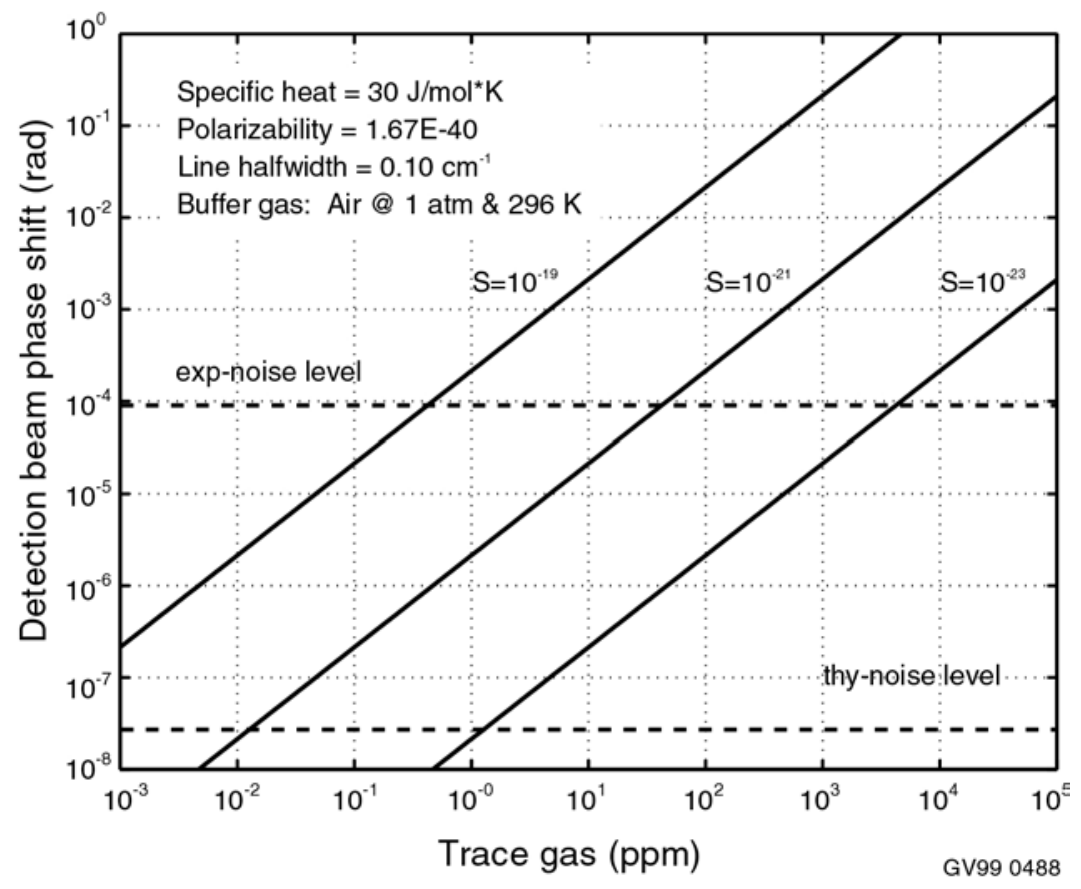

Figure 4. Theoretical detection limits calculated from the model using the data indicated for different absorption line strengths.

\section{Containerless Photoacoustic Measurements Summary}

This work has shown that containerless photoacoustic measurements can be made over beam paths of less than one meter. Model calculations agree well with the experimental measurements and have yielded a minimum detectable concentration of about 1,000 ppm of water vapor in 1 atmosphere of $\mathrm{N}_{2}$ at room temperature using a single beam pass of about $60 \mathrm{~cm}$. Several factors have been identified and quantified to improve this detectability including: improving the phase noise immunity of the photorefractive interferometric process, reducing laser and environmental phase noise, using faster and more sensitive photorefractive materials, using higher laser powers and employing stronger absorption lines of water in the infrared region of the spectrum. Efforts are underway to extend the process to that of shot-noise limited detection, which would improve the minimum detectability by at least three orders of magnitude. Suitable scaling of these parameters will allow the extension of this measurement technique to wide area spectroscopic imaging of trace gas concentrations in future work.

\section{Photorefractive Material Development}

Optical processing of information performed by interfering two waves inside a nonlinear material exhibiting photorefractivity is at the heart of the research being performed on this project. Optimization of the sensing material for this process is of the utmost importance. Several reviews have been recently published on both the physical effects of two-wave mixing in several photorefractive materials and its many applications. ${ }^{2,3,4,5}$ Photorefractivity has made an impact on the interferometric measurement process through its ability to perform optical phase demodulation through homodyne interferometry from rough and diffusely scattering surfaces ${ }^{6,7}$ The photorefractive process is employed to produce a diffracted reference wavefront with the spatial characteristics of and coaxial with the probe wavefront. Subsequent homodyne interference produces a demodulation of the phase information at frequencies greater than the 
photorefractive cutoff frequency and the mixing process itself can perform heterodyne demodulation as is employed on this project. Recently, this process has been shown to be able to demodulate and image nonstationary phase modulations also. ${ }^{8}$

Most photorefractive materials are crystalline exhibiting anisotropic behavior. Two-wave mixing of these materials is inherently complicated by the tensorial character of the optical properties of these materials. Nevertheless, two-wave mixing can occur in these materials and produce interesting effects, such as cross-polarization coupling that can be used to advantage in situations where polarizing components are used to select particular beams from the output. ${ }^{9}$ There is a need to quantitatively understand the anisotropic-diffraction process in crystalline photorefractive materials in order to take full advantage of this effect for demodulation of static and dynamic phase information. A nonlinear model of the static photorefractive two-wave mixing in cubic crystals has been developed. ${ }^{10}$ Gallium Arsenide (GaAs) is a cubic photorefractive material being studied for application to environmental sensing. Although GaAs has a smaller coupling constant than other photorefractive materials, such as bismuth silicon oxide, its response time is much faster making it particularly suitable for dynamic phase measurements. Even though GaAs is optically isotropic, the tensor nature of the electro-optic effect in GaAs crystals allows cross-polarization two-wave coupling. Experiments were performed to compare the results of two-wave mixing in a GaAs crystal with the predictions of the model developed by Yeh for the static case and to provide data to understand the dynamic response at frequencies both above and below the phase modulation frequency for which no model is yet available. Good agreement was found between the two-wave mixing results and the model for the static case and unusual cross-polarization coupling was observed for the dynamic modulation detection.

\section{Experimental Setup}

Two-wave mixing measurements were performed in a GaAs crystal using a continuous solid state laser operating at $1,064 \mathrm{~nm}$. The laser beam was split using a polarizing beam splitting cube into signal and reference beams. The signal beam was reflected from a piezoelectrically driven mirror in order to produce a calibrated phase modulation simulating ultrasonic motion. After exiting the crystal, the beam passed through a polarization analyzer and filter before entering the photodetector. The reference beam passed through an electro-optic modulator (EOM), an electronically controlled shutter before mixing with the signal beam in the crystal. Half-wave plates were used to control the polarizations of both beams before entering the GaAs crystal. The EOM and vibrating mirror allowed each beam to be phasemodulated so that the dynamic signal resulting from the coupling in the crystal could also be measured.

Three lock-in amplifiers were used to measure both the static and dynamic aspects of the two-wave mixing process. The output signal from the photodetector was connected to all three lock-in amplifiers. Each lock-in amplifier measured three signals: the static or dc signal, the dynamic signal at a particular reference frequency, and the phase of the dynamic signal relative to the reference frequency. The reference frequencies for the lock-in amplifiers corresponded to the frequency of (1) the vibrating mirror, (2) the frequency of the EOM, and (3) the difference between the vibrating mirror and EOM frequencies.

In these experiments the vibrating mirror was driven by a $15 \mathrm{kHz}$ sine wave and the EOM was driven by a $15.25 \mathrm{kHz}$ sine wave. The driving signals were mixed and filtered to provide the difference frequency of $250 \mathrm{~Hz}$.

The crystal orientation and rotation angle, $\psi$, in the laboratory coordinate system are displayed with respect to the optical beams in Figure 5. Definitions of the signal $\left(B_{s}, B_{p}\right)$ and reference $\left(A_{s}, A_{p}\right)$ beam polarizations and the crystal coordinates are also shown. Zero rotation angle for the crystal corresponds to the s-polarization being parallel to the (001) axis. 


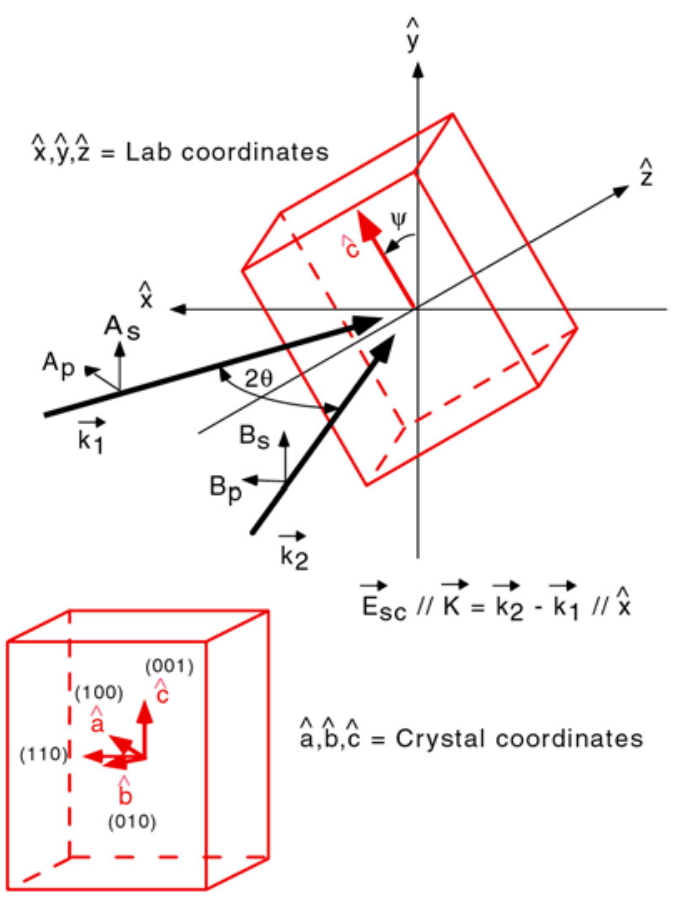

GV99 0489

Figure 5. Crystal orientation and coordinates for the anisotropic diffraction measurements.

\section{Theoretical Model}

An anisotropic diffraction model for co-directional two-wave mixing, as developed by $\mathrm{Yeh},{ }^{10}$ was used to predict the results from the static measurements. The general equations for the coupling constants at any crystal orientation angle were developed by considering the geometry displayed in Figure 5 . The spatial intensity distribution within the crystal is given by

$\mathrm{I}(\overrightarrow{\mathrm{r}})=\left|\overrightarrow{\mathrm{E}}_{1}+\overrightarrow{\mathrm{E}}_{2}\right|^{2}=\mathrm{I}_{0}\left[1+\frac{\left[\left(\mathrm{A}_{\mathrm{s}}^{*} \mathrm{~B}_{\mathrm{s}}+\mathrm{A}_{\mathrm{p}}^{*} \mathrm{~B}_{\mathrm{p}} \cos (2 \theta)\right)^{-\mathrm{i} \cdot \overrightarrow{\mathrm{r}}}+\text { c.c. }\right]}{\mathrm{I}_{0}}\right]$,

where the "fringe contrast ratio" $M(\vec{r}) \equiv \frac{\left\lfloor\left(A_{s}^{*} B_{s}+A_{p}^{*} B_{p} \cos (2 \theta)\right)^{-i \vec{K} \cdot \vec{r}}+\text { c.c. }\right\rfloor}{I_{0}}$ and $I_{0} \equiv A_{s}^{2}+A_{p}^{2}+B_{s}^{2}+B_{p}^{2}$.

The electromagnetic coupled wave description was used with the slowly varying amplitude approximation to model the transfer of amplitude and phase between the various polarization components of the signal and reference beams as they undergo the two-wave mixing process from 


$$
\begin{aligned}
& \frac{\mathrm{d}}{\mathrm{dz}} \mathrm{A}_{\mathrm{s}}=\frac{\mathrm{i}}{2 \beta} \mathrm{e}^{\mathrm{i} \phi}\left[\Gamma_{\mathrm{ss}} \cdot \mathrm{B}_{\mathrm{s}}+\Gamma_{\mathrm{sp} 2} \cdot \mathrm{B}_{\mathrm{p}}\right] \cdot \mathrm{M}^{*}(\overrightarrow{\mathrm{r}}) \mathrm{C} \\
& \frac{\mathrm{d}}{\mathrm{dz}} \mathrm{B}_{\mathrm{s}}=\frac{\mathrm{i}}{2 \beta} \mathrm{e}^{-\mathrm{i} \phi}\left[\Gamma_{\mathrm{ss}} \cdot \mathrm{A}_{\mathrm{s}}+\Gamma_{\mathrm{sp} 1} \cdot \mathrm{A}_{\mathrm{p}}\right] \cdot \mathrm{M}(\overrightarrow{\mathrm{r}}) \mathrm{C} \\
& \frac{\mathrm{d}}{\mathrm{dz}} \mathrm{A}_{\mathrm{p}}=\frac{\mathrm{i}}{2 \beta} \mathrm{e}^{\mathrm{i} \phi}\left[\Gamma_{\mathrm{p} 1 \mathrm{~s}} \cdot \mathrm{B}_{\mathrm{s}}+\Gamma_{\mathrm{p} 1 \mathrm{p} 2} \cdot \mathrm{B}_{\mathrm{p}}\right] \cdot \mathrm{M}^{*}(\overrightarrow{\mathrm{r}}) \mathrm{C} \\
& \frac{\mathrm{d}}{\mathrm{dz}} \mathrm{B}_{\mathrm{p}}=\frac{\mathrm{i}}{2 \beta} \mathrm{e}^{-\mathrm{i} \phi}\left[\Gamma_{\mathrm{p} 2 \mathrm{~s}} \cdot \mathrm{A}_{\mathrm{s}}+\Gamma_{\mathrm{p} 2 \mathrm{p} 1} \cdot \mathrm{A}_{\mathrm{p}}\right] \cdot \mathrm{M}(\overrightarrow{\mathrm{r}}) \mathrm{C}
\end{aligned}
$$

where $\mathrm{C}=\left(\frac{2 \pi}{\lambda}\right)^{2} \mathrm{n}^{4} \mathrm{r}_{41} \mathrm{E}_{\mathrm{q}}$ and $n$ is the index of refraction, $r_{41}$ is the electro-optic coefficient and $\phi$ is approximately 90 degrees in the diffusive regime for GaAs. With suitable evaluation of the coupling coefficients as a function of the rotated coordinates, these equations were numerically integrated to produce theoretical results for comparison with the static measurement results.

\section{Static Results}

Figure 6a shows the static output beam without two-wave mixing, represented by a solid line, and the static output beam with two-wave mixing data, represented by a dashed line, for the input polarizations at 45 degrees to the vertical. In this configuration, the mixing resulted in energy being transferred from the reference beam to the signal beam (i.e., two-wave gain). Figure 7a shows the static results for the input polarizations at 0 degrees to the vertical. In this case, no energy transfer (i.e., two-wave gain) occurs but a shift or transfer of polarization was observed. The theoretical calculations and that data were found to be in good agreement for many rotation angles of the crystal using the measured values for the attenuation $\alpha$ and the two-wave gain coefficient $\Gamma_{\mathrm{B}}$ indicated. The theory accurately predicted the polarization shift of the output beam as well as the two-wave intensity ratio $\eta \equiv \mathrm{I}_{\text {Bout }}$ (with mixing) $/ \mathrm{I}_{\text {Bout }}$ (without mixing)

\section{Dynamic Results}

The dynamic signal at the difference frequency results from the mixing of the signal and reference beams inside the GaAs crystal. This process is described in more detail in previous work describing the dynamic phase demodulation process for detecting traveling phase modulations in materials. ${ }^{8}$ Figure $6 \mathrm{~b}$ shows a corresponding large dynamic differential signal at $250 \mathrm{~Hz}$ was recorded with a shifted polarization from the resulting static beams for the 45 degree configuration. Somewhat unexpected, Figure $7 \mathrm{~b}$ shows that a similarly large dynamic differential signal at $250 \mathrm{~Hz}$ was recorded from the resulting static beams for the 0 degree configuration too. It is interesting to note that the differential signal is always proportional in amplitude and phase to the difference in the static baseline and static coupled signals. The effect of producing a difference frequency signal of significant amplitude when there is little or no exchange of beam energy appears to be a unique consequence of the anisotropic diffraction process.

\section{Anisotropic Diffraction Measurement Summary}

The anisotropic diffraction process has been studied in detail for the GaAs cubic crystal in both the static and dynamic phase modulation configurations. A detailed theoretical model of the diffraction process has been developed for any crystal orientation from the previously published work of $\mathrm{Yeh}^{10}$ and 


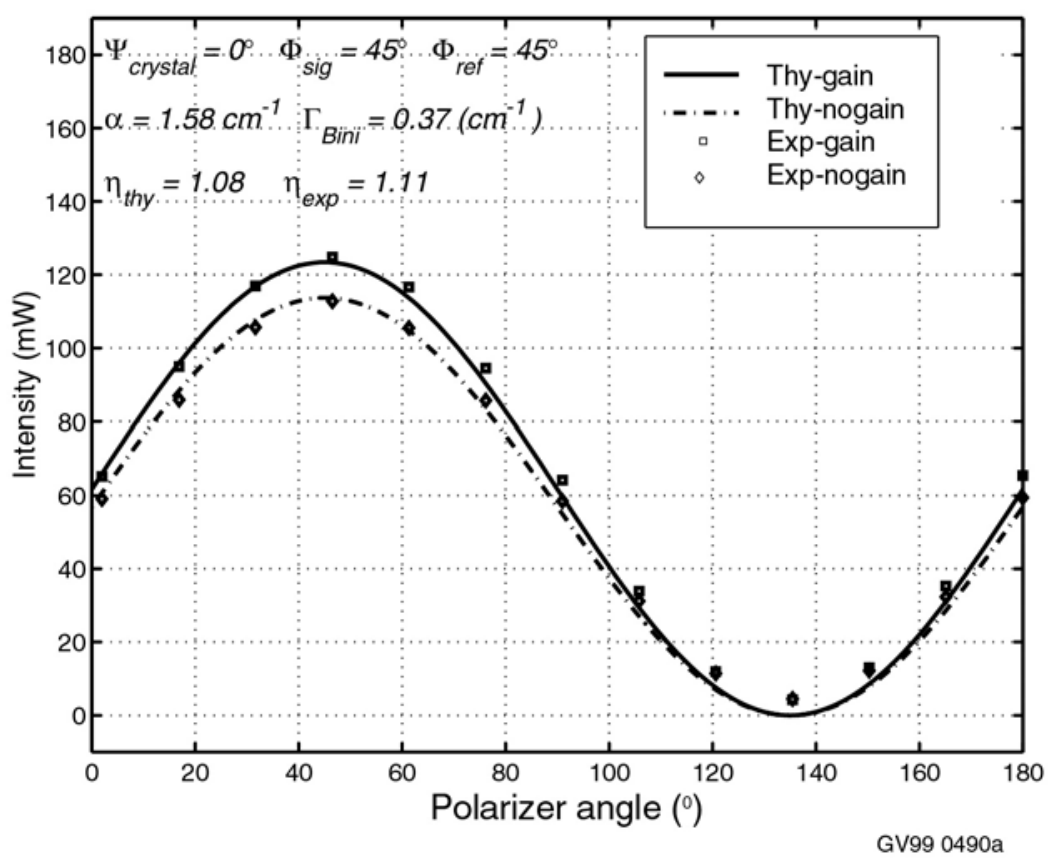

Figure 6a. Static two-wave mixing results for the 45 degree orientation in GaAs.

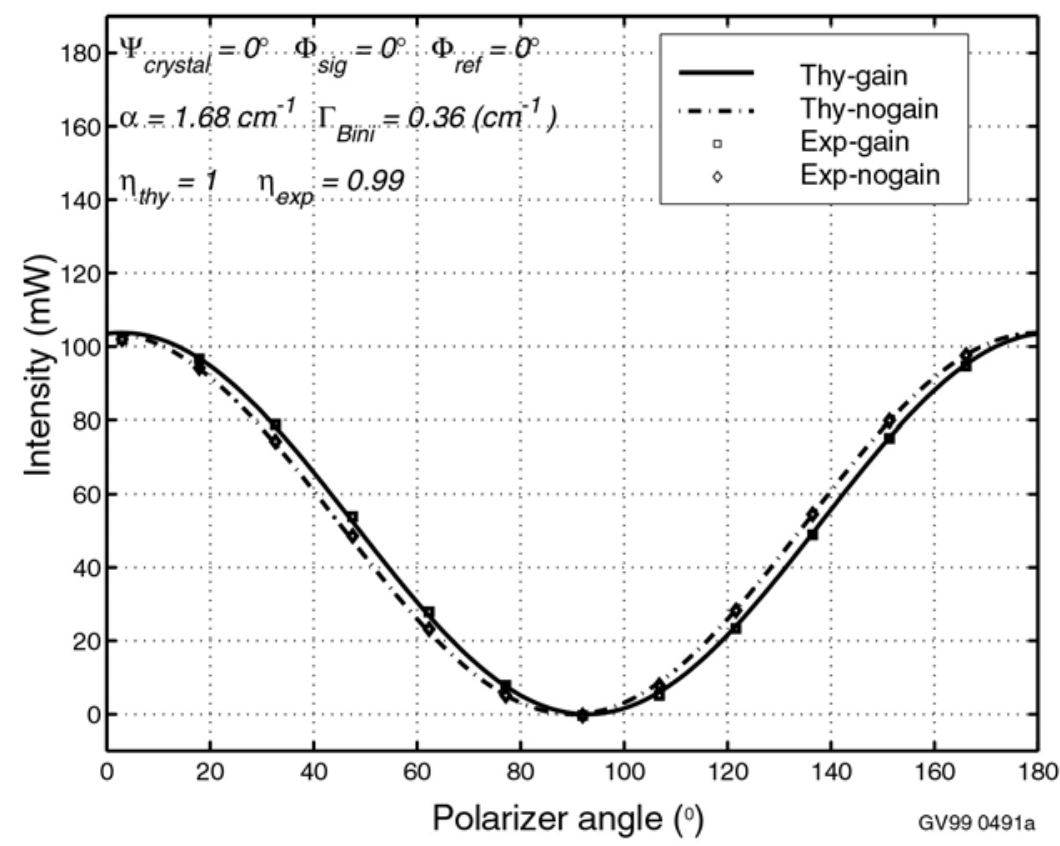

Figure 7a. Static two-wave mixing results for the 0 degree orientation in GaAs. 


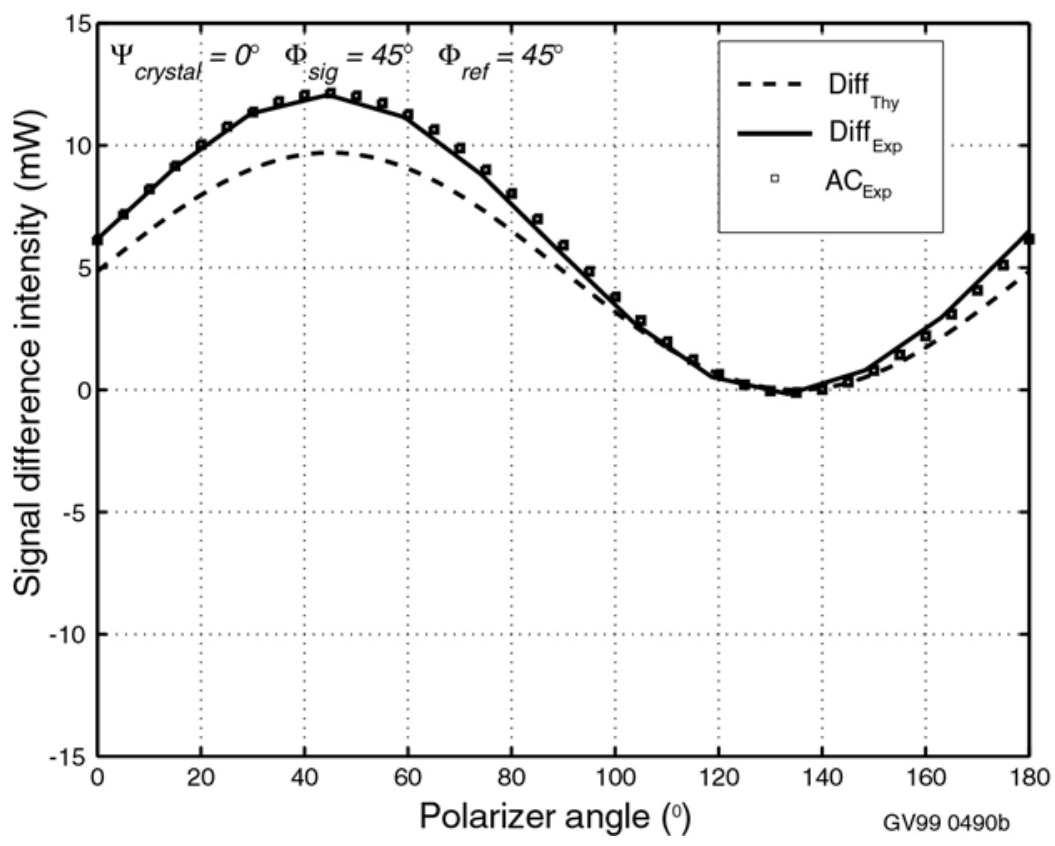

Figure 6b. Differential two-wave mixing signal for the 45 degree orientation: a configuration that exhibits energy transfer from one beam to the other.

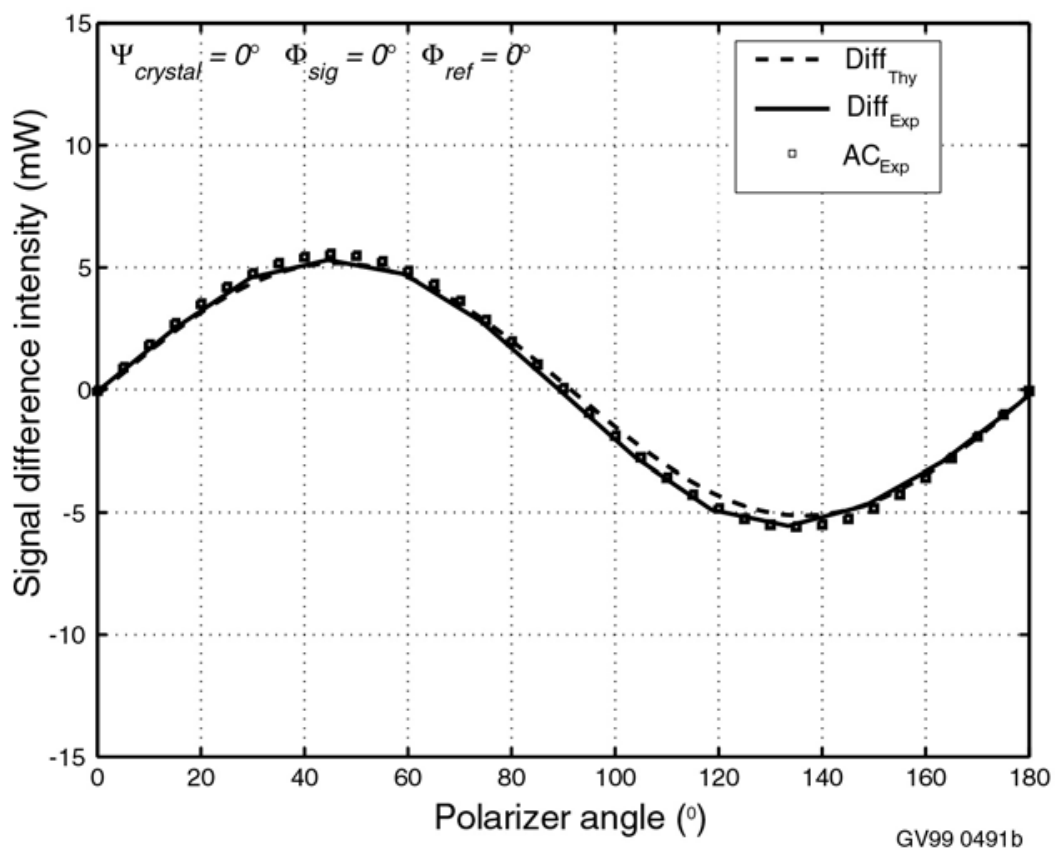

Figure 7b. Differential two-wave mixing signal for the 0 degree orientation: a configuration that exhibits only phase but no energy transfer from one beam to the other. 
compared with experimental measurements for several significant configurations of crystal orientation and input beam polarizations. Excellent agreement between numerical integration of the theoretical model and experimental measurements of the static modulation results were obtained for all the configurations tested. No extension of this model yet exists to predict the dynamic modulation results. Experimental measurement of the dynamic modulation diffraction process shows that the diffracted beam at the difference frequency can result with amplitude and polarizations determined essentially from the difference between the static beams with and without two-wave mixing. A significant differential signal was recorded for both the case when two-wave mixing gain occurs with resultant energy transfer between the beams and also when only a polarization shift results. The appearance of this latter differential signal due only to the polarization shift is a new property due to the anisotropic nature of the diffraction process in photorefractive crystals. These results have been submitted for journal publication.

\section{Phase Imaging with Photorefractive GaAs}

Dynamic holographic imaging with infrared light was accomplished using the 1,064 $\mathrm{nm}$ continuous wave laser purchased on this project. Gallium arsenide was used as its photorefractive response is much faster $(<1 \mathrm{msec})$ than bismuth silicon oxide $(>10 \mathrm{msec})$. The laser failed early in the year and took three months to fix. The system was configured with existing parts and a conventional CCD camera. Both EOM generated phase changes and resonant mode vibrations of a specular object were detected, but with low dynamic range. The system was reconfigured with components optimized for 1,064 $\mathrm{nm}$ and a better scientific CCD camera was used with 12 bit grayscale and significantly better quantum efficiency at $1,064 \mathrm{~nm}$. This greatly improved the quality of the imaged data. Spatial details of the object were more readily resolved. Some evidence of phase shifts induced in the air path, perhaps by thermal effects, were also seen.

A new electronic control scheme has been designed for scanning the interferometer system that will allow much faster measurements than the current computer-activated control scheme. This new scheme has been fabricated into a prototype electronics unit. Initial testing of the new scheme has proven successful, this method will allow the electronic lock-in detector to be always fully in lock and control the detection part of the process independent of the experimental measurement frequencies used.

\section{Materials Research Collaborations}

A collaboration was established last year on this project to investigate the capabilities of polymer materials with Professor W. E. Moerner of the Chemistry department at the University of California at San Diego (now at Stanford University) titled "The Development of Photorefractive Polymers with Improved Speed of Response." High diffraction efficiency polymers with fast response times were fabricated and sent to the INEEL for tests in the phase imaging experiments. These tests have begun and the high diffraction efficiency verified. Further tests including the modulation schemes employed in the imaging experiments are in the design stage. A final report describing work performed this past year by Professor Moerner has been sent to the INEEL and submitted for journal publication: "Trapping Studies On Photorefractive Polymers" by W. E. Moerner, D. Wright, A. Grunnet-Jepsen, and M. A. Diaz-Garcia. Collaborations are also being established with Washington State University for the investigation of semiconductor photorefractive materials.

\section{Time-Domain Nonlinear Optical Techniques for Adaptive Sensing in Environmental Monitoring}

A new research team member, Dr. Napoleon Thantu, has come to the INEEL and started work on this project. Dr. Thantu has experience in nonlinear optics and spectroscopy and will aid the photoacoustic and other spectroscopic measurement methods using adaptive interferometry. A new task 
has been started by Dr. Thantu designed to investigate the utility of the time-resolved nonlinear optical techniques using high intensity, ultrashort pulsed lasers for adaptive, environmental sensing and imaging instrumentation. A laser system capable of producing picosecond or femtosecond laser pulses and most of the necessary material components have been purchased. Ultrashort pulsed laser capability will allow unique investigations on a variety of nonlinear materials (perhaps using the optical nonlinearity of the environmentally sensitive molecular species itself) for detection, characterization, and imaging. This pulsed laser system will be a resource to other ESR projects for investigations requiring time-resolved measurements of ultrafast events or characterization of material nonlinear optical properties in condensed phases and gases. Further, this high repetition-rate laser system will benefit those measurements that require high peak, but modest average, powers and employ fast signal averaging techniques. In particular, the pump-probe nonlinear optical experimental techniques being developed offer significant utility for characterizing dynamic events, such as ionic/charge transport and metastable chemical species in natural and artificially engineered materials. The time-resolved nonlinear optical techniques also are central to nonlinear optical material investigations being conducted through a joint effort with a number of academic institutions.

The new task's immediate research goal is to establish time-domain nonlinear laser spectroscopic techniques at the INEEL and, ultimately, to develop a class of environmental sensors or sensing methodologies that are noncontacting, extremely fast, highly selective and provide imaging capability with high contrast. Nonlinear optical techniques invariably rely upon materials that exhibit a nonlinear dependence of the induced polarization on the incident light fields. The large incident light fields (although much less than typical interatomic or crystalline fields) for accessing the nonlinear optical regime (in the weak-response limit) of current experiments are attainable with commercially available ultrashort pulsed lasers. Adaptive features in this approach are manifest in the ability of the sensing process to correct for environmental perturbations. The environmental perturbations can include, for example, atmospheric turbulence, sensor motion, extraneous optical radiation fields, and electronic noise that can interfere with the sensing process and ultimately limit the minimum detectable parameters. Atmospheric turbulence can lead to a phase front distortion of the laser beams being used as an integral part of the sensor. In principle, this phase distortion can be corrected through a nonlinear optical material's intensity dependence of the index of refraction, just as deformable mirrors are employed to correct the phase-front distortion of the distant starlight by the Earth's atmosphere in astronomy. If the material nonlinear optical response is derived from subpicosecond ultrafast processes, then the environmental sensor based upon this optical nonlinearity can process the desired information on a similar time scale, ${ }^{11}$ well before the much slower environmental perturbations can adversely affect the desired information. Furthermore, nonlinear optical techniques typically use intense, large-bandwidth, pulsed laser fields that dwarf normally weak background optical radiation fields. Therefore, adaptivity to environmental conditions is inherent in ultrashort time-domain nonlinear optical measurement techniques.

\section{Analysis of Benefits of the Ultrashort Time-Domain Optical Technique}

The following analysis exhibits some of the reasoning behind the development of the ultrashort pulsed laser approach to spectroscopic measurements. In the weak field limit, the polarization induced by interaction between an intense optical field and a nonlinear optical material is given by

$$
P=\varepsilon_{0}\left(\sum_{j}^{3} \chi_{i j}^{(1)} E_{j}+\sum_{j k}^{3} \chi_{i j k}^{(2)} E_{j} E_{k}+\sum_{j k l}^{3} \chi_{i j k l}^{(3)} E_{j} E_{k} E_{1}+\ldots \ldots \ldots\right),
$$

where $\chi^{(\mathrm{n})}$ is the material susceptibility of which superscript indicates the order of the nonlinearity in the optical field, $E$, and $\varepsilon_{0}$ is the vacuum electric permittivity. In general, the susceptibility is a tensor, and, therefore, the polarization $\mathbf{P}$ does not point in the same direction as $\mathbf{E}$. The susceptibility is also, in 
general, a complex quantity of which the real part is associated with the index of refraction and the imaginary part contains information on the material absorption.

Second-harmonic generation (SHG), sum-frequency generation (SFG) and the linear Pockels effect are examples of the second-order nonlinear $\chi_{\mathrm{ijk}}^{(2)}$ processes. An example of the Pockels effect is the fielddependent index of refraction in photorefractive materials that results from a space-charge field generated by an optical excitation, and subsequent diffusion and trapping, of charge carriers that is the primary focus of the frequency-domain approach described earlier. In reference to SHG and SFG, environmentally relevant molecules adsorbed on surfaces of certain bulk materials can be spectroscopically analyzed and detected by use of these frequency conversion techniques sensitive only to the surface adsorbates, or certain interfaces, and not to the bulk, due to symmetry considerations discussed below. SHG and SFG methods employing ultrashort pulsed lasers can be used by environmental scientists to probe interfacial dynamics (air/water, soil/water, etc.). ${ }^{12}$ These techniques can selectively probe interfacial properties without interference from bulk species, whereas traditional spectroscopic techniques (absorption, fluorescence, Raman scattering) are not able to distinguish optical signals originating in the bulk and interfacial molecular species. The latter is especially true if the molecular species of interest is present in the bulk. However, the conversion efficiency for SHG and SFG scales as the peak input power implying that the use of high intensity lasers are usually needed to achieve adequate signal to noise ratio in the measurement process.

The current INEEL research and development effort on adaptive sensors relies upon the linear electro-optic or $\chi_{\mathrm{ijk}}^{(2)}$ processes of semiconductor and polymeric materials. Much of this work is associated with using photorefractive materials to image environmental information contained in the phase and amplitude characteristics of the sensing optical fields. Imaging is achieved through the refractive index grating that is created when two continuous wave (CW) optical fields overlap inside the photorefractive material. The induced refractive grating can be described by ${ }^{13}$

$$
\mathrm{n}=\mathrm{n}_{0}+\frac{\mathrm{n}_{1}}{2} \mathrm{e}^{\mathrm{i} \varphi}\left(\frac{\mathrm{E}_{1} \mathrm{E}_{2}}{\mathrm{I}_{0}}\right) \exp (-\mathrm{i} \mathbf{K} \cdot \mathbf{r})+\text { c.c. }
$$

where $n_{0}$ is the uniform index of refraction, $\mathrm{n}_{1}$ is the index of refraction induced through the photorefractive effect, $I_{0}$ is the sum of the two optical field intensities, $\varphi$ is the spatial phase shift of the index grating with respect to the intensity grating resulting from interference of the two optical beams, and $\mathbf{K}$ is the grating wave vector. Equation 2 indicates that the index modulation is dependent on the contrast of the interference fringes, or the ratio of beam intensities. In the photorefractive process, an energy transfer from one beam to the other beam occurs only if the phase shift is nonzero. A nonzero phase shift characterizes a nonlocal response.

On the other hand, Raman scattering, two-photon absorption (TPA), third-harmonic generation (THG), and the optical Kerr effect (OKE) are examples of the third-order nonlinear processes mediated by $\chi_{\mathrm{ijkl}}^{(3)}$. Due to inversion symmetry and the fact that the induced polarization changes sign upon a reversal of the electric field $\mathbf{E}$, isotropic media and crystals belonging to centrosymmetric space groups are not $\chi_{\mathrm{ijk}}^{(2)}$-active. Many molecular liquids and gases fall under this category, and their nonlinear optical response instead is of the $\chi_{\mathrm{ijkl}}^{(3)}$ origin. However, many semiconductor materials such as $\mathrm{ZnSe}, \mathrm{CdTe}$, and GaAs, to name a few, and polymers exhibit photorefractivity and the third-order optical nonlinearity simultaneously. Many specific optical nonlinearities of the targeted species are manifest only at high 
intensities and become available to be used for detection when ultrashort pulsed lasers are employed. This feature can lead to high selectivity in the optical spectroscopic process. Third-order nonlinear optical, or Kerr, media, in general, are characterized by a local response, and the induced index grating when two beams overlap in a Kerr medium is ${ }^{13}$

$$
\mathrm{n}=\mathrm{n}_{0}+\mathrm{n}_{1}^{\prime} \mathrm{I}_{0}[1+\cos (\mathbf{K} \cdot \mathbf{r})]
$$

where $I_{0}$ is the sum of the square of the field amplitudes of each beam (here, the amplitudes are taken to be equal). Note that the induced index of refraction is directly proportional to the beam intensities allowing the use of high intensity lasers to enhance the measurement signal to noise ratio and is not phase-shifted from the resultant optical interference, given by the second term in Equation 3. The thirdorder response, in general, can be regarded as being local, and, in principle, cannot lead to two-beam coupling (although four-wave mixing is possible). As mentioned above, the third-order nonlinear optical materials constitute a larger class of materials (in terms of cost and applications in the environmental imaging arena). Furthermore, the third-order nonlinear optical response is very fast and potentially can be on the same time scale (femtoseconds) as that of the optical pulses. Beam coupling efficiency can be enhanced by high peak powers that are routinely achievable with commercial ultrafast lasers while keeping the average power to levels that do not lead to deleterious thermal effects on the material.

\section{The Pump-Probe Experimental Configuration}

A pump-probe configuration is employed for the nonlinear optical experimental measurements. In a typical experiment, an intense, polarized optical pump pulse interacts with a nonlinear optical material medium. Depending on the specific nature of the experiment, this interaction can lead to excitation processes, such as electronic excitation and/or Raman scattering. The resultant material optical response manifests itself as an induced material polarization that radiates an optical field. This induced polarization, characterized by specific underlying physical processes, is interrogated by a much weaker, polarized probe pulse. Variably delaying the probe pulse with respect to the pump pulse maps out the temporal evolution of the induced polarization. This approach enables complete characterization of the temporal nature of the response, without having to rely upon the time resolution of the detector (the resolution here is limited only by the duration of the optical pulse), and the magnitude of the nonlinear optical coupling strength. Furthermore, the determination of each tensor component of $\chi_{\mathrm{ijkl}}^{(3)}$, and its real and imaginary parts, is possible. The pump and the probe beams function, respectively, as the reference and object beams when performing holographic recording and imaging. In this case, the two beams are made to overlap in space and time inside the nonlinear medium, and the resulting hologram is recorded by detecting the diffracted beam with a CCD camera. A third beam also can be used and scattered from the induced grating, as in the transient grating or optical phase conjugation geometry. In the two-beam coupling geometry, the energy loss in the probe beam is given by $\operatorname{Im}\left[\mathbf{E}_{2}^{*} \cdot \mathbf{P}^{(\mathbf{3})}\right]^{14}$ (where $\mathbf{E}_{2}$ is the probe field vector and $\mathbf{P}^{(3)}$ is the induced polarization) at each point in the third-order nonlinear optical medium. Suppose that both pump and probe are polarized in, say, the $\mathrm{x}$ direction, and the response is given by

$\chi_{\text {effective }}^{(3)}=\chi_{\mathrm{xxxx}}^{\prime}+\mathrm{i} \chi_{\mathrm{xxxx}}^{\prime}$.

The real part is proportional to the index of refraction that contributes to the phase grating and the imaginary part characterizes the nonlinear absorption that contributes to the amplitude grating. The change in the probe energy can be written as 


$$
\begin{aligned}
& \operatorname{Im}\left[\mathbf{E}_{2} \cdot \mathbf{P}^{(3)}\right]=\operatorname{Im}\left\{\int_{-\infty}^{\infty} E_{2}^{*}(t) E_{1}(t) \int_{-\infty}^{\infty} E_{1}^{*}\left(t^{\prime}\right) E_{2}\left(t^{\prime}\right) \times\left[\chi_{x x x x}^{\prime}\left(t-t^{\prime}\right)+i \chi^{\prime \prime}{ }_{x x x x}\left(t-t^{\prime}\right)\right] d t^{\prime} d t\right. \\
& \left.+\int_{-\infty}^{\infty} E_{2}^{*}(t) E_{2}(t) \int_{-\infty}^{\infty} E_{1}^{*}\left(t^{\prime}\right) E_{1}\left(t^{\prime}\right) \times\left[\chi_{x x x x}^{\prime}\left(t-t^{\prime}\right)+i \chi^{\prime}{ }_{x x x x}\left(t-t^{\prime}\right)\right] d t^{\prime} d t\right\}
\end{aligned}
$$

Since the pump and probe pulses are derived from the same optical source with the probe being delayed with respect to the pump, their electric field amplitudes can be written as $E_{1}=E(t)$ and $\mathrm{E}_{2}(\mathrm{t})=\mathrm{E}(\mathrm{t}-\tau) \mathrm{e}^{\mathrm{i} \omega \tau}$. Then Equation 11 can be separated into three components:

$\gamma(\mathrm{t})=\int_{-\infty}^{\infty}|\mathrm{E}(\mathrm{t}-\tau)|^{2}\left|\mathrm{E}\left(\mathrm{t}^{\prime}\right)\right|^{2} \chi^{\prime \prime}{ }_{\mathrm{xxxx}}\left(\mathrm{t}-\mathrm{t}^{\prime}\right) \mathrm{dt} \mathrm{t}^{\prime} \mathrm{dt}$

$\beta^{\prime}(t)=\operatorname{Im}\left\{\int_{-\infty}^{\infty} \int_{-\infty}^{\infty} E^{*}(t-\tau) E(t) E^{*}\left(t^{\prime}\right) E\left(t^{\prime}-\tau\right) \chi_{x x x x}^{\prime}\left(t-t^{\prime}\right) d t^{\prime} d t\right\}$,

and

$$
\beta^{\prime \prime}(\mathrm{t})=\operatorname{Re}\left\{\int_{-\infty}^{\infty} \int_{-\infty}^{\infty} \mathrm{E}^{*}(\mathrm{t}-\tau) \mathrm{E}(\mathrm{t}) \mathrm{E}^{*}\left(\mathrm{t}^{\prime}\right) \mathrm{E}\left(\mathrm{t}^{\prime}-\tau\right) \chi^{\prime \prime}{ }_{\mathrm{xxxx}}\left(\mathrm{t}-\mathrm{t}^{\prime}\right) \mathrm{dt} \mathrm{t}^{\prime} \mathrm{t}\right\} .
$$

Equation 12a is an incoherent term that is a convolution of intensity autocorrelation function with the material response function. This contribution persists as long as the response function does. Equations $12 \mathrm{~b}$ and $12 \mathrm{c}$, on the other hand, are coherent contributions to the diffracted beam that are nonzero only when the two beams are temporally and spatially overlapped inside the nonlinear medium so as to form a spatially modulated index grating. These contributions are pertinent to diffraction experiments that will be performed for demonstrating the significant utility of the time-domain approach for holographic imaging and recording. In the experiment, the contributions stemming from Equations $12 \mathrm{~b}$ and $12 \mathrm{c}$ can be distinguished from that of Equation 12a by modulating both pump and probe beams at certain frequencies and detecting the diffracted beam at the difference frequency. This analysis shows the various contributions to signal: two coherent contributions that are the focus when performing diffraction experiments and the other contribution that is relevant when characterizing the response in terms of its temporal nature using a two-beam setup.

\section{Experimental Setup}

A schematic of a pump-pump probe experimental configuration is given in Figure 8. Although this configuration is particularly suitable for two-beam diffraction and pump-probe OKE experiments, with slight modifications this setup can accommodate time-resolved transient grating and optical phase conjugation experimental configurations. Figure 9 is an illustration of a pulse sequence that would be employed in a diffraction experiment to read out the volume hologram recorded by a third-order nonlinear optical medium.

Initial experiments will employ $\mathrm{CS}_{2}$ and other molecular liquids such as benzene as the nonlinear medium. The experiments will be performed to explore optimal nonlinear optical experimental configuration (two beams as opposed to three beams) for holographic reading and recording. $\mathrm{CS}_{2}$ exhibits sufficiently large third-order optical nonlinearity, ${ }^{15}$ is readily available, and is often used as a calibration standard in nonlinear optical experiments designed to measure $\chi_{\mathrm{ijkl}}^{(3)}$. Figure 10 shows a nonlinear optical response of $\mathrm{CS}_{2}$ as a function of probe pulse delay with respect to the pump pulse. The experimental configuration used to measure this response is similar to the one depicted in Figure 8. Figure 10 shows the instantaneous electronic response at the zero probe delay followed by the inertial, nuclear response with its peak near $\sim 250 \mathrm{fs}$. The inertial, nuclear response is essentially nonlocal and permits transfer of energy in a two-beam coupling experiment. 


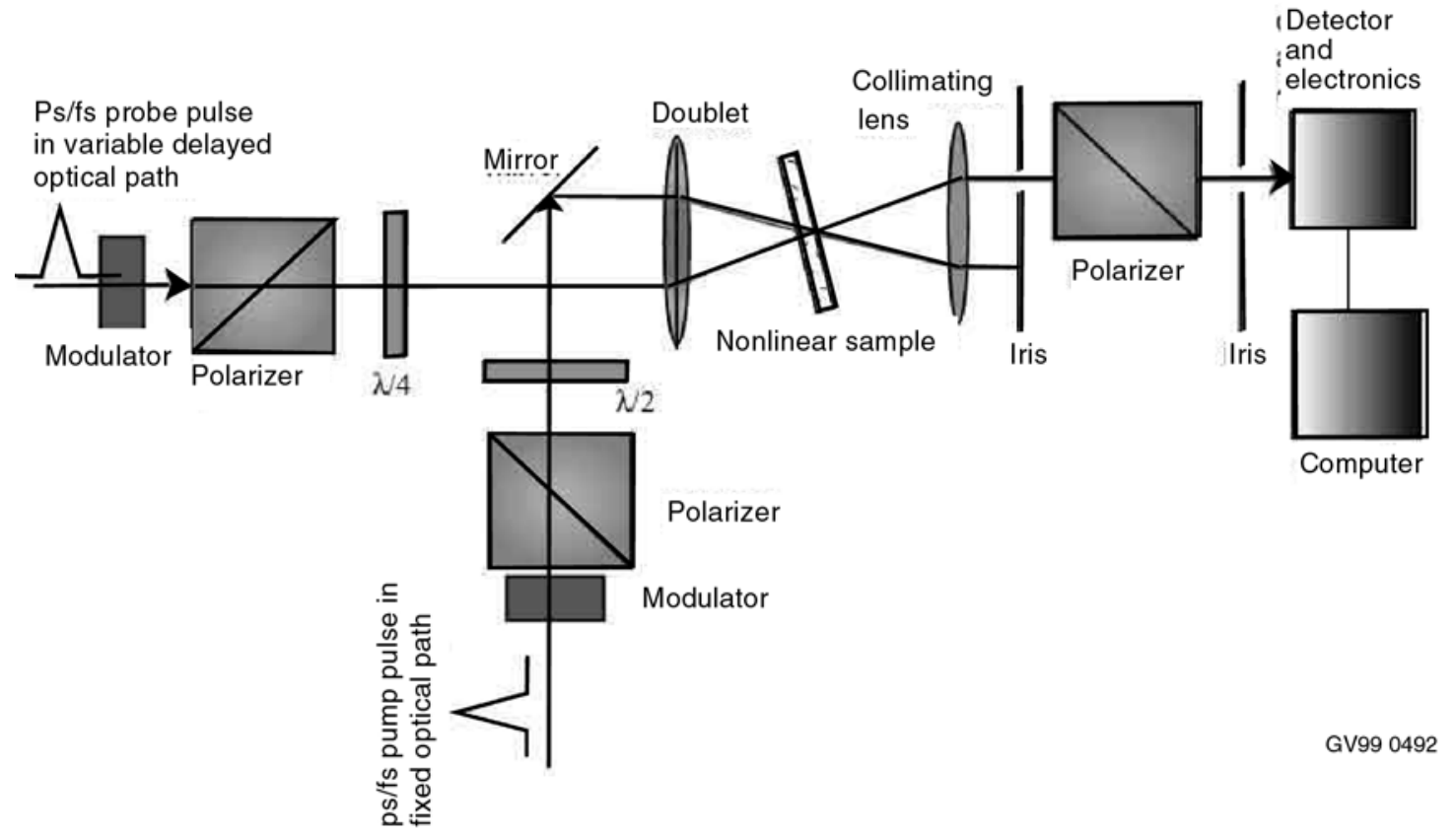

Figure 8. Schematic of the time-domain ultrashort pulse pump-probe experimental configuration.

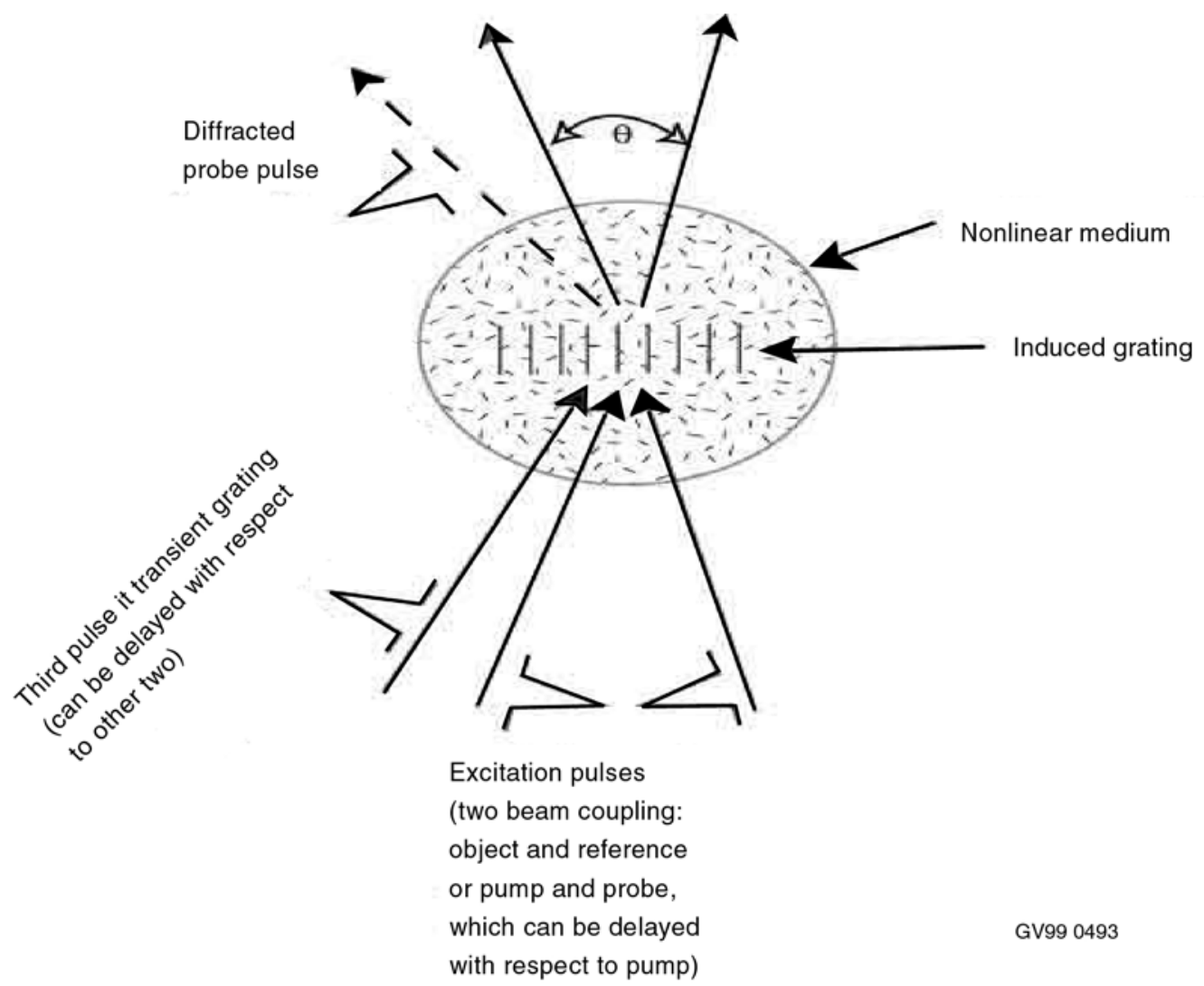

Figure 9. Pulse sequence in two-and three-beam diffraction experiments. 


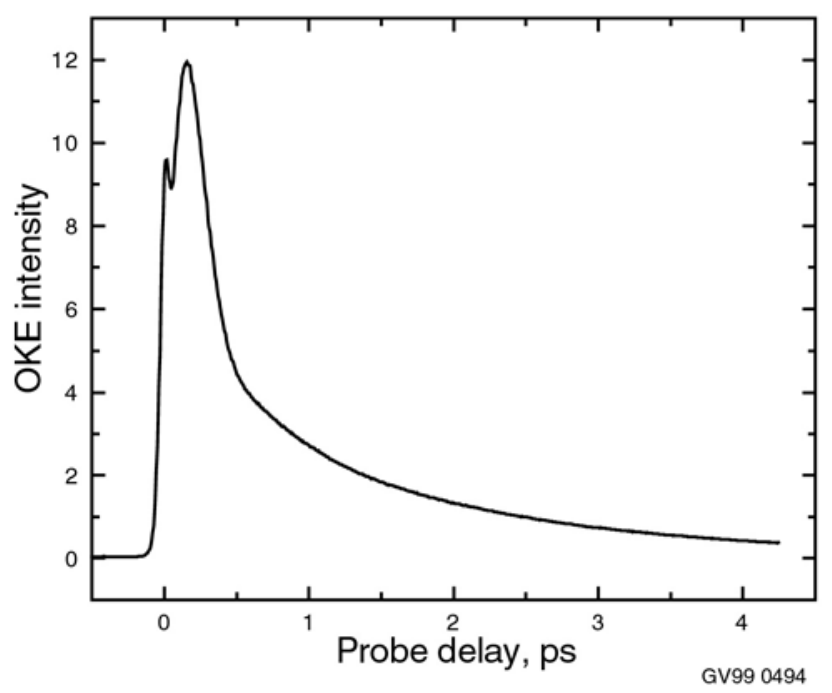

Figure 10. Real part of the third-order nonlinear response of $\mathrm{CS}_{2}$.

\section{ACCOMPLISHMENTS}

This project successfully performed the first proof of principle experimental "containerless" photoacoustic measurements along laser beam of less than one meter length and confirmed that the results were as expected from theoretical predictions. The minimum detectable trace gas concentration was determined based on the absorption line cross-section employed and the detection noise level. Methods and materials were identified to implement this spectroscopic method with ppm detectability at the shot noise level. Future work will demonstrate the performance of this technology for trace gas detection of environmentally sensitive molecules and determine the imaging limitations.

A complete experimental and theoretical analysis has been made of the anisotropic diffraction properties of an important candidate photorefractive material, GaAs. These results have been shown to agree with predictions for the static two-wave mixing case and to produce a differential signal for dynamic phase modulation even when no two-wave mixing gain is present. This is a new resultant property of anisotropic diffraction and the subject of a journal paper submission. Imaging of phase modulations has been accomplished with GaAs beginning the process of extending the photoacoustic measurements to an imaging mode.

A new researcher experienced in ultrafast laser techniques has joined this project and has established an ultrafast laser capability at the INEEL with purchase of a new femtosecond/picosecond laser system. A new task has begun to the use of ultrafast optical techniques for developing new environmental monitoring technology through highly selective optical nonlinearities that exist in selected molecular species.

\section{REFERENCES}

1. HITRAN 1996, CD-ROM available from ONTAR Corp. North Andover, MA, 01845-2000.

2. Electro-optic and Photorefractive Materials, P. Günter, editor, Springer-Verlag, New York, 1987.

3. S. I. Stepanov, International Trends in Optics, Academic Press, New York, 1991, Ch. 9. 
4. P. Yeh, Introduction to Photorefractive Nonlinear Optics, John Wiley, New York, 1993.

5. Selected Papers on Photorefractive Materials, F. M. Davidson, editor, SPIE Milestone Series Volume MS 86, SPIE Bellingham, WA, 1994.

6. G. Hamel de Monchenault and J. P. Huignard, "Two-wave mixing with time-modulated signal in $\mathrm{Bi}_{12} \mathrm{SiO}_{20}$ theory and application to homodyne wave-front detection," J. Appl. Phys. 63 (3), pp. 624-627, 1988.

7. R. K. Ing and J.-P. Monchalin, Appl. Phys., Lett. 59, 3,233, 1991.

8. K. L. Telschow, V. A. Deason, R. S. Schley and S. M. Watson, "Direct Imaging of Lamb Waves in Plates using Photorefractive Dynamic Holography," J. Acoust. Soc. Am., in press.

9. R. C. Troth and J.C. Dainty, "Holographic interferometry using anisotropic self-diffraction in $\mathrm{Bi}_{12} \mathrm{SiO}_{20}$, , Opt. Lett., 16 (1), 53, 1991.

10. P. Yeh, "Photorefractive two-beam coupling in cubic crystals," J. Opt. Soc. Am B 4(9), pp. 1,3821,386, 1987.

11. N. Thantu, J. S. Melinger, D. McMorrow and B. L. Justus, "Ultrafast nonlinear optical response of $\mathrm{CuCl}$ nanocrystallite-doped glass in the transparent region," Nonlinear Optics: Principles, Materials, Phenomena and Devices, in press.

12. K. B. Eisenthal, "Liquid interfaces probed by second-harmonic and sum-frequency spectroscopy," Chem. Rev., 96, 1,343, 1996.

13. P. Yeh, “Two-wave mixing in nonlinear media,” IEEE J. Quant. Electronics 25, 484 (1989).

14. S. L. Palfrey ad T. F. Heinz, "Coherent interactions in pump-probe absorption measurements: the effect of phase gratings," J. Opt. Soc. Am. B 2, 674, 1985.

15. W. T. Lotshaw, D. McMorrow, N. Thantu, J. S. Melinger and R. Kitchenham, "Intermolecular vibrational coherence in molecular liquids," J. Raman Spectrosc., 26, 571, 1995.

\section{APPENDIX}

\section{Peer Reviewed Publications}

1. K. L. Telschow, V. A. Deason, R. S. Schley and S. M. Watson, "Imaging of Lamb Waves in Plates for Quantitative Determination of Anisotropy using Photorefractive Dynamic Holography," Reviews of Progress in Quantitative NDE, Vol. 18A, edited by D. O. Thompson and D. E. Chimenti, Plenum Press, New York, 1998, pp. 999-1,005.

2. D. Wright, A. Grunnet-Jepsen, M. A. Diaz-Garcia, J. D. Casperson, B. Smith, M. S. Bratcher, M. S. DeClue, J. S. Siegel, W. E. Moerner, and R. J. Twieg, "Trapping Studies on Photorefractive Polymers," Proc. Soc. Photo-Opt. Instrum. Engr, 3,471, 60-71, 1998.

3. R. S. Schley, K. L. Telschow and J. Holland, "Static and Dynamic Two-Wave Mixing in GaAs," submitted for publication in Applied Optics. 


\title{
Integrated Instruments for In Situ Chemical Measurements
}

\author{
P. M. Castle, J. M. Slater, J. K. Partin, M. L. Stone, F. F. Roberto, \\ V. A. Deason, W. F. Bauer, D. A. Atkinson
}

SUMMARY

The Integrated Instruments for In Situ Chemical Measurements tasks are carrying out research to address EM's field chemical analysis and long-term monitoring needs associated with long-term stewardship. In spite of many advances in chemical analysis instrumentation, there continue to be needs associated with characterization of remediation sites as well as long-term chemical monitoring at waste disposal facilities or after closure of sites. This task is directed at several of the important issues associated with environmental characterization and monitoring, i.e., measurement sensitivity, selectivity, sampling, and portability. Six tasks address various aspects of these issues. Two tasks-detection of low-energy ions for ion-mobility spectroscopy (IMS) and laser-enhanced IMS instrumentation-address the enhancement of sensitivity. One task-microarray biosensor and microdevices-addresses selectivity. One task-membrane development/characterization utilizing MIMS — deals with sampling issues. Portability is addressed by the last two tasks: Microsampler/Surface Acoustic Wave (SAW) detector and a two-dimensional spectral imaging spectrometer using digital micromirrorarray and a single photomultiplier tube.

Detection of low energy ions for IMS and laser-enhanced IMS Instrumentation take advantage of the Idaho National Engineering and Environmental Laboratory's (INEEL's) capabilities in IMS. Research on these topics is intended to enhance both the sensitivity and breadth of applicability of IMS in the environmental arena. Progress in these two tasks will enable the fabrication of smaller, more sensitive, field portable instruments. The microarray biosensor and microdevices task is investigating the use of genetically engineered biosystems for selective probes of environmental contaminants. The membrane development/characterization utilizing MIMS task addresses both sampling and selectivity issues, in that its objective is to identify membranes that selectively pass individual compounds or classes of compounds for subsequent detection by any of several methods. This task is related to the Microsampler/Surface Acoustic Wave (SAW) detector task that uses information from the membrane studies to design a membrane sampler for sample introduction to a coated SAW array that will lead to a very compact field analytical system. Anticipated work for the next year will investigate membrane sampling interfaces for the IMS. The two-dimensional spectral imaging spectrometer using digital micromirrorarray and a single photomultiplier tube task is devoted to demonstration of a very compact spectral imaging instrument for field application.

\section{PROJECT DESCRIPTION}

\section{Detection of Low-Energy lons for Ion-Mobility Spectroscopy}

One of the objectives of this program is to enhance the sensitivity (lower the detection limits) of instrumentation that can be used for environmental measurements. The standard detection method utilized for detecting analyte ions in IMS is a Faraday cup. A Faraday cup has no ion amplification capabilities such as an electron multiplier, Channeltron, or microchannel plate. These ion amplification devices can provide amplification (gain) of orders of magnitude but are not useful at atmospheric pressure, the regime in which IMS operates. This task is exploring a new technique for ion amplification 
that operates at atmospheric pressure. Success in this research will lead to instrumentation that will have detection limits for important environmental contaminants that are lower than any other technique.

We have initiated an effort to develop a high-bandwidth, high-sensitivity ion detection system for use as a detector for IMS. The overall goal is to replace today's Faraday cup detection with a high-gain, high-bandwidth detector that would provide significant improvement over the current state-of-art in IMS performance. Existing ion detector technology (excluding Faraday cups), both gaseous and solid state, is oriented to detection of highly energetic ions that can generate multiple ion-electron (or electron-hole) pairs in the detection media. The free charge thus generated is detected as an electric current, or an avalanche multiplication step may be used for higher gain. In the latter case, gains of $10^{4}$ are common. The multiplication is necessary simply because electronic noise in the preamplifier limits pulse detection to roughly $10^{2}$ or $10^{3}$ charged particles as a minimum.

The challenge in the IMS case is to generate gain starting from a nearly thermal positive or negative ion. Our strategy is to employ atmospheric pressure gas as a detector component and to use this gas under conditions similar to those found in the cathode fall region of a glow discharge. In a conventional glow discharge, the cathode fall occupies a thin gas layer directly above the cathode. This is the only area of the discharge in which ions produce additional charged particles. At all other locations, electron impact on neutrals is the dominant method of charge carrier production. The fall region has much higher E-field than elsewhere in the discharge because of the fall's high electrical impedance, and ions entering the fall region from the anode side are accelerated into the cathode. Some ions produce secondary electron emission from the cathode; these electrons accelerate toward the anode and produce additional positive ions. In a self-sustained discharge, exactly one new positive ion is produced in this way for each ion entering the fall.

In the case of developing an ion detector we do not wish to provide a self-sustaining discharge, but rather to generate multiple charge carriers each time a single positive ion enters our fall-like region. This opportunity to provide charge gain in this manner can be understood as follows: consider a gaseous volume stressed by an E-field nearly identical to that of the cathode fall, but that no discharge is occurring. Suppose further that a positive ion enters this region, but the E-field is such that only 0.99 of an ion is produced from the initial ion on average (i.e., this discharge does not self-sustain). Then that 0.99 ion produces about 0.98 ions in turn, that produce about 0.97 ions, etc. The current gain of this system would be on the order of 100; i.e., a single ion inputted would cause roughly 100 ions to be generated before the activity ceases. In a somewhat analogous situation, gas proportional counters can produce gains of about $10 .^{4}$ This is one gain mechanism we will be exploring. These comments are only for positive ions entering the fall region. The concept for negative ion detection is less developed.

The second gain mechanism utilizes a resistively ballasted discharge. The small capacitance between the anode and cathode provides an energy reservoir that can drive a discharge that will quickly terminate, then recharge and is ready for the next ion. The gain per ion can be exceeding high, on the order of $10^{7}$ for each pf of capacitance but linearity is lost if multiple ions arrive during a single discharge cycle.

Further insight as to how an ion may trigger a discharge is to understand how ions produce charge carries in a typical glow discharge. Figure 1 shows typical electrical characteristics of a glow discharge, where $\mathrm{E}$ is electric field, $\mathrm{V}$ is potential, $\mathrm{j}$ is current density, and $\rho$ is charge density. 


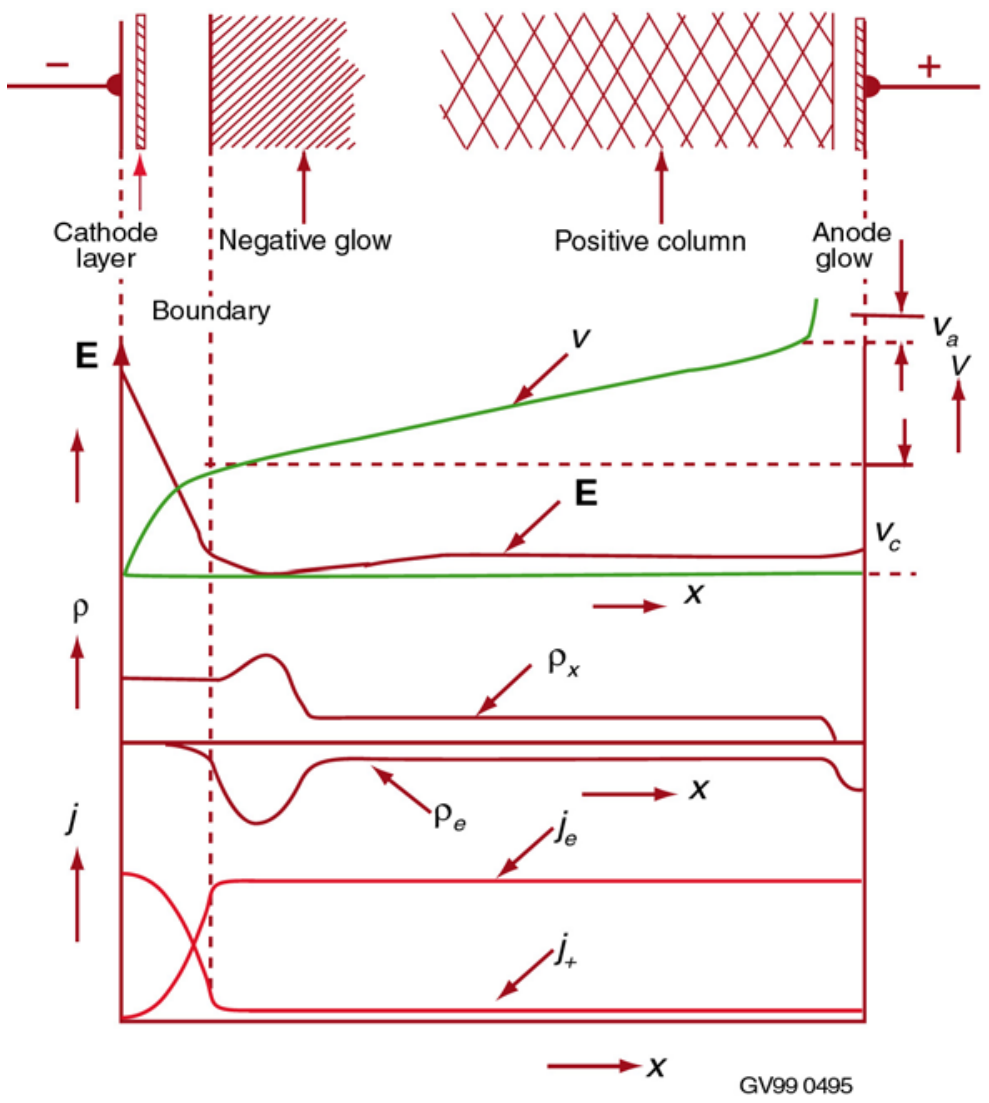

Figure 1. Electrical properties of a typical glow discharge. ${ }^{1}$

For this discussion, the interesting part of the discharge is the cathode side (left edge). Since electrons are swept toward the anode (right edge), an area of low electron density exists at the cathode end of the discharge. This is therefore a high impedance region and it carries a substantial potential drop. This drop accelerates ions into the cathode to produce secondary electrons. At equilibrium, the drop satisfies the relation: ${ }^{2}$

$$
\left(1+\frac{1}{\gamma}=\mathrm{e}^{\int_{0}^{\mathrm{d} \alpha \mathrm{d} x}}\right)
$$

where $\gamma$ is the average number of electrons emitted from the cathode surface for each bombarding positive ion, $d$ is the thickness of the cathode fall region, and $\alpha$ represents the number of ionizing collisions by electron per unit path length. We note that $\alpha$ is generally known as the first Townsend coefficient. The thickness of the cathode fall region is inversely proportional to the gas pressure, i.e., the product pd, where $\mathrm{p}$ is the gas pressure tends to be constant over large pressure ranges.

Now consider a positive ion migrating into an E-field stressed gas. The large mass of the ion guarantees that it will lose momentum in collisions with neutrals. Thus it remains nearly thermal even in the presence of the E-field and cannot cause further ionization. Note that this is markedly different from the case of an electron introduced to a field-stressed gas. The electron has low probability for momentum loss to neutrals and under realistic fields easily gains enough energy to cause secondary ionization. Radiation measurement instruments, e.g., gas proportional counters and Geiger-Mueller counters, rely on this principle. 
While the low-energy ion is not detectable in the bulk gas for lack of a multiplication mechanism, the glow discharge demonstrates that there is a mechanism for ions to lead to charge multiplication, namely that found in the cathode fall region. If one can reproduce similar conditions in a fully neutral gas, the introduction of a single ion may cause a discharge to initiate. Detection of the discharge then constitutes detection of the ion. That is the underlying basis of the detector development of this program.

The basic concept for the case of positive ions in an IMS-like geometry is shown in Figure 2. Here ions flow at atmospheric pressure toward the detection plane, the front surface of which is a single conducting anode plate with a large number of holes. Inside each hole is a pointed cathode, as shown in the breakout drawing. Ions are attracted to the cathode pins, initiating a discharge between the cathode and anode that is detected as a voltage spike on the low impedance (z) output resistor. This is inherently a high speed device because the plasma activity is fast at high pressures and the impedance of this geometry (essentially two closely spaced conducting planes) can be quite low. The individual discharges, or pixels, may not operate in a linear mode, but some degree of linearity (i.e., the ability to distinguish a high from low ion beam currents) may result simply by noting the number of pixels triggered and the trigger rate.

The specific cathode-anode geometry will be quite important. The cathode in the conceptual design of Figure 2 is pointed for two reasons. First, the field intensification around the tip produces a confined region of high field similar to that of a glow-discharge cathode-fall region. The confinement of this region is important to avoid self-breakdown, as is well known from the Paschen breakdown relationship, which shows that high E-field values must be confined to small distances to avoid breakdown. The second reason for the narrow tip concerns the path of electrons leaving the cathode. Neglecting collisions, ions striking the cathode have moved along field lines that come from the ion source. Also neglecting collisions, electrons leaving the cathode from ion bombardment will retrace these field lines, thus missing the anode. Now consider the field lines at the cathode tip. Because the tip is small, field lines arriving from the ion source nearly coalesce with those arriving from the anode. Thus collisions will provide that some of the secondary electrons and those from the avalanche can drive current in the cathode-anode circuit and produce an output pulse. If even one electron establishes an avalanche leading toward the anode, a detectable signal will result. We are working with calculation of static fields in neutral gas to better understand the details of the cathode-anode geometry.

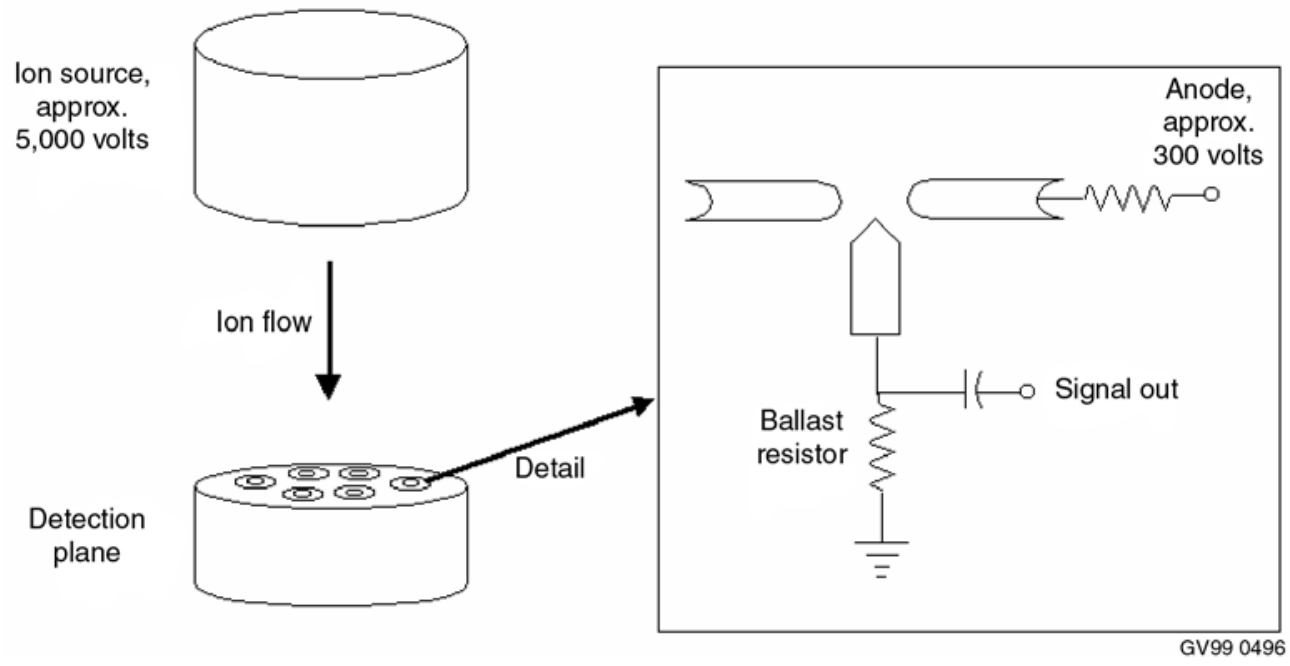

Figure 2. Conceptual diagram of IMS system with high-gain and high-bandwidth ion detection. 


\section{Experimental and Results}

The program this year had two elements: first, development of the concept, which is outlined above; second, we are unaware of any literature regarding ion-triggered discharges, so we have worked to demonstrate that ion triggering is possible.

The laboratory work was directed entirely to demonstrating that a discharge could be triggered by near-thermal ions. To do this we constructed the discharge circuit depicted in Figure 3. The circuit is in a vacuum housing maintained at 2 torr pressure with a gas composition identical to that of the ambient atmosphere. Figure 4 is a photograph of the apparatus, with the ion gun on the left and the detector on the right.

The top part of the circuit (Figure 3) is a free-running glow discharge that acts as an ion source. The detector consists of the anode-cathode wire pair at the bottom. These wires protrude roughly $5 \mathrm{~mm}$ from an insulating plate and are wired as indicated. Both electrodes are copper, with the cathode 75 microns diameter and the anode $0.7 \mathrm{~mm}$ diameter. The $+550 \mathrm{~V}$ on the anode was varied for some data as discussed below. The general methodology is to maintain all potentials of the ion gun positive relative to all potentials of the detector, so as to ensure that only positive ions can migrate to the detector end. The initial data was taken with the potentials as shown in Figure 3, which clearly showed rapid triggering of a discharge in the anode-cathode circuit when the $+1,370 \mathrm{~V}$ was applied to the ion gun and no triggering when that electrode was allowed to float. These individual events produce voltage spikes as shown in Figure 5. Since under these conditions (with $+1,370 \mathrm{~V}$ applied) only ions will migrate toward the detector, the result shows that near-thermal ions have triggered a discharge and were thus detected.
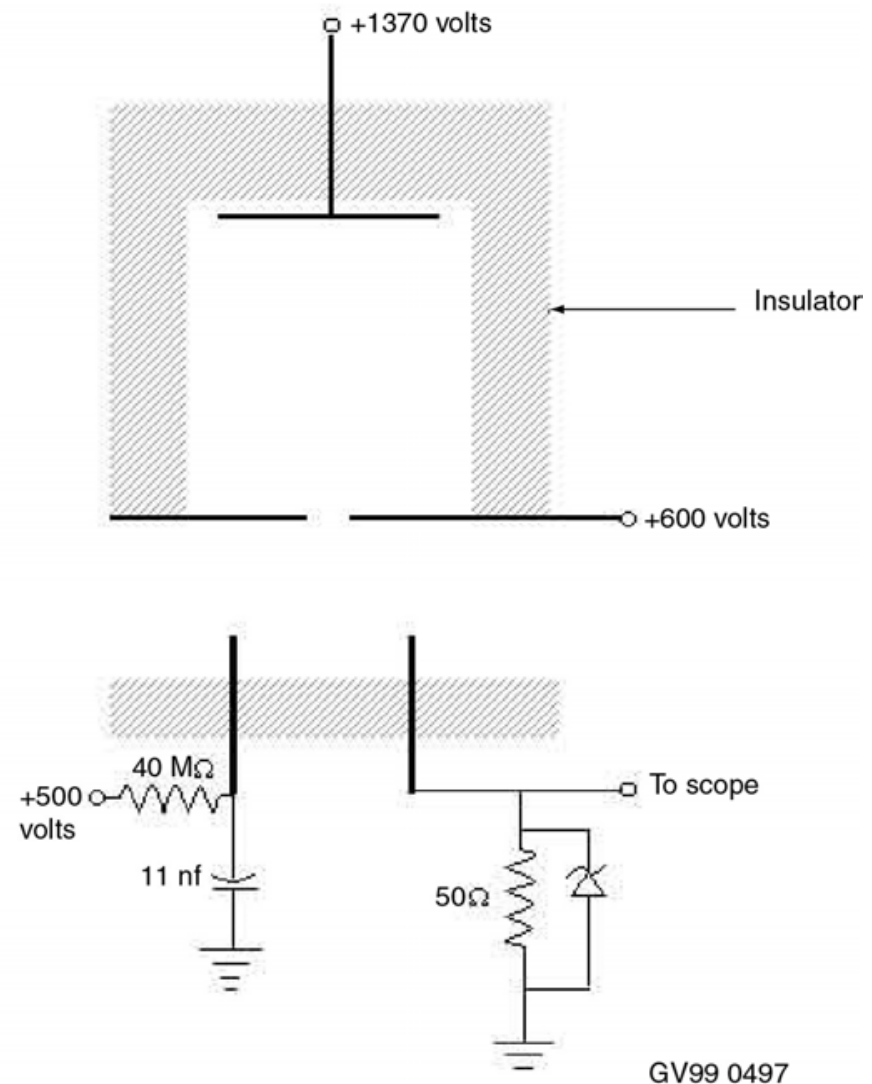

Figure 3. Discharge circuit used for ion-triggering demonstration. 


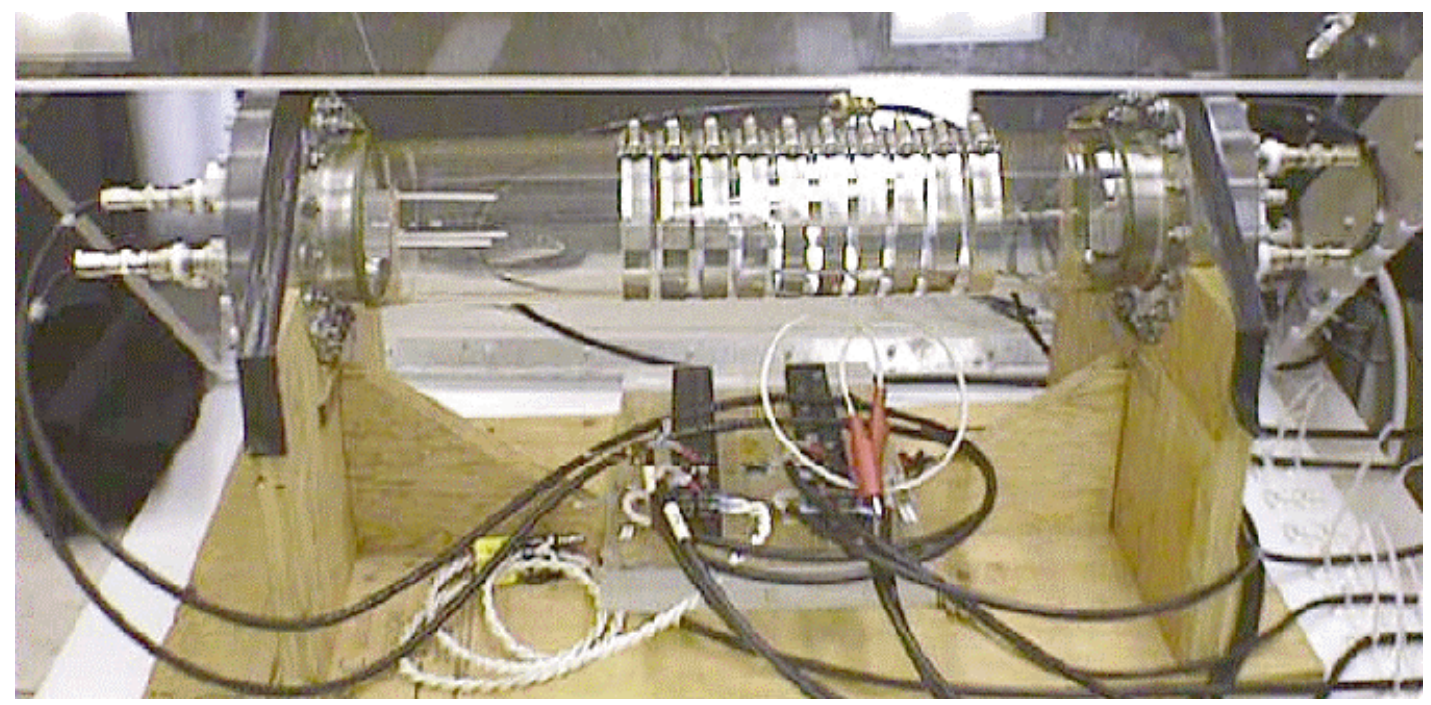

Figure 4. Photograph of vacuum housing with ion gun at left and detector at right. Metal rings over the center of the vacuum housing are not used.

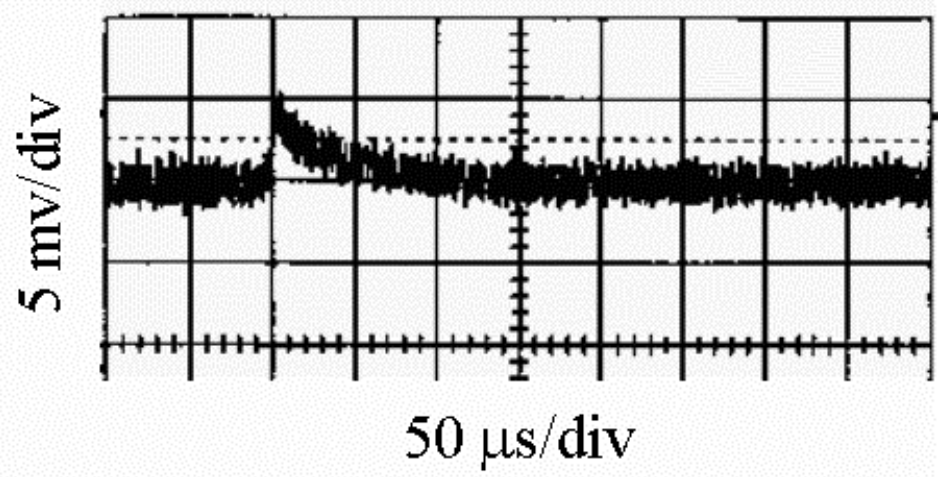

Figure 5. Discharge pulse from detector for a single event.

The anode voltage $+\mathrm{V}$ (Figure 3 ) could be increased to the point where a discharge between the detector anode and cathode is triggered, either with the presence of ions (from the ion source) or without. Other data taken this year consisted of examining the triggering of the detector discharge with and without the presence of ions. Figure 6 shows two such traces, both of which are made with the ion source powered not as indicated in the Figure 3, but with the topmost electrode at $+600 \mathrm{~V}$ and the lower ion source electrode at $+100 \mathrm{~V}$.

Both traces indicate the relative frequency of triggering of the detector discharge, i.e., current flowing between the discharge cathode and discharge anode. This frequency was measured by means of a counter that recorded the number of events (Figure 5) during a set time period. The left-hand trace is made under the condition of no power on the ion source. It shows a self-breakdown beginning at $800 \mathrm{~V}$ and increasing in frequency as the voltage increases. Conditions are identical for the right-hand trace except that the ion source is powered and ions are present in the detector area. It shows that at $+750 \mathrm{~V}$ the discharge is triggered, which is presumably due to ions. For this particular data set, there is the possibility that the triggering is actually due to electrons because one of the detector electrodes is at a more positive potential than the ion source electrodes. However, the data referred to further above does not suffer from this problem and definitively shows that ions triggered the detector. 

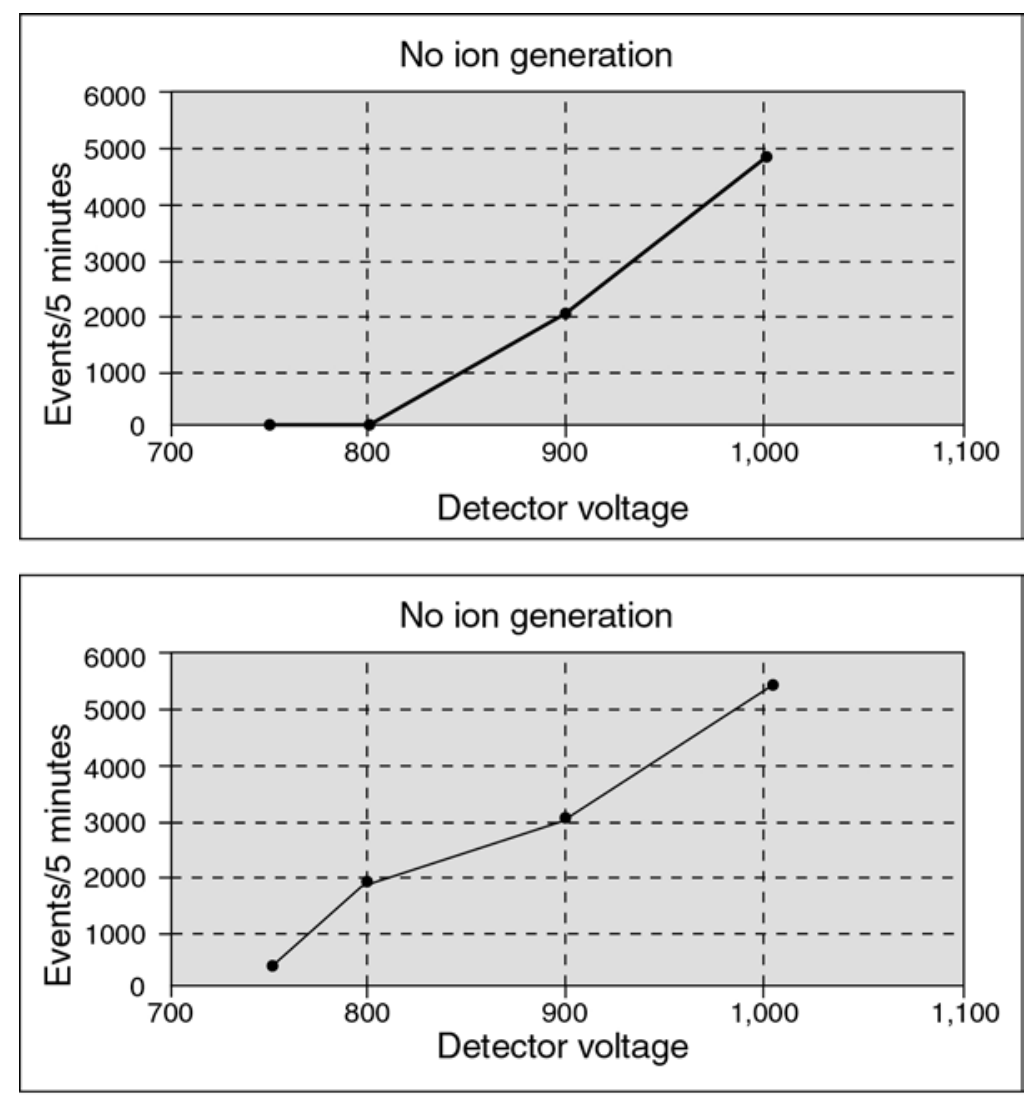

GV99 0500

Figure 6. Frequency of discharge triggering with and without ions present.

All of the data was hampered by variation of signal over time periods of several minutes. It appears at this time that charge buildup on the insulator surfaces either near the electrodes or on the glass vacuum vessel have caused this problem. If so, this is correctable with shields over the insulators. It will be examined next year.

\section{The Path Forward}

Having demonstrated ion triggering, there are several basic development steps required to move toward a viable detector. First, a workable geometry must be demonstrated, even at the low few-torr pressures of these experiments. The current geometry demonstrated only ion triggering, and further testing will likely show that it is inefficient. As was mentioned above, the shape of the electrodes will determine how well ions can find the cathode and how well secondary electrons leaving the cathode can develop current in the cathode-anode circuit, which leads to detection. When the geometry is optimized, there is good chance it can be made to work at much higher pressures. Pressure scaling laws for the cathode fall of a glow discharge show that the thickness falls as $1 / \mathrm{P}$, where $\mathrm{P}$ is pressure.

A further issue, then, is to demonstrate pressure scaling. The cathode fall thickness is roughly 3 $\mathrm{mm}$ at 2 torr, which sets the geometric scale size of the anode-cathode geometry. Moving to 1 atmosphere shrinks the scale size to more nearly 3 microns. While this may at first appear to be unworkable, it is useful to realize that field-emission displays for computers employ a similar geometry to that of Figure 2, and area already working with scale sizes of microns and smaller. They are made with lithographic techniques. Thus one should be optimistic regarding manufacturability. It is worth noting field emission may limit the high-pressure scaling of the ion detection technique described here. 
A third issue is the isolation of the individual discharges, since the pressure scaling dictates a large number of separate, but closely packed, detection pixels as indicated very roughly in Figure 2. Isolation refers to the issue of triggering one discharge without causing a spontaneous triggering of neighboring discharges. One potential cause of cross-talk is ultraviolet (UV) photons. If this problem develops, it might be solved through geometry adjustment or even addition of an organic vapor with UV absorption properties. The latter strategy has been used successfully in gas proportional counters.

The plan for next year addresses these items. The geometry scaling will proceed at several torr pressures and be guided by electrostatic calculations. This is the first priority. Then pressure scaling will move first to roughly the 25 torr region, where conventional wire sizes of several thousandths of an inch will just be sufficient for the cathode. This is the second priority. For these and higher pressures a radioactive ion source will be used, since the glow discharge source becomes increasingly unstable with pressure. An attempt was made this year to use a radioactive Ni source, but the purchased source did not meet DOE radiation standards for leakage. For later program steps to higher pressures, lithographic techniques will be required for fabrication of single pixels and arrays. Some preliminary work on manufacturability is planned at the paper-study level.

\section{Laser-Enhanced IMS Instrumentation}

The radioactive nickel source, which is used to produce the ion chemistry that creates the analyte ions, has several drawbacks. Because the gas phase ion chemistry depends on the transfer of a proton from an ionized water cluster, the analyte molecules must have a higher proton affinity than the water cluster. Not all molecules have high proton affinities. This limits the range of molecules that can be analyzed by this technique. Additionally, various competing reactions, such as recombination, in the gas phase ion chemistry limit the concentration of protons that can be produced. This limits the upper end of the dynamic range of the technique.

Using a laser to photoionize analyte molecules offers an expansion of the types of molecules that can be analyzed by IMS. The ionization process would no longer depend on proton affinities. There are two principal processes by which photoionization can occur. Simple single photon ionization requires a laser with a short enough wavelength (high enough energy photon) to exceed the ionization energy of the molecule. Since the shortest wavelength lasers in common use (excimers) only produce $193 \mathrm{~nm}$ photons, this places a limit on the analytes that could be addressed. Multiphoton ionization, however, can overcome this difficulty by supplying more than one energetic photon to a molecule in order to exceed its ionization energy. In addition to producing short wavelength photons, Excimer lasers can produce high peak power density pulses $\left(100 \mathrm{~s}\right.$ of $\left.\mathrm{MW} / \mathrm{cm}^{2}\right)$. These power densities are sufficient to ionize many different molecules. High power densities can produce large concentrations of ions up to plasma densities.

An additional benefit that may be gained from the use of an excimer laser is enhanced instrumental resolution. The current gating method for allowing ions into the drift region is an electronic gate with a fairly slow rise time. Since the ions will be created only by the photoionizing pulse, there will be no need to gate the ions into the drift region as is needed with the nickel source. The photoionization pulse has a time pulse width of 10-20 ns, much narrower than the electronic gate. Greater resolution will lead to the possibility of creating a more compact instrument. Eliminating the fragile electronic gate will lead to a more rugged, portable instrument.

An old Questek Model 2620 Excimer laser system was refurbished for use in these experiments. The laser system emits 500 millijoules of energy in a 20 ns pulse with a repetition rate of $25 \mathrm{~Hz}$ at $248 \mathrm{~nm}$ using a krypton fluoride gas mixture. The radiation is emitted into a $10 \times 20$-millimeter beam. The irradiance required for desorption is on the order of $10^{6}-10^{7} \mathrm{~W} / \mathrm{cm}^{2}$, while ionization generally requires 
on the order of $10^{8}-10^{9} \mathrm{~W} / \mathrm{cm}^{2}$. The peak energies of the unfocused excimer pulses are approximately $25 \mathrm{MW}$ and can be readily focused to the energy densities required for ionization.

Mechanical and electrical interfacing issues between the laser and IMS instrument have been evaluated and the necessary systems designed and fabricated. In addition, an interlocked enclosure has been built to address the safety issues of shielding personnel from ultraviolet irradiation from the laser or accidental contact with high voltage from the IMS. In addition, a quartz tube was specified and procured to replace the glass tube in the existing instrument, allowing the 248-nanometer radiation to be focused into the device. This tube, which has recently been delivered, is currently being installed into IMS instrument and testing is expected to begin in the next few weeks.

\section{Microarray Biosensor and Microdevices}

Environmental samples are often not clean, well behaved samples. The matrix may be complex and contain species that interfere with routine measurements of a contaminant of interest. The strategies for dealing with this situation are to provide sample preconditioning that removes the interferences or develop a highly selective probe for the contaminant. The technical objective of this year's effort has been to construct, using recombinant DNA methodologies, two different arsenic responsive reporter gene systems, and test the whole bacterial cells carrying these reporter genes in collaboration with technical staff from the Applied Optics Department.

Construction of the recombinant plasmids, carrying either a light-producing reporter gene (bacterial luciferase, lux ), or green fluorescent protein (GFP), from a marine jellyfish, was considered to be straightforward, given the past experience of the molecular biologists performing this work. Two of these scientists had previously published a paper describing the DNA sequence of the arsenic resistance operon from the plasmid R46). The senior molecular biologist had previously used the lux reporter gene to construct a constitutively expressed lux plasmid. This plasmid is being used as a positive control to prepare the optics bench that will be used to evaluate the eventual arsenic biosensors.

The general strategy decided upon was to place the arsR gene (arsenic repressor protein gene that controls induction of the resistance genes in the native arsenic resistance system from R46) in front of the lux or GFP reporter genes. In this way, exposure to arsenic would interact with the arsR gene product, leading to its removal from the transcriptional start for lux or GFP, leading to the induction of either of these reporter genes.

Initial constructs utilized the previously sequenced arsenic resistance operon cloned into the high copy number plasmid, pUC19 (plasmid designated pIRC120). For construction of the lux reporter system, an SphI/PstI digest was performed on this plasmid, and that fragment was then inserted into the promoterless lux-carrying plasmid, pUCD615 provided by Dr. Clarence Kado, University of California, Davis). Exposure of bacterial cells carrying this construct to differing levels of arsenic failed to produce light.

At the same time, the GFP reporter gene was amplified from a control plasmid, pGFPuv (also being used as a positive control for optics bench preparation), and cloned into PstI-digested pIRC120. Attempts to observe GFP production upon exposure to arsenic with this construct also failed.

Evaluation of these results suggested that the small amount of ars $\mathrm{D}$ gene sequence remaining upon digestion of pIRC120 with PstI was interfering with correct transcription and production of the two reporter proteins.

Over the course of the last three months, an alternative strategy has been devised. The arsR gene alone has been amplified from pIRC120 using primers that provide convenient restriction sites (XbaI and $\mathrm{KpnI}$ ) to allow cloning of the arsR control element in front of either lux or GFP reporter genes. 
A bacterial biosensor responding to $1 \mathrm{ppm}$ arsenite was successfully constructed and tested at the end of the fiscal year. Construction of the recombinant DNA plasmid carrying bacterial luciferase (lux) genes under the control of a bacterial arsenic response element (arsR) was accomplished by first subcloning the lux genes from the plasmid, pUCD615 (obtained from the laboratory of Dr. C. Kado at UC Davis), into the cloning vector, $\mathrm{pUC18}$. The arsR response element was amplified from our arsenic resistant clone, pIRC120, incorporating restriction endonuclease sites for $\mathrm{XbaI}$ and $\mathrm{KpnI}$. The fragment was digested with these enzymes, and ligated into the pUC18/lux construct digested with the same enzymes.

The bacterial strains carrying the recombinant plasmid were exposed to $1 \mathrm{ppm}$ sodium arsenite and light production was driven by the addition of exogenous decaldehyde. Comparisons with control strains not carrying the plasmid demonstrated that light production was stimulated by the presence of arsenic in only the strain carrying our recombinant plasmid.

Similar tests are being completed with the green fluorescent protein (GFP) contructs in the presence of arsenite, looking for the production of fluorescent material in the recombinant bacterial strains.

Another thrust in this activity has been to examine the possibility of using bio-self-assembly techniques to fabricate microdevices. The initial effort completed this FY has been the design and fabrication of micro-components that can be used later to evaluate self-assembly techniques under consideration at the INEEL. To this end, a contract was developed with Kelvin Lynn at Washington State University to assist in this effort. A preliminary design and specification list was prepared at the INEEL, and a student at WSU prepared the detailed template required by a silicon foundry. These components consisted of a series of "boxes" of various sizes and shapes and other components that will ultimately be inserted in the boxes (Figure 7). The boxes consist of walls and a closed bottom (Figure 8). Finally, a pair of boxes will be assembled to provide a closed container. Some of the boxes have a central spindle standing up from the bottom (Figure 9). This spindle can be used to mount and hold a rotating component such as a gear. This was intended to simulate the assembly of a gear box.

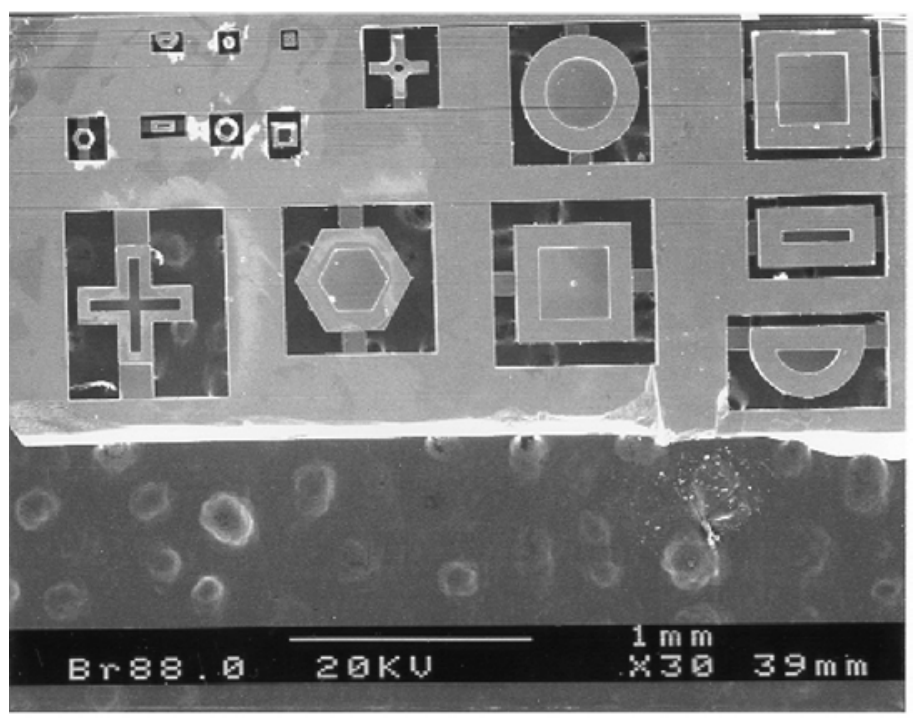

GV99 0501

Figure 7. Some of the shapes fabricated on a silicon wafer by deep ion etching. 


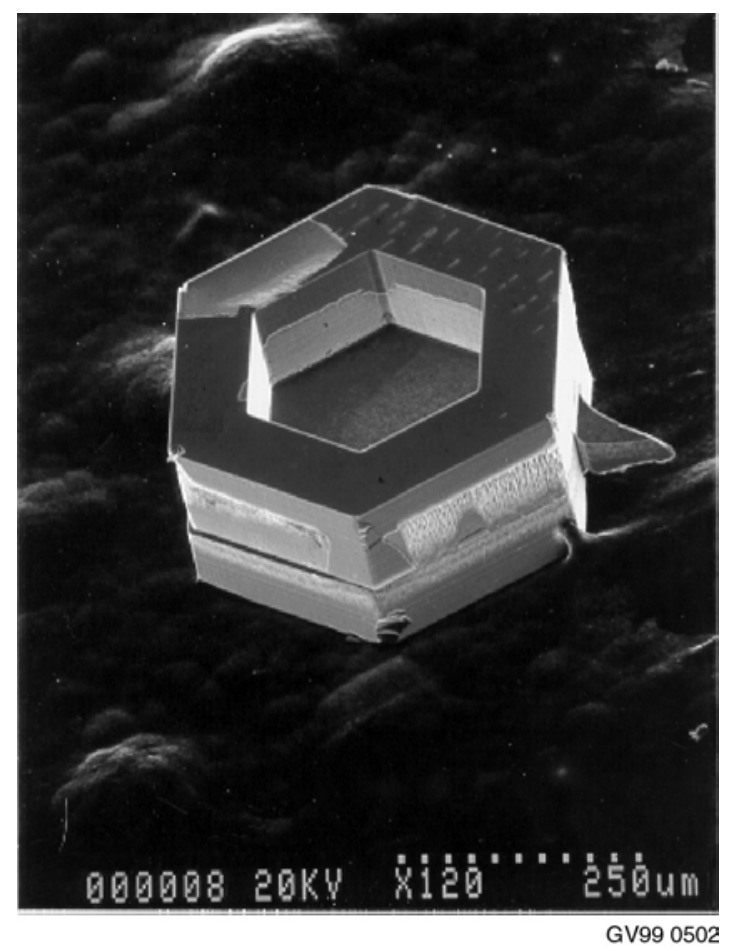

Figure 8. Close up view of one of the box forms after separation from the wafer.

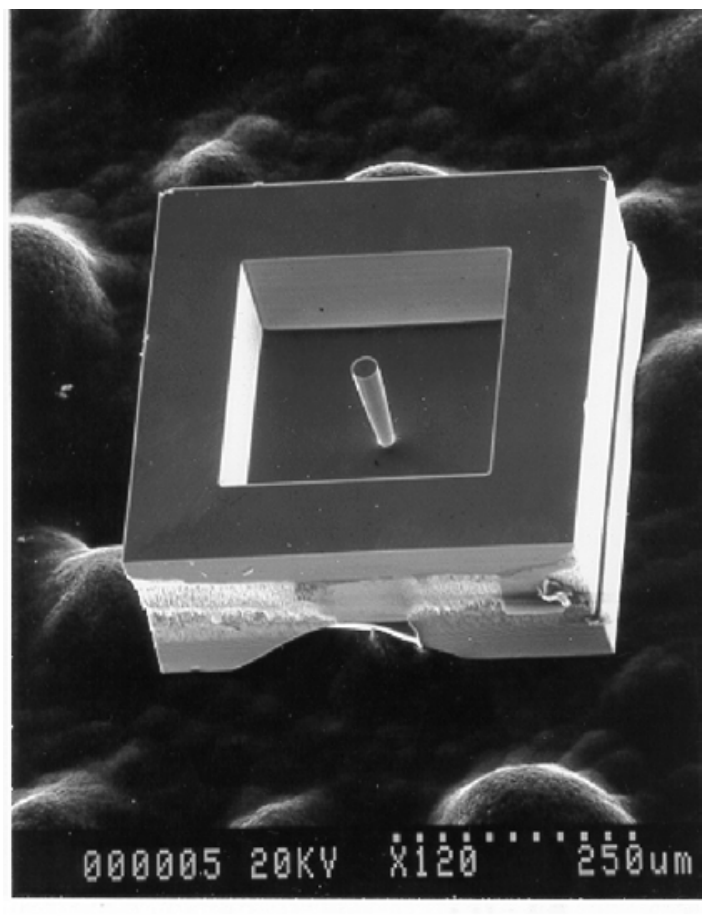

GV99 0503

Figure 9. A box form with a central spindle rising from the bottom. 
Dr. Lynn located a foundry capable of deep ion etching, the preferred fabrication process for our particular type of component. Very high aspect ratios for etched structures are possible with this technique (Figure 10). The initial 4 inch silicon wafer fragmented during fabrication due to residual stresses in a mounting adhesive. The foundry agreed to produce a second wafer. Meanwhile, a series of electron micrographs of components from the first wafer were made. These showed that the process appears to be working. The second wafer is now at the INEEL and will be held in reserve for future tests.

The contract with WSU is now completed.

\section{Membrane Development/Characterization Utilizing MIMS}

Membrane Introduction Mass Spectrometry (MIMS) has made large strides in capability improvement since its introduction. The main progress has been in systematic design changes that have allowed this technique to measure analytes in the parts-per-trillion range with samples that have had no special handling or pretreatment. In spite of these improvements, however, the most important component of MIMS - the membrane-has not been optimized. In this task, membranes have been selected, manufactured, tested, and evaluated as candidates in applications involving chlorocarbon analysis. The results showed interesting responses by different polymers that may make them good candidates for all of the EM-relevant techniques being considered/developed.

The identification of organic compounds, especially in aqueous media, is considerably important in many environmental applications as well as in medical and process applications. ${ }^{1}$ Many techniques are available, and the performance of each can be enhanced by configurations that exclude unwanted (corrosive, interfering, diluent) chemicals. One of the most useful techniques is mass spectroscopy due to its specificity. MIMS is a technique that allows preconcentrated, selective analyte introduction directly into a mass spectrometer. ${ }^{2}$

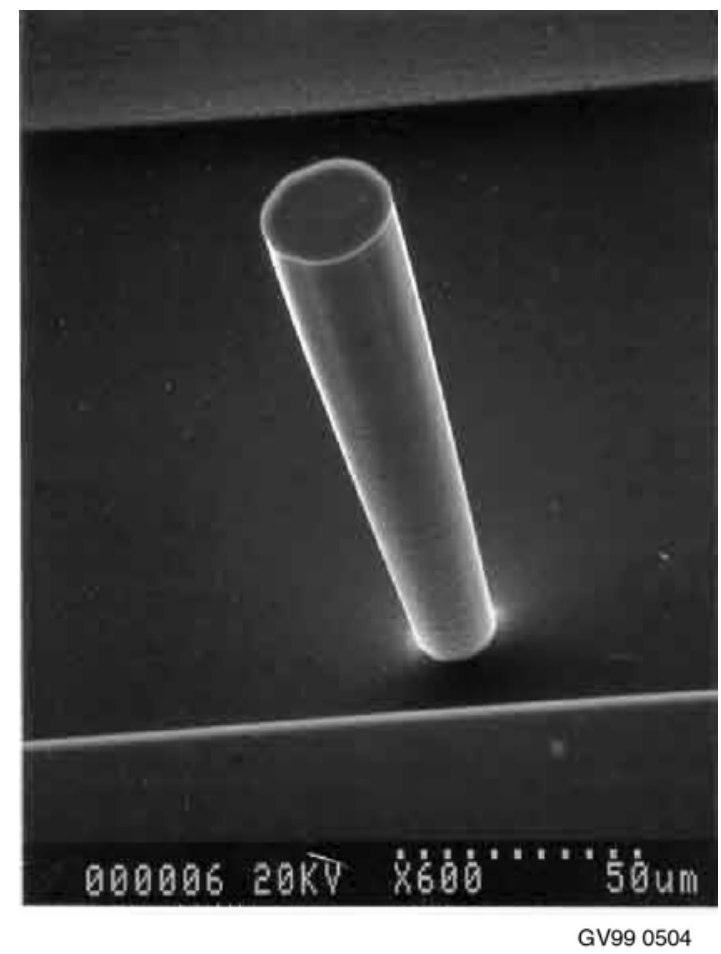

Figure 10. The central spindle of Figure 9 shows the excellent definition and high aspect ratio provided by deep ion etching. 
MIMS has become an important analytical technique for a variety of analytes including chlorinated hydrocarbons. A number of papers report modifications in hardware to improve performance. ${ }^{3}$ Near realtime process monitoring has also become an important application area of MIMS. ${ }^{4}$ Many types of analytes ranging from volatile organics to dissolved ions have been detected at very low concentrations using this technique. ${ }^{3,5}$ MIMS has been demonstrated to be equal to or superior to purge-and-trap GC/MS, and static headspace-gas chromatography. ${ }^{6}$ Gases of physiological interest have been analyzed. ${ }^{7}$ Far less extensively studied are the membrane materials, which act as the selective interface. Several authors have performed extensive testing on silicone membranes, both as sheet and hollow fibers. ${ }^{8}$

Figure 11 shows a schematic of how the membrane is mounted at the tip of the probe. The membrane is positioned between supporting screens and mounted with screws to the end of the probe. The picture shows the two openings that allow the test solution to pass across the membrane and then back out of the probe.

Hydrophobic silicone membranes are selective for organic compounds and they have been used extensively as a selective interface between samples containing volatile organic compounds (VOCs) and the vacuum of the mass spectrometer. As a result, the permeate stream reaching the mass spectrometer is enriched in VOCs. Contaminants in water samples have been detected at the sub-parts-per-trillion level using silicone rubber membranes, whereas contaminants in air have been detected at the parts-per-trillion level. Direct analysis of semivolatile organic compounds without preconcentration steps has also been demonstrated. ${ }^{8}$

Figure 12 is a spectrum that shows three consecutive injections of a 1,000 ppm TCE in water solution into the test stream. The injection to injection repeatability is good. By injecting a set of known standards, an unknown concentration can be determined.

For more than a decade, the separations group at the INEEL have been involved in developing membranes that a) can have their chemical, mechanical, and thermal properties tailored for specific applications, and b) can survive harsh conditions. This task will benefit from all of the existing in-house membrane capabilities. Membranes were both bought and synthesized at the INEEL. Figure 13 shows a

\section{Sheet membrane MIMS probe}

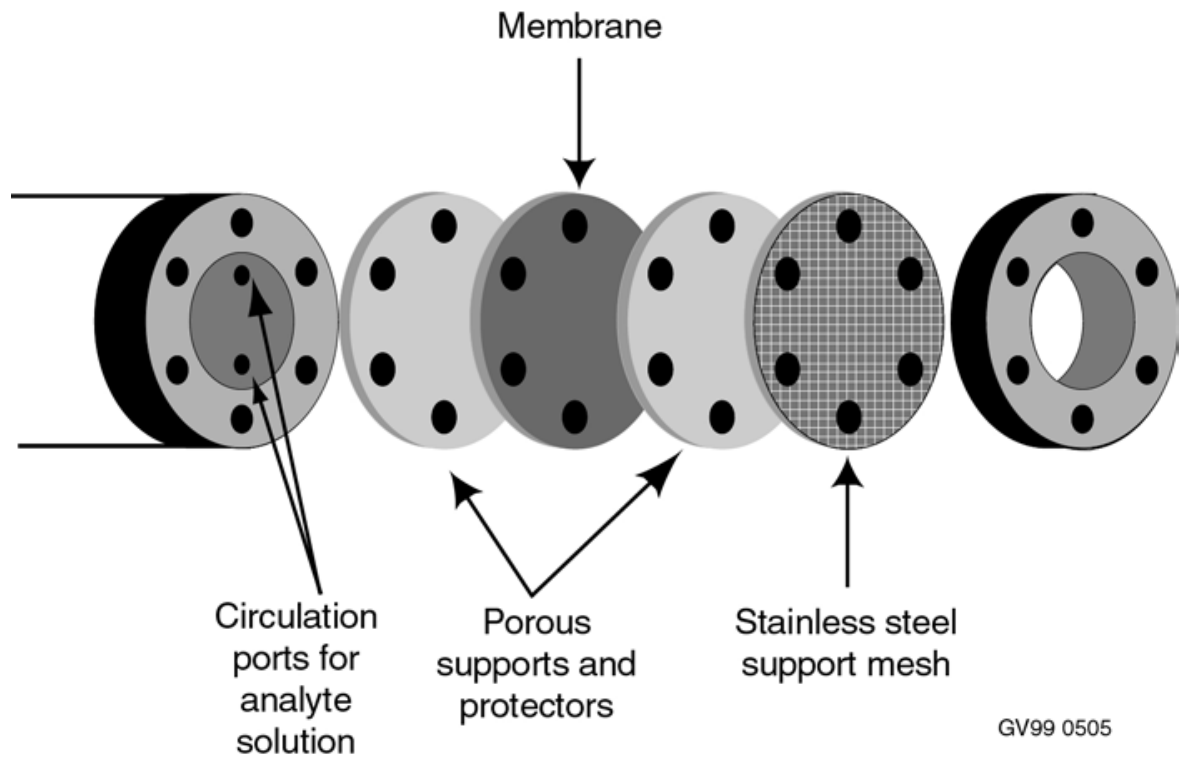

Figure 11. Sketch of how the membrane is mounted on the end of the MIMS probe. 
MIMS: TCE through polyphosphazene membrane

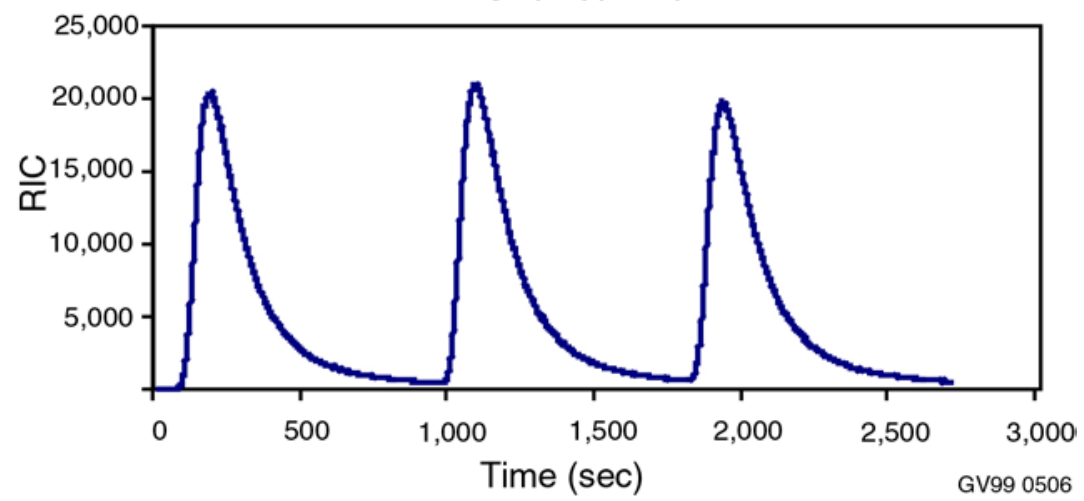

Figure 12. The results of three consecutive $1 \mathrm{ml}$ injections of a 1,000 ppm TCE solution across a polyphosphazene membrane.

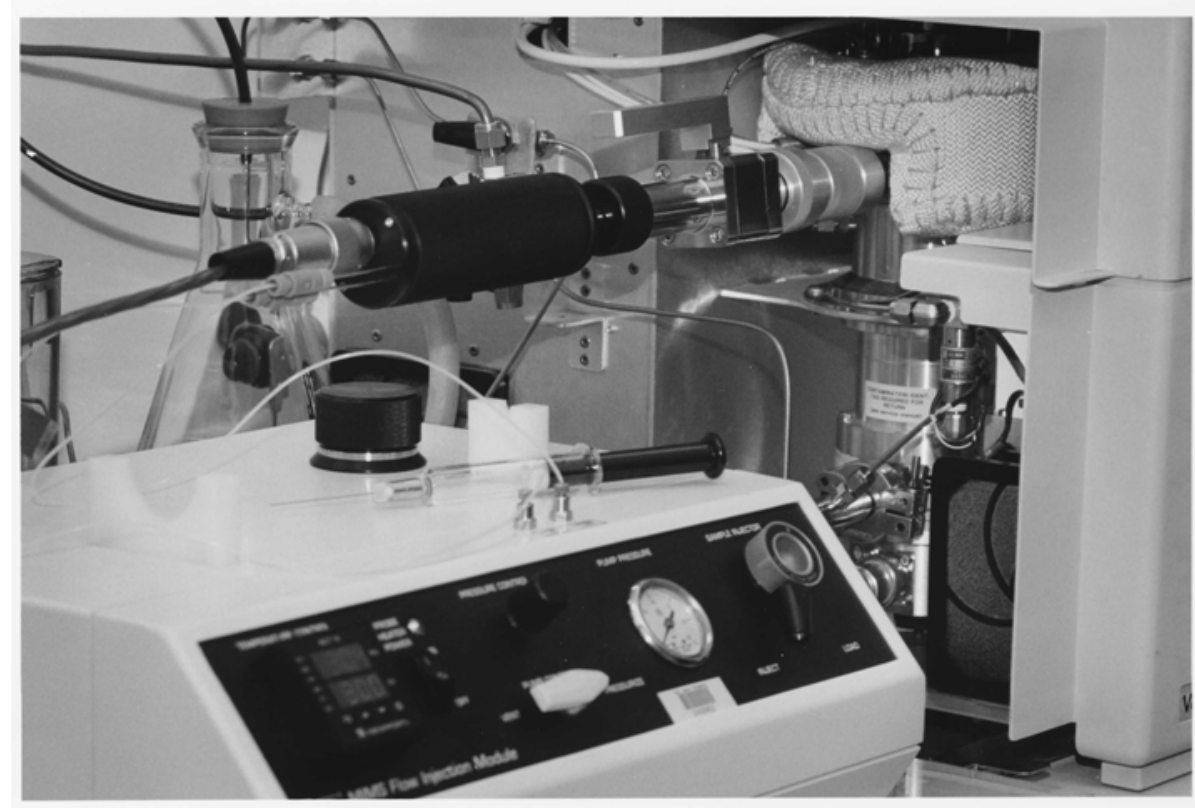

Figure 13. Close up view of the probe that has been inserted into the side of the mass spectrometer.

close up of how the probe is positioned directly into the side of the mass spectrometer. This way, as permeates pass through the membrane they are transported directly into the ion trap of the spectrometer, giving extremely good sensitivity to the whole scheme.

\section{Microsampler/SAW Device}

Sample conditioning is an important aspect of field characterization and monitoring activities. For measurements of low concentrations of contaminants under varying matrix conditions some sort of sample preconditioning is likely to be necessary. As discussed in the membrane development/characterization utilizing MIMS task, the INEEL has a significant background in diffusion membrane materials, particularly in polyphosphazene materials. The emphasis in this task has been the development of small analytical instruments based on surface acoustic wave (SAW) detectors coupled with selective membrane sampling. The membranes to be used in these studies are those identified in the membrane development/characterization utilizing MIMS task as showing selectivity in the passage of 
environmental contaminants of interest, e.g., trichloroethylene, carbon tetrachloride, and other halogenated solvents.

SAW technology is not new to the DOE system; the PAWS (portable acoustic wave sensor) was demonstrated with support from EM. The technique involves creating a resonant wave at a specific frequency (in the range of $200 \mathrm{MHz}$ for current devices) on the surface of a piezoelectric substrate. The resonance frequency of an individual device is dependant on a number of physical parameters of the crystal, one of which is the mass that is being moved by the surface wave. A change in that affected mass changes the resonance frequency of the crystal. Any chemical that adsorbs on the surface of the SAW will therefore change the resonance frequency of the SAW by changing the affected mass. The amount of change in frequency is a function (not necessarily linear ${ }^{1}$ ) of the additional mass of the adsorbed species. Sub-ppm sensitivities have been demonstrated for SAW detectors.

While SAWs have reasonable sensitivity for many volatile and semivolatile organics, they are not selective. Anything that has an appreciable sticking coefficient for the SAW surface will give a signal. One means of getting around this lack of selectivity is preconditioning the sample such that the SAW sees only the species of interest in the absence of the sample matrix or other competing species. One method of using the selective diffusion data from the membrane development/characterization utilizing MIMS task is to take advantage of the materials that show some selectivity by employing them as is done in the MIMS system. Use the membrane as a barrier in front of the SAW. Figure 14 is a schematic of the test apparatus that has been assembled to demonstrate the membrane/SAW system.

The system currently is designed for gas phase experiments but with modification could be used to analyze liquid samples. In Figure 14 the part of the system to the left of the membrane is the sample introduction piece. To the right of the membrane is the analyzer portion of the apparatus. Samples can be introduced in two ways, by injection through the septum (pulsed mode) or by blending a measured flow of nitrogen saturated with sample into the main nitrogen stream (continuous mode). The sample laden nitrogen flow passes through one half of a small chamber (about one $\mathrm{ml}$ ) that is divided by the diffusion membrane. The sample passes over the circular diffusion membrane (about one $\mathrm{cm}$ in diameter). On the other side of the membrane, a stream of pure nitrogen sweeps through a similar chamber volume carrying anything that diffuses through the membrane to SAW detector.

\section{Membrane Microsampler/SAW}

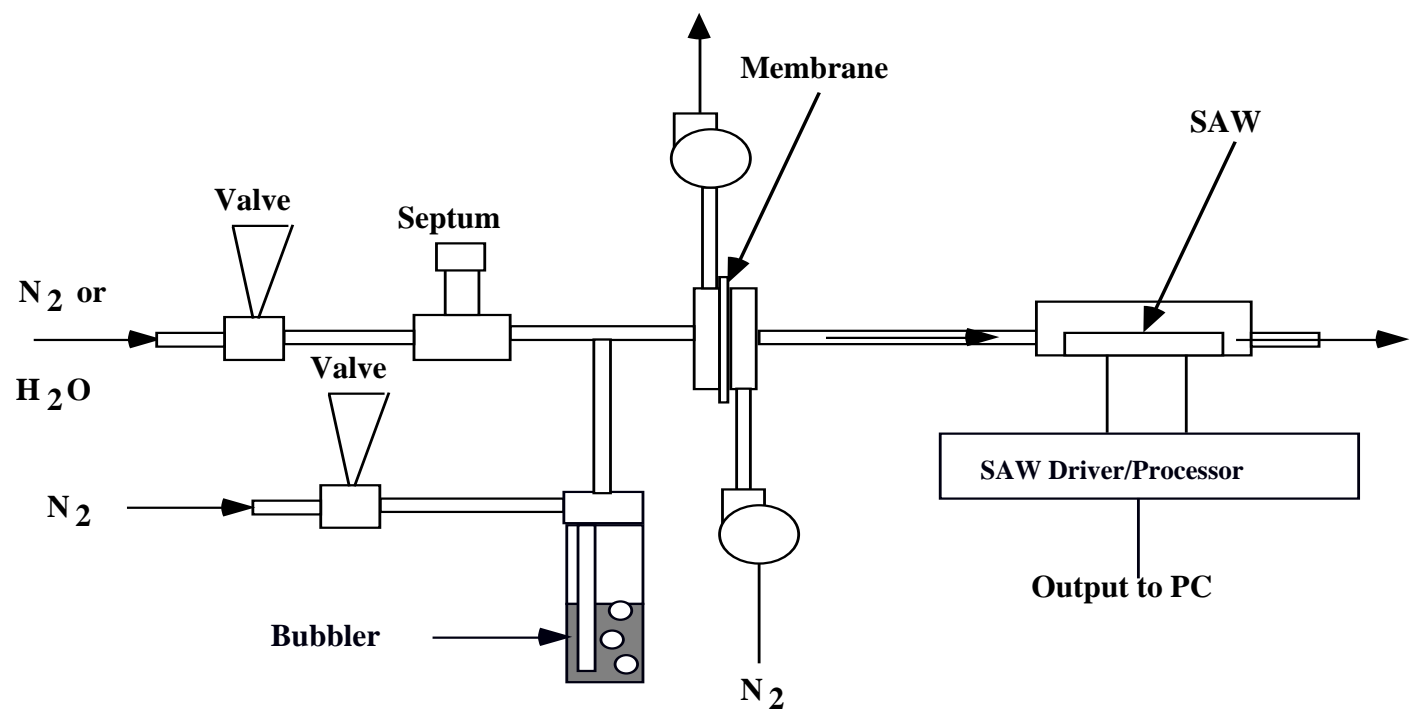

Figure 14. System to test the performance of selective diffusion membranes with a SAW detector. 
The SAW is a commercial unit (SAW Pro-250) purchased from Microsensor Systems, Inc. The device is a dual SAW configuration in which one SAW is the sample sensing resonator and the other provides a reference frequency. The frequency of the sample side is mixed with the reference side. Any change in the sample side frequency produces a low frequency difference signal that is a function of the amount of sample adsorbing on the surface of the sample SAW. The SAW Pro- 250 can be a stand-alone system. However, it is configured to be run from a PC using software supplied with the unit.

In many applications where there is a need to identify and quantitate some species in the presence of others, coated arrays of SAWS have been used. Differing responses of SAWs coated with different substances to various target analytes can be related to the solubilities of the analytes in the coatings. ${ }^{2}$ Using libraries of analyte versus coating responses and chemometrics has allowed some successful applications of SAW technology in analytical applications. However, they are not often good at sorting out complex mixtures at low concentrations. In spite of this the appropriate coating will enhance the sensitivity of the response to an analyte that is soluble in the coating.

Preliminary experiments to test the responsiveness of the SAW device to two volatile organics, acetone and methanol, have been carried out with both coated and uncoated SAW devices. A SAW resonator was coated with a thin acrylate film deposited by evaporation of a dilute solution of the acrylate in acetone. Preliminary experiments to test the response to acetone and methanol were carried using the sampling system shown above without a membrane in place. It was thought that the acrylate coated SAW would show a stronger response to acetone than to methanol based on solubility considerations. ${ }^{2}$ However, the responses were similar for concentrations in the vicinity of $300 \mathrm{ppm}$. This is not yet adequate sensitivity for the types of applications envisioned for this system. Additional work needs to be done to optimize the resonator coatings. Promising materials from the membrane development/characterization utilizing MIMS task will be used both as resonator coatings and separation membranes.

\section{A Two-Dimensional Spectral Imaging Spectrometer Using a Digital Micromirror Array and a Single Photomultiplier Tube}

There are many EM needs that would benefit from highly versatile optical instruments for spectroscopy, imaging and spectroscopic imaging with essentially no moving parts (i.e., rugged) that can be used in field or research tasks. Field portable instrumentation and methods for UV-Vis, NIR and Raman spectroscopy are continuously being developed. New methods for microbial identification in the field using fluorescent in situ hybridization (FISH), immunoassays for contaminant species, mapping U or $\mathrm{Pu}$ surface contamination via their fluorescence spectrum, etc., would all be methods that could benefit from this type of instrumentation development.

Two-dimensional spectral imaging, sometimes referred to as hyperspectral imaging, has a variety of diverse applications spanning the micro- to macroscopic scale and a variety of scientific fields, e.g., microbiology, chemistry, geochemistry, forensics, environmental, astronomy and agriculture. An example of the value of true two-dimensional spectral imaging is given by the comparison of the Landsat and AVIRIS satellites. Both cover the spectral range of approximately 350-2500 $\mathrm{nm}$ and both use their motion to image the surface in a push broom like technique. The Landsat satellites cover this range with seven broad band filters while the AVIRIS uses a grating to disperse it onto CCDs to get 244 wavelengths (10 $\mathrm{nm}$ bandpass) of the same spectral region. The Landsat can identify vegetation in a particular field on the earth's surface. The AVIRIS can not only identify vegetation in the field but can use the spectrum to identify the specific crop growing in that field. ${ }^{1}$

Unfortunately, the common laboratory instrumentation for two-dimensional spectroscopic imaging is not typically so versatile as the AVIRIS. Most laboratory "spectral imaging" systems depend upon a very limited set of bandpass filters for spectral selection and either a CCD camera or mechanical devices 
such as a "moving stage" or raster scanning system for spatial selection. The filters incorporated into these spectroscopic imaging systems severely limit the number of applications for which the instrument can be used. Not only is the wavelength selection limited, but the bandpass is frequently too large for optimum selectivity or to successfully employ mathematical techniques to extract the information. In fluorescence microscopy, the bandpass of most filters utilized is on the order of 10-40 nm. Liquid crystal tunable filters (LCTF) and acousto-optical tunable filters (AOTF) are commercially available and can be used for more versatile wavelength selection in imaging applications. ${ }^{2,3}$ However, the light throughput, relatively wide bandpasses, requirement for an expensive CCD detector, and price of these devices may still be limiting for many applications. LCTFs range in price from as little as $\sim \$ 5 \mathrm{~K}$ for a wide bandpass model to more than $\$ 30 \mathrm{~K}$ for a model with resolution sufficient for Raman imaging. Grating or prism instruments are much cheaper but require a "moving stage" to acquire an image at multiple wavelengths unless only 1D spatial information is required (i.e., the spatial information along the entrance slit of the spectrometer if a $2 \mathrm{D}$ array detector such as a CCD is used).

Two-dimensional spectral imaging systems employing a "moving stage" are simply too slow to be used for most routine applications. Collection of a single two-dimensional spectral image can take several hours using this technique. With the use of a CCD camera, an image can be obtained in much less than one second or by integrating for several seconds to enhance the signal-to-noise ratio. Spectral images with a CCD can be obtained but a filter wheel, AOTF, LCTF or moving stage with a slit entrance to a polychrometer are necessary. Acquisition time using the CCD cameria is generally on the order of minutes. While CCD cameras are readily available, their sensitivity is limited for many low-light applications like fluorescence or Raman imaging. Intensified CCD cameras appropriate for many lowlight applications are commercially available but cost on the order of $\$ 15 \mathrm{~K}-\$ 25 \mathrm{~K}$. These high sensitivity CCD cameras are still as much as 100x less sensitive than a photomultiplier tube, which can have a cost of less than $\$ 2 \mathrm{~K}$, including electronics.

Hadamard spectroscopy or imaging is performed by making a series of $\mathrm{n}$ measurements of $\mathrm{n}$ masks of $\mathrm{n}$ elements. The individual responses at the $\mathrm{n}$ elements are then calculated via a set of simultaneous equations. In its simplest terms, this is a least squares solution described by $M=S \cdot I$ where $M$ represents a vector of $n$ measurements. $\mathrm{S}$ is a Sylvester matrix derived from a Hadamard matrix, describing which of the $n$ elements are on or off during each of the $n$ measurements, and $I$ is the vector of the actual response at each of the $n$ elements that is to be determined. Because multiple elements are on during each measurement, the Hadamard technique provides a multiplex advantage that can significantly increase the signal-to-noise ratio $(\mathrm{S} / \mathrm{N})$.

Hadamard encoding using either mechanical, ${ }^{4} \mathrm{AOTF}^{2}$ or liquid crystal devices (LCD) ${ }^{5,6}$ as masks has been used to allow otherwise common spectroscopic and microscopic equipment to be used to collect spectra and images with either single or multiple detector elements. The multiplex advantage associated with the Hadamard encoding has improved the signal-to-noise for these imaging and spectroscopic applications. The latest developments in Hadamard spectrometers and spectral imaging devices have employed digital micromirror array devices (DMA) ${ }^{7,8}$ DMAs are a two dimensional array of micromirrors $(16 \mu \mathrm{m} 2$ separated by $1 \mu \mathrm{m})$ mounted on a SRAM chip. The development of DMAs is being driven by the digital television and projection market. Therefore, it is expected that these devices will become common place and even less expensive in the future. For spectroscopy applications, these devices hold much promise because they can be addressed rapidly ( $<20 \mu \mathrm{sec}$ compared to $\sim 10 \mathrm{msec}$ on a LCD), do not suffer from light leakage or photodegradation like $\mathrm{LCDs}^{9}$ and are ultimately much cheaper than CCDs. Currently, a projector with a single DMA is $\sim \$ 3 \mathrm{~K}$. The use of DMAs for other spectroscopic applications involving atmospheric chemistry has also been suggested. ${ }^{10}$ 


\section{The Instrument}

The DMA spectroscopic and two-dimensional spectroscopic imaging applications to date have employed a single DMA. We will be building a two-dimensional spectral imaging instrument employing two DMA devices. This instrument will be highly versatile and will ultimately cost $<\$ 20 \mathrm{~K}$. Figure 15 is a highly simplified schematic of the two-dimensional spectral imaging system design generated using OSLO LT 5.4 optical design software. The imaging spectrometer will consist of an input source from a microscope, telescope, sensor array, etc., that will be focused onto the first DMA device, the image DMA. The beam will be reflected from the first device onto an appropriate concave grating that will disperse the beam onto the second DMA, the spectrometer DMA, which will be in the focal plane of the spectrometer. The light from the second DMA will be focused onto the input window of a PMT. The spectrometer design in Figure 15 shows a collimated input beam, but other beams can be used with appropriate modification to the input beam so that the desired image focused onto the first DMA.

The proposed two-dimensional spectral imaging spectrometer is highly versatile and will ultimately be operable in a variety of modes:

- As a simple spectrometer where either all or a user-selected set of the imaging DMA elements are "on" and the spectrometer DMA is scanned as a moving slit to record spectra. Spectra of the entire image field or some area of interest will be obtained.

- $\quad$ As a Hadamard transform spectrometer where either all or a user selected set of the imaging DMA elements are "on" and the spectrometer DMA is "scanned" as a Hadamard mask. Again, spectra of the entire image field or some area of interest will be obtained.

- As an imaging system where either all or some portion of the elements of the spectrometer DMA are "on" and the imaging DMA is raster scanned either with individual or "binned" pixels. In this way, either bright-field images or images at specific wavelengths with adjustable bandpasses can be obtained.

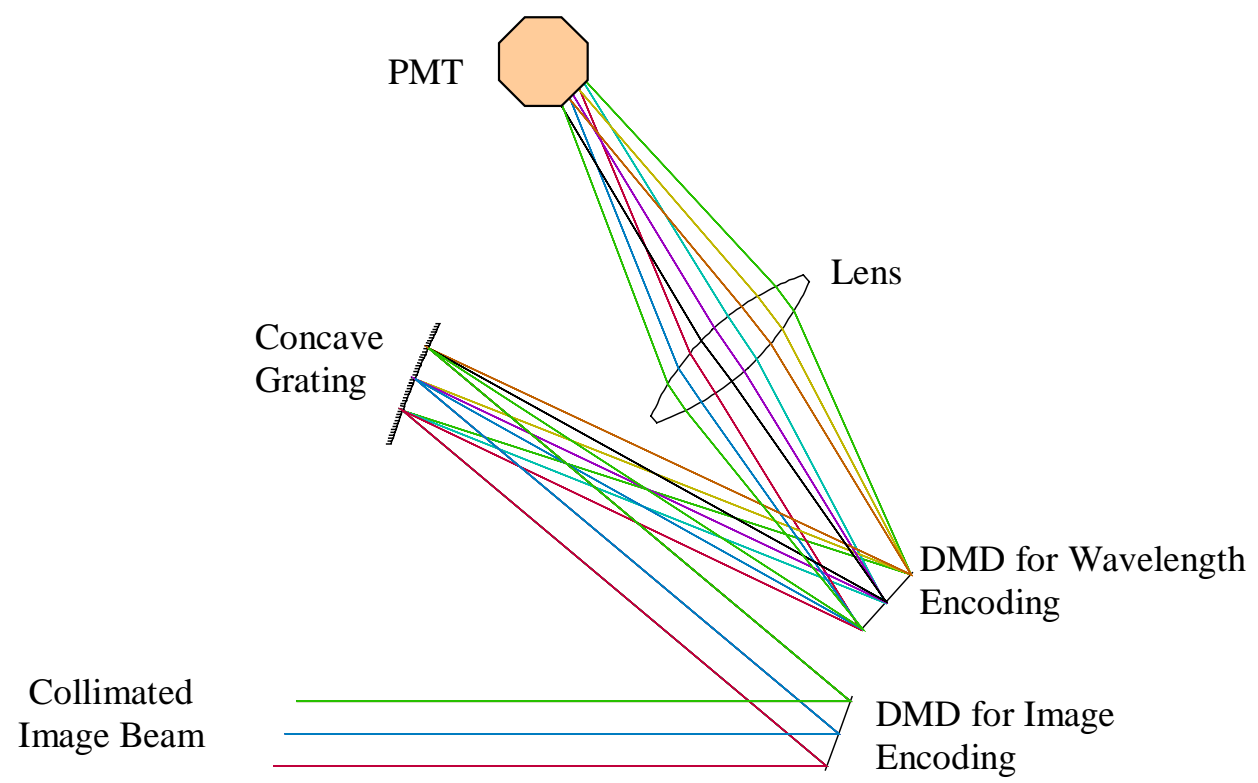

Figure 15. Schematic of proposed imaging spectrophotometer utilizing two DMAs. 
- As a Hadamard imaging system where either all or some portion of the elements on the spectrometer DMA are "on" and the imaging DMA is "scanned" as a Hadamard mask. Pixels, and subsequently the Hadamard encoding, may be binned to produce lower resolution images. Again, in this way, either bright-field images or images at specific wavelengths with adjustable bandpasses can be obtained.

- $\quad$ As an imaging spectrometer where the imaging DMA is raster scanned either as individual pixels binned sets of pixels and at each step the spectrometer DMA is scanned as a slit to record the spectrum. Alternatively, the spectrometer DMA may be "scanned" (like peak jumping) as a series of filters of any desired wavelength or bandpass.

- As a Hadamard imaging spectrometer where all or some portion of the imaging DMA is "scanned" as Hadamard encoding mask and the spectrometer DMA is scanned either as a slit or series of filters to record the spectral information.

- Various other combinations such as scanning the imaging DMA as either a horizontal or vertical slit and utilizing the spectrometer DMA as a Hadamard mask or application of various other encoding schemes using one or more of the DMAs.

- Lifetime imaging and spectroscopy using any of the configurations above and recording the signal as a function of time with a lock to an appropriately modulated source.

There are several inherent advantages and disadvantages to the DMA-based imaging spectrometer design.

\section{Advantages of the DMA-Based Imaging Spectrometer}

- $\quad$ Cost. The market for digital television/projection is driving the development of the DMA devices. Because of this, the price of the available DMA devices (sold as a DLP package with necessary electronics) will be dropping from the current range of $\$ 1.5-3.0 \mathrm{~K}$. Photomultiplier tubes with housings, readout electronics, and a power supply can be obtained for under $\$ 5 \mathrm{~K}$. For applications where a photomultiplier tube is not necessary, the detection system can be considerably cheaper. With a high sensitivity PMT and a single DMA, the cost will be comparable to the cost of a single CCD suitable for most spectroscopic applications. As a two-dimensional spectral imaging system with a total component cost of $<\$ 20 \mathrm{~K}$, the system will be capable of performing as well or better than other imaging spectroscopic systems for Raman and fluorescence applications where package costs run from as little as $\$ 60 \mathrm{~K}$ to as much as $\$ 200 \mathrm{~K}$.

- $\quad$ Sensitivity. PMTs respond more quickly and are as much as $100 x$ more sensitive than the best CCD. Coupled with an encoding scheme to take advantage of the multiplex capability, the system could well achieve sensitivities of 1,000x greater than CCD-based imaging systems.

- Versatility. With a few exceptions, this system should be capable of performing all of the functions of several other designs at a lower cost. Even without the imaging DMA, the addition of a grating/prism and a single DMA to a device such as a confocal microscope would make a variable bandpass filter that would be considerably more versatile than the current systems. Eliminating undesirable laser lines or optimizing the bandpass for each application will be easy. "Binning" for variable resolution images and user selection of areas of interest for more detailed imaging and spectroscopic analysis are additional examples of the versatile range of potential operation modes. 
The same basic design but with different gratings and detectors can be used for absorption, Raman, or fluorescence two-dimensional spectroscopic imaging.

\section{Disadvantages of the DMA-Based Imaging Spectrometer}

The major disadvantage, speed, stems from the fact that DMAs were developed primarily for visual applications and currently may have limited utility in the UV range.

While the DMA speed itself is more than adequate for most applications, the use of a single detector will limit the available speed of collecting an image, spectrum, or spectral image. For large format DMAs, several seconds will be required to record the intensity of each pixel separately. For current DMAs, the switching rate is less than a few $\mu$ seconds. Therefore, depending upon the array size, as few as 6 seconds and as many as 26 seconds may be required to collect a full image at a single wavelength/bandpass. For low light applications this is comparable to what may be required for a CCD, but for "bright" applications this may be excessively long. In these cases, it may be preferable to replace the PMT with an appropriate linear array detector and speed the process by scanning both DMAs as slit arrays, one in the vertical direction and one in the horizontal direction for full two-dimensional spectral imaging.

For ease and simplicity, the software applications to operate the instrument and process the images are being developed in LabView (National Instruments) and MatLab (The Mathworks, Inc., V5.3). To date, simple routines to operate a single DMA as a moving slit spectrometer and as an imaging unit using only randomly generated masks have been developed in LabView. A MatLab function has been developed to generate a series of binned Hadamard masks for the 600x800 element DMAs at low resolution images. These masks break the DMA down $n-1$ elements where $n$ is the size of the Hadamard matrix. The first row and column containing all ones are eliminated from the Hadamard matrix, leaving only $n-1$ elements for each mask. The number of mask elements, $n$, can be any size where n, n/12, or n/20 are powers of 2 . Figure 16 is an example where the original $600 x 800$ element image has been reduced to a 23x89 element image corresponding to a Hadamard mask of rank 2,047 (i.e., $n=2,048$ ). Because no noise has been added to the calculation, the resulting Hadamard image is exactly the same as an image that has simply been binned using $26 \times 8$ pixels from the original image. This verifies that the masks and Hadamard transform are being calculated correctly.
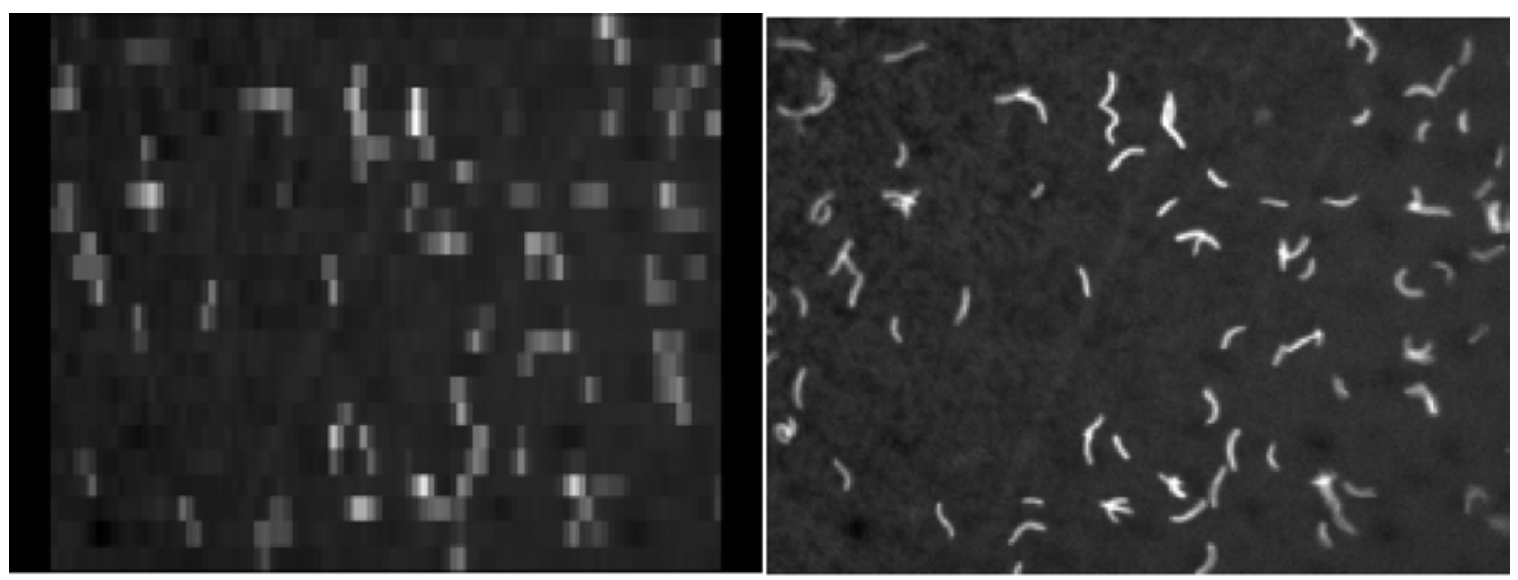

GV99 0510

Figure 16. 23x89 element Hadamard transform image (left) calculated from the original fluorescence image of bacteria on a polycarbonate filter (right). 
To apply the mask to the DMA, the mask is simply written to the computer display and this display is duplicated by the projector electronics. Unfortunately, the Windows NT-based operating system presently has a problem. There is no easy and direct way in either MatLab or LabView to create an image that covers the entire display without having the Windows box outlines also present. The only way around this at this point in time is to create a $\mathrm{C}++$ based function call to override the Windows controls and create a full screen, "presentation display window" analogous to the "picture" mode in most imaging software or the "presentation" mode in software such as PowerPoint. This work is currently in the progress.

\section{ACCOMPLISHMENTS Detection of Low-Energy lons for IMS}

- A conceptual design for a high-gain, high-bandwidth ion detector useful for IMS systems has been developed.

- $\quad$ The detector is based on the concept of an ion triggered discharge, which has been successfully demonstrated at low pressure this year.

- $\quad$ Physical principles expected to affect scaling of the device to high pressures have been identified.

- A path for continued development, involving optimization at low pressures followed by pressure scaling experiments, is planned.

\section{Laser-Enhanced IMS Instrumentation}

- $\quad$ The excimer laser was refurbished and is ready for use.

- A new quartz tube for the IMS has been acquired to allow the $248 \mathrm{~nm}$ radiation into the ionization chamber.

- The mechanical and electrical interfaces between the IMS and the laser have been designed and fabricated.

\section{Microarray Biosensor and Microdevices}

- A bacterial biosensor responding to $1 \mathrm{ppm}$ arsenite was successfully constructed and tested at the end of the fiscal year.

- $\quad$ Received microfabricated silicon shapes for self-assembly experiments.

\section{Membrane Development/Characterization Utilizing MIMS}

- $\quad$ Developed the techniques/methodologies to produce high quality, thin film membranes composed of Neoprene ${ }^{\mathrm{TM}}$, EPDM rubber (Keltan $\left.{ }^{\mathrm{TM}}\right)$, Mylar ${ }^{\mathrm{TM}}$, PDMS, and two polyphosphazene materials.

- Tested all of these materials against the following series of chlorinated hydrocarbons: chloroform, carbon tetrachloride, methylene chloride, and trichloroethane. Several of the materials were tested both as thicker neat films as well as thin films cast upon porous supports. 
- $\quad$ Some of the polymers showed excellent responses, some allowed nothing through, and perhaps most interesting, some responded to only selected members of this family of halocarbons. This is the kind of difference that should make it possible to use these materials in a suite of detection schemes.

- During this year a publication based upon the data from last year was submitted to the journal Analytica Chimica Acta. It had a favorable review and will be published after minor revisions.

\section{Microsampler/SAW Device}

- $\quad$ Acquired a commercial SAW system.

- $\quad$ Designed and fabricated a membrane, sample preconditioning system.

- $\quad$ Tested the response of coated and uncoated SAW detectors in the presence of several volatile organics.

\section{A Two-Dimensional Spectral Imaging Spectrometer Using Digital Micromirror Array and a Single Photomultiplier Tube}

- $\quad$ Simple routines to operate a single DMA as a moving slit spectrometer and as an imaging unit using only randomly generated masks have been developed in LabView. A MatLab function has been developed to generate a series of binned Hadamard masks for the 600x800 element DMAs at low resolution images.

- The manufacturer of the DMAs does not directly sell them. The only way to acquire one is to purchase a video projector that contains one. Two such projectors (Davis, DL 450) have been purchased, along with a photomultiplier tube (Hitatchi, R928), including the necessary power supplies, housings and electronics (PTI, Model 814 PMT detection system), and a multifunction card for a PC (National Instruments, PCI-MIO-16E-1) to acquire the data from the photomultiplier tube, change voltages on the PMT, operate shutters, etc., as necessary.

\section{REFERENCES}

\section{Detection of Low-Energy lons for Ion-Mobility Spectroscopy}

1. Editor E. E. Kunhardt and L. H., Electrical Breakdown and Discharges in Gases, Part B Maxroscopic Processes and Discharges, Plenum Press, New York (1981) p. 121

2. J. D. Cobine, Gaseous Conductors, Theory and Engineering Applications, Dover Publication, Inc., New York, p. 219

\section{Membrane Development/Characterization Utilizing MIMS}

1. B. J. Harland, P. J. D. Nicholson, and E. Gillings, "Determination of Volatile Organic Compounds In Aqueous systems By Membrane Inlet Mass Spectrometry," Water Resources, 21(1), (1987) 107-113.

2. S. Bauer, "Membrane introduction mass spectrometry; an old method that is gaining new interest through recent technological advances," Trends in Analytical Chemistry, 14(5), (1995), 202-213. 
3. F. R. Lauritsen and R. A. Ketola, "Quantitative Determination of Semivolatile Organic Compounds in Solution Using, Trap-and-Release Membrane Inlet Mass Spectrometry," Analytical Chemistry, 69, (1997) 4,917-4,922.

4. N. Kasthurikrishnan, R. G. Cooks, M. J. Thompson, "Sampling of Aryldiazonium, Anilino, and Aryl Radicals by Membrane Introduction Mass Spectrometry: Thermolysis of Aromatic Diazoamino Compounds," American Society for Mass Spectrometry, 9(3), (1998) 234-241.

5. N. Kasthurikrishnan, and R. G. Cooks, "Thin Pervaporation Membranes for Improved Performance in On-line Flow Injection Analysis Membrane Introduction Mass Spectrometry," Rapid Communications in Mass Spectrometry, 10, (1996), 551-756.

6. R. A. Ketola, V. Virkki, M. Ojala, V. Komppa, and T. Kotiaho, "Comparison of different methods for the determination of volatile organic compounds in water samples," Talanta, 44, (1997) 373-382.

7. J. E. Baumgardner and G. R. Neufeld, "Countercurrent Extraction of Sparingly Soluble Gases for Membrane Introduction Mass Spectrometry," Annals of Biomedical Engineering, 25, (1997) $858-869$.

8. M. E. Cisper and P. H. Hemberger, "Organometallic Analysis by Membrane Introduction Mass spectrometry," Rapid Communications in Mass Spectrometry, 11, (1997) 1,454-1,456.

\section{Microsampler/Surface Acoustic Wave (SAW) Detector}

1. J. W. Grate, S. L. Rose-Pehrsson, D. L. Venezky, M. Klusty, and H. Woltjen, "Smart Sensor System for Trace Organophosphorous and Organosulfur Vapor Detection Employing a Temperature Controlled Array of Surface Acoustic Wave Sensors, Automated Sample Preconcentration, and Pattern Recognition," Anal. Chem. 1993, 65, 1,868-1,881.

2. D. S. Ballantine, S. L. Rose, J. W. Grate, and H. Woltjen, "Correlation of Surface Acoustic Wave Device Coating Properties with Solubility Properties and Chemical Structure Using Pattern Recognition," Anal. Chem. 1986, 58, 3,058-3,066.

\section{Two-Dimensional Spectral Imaging Spectrometer Using Digital Micromirrorarray and a Single Photomultiplier Tube}

1. N. Short, "Remote Sensing and Image Interpretation \& Analysis in Remote Sensing Tutorial (An Online Handbook)," Jon Robinson, ed., Applied Information Sciences Branch at NASA's Goddard Space Flight Center, http://rst.gsfc.nasa.gov/, 1999.

2. J. F. I. Turner, and P. J. Treado, "Near-Infrared Acousto-Optic Tunable Filter Hadamard Transform Spectroscopy," Applied Spectroscopy, 50, 2, 1996, 277.

3. P. J. Treado, I. W. Levin, and E. N. Lewis, "Indium Antimonide (InSb) Focal Plane Array (FPA) Detection for Near-Infrared Imaging Microscopy," Applied Spectroscopy, 48, 5, 1994, 857.

4. M. K. Bellamy, A. N. Mortensen, R. M. Hammaker and W. G. Fateley, "Chemical mapping in the mid- and near-IR spectral regions by Hadamard Transform/FT-IR spectrometry," Applied Spectroscopy, 51, 4, 1997, 477-486. 
5. W. G. Fateley, R. M. Hammaker, J. V. Paukstelis, S. L. Wright, E. A. Orr, A. N. Mortensen and K. J. Latas, "Application of a two-dimensional Hadamard encoding mask for the imaging of thinlayer chromatography plates by laser-induced fluorescence or surface-enhanced Raman scattering and for use with a photoacoustic detector to generate three-dimensional photoacoustic images," Applied Spectroscopy, 47, 9, 1993, 1,464-1,470.

6. Q. S. Hanley, P. J. Verveer, and T. M. Jovin, "Spectral imaging in a programmable array microscope by Hadamard transform fluorescence spectroscopy," Applied Spectroscopy, 53, 1, 1999, 1-10.

7. Q. S. Hanley, P. J. Verveer, and T. M. Jovin, "Optical sectioning fluorescence spectroscopy in a programmable array microscope," Applied Spectroscopy, 52, 6, 1998, 783-789.

8. R. A. DeVerse, R. A. Hammaker, and W. G. Fateley, "Hadamard transform Raman imagery with a digital micro-mirror array," Vibrational Spectroscopy, 19, 1999, 177-186.

9. L. J. Hornbeck, "From cathode rays to digital micromirrors: a history of electronic projection display technology," TI Technical Journal, July-September, 1998, 7-46.

10. S. Chakrabarti, "Ground based spectroscopic studies of sunlit airglow and aurora," Journal of Atmospheric and Solar!Terrestrial Physics, 60, 1998, 1,403-1,423.

\section{APPENDIX}

\section{Peer Reviewed Publications:}

Mark L. Stone, Garold L. Gresham, and Linda N. Polson, "Characterization of Two Pholyphosphazene Materials as Membranes in MIMS," accepted for publication in Analytica Chimica Acta.

\section{Presentations:}

None. 


\title{
Intelligent Nonintrusive Methods for Characterization
}

\author{
T. J. Roney, J. C. Determan, D. C. Kunerth, T. A. White, and D. M. Tow \\ SUMMARY
}

This project addresses measurement interpretation and advanced measurements for use in characterization of nuclear waste and industrial inspection problems. Three tasks are emphasized: interpretation and processing of nuclear waste characterization data, material characterization using electromagnetic methods, and three-dimensional imaging in constrained environments. These three areas of research pose challenges of a basic nature but also hold promise for useful application in several Department of Energy (DOE) programs, including transuranic waste characterization and spent nuclear fuel characterization.

Work in intelligent processing centers on the development of an expert system to validate nuclear assay data from systems installed at the Idaho National Engineering and Environmental Laboratory (INEEL) was performed. The objective for FY-99 was to develop a prototype expert system to automatically process neutron assay data and to develop methods for automatic generation of the rules that make up the expert system. The use of genetic algorithms was employed for automatic rule generation and formed the basis of the expert system. The prototype expert system was provided to INEEL data validation physicists for review and comment. The response from these potential end users of the technology was highly encouraging.

For the effort in electromagnetic material characterization, the focus is on real-time pulsed (multifrequency) eddy current data inversion. Pulsed electromagnetic methods may allow for real-time analysis of critical DOE structures such as spent nuclear fuel containers, waste storage containers, protective metal coatings, and composite or graded metal structures. In FY-99, the main objective was to develop real time neural networks to process pulsed eddy current data and to test neural-network-based data processing techniques. To achieve this objective, an eddy current, data acquisition system was constructed and used to acquire training and test data sets for the neural network processor. The network was successfully trained and used to simultaneously determine the wall thickness of a sample under the influence of probe lift-off.

For the 3-D imaging task, the objective is to develop capabilities to acquire and process 3-D images on objects that are difficult to access from all sides. This situation arises in spent nuclear fuel storage and many industrial-processing situations. In FY-99, the goal was to develop imaging capabilities that would enable the development of algorithms required to process limited data. A laboratory system has been designed and will be completed in the first quarter of FY 2000. To support the algorithm development, an effort was made to acquire and install 3-D modeling codes. Two simulation packages were acquired and installed on INEEL computers. Both codes will be used to answer system design questions and to address specific imaging situations. Finally, questions regarding system alignment and scatter for 2-D and 3-D x-ray imaging systems were addressed in collaboration between INEEL and the University of Utah. Understanding and correcting errors caused by alignment and scatter is critical for obtaining highquality tomographic images. Results of both of these studies proved to be very useful in improving image quality on existing imaging systems at the INEEL. 


\section{PROJECT DESCRIPTION}

This section is split into three parts reflecting the three major tasks within the project: expert systems processing of nuclear waste characterization data, multifrequency eddy current system development and data analysis, and three-dimensional imaging.

\section{Expert Systems Processing of Nuclear Waste Characterization Data}

There were two thrusts to the expert systems research in FY-99. One was the continued development of an expert system for automatically generating classification rules from data. The goal with respect to this work for FY-99 was to improve both the classification accuracy and the speed of the rule generation process. Both of these objectives were met. Increasing the speed of the rule generation process is important because the speed of the process is a function of the number of features involved in classification, and this means that accelerating the process leads to a wider range of practical, potential applications. Automatic rule generation is of little use if the generated rules perform poorly, so it is always good to seek improved classification accuracy. Genetic algorithms were used instead of backpropagation to refine initial rule estimates to better fit the training data. A method known as genetic data clustering (GDC) was developed that achieved better classification accuracy than the existing system due to the use of probabilistic rather than deterministic clustering. Avoiding the highly iterative, and therefore costly, step of backpropagation also helped to improve the rule generation speed. In addition, various sources of inefficient processing were isolated and corrected.

The second thrust of this work was to continue development of a prototype expert system for review/validation of nondestructive waste assay data. This work has proceeded through manual elicitation and coding of rules through interviews with application experts. Rules for validating data from the neutron assay system had been gathered and coded in the first year of the effort. In FY-99, rules relating to data from the gamma assay system were also elicited and coded. Initial prototype testing was also begun. This work, while not yet complete, has already demonstrated the feasibility of expert system technology to a real world application.

\section{Automatic Rule Generation}

Knowledge to be encoded in an expert system can come in many forms. It may be that an expert has formulated a set of precise rules based on independent observations, and these can be elicited and encoded. It may also be that an expert must examine a set of features and make a more integrated judgement. In the latter case, the rules may be so interrelated that they can not be easily extricated from one another and encoded in a simple fashion. The expert, however, can easily apply his knowledge to accurately classify a data set, and may even give rationales for his judgements based on relative magnitudes of various features in the data. The interrelatedness of features in the data means that the relationship from features to classification is highly non-linear, such that it would be difficult, even impossible, to identify hard numerical limits on the features that lead to an accurate classification. However, there are mathematical procedures that can be followed to generate a set of interrelated rules from expert classified training data. These rules employ fuzzy logic, and accurately model a nonlinear decision-making process.

Generation of expert system rules from training data is a two-step process. The first step is to locate data clusters in the training data. The second step is to use parameters derived from the data clusters to form fuzzy rule membership functions. Figure 1(JCD-1) is an overview of the rule generation process. In data clustering, the training data are viewed as input/output vectors in a hyperspace, the 


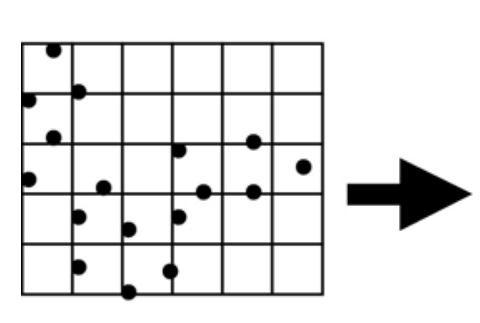

Training data

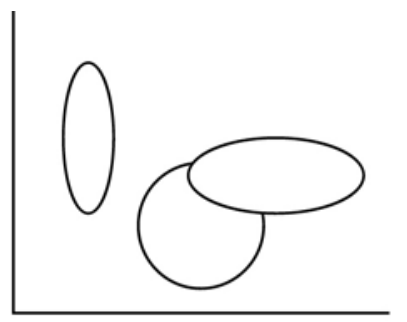

Data clusters

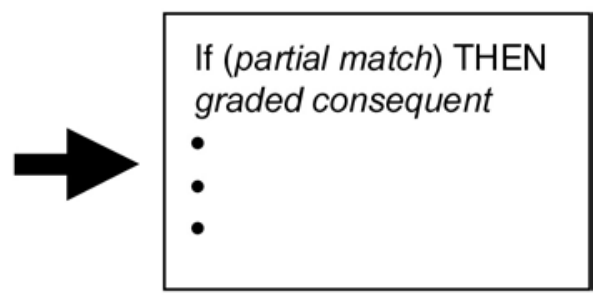

Fuzzy rules

Figure 1. Overview of rule generation process.

dimension of which is the total number of input and output elements. As indicated in Figure 1, the rule generation process involves identifying data clusters within the training data. A cluster of data points represents an approximate relationship from input to output, and this relationship can be represented as a rule using fuzzy logic (Zadeh 1965)1. Fuzzy logic naturally captures the approximate nature of this relationship.

Consider a simple example. A precisely known relationship could be represented as a single data point $\left(\mathrm{x}_{1}{ }^{*}, \mathrm{x}_{2}{ }^{*}, \mathrm{x}_{3}{ }^{*}, \mathrm{y}^{*}\right)$, rather than a cluster of data points. This data point could be interpreted as a rule of the form:

$\operatorname{IF}\left(\mathrm{x}_{1}=\mathrm{x}_{1}{ }^{*}\right) \operatorname{AND}\left(\mathrm{x}_{2}=\mathrm{x}_{2}{ }^{*}\right) \operatorname{AND}\left(\mathrm{x}_{3}=\mathrm{x}_{3}{ }^{*}\right)$ THEN y IS $\mathrm{y}^{*}$

In words, any data point $\left(\mathrm{x}_{1}, \mathrm{x}_{2}, \mathrm{x}_{3}, \mathrm{y}\right)$ with features that exactly match the features of the known data point $\left(\mathrm{x}_{1}{ }^{*}, \mathrm{x}_{2}{ }^{*}, \mathrm{x}_{3}{ }^{*}, \mathrm{y}^{*}\right)$ has the same output as that data point. While we have simplified the concept to the point of triviality, we can use this example to generalize to a more realistic situation. When dealing with training data, there is typically not any one single data point that represents an exact relationship, but there exist clusters of related data points, and a cluster centroid can be viewed as an abstracted data point. This abstracted data point forms a rule in the same manner as the single data point did above, except that the relationship is no longer exact. A cluster centroid is found by averaging the values of training data features and known classification values overall training points within a cluster. Thus, clusters of similar data points in training data define rules, but these rules are not exact and are most naturally represented as fuzzy rules. A concept that is inextricably bound with averaging is that of variation. Cluster centroid coordinates and standard deviations with respect to these coordinates are used to define fuzzy rule membership function parameters. form:

For $\mathrm{n}$ input and one output element in each datum, the generated fuzzy rules take the following

IF $\left(x_{k 1}\right.$ matches $\left.A_{i 1}\right)$ AND ... ( $x_{k n}$ matches $\left.A_{i n}\right)$ THEN $\left(y_{i}\right.$ is $\left.B_{i}\right)$, where

$i$ indicates the $i^{\text {th }}$ rule,

$\mathrm{x}_{\mathrm{k} 1} \ldots \mathrm{x}_{\mathrm{kn}}$ are normalized rule input values for the $\mathrm{k}^{\text {th }}$ data point, $\mathrm{x}_{\mathrm{k}}$,

$\mathrm{y}_{\mathrm{i}}$ are rule output values,

$A_{i 1} \ldots A_{\text {in }}$ are exponential membership functions, and

$B_{i}$ is a symmetric membership function. 
The use of a symmetric output function allows for a simpler solution, in that only the centroid of the output membership functions need be determined. The input membership functions for the $\mathrm{i}^{\text {th }}$ rule are expressed as follows:

$\mathrm{A}_{\mathrm{ij}}\left(\mathrm{x}_{\mathrm{kj}}\right)=\exp \left(-.5\left(\left(\mathrm{x}_{\mathrm{kj}}-\mathrm{x}_{\mathrm{ij}}{ }^{*}\right) / \sigma_{\mathrm{ij}}\right)^{2}\right), 1<=\mathrm{j}<=\mathrm{n}$, where

$\mathrm{x}_{\mathrm{ij}}{ }^{*}$ and $\sigma_{\mathrm{ij}}$ are estimated cluster means and standard deviations, respectively, and are derived as discussed below.

The result of the calculation $\mathrm{A}_{\mathrm{ij}}\left(\mathrm{x}_{\mathrm{kj}}\right)$ is referred to as the match strength of the input $\mathrm{x}_{\mathrm{kj}}$ with the associated membership function $\mathrm{A}_{\mathrm{ij} \text {. }}$ The match strength of a rule with multiple inputs is the product of the component match strengths. The rule strength of rule $\mathrm{i}$ with respect to point $\mathrm{k}$ is given the symbol $\mu_{\mathrm{i}}$ and is expressed as:

$\mu_{\mathrm{i}}\left(\mathrm{x}_{\mathrm{k}}\right)=\exp \left(-.5 \sum\left(\left(\mathrm{x}_{\mathrm{kj}}-\mathrm{x}_{\mathrm{ij}}{ }^{*}\right) / \sigma_{\mathrm{ij}}\right)^{2}\right), \mathrm{j}=1 \ldots \mathrm{n}$.

In fuzzy inferencing, the rule match strength modifies the rule output. Greater rule match strength lends greater weight to the consequence of the rule. This is typically accounted for in a defuzzification algorithm. Using a standard method of defuzzification (the center of gravity algorithm), the output of the system is found from:

$\mathrm{y}_{\mathrm{k}}=\Sigma_{\mathrm{i}=1}\left(\mu_{\mathrm{i}} \mathrm{y}_{\mathrm{i}}^{*}\right) / \Sigma_{\mathrm{i}=1} \mu_{\mathrm{i}}=\Sigma_{\mathrm{i}=1}\left(\left(\mu_{\mathrm{i}} / \Sigma_{\mathrm{i}=1} \mu_{\mathrm{i}}\right) \mathrm{y}_{\mathrm{i}}^{*}\right), \mathrm{i}=1 \ldots \#$ of rules, where

$\mathrm{k}$ represents the $\mathrm{k}^{\text {th }}$ data point,

$\mathrm{y}_{\mathrm{i}}{ }^{*}$ is the output membership function centroid for the $\mathrm{i}^{\text {th }}$ rule,

and $\left(\mu_{\mathrm{i}} / \Sigma_{\mathrm{i}=1} \mu_{\mathrm{i}}\right)$ represents the normalized weight of each rule.

In words, the rule set output is the weighted sum of the individual rule outputs, where the weight on each rule is the rule match strength, normalized by the sum of all rule match strengths. The role of data clustering in the generation of fuzzy rules is to derive meaningful values for the membership function parameters $\mathrm{x}_{\mathrm{ij}}{ }^{*}, \mathrm{y}_{\mathrm{i}}{ }^{*}$ and $\sigma_{\mathrm{ij}}$, as discussed below. These parameters correspond to cluster centroid and standard deviation values.

There are many data clustering algorithms (Bezdek, Li, Attikiouzel \& Windham 1997).2 Our work in automatic rule generation began by building on the subtractive clustering method (Chiu 1994). 3 A potential function estimates the density of data points in the region of a node. The node with the greatest value of the potential function is chosen as the first cluster center. The potential function at the remaining nodes is then revised to remove the influence of the selected node. Successive cluster centers are chosen as the node with the greatest value of the potential function at each cycle, and the function values are again revised. The number of clusters to be found is not directly specified; instead, stopping criteria are specified. When too few data points exist in the vicinity of the currently selected node, cluster center selection ceases.

In subtractive clustering, each cluster is initially characterized by estimates of the centroid coordinates $\left(\mathrm{x}_{\mathrm{ij}}{ }^{*}, \mathrm{y}_{\mathrm{i}}{ }^{*}\right)$ and standard deviations $\left(\sigma_{\mathrm{ij}}\right)$. Gradient descent optimization is performed on the variables $\mathrm{x}_{\mathrm{ij}}{ }^{*}, \mathrm{y}_{\mathrm{i}}{ }^{*}$, and $\sigma_{\mathrm{ij}}$ to fit them to the training data. This process is analogous to backpropagation training of neural networks. Convergence is achieved when the root-mean-square (RMS) error of the system with respect to the training data becomes relatively constant; i.e., the difference in successive iteration-averaged RMS values is below a specified criterion). The optimized parameters are used to form 
fuzzy rules, as discussed above. Figure 2 graphically depicts the relationship between clusters and fuzzy membership function parameters.

The automatic rule generation process outlined above has been modified to improve both the speed of the rule generation algorithm and accuracy of the resulting rules. A genetic data clustering algorithm has been developed to replace the combined steps of subtractive clustering and backpropagation. Genetic algorithms (Goldberg 1989)4 are a class of optimization techniques that are broadly applicable to many types of problems. Their usefulness derives from the fact that they do not rely on gradient information, but instead attempt to imitate the forces of natural selection in seeking out superior solutions to problems. When using genetic algorithms, a data structure ("chromosome") to represent the problem and an objective function that measures the performance level ("fitness") of a solution to the problem must be specified. The genetic algorithm generates and evaluates a large number of these data structures ("population") and employs the objective function to select superior solutions. Initial populations are typically generated through some random process specific to the problem at hand. The population of solutions is evolved by recombination of superior solutions in a manner that simulates the genetic processes of crossover (sharing of genetic material from parent chromosomes to create a child chromosome) and mutation (modification of an isolated chromosome). Algorithms that simulate these processes are known as genetic operators. In general, the effect of the mutation operator is to discover new solutions, and the effect of the crossover operator is to discover new combinations of existing data, and to share newly discover data and combinations of data throughout the population. While such discoveries may be either beneficial or harmful, the selection process ensures that only the beneficial discoveries will propagate (in the long run).

Subtractive clustering is used only in the initialization step of GDC, and backpropagation has been eliminated entirely. Genetic operators designed specifically for the GDC algorithm were developed, and an objective function based on the Xie-Beni fuzzy clustering index, discussed below, (Xie and Beni 1991)5 was used in the GDC algorithm. Once a set of clusters has been found in the training data, means and standard deviations are calculated in each dimension of the training data; fuzzy rules are then constructed from these values in the manner described above. This section discusses the GDC algorithm and its relationship to the process of automatic rule generation. Although fuzzy data clustering methods were initially employed for automatic rule generation, the GDC is actually a crisp data clustering algorithm that nonetheless allows determination of fuzzy rules.

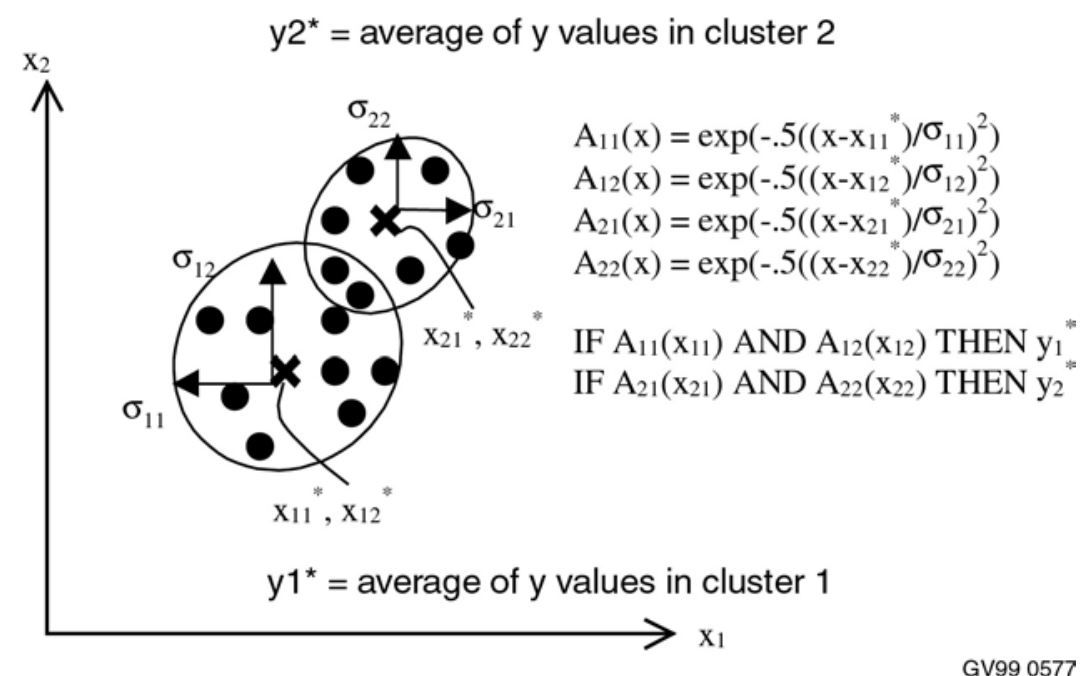

Figure 2. JCD-2. 
The genetic representation of a solution is discussed first. The building blocks of a solution are clusters of data points, thus, a good representation will allow members of a cluster to be contiguous genes. The genetic operators will be designed to operate on these building blocks, thus they need to be clearly distinguished in the representation. Figure 3 (JCD-3) shows the representation used in the GDC algorithm. The representation is a simple one-dimensional list of integers, where data points belonging to a given cluster are neighboring list elements. Following the data point identifiers are integers representing the number of clusters and the size of each cluster.

Two primary factors are considered in designing an objective function for data clustering: training set performance and cluster validity. Training set performance, $\mathrm{P}_{\mathrm{TS}}$, should be a component of the objective function. However, as the correct number of clusters is generally unknown, an unrestrained pursuit of performance will tend to lead to subdivision of proper clusters and overfitting of the training data. Therefore, a cluster validity index should also be included in the objective function. The Xie-Beni index, $\mathrm{S}_{\mathrm{XB}}$, has been proposed specifically for evaluating the validity of fuzzy clusters and has proven particularly useful in the present application. Numerically, $S_{\mathrm{XB}}$ is the ratio of a number that represents the "compactness" of a cluster to the cluster separation.

Summing terms based on $\mathrm{S}_{\mathrm{XB}}$ and $\mathrm{P}_{\mathrm{TS}}$ produced good performance on one data set, but better performance generally was obtained by forming a "tacking" function. A tacking function is an objective function that returns alternate values on successive generations, much like a sailboat sailing into the wind with a zigzag motion,. In essence, the tacking function provides selective pressure alternately in orthogonal directions to move the solution in the desired direction.

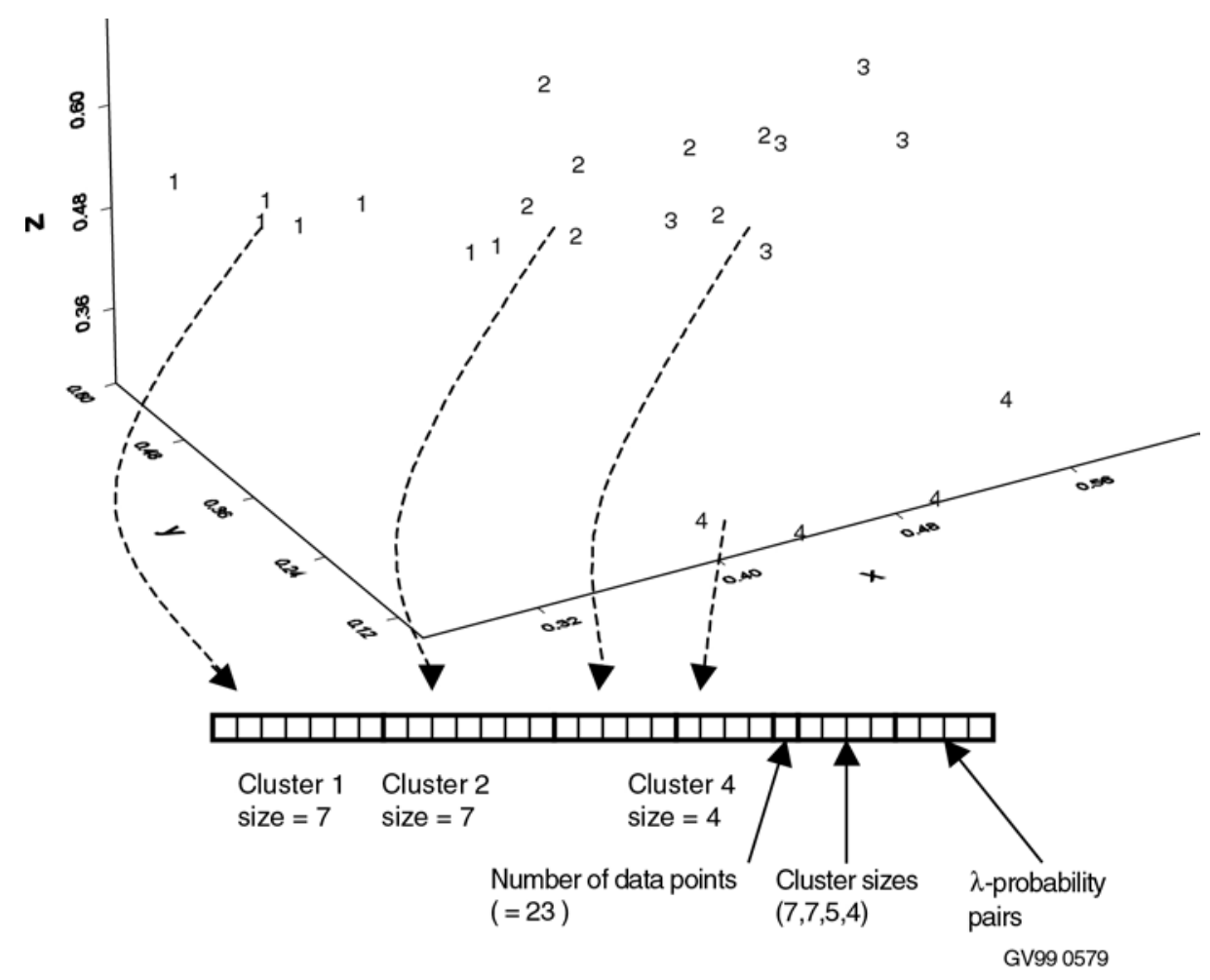

Figure 3. (JCD-3). 
An initialization routine was developed that used subtractive clustering to provide an initial estimate of cluster membership for each proposed solution. The estimated cluster means were used to calculate the distance of each point from the estimated cluster centroids. Points were assigned to each cluster probabilistically, with the probability of assignment to a cluster proportional to the square of a point's distance from the cluster centroid. Any given point had the greatest probability of being associated with the closest cluster centroid, but had some probability of being associated with any cluster. A population of proposed solutions initialized in this manner will tend to include varied estimations of the membership partition of the data points, each close to the correct solution, and each differing from each other primarily in the membership of questionable points from regions of overlap.

Data clustering is a combinatorial problem; therefore order and cycle crossover (Goldberg 1989)3 were tested on this problem, but found to yield neutral results. Order and cycle crossover are textbook methods for performing crossover under the constraints of combinatorial problems; i.e., that all genes must be present in the child chromosome, and none duplicated. What is needed in data clustering is a crossover method that, to the extent possible, picks whole clusters from two parents, resolves membership conflicts between clusters from different parents that possess a common data point, and ensures that the resulting child genome represents a valid and complete set of data points. Basically, clusters are randomly selected from two parents and copied into a child. With each cluster selection, the cluster is removed from the donor parent and the points of this cluster are removed from the other parent. This algorithm ensures that only unused data points are available for inclusion in the child at each step, and that all data points are included in the child.

The mutation operator was designed to complement the initialization operator. Some form of swap mutation, to swap data points between clusters of a given solution, seemed the most beneficial. The particular method chosen was to select two clusters at random between which a swap would occur, and to pick a data point from each cluster probabilistically, where the probability of selection was based on squared distance from the cluster centroid. A small chance of selecting no point from a cluster was included in the swap procedure to allow clusters to change size as well as membership; a one-sided swap moves a point from one cluster to another.

Chaos theory was also used in designing the mutation operator. Chaos underlies many natural phenomena, from turbulent fluid flow to global weather patterns (Gleick 1988), 6 from healthy heart rhythms (Goldberger, Rigney \& West 1990) 7 to DNA coding sequences (Ohno 1988).8 Complex dynamic systems with intricate feedback paths may often be understood in terms of chaos theory. Therefore it is natural to speculate that evolutionary processes may also be understood in terms of chaos. Beyond Natural Selection (Wesson 1981)9 thoroughly motivates and discusses this speculation. The application of chaos theory to genetic algorithms was explored in Determan \& Foster (1999)10 by employing the logistic equation, and results of that work were applied to the swap mutation operator.

The logistic equation (Moon 1992)11 is:

(2.1.1) $x_{n+1}=\lambda x_{n}\left(1-x_{n}\right)$

The properties of the logistic function are well known, but we briefly discuss them here. For values of $\lambda$ in $(0,3)$, (1) will converge to some value $x$. For $\lambda$ between 3 and about 3.56 , the solution to (1) bifurcates into two, then four, then eight (and so on) periodic solutions. For $\lambda$ between 3.56 and 4, the solutions to (1) become fully chaotic: neither convergent nor periodic, but variable with no discernable pattern. As $\lambda$ approaches 4 , the variation in solutions to (1) appears increasingly random. We refer to these three regimes as convergent, bifurcating, and chaotic, respectively. Note that the logistic equation 
is ideally suited to the task of mutating probability values, as the $x_{n}$ and $x_{n+1}$ are required to be real numbers in $(0,1)$.

The swap mutator described above is applied with a frequency determined by the probability of mutation, $\mathrm{P}_{\mathrm{m}}$. While the usual value of .001 is so low that the effect of mutation would be negligible, any large, constant value will likely prevent convergence by continually modifying the entire population. Therefore, $\mathrm{P}_{\mathrm{m}}$ must change as the problem progresses, so the best course is to let it be determined genetically. The logistic equation was employed to mutate $\mathrm{P}_{\mathrm{m}}$ within the swap mutator. To allow comparison to a nonchaotic method, a nonchaotic swap mutator that also modified $\mathrm{P}_{\mathrm{m}}$ was also developed, as described below.

In nonchaotic swap mutation, the genome includes an integer gene value representing the mutation probability. The allele values for this gene range between 1 and $10^{5}$, such that mutation probabilities between $10^{-5}$ and 1 are possible. The swap mutator function is extended to modify this parameter. Conceptually, the method is designed to reduce the mutation probability for genomes that are achieving good results (to encourage their survival and propagation); the remaining genomes should either improve or disappear. The method chosen is to select any genome with an objective score within $5 \%$ of the maximum score in the population and reduce its $\mathrm{P}_{\mathrm{m}}$ with the following formula:

$$
\mathrm{P}_{\mathrm{m}+1}=\mathrm{P}_{\mathrm{m}}-\min \left(\mathrm{P}_{\mathrm{m},}\left(1-\mathrm{P}_{\mathrm{m}}\right)\right) / 4
$$

The effect is to produce small changes in the mutation probability near one or zero and larger changes in the midrange, while preventing $\mathrm{P}_{\mathrm{m}}$ from becoming invalid.

The chaotic swap mutator is similar to that just described, except for the introduction of chaos. In this method, the genome is extended to represent both $\mathrm{P}_{\mathrm{m}}$ and $\lambda$. Like $\mathrm{P}_{\mathrm{m}}, \lambda$ is represented as an integer, with allele values between 1 and $2^{16}$, such that when divided by $2^{14}$ a maximum value of 4 results. Instead of directly modifying $\mathrm{P}_{\mathrm{m}}$, however, the chaotic method modifies $\lambda$ in a manner analogous to $\mathrm{P}_{\mathrm{m}}$ above (slowly near 0 and 4, faster in the midrange, and is applied to only those genomes achieving a high objective score). The logistic function, using the genetically represented $\lambda$, is applied to mutate $\mathrm{P}_{\mathrm{m}}$.

Tests have shown that some slight modifications to (2) above yield improved results. The divisor of 4 has been replaced with an integer variable that is randomly chosen from the interval $(2,8)$. The initial choice of 4 was arbitrary, so it seemed better not to be rigid on this value. Also, although reducing $\mathrm{P}_{\mathrm{m}}$ over time has conceptual appeal, improved results were obtained by allowing the terms of (2) to be randomly added or subtracted, not just subtracted.

\section{Test Results}

To test the performance of the rule generation system, a set of waste container assays with known validity confidence ratings was generated. To do this, a panel of three waste nondestructive assay (NDA) experts was assembled and given a set of waste assays to assign validity confidence ratings on a scale from 0 to 10. They rated their confidence in each of the three measurement modes of the system (one active and two passive neutron measurement modes) for each assay. The set contained 99 assays evenly selected from the waste types: graphite, combustibles, and glass. Disagreement existed between the experts as to the proper classification of each assay, due partly to differing scales of judgment used by each expert, and partly to the uncertainty inherent in the interpretation of NDA results. A normalization procedure was used to reduce the scaling bias of each expert. Assays with normalized scores that still did not agree between the experts were removed from the test set. The final data set contained 67 assays. Three figures of merit derived from the assay measurements were determined for each measurement 
mode of the assay system and were used as training input. Thus, there were three data sets, each consisting of three input and one classification.

Results of previous and current work were compared. The GDC algorithm produces a distribution of answers when performed repeatedly on a given training set. While the distribution is reasonably tight, maximal performance is achieved by performing five calculations and keeping the best set of resulting rules. Good performance was obtained with the GDC algorithm on all of the waste assay data. Improved performance was obtained on each data set, compared to results obtained in earlier work, based on subtractive clustering. Table 1 compares the results of earlier work with results obtained with the GDC algorithm. No specific timing comparison data was available for these data sets, but tests of both systems on the same machine indicate that the GDC algorithm is about $50 \%$ faster than the initial rule generation algorithm. This estimate accounts for the fact that five calculations must be performed with the GDC algorithm.

GDC classification accuracies are also presented in Table 1 for rules generated with the nonchaotic version of the swap mutator. The best-of-five scores are seen to be several points lower for every data set. This is due to reduced probability of achieving a given performance level. In summary, our automatic rule generation system has been improved to be both more efficient and more accurate.

The explanation of why chaos has improved GDC performance is still under investigation, but is believed to be due to the effect of chaos on the genetic search process. Genetic algorithms are commonly considered to occur in two phases: exploration and exploitation. Initially, the genetic search must be exploratory, such that good solution building blocks can be discovered. Later, the genetic search must exploit these good building blocks and spread them through out the population without destroying them through excessive mutation. Note that these are not mutually exclusive phases, but that both will overlap each other with emphasis gradually switching from one to the other as the calculation proceeds. Each solution carries its own value of the probability of mutation, $\mathrm{P}_{\mathrm{m}}$, and its own value of the logistic parameter $\lambda$. High $\mathrm{P}_{\mathrm{m}}$ solutions will explore to a greater degree and low $\mathrm{P}_{\mathrm{m}}$ solutions will exploit to a greater degree. Solutions with convergent $\lambda$ will not change the value of $\mathrm{P}_{\mathrm{m}}$ in successive generations very much, whereas solutions with chaotic $\lambda$ will produce children with unpredictably varying values of $\mathrm{P}_{\mathrm{m}}$. Solutions with high $\mathrm{P}_{\mathrm{m}}$ and convergent $\lambda$ are likely to suffer from excessive mutation through the generations, and ultimately become extinct. Solutions with low $\mathrm{P}_{\mathrm{m}}$ and convergent $\lambda$ will tend to be more exploitive, and therefore only propagate if they are paired with an otherwise good solution; i.e., contain proper clusters. These considerations imply that chaotic $\lambda$ is advantageous initially when good building blocks are still being formed, that convergent $\lambda$ is advantageous once good building blocks have been discovered, and that having both values in the initial population is a necessary condition. The processes of selection and crossover will tend to combine good building blocks discovered by explorers with the low $\mathrm{P}_{\mathrm{m}}$ and convergent $\lambda$ values of exploitive individuals. To aid the transition from chaotic to convergent

Table 1. Comparison of rule set classification accuracies between GDC generated rules and results obtained in previous work.

Generated Rule Set Classification Accuracy (\%)

\begin{tabular}{lccc}
\cline { 3 - 3 } \multicolumn{1}{c}{ Data Set } & $\begin{array}{c}\text { GDC Execution } \\
\text { Time(s) }\end{array}$ & $\begin{array}{c}\text { GDC } \\
\text { (Chaotic/Nonchaotic) }\end{array}$ & Previous Work \\
\hline Active Mode Neutron Data & 187 & $82 / 75$ & 78 \\
Passive Shielded Neutron Data & 210 & $84 / 80$ & 80 \\
Passive System Neutron Data & 190 & $93 / 86$ & 90 \\
\hline
\end{tabular}


$\lambda$ values in good solutions, the mutation operator also includes the effect of mutating $\lambda$ itself for solutions with high fitness. High fitness solutions will tend to decrease $\lambda$ from the chaotic regime to the convergent regime through the generations.

Sensitivity calculations and measurements are suggested by the above considerations that could help isolate the effects of chaos on genetic algorithms. It would be informative to track percentages of convergent and chaotic solutions and confirm that these percentages vary as speculated above.

Eliminating convergent, high $\mathrm{P}_{\mathrm{m}}$ solutions from the initial population may also be either beneficial or neutral, since these are expected to become extinct. On the other hand, ensuring that such solutions are in the initial population, and confirming their extinction would support the above speculations. Initializing populations to be entirely convergent or entirely chaotic would also be informative. Replacing chaotic variation with random variation, combined with a $\lambda$-like parameter for turning this behavior on and off would likewise be informative.

\section{Nondestructive Assay Expert System}

The objective for the second task in this project was to finish acquiring data review rules for the nondestructive waste assay data review expert system, implement these newly-acquired rules, and confirm/correct previously acquired rules. With the exception of a set of rules to verify that proper $\mathrm{Pu}$ isotopics had been measured, these goals were accomplished. In a series of meetings with expert NDA personnel, previously acquired rules were verified or modified as needed. The modified rules were implemented within the review system, and a prototype system installed at the Stored Waste Examination Pilot Plant (SWEPP) facility for testing. Testing was performed by reviewing data manually and automatically in parallel. No specific feedback has been obtained as of this writing, but general satisfaction with the results has been expressed. Rules pertaining to the gamma assay data were also developed. These rules verified that correct data tables were in use and that peak fit errors for key peaks were acceptable. The newly acquired rules have also been implemented within the review system, but have not yet been tested. Finally, all the rules of the review system have been reformulated within a generic framework for NDA data review. The generic framework was developed under separate funding by working with two distinct NDA organizations and analyzing the similarities and differences between their data review procedures. This work has demonstrated the feasibility of employing expert system technology for a real application in the realm of nondestructive assay.

\section{New Work}

Several tasks are planned for FY 2000. The review system prototype will be completed, and reinstalled at SWEPP for final testing. New applications for the review system and for the rule generation system will be sought. Work will also be initiated on applying expert system technology to problems faced in the design of the Advanced Mixed Waste Treatment Facility (AMWTF). In the AMWTF, several radiological constituent characterization systems will be in operation (neutron based box systems, tomographic gamma systems, traditional passive active neutron [PAN] systems), at high throughput, such that a large amount of diverse data will be generated on each waste batch. Each individual measurement system would benefit from the application of expert system technology in order to minimize data validation expense and execution time. Additionally, expert system techniques will be useful in the reduction and interpretation of assay system data from multiple process assay systems. The expert system would acquire and validate assay data from each individual process assay system and subsequently integrate the data set making online decisions regarding the plant process state in terms of fissile material throughput and inventory. Also, there is no prospective online evaluation of fissile material holdup based on the input from the set of assay systems that would allow prediction of future states. This results in unanticipated operational limit trips that stop facility operations. Expert systems can recognize such patterns and alert operators of impending events before expensive plant shutdowns. Expert system 
techniques currently used for data validation would be extended to work with the plant control software to integrate fissile material concerns into facility operations in a more efficient manner. In particular, efficiency would be increased by reduced facility downtime and improved response time to shutdown events.

\section{Multifrequency Eddy Current System Development and Data Processing}

The objective of this task is to develop real-time, multifrequency, eddy current, data inversion technologies for the purpose of automated material characterization and nondestructive evaluation. The significance of the work is the development of data processing tools that permit the use of pulsed/multifrequency, eddy current technologies for material characterization, including automated/realtime applications for various complex structures. Some of these complex structures of specific interest to DOE-EM included spent fuel, waste storage containers, protective metal coatings, and composite or graded metal structures. Specific objectives for FY-99 included evaluation of neural networks as a means to process pulsed/multifrequency, eddy current data, implementation of neural networks in a data acquisition system, and investigation of model-based, eddy current data inversion. FY-99 efforts concentrated on the development and testing of neural network based data inversion/processing techniques. The pulsed, eddy current inspection of 55-gallon waste drums was selected as the test case. The objective of the inspection is to determine wall thickness as a means of characterizing structural integrity. Complicating the inspection are geometry, rust, labels, and dents. Various neural networks were developed and tested for the purpose of determining wall thickness and probe lift-off. Probe lift-off is indicative of labels, surface rust, and probe misalignment that decouple the probe from the test piece. The effort demonstrated that neural networks could be successfully trained and applied to the problem of processing the multifrequency data to simultaneously obtain wall thickness and lift-off. Also demonstrated was the ability to implement the neural network processing capability in an eddy current, data acquisition system.

\section{FY-99 Multifrequency Eddy Current Activities}

Work was performed to develop a basic understanding of the capabilities of neural networks to process-pulsed, eddy current responses. Various networks were developed and tested for functionality. Work was performed in collaboration with Dr. Lloyd Allred of Hill AFB, Utah, now at Los Alamos National Laboratory.

Network Training and Testing. The neural nets were trained using data collected from a carbon steel calibration strip that consisted of 11 steps, ranging from 20 to 70 mils. Lift-off was introduced into the measurement process via 12 nonconducting shims ranging from 0.5 to 30 mils. Initial testing performed in collaboration with Dr. Allred identified several features of the eddy current response that potentially could be used to train a network. Nine features believed to be the most relevant were chosen. Figure 4 $(D C K-1)$ shows the definitions of each feature. These features have enough information about the signal that variations in material thickness and probe lift-off can be determined. An investigation using features R1 through R4 and G1 through G3 proved that a neural net could be used with reasonable accuracy to determine thickness and lift-off variations. However, features T1 and T2 were later added in an attempt to reduce the errors in the neural net determination of material thickness and lift-off. The neural networks and training found to be functional used three-layer, back propagation with nine nodes in the input layer, five nodes in the hidden layer, and one output node. Typically, the nine feature inputs are first scaled by a sigmoid-squashing function (1) to obtain outputs between 0 and 1 . 


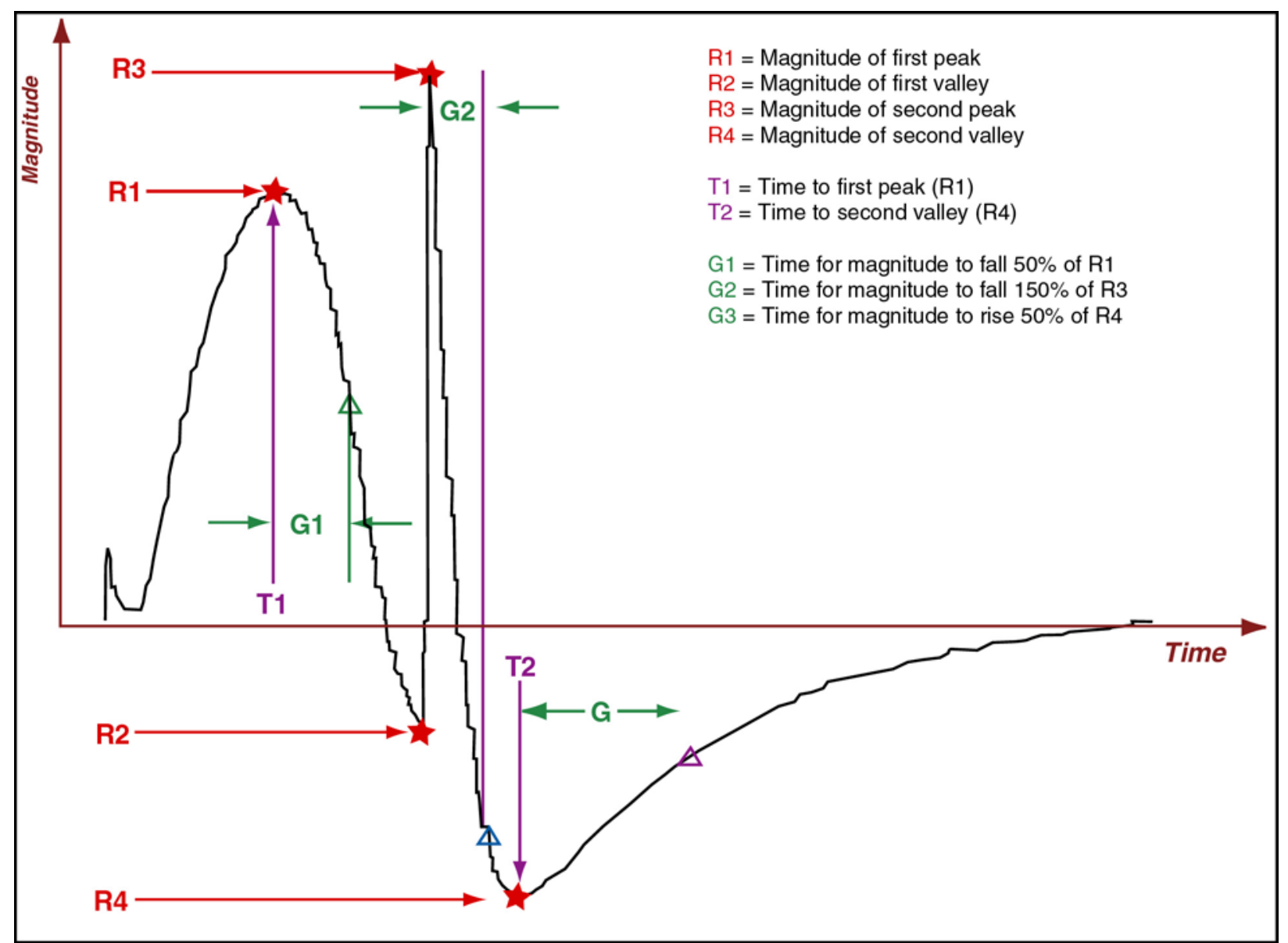

GV99 0579

Figure 4. Feature definitions.

The sums of the weighted inputs are taken in the hidden layer and weighted and summed again in the output layer. The value is then squashed by the sigmoid function and the thickness/lift-off is output in percent of the trained range. There are two networks used, one for the thickness determination and one for the lift-off determination. A schematic of the basic neural network structure is illustrated in Figure 5 (DCK-2).

The test results from one specific set of neural networks and data set is provided in Figure 6 (DCK-3). Presented are the plots of determined/calculated thickness versus actual thickness at different lift-offs. For this test, 132 waveforms were acquired using various lift-off values and plate thicknesses. Random samples of three-fourths of these waveforms were used to train the network. The other quarter was used to test the accuracy of the network. The thickness prediction closely follows the actual values until it reaches the upper range of the thickness where increased error is observed. Whether this is from lack of electromagnetic field penetration or inadequate network training has not been determined. The significance of the results obtained is that neural networks can be used as an efficient data processing tool for pulsed eddy current responses.

Data Acquisition System. A LabView ${ }^{\mathrm{TM}}$ controlled data acquisition system having neural network processing capabilities was also developed and tested. Figure 7 is block diagram of major system components. This system was used to acquire and process the data presented in Figure 6. Although 


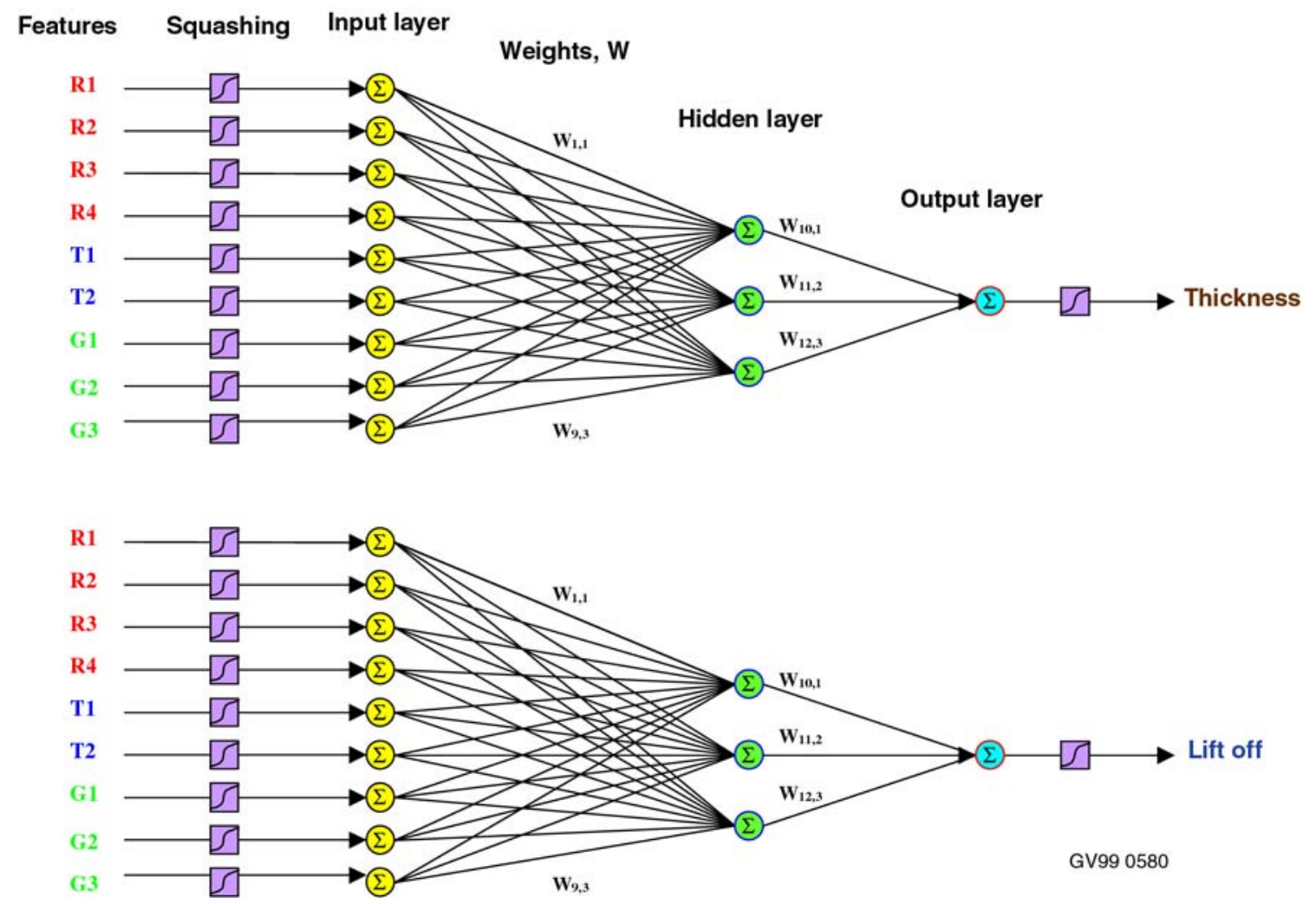

Figure 5. Neural network structure.
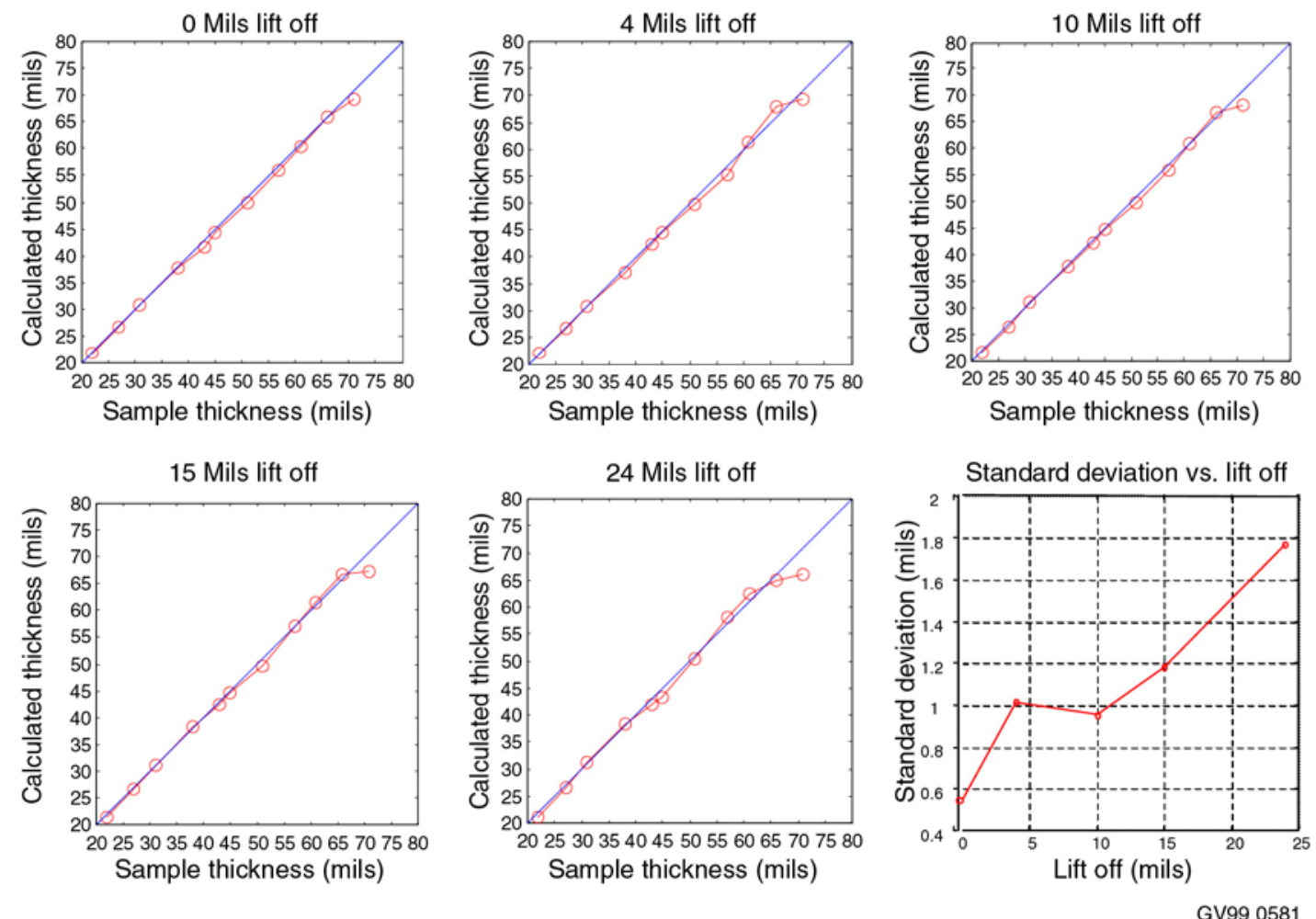

Figure 6. Neural network determined thickness with increasing lift-off. 


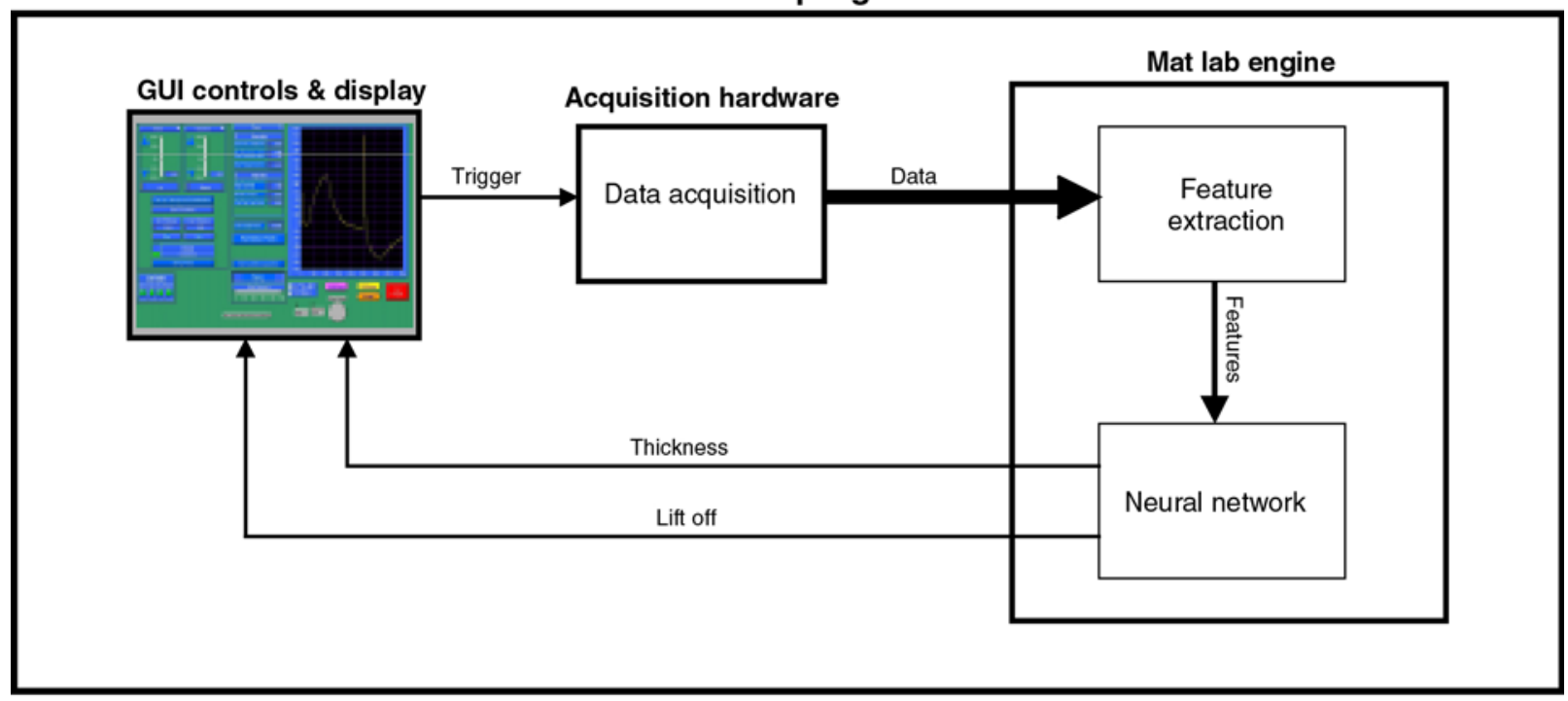

GV99 0582

Figure 7. Data acquisition system block diagram.

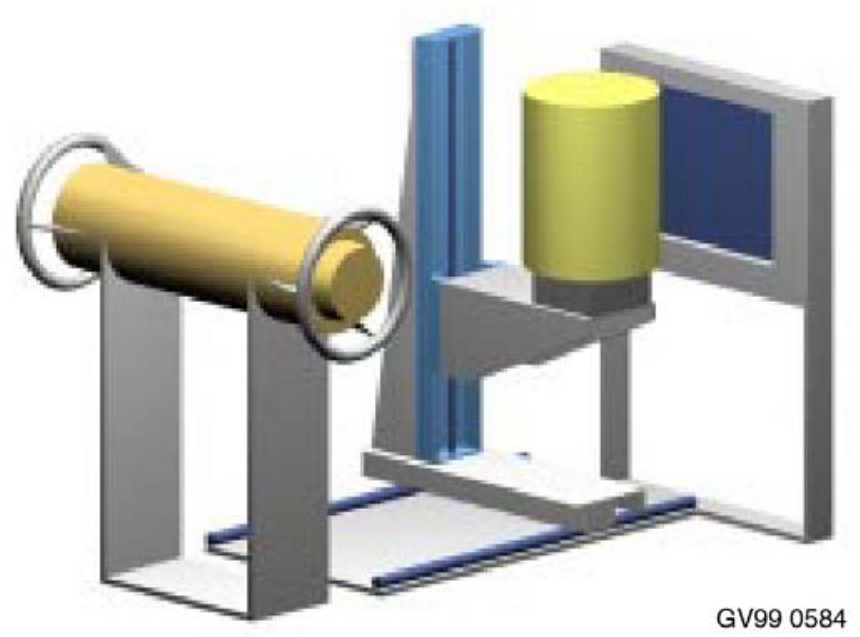

Figure 8. A conceptual drawing of the imaging system. The source is in the foreground on the left. The object manipulation stages (vertical translate, horizontal translate, and rotate) are shown in the center and the area detector is on the right.

functional, the interpreted nature of LabView ${ }^{\mathrm{TM}}$ and embedded Matlab ${ }^{\mathrm{TM}}$ subroutines resulted in slow data rates. An evaluation of different methodologies to improve data rates suggested that separation of the data acquisition and data processing tasks on different processors may be the best solution to the problem. A neural net, hardware-based approach would significantly increase data rates, but is believed to be less versatile for laboratory development tasks.

Model Based Data Inversion. The evaluation of model based data inversion to obtain electrical conductivity profiles continued via discussions with potential university collaborators. Topics being discussed include the objective of the data inversion effort, as well as collaborator interest and 
capabilities. Presently, no collaborative work has been initiated. The proposed effort will limit the inversion problem to conductivity in an effort to address bulk material characterization problems such as microstructure variations, structure (e.g., thickness of coating or laminations), and process parameters (e.g., temperature gradient profiles). The intent is to develop a real time/near real time inversion algorithm.

In FY 2000, emphasis will be placed on the demonstration of the neural network technology for actual 55-gallon drum test specimens. The significance of the effort is the eventual need to determine drum integrity for the continued safe storage of waste drums. The measurement problem consists of extracting the wall thickness from a pulsed, eddy current, time domain response containing various contributions, including probe lift-off/wobble, surface rust, magnetic permeability variations, and electrical conductivity variations. FY-99 efforts resulted in the demonstration of a neural network having the ability to determine wall thickness of carbon steel test samples with varying degrees of probe lift-off. FY 2000 efforts will include the integration of the neural network into a functional scanning system that can be used to demonstrate neural network, drum measurement capabilities. Additional test variables that need to be addressed include surface rust, labels, dents, seams, and pinholes. The existing neural network will be initially tested to determine its ability to address the added variables and modified as needed to overcome inadequacies.

\section{Three Dimensional Imaging}

The objective for this task is to develop capabilities to acquire and process 3-D images on objects that are difficult to access from all sides. This situation arises in spent nuclear fuel storage and many industrial-processing situations. The goals of the x-ray-imaging task in FY-99 were twofold. The first was to design and build a transportable imaging system capable of digital radiography (DR) and threedimensional (3-D) computed tomography (CT). The imaging system has been designed, and the components necessary to build it have been acquired. It will be assembled, and software to control motion, data acquisition and image display will be written in early in FY 2000. In the meantime, a prototype system has been used to collect initial conebeam CT data. These two imaging systems will be discussed in more detail in the Imaging Systems section below.

The second goal of this task was to conduct a set of experiments and simulations that will improve our ability to design efficient and effective imaging systems for use in challenging imaging situations. The prototype system described above has been used to investigate the effects of Compton scatter (White et al.) 12 and system misalignment (Noo et al. 1999)13 on image quality. Details of these two studies will be discussed in the sections Compton Scatter and System Misalignment below. Computer modeling experiments have been used to verify some of the results of the Compton scatter experiments. Another simulation package developed by collaborators at the University of Utah has been used to investigate system misalignment issues.

\section{Imaging Systems}

A conceptual drawing of the conebeam CT system that is being is shown in Figure 9. The system has been designed to be built on a desktop, but it is also meant to be easily transported and reassembled at other locations. An amorphous, silicon, flat-panel imager (FPI) will be used as the x-ray detector. This detector has the advantage of large area $\left(300 \times 400 \mathrm{~mm}^{2}\right)$, high spatial resolution $(0.127-\mu \mathrm{m}$ pixel pitch), and good dynamic range (12-bits/pixel). Initially, the system will use x-ray sources already available. Additional sources can be accommodated with minimum system modifications. 

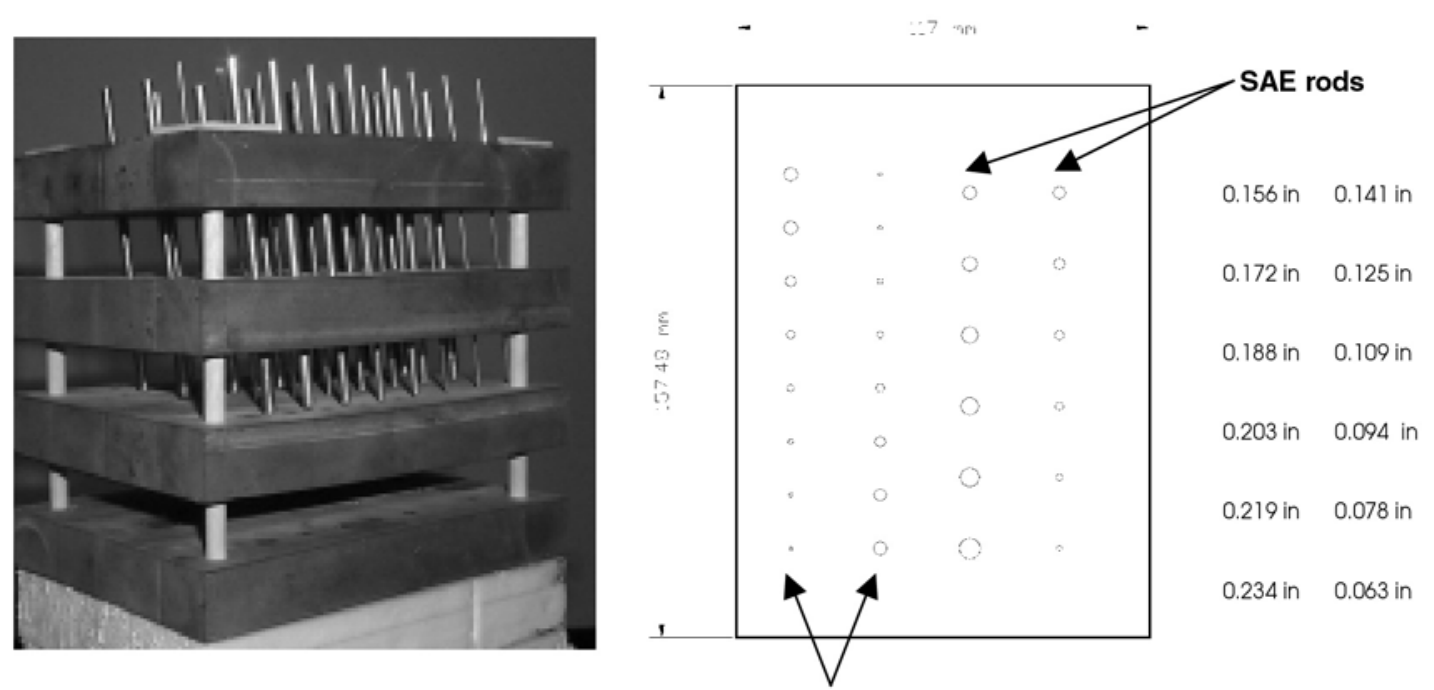

SI rods: $1.0,1.2,1.5,2.0,2.5,3.0,3.5,4.0 \mathrm{~mm}$

GV99 0584

Figure 9. The scattering object used in the comparison of scattering effects for fan and conebeam systems. The solid layers are a polyester resin with an array of 28 holes through each. The hole diameters vary from 1-mm to 6-mm. The upper two resin slabs have brass and stainless steel rods through the holes. The rods extend above the top slab and below the bottom of the second slab.

The imaging system will have three motorized axes of motion; the object stage can be rotated, translated vertically, and translated between the source and detector. Additionally, the source and detector can be closer to or further from each other. The rotation motion is essential for collecting radiographs of an object at multiple angles to be used for conebeam CT reconstruction. The vertical translate motion allows for data from helical trajectories to be collected. In the object's frame of reference, if the object is rotated and vertically translated as radiographs are collected, then the source and detector map out a helix. This motion is necessary for CT imaging if the object is taller than the maximum height of the detector. Also, data from a helical trajectory results in a more accurate reconstruction than data from a circular trajectory (where no vertical translation as data is collected) even for small objects. Translation of the object between source and detector allows for magnification of small objects onto the full area of the detector.

In order to perform initial CT experiments as the dedicated system was being developed, we used an existing prototype imaging system under development for a separate project. This system is highly configurable. It is capable of using $300-\mathrm{kVp} / 3 \mathrm{~mA}$ x-ray source with a 2-D FPI or a 1D linear diode array (LDA) as the detector. The system has a vertical translate stage for the source and detector and a rotate stage for the object and can thus collect 3-D CT data with either detector with either circular or helical orbits. (When using the LDA, the term spiral instead of helical is often used to describe data collected, using both vertical and rotational motions.) The system can also be configured with an isotopic source and single-element high-purity germanium detector to collect first-generation CT data. This system has been used to develop data collection and processing software that will be used on the dedicated system. All of the experiments described in this section have been performed on this system. 


\section{Compton Scatter}

Detection of Compton-scattered photons degrades radiographic and CT image quality. In a Compton-scatter event, an x-ray photon interacts with a free electron, imparting some of the photon energy to the electron; the photon leaves the interaction site at some angle relative to its incident direction. If this photon is detected, it reduces the contrast and image quality of the resulting radiograph or CT reconstruction. Compton scatter is a 3-D phenomenon-the photon can scatter into any direction.

One method used to minimize the detection of scattered photons is to collimate the x-ray beam and use a linear array of detector elements, as opposed to the area array discussed above. A high-density material is used to both limit the extent of the source to a planelike region and limit the field of view of the detector to the same planar region. Thus, only a small portion of the object is irradiated at a time; a smaller irradiated region means that fewer x-ray photons are scattered and a smaller percentage of the detected photons have been scattered. However, collecting a radiograph or projection data with a 1-D array of detectors takes longer and requires more motion and time than collecting data with a 2-D array. Thus, a fundamental question of system design involves the tradeoff between data collection time and the effects of image quality due to detecting Compton-scattered photons.

A set of experiments were performed (using the prototype imaging system) to investigate this tradeoff for 3-D CT imaging. In the first experiment, data were collected on a spiral orbit with the LDA. In the second experiment, the area detector was used and conebeam data were collected on a circular orbit. These two experiments provide the limiting cases for the effects of scatter: with the LDA, very few scattered photons are detected, while with the area detector, the number of detected scattered photons is much higher. In a third experiment, the area detector was collimated such that only a thin plane through the object was imaged. The third experiment verified that differences in the CT images from the first two experiments were due to scatter and not other detector differences (such as sensitivity or dynamic range).

The object that was imaged in these experiments was the scattering phantom shown in Figure 10. The phantom consists of six layers of polyester resin $\left(117 \times 157 \times 21 \mathrm{~mm}^{3}\right)$ separated by 21 -mm thick air regions. Each resin layer has an array of 28 different-sized holes; in the upper three layers, the holes are filled with stainless steel or brass rods. The array of holes or rods serves as a resolution test-what is the smallest-sized hole that can be imaged? The resin is a scattering medium; in the presence of scatter (when using an area detector instead of a linear array), the ability to resolve the smaller holes/rods should be diminished.

Slices of a 3-D reconstruction of the scattering phantom from experiments one and two are shown in Figure 10 and profiles through one line of the holes is shown in Figure 11. The slice from the fanbeam case (Experiment 1) shows better detail at the edges and better contrast resolution of the array of holes. This was an expected result - the images that include scatter are of inferior quality compared to scatterfree images. However, to collect data sufficient for a 3-D volume reconstruction requires much more time with an LDA, which only "sees" a plane through the object, than with an area detector. For these experiments, the conebeam data collection took longer because readout time for each image is about 6-sec; current FPI detectors have readout rates approaching 30-frames/sec.

These experiments represent two extremes for transmission CT, a 38-degree cone angle for the area-detector experiment compared to a 0.05 -degree cone angle for the fanbeam case. There is most likely a compromise system, a smaller cone angle, for which the detection of scattered photons does not compromise image quality. Researchers at the University of Utah and elsewhere have developed reconstruction algorithms for systems that collect data on a helical orbit, but for which the projection of the entire object does not fall upon the detector at one time. In FY 2000, experiments will be undertaken that will explore "opening up" the cone angle from the fanbeam case and using these reconstruction algorithms to attempt to optimize the collection-time versus scatter-degradation tradeoff. 


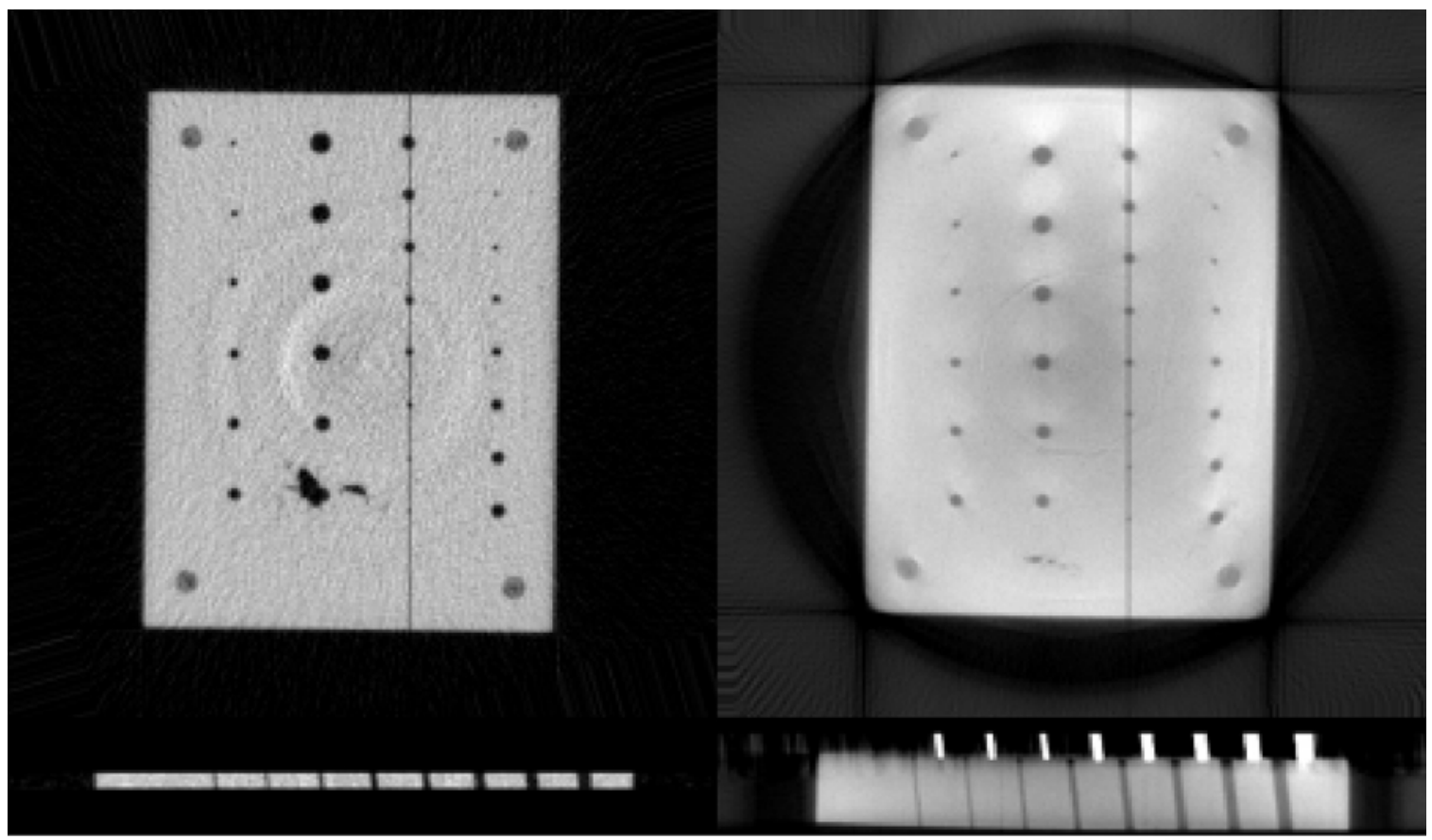

GV99 0585

Figure 10. Reconstructions of a slice of the scattering phantom from data acquired with the LDA (left) and the area detector (right). The upper part of the figure is a slice transverse to the rotation axis and at the bottom are slices parallel to the rotation axis. The edges of the object are better defined and the holes better resolved in the fan beam data.

\section{Profiles through holes}

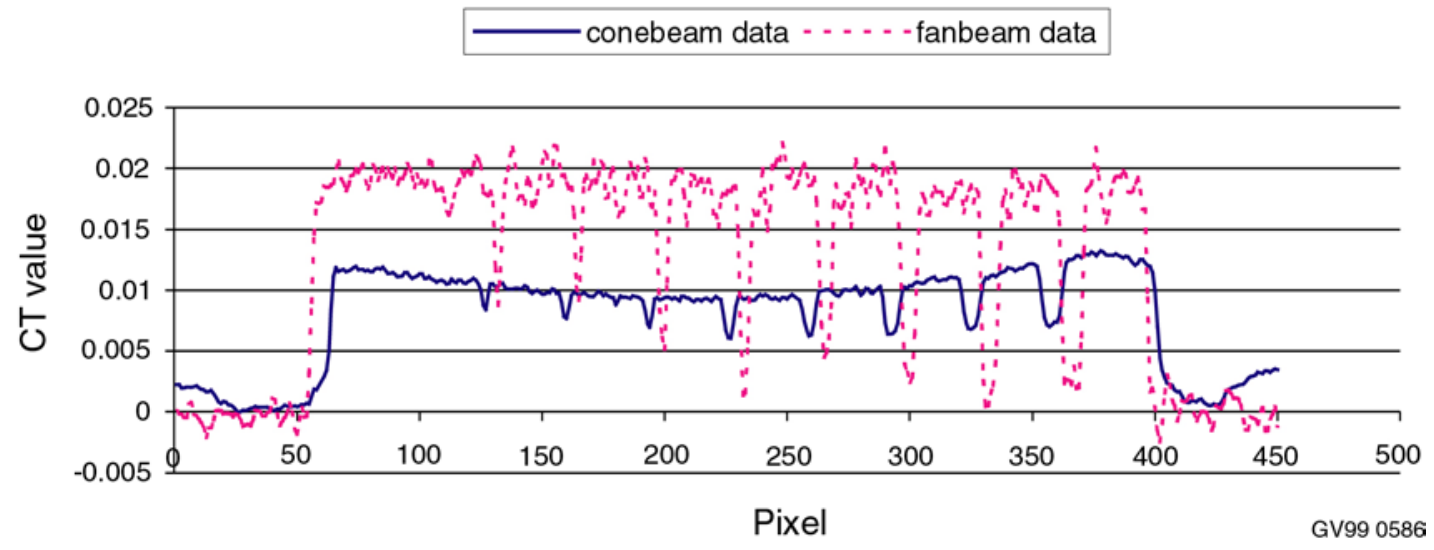

Figure 11. Profiles along a line containing holes in the two transverse slices of Figure 3 . Note that the contrast of the holes is improved for the fanbeam as compared to the conebeam case. 


\section{System Misalignment}

A CT imaging system consists of a source, detector, and a manipulation system for either the source/detector pair, object, or both. Exact system alignment is essential for high-quality CT imaging. For example, the axis of rotation is assumed to be parallel to the detector and perpendicular to the line from the source to the closest point on the detector. It is difficult and time consuming to build a perfectly aligned system, especially a system that is reconfigurable. However, if the relative positions of the components are known to a high accuracy and these positions are accounted for in the reconstruction algorithm, then high-quality reconstructions can still be achieved. Characterization of the alignment of CT systems and compensatory reconstruction algorithms are the focus of an ongoing collaboration with the Medical Imaging Research Laboratory (MIRL) at the University of Utah.

A conebeam imaging system that collects data on a circular orbit has seven parameters that describe the relative position of its components (see Figure 12):

- $\quad R$-the distance between the source and the center of rotation,

- $\quad D$-the distance between the source and the detector,

- $\left(u_{0}, v_{0}\right)$ - the point on the detector closest to the source,

- $\quad(\theta, \phi, \eta)$ —angles that describe the detector's orientation in space.

Researchers at MIRL have developed an alignment procedure that determines six of these seven parameters; the seventh parameter is fairly easy to align mechanically. The alignment algorithm is well conditioned mathematically. This is significant—all previous published alignment techniques have been ill conditioned and dependent upon a good initial estimate of the system parameters to converge on the correct solution.

The alignment procedure consists of collecting 20 to 80 projections of an object (phantom) that consists of two small steel spheres. As the object is rotated, the projections of the spheres map out two ellipses on the detector. The parameters of these ellipses are used to determine the system alignment parameters. The phantom and data collection are simple, and the algorithm takes only a few seconds to run, enabling this to be a routine procedure.

As an example of the need for system alignment, consider the images shown in Figure 13. These are reconstructed slices from the scattering phantom shown in Figure 10; the slices are near the top of the object where the metal rods extend above the top resin slab. In the image on the left, all system alignment parameters have been accounted for except the rotation of the detector in its plane $(\eta)$. In the image on the right, $\eta$ has been correctly accounted for. The cross sections of the rods, which should be small disks, are reconstructed as small donuts. The artifact is due to a misalignment in $\eta$ of $0.23^{\circ}$. It would be difficult to measure and mechanically correct for such a small angle in the laboratory.

Recently, the MIRL group has derived an algorithm for alignment of a conebeam system that collects data on a helical orbit. Movement of the source and detector vertically during data acquisition requires that two new parameters be added to the description of the system alignment. These two parameters describe the orientation of the axis of rotation relative to the source and detector. The algorithm requires a modification of the 2 -sphere alignment phantom to include a third sphere. Experiments to test this alignment procedure will be performed in FY 2000. 

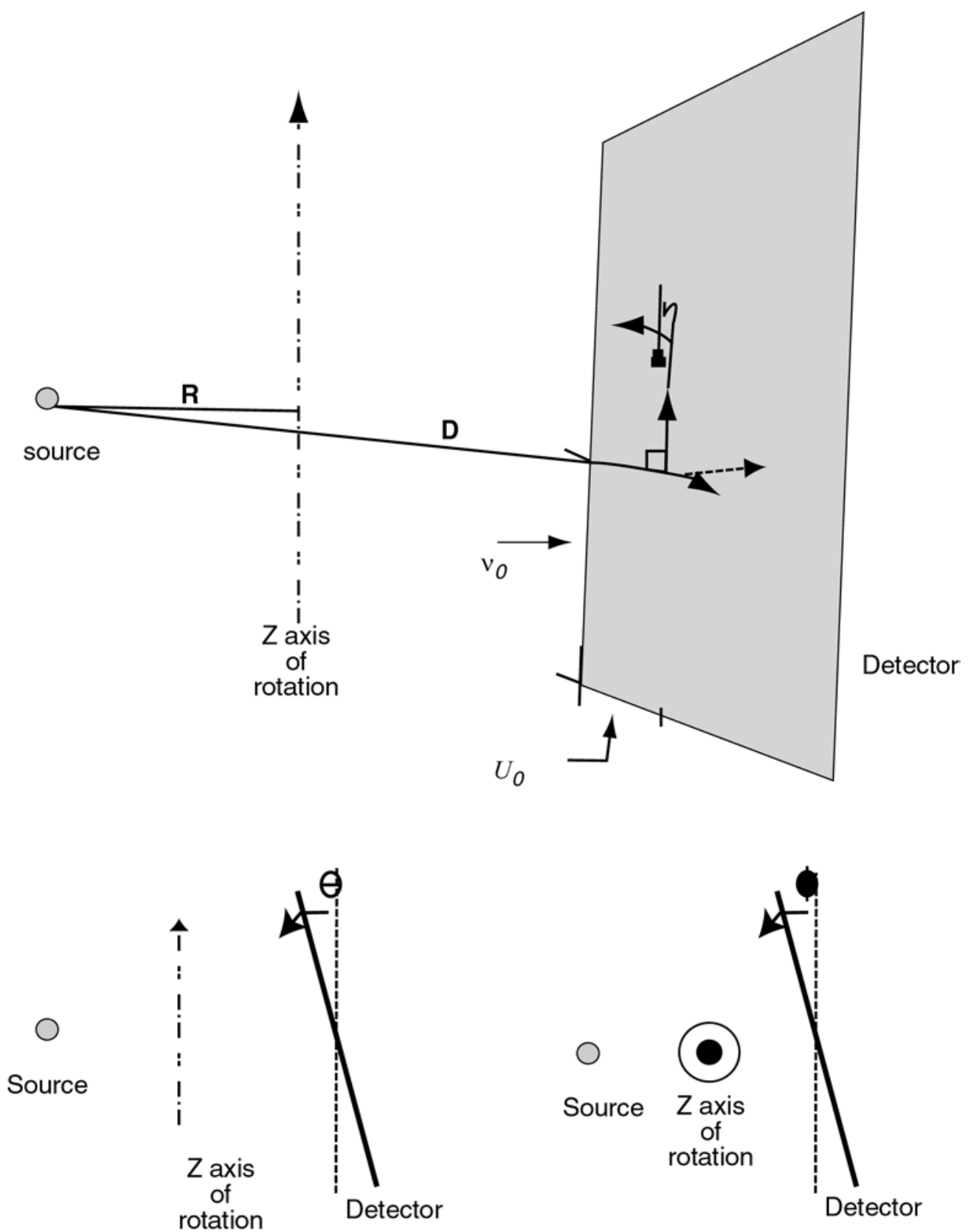

Side View

Top View

GV99 0587

Figure 12. The system alignment parameters for a conebeam system collecting data on a circular orbit only. 


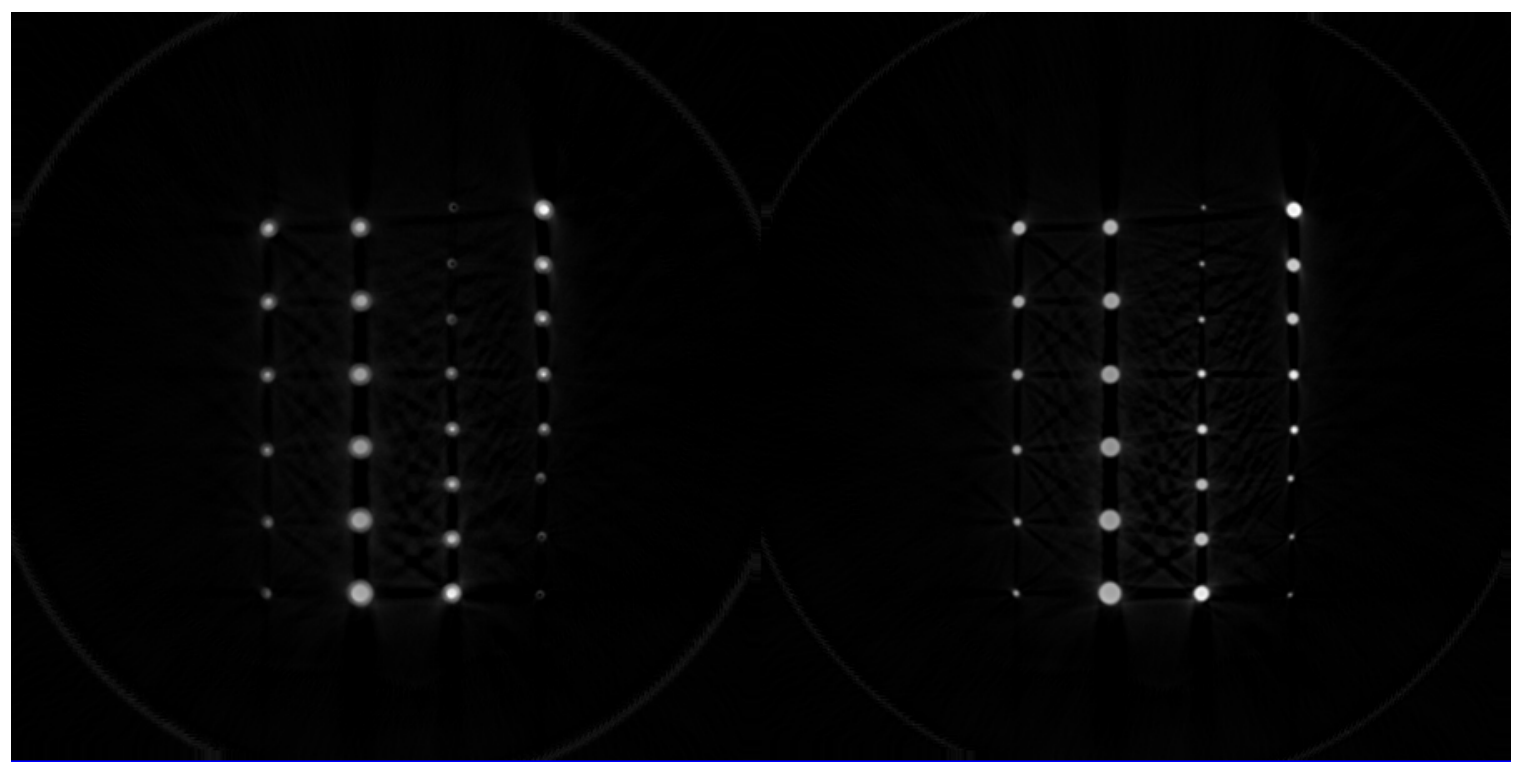

Figure 13. CT slices through the top part of the scattering phantom. The image on the left was reconstructed with incorrect alignment parameters and the effect is seen in the cross-sections of the rods appearing as "donuts" instead of disks. The image on the right was reconstructed using the correct alignment parameters.

Progress has also been made describing the alignment of a spiral CT system that uses an LDA. At first glance, this is a simpler alignment procedure, since there are fewer parameters (four instead of seven); however, it turns out to be a trickier problem. A phantom object, three spheres in a plane transverse to the axis of rotation, and an algorithm have been developed to address this problem.

\section{Development of 3-D Modeling-MCNP}

Concurrent with the need to develop good imaging systems is a need to develop computer models capable of simulating imaging situations. This capability is critical for the design of the imaging systems and the planning and interpretation of imaging experiments. The two programs we have used this year are MCNP and XRSIM. They are described below.

MCNP is a Monte Carlo particle transport program that can be used to simulate photon, neutron, and electron transport. Its code can be run in parallel and can be configured to run in either single processor or multiprocessor mode. The program was obtained for its capability to fully model the physics of x-ray transport, but had to be modified for use in modeling imaging problems as opposed to single particle ray tracing (or nonimaging problems).

MCNP source code was obtained from Oak Ridge National Laboratory and installed first on a PC, then on an SGI Origin2000, and then on the INEEL Beowulf Alpha cluster. Numerous modifications to the source code and the environmental variables were required for each installation. Many of the modifications were due to compiler variations. We could not obtain the recommended PC Lahey Fortran compiler because it is obsolete and no longer available. The other machines each had a different flavor of the GNU C compiler. Some changes were required by the operating system; the PC runs under NT, the SGI runs IRIX, and the Beowulf cluster runs LINUX. 
A radiography patch, developed at the Los Alamos National Laboratory (LANL) to simulate area $\mathrm{x}$-ray detectors, was obtained and integrated into each MCNP installation. Many test cases were run employing the capabilities provided by the patch. We reached the conclusion that the available singleprocessor computers were too slow to accurately simulate $\mathrm{x}$-ray scattering on an area detector.

An effort was made to configure MCNP to run in multiprocessor mode on the SGI Origin2000. This effort consumed a great amount of time and is yet to be successful. The conclusion drawn is that the parallel-processing enabling software, Parallel Virtual Machine (PVM), which is used by MCNP for message passing in multiprocessor mode, was incorrectly installed. There is no local support to resolve problems with PVM because it is considered obsolete. We moved on to install the program in multiprocessor mode on an available Beowulf Alpha cluster. With 16 nodes, the cluster is expected to be much faster than the SGI.

The Beowulf cluster presented a host of new problems. MCNP now seems to be installed correctly, but message passing between nodes is so slow that it makes the program useless. Again, the problem is with PVM, and there is no local support to help resolve the problem. An Oak Ridge PVM expert seems certain that the problem is with the ethernet connecting the nodes. The Beowulf system administrator doubts this because the cluster seems to work fine when MPI, a different message passing program, is used in other applications.

A patch was developed to permit the use of a fan beam x-ray source with MCNP. The standard MCNP version permits only x-ray sources that are isotropic within a conical region extending from the photon source. When a linear detector array is used, the vast majority of emitted photons never gets near the detector array, and thus has a negligible effect on the number of detected $\mathrm{x}$-rays. This is especially true when collimation is employed. MCNP is thus very inefficient and takes so long to simulate realistic problems that it is practically useless for modeling a linear detector array. The patch that was developed permits the simulation of a thin fan beam. Since most x-rays are emitted in the plane of the detector array, simulations are much more efficient because each photon now has a much greater chance of being detected.

Numerous simulations were run to study MCNP performance. Comprehensive models usually execute too slowly to be of use. Judgement is usually required in knowing what elements of the model can be omitted without seriously affecting the simulation outcome. For example, when a collimator is placed in front of a linear detector array, it usually suffices to restrict the vertical extent of the fan beam so that it just barely illuminates the collimator slit. In reality, an actual fan beam may illuminate a larger area of the collimator, but photons that strike the front surface of the tungsten collimators are almost never detected and can be omitted from the model. Omitting these unimportant photons speeds up execution.

True 3-D simulations of an area detector are impractical for all but the simplest of geometries. However, the magnitude of the scattering to be expected can be estimated quite accurately by using a cone beam and a linear array of detectors. Properly oriented, a linear array can provide a pretty clear understanding of the scattering that would occur if an area detector were used.

There are a lot of "wasted" photons in a cone beam. A fan beam with suitably limited vertical extent can closely approximate the effect of a cone beam while executing much more efficiently. Numerous simulations were conducted to study the tradeoffs between fan beam vertical extent, accuracy, and execution speed.

Another program, XRSIM, was obtained from Iowa State University. This program does not simulate scattering, but it executes much faster and has a much nicer user interface. In addition, it can 
model actual x-ray sources and actual detectors. With MCNP, it is difficult to correlate the number of photon histories with exposure by an actual x-ray source. Likewise, it is difficult to correlate the number of photon histories with the response of a real detector array or photographic film. XRSIM is currently installed on a SUN workstation and we are developing models of objects of interest to use in the simulations.

\section{ACCOMPLISHMENTS}

\section{Expert Systems}

Completed incorporation of all rules were relevant to the validation of the neutron assay system currently in use at the Radioactive Waste Management Complex (RWMC).

Completed prototype expert system and provided it to RWMC data validation physicist for review and use on real data.

\section{Electromagnetic Material Characterization}

Neural networks were developed, trained, and tested for the purpose of processing pulsed, eddy current data.

An eddy current data acquisition system, having neural network, data processing capabilities, was developed and tested.

The success of neural networks for determining wall thickness and probe lift-off was demonstrated.

\section{3-D Imaging in Constrained Environments}

Alignment parameters were successfully determined for the 2-D fan-beam and the 3-D cone-beam $\mathrm{x}$-ray imaging systems and were applied to laboratory systems with positive results.

The design for the 3-D x-ray imaging system was completed and components were acquired.

MCNP was successfully installed, modified, and used for radiographic projection simulation on a single processor UNIX machine.

\section{REFERENCES}

1. L. A. Zadeh, "Fuzzy Sets,” Information Control, Vol. 8, 1965, pp. 338-353.

2. J. C. Bezdek, W. Q. Li, Y. Attikiouzel, and M. Windham, "A Geometric Approach to Cluster Validity for Normal Mixtures," Soft Computing, Vol. 1, 1997, pp. 166-179.

3. S. L. Chiu, "Fuzzy Model Identification based on Cluster Estimation," Journal of Intelligent and Fuzzy Systems, Vol.2, 1994, pp. 267-278.

4. D. Goldberg, Genetic Algorithms in Search, Optimization, and Machine Learning, Reading, Massachusetts: Addison-Wesley Publishing Company, Inc., 1989. 
5. X. Xie, and G. Beni, “A Validity Measure for Fuzzy Clustering,' IEEE Transactions on Pattern Analysis and Machine Intelligence, Vol. 13, No. 8, 1991, pp. 841-847.

6. J. Gleick, Chaos: Making a New Science, New York: Viking, 1988.

7. A. L. Goldberger, D. R. Rigney, and B. J. West, "Chaos and Fractals in Human Physiology," Scientific American, Vol. 262, February 1990, pp. 42-49.

8. S. Ohno, "Codon Sequence is But an Illusion Created by the Construction Principle of Codon Sequence," Proceedings of the National Academy of Science 85 USA, 1988, pp. 4378-4386.

9. R. G. Wesson, Beyond Natural Selection, Cambridge, Massachusetts: The MIT Press, 1981.

10. J. Determan and J. Foster, "Using Chaos in Genetic Algorithms," Proceedings of the 1999 Congress on Evolutionary Computation, Vol. 3, pp. 2094-2101, Piscataway, New Jersey: IEEE Press, 1999.

11. F. C. Moon, Chaotic and Fractal Dynamics, an Introduction for Applied Scientists and Engineers, New York: John Wiley \& Sons, Inc., 1992.

12. T. A. White, T. J. Roney, R. J. Pink, F. Noo, R. Clackdoyle, M. Smith, and W. F. Jones, "Comparison of Fan- and Cone-Beam Imaging Capabilities on a Portable X-Ray Imaging System" in Developments in X-Ray Tomography II, Ulrich Bonse, Editor, Proceedings of SPIE, Vol. 3772, pp. 138-146.

13. F. Noo, C. Mennessier, R. Clackdoyle, T.A. White, and T.J. Roney, "Non-Iterative Methods for Scanner Calibration in Cone-Beam Tomography," Proceedings of the 1999 International Meeting on Fully Three-Dimensional Image Reconstruction in Radiology and Nuclear Medicine, Egmond aan Zee, The Netherlands, June 23-26, 1999, pp. 155-158.

\section{APPENDIX}

\section{Peer Reviewed Publications}

1. F. Noo, R. Clackdoyle, C. Mennessier, T. A. White, and T. J. Roney, "A direct analytic method for scanner calibration in cone-beam tomography," J. of Optical Society of America A (submitted September 1999).

2. J. C. Determan, G. K. Becker, "Automatic Expert System Rule Generation on Nondestructive Waste Assay Data at the Idaho National Engineering and Environmental Laboratory," Journal of Evolutionary Computation (submitted July 1999).

3. F. Noo, R. Clack, T. J. Roney, and T. A. White, "The dual-ellipse cross vertex path for exact reconstruction of long objects in cone-beam tomography," Phys. Med. Biol. 43, 1998, 797-810.

\section{Presentations}

1. J. T. Johnson, D. C. Kunerth, C. R. Tolle, and L. G. Allred, "Neural Network Processing of Pulsed Eddy Current Responses," ASNT Fall Conference, Phoenix, AZ, Oct 11-15, 1999.

2. J. C. Determan and J. Foster, "Using Chaos in Genetic Algorithms," 1999 Congress on Evolutionary Computation, Washington D. C., July 7, 1999. Proceedings of the 1999 Congress on Evolutionary Computation, Vol. 3, 2094-2101, Piscataway, New Jersey: IEEE Press 
3. J. C. Determan, "A Generic Expert System for Data Review/Validation of Nondestructive Waste Assay Data," $40^{\text {th }}$ Annual Meeting of the Institute for Nuclear Materials Management and Interface Working Group and Neutron Users Group (joint meeting), Phoenix, AZ, July 27-30, 1999.

4. F. Noo, R. Clackdoyle, T. A. White, and T. J Roney, "Image reconstruction from misaligned truncated helical cone-beam data," 1999 IEEE Nuclear Science Symposium and Medical Imaging Conference, Seattle, WA, October 1999.

5. T. A. White, T. J. Roney, R. J. Pink, M. Smith, R. Clackdoyle, and F. Noo, "Comparison of fanand cone-beam imaging capabilities on a portable x-ray imaging system," SPIE $44^{\text {th }}$ Annual Meeting, Denver, CO, July 1999. Proceedings of the SPIE, Vol. 3772-14 (to be published).

6. T. J. Roney, T. A. White, R. J. Pink, and M. Smith, "Field-Portable Gamma Ray and X-Ray Inspection Systems," $37^{\text {th }}$ Weapons Agency Nondestructive Testing Organization (WANTO) Meeting, Sandia National Laboratories, Albuquerque, NM, Feb 22-26, 1999.

7. D. C. Kunerth, C. R. Tolle, J. T. Johnson, L. G. Allred, "Neural Network Processing of Pulsed Eddy Current Responses," $37^{\text {th }}$ Weapons Agency Nondestructive Testing Organization (WANTO) Meeting," Sandia National Laboratories, Albuquerque, NM, Feb 22-26, 1999. 


\title{
Nondestructive Assay
}

\author{
Y. D. Harker, J. D. Cole, R. J. Gehrke, and J. W. Mandler \\ SUMMARY
}

The overall objective of this project is to advance the state-of-the-art in nondestructive assay (NDA). The main thrust is research on developing new signatures that will improve NDA in two ways: (1) provide more detail concerning specific nuclide concentrations and (2) improve the overall accuracy of the data. The scope of work for FY 1999 includes prompt fission fragment signature measurements, actinide gamma-ray spectrometry, prompt gamma neutron activation analysis, and advanced methods development for analyzing passive gamma-ray based NDA data. There are four highlights for FY 1999.

First, the correlation matrix for ${ }^{235} \mathrm{U}$ fission fragment decay data has been developed and is now out for peer review. Raw data to produce a similar matrix for ${ }^{239} \mathrm{Pu}$ fission fragment decay has been accumulated. Several journal publications and presentations have been produced about the physics of fission, the decay of prompt fission products, and the use of prompt fission data in NDA.

Second, reference quality actinide gamma-ray spectral data have been produced. These data are now included in the U.S. Evaluated Nuclear Decay Scheme File and the INEEL Gamma-Ray Spectrum Catalogue. Gamma-ray spectra, taken by this project, from aged sources of ${ }^{233} \mathrm{U}$ and ${ }^{241} \mathrm{Am}$ have led to improvements is analyzing gamma-ray spectra from real transuranic waste. This information has been shared with the assay group at Radioactive Waste Management Complex (RWMC).

Third, the Monte Carlo radiation transport code, CEARPGA, which was developed by North Carolina State University (NCSU) for use in analyzing prompt gamma neutron activation analysis (PGNAA) data, was modified for use in INEEL (and DOE complex) applications.

Fourth, an analytical gamma-ray transport code was developed and tested for use in real-time analysis of gamma-ray based NDA. The techniques developed here have been shared with the assay group at the RWMC and their analysis code is being modified, by their software development group, to accommodate the new analysis methods.

\section{PROJECT DESCRIPTION}

\section{Fission Research}

Fission research studies are in progress using the IPNS at ANL-E. These studies support the development of a new concept in NDA where the $\gamma-\gamma, \gamma-\gamma-\gamma$, and $\gamma$-n coincidence identifies the occurrence of individual fission events. Because of the coincidence nature of the measurement process, the prompt gamma-ray spectra can be used to uniquely identify the prompt fission fragments. The sum of the prompt fission fragment atomic numbers indicates directly the atomic number (i.e., element) of the fissile nucleus. Through an accumulation of data from several individual fission events, neutron multiplicity and isotopic distributions are determined as well. Since this approach is very new, the data to support its development and use is virtually nonexistent. During FY 1998, the fission experiment at IPNS commenced with ${ }^{235} \mathrm{U}$ fission being the reaction of interest. The apparatus to perform those measurements is shown in Figure 1. In this experiment there are up to eight Compton suppressed highpurity germanium (HPGe) detectors and liquid scintillation neutron detectors surrounding the fission target, which is located in an IPNS neutron beam line in the center of the detector array. 
The data acquisition phase of the ${ }^{235} \mathrm{U}$ fission experiment was concluded in December 1998. The monumental task of data analyses was initiated in FY1999 and at this point a correlation matrix for use in fissile nuclide identification is nearing completion. In addition to the correlation matrix, these fission fragment signature measurements are providing new data about the physics of fission. Meanwhile, new measurements were initiated using the IPNS experimental apparatus with a target of ${ }^{239} \mathrm{Pu}$. Data acquisition for neutron induced fission of ${ }^{239} \mathrm{Pu}$ was completed in August 1999. For FY 2000, the next fissile nuclide to be studied at IPNS is ${ }^{233} \mathrm{U}$.

Analysis of the ${ }^{252} \mathrm{Cf}$ data from the Gammasphere continues as a part of this research because the quality of these data are very good and it supports our current emphasis on neutron induced fission. Recent examination of the raw data from this experiment using the new methods to correct electronic drifts and screen bad events shows that the statistics in the correlation matrix can be increased by possibly as much as a factor of three.

The contracts for the new detectors and Compton suppression shields were placed in FY 1999. These units will replace the detectors and shields that were borrowed from ANL/Physics at the beginning of these studies. The new detectors and shields will be delivered in FY 2000. EG\&G Ortec and Bicron are the suppliers of the detectors and shields, respectively. The new detector installation will be a permanent part of the IPNS facility. This is seen as a difficult task due to the restricted budgets available in DOE.

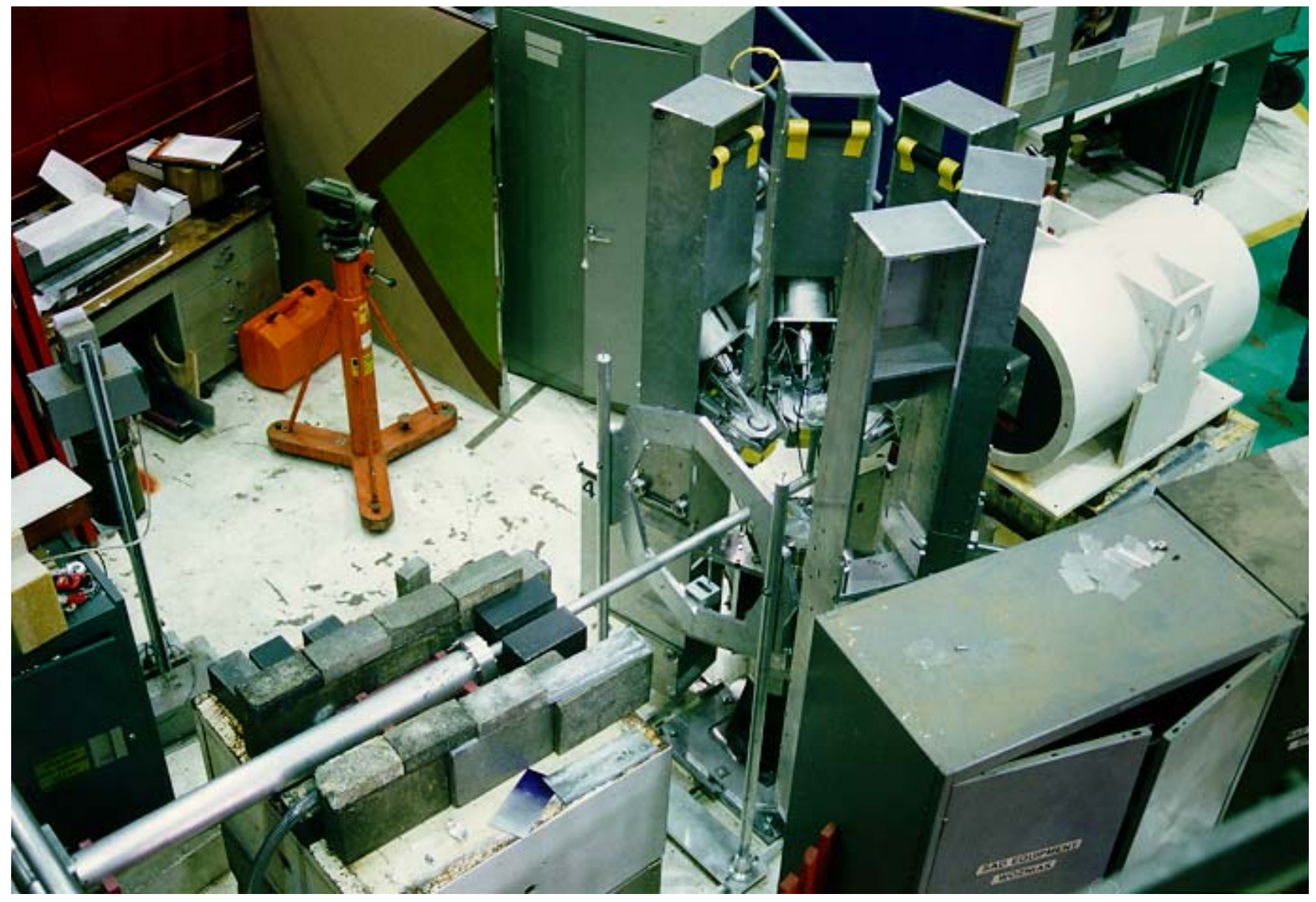

Figure 1. INEEL fission experiment at the intense pulsed neutron source. 
The archiving of the raw data sets for all the fission experiments (this represents approximately 600 gigabytes of data) began in FY 1999. These data are being written to 19-mm tapes by a special tape drive. This media has a minimum 10-year retention life.

The DOE/IPP program money was received at INEEL for funding the Russian scientists as part of the international fission physics collaboration. The work to put a contract in place is long and involved and it is hoped that this will be finished in early FY 2000.

The ${ }^{233} \mathrm{U}$ target for the IPNS experiment was obtained in FY 1999 and shipped to IPNS. This is 3.2 gm of $90 \%$ plus enriched material. This will be the target for most of FY 2000. The next target element after ${ }^{233} \mathrm{Ci}$ is ${ }^{237} \mathrm{~Np}$. A design and requirements for a $\mathrm{Np}-237$ target was sent to Scientific Research Institute for Atomic Reactors (Dmitrovgrad) - also known as the All-Russian Institute of Atomic Reactors (NIIAR) — as they can fabricate the neptunium disk for a low cost.

\section{Significant Fission Experiment Results}

Low-energy fission, i.e., spontaneous fission or induced fission by thermal or low energy neutrons, is an important area to the study of nuclear physics. Although the study of fission has been overshadowed to some extent by advances in accelerators and in-beam studies, fission remains an important method to study the rearrangement of large groups of nucleons from one nucleus into two nuclei.

Fission Yields. There is now renewed interest in remeasuring fission yields. This is a result of largescale improvements in detectors, electronics, and automated data processing techniques that have occurred since the yields were originally measured via fast chemistry and beta decay. The new measurements have yielded new detailed information about the prompt fission fragment production. With the use of large arrays of Compton suppressed high-purity germanium detectors, coincidence measurements have been made and matrices of the prompt $\gamma-\gamma$ and $\gamma-\gamma-\gamma$ coincidences emitted from the fission fragments have been built. An early paper from our collaboration ${ }^{1}$ presented the first such results and drew attention to these data for the theoretical community. ${ }^{2}$ With this interest, a more detailed analysis was made by the collaboration and yield data of the new form was presented ${ }^{3}$ for 139 fragment pairs from the fission of ${ }^{252} \mathrm{Cf}$. Table 1 is the result of this for the Ba-Mo pair.

Table 1. Yields of correlated fragment pairs of Mo-Ba in the spontaneous fission of ${ }^{252} \mathrm{Cf}$. Yields are given in percent, i.e., in number of pairs per 100 fission events. The last line of the table (i.e., $\bar{v}$ ) lists the mean number of neutrons evaporated from the primary fragments. Data in this table are from

Reference 3.

\begin{tabular}{|c|c|c|c|c|c|c|c|c|c|c|}
\hline & ${ }^{138} \mathrm{Ba}$ & ${ }^{140} \mathrm{Ba}$ & ${ }^{141} \mathrm{Ba}$ & ${ }^{142} \mathrm{Ba}$ & ${ }^{143} \mathrm{Ba}$ & ${ }^{144} \mathrm{Ba}$ & ${ }^{145} \mathrm{Ba}$ & ${ }^{146} \mathrm{Ba}$ & ${ }^{147} \mathrm{Ba}$ & ${ }^{148} \mathrm{Ba}$ \\
\hline${ }^{102} \mathrm{Mo}$ & & & & & $0.02(2)$ & $0.04(3)$ & $0.09(6)$ & $0.13(5)$ & $0.10(7)$ & $0.06(4)$ \\
\hline${ }^{103} \mathrm{Mo}$ & & $0.05(3)$ & $0.07(2)$ & $0.02(2)$ & $0.13(9)$ & $0.67(10)$ & $0.86(20)$ & $0.46(8)$ & $0.40(30)$ & $0.12(9)$ \\
\hline${ }^{104} \mathrm{Mo}$ & $0.08(3)$ & $0.18(4)$ & $0.34(4)$ & $0.36(4)$ & $0.48(10)$ & $1.14(4)$ & 0.74 & $0.39(4)$ & $0.23(17)$ & $0.04(3)$ \\
\hline${ }^{105} \mathrm{Mo}$ & $0.02(2)$ & $0.07(5)$ & $0.11(4)$ & $0.65(10)$ & $1.05(25)$ & $1.30(11)$ & $0.59(17)$ & $0.13(7)$ & $0.23(15)$ & \\
\hline${ }^{106} \mathrm{Mo}$ & $0.01(1)$ & $0.12(3)$ & $0.44(3)$ & $0.92(4)$ & $0.88(10)$ & $0.65(4)$ & $0.16(8)$ & $0.08(5)$ & & \\
\hline${ }^{107} \mathrm{Mo}$ & $0.02(2)$ & $0.12(4)$ & $0.11(3$ & $0.35(16)$ & $0.14(8)$ & $0.13(8)$ & $0.15(7)$ & & & \\
\hline${ }^{108} \mathrm{Mo}$ & $0.02(1)$ & $0.06(3)$ & $0.10(3)$ & $0.14(5)$ & $0.12(10)$ & $0.06(5)$ & & & & \\
\hline$\sum Y_{\mathrm{Ba}}$ & $0.15(6)$ & $0.60(9)$ & $1.17(10)$ & $2.44(20)$ & $2.82(29)$ & $3.99(19)$ & $2.58(32)$ & $1.21(14)$ & $0.96(37)$ & $0.22(10)$ \\
\hline $\bar{v}$ & $9.5(2.0)$ & $6.5(1.0)$ & $5.6(6)$ & $4.3(4)$ & $3.7 .(5)$ & $3.4(2)$ & $2.9(4)$ & $2.3(3)$ & $1.4(5)$ & $1.1(5)$ \\
\hline
\end{tabular}


This table presents entirely new information giving details on every combination of Ba-Mo pairs and neutron multiplicity directly. In addition, when this information is coupled to the $\gamma$-ray spectroscopy information, specific results are obtained on deformation of the fragments, the angular momentum distribution on each charge pair, excitation energy of the fragments, and a better determination of the total kinetic energy for the fragments. These results are presented in detail in Reference 3.

Another interesting and unexpected result has to do with "cold fission" or "cold compact fission." These are cases where the fragments are formed near their ground state configuration and no neutrons are emitted. The data for these cases are so far rather sparse so that it is difficult to draw general conclusions about the fission process.

Nuclear Molecules. An excellent survey ${ }^{4}$ of the concept of nuclear molecules was done in 1997 that can be used as a reference, so this report will not repeat the detail of that general survey. The basic concept of a nuclear molecule is that there are two or more centers of charge of nuclear material that are separated by less than a nuclear radius. This results in two nuclei that seem to behave as one, yet they display properties, such as $\gamma$-ray emission, of independent nuclei. This structure is generally seen in scattering experiments like those described in the review paper cited. ${ }^{4}$ We have reported ${ }^{5}$ ternary fission from the ${ }^{252} \mathrm{Cf}$ studies, but with an unusual aspect. The third fragment, ${ }^{10} \mathrm{Be}$, emits a $\gamma$ ray that is not Doppler-broadened. When ternary fission occurs, the lightest fragment moves away from the two massive fragments with speed sufficient to cause the observed $\gamma$-ray line widths to appear wider in the spectra than those lines emitted from a stationary nucleus. In addition to the primary $\gamma$ ray in the ${ }^{10} \mathrm{Be}$ that is the $2^{+} \rightarrow 0^{+}$transition, we also observe a very weak $\gamma$ ray with an energy close to the $2^{+} \rightarrow 0^{+}$transition energy and in coincidence with the other radiation from the fission. The statistics of these particular events are very low and this makes for a weak case. A paper has been prepared and submitted for publication with the claim that we are observing a nuclear molecule.

Figure 2 is a representation of the configuration proposed for the nuclear molecule and the potential energy well for the ${ }^{10} \mathrm{Be}$. The nuclear potential of the beryllium is deformed by the presence of the two larger fragments and the energy of the ground state transition is reduced. The solid curve in the figure represents the case for the beryllium not in the molecule and the dashed curve represents the case in the molecule.

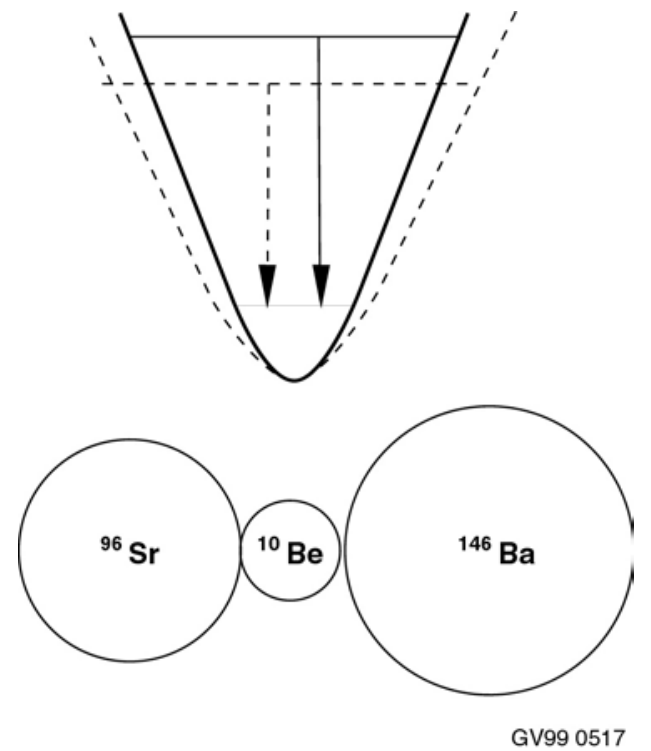

Figure 2. Fragment arrangement and nuclear potential. 
There are several significant points to notice in this claim. The three fragments must stay close together for approximately 1 picosecond for this to occur. The nuclear and electric fields of the large fragments must perturb the nuclear potential of the ${ }^{10} \mathrm{Be}$ to cause the shift in energy. The nuclear molecule is a linear molecule with the ${ }^{10} \mathrm{Be}$ being formed in the neck of the fissioning nucleus. This is a large expansion on the previously reported examples of nuclear molecules and is the first such case reported in fission. A significant point to the nuclear molecule explanation is that it supports multiple charge centers in the nucleus. This approach has been critical in explaining fission and is an important basis for cluster nuclei, i.e., nuclei composed of clustering or building blocks of lighter nuclei.

Future of the Experiments-The fission experiments will yield detailed information on the fission process to produce a much more complete fundamental picture of fission and nuclear structure. These results will provide better information in applied areas such as NDA methods currently being developed and for the use in nuclear engineering by providing more accurate and detailed parameters for use in modeling and designing nuclear systems.

The data sets that are being obtained from the fission experiments are self-consistent studies that allow direct comparison of all observed fission channels for a particular fissile isotope. Although the results of 139 product pairs are presented in Reference 3, this represents the results from only five sets of fragment pairs of Pd-Te, Ru-Xe, Mo-Ba, Zr-Ce, and Sr-Nd. This is a small number when you consider all the pairs in the fission of ${ }^{252} \mathrm{Cf}$. There are many more pairs yet to be analyzed. The induced fission analysis is just beginning on this phase.

Particular signatures for use in NDA can be extracted relatively quickly, but the detailed analysis of these induced fission experimental data will require the continued work of the current members of the collaboration for several years.

\section{Actinide Gamma-Ray Spectrum Measurements}

In the DOE complex there are needs for better data to support NDA focused on transuranic waste and spent nuclear fuel characterization. To meet this critical need, this project has undertaken a task to evaluate actinide gamma-ray spectra and decay data and to perform new spectrum measurements using current state-of-the-art spectrometers. The collection of pure actinide gamma-ray spectra covering the energy range from 10 to $1,500 \mathrm{keV}$ has been the focus of this research task and the emphasis is on identifying all peaks, including artifact peaks. Spectra of actinides with and without their progeny are collected. Those actinides commonly found in TRU and other radioactive waste, as well as actinides and their progeny being considered for medical applications, are being studied. Because of this project's close ties to the TRU waste and spent nuclear fuel characterization activities ongoing at INEEL, there have been several opportunities to transfer technology to the production programs at INEEL using NDA (and to a lesser extent to the rest of the DOE complex). As an example, the measurement of ${ }^{233} \mathrm{U}$ gammaray spectra (for fresh and aged ${ }^{233} \mathrm{U}$ ) has produced an improved method for ${ }^{233} \mathrm{U}$ analysis. This method was made available to the INEEL 3100 Cubic Meter project and it is now part of the analysis code used to assay TRU waste barrels at the Stored Waste Examination Pilot Plant (SWEPP). Currently, this project is working on a method to measure the age of the ${ }^{241} \mathrm{Am}$ in TRU waste and/or determine the presence of ${ }^{237} \mathrm{~Np}$ in excess of that from ${ }^{241} \mathrm{Am}$ decay.

An early accomplishment for this task in FY 1999 occurred during the systematic acquisition of gamma-ray spectra taken on aged ${ }^{233} \mathrm{U}$. The presence of ${ }^{233} \mathrm{U}$ progeny were observed, including all gamma-ray emitting members of the decay chain to ${ }^{213} \mathrm{Po}\left({ }^{209} \mathrm{~Pb}\right.$, the daughter of ${ }^{213} \mathrm{Po}$, is a pure beta emitting radio-nuclide decaying to stable ${ }^{209} \mathrm{Bi}$ ). Of particular interest is a very strong ${ }^{213} \mathrm{Bi}$ peak at $440 \mathrm{keV}$. These data have been valuable in helping analysts involved in data review at the SWEPP to interpret TRU waste drum assay data. When the presence of ${ }^{233} \mathrm{U}$ is suspected in the waste, the protocol 
now incorporates looking for the $440-\mathrm{keV}$ gamma-ray peak from the decay of the daughter ${ }^{213} \mathrm{Bi}$. This gamma ray does not appear to have any interference from other radioactinides and it is strongly present in spectra of waste where the progeny of U-233 have had an opportunity to grow in. After several years of decay, the ${ }^{213} \mathrm{Bi}$ gamma ray dominates the region of the spectrum above $\sim 400 \mathrm{keV}$.

A second significant accomplishment during FY 1999 was the upgrade, installation, and calibration of a new HPGE gamma-ray spectrometer for the acquisition of high-quality gamma-ray spectra. A 50-mm diameter by 20 -mm thick coaxial, $\mathrm{n}$-type, Ge detector was chosen so that gamma rays covering an extended energy range from $\sim 8 \mathrm{keV}$ to over $1,500 \mathrm{keV}$ could be acquired with one spectrometer. To reduce the thickness of the diode junction so that the detector can be used to detect the low-energy photons common in the decay of many actinides, the junction is ion implanted. Use of the portable InSpector $^{\text {TM }}$ analyzer was replaced by a laboratory-grade multichannel analyzer consisting of a Canberra Acquisition Interface Module (AIM) and a Model 8713 analogue-to-digital converter equipped with an INEEL pulser control logic circuit and an INEEL low-power pulser to provide pulse injection with subsequent removal technology. An EG\&G Ortec 672 amplifier was installed along with a new workstation for controlling the low-energy germanium (LEGE) spectrometer and for archiving data. These upgrades improved the system stability and spectrum quality, but not without much time and effort to identify and fix some large gain and zero shifts and later some small gain instabilities that would not have been detectable if we had not installed the pulser system. This "permanent" installation also simplifies the use of the Ge spectrometer. PCGAP, the Ge spectrometer control and gamma-ray analysis program, was modified to control the AIM and allow interactive analysis of the spectra and storage of the data. Finally, an artifact peak at $61.5 \mathrm{keV}$ did not leave the spectrum until the time constant was changed from 4 to 2 microseconds.

A second Ge spectrometer is being set up so that spectral data can be collected on two sources simultaneously. This spectrometer should be operational by the end of the first quarter of FY 2000.

Third, over the course of the year spectra were acquired and ENSDF $\gamma$-ray energies and intensities were downloaded for the following radioactinides: ${ }^{233} \mathrm{U},{ }^{239} \mathrm{Pu},{ }^{240} \mathrm{Pu},{ }^{242} \mathrm{Pu}$, and ${ }^{241} \mathrm{Am}$. In addition, some data were acquired on ${ }^{235} \mathrm{U},{ }^{237} \mathrm{~Np},{ }^{238} \mathrm{Pu}$, and ${ }^{243} \mathrm{Am}$. Difficulties are being encountered in the preparation of a quality ${ }^{237} \mathrm{~Np}$ source due to the physical and chemical form of the source material.

As a result of a number of cleanup campaigns, high-purity sources of many important radioactinides are no longer available at the INEEL. Therefore, discussions have been held with Charles Alexander and after a visit to Alexander and Bigelow at Oak Ridge National Laboratory (ORNL) it was decided that it would be more efficient to collect many of these less common radioactinides at ORNL. It was suggested that we could minimize cost by only using source material from their supply and returning it in suitable chemical form after collecting the spectra. The radionuclides of interest are ${ }^{231} \mathrm{~Pa},{ }^{234} \mathrm{U},{ }^{236} \mathrm{U}$ ${ }^{244} \mathrm{Pu},{ }^{243} \mathrm{Cm},{ }^{244} \mathrm{Cm},{ }^{245} \mathrm{Cm},{ }^{249} \mathrm{Cf}$, and ${ }^{249} \mathrm{Bk}$. In addition, we may wish to acquire spectra of ${ }^{227} \mathrm{Ac}$ and ${ }^{237} \mathrm{~Np}$ if we cannot obtain quality spectral data at the INEEL. Alexander and Bigelow would also be interested in a collaboration to develop ${ }^{245} \mathrm{Cm}$ as a very good neutron dosimeter and in the production of ${ }^{236} \mathrm{Pu}$ from ${ }^{237} \mathrm{~Np}$ via a $(\gamma, \mathrm{n})$ reaction.

Information and source preparation materials to be used for the preparation of radioactinide sources for the acquisition of high-quality actinide spectra to be acquired at ORNL this fall/winter have been sent to Alexander at ORNL. Discussions on the best path forward for the preparation and acquisition of spectra of ${ }^{237} \mathrm{~Np}$ have taken place. Specifically, discussions focused on whether to continue efforts to

\footnotetext{
${ }^{\text {TM }}$ Canberra Industries, Inc., 800 Research Parkway, Meriden, CT 06450.
} 
dissolve a high-purity bead of calcined $\mathrm{Np}_{2} \mathrm{O}_{3}$ or to identify another source of ${ }^{237} \mathrm{~Np}$ at INEEL or at ORNL. Present thinking is to obtain a quality spectrum of ${ }^{237} \mathrm{~Np}$ with daughters at ORNL in FY 2000.

Efficiency calibration of the two Ge detectors to be used to study actinides will be measured for two distances: 10- and 20-cm. Specially prepared point-source standards of ${ }^{155} \mathrm{Eu},{ }^{241} \mathrm{Am}$, and ${ }^{57} \mathrm{Co}$ are being purchased to allow efficiency calibration below $122 \mathrm{keV}$ (i.e., from $\sim 10$ to $122 \mathrm{keV}$ ).

Finally, a method for deducing the "age" of actinide waste containing ${ }^{241}$ Am was developed during the past summer while collecting quality spectra of ${ }^{241} \mathrm{Am}$ and its progeny. An Excel spreadsheet calculating the grow-in of ${ }^{233} \mathrm{~Pa}$ from a ${ }^{241} \mathrm{Am}$ parent shows that the elapsed time from being freshlyseparated can be determined from a several hour count of a 10 to 20 microcurie source of ${ }^{241} \mathrm{Am}$ at $20 \mathrm{~cm}$ from a $\sim 15 \%$ LEGE detector. The ratio of the $322-\mathrm{keV}$ and $312-\mathrm{keV}$ peak areas of ${ }^{241} \mathrm{Am}$ and ${ }^{233} \mathrm{~Pa}$, respectively, can be measured to an accuracy of $\sim 2$ to $4 \%$ (one sigma) for a 10-hour count and converted to a grow-in time. This grow-in time can be calculated to an accuracy of about 1 year for a $\sim 20$ year grow-in of the ${ }^{233} \mathrm{~Pa}$ granddaughter. It should also be noted that the parent:daughter ratio of emission rates is a very effective way to determine if the ${ }^{237} \mathrm{~Np}$ present is in excess of the amount from grow-in. The Rocky Flats Environmental Site could use this technique right now.

A draft of a paper is being written that applies this finding to real TRU waste drums. Most of the TRU waste from the Rocky Flats Plant contains several other actinide radionuclides, including ${ }^{239} \mathrm{Pu},{ }^{235} \mathrm{U}$, ${ }^{238} \mathrm{U}$, and other actinides in smaller activity concentrations. The paper being written will suggest how the various potential contaminating radioactinides with their interfering $\gamma$ rays can be removed from the ${ }^{233} \mathrm{~Pa}$ and ${ }^{241} \mathrm{Am} \gamma$ ray peaks of interest.

During FY 1999, R. J. Gehrke was appointed to the Transuranium Research Isotope Users Committee, which provides input and guidance for the production, distribution, and use of transuranium isotopes for research purposes. Membership on this committee is a valuable asset to the INEEL NDA and nuclear structure research since it keeps us informed on the transuranium isotopes in the ORNL inventory, the isotopes being produced, and the schedule of their availability. Radioisotopes from ${ }^{238} \mathrm{Pu}$ to ${ }^{257} \mathrm{Fm}$ are prepared and available through this committee. R. J. Gehrke organized the Russell Heath Memorial Session, "Advances in X- and Gamma-Radiation Detectors" for the ANS 1998 Winter Meeting in Washington, DC, held November 12-17, 1998.

\section{Prompt Gamma Neutron Activation Analysis}

The Portable Isotopic Neutron Spectroscopy (PINS) technique has been successfully developed by INEEL as an in-field chemical agent (weapons) identification system. There is a thrust in this project to take the PINS concept one step further and make it a true field deployable NDA technique. That is, make the PINS concept capable of doing quantitative elemental analysis in the field. The general process on which PINS is based is the prompt gamma neutron activation analysis (PGNAA). The major concerns in determining quantitative elemental concentrations using PGNAA are (1) inadequate data on $\gamma$-ray production yields, (2) uncertainty concerning attenuation of the interrogating neutrons, and (3) uncertainty concerning the attenuation of activation gamma rays. The current emphasis in this area is on modeling the interrogation neutron transport and activation-gamma-ray transport for materials and geometry applicable to DOE site characterization and industrial process control applications. From these transport studies, analysis methods will be developed that equate measured signals (gamma spectrum peak areas) to elemental concentration. This project has teamed with North Carolina State University (NCSU), who have been working in this area for some time and have developed transport codes (models) that can be extended to the applications of interest to our program. 
NCSU developed modifications to their CEARPGA computer program to allow additional geometries, including a large planar sample. They also initiated (and nearly completed) modifications to add the INEEL PINS geometry. Modifications to implement this geometry are difficult because the axis of the sample is normal to the axis of the detector, which is a configuration nonstandard for the CEARPGA program.

PGNAA $\gamma$-ray spectra from a suite of individual elements and compounds (such as $\mathrm{Fe}, \mathrm{SiO}_{2}, \mathrm{Al}_{2} \mathrm{O}_{3}$, $\mathrm{CaO}$, and $\mathrm{H}_{2} \mathrm{O}$ ) and from mixtures and samples of various phosphate ores were obtained using both a ${ }^{252} \mathrm{Cf}$ neutron source and a ${ }^{241} \mathrm{Am}-\mathrm{Be}$ neutron source. These spectra were measured for purposes of evaluating the CEARPGA program. Gamma-ray spectra to be used for the determination of Ge detector response functions were also obtained.

The CEARPGA program was implemented at INEEL during FY 1999. NCSU moved CEARPGA to a PC platform for use at INEEL, and a copy of the program was given to INEEL for exclusive use by INEEL staff members only. A short course was developed together with a user's manual. Both were given to selected INEEL staff members.

Results of the first round of evaluations of the CEARPGA program indicate that CEARPGA has a number of limitations and drawbacks that affect its ability to accurately predict PGNAA $\gamma$-ray spectra. First, and foremost, the accuracy of the CEARPGA results depend on the quality of the detector response functions. This is not a problem if the detector is NaI, where the resolution is only mediocre, detectors are of standard sizes, and the response functions have been well-known for many years (for about 40 years). However, when the detector is $\mathrm{Ge}$ (which possesses very high resolution, exists in a wide variety of sizes, and has response functions that are less well known), accurate measurements must be performed to determine the response functions for each specific detector used. The job is made more difficult because mono-energetic high energy $\gamma$-ray sources are very difficult to find. It would be better if CEARPGA would be modified to use a Monte Carlo method to calculate detector response rather than rely on detector response functions. This will be addressed during FY 2000.

Another limitation to CEARPGA (and all current methods used to predict PGNAA $\gamma$-ray spectra) is actually a limitation of our present knowledge of the $\gamma$ rays emitted by neutron-induced reactions and not the program itself. Adequate knowledge of neutron capture $\gamma$-ray spectra is available only for thermal neutrons. Not enough is known about neutron capture $\gamma$ rays from the resonance energy and higherenergy neutrons. Therefore, CEARPGA assumes that the capture of a neutron of any energy results in the $\gamma$-ray spectrum produced by capture of a thermal neutron. In addition, again because of the lack of basic nuclear data, CEARPGA assumes that every neutron inelastic scattering event, no matter what the energy of the neutron, results in the $\gamma$-ray spectrum produced by inelastic scattering of fission spectrum neutrons. This assumption leads to serious problems for higher-energy neutron sources (such as 14-MeV neutrons or those emitted by a ${ }^{241} \mathrm{Am}$-Be source). For these neutron sources, the PGNAA $\gamma$-ray spectrum contains prominent peaks from neutron inelastic scattering in oxygen and carbon and from the (n,p) reaction on oxygen. However, these are absent from the spectra predicted by CEARPGA. Finally, sum peaks due to $\gamma$ rays in coincidence are not taken into account by CEARPGA because of the lack of information concerning coincident $\gamma$ rays.

NCSU and INEEL personnel performed a relatively detailed investigation of what basic nuclear data are needed in order to enhance the ability of the NCSU method to PGNAA $\gamma$-ray spectra produced when bulk material is bombarded with high-energy neutrons. The LANSCE facility at Los Alamos National Laboratory (LANL) possesses some data (but not for all elements of interest), but they must first be retrieved and analyzed. The National Institute of Standards and Technology (NIST) and the Institute of Isotope and Surface Chemistry in Budapest, Hungary, possess a large amount of capture $\gamma$-ray spectra 
from the capture of cold and thermal neutrons. However, a large amount was obtained using antiCompton detectors (which have not been used for PGNAA applications), no $\gamma-\gamma$ coincidence measurements were made, and no data were obtained using higher-energy neutrons (resonance region and higher). It is the intent of this task to modify the CEARPGA program to address the identified shortcomings when the basic nuclear data are available (a sizeable fraction of which is expected to be obtained by the E\&R Nuclear Structure Research Project, which was initiated during the middle of FY 1999).

\section{Passive Gamma-Ray Based NDA Analysis}

Passive gamma-ray spectrometry is a standard NDA technique used to assay barrels and boxes containing radioactive contaminated materials. As with most nuclear-based NDA, the accuracy of assay results depend on how well the assay analysis can correct for signal loss due to attenuation and geometry. The most common type of gamma-ray based assay system is the segmented gamma system (SGS) and adaptations of it; e.g., Canberra IQ-3 ${ }^{\text {TM }}$, Active \& Passive Computed Tomography (developed at Lawrence Livermore National Laboratory). These systems rely on the use of external source(s) to determine the attenuation corrections and from the two examples given, the analysis algorithms do range in complexity from simple attenuation correction to computed tomography. As a general rule, the more complex the correction technique, the more accurate the result. However, the degree of accuracy comes with a cost in terms of equipment expenditure and/or assay time. Based on our experience at INEEL involving assaying real TRU waste, there are many applications where the accuracy requirements can not justify the added expense and it is our expectation that simple gamma-ray spectrometers coupled with simple correction algorithms can satisfy most assay accuracy requirements. As a result, this project is working to determine the accuracy limitations of simple passive gamma-ray spectrometers (i.e., without external sources). The approach here is to develop an attenuation correction algorithm that relies on the inherent data available from the basic gamma-ray spectra. The next step is to test this analysis methodology using data obtained from assays involving surrogate and real waste drums using the SWEPP Gamma Ray Spectrometry system (a modified Canberra $\mathrm{Q}^{2}$ system).

A transport code called response matrix with in-scattering, RESPMATS, was developed to account for gamma-ray attenuation and in-scattering for a simple gamma-ray based NDA system. This code is the first element in an analysis routine that will perform real-time automated analysis of gamma-ray spectral data to arrive at absolute estimates of radionuclide contents, particularly ${ }^{239} \mathrm{Pu}$ and ${ }^{241} \mathrm{Am}$. This transport code uses only the information provided by the gamma-ray spectrum (or multiple spectra) combined with net matrix weight and fill height to correct for matrix attenuation of the gamma-ray signals. It was written explicitly for a rotating drum/multiple detector array configuration that is common to many NDA applications. To simplify the analysis so that the number of unknown parameters fit within the constraints of a simple assay system, this code assumes uniform matrix density, but does take into account different fill heights. The density is determined by dividing the net matrix weight by the matrix volume (defined by the inner diameter of the drum liner and the fill height). The RESPMATS code is also able to account for different nonuniform source distributions within a drum.

RESPMATS Code Testing-To test the RESPMATS code, a series of surrogate drum measurements were conducted using the SWEPP Gamma-Ray Spectrometer (SGRS). The SGRS is a modified Canberra Q-2 assay system with four HPGe detectors located vertically along side of a rotating 208 liter drum. This assay system is currently being used to determine the relative isotopic ratios to support isotopic identification in the INEEL TRU Waste Characterization Program. The surrogate drums and sources

\footnotetext{
${ }^{\text {TM }}$ Canberra Industries, Inc. 800 Research Parkway, Meriden, CT 06450.
} 
used in these tests are those developed for the INEEL TRU characterization program in support of "INEEL 3100 Cubic Meter program." Listed in Table 2 are the results of surrogate drum tests. In this table the recovery factor, $\mathrm{R}$, is defined as the measured ${ }^{239} \mathrm{Pu}$ mass divided by the known ${ }^{239} \mathrm{Pu}$ mass. For these tests the source placement was exactly modeled in the RESPMATS source distribution. The waste categories represented in these results covers a wide range of matrix properties, from zero matrix to inorganic sludge. The excellent agreement shown in Table 1 demonstrates that under known matrix and source conditions, the RESPMATS attenuated efficiency calculation is working properly and is capable of handling the standard assay geometry of a the drum and multiple detector array.

The next step in RESPMATS testing was to try it on real waste assay data. The first phase of real waste tests was done using gamma-ray spectrum data taken using the SGRS on a select set of inorganic sludge waste drums. This particular set of drums was chosen because they had been cored at Argonne National Laboratory-West and radiochemistry analysis was done on the core samples. (Once again this project is taking advantage of the wealth of data available from the INEEL 3100 Cubic Meter program.) The radiochemistry results were then used as the bases to compare with the RESPMATS results derived from the corresponding gamma-ray spectrum data. Listed in Table 3 are the results of those tests. In this table, the comparisons are again based on recovery factors where the recovery factor, in these cases, is defined as the RESPMATS derived measured ${ }^{239} \mathrm{Pu}$ mass divided by the radiochemistry derived ${ }^{239} \mathrm{Pu}$ mass. In contrast to the surrogate tests described above, there are errors associated with both the RESPMATS data and the radiochemistry data.

There were three mass distribution models used in these tests; i.e., one radial zone where the same distribution is uniform, two radial zones, and a radial distribution where some distribution is linear with respect to the radial distance. With each mass distribution model and drum assay, the parameters within the model were adjusted by least squares regression to achieve the maximum consistency between measured mass values from four prominent lines in the ${ }^{239} \mathrm{Pu}$ decay. The gamma spectrum data used in these tests were sum spectra where the individual detector spectra are summed into one spectrum for each assay. Because sum spectra were used, there was only one axial zone used in the mass distribution models, which extends from the bottom of the waste matrix to the top of the waste matrix. The data listed in Table 2 display a moderate range of recovery factors. It is not possible to determine from these data whether the gamma spectrum results are in error or the radiochemistry results are in error. Nevertheless, most recovery factors are within the corresponding acceptable ranges based on the Waste Isolation Pilot Plant Quality Assurance Program Plan ${ }^{6}$ (WIPP/QAPP) bias acceptance criteria.

Table 2. Pu-239 recovery factors from the surrogate drum measurements.

\begin{tabular}{|c|c|c|c|c|c|c|c|}
\hline Surrogate & $\begin{array}{c}129 \mathrm{keV} \\
\mathrm{R}\end{array}$ & $\begin{array}{c}203 \mathrm{keV} \\
\mathrm{R}\end{array}$ & $\begin{array}{c}345 \mathrm{keV} \\
\mathrm{R}\end{array}$ & $\begin{array}{c}375 \mathrm{keV} \\
\mathrm{R}\end{array}$ & $\begin{array}{c}413 \mathrm{keV} \\
\mathrm{R}\end{array}$ & $\begin{array}{c}\text { Average } \\
\text { R }\end{array}$ & $\begin{array}{c}\text { Std. Dev. } \\
\text { R }\end{array}$ \\
\hline Zero matrix & 0.9584 & 0.9832 & 0.9581 & 0.9675 & 0.9884 & 0.9712 & 0.0142 \\
\hline Inorganic sludge & 1.006 & 1.007 & 0.9782 & 1.004 & 1.016 & 1.002 & 0.0143 \\
\hline Organic sludge & 1.003 & 0.9921 & 0.9554 & 0.9649 & 0.979 & 0.9788 & 0.0194 \\
\hline Mixed metals & 1.049 & 0.9662 & 0.9875 & 1.011 & 1.028 & 1.008 & 0.0326 \\
\hline Filters & 1.013 & 1.012 & 0.9755 & 0.9972 & 1.006 & 1.001 & 0.0154 \\
\hline Combustibles & 1.014 & 1.022 & 0.9917 & 1.008 & 1.012 & 1.009 & 0.0112 \\
\hline Glass & 1.01 & 1.018 & 0.9946 & 0.9927 & 1.017 & 1.066 & 0.0121 \\
\hline Graphite & 1.001 & 1.024 & 0.9587 & 1.007 & 1.031 & 1.004 & 0.0283 \\
\hline
\end{tabular}


Table 3. Pu-mass recovery factors for real inorganic sludge waste.

\begin{tabular}{|c|c|c|c|c|c|c|c|c|}
\hline Bar Code & $\begin{array}{c}\text { Acceptable } \\
\text { Recovery } \\
\text { Range }\end{array}$ & $\begin{array}{c}\text { Radiochemistry } \\
\text { Pu Mass }\end{array}$ & $\begin{array}{c}\text { One } \\
\text { Zone } \\
\text { Pu Mass }\end{array}$ & $\mathrm{R}$ & $\begin{array}{c}\text { Two } \\
\text { Zone } \\
\text { Pu Mass }\end{array}$ & $\mathrm{R}$ & $\begin{array}{c}\text { Linear } \\
\text { Dist. } \\
\text { Pu Mass }\end{array}$ & $\mathrm{R}$ \\
\hline 000513 & $0.4-1.75$ & 0.197 & 0.142 & 0.72 & 0.122 & 0.62 & 0.26 & 1.32 \\
\hline 003325 & $0.3-2.0$ & 0.282 & 0.165 & 0.59 & 0.214 & 0.76 & 0.338 & 1.20 \\
\hline 004221 & $0.3-2.0$ & 2.001 & 0.942 & 0.47 & 0.94 & 0.47 & 1.4507 & 0.72 \\
\hline 005698 & $0.4-1.75$ & 0.291 & 0.114 & 0.39 & 0.398 & 1.37 & 0.74 & 2.54 \\
\hline 010549 & $0.3-2.0$ & 0.968 & 0.689 & 0.71 & 0.601 & 0.62 & 0.9623 & 0.99 \\
\hline 012287 & $0.3-2.0$ & 5.712 & 4.725 & 0.83 & 4.074 & 0.71 & 6.886 & 1.21 \\
\hline 024498 & $0.3-2.0$ & 1.813 & 1.701 & 0.94 & 1.825 & 1.01 & 1.953 & 1.08 \\
\hline 030186 & $0.4-1.75$ & 0.319 & 0.179 & 0.56 & 0.117 & 0.37 & 0.1409 & 0.44 \\
\hline 031166 & $0.3-2.0$ & 1.869 & 1.191 & 0.64 & 1.475 & 0.79 & 1.6267 & 0.87 \\
\hline 032674 & $0.4-1.75$ & 0.376 & 0.213 & 0.57 & 0.364 & 0.97 & 0.4112 & 1.09 \\
\hline
\end{tabular}

Transform Method for Efficiency Curve Interpolation-As part of the development of the gamma-spectrum analysis routine, RESPMATS, a new approach for interpolating gamma spectrometer efficiency data was developed. Interpolating the efficiency data is necessary because the gamma-ray energies used in calibration are not the same as those used in assay analysis. At high energies, interpolation is straightforward because the efficiency curve is monotonically decreasing with gamma-ray energy. At lower energies, the efficiency curve is peaked between 100 and $200 \mathrm{keV}$. Interpolating at these lower energies is problematic because it is difficult to represent the peak in the efficiency curve. Often this interpolation process requires an interactive routine where the judgement on the quality of the interpolated efficiency curve is based on the decisions (and expertise) of the one performing the interpolation. For normal HPGe detectors, the peak in the efficiency curve is near $100 \mathrm{keV}$. In TRU waste characterization using gamma-ray based NDA, the HPGe detectors are often shielded from the low energy radiation arising from the decay of ${ }^{241} \mathrm{Am}$. The peak in the effective efficiency curve is now an energy of approximately $200 \mathrm{keV}$. The higher energy for the peak of the efficiency curve is therefore a concern because the peak is now right in the range used in most TRU assay analyses. To eliminate the need for interactive interpolation, a transform method of interpolation was developed by this project. In this approach, the efficiency data measured during calibration are transformed into a form that can be fit by simple functions and then the resulting fit function is transformed back to the original form. This technique was applied to the SWEPP calibration data and the results were compared with original calibration data. Shown in Figure 3 is the transform of the SWEEP efficiency calibration data, where the transform function is related to the efficiency function by the following expression:

$T(E)=\varepsilon(E) \exp \left\{\left[\frac{\mu(E)}{\rho}\right] \times Z\right\}$

where

$$
T(E)=\text { transform function }
$$




$$
\begin{array}{ll}
\varepsilon(E) & =\text { original efficiency function } \\
\frac{\mu(E)}{\rho} & =\text { mass attenuation coefficient } \\
E & =\text { gamma }- \text { ray energy. }
\end{array}
$$

The transform function shown in Figure 3 is transformed from the efficiency function using the mass attenuation coefficient tables for cadmium and a value of $Z=1.538 \mathrm{~g} / \mathrm{cm}^{2}$. The cadmium mass attenuation coefficients were used because there are cadmium filters placed in front of the SWEPP HPGe detectors to reduce the count rate from the $60 \mathrm{keV}$ gamma ray produced by the decay of ${ }^{241} \mathrm{Am}$. The value of $Z$ was adjusted to produce a simple monotonic decreasing transform function with respect to the gamma-ray energy. In Figure 3 the fit function to the transform data is a sixth order power series in gamma-ray energy.

SGRS efficiency (transform)

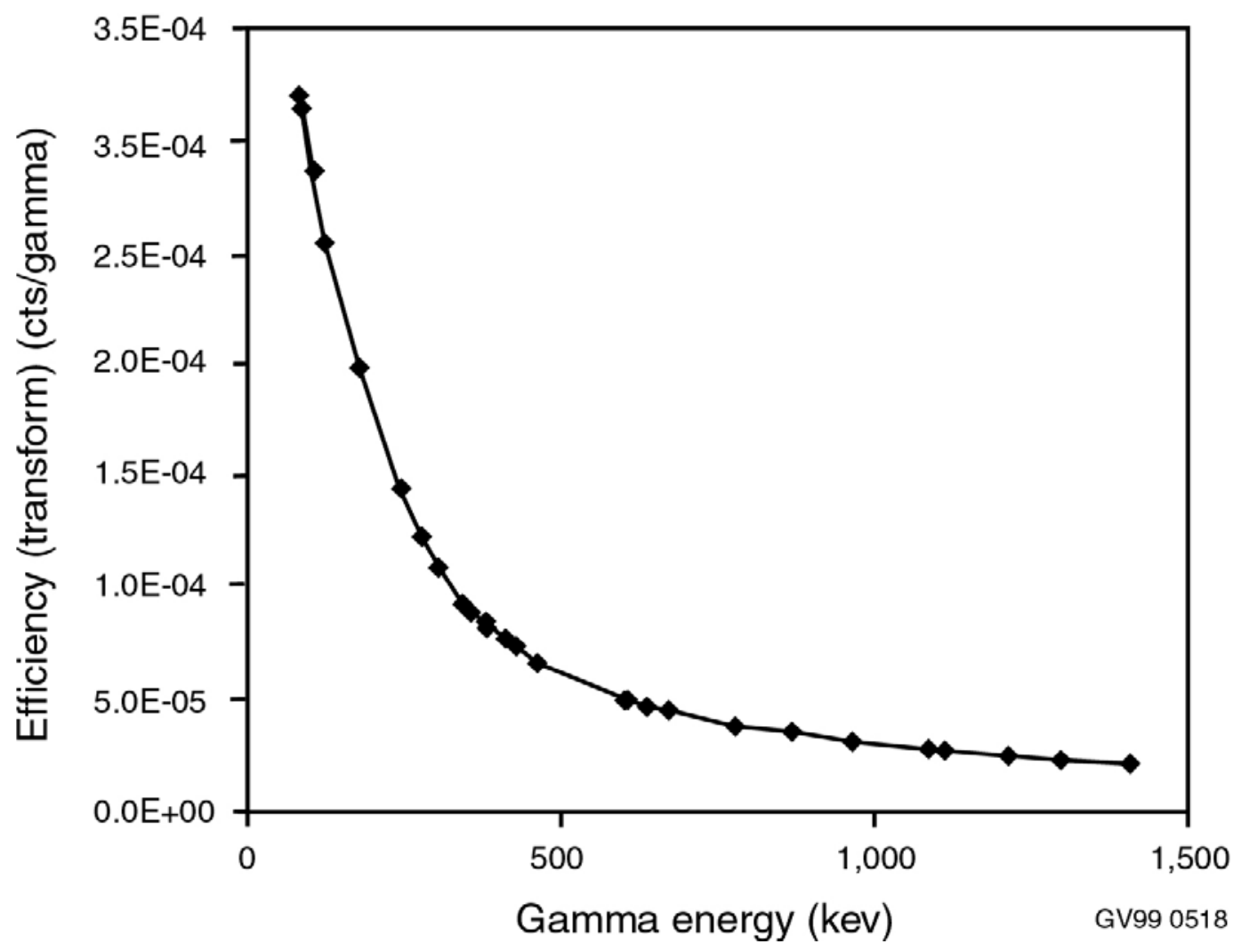

Figure 3. Transform efficiency function versus gamma-ray energy. 
The transform back to the regular efficiency curve is shown in Figure 4. The inverse transform is given by the following expression:

$$
\langle\varepsilon(E)\rangle=\langle T(E)\rangle \exp \left\{-\left[\frac{\mu(E)}{\rho}\right] \times Z\right\}
$$

where

$$
\langle\rangle=\text { estimated fit data. }
$$

Listed in Table 4 are tabulated results comparing the measured efficiency data with the estimated efficiency data derived from the transform fit function. As can be seen from this table, the differences are on the order of $1-2 \%$ and the maximum difference is $2.2 \%$. The success using the transform method in this project's research has lead to it being implemented by the SWEPP assay group for use in the TRU characterization program. Its use by the SWEPP group is expected to reduce downtime at the SGRS due to detector replacement and calibration from 1 week to less than 1 day.

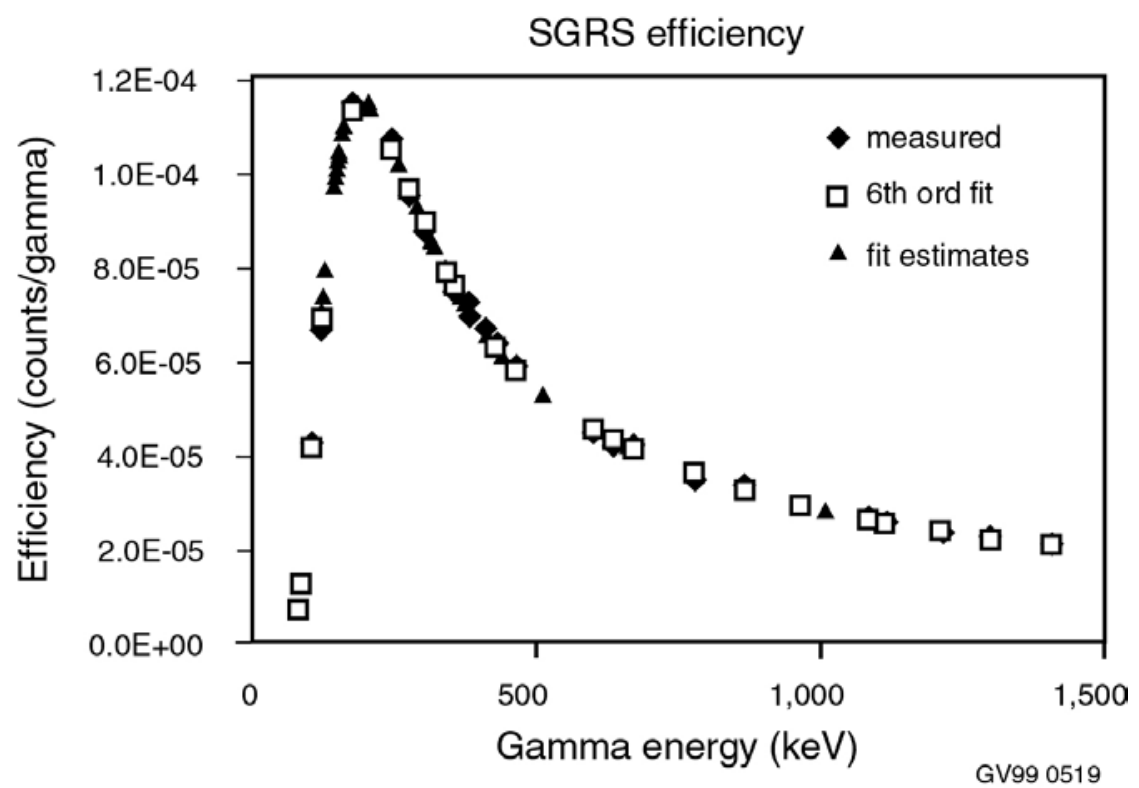

Figure 4. SWEPP gamma-ray spectrometer efficiency versus gamma-ray energy.

\section{ACCOMPLISHMENTS}

This section contains a short synopsis of the major accomplishments of this project. Details and other noteworthy progress items are given in the Project Description Section above. Because of the close relationship between the research persons of this project and those involved in the waste management programs, particularly at the INEEL, this project and INEEL programs have benefited by the flow of information and data both ways.

- The collection of fission fragment and related neutron multiplicity data using ${ }^{235} \mathrm{U}$ as a target was completed. Correlated gamma-ray spectrum and neutron data were accumulated for approximately $2 \times 10^{9}$ fission events using the IPNS fission experiment system. 
Table 4. Comparison of fit efficiency data with measured efficiency data.

\begin{tabular}{|c|c|c|c|}
\hline $\begin{array}{c}\text { Gamma-Ray Energy } \\
(\mathrm{keV})\end{array}$ & $\begin{array}{c}\text { Measured Efficiency } \\
\text { (counts/gamma) }\end{array}$ & $\begin{array}{c}\text { Estimated Efficiency } \\
\text { (counts/gamma) }\end{array}$ & $\begin{array}{c}\text { Relative Difference } \\
(\%)\end{array}$ \\
\hline 80.99 & $6.55 \mathrm{E}-06$ & $6.59 \mathrm{E}-06$ & 0.56 \\
\hline 86.54 & $1.22 \mathrm{E}-05$ & $1.21 \mathrm{E}-05$ & -0.63 \\
\hline 105.31 & 4.19E-05 & $4.11 \mathrm{E}-05$ & -1.24 \\
\hline 121.78 & 6.67E-05 & $6.82 \mathrm{E}-05$ & 2.22 \\
\hline 176.32 & $1.14 \mathrm{E}-04$ & $1.13 \mathrm{E}-04$ & -1.01 \\
\hline 244.70 & $1.06 \mathrm{E}-04$ & $1.04 \mathrm{E}-04$ & -1.69 \\
\hline 276.39 & $9.52 \mathrm{E}-05$ & $9.59 \mathrm{E}-05$ & 0.74 \\
\hline 302.85 & $8.75 \mathrm{E}-05$ & 8.89E-05 & 1.54 \\
\hline 344.28 & $7.70 \mathrm{E}-05$ & 7.82E-05 & 1.52 \\
\hline 356.00 & $7.46 \mathrm{E}-05$ & 7.55E-05 & 1.16 \\
\hline 380.42 & $7.15 \mathrm{E}-05$ & $7.05 \mathrm{E}-05$ & -1.40 \\
\hline 383.84 & $6.94 \mathrm{E}-05$ & $6.99 \mathrm{E}-05$ & 0.62 \\
\hline 411.13 & $6.59 \mathrm{E}-05$ & $6.50 \mathrm{E}-05$ & -1.30 \\
\hline 427.88 & $6.32 \mathrm{E}-05$ & $6.24 \mathrm{E}-05$ & -1.27 \\
\hline 463.37 & $5.78 \mathrm{E}-05$ & $5.75 \mathrm{E}-05$ & -0.58 \\
\hline 600.50 & $4.46 \mathrm{E}-05$ & $4.51 \mathrm{E}-05$ & 1.11 \\
\hline 606.63 & $4.50 \mathrm{E}-05$ & 4.49E-05 & -0.73 \\
\hline 635.89 & $4.21 \mathrm{E}-05$ & $4.28 \mathrm{E}-05$ & 1.64 \\
\hline 671.38 & $4.14 \mathrm{E}-05$ & $4.08 \mathrm{E}-05$ & -1.42 \\
\hline 778.90 & $3.48 \mathrm{E}-05$ & $3.55 \mathrm{E}-05$ & 2.11 \\
\hline 867.39 & $3.27 \mathrm{E}-05$ & $3.20 \mathrm{E}-05$ & -2.10 \\
\hline 964.11 & $2.87 \mathrm{E}-05$ & $2.88 \mathrm{E}-05$ & 0.38 \\
\hline $1,085.84$ & $2.58 \mathrm{E}-05$ & $2.58 \mathrm{E}-05$ & -0.17 \\
\hline $1,112.09$ & $2.53 \mathrm{E}-05$ & $2.53 \mathrm{E}-05$ & -0.05 \\
\hline $1,212.97$ & $2.30 \mathrm{E}-05$ & $2.32 \mathrm{E}-05$ & 0.99 \\
\hline $1,299.15$ & $2.17 \mathrm{E}-05$ & $2.16 \mathrm{E}-05$ & -0.76 \\
\hline $1,408.02$ & $2.01 \mathrm{E}-05$ & $2.02 \mathrm{E}-05$ & 0.14 \\
\hline
\end{tabular}


- $\quad$ The correlation matrix for the ${ }^{235} \mathrm{U}$ data was constructed and sent to our collaborators for further analysis. A graduate student from Vanderbilt University spent a month at INEEL beginning the building of this matrix.

- $\quad$ The induced fission experiment for ${ }^{239} \mathrm{Pu}$ was started and completed at IPNS during FY 1999. Approximately $3 \times 10^{9}$ fission events were collected. The correlation matrix and analysis of this data will occur in FY 2000.

- A method of corroborating the presence of trace amounts of ${ }^{233} U$ in transuranic waste was developed. The presence of ${ }^{233} \mathrm{U}$ progeny was observed, including all gamma-ray emitting members of the decay chain to ${ }^{213} \mathrm{Po}\left({ }^{209} \mathrm{~Pb}\right.$, the daughter of ${ }^{213} \mathrm{Po}$, is a pure beta emitting radio-nuclide decaying to stable ${ }^{209} \mathrm{Bi}$ ). Of particular interest is a very strong ${ }^{213} \mathrm{Bi}$ peak at $440 \mathrm{keV}$. The presence of this peak at $440 \mathrm{keV}$ can be used to corroborate the presence of ${ }^{233} \mathrm{U}$. This method is now used by analysts involved in data review at the SWEPP to interpret TRU waste drum assay data.

- A method for deducing the "age" of actinide waste containing ${ }^{241}$ Am was developed and an article describing this technique has been submitted to the Waste Management Journal.

- $\quad$ North Carolina State University (NCSU), under contract to the INEEL in support of PGNAA task, developed modifications to their CEARPGA neutron and gamma transport computer program to allow additional geometries, including a large planar sample. They also initiated (and nearly completed) modifications to add the INEEL PINS geometry.

- $\quad$ The CEARPGA program was implemented at INEEL during FY 1999. NCSU moved CEARPGA to a PC platform for use at INEEL, and a copy of the program was given to INEEL for exclusive use by INEEL staff members. A short course was developed together with a user's manual. Both were given to selected INEEL staff members.

- A transport code called response matrix with in-scattering, RESPMATS, was developed to account for gamma-ray attenuation and in-scattering for a passive gamma-ray based NDA system. This code is the first element in an analysis routine that will perform real-time automated analysis of gamma-ray spectral data to arrive at absolute estimates of radionuclide contents, particularly ${ }^{239} \mathrm{Pu}$ and ${ }^{241} \mathrm{Am}$.

- $\quad$ As part of the development of the gamma-spectrum analysis routine, RESPMATS, a new approach for interpolating gamma spectrometer efficiency data was developed. This simple interpolation routine has also been implemented in the gamma spectrum analysis code used by the SWEPP Gamma Ray Spectrometer (SGRS) at the INEEL RWMC. An abstract of a paper describing this technique has been submitted for presentation at the $7^{\text {th }}$ Nondestructive Assay Waste Characterization Conference.

\section{REFERENCES}

1. J. Kliman, V. Polhorsky, M. Morhac, G. M. Ter-Akopian, Yu. Ts. Oganessian, G. S. Popeko, A. V. Daniel, J. H. Hamilton, K. Butler-Moore, A. V. Ramayya, W.-C. Ma, X. Zhao, Q. Lu, D. Shi, J. K. Deng, J. Kormicki, S .Zhu, J. D. Cole, R. Aryaeinejad, R. C. Greenwood, S. S. Harrill, N. D. Lohstreter, I. Y. Lee, N. R. Johnson, and F. K. McGowan, "Correlated Yields and the Emission of Neutrons of Mo/Ba Fragments from ${ }^{252} \mathrm{Cf}(\mathrm{SF})$, , Yad. Fiz. 57, No 7, 1174, 1994; Phys. Atomic Nuclei 57, 1108, 1994. 
2. A. Sandulescu, A. Florescu, F. Carstoiu, W. Greiner, J. H. Hamilton, A. V. Ramayya, and B. R. S. Babu, "Isotopic Yields for the Cold Fission of 252Cf," Phys. Rev. C54, 258, 1996.

3. G. M. Ter-Akopian, J. H. Hamilton, Yu. Ts. Oganessian, A. V. Daniel, J. Kormicki, A. V. Ramayya, G. S. Popeko, B. R. S. Babu, Q.-H. Lu, K. Butler-Moore, W.-C. Ma, E. F. Jones, J. K. Deng, D. Shi, J. Kliman, M. Morhac, J. D. Cole, R. Aryaeinejad, N. R. Johnson, I. Y. Lee, and F. K. McGowan, "Yields of Correlated Fragment Pairs in Spontaneous Fission of ${ }^{252} \mathrm{Cf}$," Phys. Rev. C55, 1146, 1997.

4. $\quad$ R. R. Betts and A. H. Wuosmaa, "Nuclear Molecules," Rep. Prog. Phys. 60, 819, 1997.

5. A. V. Ramayya, J. H. Hamilton, J. K. Hwang, L. K. Peker, J. Kormicki, B. R. S. Babu, T. N. Ginter, A. Sandulescu, A. Florescu, F. Carstoiu, W. Greiner, G. M. Ter-Akopian, Yu. Ts. Oganessian, A. V. Daniel, W. C. Ma, P. G. Varmette, J. O. Rasmussen, S. J. Asztalos, S. Y. Chu, K. E. Gregorich, A. O. Macchiavelli, R. W. Macleod, J. D. Cole, R. Aryaeinejad, K. Butler-Moore, M. W. Drigert, M. A. Stoyer, L. A. Bernstein, R. W. Lougheed, K. J. Moody, S. G. Prussin, S. J. Zhu, H. C. Griffin, and R. Donangelo, "Cold (Neutronless) alpha Ternary Fission of ${ }^{252}$ Cf," Phys.Rev. C57, 2370, 1998.

6. U.S. Department of Energy, TRU Waste Characterization Quality Assurance Program Plan, Interim Change, CAO-94-1010, 1996.

\section{APPENDIX}

\section{Peer Reviewed Publications}

1. J. H. Hamilton, A. V. Ramayya, J. K. Hwang, W. Greiner, S. J. Zhu, A. Sandulescu, A. Florescu, J. Kormicki, G. Ter-Akopian, Y. Oganesyan, A. V. Daniel, G. S. Popeko, J. Kliman, M. Morhac, J. D. Cole, R. Aryaeinejad, M. W. Drigert, W. E. Collins, W. C. Ma, E. F. Jones, L. K. Peker, P. M. Gore, G. Drafta, B. R. S. Babu, G. Wang, and J. K. Deng, "Cold Spontaneous Fission Processes of ${ }^{252} \mathrm{Cf}$ and the Structure of Neutron Rich Ba and La Nuclei," Acta Phys. Slovaca 49, 31, 1999.

2. S. J. Zhu, J. H. Hamilton, A. V. Ramayya, M. G. Wang, J. K. Hwang, E. F. Jones, L. K. Peker, B. R. S. Babu, G. Drafta, W. C. Ma, G. L. Long, L. Y. Zhu, M. Li, C. Y. Gan, T. N. Ginter, J. Kormicki, J. K. Deng, D. T. Shi, W. E. Collins, J. D. Cole, R. Aryaeinejad, M. W. Drigert, J. O. Rasmussen, R. Donangelo, J. Gilat, S. Asztalos, I. Y. Lee, A. O. Macchiavelli, S. Y. Chu, K. E. Gregorich, M. F. Mohar, M. A. Stoyer, R. W. Lougheed, K. J. Moody, J. F. Wild, S. G. Prussin, G. M. Ter-Akopian, A. V. Daniel, and Yu. Ts. Oganessian, "Octupole Correlations in Neutron-Rich ${ }^{145,147}$ La Nuclei: Coriolis-Limit-Coupling Bands with Aligned $\mathrm{h}_{11 / 2}$ Proton," Phys.Rev. C59, 1316, 1999.

3. S. -J. Zhu, M. -G. Wang, J. H. Hamilton, A. V. Ramayya, B. R. S. Babu, W. C. Ma, G-L. Long, L. -Y. Zhu, M. Li, M. Sakhaee, C. -Y. Gan, L. -M. Yang, J. Kormicki, J. D. Cole, R. Aryaeinejad, M. W. Drigert, J. O. Rasmussen, M. A. Stoyer, S. Y. Chu, K. E. Gregorich, M. F. Mohar, S. G. Prussin, I. Y. Lee, Yu. Ts. Oganessian, G. M. Ter-Akopian, and A. V. Daniel, "Octupole eformation ands of $\quad \pi \mathrm{h}_{11 / 2}$ in Neutron-Rich ${ }^{145,147}$ La Nuclei," Chin.Phys.Lett. 16, 169, 1999.

4. J. H. Hamilton, E. F. Jones, P. M. Gore, A. V. Ramayya, R. S. Dodder, C. J. Beyer, J. K. Hwang, X. Q. Zhang, S. J. Zhu, A. P. de Lima, J. Kormicki, J. D. Cole, R. Aryaeinejad, W. C. Ma, G. M. Ter-Akopian, Yu. Ts. Oganessian, A. V. Daniel, J. O. Rasmussen, S. J. Asztalos, I. Y. Lee, 
A. O. Macchiavelli, M. A. Stoyer, R. W. Lougheed, S. G. Prussin, and R. Donangelo, “A New Phenomenon-Shifted Identical Bands and New Region of Stable Octupole," Nuovo Cim. 111A, 633, 1998.

5. J. K. Hwang, A. V. Ramayya, J. H. Hamilton, L. K. Peker, J. Kormicki, B. R. S. Babu, T. N. Ginter, C. J. Beyer, G. M. Ter-Akopian, Yu. Ts. Oganessian, A. V. Daniel, W. C. Ma, P. G. Varmette, J. O. Rasmussen, S. J. Asztalos, S. Y. Chu, K. E. Gregorich, A. O. Macchiavelli, R. W. Macleod, J. Gilat, J. D. Cole, R. Aryaeinejad, K. Butler-Moore, M. W. Drigert, M. A. Stoyer, L. A. Bernstein, R. W. Lougheed, K. J. Moody, S. G. Prussin, H. C. Griffin, and R. Donangelo, "Close Doublet Structures in ${ }^{103} \mathrm{Mo},{ }^{109,111} \mathrm{Ru}$, and Neighbours: Rotation-Alignment for the Half-Filled $\mathrm{h}_{11 / 2}$ Subshell," J.Phys.(London), G24, L9, 1998.

6. J. K. Hwang, A. V. Ramayya, J. H. Hamilton, L. K. Peker, J. Kormicki, B. R. S. Babu, T. N. Ginter, C. J. Beyer, J. O. Rasmussen, J. Gilat, S. J. Asztalos, S. Y. Chu, K. E. Gregorich, A. O. Macchiavelli, R. W. Macleod, G. M. Ter-Akopian, Yu. Ts. Oganessian, A. V. Daniel, W. C. Ma, P. G. Varmette, J. D. Cole, R. Aryaeinejad, K. Butler-Moore, M. W. Drigert, M. A. Stoyer, L. A. Bernstein, R. W. Lougheed, K. J. Moody, S. G. Prussin, H. C. Griffin, R. Donangelo, and S. J. Zhu, "Identification of the Gamma Transitions in Tc and Cs Products of ${ }^{252}$ Cf Fission and Possible (7/2)+ [413] Bands in ${ }^{105-109}$ Tc Isotopes," Phys.Rev. C57, 2250, 1998; Erratum Phys.Rev. C58, 3013, 1998.

7. J. K. Hwang, A. V. Ramayya, J. Gilat, J. H. Hamilton, L. K. Peker, J. O. Rasmussen, J. Kormicki, T. N. Ginter, B. R. S. Babu, C. J. Beyer, E. F. Jones, R. Donangelo, S. J. Zhu, H. C. Griffin, G. M. Ter-Akopyan, Yu. Ts. Oganessian, A. V. Daniel, W. C. Ma, P. G. Varmette, J. D. Cole, R. Aryaeinejad, M. W. Drigert, and M. A. Stoyer, "Rotational Bands in ${ }^{101-103} \mathrm{Nb}$ and ${ }^{98,100} \mathrm{Y}$ Nuclei and Identification of Yrast Bands in ${ }^{146} \mathrm{La}$ and ${ }^{149} \mathrm{Pr}, "$ Phys.Rev. C58, 3252, 1998.

8. A. V. Ramayya, J. H. Hamilton, J. K. Hwang, L. K. Peker, J. Kormicki, B. R. S. Babu, T. N. Ginter, A. Sandulescu, A. Florescu, F. Carstoiu, W. Greiner, G. M. Ter-Akopian, Yu. Ts. Oganessian, A. V. Daniel, W. C. Ma, P. G. Varmette, J. O. Rasmussen, S. J. Asztalos, S. Y. Chu, K. E. Gregorich, A. O. Macchiavelli, R. W. Macleod, J. D. Cole, R. Aryaeinejad, K. Butler-Moore, M. W. Drigert, M. A. Stoyer, L. A. Bernstein, R. W. Lougheed, K. J. Moody, S. G. Prussin, S. J. Zhu, H. C. Griffin, and R. Donangelo, "Cold (Neutronless) Alpha Ternary Fission of ${ }^{252}$ Cf," Phys.Rev. C57, 2370, 1998.

9. A. V. Ramayya, J. K. Hwang, J. H. Hamilton, A. Sandulescu, A. Florescu, G. M. Ter-Akopian, A. V. Daniel, Yu. Ts. Oganessian, G. S. Poeko, W. Greiner, L. K. Peker, J. Kormicki, B. R. S. Babu, T. N. Ginter, F. Carstoiu, W. C. Ma, P. G. Varmette, J. O. Rasmussen, S. J. Asztalos, S. Y. Chu, K. E. Gregorich, A. O. Macchiavelli, R. W. Macleod, J. D. Cole, R. Aryaeinejad, K. Butler-Moore, M. W. Drigert, M. A. Stoyer, L. A. Bernstein, R. W. Lougheed, K. J. Moody, S. G. Prussin, S. J. Zhu, H. C. Griffin, and R. Donangelo, "Observation of ${ }^{10} \mathrm{Be}$ Emission in the Cold Ternary Spontaneous Fission of ${ }^{252}$ Cf," Phys.Rev.Let. 81, 947, 1998.

10. X. Q. Zhang, J. H. Hamilton, A. V. Ramayya, L. K. Peker, J. K. Hwang, E. F. Jones, J. Kormicki, C. J. Beyer, P. M. Gore, B. R. S. Babu, T. N. Ginter, S. J. Asztalos, S. Y. Chu, K. E. Gregorich, I. Y. Lee, A. O. Macchiavelli, R. W. Macleod, J. O. Rasmussen, J. Gilat, G. M. Ter-Akopian, Yu. Ts. Oganessian, A. V. Daniel, W. C. Ma, P. G. Varmette, J. D. Cole, R. Aryaeinejad, K. Butler-Moore, Y. X. Dardenne, M. W. Drigert, M. A. Stoyer, J. F. Wild, J. A. Becker, L. A. Bernstein, R. W. Lougheed, K. J. Moody, R. Donangelo, S. G. Prussin, H. C. Griffin, and S. J. Zhu, "Identification of New Negative-Parity Levels in ${ }^{152,154}$ Nd," Phys.Rev. C57, 2040, 1998; Erratum Phys.Rev. C58, 3012, 1998. 
11. S. -J. Zhu, C. -Y. Gan, J. H. Hamilton, A. V. Ramayya, B. R. S. Babu, M. Sakhaee, W. C. Ma, G. -L. Long, J. -K. Deng, L. -Y. Zhu, M. Li, L. -M. Yang, J. Kormicki, J. D. Cole, R. Aryaeinejad, Y. K. Dardenne, M. W. Drigert, J. O. Rasmussen, M. A. Stoyer, S. Y. Chu, K. E. Gregorich, M. F. Mohar, S. G. Prussin, I. Y. Lee, N. R. Johnson, and F. K. McGowan, "Collective Band Structures in the Neutron-Rich ${ }^{107,109}$ Ru Nuclei," Chin.Phys.Lett. 15, 793, 1998.

12. R. J. Gehrke, L. V. East, and Y. D. Harker, "Information in Spectra From Sources Containing "Aged" ${ }^{241}$ Am As From TRU Waste," to be submitted to the Journal of Waste Management.

\section{Presentations}

1. J. D. Cole, M. W. Drigert, R. Aryaeinejad, E. L. Reber, J. K. Jewell, J. H. Hamilton, A. V. Ramayya, C. J. Beyer, G. M. Ter-Akopian, Ts. Yu. Oganessian, G. S. Popeko, and A. V. Daniel, "Induced Fission and Signature Studies of Actinide Isotopes Using ANL/IPNS Thermal Neutrons and an Array of Detectors," Winter Meeting of American Nuclear Society, Boston, Massachusetts, November 1998.

2. J. D. Cole, M. W. Drigert, R. Aryaeinejad, E. L. Reber, J. K. Jewell, J. H. Hamilton, A. V. Ramayya, C. J. Beyer, G. M. Ter-Akopian, Ts. Yu. Oganessian, G. S. Popeko, and A. V. Daniel, "Using New Fission Data with the Multi-Detector Analysis System for Spent Nuclear Fuel," International Conference on Perspectives in Nuclear Physics, Paradise Island, Bahamas.

3. J. W. Mandler, "Application of PGNAA to Ore Mining and Processing," 1999 Industrial Radiation and Radioisotope Methods and Applications (IRRMA 99) Topical Meeting, Raleigh, NC, October 3-8, 1999.

4. R. J. Gehrke and R. G. Helmer, "Photon Spectra of Radionuclides Acquired on Large-Area, Thin-Window HPGE Spectrometers," Proceedings of the $6^{\text {th }}$ Nondestructive Assay Waste Characterization Conference, Salt Lake City, UT, November 17-19, 1998.

5. R. J. Gehrke, "The Role of the INEEL in Ionizing Radiation Measurements and Standards," Presented by invitation at the Annual Meeting of the Council on Ionizing Radiation Measurements and Standards, National Institute of Standards and Technology, Gaithersburg, MD, October 19-22, 1998.

6. R. G. Helmer, J. R. Davidson, and R. J. Gehrke, "Gamma-Ray Spectrum Catalogue," Presented at the R. L. Heath Symposium Sessions of the Winter Meeting of the American Nuclear Society held Nov. 12 to 17, 1998, in Washington DC, ANS Transactions, 79, (1998) 114.

\section{Organized Technical Sessions}

"Russell Heath Memorial Session: Advances in X- and Gamma-Radiation Detectors," organized by R. J. Gehrke for the ANS 1998 Winter Meeting in Washington D C held November 12 to 17, 1998. 


\title{
Nuclear Structure Research
}

\author{
J. W. Mandler, R. J. Gehrke, R. G. Helmer \\ SUMMARY
}

The objective of this project is to perform nuclear structure research that will build on and expand the INEEL's current nuclear physics expertise in support of DOE EM. Five areas are being pursued that will result in furthering the knowledge of nuclear structure, expanding the database that is required for modeling (development of new models and improvement of existing models), providing data necessary for practical applications using nuclear techniques, and building the INEEL's capabilities in $\gamma$-ray metrology. These five research areas are (1) high accuracy $\gamma$-ray intensity measurements, (2) decay schemes for heavy-mass and short-lived nuclides, (3) nuclear data measurements for prompt reactions, (4) measurement of additional $\gamma$-rays in $(\mathrm{n}, \gamma)$ reactions, and (5) very-low-level radiation detection.

In Research Area 1, Monte Carlo calculated peak efficiencies for a Ge detector were compared with measurements made with the University of Michigan Ge detector for nuclides that have $\gamma$-rays with very well-known relative intensities, and these data were used to expand the range of the high-accuracy efficiency curve to cover the energy range from 433 to $2,754 \mathrm{keV}$. This resulted in a relative efficiency with an uncertainty of about $0.1 \%$ over this energy range, compared to the previous best of $\sim 0.5 \%$. In Research Area 2, a very careful measurement of the $\gamma$-ray spectrum and decay of ${ }^{49} \mathrm{Ca}$ was performed. By using the high-accuracy efficiency curve (above), $\gamma$-ray intensities that are from 10 to 100 times more accurate than current data were obtained. In Research Areas 3 and 4, a review of the various neutron facilities around the world was conducted, and we have established collaborations with LANSCE at Los Alamos National Laboratory (LANL), National Institute of Standards and Technology (NIST), and the Institute of Isotope and Surface Chemistry in Budapest, Hungary. Finally, in Research Area 5, a special radiochemical separation method was developed and used to remove trace quantities of natural radioactive materials from 680 grams of mass-separated stable ${ }^{100} \mathrm{Mo}$. The ${ }^{100} \mathrm{Mo}$ was ultrapurified to a level never before attained; a level acceptable to the NEMO-3 double beta decay experiment. Other collaborations were established with Idaho State University, Texas A\&M University, and North Carolina State University.

\section{PROJECT DESCRIPTION}

\section{Introduction}

The objective of this project is to perform nuclear structure research that will build and expand the INEEL's current nuclear physics expertise. The studies involve five related research areas. All five address the need to improve the known nuclear data to either: (1) verify and validate our present understanding of nuclear structure and the interactions of radiation with matter or (2) to provide a foundation to be used to improve our current understanding of and ability to predict nuclear parameters such as level energies, transition probabilities, etc. Pursuit of these five areas will result in furthering the knowledge of nuclear structure, expanding the database that is required for modeling (development of new models and improvement of existing models), providing data necessary for practical applications using nuclear techniques, and building the INEEL's capabilities in $\gamma$-ray metrology.

Research Areas 1 and 4 will result in improvement in the known accuracy of nuclear parameters and better understanding of nuclear structure models as well as enhance the INEEL's $\gamma$-ray metrology capability. Completion of Research Area 4 will verify whether all uniquely defined prompt $\gamma$-ray 
transitions following neutron capture have been identified and correctly placed in the level scheme. This exercise will verify the correctness and completeness of the level scheme or the need to reinvestigate it.

Research Area 2 will provide new and better nuclear structure information for high-mass and also for short-lived nuclides. The decay schemes for some of these nuclides have never been adequately studied, and in some cases there are inconsistencies in $\gamma$-ray emission probabilities.

As a result of Research Area 3, new nuclear structure information (especially of the higher-energy levels) will be obtained. This information will be useful in testing evaluated cross sections and in testing nuclear models relating to nuclear states, transition multipolarities, and level densities. In addition, our knowledge of the $\gamma$-rays emitted upon neutron capture and neutron inelastic scattering will be greatly enhanced, especially as to how these $\gamma$-rays vary with neutron energy. This information is very important in attempts to accurately model the $\gamma$-rays emitted when a neutron interacts with matter. Current application models are relatively crude. They make assumptions which, while admittedly not totally correct, are currently made because of lack of suitable theoretical models due in turn to lack of data concerning neutron capture and inelastic scattering at different neutron energies. When adequate data are obtained for a sufficient number of neutron energies, a theoretical model for $\gamma$-ray yields resulting from $(\mathrm{n}, \gamma)$ and $\left(\mathrm{n}, \mathrm{n}^{\prime} \gamma\right)$ reactions at varying neutron energies can be developed and validated.

Research Areas 2 and 3 focus upon expanding the present knowledge of nuclear structure. Research in these areas will expand the knowledge of prompt $\gamma$-rays emitted for reactions initiated by a wider range of neutron energies than is presently in the database and improve the quality of the present prompt and decay data. The results of these tasks have immediate application to radioanalysis. Accurate radioanalysis depends directly upon accurate $\gamma$-ray energies, emission probabilities, and level schemes. The currently available tables of prompt $\gamma$-ray energies and intensities in units of $\gamma$ rays per 100 captures are limited mainly to thermal neutron energies, and even these have many errors, including wrong assignments, inaccurate energies, and poorly measured intensities. The same is the case for $\gamma$ rays from inelastic scatter of fast neutrons. The new fundamental understanding of nuclear structure obtained from Research Areas 2 and 3 is expected to result in new practical applications using radioanalysis. For example, with a more detailed set of data from multiple neutron energies, prompt gamma neutron activation analysis (PGNAA) can be expanded from a qualitative to a quantitative technique for more matrices and for more elements and isotopes, allowing it to be applied to a wider variety of mining and industrial applications.

Research Area 5 will focus on furthering our present knowledge of very-low-level radiation detection. Very-low-level radiation detection is required for basic nuclear physics research such as studies of low intensity nuclear transitions and studies to determine whether the neutrino has mass. These areas are important in that they directly affect nuclear models and our knowledge of nuclear parameters. Very-low-level radiation detection is also important in applications such as measuring radioactivity at and below the environmental level, reducing the radioactivity in ultrapure materials (such as semiconductors), measuring radioactive uptake by plants, performing tracer studies, and determining the radioactivity in the body. Our goal is to develop the capability for the INEEL to reduce background levels during counting so that smaller, less radioactive samples can be assayed to currently-required accuracy, thereby enhancing the signal and reducing the amount of waste to be disposed.

The specific areas of research are discussed in more detail in the following sections. 


\section{Research Area 1: High Accuracy $\gamma$-Ray Measurements}

\section{Background}

Up to the current time, the most precise measurements of relative $\gamma$-ray intensities with Ge semiconductor detectors have been $\sim 0.5 \%$ even at the national standards laboratories. Some recent work at the University of Michigan has suggested that it is possible to push this precision down to $\sim 0.1 \%$ over a limited energy range, namely 661 to $1,332 \mathrm{keV}$. Since their work was published, we have initiated a cooperative effort to significantly expand the energy range over which this precision can be achieved and to probe the extent to which modeling codes can be used to greatly improve the accuracy of efficiency curves.

\section{Method}

This effort will include measurements on a University of Michigan HPGE detector for additional nuclides that have $\gamma$ rays with very well-known relative intensities and energies, thereby expanding the energy range. At the same time, we will carry out Monte Carlo photon and electron transport calculations of the detector efficiencies at the same energies as the measured values. A comparison of the measured and Monte Carlo data will (1) verify the validity of the physics modeling within the Monte Carlo code, (2) provide a check for any unidentified problems with the measured data, (3) provide guidance in improving the modeling of the detector geometry (certain information about the construction of these detectors and their mounting are considered proprietary and must be deduced by such comparisons), and (4) then, if needed, deduce an energy-dependent correction to the Monte Carlo results. Finally, the Monte Carlo results will be used to compute relative efficiency values at intermediate $\gamma$-ray energies and, thereby, generate a dense enough set of precise efficiency values so that a numerical interpolation method can be used to determine the efficiency at any energy in the range of the data.

Absolute detector efficiencies may then be obtained by measurement of a few high-accuracy $(0.1 \%)$ calibrated sources (produced by a radioactivity standards laboratory) and computing a normalization factor to convert the relative efficiency to an absolute efficiency curve.

This method of efficiency calibration could then be performed on a detector at the INEEL for high accuracy measurements

\section{Use}

An experiment has been proposed (by a Texas A\&M professor) to determine one of the parameters of $\beta$ decay by a very precise relative $\gamma$-ray intensity measurement. This experiment cannot be done until the efficiency determination methodology discussed here is fully developed at energies down to $74 \mathrm{keV}$.

These highly-calibrated Ge detectors can then be used to obtain better $\gamma$-ray intensities for various radionuclides. Besides directly improving the database of $\gamma$-ray data at the National Nuclear Data Center at the Brookhaven National Laboratory (BNL), such measurements can be used to improve the data that are deduced from such $\gamma$-ray intensities. This includes the $\log f t$ values, which are a measure of the 'speed' of the $\beta$ decay branches from the parent to the daughter nuclide. These $\log f t$ values are often used by evaluators to deduce the spins and parities of the daughter levels and determine information of interest for nuclear structure studies.

Internal-conversion coefficients give the ratio of the probability of the nucleus emitting an atomic electron, to that for a $\gamma$-ray photon for a $\gamma$-ray transition. Historically, the best measurements of these 
coefficients have uncertainties of about $1 \%$. In many radionuclide decays, it is possible to deduce the $\mathrm{K}$ conversion coefficients from a measurement of the relative intensities of the $\gamma$ ray and the K x-ray fluorescence yield.. If the precise relative efficiency curve can be extended down to the region of the $\mathrm{K} \mathrm{x}$-rays, it may be possible to make some very precise measurements of internal-conversion coefficients. These values can then be used to test the results of theoretical model calculations of the coefficients. This is an issue of current interest since a new code to make these calculations is being prepared (cooperative effort of Russian scientists and those at Oak Ridge National Laboratory [ORNL]) and it will be of interest to show that its results are better than the previous calculations.

\section{Collaborations}

We expect to establish collaborations with several university researchers.

\section{FY 1999 Accomplishments}

Monte Carlo calculations of the efficiency for the University of Michigan 100\% p-type Ge detector were performed. Comparison of these Monte Carlo results with precise measurements performed by the University of Michigan verified, at the $0.1 \%$ level, the validity of the physics modeling within the Monte Carlo code and thereby indicated that the Monte Carlo method is able to produce accurate predictions (with some adjustment of the dead layers of the crystal). The Monte Carlo results were then used to compute relative efficiency values at intermediate $\gamma$-ray energies and aid in the numerical interpolation of the efficiency at any desired energy within the range of the data.

These Monte Carlo results were used along with the measurements made with the University of Michigan Ge detector for nuclides that have $\gamma$ rays with very well-known relative intensities to expand the efficiency curve range to 433 to $2,754 \mathrm{keV}$. This resulted in a relative efficiency with an uncertainty of about $0.1 \%$ for this energy range. A paper summarizing this work was written and submitted for publication. In addition, this work was presented to and discussed with international colleagues and collaborators.

Plans have been made for the next phase of this Monte Carlo simulation work that will be to compare the efficiencies computed with several different Monte Carlo photon and electron transport codes. Preliminary contacts were made with researchers at the University of Michigan, North Carolina State University, Lawrence Berkeley National Laboratory, and the University of Bucharest, Bucharest, Romania, to solicit their future participation in this effort. All indicated a strong interest to participate.

A desired improvement to our knowledge of this high-accuracy relative efficiency curve is to convert it to a high-accuracy absolute efficiency curve. In order to accomplish this, accurately-known (having an uncertainty of about $0.1 \%$ compared to those currently available that have an uncertainty of about $1.5 \%$ ) efficiency calibration sources are required. These sources would be used to convert our precise relative efficiencies to absolute efficiencies. Discussions were held with the French and German radioactivity standards laboratories, Laboratorie Primaire des Rayonnements Ionisants (LPRI) and Physikalisch-Technische Bundesanstalt (PTB), respectively, concerning the possibility of providing these high-accuracy efficiency calibration sources. Both laboratories expressed interest in this project and PTB has provided a price quote for such a source of ${ }^{60} \mathrm{Co}$. These sources will have to be made especially for us.

Discussions were held with Texas A\&M University concerning a collaboration to establish a precision efficiency curve for a Ge detector of theirs in the $74-600 \mathrm{keV}$ region. Texas A\&M University needs this energy range to support their work in determining one of the parameters of $\exists$ decay by a very precise relative $\gamma$-ray intensity measurement. Although this is much lower and much harder than the 
range of 433 to $2,754 \mathrm{keV}$, which we recently attained, we believe that it may be possible. Monte Carlo calculations have been made in order to aid in the determination of the position of the Texas A\&M detector in its housing. The output of the CYLTRAN Monte Carlo code was modified to give additional information required for the Texas A\&M work, and efficiencies have been calculated at the energies of the $\gamma$ rays from ${ }^{133} \mathrm{Ba}$, which may be useful for precise measurements of the relative efficiency in this energy region.

Researchers at the Institute of Isotope and Surface Chemistry in Budapest, Hungary, have made careful measurements of the efficiency of a Ge detector over an energy range that extends up to $11 \mathrm{MeV}$. We have established a collaboration in which we will make Monte Carlo calculations of some efficiencies for this detector system, which includes a collimator. This work, together with our other collaborations, will give us experience from $50 \mathrm{keV}$ to $11 \mathrm{MeV}$.

A professor at the University of Cincinnati has contacted us and suggested a collaboration in the area of high precision $\gamma$-ray spectrometry.

\section{Research Area 2: Decay Schemes for Heavy-mass and Short-lived Nuclides}

\section{Background}

The long $\alpha$-particle decay chains of high-mass nuclides such as ${ }^{233} \mathrm{U},{ }^{235} \mathrm{U}$, and ${ }^{238} \mathrm{U}$ have some short-lived isotopes (e.g., ${ }^{221} \mathrm{Fr},{ }^{217} \mathrm{At},{ }^{213} \mathrm{Bi}$ ) for which the decay schemes have not been well studied. In other cases, due to the lack of accurately measured emission probabilities, the intensities of the $\gamma$-rays emitted from one member of the chain do not agree with those from another member of the chain (e.g.,

${ }^{226} \mathrm{Ra}$ chain). In addition to the high-mass region, there are some short-half-life radionuclides throughout the Chart of the Nuclides of theoretical or practical importance whose decay data are not well measured.

\section{Method}

We will (1) develop the necessary radiochemistry methods to give pure sources of the desired elements (some of which have short half-lives) and (2) measure the $\gamma$-ray energies and intensities for the individual nuclides, as well as groups of nuclides that occur together as a chain due to a longer half-life parent.

Use

The results of these studies will provide new and better nuclear structure information for these nuclides. In addition, these decay chains are of interest in various practical applications, such as the assay of residue from the production of weapons, reactor fuel and radwaste, but have not been used because the above inconsistencies limit the use of the daughter products in the radioanalysis of the parent actinide.

\section{Collaborations}

Some of this work will be conducted at the University of Michigan.

\section{FY 1999 Accomplishments}

Researchers at the University of Michigan have performed a very careful and lengthy measurement of the gamma-ray spectrum from the decay of ${ }^{49} \mathrm{Ca}(8.7 \mathrm{~m}$ half-life). Since they used the same detector as used in the Research Area 1 study (but at a different source distance), we are able to provide accurate 
relative efficiencies for the ${ }^{49} \mathrm{Ca} \gamma$-ray energies. As a result, this work will provide $\gamma$-ray intensities for this decay that are from 10 to 100 times more accurate than the currently available data. These highaccuracy intensities will then be used to develop high-accuracy decay scheme information (i.e., significantly more accurate values than currently known). One facet of the University of Michigan work is to compute the coincidence-summing corrections for their measured $\gamma$-ray intensities. In support of this work, we computed, using Monte Carlo methods, the total efficiency of their detector at $\gamma$-ray energies for the source-detector geometry used in their ${ }^{49} \mathrm{Ca}$ measurements.

The University of Michigan performed high-precision measurements of $\gamma$-ray spectra from shortlived radionuclides with very well-known relative intensities and energies (e.g. ${ }^{24} \mathrm{Na}$ and ${ }^{108 \mathrm{~m}} \mathrm{Ag}$ ) for use in the development of high-accuracy efficiency values (Research Area 1). They also initiated work on the measurement of the $\gamma$-ray spectra from ${ }^{233} \mathrm{U}$ and ${ }^{229} \mathrm{Th}$ and their short-lived daughters. In order to accomplish this, they had to develop separations techniques to isolate specific radionuclides. This work has been delayed somewhat due to difficulties in obtaining sufficient acceptable ${ }^{229} \mathrm{Th}$ material.

\section{Research Area 3: Nuclear Data Measurements for Prompt Reactions and Radionuclides}

\section{Background}

The $\gamma$-ray energies and intensities for many multi-isotope elements produced from prompt reactions are fragmentary, with data measured in great detail for some of the isotopes and minimal or no data for other isotopes. There still remain some $\gamma$-ray transitions that have not been well measured or placed in their level schemes or decay schemes and have important nuclear structure implications or limit radioanalysis results.

\section{Method}

Using highly-calibrated Ge detectors, we will measure the $\gamma$-ray energies and intensities (in $\gamma$ 's per particle interaction) from selected high-purity elemental samples at a variety of neutron energies from cold neutrons to $14-\mathrm{MeV}$. Similar measurements will be performed on specific isotopes of each element.

\section{Use}

This information will provide new nuclear structure information (especially of the higher-energy levels), to test evaluated cross sections, and to test theoretical nuclear models for their ability to identify various types of states, transition multipolarities, and level densities. In addition, our knowledge of the $\gamma$ rays emitted upon neutron capture and neutron inelastic scattering will be enhanced. For example, very little is known about how the spectrum of emitted $\gamma$ rays changes as the neutron energy changes. When this information is obtained, a theoretical model can be developed to predict how this $\gamma$-ray emission changes with neutron energy. Such a model is very important in the application arena. If prompt gamma neutron activation analysis is to be developed for expanded use in quantitative elemental analysis, it will be necessary for Monte Carlo modeling codes to have access to accurate reaction data for neutron $/ \gamma$ ray/electron transport so that all modes of interaction with matter, including those occurring in the detector can be properly handled.

The information gained from this study is of interest in astrophysics where high-energy reactions (e.g., reactions initiated by high-energy neutrons resulting from the fusion process in stars) result in the emission of prompt $\gamma$ rays. The results of this study will also be useful in identification and quantification of short half-life radionuclides produced by high-energy accelerators as in the case of those produced by 
the Accelerator Produced Tritium experiments being performed at LANSCE. This information is required to ensure that cleanup systems associated with the accelerator-produced tritium program will allow tritium handling to be done in a glove box instead of in a hot cell where operational costs of production are greater.

There will be many practical applications for the database, especially in the area of elemental analysis via PGNAA. PGNAA is potentially a very powerful technique for determining elemental content, the minor impurities in a material, and the presence of RCRA elements in an ore or waste that indicate the need for special handling or disposal. However, the lack of an adequate database is one missing link that is delaying the full realization of the usefulness of this technique. The data for neutron energies up to14 $\mathrm{MeV}$ will be useful additions to databases that can be used in practical applications such as elemental analysis and the associated design of experimental arrangements (e.g., in the design of the next generation PINS system). The data for high-energy neutrons can be used to determine the relative contributions of various neutron induced reactions (e.g., $[n, \alpha],[n, p],[n, s p a l l a t i o n])$.

\section{Collaborations}

This work will involve facilities, data, personnel at LANL and NIST and possibly other laboratories.

\section{FY 1999 Accomplishments}

Investigations of the state-of-the-art of nuclear data relating to prompt $\gamma$ rays from neutron-induced reactions were performed. We concluded that the thermal neutron cross sections need to be improved since the uncertainty (1- $\Phi$ ) for about half of the elements is greater than $10 \%$. For quality applications using PGNAA, better cross-section values are required. Detailed information concerning $\gamma$-ray intensities from neutron inelastic scattering is only known for a fission-neutron spectrum. Very little is known for discrete neutron energies, although there is some information for $14-\mathrm{MeV}$ neutrons. In order to accurately model nondestructive assay (NDA) techniques using fast neutron reactions, the $\gamma$ rays from neutron inelastic scattering need to be known as a function of neutron energy. In addition, the Evaluated Nuclear Structure Data File (ENSDF) energies for capture $\gamma$ rays are reasonable, but the intensities (i.e., [rays per 100 captures]) have problems.

Over the past 6 months, a concerted effort has been made to identify facilities that are adequate for carrying out our scope of work. Capture $\gamma$-ray spectra were obtained from NIST and from the Institute of Isotope and Surface Chemistry, Budapest, Hungary. These facilities are currently leaders in capture $\gamma$-ray studies. Both have operating neutron beams we could use. The Institute of Isotope and Surface Chemistry has a cold neutron beam, and NIST has both thermal and cold beams. Computer routines were developed to read these data files so that the spectra can be analyzed with PCGAP's GINA interactive analysis program for gamma-ray energies and peak areas. A comparison of the results of the analysis of the spectra obtained from the two facilities indicated that those from the Institute of Isotope and Surface Chemistry are of higher quality. This is due to the cleaner characteristics of the neutron beam at the Institute of Isotope and Surface Chemistry and the fact that they routinely use Compton-suppressed detectors. It was concluded that we could use the NIST facility for our work with thermal neutrons, but at present the Institute of Isotope and Surface Chemistry is superior for our cold neutron work. Our work will consist of performing high-quality singles measurements that have not yet been done with large ( $>50 \%$ relative efficiency) Ge detectors and to use (-( coincidence techniques to: (1) study ( cascades not otherwise discernable in singles measurements or to (2) place the $\gamma$ rays in the level scheme. 
For high-energy neutron studies, the LANSCE facility at LANL is currently a leader. At this facility we will measure $\gamma$ rays from high-energy neutron reactions. LANSCE has obtained $\gamma$-ray spectra from $(n, x \gamma)$ reactions for a small set of elements and neutron energies ranging from 2 or $4 \mathrm{MeV}$, depending on the element, to $100 \mathrm{MeV}$. A few preliminary $\gamma$-ray spectra were obtained from LANSCE. Analysis of these spectra indicated that the LANSCE data are of interest to us. Data currently exist for some of the elements of interest to us (i.e., those that are of use in PGNAA applications), but not for all. In addition, it will take some effort to retrieve and use the existing data. Therefore, a collaboration was developed with LANSCE for the purpose of: (1) retrieving all existing data of interest to us, (2) providing assistance in analyzing these data, and (3) performing measurements to collect new data.

Work on retrieving the first set of existing data (those for carbon, oxygen, and silicon), including neutron binning of the data and measurement of fluences, was initiated by LANSCE personnel. When these data are available to us, we will analyze them, identifying $\gamma$-ray peaks, calculating $\gamma$-ray intensities, and generating level diagrams. A proposal to measure $\gamma$ rays from $(n, x \gamma)$ reactions for other elements of interest (e.g., phosphorus and other elements of interest to PGNAA users) during FY 2000 is under consideration.

\section{Research Area 4: Measurement of Additional $\gamma$ Rays in $(n, \gamma)$ Reaction}

\section{Background}

If one observes all of the prompt $\gamma$ rays following thermal neutron capture, the sum of the products of the $\gamma$-ray energies and intensities (in $\gamma$ 's per decay) should add up to the energy of the neutron capture state. For low-mass nuclides, the $\gamma$-ray spectra are simple enough that existing experimental data are complete and this relationship holds. But, for medium and heavy mass nuclides the spectra are very complex with a significant number of $\gamma$ rays appearing in the continuum due to the very high density of nuclear levels. Under these conditions, the historical peak analysis techniques only extract the data for a fraction of the $\gamma$ rays. Therefore, the above sum is much less than the energy of the capture state.

\section{Method}

The concept here would be to develop and apply a method to extract the data for a continuous spectrum of $\gamma$ rays, in addition to the data for the discrete lines that are normally observed. This methodology will require some spectrum deconvoluting software, in addition to the determination, from a mixture of measured spectra for simple radionuclide decays and Monte Carlo calculations of the detector response to monoenergetic photons. (This type of process was used a few years ago on data measured at the mass separator at the Test Reactor Area [TRA].)

\section{Use}

This work will result in a more complete understanding of the statistical nature of $\gamma$-ray emission from high-energy states. (We recently received a request for advice on how to compensate for the lack of completeness in these data.)

\section{Collaborations}

Work would be done at a reactor with appropriate neutron facilities in cooperation with their staff. 


\section{FY 1999 Accomplishments}

Data from ENSDF for selected thermal neutron (n,() reactions were obtained and analyzed to determine if all the energy resulting from thermal neutron capture can be accounted for by the known $\gamma$ rays. Results indicated that for the low-Z isotopes (e.g., thermal neutron capture in ${ }^{28} \mathrm{Si}$ and ${ }^{34} \mathrm{~S}$ ), both the energy resulting from thermal neutron capture and the (transitions from the capture state are adequately accounted for. For the medium- $\mathrm{Z}$ and high- $\mathrm{Z}$ isotopes, however, only a small fraction of the neutron capture energy and the ( transitions from the capture state are accounted for. For example, for thermal neutron capture in ${ }^{113} \mathrm{Cd}$, only about $32 \%$ of the neutron capture energy and about $11 \%$ of the transitions from the capture state are accounted for. For thermal neutron capture in ${ }^{155} \mathrm{Gd}$, only about $31 \%$ of the neutron capture energy and about $20 \%$ of the transitions from the capture state are accounted for. This is due to the fact that only a limited fraction of the discrete gamma rays are seen in capture $\gamma$-ray spectra. A large fraction of the $\gamma$ rays are in the unresolved continuum.

A study of methods for extracting the $\gamma$ rays from the continuum was initiated. To date, only one potential candidate method has been identified. Work was initiated to explore the use of this method, but it does not look like this method will be successful. It does not provide results with the accuracy we would like. It appears that the most appropriate way to obtain additional data is to use advanced experimental methods to measure and identify additional discrete $\gamma$ rays from neutron capture. By a combination of using the cleanest neutron beams available (e.g., the advanced cold neutron beam with a chopper currently being built at the Institute for Isotope and Surface Chemistry in Budapest, Hungary) together with INEEL's new, large Ge detectors and anti-Compton and (-( techniques, we expect to obtain information on additional $\gamma$ rays from neutron capture reactions. Development of a collaboration with the Institute of Isotope and Surface Chemistry to accomplish this is under way. Measurements are expected to commence during the second half of FY 2000, depending on the completion of and the availability of the new cold neutron beam facility in Budapest. Measurements using the thermal neutron beam facility at NIST is also being contemplated. A collaboration with NIST has been set up for this purpose and also for the purpose of obtaining their available data.

The measurements in Research Areas 3 and 4 require the availability of large high-purity Ge $\gamma$-ray detectors. To this end, we have obtained an existing $74 \%$ Ge detector for use on the project and sent it back to the manufacturer for rework. We also ordered a new $100 \%$ Ge detector. Reworking of the $74 \%$ Ge detector has been completed, and the new $100 \%$ Ge detector has been received from the manufacturer.

Our involvement in technical societies and in organizing sessions for technical meetings has been very useful to the Nuclear Structure Research Project (especially Research Areas 3 and 4), the Environmental Systems Research Program, and the INEEL during the year. Not only does it give the Nuclear Structure Research Project's personnel visibility, it has allowed us to invite papers from other INEEL personnel performing research on the Environmental Systems Research Program (such as the Nondestructive Assay Project) and other INEEL projects. In addition, it brought us into contact with researchers with whom we expect to collaborate in the future. It also brought us into contact with a recent $\mathrm{Ph} . \mathrm{D}$. on a postdoctoral assignment at LANSCE who accepted an employment offer from us and will be joining our staff in November 1999.

\section{Research Area 5: Very Low-Level Radiation Detection}

\section{Background}

The ability to detect and quantatively measure very low-level radiation is becoming more important to both the basic and applied nuclear physics communities. For example, very low-level radiation 
detection is required in the study of low intensity nuclear transitions and the study of double beta decay (which can also determine whether the neutrino has mass). For the double beta decay experiment, advances are required not only in radiation measurement techniques, but also in separations methods for ultrapurification of mass-separated ${ }^{100} \mathrm{Mo}$. The applied nuclear physics community needs new instruments and techniques for the measurement of very low levels of radioactivity in the environment, biological media, and ultrapure materials. Not only must the lower limit of detection be reduced, the accuracy of the quantative results must also be improved. This can be achieved either by increasing the efficiency of the radiation detection technique or by using advanced methods to reduce the background. In many cases, a given sensitivity can be achieved either with less radioactivity (smaller samples) or shorter count times. Therefore, achievement of lower backgrounds can have a far-reaching implication even with relatively routine counting. Development of these instruments and methods requires research in radiation detection, radiation transport, detector materials and design, and active and passive shielding.

\section{Method}

Since there is a commonality between the needs of the double beta decay experiment and our needs for very low-level radiation detection and new separations techniques, we will continue our involvement in the international collaboration performing this experiment. We will develop radiochemistry separation methods to remove the natural radioactivity from mass-separated ${ }^{100}$ Mo for the double beta decay experiment, and we will participate in the design and implementation of the very-low-level radiation measurements. Research in very low-level radiation detection that we will perform include areas such as large-area proportional counters, liquid scintillation counters that can discriminate between gamma and particle radiation, and advanced coincidence techniques. We will also perform research in radiation transport, radiation detector design, and active and passive shielding. This research will lead to improved methods and instruments for very low-level detection of radionuclides in materials and for qualitatively measuring very low-level radionuclide concentrations in the environment and in humans.

\section{Use}

The work will result in a better understanding of the dynamics of the universe via knowledge of the mass of the neutrino. It will also result in new separations technology that can be applied not only to the manufacture of ultrapure materials, but also in isotope production (e.g., isotope production at the Advanced Test Reactor [ATR]). With the new radiation measurement instruments and techniques, our ability to study low intensity nuclear transitions and to accurately determine the radioactivity in the environment, materials, and biological media will be enhanced. In particular, the lower limit of detection will be reduced and the accuracy of measurements will be improved. We intend to develop this capability for the INEEL.

\section{Collaborations}

The double beta decay experiment involves an international collaboration of U.S., French, Russian, and Hungarian scientists.

\section{FY 1999 Accomplishments}

During this year, we: (1) continued our involvement in the NEMO-3 international collaboration that is performing this experiment, (2) developed radiochemistry separation methods to remove the natural radioactivity from mass-separated stable ${ }^{100}$ Mo for the double $\exists$-decay experiment, (3) developed an improved method to minimize the particle size of the Mo (to less than 10 microns), and (4) separated a sample of natural Mo that, when tested, proved to be the purest ever produced and of sufficient purity to meet the needs of the double $\exists$-decay experiment. We then scaled up the apparatus and method to 
separate gross (i.e., kilogram) amounts of Mo isotopes. This included the assembly, testing, and implementation of a new setup that allows the reduction of larger batches of $\mathrm{MoO}_{3}$. By the end of FY 1999 we accomplished the separation of 680 grams of ${ }^{100}$ Mo. Almost as important as purifying the ${ }^{100} \mathrm{Mo}$, our procedure resulted in the loss of only 5 grams of ${ }^{100} \mathrm{Mo}$. This is very important due to the very high value of ${ }^{100} \mathrm{Mo}\left(\$ 6,700\right.$ per gram in the U.S.). Our losses of ${ }^{100} \mathrm{Mo}$ were well below the requirements of the NEMO-3 collaboration, which was $5 \%$ or $\sim 34$ grams.

This purified ${ }^{100}$ Mo was hand-carried to France, mixed with resin and converted into a ribbon shape, and installed in the experimental apparatus at Mondane, where the measurements will be conducted. We also continued our participation in the design and implementation of the very-low-level radiation measurements required for the double $\exists$ decay experiment.

Through the INEEL Academic Exchange Program, we awarded a faculty fellowship to Prof. S. Sutton of Mount Holyoke College and an undergraduate fellowship to Ms. C. Riddle of Idaho State University. Both individuals worked on the ${ }^{100}$ Mo separation task. At the end of summer, there was a contest at each of the INEEL facilities where fellowship students presented their summer accomplishments. Ms. Riddle was chosen as the TRA's representative to present her work on ${ }^{100}$ Mo purification to the complete laboratory. Prof. Sutton presented our work at a NEMO-3 experimenters' meeting at Prague, Czech Republic. He will also discuss the NEMO-3 instrument at the Winter Meeting of the American Nuclear Society in Long Beach, CA.

\section{Staffing}

A number of candidates possessing applicable nuclear physics, nuclear chemistry, and nuclear engineering expertise were interviewed for potential positions in this research project. Two offers were extended. One was to a Ph.D. from the University of Kentucky and currently serving a postdoctoral appointment at LANSCE. He accepted our offer and will join our staff in early November, 1999. The other was to a Ph.D. candidate in nuclear engineering at North Carolina State University who has a background in radiation transport, modeling, and prediction of gamma-ray spectra from high energy neutron-induced reactions. Because the obtaining of his Ph.D. has been delayed a few months, he has not yet either accepted or rejected our offer. If he accepts, he will probably join our staff in spring of 2000.

\section{Collaborations}

Numerous collaborations with universities, other national laboratories, and foreign facilities have been established to accomplish the goals of the Nuclear Structure Research Project. The following collaborations have been set up:

Idaho State University (ISU) and INEEL. This collaboration brings the expertise of Dr. R. G. Helmer (who recently retired from our staff) to the project and access to the accelerator facilities and scientific staff at ISU for neutron-induced reaction experiments. These resources are applicable to Research Areas 1, 2, 3, and 4.

University of Michigan, Albion College, and INEEL. This collaboration brings capabilities of the University of Michigan and the personnel (primarily Dr. H. C. Griffin) at the university and at Albion College (Dr. M. A. Ludington) to the measurements in Research Areas 1 and 2.

Texas A\&M University and INEEL. This collaboration involves working with Texas A\&M (Dr. J. Hardy) in experimental measurements to test new $\exists$-decay theories and supports Research Area 1. 
National Institute of Standards and Technology (NIST) and INEEL. This collaboration brings the capabilities of NIST personnel (primarily Dr. R. Lindstrom), data that NIST has measured, and access to the NIST thermal and cold neutron beam facilities. It supports Research Areas 3 and 4.

Institute of Isotope and Surface Chemistry, Budapest, Hungary, and INEEL. This collaboration brings the capabilities of the Institute of Isotope and Surface Science personnel (primarily

Drs. G. L. Molnar and Zs. Revay), data measured by the Institute, and access to their cold neutron beam facility. It supports Research Areas 1, 3, and 4.

LANL and INEEL. This collaboration involves the LANSCE facility at LANL and brings the expertise of LANSCE personnel (primarily Dr. R. Nelson), data obtained at LANSCE, and access to the LANSCE neutron beam. It supports Research Areas 3 and 4.

North Carolina State University (NCSU) and INEEL. This collaboration brings the radiation modeling capabilities of NCSU (primarily Dr. R. Gardner), access to NCSU's neutron irradiation facilities, and information concerning the needs of the applied community. It supports Research Areas 1, 3 , and 4 .

Neutrino Experiment-Molybdenum-3 (NEMO-3). This is an international collaboration involving researchers from the France, the U.S. (INEEL and Mount Holyoke College), Russia, and Hungary. It supports Research Area 5 by giving us access to and involvement with measurements and techniques in the forefront of very low-level radiation detection.

\section{ACCOMPLISHMENTS}

The Nuclear Structure Research Project was initiated during the middle of FY 1999, and initial activities were performed. These initial activities consisted of: (1) reviews of existing nuclear data and discussions with national and international researchers to determine the state-of-the-art of the nuclear structure data relevant to the objectives of the project, (2) identification of facilities and development of contacts and collaborations needed for obtaining existing data and for performing the required experiments, (3) initiation of measurements that could be started in the short term and planning for longer-term measurements, (4) obtaining additional staff members with expertise required for the program, and (5) procurement of detectors and other equipment required for the planned measurements. Even though this project has been funded for only 6 months, the following significant accomplishments have been achieved.

The range over which very accuracy $(\sim 0.1 \%)$ relative $\gamma$-ray intensities can be measured with Ge semiconductor detectors was successfully extended from 433 to $2,754 \mathrm{keV}$. This is a major enhancement over the previous most accurate relative intensities $(\sim 0.5 \%)$. This was accomplished by performing Monte Carlo calculations of the efficiency for a Ge detector and then comparing the results with precise measurements. These comparisons verified, at the $0.1 \%$ level, the validity of the physics modeling within the Monte Carlo Code itself and thereby indicated that the Monte Carlo method is able to produce accurate predictions. This work was well received by the technical community and has generated considerable interest both nationally and internationally.

Researchers at the University of Michigan have preformed a very careful and lengthy measurement of the $\gamma$-ray spectrum from the decay of ${ }^{49} \mathrm{Ca}(8.7 \mathrm{~m}$ half-life). Our new relative efficiency method is providing high-accuracy relative efficiencies for the ${ }^{49} \mathrm{Ca} \gamma$-ray energies, and as a result, this work will provide $\gamma$-ray intensities for this decay that are from 10 to 100 times more accurate than the currently available data. 
Gamma-ray spectra from high-energy neutron-induced reactions on carbon, oxygen, and silicon have been measured at LANSCE, but never retrieved or analyzed. These data have been identified and located, and retrieval of the data (including neutron binning) was initiated. When analysis of these data is completed, $\gamma$-ray spectra as a function of neutron energy for the neutron energy range 4 to $100 \mathrm{MeV}$ will be obtained. This type of nuclear information has never before been obtained.

An isotope separation method was developed and tested that yielded the purest ${ }^{100}$ Mo ever produced and of sufficient purity to meet the goals of the international double beta-decay experiment. Without ${ }^{100}$ Mo of this purity, the double beta-decay experiment cannot be conducted. Using our method, a batch of 680 grams of ${ }^{100}$ Mo was ultrapurified and transported to France for use in the experiment.

Numerous collaborations with universities, other national laboratories, and foreign facilities have been established to accomplish the goals of the Nuclear Structure Research Project. These collaborations include Idaho State University, University of Michigan, North Carolina State University, Texas A\&M University, Albion College, Mount Holyoke College, NIST, the LANSCE facility at LANL, the Institute of Isotope and surface Chemistry in Budapest, Hungary, and the international NEMO-3 collaboration (which involves researchers from France, the U.S., Russia, and Hungary).

\section{APPENDIX}

\section{Peer Reviewed Publications}

1. R. G. Helmer (Idaho State University), R. J. Gehrke, J. R. Davidson, and J. W. Mandler, "Scientists, Spectrometry, and $\gamma$-Ray Spectrum Catalogues 1957-2007," invited by editor to appear in the Journal of Radioanalytical and Nuclear Chemistry.

2. M. A. Ludington (Dept. of Physics, Albion College) and R. G. Helmer (currently at Idaho State University), "High-Accuracy Measurements and Monte Carlo Calculations of the Relative Efficiency Curve of an HPGE Detector from 433 to 2,754 keV," submitted for publication in Physics Research in Nuclear Instruments and Methods.

\section{Presentations}

1. R. J. Gehrke, J. K. Hartwell, R. G. Helmer, and B. D. Harlow, "ISOCS Results for Test Geometries," 1999 Canberra Users' Meeting, Boulder, CO, May 24-28, 1999.

2. R. G. Helmer and C. van der Leun (University of Utrecht, The Netherlands), "Recommended Standards for Gamma-Ray Energy Calibration," invited presentation at the Tenth International Symposium on Capture Gamma-Ray Spectroscopy and Related Topics, Santa Fe, NM, August 30-September 3, 1999.

3. R. J. Gehrke, "The Role of the INEEL in Ionizing Radiation Measurements and Standards," presented at the Idaho State University, College of Engineering Graduate Seminar Series, September 10, 1999.

\section{Organized Technical Sessions}

1. "Prompt Gamma Neutron Activation Analysis," organized by R.J. Gehrke for the 1999 Industrial Radiation and Radioisotope Methods and Applications (IRRMA 99) Topical Meeting, 0ctober 3-8, 1999, Raleigh, NC. 
2. "Radioanalysis Employing Multidetector Systems," organized by R.J. Gehrke for the American Nuclear Society 1999 Winter Meeting to be held November 14-18, 1999, Long Beach, CA.

\section{Standards}

1. "American National Standard for Calibration and Use of Germanium Spectrometers for the Measurement of Gamma-Ray Emission Rates for Radionuclides," N42.14-1999, July 1999. 


\section{Science and Technology Foundation}

The Science and Technology Foundations portion of the program provides research integration, research portfolio analysis, and overall management functions. The Science and Technology Integration (STI) activity has the responsibility for monitoring the EM Complex-wide technology and science needs in such databases as the OST Needs Management System. An understanding of these needs is necessary to insure that the ESR research portfolio is addressing issues that are of importance to EM. An additional function within the STI activity is maintaining connections with the Focus Areas in order to provide transition avenues for ESR science and technology into operations and to facilitate the transition of the some of the investment in the EM Science Program (EMSP) into operations. The ESR portfolio technical content and the technical content of the collaborative efforts between ESR and EMSP are subjected to peer reviews that are arranged through this portion of the ESR program. 


\title{
Science and Technology Integration
}

\author{
Peter Castle (PI) \\ SUMMARY
}

The Science and Technology Integration (STI) task of the STI project in the Environmental Systems Research (ESR) program provides analysis and interface capabilities. The types of analysis carried out in this project are directed at understanding the science and technology development needs of the Environmental Management (EM) Program complex wide. This understanding is used in the evaluation of the ESR portfolio to ensure that the research is relevant to EM problems. EM funds a variety of research efforts complex wide. This analysis focuses on the research programs funded by the EM Science Program (EMSP) and the EM Focus Areas to avoid duplication of ongoing EM-funded work. Also, STI has been responsible for arranging for external reviews of two of the five Environmental Systems Research Areas each year.

The ESR program has made a commitment to act as a bridge to help some mature EMSP progress from their basic science considerations to a greater applications orientation. This will assist the Focus Areas in their attempt to harvest the results of the EMSP-funded research. STI serves as the interface with the Focus Areas and ESR. STI works with the Focus Areas to identify EMSP projects of future value to the Focus Areas and to the capabilities of the INEEL that need further development before they can be adopted by the Focus Areas. Since the INEEL is the designated EM laboratory, the ESR program is responsible for deploying its research and making its capabilities available complex wide, as well as at the INEEL. The interfaces to accomplish these activities are being established with the EMSP and the Focus Areas.

The purpose of the Organizational Development for Science and Technology Integration task for the STI project is to provide organizational development support to the STI workscope in its objective to optimize the success of EM efforts to develop and implement its projects. This project uses INEEL capabilities in human factors science. The project focuses on organizational development skills such as making decisions, managing meetings, tracking the content of meetings, defining the problem, identifying the solution, building consensus, forming collaborations, avoiding unproductive defensive strategies, assuming responsibility, communicating openly, mediating opposing views, and arbitrating disputes. Project funds were used to perform research; acquire tools, training, and software; implement demonstration projects with EM personnel; and prepare papers and reports.

\section{PROJECT DESCRIPTION}

\section{Task 1: STI (Peter Castle)}

The STI task has four main objectives:

1. Analyzing the ESR research portfolio against the complex-wide identified technology needs, science needs, and EM-funded research activities.

2. Disseminating ESR research results to INEEL operations and to EM operations.

3. Providing a bridge for ESMP projects to move from basic science to focus area adoption.

4. Providing annual external reviews for the Environmental Systems research areas. 
The analysis of the ESR portfolio against complex-wide needs and research activities is carried out using various EM-managed databases. The EMSP maintains a database, accessed through their homepage (http://www.em.doe.gov/science/), that contains information concerning all funded EMSP projects. The Needs Management System (NMS) is maintained by EM-50 and accessed through their homepage (http://EM-Needs.em.doe.gov/Home/Entry.asp). The NMS contains a list with descriptions of both basic science and technology development needs. Individual Site Technology Coordination Groups (STCGs), associated with many of the Department of Energy (DOE) Operations Offices, supply the entries for this database. The data in NMS describes technology and science gaps or opportunities associated with ongoing or anticipated operations uncovered by the STCGs during their interactions with operations personnel. The research activities within the ESR portfolio can all be linked to one or more of the needs in the NMS.

STI attempts to establish links between the Idaho National Engineering and Environmental Laboratory (INEEL) and EM operational needs by identifying potential end users of the research in the ESR program. An example of this activity is the initiation of a meeting between the Idaho Nuclear Technology and Engineering Center (INTEC), the Waste Experimental Reduction Facility (WERF), the Mixed Waste Focus Area, and researchers from the Adsorption and Absorption Materials for Molecular Separations task in the Transport Aspects of Selective Mass Transport Agents research area. INTEC and WERF both have issues with mercury emissions from their stacks that will exceed the upcoming new Environmental Protection Agency (EPA) maximum achievable control technology (MACT) standards. The research, carried out in the above research task, deals with the ability of copper sulfide $(\mathrm{CuS})$ to take up and bind mercury from both the solution and the gas phase. Discussions were held among the researchers, the operations people, and the focus area personnel.

Approximately 136 EMSP projects came to the end of their funding on 9/30/99. Twelve of those projects successfully competed for new funding in the most recent call for proposals. In the remaining 124 projects, there is much research that will be valuable to EM, provided the Focus Areas are able to harvest it. Because of constraints for FY 2000, the Focus Areas will not be able to bring EMSP research projects forward. Additionally, many of the research projects are not far enough into the development pathway to be adopted by the Focus Areas. To be of interest, many need to move through the applied research stage.

ESR has proposed to work with the Focus Areas to determine which of the 1996 EMSP projects, being carried out at universities, matches needs identified in their baseline planning. When the Focus Areas define their prioritized list of EMSP projects, ESR will evaluate the projects to determine the ultimate value to the INEEL and to strengthen the ESR research capabilities. When suitable matches are found, ESR intends to co-fund researchers at both the INEEL and the universities. Alternatively, there is the possibility of offering postdoctoral appointments and/or sabbaticals to the research groups.

While initial contacts to begin this process have been made with three of the Focus Areas (Mixed Waste, Tanks, and Subsurface Contaminants), the intent for FY 2000 is to concentrate on Subsurface Contaminants (SubCon) activities. A small workshop was held in Germantown, Maryland, in July with field and EM-50 HQ people representing SubCon, EMSP, and ESR. The purpose was to discuss the process for identifying EMSP projects of interest to SubCon. These negotiations are ongoing. SubCon is in the process of codifying its prioritization process for selecting projects.

The Environmental Systems Research activity views external reviews of the research and research directions as an important part of maintaining a viable and credible research portfolio. In FY-98 and FY99 two of the research areas, selected from five each year, have been reviewed by a panel of individuals chosen for their expertise and reputations in fields of research related to the ESR Research Areas. For the FY-99 reviews, the research areas Chemistry of Environmental Surfaces and Materials Dynamics were 
chosen for review. The following reviewers were chosen for the Chemistry of Environmental Surfaces Research Area:

- $\quad$ Prof. Joel Harris, Chemistry Department, University of Utah

- Prof. Ronald Crawford, Co-Director, Center for Hazardous Waste Remediation Research, University of Idaho

- $\quad$ Prof. Timothy Ginn, Associate Professor of Civil and Environmental Engineering, University of California at Davis.

The following reviewers were chosen for the Materials Dynamics research area:

- $\quad$ Prof. Daniel Sordelet, Department of Materials Science and Engineering, Iowa State University

- $\quad$ Prof. Muhamad Sahimi, Department of Chemical Engineering, University of Southern California

- $\quad$ Prof. James C. Earthman, Department of Chemical and Biochemical Engineering and Materials Science, University of California, Irvine.

On August 12, 1999, the panel viewed the following presentations of the project activities for Chemistry of Environmental Surfaces research area:

- $\quad$ Advanced Strategies for Probing Structure and Properties at the Top Monolayer

- $\quad$ Chemical and Biological Reactions at Environmental Surfaces

- $\quad$ Reactive Transport in Variably Saturated Heterogeneous Media

- $\quad$ Transport Phenomena in Geologic Porous Media.

The panel was active in questioning the presenters and the content of the questions indicated a high degree of understanding of the intent, context, and progress of the research activities being described. The comments, conclusions, and recommendations of the panel members were circulated in draft form among them and then submitted to STI for collation and summation.

The following one-on-one presentations of the Materials Dynamics research area were made to individual members of the panel on different days in late August and early September:

- $\quad$ Corrosion and Aging

- $\quad$ Coatings for Environmental Applications

- $\quad$ Transport in Solid and Liquid Media.

The comments, conclusions, and recommendations of the panel members were circulated in draft form among them and then submitted to STI as below. 


\section{Conclusions and Recommendations: Chemistry of Environmental Surfaces Research Area}

Overall comments from the reviewers were positive, indicating that many of the goals of the program are being achieved, such as strengthening the INEEL's capabilities by adding new staff, addressing research issues of importance to EM, and making significant technical progress in a relatively short time. The panel did identify a need for greater communication among the four activities that comprise the Chemistry of Environmental Surfaces research area. They felt that an increase in communication among the researchers would lead to a clearer definition of research tasks that need to be carried out from the molecular scale up to the field scale. The following comments are directed at specific projects.

Advanced Strategies for Probing Structure and Properties at the Top Monolayer. The comments concerning this task were complimentary in general. There was some concern about the relevance of the gas phase ion chemistry to real world surface chemistry. However, the panel recognized that the PI was aware of these problems and that the studies do generate useful data to begin the adsorption modeling. It was recommended that some means of varying the ion trap temperature would provide a way to determine the enthalpy and entropy contributions to the desorption rate. It was also suggested that infrared cavity ring-down spectroscopy might be a better choice than fluorescence spectroscopy for determining ion structures in the Fourier transform mass spectrometry (FT MS). While fluorescence is a sensitive means for optical detection, it usually does not provide a great deal of structural information.

Chemical and Biological Reactions at Environmental Surfaces. The panel felt that this task would benefit from a consolidation of activities. A greater focus on fewer tasks would provide better results. Several changes in research strategy were suggested. Deposition of an optically thin layer of the iron oxide on a transparent substrate would provide a means of spectroscopically probing the interaction of the microbes with the iron oxide at the microbe/oxide interface. All of the current techniques being applied cannot gather information from this interface. The suggestion was made to study Fe(III) reducers of greater environmental significance, such as the geobacter species, once the diagnostic skills have been honed using shawanella putrefaciens as the model. It was also suggested that determination of the priority of physical versus chemical processes for the microbial attachment needs to be sorted out by conducting flow experiments in tortuous porous media with non-metallic controls, such as glass beads. This would help in understanding what might happen in geologic media.

Reactive Transport in Variably Saturated Heterogeneous Media. This task received some of the strongest positive comments. It was suggested that near infrared Raman spectroscopy could be used to monitor changes in soil surface chemistry as a function of $\mathrm{pH}$ in order to understand the wide range in proton capacities and equilibration times for varying soils. Overall, the modeling efforts received high marks.

Transport Phenomena in Geologic Porous Media. The reviewer(s) were excited about the tensiometer measurements that appear to show effects of tidal pressures on soil water tensions. The PIs are encouraged to publish this work quickly. It is suggested that the actinide mobility work be more closely tied to activities in the second and third tasks. Additionally, it has been suggested that more attention be paid to the uranium mass balance (recovery) in future experiments, a point mentioned by all three reviewers. 


\section{Conclusions and Recommendations: Materials Dynamics}

Corrosion and Aging. In summary, this task is extremely important and presents many formidable challenges. Many of the challenges have already been overcome through an interdisciplinary team effort by the INEEL researchers. Based on the technical capabilities of the team members and their willingness to collaborate with colleagues at other institutions, it is anticipated that they will successfully complete the remaining tasks. This group has demonstrated their ability to perform complex corrosion experiments with selected microbes from SNF storage sites. They have also achieved much expertise with state-ofthe-art diagnostic techniques that are aimed at investigating the localized corrosion processes that typically result from microbial activity. The group is encouraged to extend their research via the planned SECM development and to consider the incorporation of other electrochemical methods that could lead to a better understanding of the aging processes under investigation.

Coatings for Environmental Applications. In addition to the present facilities, detection and analysis of scattered laser light from the surface of the coatings should be considered as an augmentation to the phase Doppler and laser-acoustic NDE techniques already employed. This diagnostic method could allow the researchers to monitor the formation of surface defects that are difficult to detect using other NDE techniques. Moreover, much of the expertise and equipment needed for this technique already exist in the coating laboratories.

The researchers have overcome a number of formidable challenges in their development of capabilities that establish a more scientific basis for relating the properties of coatings with controllable parameters in thermal spray processing. In particular, the measurement of residual stresses, using a unique instrument that combines curvature measurements and laser-acoustic nondestructive evaluation (NDE), deserves considerable recognition. This development is providing important information that reveals the relationships between residual stress and the observed adhesive strength of coatings deposited with a systematic variation in process conditions.

The planned future work for this task is reasonable and appropriate for the goals identified for EM operations. The researchers have chosen an excellent model ceramic, $\mathrm{MgAl}_{2} \mathrm{O}_{4}$, for the present research that undergoes a relatively simple solidification and cooling history during deposition. In addition to this material, the researchers are encouraged to consider other ceramic, metallic, and intermetallic coatings once a good understanding of the coating currently under investigation is achieved.

Transport in Solid and Liquid Media. The work to date is impressive in that it has gone beyond treating transport as a continuum process and is more appropriately addressing transport along defects in rock and concrete. In particular, the research is aimed at examining the effects of flow path tortuosity and the structure of cracks. The results of this effort have been the development of models that perform numerical determinations of transport coefficients for computer-generated composite materials. The required input information for these models, such as pore density and crack face structure, is rigorous. However, high-resolution techniques now exist that can provide this information at target sites that are critical for the INEEL. This research should provide a basic understanding of important processes relevant to other Environmental Management ESR projects including computer modeling performed by the hydrology group and the detailed characterization of the unsaturated region between the surface and water table.

The present plans for future work are appropriate and should lead to a better understanding of critical transport processes. In addition to the planned work, the effects of seismic activity might be considered once transport through static media is adequately understood. Currently available computerbased methods for determining fractal dimensions may also be applied in the future to measured crack surfaces and pore structures for predicting transport at INEEL target sites using the models developed in 
the present work. The proposed addition of a postdoctoral researcher for this work is supported, as well as the strengthening of collaborations with other researchers.

The panel, as a whole, is impressed by the Materials Dynamics research area activities and is in agreement that expanding research activities and adding staff could expand the program in several areas. Positive suggestions were made concerning additional diagnostics, such as electrochemical impedance spectroscopy and electrochemical noise analysis, which could be used to advantage in the Corrosion and Aging studies. The planned development of a scanning electrochemical microscope in collaboration with University of Utah researchers is commended. Suggestions are provided concerning the surface preparation and cleaning that are important in the studies of adhesive and cohesive bonding in the preparation of coatings. It is suggested that the issue of oxygen depletion in the coatings and its relationship to defect-assisted coating degradation be investigated.

\section{Task 2: Organizational Development for Science and Technology Integration (Bruce Kaplan)}

The following discussion describes the three activities that made up this task with a subsection for each of these activities.

\section{Electronic Meeting Software}

This activity consists of selecting and demonstrating electronic meeting software. Studies and personal experience show that the use of electronic meeting systems can cut meeting times in half, provide a better record of the meeting, and promote better acceptance of the results by the participants. In meetings where the tools were used, participants strongly agreed (4.57 on a 5-point scale) that the tool made a positive contribution to the process and product of the meeting. The electronic nature of the record also means that the meeting record can be published almost immediately after the meeting, rather than the weeks it may take to transcribe traditional flip charts.

Electronic meeting software packages have traditionally been implemented in a meeting room setting where several personal computers are located in a room, all connected to a central server. The computer system supplements the normal meeting process and automates traditional low-tech meeting techniques such as brain-storming, voting, reduction, and others.

Our FY-99 effort built upon work from the previous year. During FY-98 we evaluated four electronic meeting software packages and a facility against a set of objective selection criteria. These were:

- GroupSystems, originally developed at the University of Arizona and now marketed by the Ventana Corporation

- Consensus@anyWARE, originally developed at the University of Georgia under the name TCBWorks and now marketed by the Soft Bicycle Company

- $\quad$ MeetingWorks, marketed by Enterprise Solutions, Inc.

- $\quad$ Facilitate.Com, marketed by Facilitate.com, Inc.

- The Simplot Center, a decision room equipped with electronic meeting room hardware and software, is a facility operated by Idaho State University at Pocatello, Idaho. The facility 
can be rented, but the software is not available for commercial purchase, since the software is proprietary to the University.

Using the results of the evaluation, we selected and purchased GroupSystems and installed it on ten laptop computers. It provides portable meeting support under the title: Environmental Decision Support Center (EDSC). The system/service is available to any INEEL projects or programs.

The system provides several advantages to a traditionally facilitated meeting:

- Anonymity - the use of the electronic tools does not identify the participant

- $\quad$ Focus on content, not personality

- $\quad$ Equal participation-ideas are not inhibited by dominant personalities; all participants have equal access to the tools

- $\quad$ Parallel and simultaneous communications-accomplished through a Local Area Network

- $\quad$ Time saving

- $\quad$ Creative and innovative environment

- $\quad$ Complete written record of the meeting-each participant can leave the meeting with a record of the meeting accomplishments in the form of a paper copy, a computer diskette, or both.

During FY-99, in response to increased demand for the system, we installed it on ten additional laptop computers. Since its beginning in May 1998, the EDSC has supported 35 meetings.

We have used and demonstrated the EDCS at the capabilities ranking session for the INEEL Capabilities Assessment Report. The huge task of evaluating the data and ranking INEEL research and development capabilities was accomplished in only six hours. Reviews from other meetings where we used GroupSystems have likewise been favorable. GroupSystems is being used at EM meetings and other meetings at the INEEL without our supervision or participation. This is evidence that this effort to select and implement a useful tool has been successful.

\section{Human Interaction Workshops}

Our FY-98 research determined that doing complex wide issues analysis and managing organizational conflicts of interest can require substantial organizational development abilities, including those we called "honest broker." For this reason, our research has focused heavily on defining what those abilities are and how to put them into action. We found that such work is a value-driven activity. As such, it is necessary that the values involved in implementing organizational development efforts be made explicit and that the competencies involved in putting the values into action to be identified and learned.

The two workshops included in our FY-99 effort were a step in that direction. They tested out preliminary ideas we were beginning to formulate and identified challenges and opportunities for moving forward. The workshops were designed to be equally relevant to researchers in these areas and to those wishing to continue building their competencies as facilitators, mediators, conflict managers, and the like. 
These workshops focused on the organizational skills and capabilities required for science and technology integration. They were built on the idea that talk is not cheap. INEEL staff talk with one another, with customers, regulators, and researchers from around the DOE complex and from universities and research institutions world-wide. How well we reason together with these people determines the value we create. The workshops were designed to help participants learn ways to make business dialogue work by capturing the logic behind business arguments, helping people understand one another's logic, and asking questions that help move things forward.

The quality of such dialogue depends on the working relationships among individuals and groups. Reactive relationships form a relationship system that inhibits progress on business issues. The workshops were designed to show participants how to build more productive relationships by mapping patterns of interaction that limit effectiveness and by designing and practicing a range of interventions to alter those patterns and the systems that maintain them.

Each of us has characteristic ways of responding that both enable and constrain our effectiveness. When we are in relationship systems, we can unknowingly become part of the problem rather than the solution. The workshops were designed to show participants how to increase their freedom of action in difficult situations by identifying the conditions that trigger counterproductive reactions, designing and practicing alternative actions, and creating action plans for continued development.

In preparation for these workshops, participants wrote up individual case studies describing a work incident in which the person failed to get the outcomes he'd hoped for as a result of his work with others. These case studies became a focus for the seminar and helped participants learn the use of the tools and methods that lead to more productive human interactions. Problem areas included providing constructive feedback, obtaining the information needed for decision making, and dealing with interpersonal conflicts.

\section{Group Decision-Making Tools}

Two obstacles preventing effective outcomes from meetings are (1) groups often don't know what the true problem is that prompted the meeting and (2) groups tend to jump right to the solution before clearly identifying the problem. Every meeting takes a little free-form discussion time at the start for groups to form a clear understanding of the purpose and objective of the meeting. The key to this freeform time is to allow the group to easily explore their options while still capturing for the record the flow, pattern, and energy of the discussion. This free-form discussion does not always work well with traditional facilitation techniques of flip charts and nominal group processes. Using an Issue Based Information Systems (IBIS) notation combined with the Visual Issue Mapping System (VIMS) and QuestMap software helps groups to explore issues. The VIMS combines the IBIS notation with an effective visual mapping technique, providing a practical tool for building shared understanding. VIMS improves ownership, understanding, and documentation on a project. The tools provide a record and logical flow of the discussion that can be used to provide focus to the rest of the meeting and assist stuck groups in getting unstuck.

We trained in IBIS notation and VIMS meeting management and procured the QuestMap software. We implemented these meeting management tools in demonstration projects at several meetings. This activity served to support Science and Technology Integration objectives to optimize the effectiveness of EM meetings and decision making. It also serves to contribute to the INEEL's core human factors capability.

Good systems engineering practices dictate that a problem be fully understood and all requirements be identified before any solution is suggested. Group meetings, however, are seldom patient enough to follow this step-by-step process to a solution. This may be due to different levels of technical 
understanding by group members or a lack of a clear definition of the problem. The reality is for a group to alternate between problem definition and solution development as the group members' understanding reaches a common level. This process of jumping from problems to solutions and back can be very frustrating to a group and is not consistent with good systems engineering process. By explaining the nature of such problems to a group at the outset, we can help to ease some of the frustration associated with the process. Explanations improve the group's satisfaction with the meeting and provide an understanding that this switching back and forth is normal and not the result of a dysfunctional meeting.

Traditional thinking, cognitive studies, and existing design methods all predict that the best way to work on a problem is to follow an orderly and linear process, working from the problem to the solution. You begin by understanding the problem, which can include gathering and analyzing data. Once you have specified the problem and analyzed the data, you are ready to formulate and then implement a solution. This is known as the waterfall model, illustrated in Figure 1, because it suggests a waterfall as the design flows down the steps.

A study was conducted on a group of designers who were given an engineering problem to solve. They would start by trying to understand the problem, but would immediately jump to formulating potential solutions. Then they would go back to refining their understanding of the problem. Rather than being orderly and linear, the line plotting the course of their thinking looked more like a seismograph for a major earthquake, as illustrated in Figure 2. This pattern is both chaotic, for obvious reasons, and opportunity-driven, because in each moment the designers are seeking the best opportunity to progress toward a solution. What drove the flow of their thoughts was an internal drive to make the most headway possible, regardless of where it occurred, by making opportunity-driven leaps in the focus of their attention.

This type of problem solving process is often employed when trying to solve a "wicked problem." A wicked problem is defined as having the following characteristics:

- The problem is an evolving set of interlocking issues and constraints. Indeed, there is no definitive statement of the problem. You don't understand the problem until you have developed a solution.

- There are many stakeholders, people who care about or have something at stake in how the problem is resolved. This makes the problem solving process fundamentally social. Getting the right answer is not as important as having stakeholders accept whatever solution emerges.

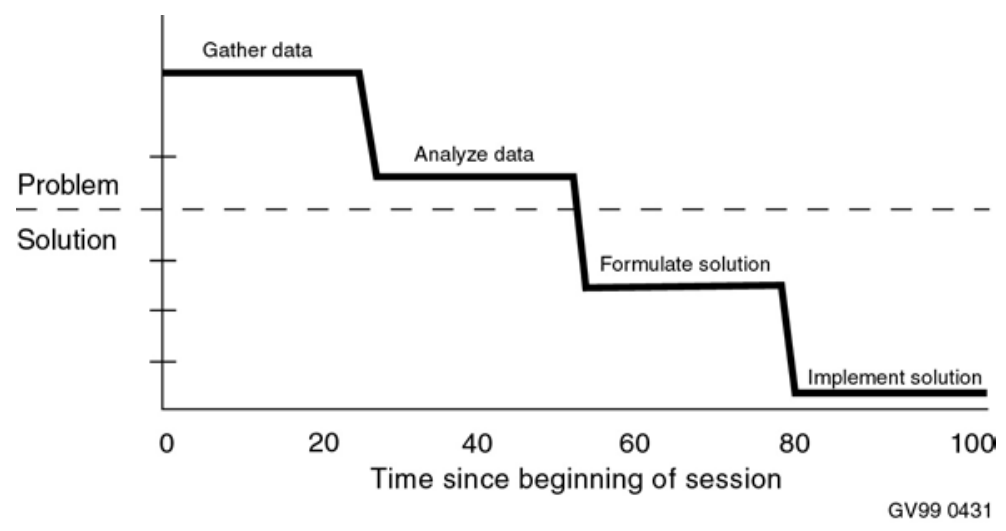

Figure 1. Waterfall model. 


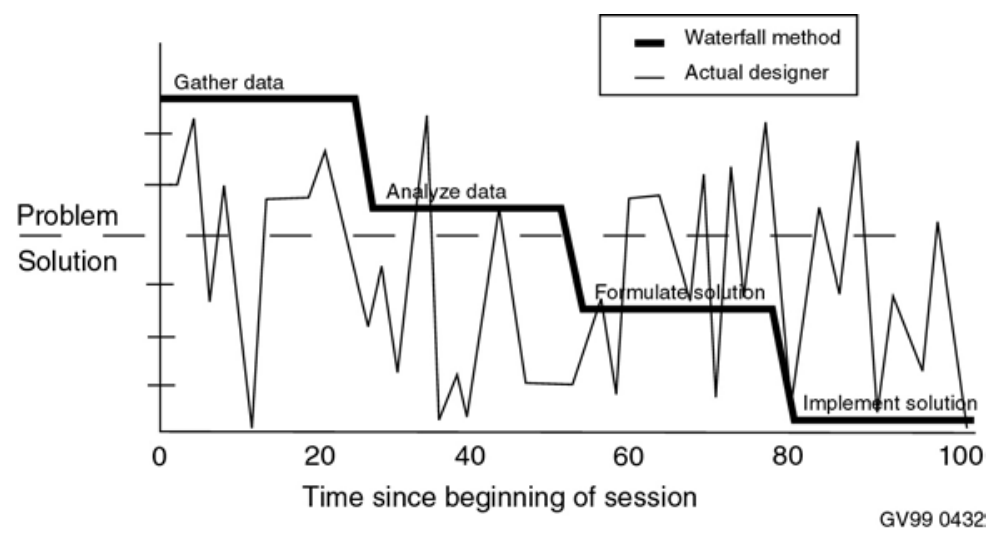

Figure 2. Waterfall model compared with an actual problem-solving session.

- The constraints on the solution, such as limited resources and political ramifications, change over time. The constraints change, ultimately, because we live in a rapidly changing world. Operationally, they change because many constraints are generated by the stakeholders, who come and go, change their minds, fail to communicate, or otherwise change the rules by which the problem must be solved.

- $\quad$ Since there is no definitive problem, there is no definitive solution. The problem-solving process ends when you run out of time, money, energy, or some other resource, not when some perfect solution emerges.

The solution pattern is complicated when more than one designer is assigned to their study problem. While the second designer follows a seismographic pattern of problem-solving activity, just as the first designer did, the line graphing her work is different from the first. Because the second designer's background and approach are quite different from the first designer's, the patterns differ. The two problem-solving patterns may match well at times; but at other points in the project, the designers are in different places and have different views about what to do next. These patterns are shown in Figure 3.

In theory, VIMS makes a lot of sense and is easy to apply. In practice, VIMS requires a lot of skill to allow the quality of the process to come through to a group and not get bogged down in the VIMS rules and nomenclature. Application of QuestMap has been used in an INTEC Thermal Energy Options meeting and a High Level Waste (HLW) Environmental Impact Statement (EIS) Technical Evaluations meeting as a hidden tool in order to gain experience in its application. One facilitator used the tool without the knowledge of the group, while another facilitator used traditional facilitation techniques. The

tool was used during a free-form session in a Technology Deployment Center (TDC) strategic planning meeting with the group's knowledge. The results were shared with the group as a means to help focus the discussion for the rest of the meeting that used nominal group techniques. The QuestMap information was well received by the group. There were several comments that the map helped to explain and document the discussion in such a way that they could now let go of many of the side issues that that been consuming their time so they could now focus on the main issues. The meeting owner asked for copies of the map for his records because it helped him organize his thoughts about the subject.

The TDC meeting was also presented a series of slides to explain the wicked problem concept and how it applied to their meetings. Very favorable comments were received on the slides, and the group suggested that the slides should be a standard presentation at the start of any meeting. The group noted 


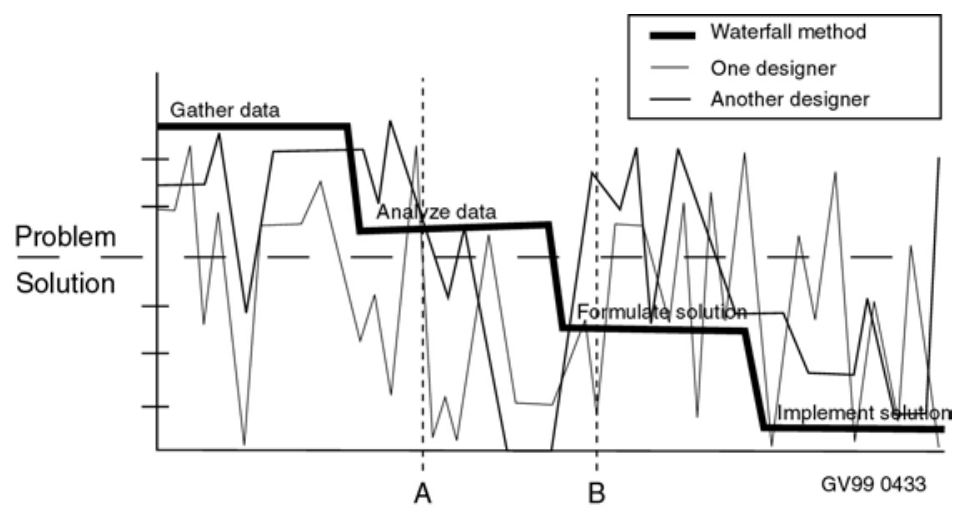

Figure 3. Waterfall model compared with two actual problem-solving sessions led by different designers.

that it helped to know that the back-and-forth process of problem solving was not abnormal or indicative of a poor group process and was not a waste of time.

\section{ACCOMPLISHMENTS}

\section{Task 1}

- $\quad$ Carried out the annual review of complex wide needs versus ESR research projects.

- Initiated the process for harvesting EMSP research in cooperation with the Focus Areas.

- $\quad$ Arranged for external reviews of two research areas.

- Initiated contact between INTEC, WERF, and the Mixed Waste Focus Area and ESR researchers.

\section{Task 2}

Human interaction workshops, meeting management training and software trial and selection contributed to this project. Demonstration activities provided practice, experience, and opportunities for encouraging more widespread use of useful meeting management and organizational development tools. Human interaction workshops built the skills used in successfully implementing the demonstration projects. These skills and abilities are now available at the INEEL to address the new science and technology integration challenges facing us as a national laboratory. 


\section{APPENDIX}

\section{Peer Reviewed Publications}

\section{Chemical and Biological Reactions at Environmental Surfaces}

W. F. Bauer, J. M. Barnes, G. Elias, and P. Pryfogle, "An Improved Method for Determination of Intracellular pH Using Fluorescence Spectroscopy," in preparation for Analytical Biochemistry.

W. F. Bauer, J. M. Barnes, G. Elias, G. Redden, P. Pryfogle, "Identification of Siderophores and Cytochromes Released by Microbial Cells," in progress.

G. Redden, J. Bargar, and R. Bencheikh-Latmani, "Citrate Enhanced Sorption of U(VI) on Goethite:

Characteristics Of A Ternary Complex Using EXAFS," submitted to Environmental Science and Technology, 1999.

\section{Reactive Transport in Variably Saturated Heterogeneous Media}

R. W. Johnson and M. D. Landon, "A B-Spline Based Collocation Method to Approximate the Solutions to the Equations of Fluid Dynamics," Paper FEDSM99-7160, Proceedings of the 3rd ASME/JSME Joint Fluids Engineering Conference, San Francisco, California, July 18-23, 1999.

R. W. Smith and A. L. Schafer, "Effective Reactive Surface Area: An Anisotropic Property of Physically and Chemically Heterogeneous Media," Materials Research Society Symposium. Proceedings, Vol. 556, in press 1999.

\section{Transport Phenomena in Geologic Porous Media}

J. L. Garland, and R. M. Lehman, "Dilution/Extinction of Community Phenotypic Characters to Estimate Relative Structural Diversity in Mixed Communities," FEMS Microbiol. Ecol., in press 1999.

R. B. Genter, and R. M. Lehman, "Metal Toxicity Ascertained by Algal Population Density, Heterotrophic Substrate Utilization, and Fatty Acid Profile in a Small Stream," Environmental Toxicology and Chemistry, in press 1999.

S. P. O'Connell, D. Lawson, M. E. Watwood, and R. M. Lehman, “A BASIC Program for Reduction of Data from Community-Level Physiological Profiling Using Biolog Microplates," submitted to Journal of Microbiological Methods following rejection from Applied and Environmental Microbiology.

\section{Selective Mass Transport in Polymers}

F. F. Stewart and M. K. Harrup, "Phosphazene Monomers from the Regiospecific Reaction of tert-Butylhydroquinone with Hexachlorocyclotriphosphazene: A New Composite Material Precursor," $J$. Appl. Poly. Sci., Vol. 72, No. 8, 1999, p. 1,085.

F. F. Stewart, R. E. Singler, M. K. Harrup, E. S. Peterson, and R. P. Lash, "Electron Beam Crosslinking of Fluoroalkoxy, Methoxyethoxyethoxy and Substituted Phenoxy Polyphosphazenes: Physical and Chemical Characterization and Comparison to a Thermally Induced Free Radical Process and Ionic Complexation," J. Appl. Poly. Sci., in press. 
F. F. Stewart, M. K. Harrup, R. P. Lash, and M. N. Tsang, "Synthesis, Characterization and Thermal Stability of Phosphazene Terpolymers with 2-(2-Methoxyethoxy)ethoxy and Diacetone D-Glucofuranosyl Pendant Groups," Polymer International, in press.

F. F. Stewart, M. K. Harrup, T. A. Luther, C. J. Orme, and R. P. Lash, "Formation of Pervaporation Membranes from Polyphosphazenes Having Hydrophilic and Hydrophobic Pendant Groups: Synthesis and Characterization," Submitted to Chemistry of Materials.

\section{Computational Simulation}

C. R. Tolle, J. L. Budzien, and R. A. LaViolette, "Do Dynamical Systems Follow Benford's Law?" Physica D, submitted and in review, 1999.

D. H. Dahl and R. A. LaViolette, "RAWKRAB: A Random-Walk Random-Byte Generator," Appl. Math. Mod., submitted and in review, 1998.

C. R. Tolle and L. Allred, "Time-Based Clustering Symbol Train Creation," NASA Tech Briefs, (submitted and in review, 1998).

R. A. LaViolette, M. E. Watwood, T. R. Ginn, and D. L. Stoner, "Spatial Disorder and Degradation Kinetics in Intrinsic Biodegradation Schemes," J. Phys. Chem., Vol. 103, 1999, p. 4480.

C. R. Tolle, and R. W. Gundersen, "Searching for Determinism in Erratic Signals: Fuzzy Time-Based Clustering of Delay Vectors," Intelligent Engineering Systems Through Artificial Neural Networks 8, Edited by C. H. Dagli, New York, NY: ASME Press, 1998.

L. R. Pratt and R. A. LaViolette, "Quasi-Chemical Theories of Associated Liquids," Mol. Phys., Vol. 94, 1998, p. 909.

J. L. Budzien, J. D. McCoy, J. G. Curro, R. A. LaViolette, and E. S. Peterson, "The Solubility of Gases in Polyethylene: Integral Equation Study of Standard Molecular Models," Macromolecules Vol. 31, 1998, p. 6669 ; erratum p. 8653.

J. L. Budzien, J. D. McCoy, D. H. Weinkauf, R. A. LaViolette, and E. S. Peterson, "Solubility of Gases in Amorphous Polyethylene," Macromolecules, Vol. 31, 1998, p. 3368.

\section{Transport in Solid and Liquid Media}

C. DeW. Van Siclen, "Walker Diffusion Method for Calculation of Transport Properties of Composite Materials," Physical Review E, Vol. 59, No. 3, 1999, pp. 2804-2807.

C. DeW. Van Siclen, "Anomalous Walker Diffusion through Composite Systems," Journal of Physics A, Vol. 32, 1999, pp. 5763-5771.

\section{Adaptive Sensors}

K. L. Telschow, V. A. Deason, R. S. Schley and S. M. Watson, "Imaging of Lamb Waves in Plates for Quantitative Determination of Anisotropy using Photorefractive Dynamic Holography," Reviews of Progress in Quantitative NDE, Vol. 18A, edited by D. O. Thompson and D. E. Chimenti, Plenum Press: New York, 1998, pp. 999-1005. 
D. Wright, A. Grunnet-Jepsen, M. A. Diaz-Garcia, J. D. Casperson, B. Smith, M. S. Bratcher, M. S. DeClue, J. S. Siegel, W. E. Moerner, and R. J. Twieg, "Trapping Studies on Photorefractive Polymers," Proc. Soc. Photo-Opt. Instrum. Engr., Vol. 3471, 1998, pp. 60-71.

R. S. Schley, K. L. Telschow and J. Holland, "Static and Dynamic Two-Wave Mixing in GaAs," submitted for publication in Applied Optics.

\section{Integrated Instruments for In Situ Chemical Measurements}

M. L. Stone, G. L. Gresham, and L. N. Polson, "Characterization of Two Pholyphosphazene Materials as Membranes in MIMS," accepted for publication in Analytica Chimica Acta.

\section{Intelligent Nonintrusive Methods for Characterization}

F. Noo, R. Clackdoyle, C. Mennessier, T. A. White, and T. J. Roney, "A Direct Analytic Method for Scanner Calibration in Cone-Beam Tomography," J. of Optical Society of America A (submitted September 1999).

J. C. Determan, G. K. Becker, "Automatic Expert System Rule Generation on Nondestructive Waste Assay Data at the Idaho National Engineering and Environmental Laboratory," Journal of Evolutionary Computation (submitted July 1999).

F. Noo, R. Clack, T. J. Roney, and T. A. White, "The Dual-Ellipse Cross Vertex Path for Exact Reconstruction of Long Objects in Cone-Beam Tomography," Phys. Med. Biol. 43, 1998, pp. 797-810.

\section{Nondestructive Assay}

J. H. Hamilton, A. V. Ramayya, J. K. Hwang, W. Greiner, S. J. Zhu, A. Sandulescu, A. Florescu, J. Kormicki, G. Ter-Akopian, Y. Oganesyan, A. V. Daniel, G. S. Popeko, J. Kliman, M. Morhac, J. D. Cole, R. Aryaeinejad, M. W. Drigert, W. E. Collins, W. C. Ma, E. F. Jones, L. K. Peker, P. M. Gore, G. Drafta, B. R. S. Babu, G. Wang, and J. K. Deng, "Cold Spontaneous Fission Processes of ${ }^{252} \mathrm{Cf}$ and the Structure of Neutron Rich Ba and La Nuclei," Acta Phys. Slovaca 49, 31, 1999.

S. J. Zhu, J. H. Hamilton, A. V. Ramayya, M. G. Wang, J. K. Hwang, E. F. Jones, L. K. Peker, B. R. S. Babu, G. Drafta, W. C. Ma, G. L. Long, L. Y. Zhu, M. Li, C. Y. Gan, T. N. Ginter, J. Kormicki, J. K. Deng, D. T. Shi, W. E. Collins, J. D. Cole, R. Aryaeinejad, M. W. Drigert, J. O. Rasmussen, R. Donangelo, J. Gilat, S. Asztalos, I. Y. Lee, A. O. Macchiavelli, S. Y. Chu, K. E. Gregorich, M. F. Mohar, M. A. Stoyer, R. W. Lougheed, K. J. Moody, J. F. Wild, S. G. Prussin, G. M. Ter-Akopian, A. V. Daniel, and $\mathrm{Yu}$. Ts. Oganessian, "Octupole Correlations in Neutron-Rich ${ }^{145,147}$ La Nuclei:

Coriolis-Limit-Coupling Bands with Aligned h ${ }_{11 / 2}$ Proton," Phys.Rev. C59, 1999, p. 1316.

S. -J. Zhu, M. -G. Wang, J. H. Hamilton, A. V. Ramayya, B. R. S. Babu, W. C. Ma, G-L. Long, L. -Y. Zhu, M. Li, M. Sakhaee, C. -Y. Gan, L. -M. Yang, J. Kormicki, J. D. Cole, R. Aryaeinejad, M. W. Drigert, J. O. Rasmussen, M. A. Stoyer, S. Y. Chu, K. E. Gregorich, M. F. Mohar, S. G. Prussin, I. Y. Lee, Yu. Ts. Oganessian, G. M. Ter-Akopian, and A. V. Daniel, "Octuple Eformation ands of $\pi \mathrm{h}_{11 / 2}$ in Neutron-Rich ${ }^{145,147}$ La Nuclei," Chin.Phys.Lett. Vol. 16, 1999, p. 169.

J. H. Hamilton, E. F. Jones, P. M. Gore, A. V. Ramayya, R. S. Dodder, C. J. Beyer, J. K. Hwang, X. Q. Zhang, S. J. Zhu, A. P. de Lima, J. Kormicki, J. D. Cole, R. Aryaeinejad, W. C. Ma, G. M. Ter-Akopian, Yu. Ts. Oganessian, A. V. Daniel, J. O. Rasmussen, S. J. Asztalos, I. Y. Lee, A. O. Macchiavelli, M. A. 
Stoyer, R. W. Lougheed, S. G. Prussin, and R. Donangelo, "A New Phenomenon-Shifted Identical Bands and New Region of Stable Octupole," Nuovo Cim. 111A, 1998, p. 633.

J. K. Hwang, A. V. Ramayya, J. H. Hamilton, L. K. Peker, J. Kormicki, B. R. S. Babu, T. N. Ginter, C. J. Beyer, G. M. Ter-Akopian, Yu. Ts. Oganessian, A. V. Daniel, W. C. Ma, P. G. Varmette, J. O. Rasmussen, S. J. Asztalos, S. Y. Chu, K. E. Gregorich, A. O. Macchiavelli, R. W. Macleod, J. Gilat, J. D. Cole, R. Aryaeinejad, K. Butler-Moore, M. W. Drigert, M. A. Stoyer, L. A. Bernstein, R. W. Lougheed, K. J. Moody, S. G. Prussin, H. C. Griffin, and R. Donangelo, "Close Doublet Structures in ${ }^{103} \mathrm{Mo}$, ${ }_{109,111} \mathrm{Ru}$, and Neighbours: Rotation-Alignment for the Half-Filled $\mathrm{h}_{11 / 2}$ Subshell," J.Phys.(London), G24, L9, 1998.

J. K. Hwang, A. V. Ramayya, J. H. Hamilton, L. K. Peker, J. Kormicki, B. R. S. Babu, T. N. Ginter, C. J. Beyer, J. O. Rasmussen, J. Gilat, S. J. Asztalos, S. Y. Chu, K. E. Gregorich, A. O. Macchiavelli, R. W. Macleod, G. M. Ter-Akopian, Yu. Ts. Oganessian, A. V. Daniel, W. C. Ma, P. G. Varmette, J. D. Cole, R. Aryaeinejad, K. Butler-Moore, M. W. Drigert, M. A. Stoyer, L. A. Bernstein, R. W. Lougheed, K. J. Moody, S. G. Prussin, H. C. Griffin, R. Donangelo, and S. J. Zhu, "Identification of the Gamma Transitions in Tc and Cs Products of ${ }^{252}$ Cf Fission and Possible (7/2)+ [413] Bands in ${ }^{105-109}$ Tc Isotopes," Phys.Rev. C57, 2250, 1998; Erratum Phys.Rev. C58, 3013, 1998.

J. K. Hwang, A. V. Ramayya, J. Gilat, J. H. Hamilton, L. K. Peker, J. O. Rasmussen, J. Kormicki, T. N. Ginter, B. R. S. Babu, C. J. Beyer, E. F. Jones, R. Donangelo, S. J. Zhu, H. C. Griffin, G. M. Ter-Akopyan, Yu. Ts. Oganessian, A. V. Daniel, W. C. Ma, P. G. Varmette, J. D. Cole, R. Aryaeinejad, M. W. Drigert, and M. A. Stoyer, "Rotational Bands in ${ }^{101-103} \mathrm{Nb}$ and ${ }^{98,100}{ }^{\mathrm{Y}}$ Nuclei and Identification of Yrast Bands in ${ }^{146} \mathrm{La}$ and ${ }^{149} \mathrm{Pr}, "$ Phys.Rev. C58, 3252, 1998.

A. V. Ramayya, J. H. Hamilton, J. K. Hwang, L. K. Peker, J. Kormicki, B. R. S. Babu, T. N. Ginter, A. Sandulescu, A. Florescu, F. Carstoiu, W. Greiner, G. M. Ter-Akopian, Yu. Ts. Oganessian, A. V. Daniel, W. C. Ma, P. G. Varmette, J. O. Rasmussen, S. J. Asztalos, S. Y. Chu, K. E. Gregorich, A. O.

Macchiavelli, R. W. Macleod, J. D. Cole, R. Aryaeinejad, K. Butler-Moore, M. W. Drigert, M. A. Stoyer, L. A. Bernstein, R. W. Lougheed, K. J. Moody, S. G. Prussin, S. J. Zhu, H. C. Griffin, and R. Donangelo, "Cold (Neutronless) Alpha Ternary Fission of ${ }^{252}$ Cf," Phys.Rev. C57, 1998, p. 2370.

A. V. Ramayya, J. K. Hwang, J. H. Hamilton, A. Sandulescu, A. Florescu, G. M. Ter-Akopian, A. V. Daniel, Yu. Ts. Oganessian, G. S. Poeko, W. Greiner, L. K. Peker, J. Kormicki, B. R. S. Babu, T. N. Ginter, F. Carstoiu, W. C. Ma, P. G. Varmette, J. O. Rasmussen, S. J. Asztalos, S. Y. Chu, K. E. Gregorich, A. O. Macchiavelli, R. W. Macleod, J. D. Cole, R. Aryaeinejad, K. Butler-Moore, M. W. Drigert, M. A. Stoyer, L. A. Bernstein, R. W. Lougheed, K. J. Moody, S. G. Prussin, S. J. Zhu, H. C. Griffin, and R. Donangelo, "Observation of ${ }^{10}$ Be Emission in the Cold Ternary Spontaneous Fission of ${ }^{252}$ Cf," Phys.Rev.Let. Vol. 81, 1998, p.947.

X. Q. Zhang, J. H. Hamilton, A. V. Ramayya, L. K. Peker, J. K. Hwang, E. F. Jones, J. Kormicki, C. J. Beyer, P. M. Gore, B. R. S. Babu, T. N. Ginter, S. J. Asztalos, S. Y. Chu, K. E. Gregorich, I. Y. Lee, A. O. Macchiavelli, R. W. Macleod, J. O. Rasmussen, J. Gilat, G. M. Ter-Akopian, Yu. Ts. Oganessian, A. V. Daniel, W. C. Ma, P. G. Varmette, J. D. Cole, R. Aryaeinejad, K. Butler-Moore, Y. X. Dardenne, M. W. Drigert, M. A. Stoyer, J. F. Wild, J. A. Becker, L. A. Bernstein, R. W. Lougheed, K. J. Moody, R. Donangelo, S. G. Prussin, H. C. Griffin, and S. J. Zhu, "Identification of New Negative-Parity Levels in ${ }^{152,154}$ Nd," Phys.Rev. C57, 1998, p. 2040; Erratum Phys.Rev. C58, 1998, p.3012.

S. -J. Zhu, C. -Y. Gan, J. H. Hamilton, A. V. Ramayya, B. R. S. Babu, M. Sakhaee, W. C. Ma, G. -L. Long, J. -K. Deng, L. -Y. Zhu, M. Li, L. -M. Yang, J. Kormicki, J. D. Cole, R. Aryaeinejad, Y. K. Dardenne, M. W. Drigert, J. O. Rasmussen, M. A. Stoyer, S. Y. Chu, K. E. Gregorich, M. F. Mohar, S. G. 
Prussin, I. Y. Lee, N. R. Johnson, and F. K. McGowan, "Collective Band Structures in the Neutron-Rich ${ }^{107,109}$ Ru Nuclei," Chin.Phys.Lett., Vol. 15, 1998, p. 793.

R. J. Gehrke, L. V. East, and Y. D. Harker, "Information in Spectra From Sources Containing "Aged"

${ }^{241}$ Am As From TRU Waste," to be submitted to the Journal of Waste Management.

\section{Nuclear Structure Research}

R. G. Helmer (Idaho State University), R. J. Gehrke, J. R. Davidson, and J. W. Mandler, "Scientists, Spectrometry, and $\gamma$-Ray Spectrum Catalogues 1957-2007," invited by editor to appear in the Journal of Radioanalytical and Nuclear Chemistry.

M. A. Ludington (Dept. of Physics, Albion College) and R. G. Helmer (currently at Idaho State University), "High-Accuracy Measurements and Monte Carlo Calculations of the Relative Efficiency Curve of an HPGE Detector from 433 to 2,754 keV," submitted for publication in Physics Research in Nuclear Instruments and Methods.

\section{Presentations}

\section{Advanced Strategies for Probing Structure and Reactivity at the Top Monolayer}

A. K. Gianotto, G. S. Groenewold, J. R. Scott, G. F. Kessinger, M. T. Benson, and J. B. Wright, "Condensation Reactions of Aluminosilicate Oxyanions With Water," Proceedings of the $47^{\text {th }}$ American Society Mass Spectrometry Conference on Mass Spectrometry and Allied Topics, Dallas, Texas, June 1317, 1999.

J. R. Scott, G. S. Groenewold, A. K. Gianotto, G. F. Kessinger, M. T. Benson, and J. B. Wright, "Reactions of Gas-Phase $\mathrm{Al}_{\mathrm{x}} \mathrm{O}_{\mathrm{y}}$ " and $\mathrm{Si}_{\mathrm{x}} \mathrm{O}_{\mathrm{y}}^{-}$Anions With Water," Proceedings of the $47^{\text {th }}$ American Society Mass Spectrometry Conference on Mass Spectrometry and Allied Topics, Dallas, Texas, June 1317, 1999.

G. S. Groenewold, G. F. Kessinger, J. E. Delmore, G. L. Gresham, and A. Shaw, "Enhancement of SIMSGenerated Oligomeric Oxyanions from Refractory Oxide Surfaces Using $\mathrm{Cs}^{+}$Substitution," Proceedings of the $47^{\text {th }}$ American Society Mass Spectrometry Conference on Mass Spectrometry and Allied Topics, Dallas, Texas, June 13-17, 1999.

G. L. Gresham, G. S. Groenewold, J. E. Olson, and A. D. Appelhans, "Formation of Alkali Halide Cluster Ions and Assessment of Their Stability," Proceedings of the 47th American Society Mass Spectrometry Conference on Mass Spectrometry and Allied Topics, Dallas, Texas, June 13-17, 1999.

\section{Chemical and Biological Reactions at Environmental Surfaces}

M. Vierkorn, P. Maurice, and W. F. Bauer, "Assessing the Importance of Microbial Attachment in Mineral Dissolution: TMAFM of Interactions Between Shewanella alga Bacteria and Fe Oxide surfaces," Meeting of the Geological Society of America, Toronto, Canada, October 26-29, 1998.

G. Redden, J. Bargar, and Per Persson, 1999, "Structure of a Ternary Goethite-Citrate-Uranyl Surface Complex," 217 $7^{\text {th }}$ ACS National Meeting, Anaheim, California, May 1999.

G. Redden, "A Sorption Experiment and Approaches to Complexation Modeling: A Discussion on Goals, Model Applications, Model Constraints, and Environmental Applications," Lecture given at 
Stanford University for CEE 373: Special Topics In Aquatic Chemistry Solute Interactions At Solid-Solution Interfaces, Stanford, California, 1999.

W. F. Bauer, J. M. Barnes, G. Elias, and P. A. Pryfogle, "Measurement of pH in Microbial Microenvironments Near Mineral Surfaces," XIV th International Symposium on Environmental Biogeochemistry, Toronto, Canada, September 26-30, 1999.

G. Redden, J. Bargar, R. Bencheikh-Latmani, and W. Bauer, "Surface Interactions Between Goethite, Citrate and Uranyl: Linking Surface Chemistry to Issues of Microbial Influences on Metal Partitioning and Bioavailability," XIV th International Symposium on Environmental Biogeochemistry, Toronto, Canada, September 26-30, 1999.

L. Hull, G. Redden, and M. Pace, "Adsorption Parameters for Raffinate Migration," to be presented at the Materials Research Society Fall Meeting, Boston, Massachusetts, November 29-December 3, 1999.

G. Redden and J. A. Davis, 1999, "Surface Complexation Models in Solute Transport: Issues of Practicality and Applicability for Transport Modeling," to be presented at the Materials Research Society Fall Meeting, Boston, Massachusetts, November 29-December 3, 1999.

\section{Reactive Transport in Variably Saturated Heterogeneous Media}

R. W. Johnson, and M. D. Landon, "A B-Spline Based Collocation Method to Approximate the Solutions to the Equations of Fluid Dynamics," 3rd ASME/JSME Joint Fluids Engineering Conference, San Francisco, California, July 18-23, 1999.

R. W. Smith and A. L. Schafer, "Effective Reactive Surface Area: An Anisotropic Property of Physically and Chemically Heterogeneous Media (ads.)," Scientific Basis for Nuclear Waste Management XXII, Materials Research Society 1998 Fall Meeting, Boston, Massachusetts, 1998.

\section{Transport Phenomena in Geologic Porous Media}

J. L. Garland, and R. M. Lehman, "Dilution/Extinction of Community Phenotypic Characters to Estimate Relative Bacterial Species Diversity in Mixed Communities," Abstract, ASM Conference on Microbial Biodiversity, Chicago, Illinois, August 1999.

M. E. Kauffman, H-Y Holman, R. M. Lehman, M. C. Martin, and W. R. McKinney, "Application of Synchrotron Radiation-Based Fourier Transform Infrared Spectromicroscopy to the Investigation of Bacterial Attachment to Aluminum and to Mineralogically-Heterogeneous Geologic Substrata," Abstract, Advanced Light Source Users Conference, Berkeley, California, October 1999.

M. E. Kauffman, H-Y Holman, R. M. Lehman, M. C. Martin, and W. R. McKinney, "Investigation of Bacterial Attachment to Distinct Mineral Phases within Heterogeneous Geologic Substratum using Synchrotron Radiation-Based Fourier Transform Spectromicroscopy," Abstract, Fall Meeting of the American Geophysical Union, San Francisco, California, December 1999.

S. P. O’Connell, F. S. Colwell, R. M. Lehman, and M. E. Watwood, "Groundwater Microbial Species Diversity as Affected by Trichloroethylene in Microcosm Experiments," Abstract, ASM Conference on Microbial Biodiversity, Chicago, Illinois, August 1999. 
M. S. Wilson, R. M. Lehman, and F. S. Colwell, "Comparison of Culture and Nonculture-Based Methods for Measuring Abundance of Methanotrophic Bacteria in a Deep Basalt Aquifer," Abstract, International Symposium on Subsurface Microbiology - 1999, Vail, Colorado, August 1999.

J. C. Ingram, M. M. Cortez, G. S. Groenwold, W. F. Bauer, R. M. Lehman, and S. P. O'Connell, 1999, Static SIMS Detection of Cell Membrane Biomolecules," Abstract, 47th American Society for Mass Spectrometry Conference on Mass Spectrometry, Dallas, Texas June 13-17, 1999.

J. M. Hubbell, "Vadose Zone Monitoring Using Advanced Tensiometers," TechnoVentions'98, University of Central Florida, Orlando, FL, December 9-12, 1998.

J. M. Hubbell, "Field Observations of Soil Water Potential in Deep Vadose Zones Using advanced tensiometers," Western Regional Soil Physics Society W-188, Henderson, NV, January 4-6, 1999.

"Water Potential to Depths of $30 \mathrm{~m}$ in Fractured Basalt and Sedimentary Interbeds," Proceedings of the International Workshop on Characterization and Measurement of Hydraulic Properties of Unsaturated Porous Media, US Salinity Laboratory, University of California, Riverside CA, summer 2000.

"Monitoring and Surveillance in Near Surface Disposal Facilities", IAEA (International Atomic Energy Agency), Vienna, Austria, summer 2000.

\section{Selective Mass Transport in Polymers}

F. F. Stewart, E. S. Peterson, M. L. Stone, M. K. Harrup, L. A. Polson, and C. J. Orme, "Membrane Separations Using New Polymers," Filtration ‘98, Atlantic City, NJ, December 1998.

J. L. Budzien, J. D. McCoy, J. G. Curro, R. A. Laviolette, "A Simple Relation for the Solubility of Gases in Polymers," poster at the American Physical Society Conference, Atlanta, GA, March, 1999.

T. A. Luther, F. F. Stewart, and M. K. Harrup, "Preparation of Hexa(Methyl Hydroquinone) Cyclotriphosphazene and Substitution Reactions with Alkyl Halides," American Chemical Society Northwest Regional Meeting, Portland, Oregon, June 1999, Paper \# 364.

F. F. Stewart, M. K. Harrup, E. S. Peterson, M. L. Stone, C. J. Orme, and L. A. Polson, "Chemically Tailored Phosphazene Polymers for Water Separations Applications: Synthesis, Characterization, and Membrane Formation." Presented to the Macromolecular Secretariat, Symposium on Polymeric Materials in Separations, $218^{\text {th }}$ National Meeting of the American Chemical Society, New Orleans, LA, August 1999, MACR 19.

\section{Computational Simulation}

M. M. Rashid and R. Roy, "Experimental Comparison and Model Assessment for a Novel Approach to Inelastic Fracture," Fifth U. S. National Congress on Computational Mechanics, U. of Colorado, Boulder, Colorado, August 4-6, 1999 (contributed).

J. L. Budzien, J. D. McCoy, J. G. Curro, and R. A. LaViolette, "A Simple Relation for the Solubility of Gases in Polymers," Centennial Meeting of the American Physical Society, Atlanta, Georgia, March 2026, 1999 (contributed).

R. A. LaViolette, "Spatial Disorder and Degradation Kinetics in Soil Restoration," Physical Chemistry Seminar, University of Oregon, Eugene, Oregon, February 15, 1999 (invited). 
R. A. LaViolette, "Spatial Disorder and Degradation Kinetics in Soil Restoration," Physical Chemistry Seminar, University of Cincinnati, Cincinnati, Ohio, January 21, 1999 (invited).

C. R. Tolle, and R. W. Gundersen, "Searching for Determinism in Erratic Signals: Fuzzy Time-Based Clustering of Delay Vectors," ANNIE '98, St. Louis, Missouri, November 1-4, 1998 (contributed).

\section{Coatings for Environmental Applications}

Microstructure Effects on Stainless Steel Substrates from Deposition of Plasma Spray Ceramic Coatings, R. N. Wright, W. D. Swank, J. R. Fincke, to be presented at the International Thermal Spray conference, 8-11 May 2000, Montreal, Canada.

Residual Stress Determination from a Laser-Based Curvature Measurement, W. D. Swank, R. A. Gavalya, J. K. Wright, R. N. Wright, to be presented at the International Thermal Spray Conference, 811 May 2000, Montreal, Canada.

\section{Corrosion and Aging}

D. F. Bruhn, C. R. Breckenridge, M. N. Tsang, C. S. Watkins, W. E. Windes, F. F. Roberto, R. N. Wright, R. R. Brey, and P. J. Pinhero, "Irradiation Of Microbes From Spent Nuclear Fuel Storage Pool Environments," Global '99, Jackson, WY, September 2, 1999.

P. J. Pinhero and C. S. Watkins, "Imaging Microbiologically-Influenced Pitting Corrosion of Spent Nuclear Fuel Cladding Material with a Scanning Reference Electrode Technique (SRET)," MRS1999, Symposium QQ18: Microbial Processes in Waste Management, Boston, Massachusetts, December 1, 1999.

\section{Intelligent Nonintrusive Methods for Characterization}

J. T. Johnson, D. C. Kunerth, C. R. Tolle, and L. G. Allred, "Neural Network Processing of Pulsed Eddy Current Responses," ASNT Fall Conference, Phoenix, AZ, Oct 11-15, 1999.

J. C. Determan and J. Foster, "Using Chaos in Genetic Algorithms," 1999 Congress on Evolutionary Computation, Washington D. C., July 7, 1999. Proceedings of the 1999 Congress on Evolutionary Computation, Vol. 3, 2094-2101, Piscataway, New Jersey: IEEE Press

J. C. Determan, "A Generic Expert System for Data Review/Validation of Nondestructive Waste Assay Data," $40^{\text {th }}$ Annual Meeting of the Institute for Nuclear Materials Management and Interface Working Group and Neutron Users Group (joint meeting), Phoenix, AZ, July 27-30, 1999.

F. Noo, R. Clackdoyle, T. A. White, and T. J Roney, "Image reconstruction from misaligned truncated helical cone-beam data," 1999 IEEE Nuclear Science Symposium and Medical Imaging Conference, Seattle, WA, October 1999.

T. A. White, T. J. Roney, R. J. Pink, M. Smith, R. Clackdoyle, and F. Noo, "Comparison of fan- and cone-beam imaging capabilities on a portable x-ray imaging system," SPIE $44^{\text {th }}$ Annual Meeting, Denver, CO, July 1999. Proceedings of the SPIE, Vol. 3772-14 (to be published).

T. J. Roney, T. A. White, R. J. Pink, and M. Smith, "Field-Portable Gamma Ray and X-Ray Inspection Systems," $37^{\text {th }}$ Weapons Agency Nondestructive Testing Organization (WANTO) Meeting, Sandia National Laboratories, Albuquerque, NM, Feb 22-26, 1999. 
D. C. Kunerth, C. R. Tolle, J. T. Johnson, L. G. Allred, "Neural Network Processing of Pulsed Eddy Current Responses," $37^{\text {th }}$ Weapons Agency Nondestructive Testing Organization (WANTO) Meeting," Sandia National Laboratories, Albuquerque, NM, Feb 22-26, 1999.

\section{Nondestructive Assay}

J. D. Cole, M. W. Drigert, R. Aryaeinejad, E. L. Reber, J. K. Jewell, J. H. Hamilton, A. V. Ramayya, C. J. Beyer, G. M. Ter-Akopian, Ts. Yu. Oganessian, G. S. Popeko, and A. V. Daniel, "Induced Fission and Signature Studies of Actinide Isotopes Using ANL/IPNS Thermal Neutrons and an Array of Detectors," Winter Meeting of American Nuclear Society, Boston, Massachusetts, November 1998.

J. D. Cole, M. W. Drigert, R. Aryaeinejad, E. L. Reber, J. K. Jewell, J. H. Hamilton, A. V. Ramayya, C. J. Beyer, G. M. Ter-Akopian, Ts. Yu. Oganessian, G. S. Popeko, and A. V. Daniel, "Using New Fission Data with the Multi-Detector Analysis System for Spent Nuclear Fuel," International Conference on Perspectives in Nuclear Physics, Paradise Island, Bahamas.

J. W. Mandler, "Application of PGNAA to Ore Mining and Processing," 1999 Industrial Radiation and Radioisotope Methods and Applications (IRRMA 99) Topical Meeting, Raleigh, NC, October 3-8, 1999.

R. J. Gehrke and R. G. Helmer, "Photon Spectra of Radionuclides Acquired on Large-Area, Thin-Window HPGE Spectrometers," Proceedings of the $6^{\text {th }}$ Nondestructive Assay Waste Characterization Conference, Salt Lake City, UT, November 17-19, 1998.

R. J. Gehrke, "The Role of the INEEL in Ionizing Radiation Measurements and Standards," Presented by invitation at the Annual Meeting of the Council on Ionizing Radiation Measurements and Standards, National Institute of Standards and Technology, Gaithersburg, MD, October 19-22, 1998.

R. G. Helmer, J. R. Davidson, and R. J. Gehrke, "Gamma-Ray Spectrum Catalogue," Presented at the R. L. Heath Symposium Sessions of the Winter Meeting of the American Nuclear Society held Nov. 12 to 17, 1998, in Washington DC, ANS Transactions, 79, (1998) 114.

\section{Nuclear Structure Research}

R. J. Gehrke, J. K. Hartwell, R. G. Helmer, and B. D. Harlow, "ISOCS Results for Test Geometries," 1999 Canberra Users' Meeting, Boulder, CO, May 24-28, 1999.

R. G. Helmer and C. van der Leun (University of Utrecht, The Netherlands), "Recommended Standards for Gamma-Ray Energy Calibration," invited presentation at the Tenth International Symposium on Capture Gamma-Ray Spectroscopy and Related Topics, Santa Fe, NM, August 30-September 3, 1999.

R. J. Gehrke, "The Role of the INEEL in Ionizing Radiation Measurements and Standards," presented at the Idaho State University, College of Engineering Graduate Seminar Series, September 10, 1999.

"American National Standard for Calibration and Use of Germanium Spectrometers for the Measurement of Gamma-Ray Emission Rates for Radionuclides,” N42.14-1999, July 1999. 


\section{Patents}

Transport Phenomena in Geologic Porous Media

J. M. Hubbell and James B. Sisson, June 29, 1999, Patent Number 5,915,476, U.S. Patent and Trademark Office, "Monitoring Well," Assignee: Lockheed Martin Idaho Technologies Company, Idaho Falls, ID, 20 claims, 4 drawing sheets.

J. M. Hubbell and James B. Sisson, June 29, 1999, Patent Number 5,915,476, US Patent and Trademark Office, "Monitoring Well," Assignee: Lockheed Martin Idaho Technologies Company, Idaho Falls, Idaho, 20 claims, 4 drawing sheets. 